DOE/RL-91-59

\title{
Closure Report for N Reactor
}

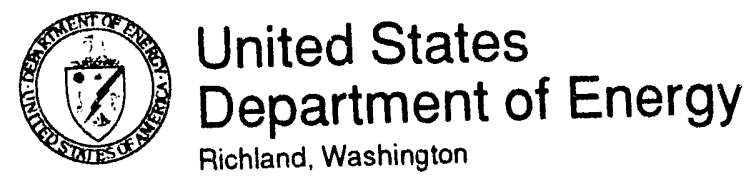

Approved for Public Release 


\section{LEGAL DISCLAIMER}

This report was prepared as an account of work sponsored by an agency of the United States Government. Neither the United States Government nor any agency thereol, nor any of their employees, nor any of their contractors, subcontractors or their employees, makes any warranty, express or implied. or assumes any legal liability or responsibility for the accuracy, completeness, or any third party's use or the results of such use of any intormation, apparatus, product, or process disclosed, or represents that its use would not infringe privately owned righis. Relerence herein to any specific commercial product, process, or service by trade name, trademark, nanufacturer, or otherwise, does not necessarily constitute or imply its endorsement, recommendation, or favoring by the United States Government or any agency thereof or its contractors or subcontractors. The views and opinions of authors expressed herein do not necessarily state or reflect those of the United States Government or any agency thereot.

This report has been reproduced from the best available copy Available in paper copy and microfiche.

Available to the U.S. Department of Energy and its contractors from

Office of Scientific and Technical Information P.O. Box 62

Oak Ridge, TN 37831

(615) 576.8401

Available to the public from the U.S. Department of Commerce National Technical Information Service

5285 Port Royal fioad

Springfield, VA 2216

(703) 487.4650

Printed in the United States of America 


\section{Closure Report for N Reactor}

Date Published

January 1994

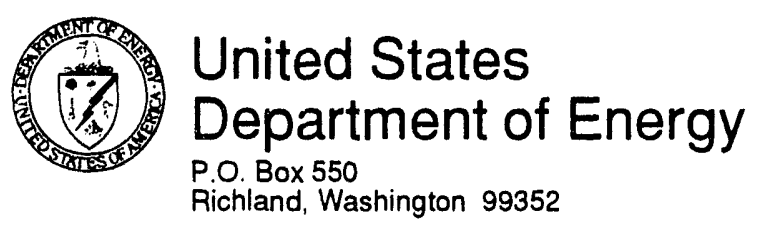

Approved for Public Release 


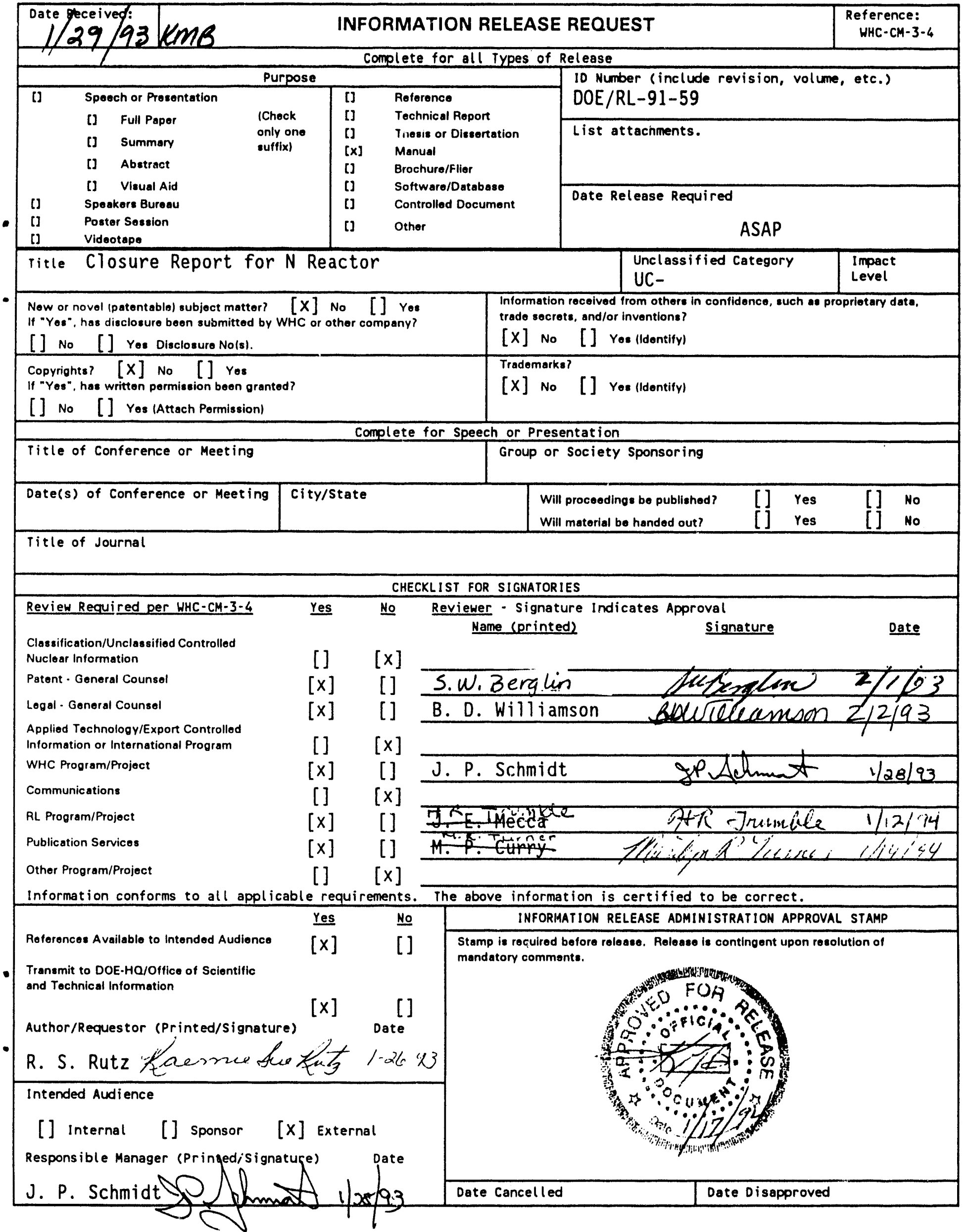




\section{EXECUTIVE SUMMARY}

This report has been prepared to satisfy Section $3156(b)$ of Public Law 101-189 (Reports in Connection with Permanent Closures of Department of Energy Defense Nuclear Facilities), which requires submittal of a Closure Report to Congress by the Secretary of Energy upon the permanent cessation of production operations at a U.S. Department of Energy (DOE) defense nuclear facility (Watkins 1991).

This closure report provides:

(1) A complete survey of the environmental problems at the facility

(2) Budget quality data indicating the cost of environmental restoration and other remediation and cleanup efforts at the facility

(3) A proposed cleanup schedule.

The cessation of production operations at the DOE's 4000-MW N Reactor and its $2.6-\mathrm{km}^{2}(640$-acre) site on the Hanford Site leaves several environmental problems requiring disposition. These include, in part, over 3,785,000 L $(1,000,000 \mathrm{gal})$ of radiologically contaminated water and $90,700 \mathrm{~kg}$ $(200,000 \mathrm{lb})$ of radiologically contaminated hardware in the reactor's spent fuel basin, over 8,000 ci of radionuclides in soil-column disposal facilities, radiologically contaminated groundwater with ${ }^{90} \mathrm{Sr}$ concentrations of up to seven times the DOE guidelines, three soil-column disposal facilities requiring closure in compliance with regulations promulgated under authority 
of the Resource and Conservation and Recovery Act of 1976, as amended, and numerous spills and releases of chemical and radiological materials to the environs.

The N Reactor site has 109 discrete sources of known environmental contamination. The nature and extent of this contamination, together with ihat found at the eight retired reactor sites on DOE's Hanford Site, formed the bases for Hanford's 100 Areas to be placed on the National Priority List (NPL) in accordance with regulations promulgated under authority of the Comprehensive Environmental Response, Compensation, and Liability Act (CERCLA) of 1980 , as amended.

The DOE will address these environmental problems through (1) stabilization of existing environmental and industrial safety hazards to preclude any future releases and to minimize surveillance and maintenance requirements, (2) remediation of contaminated soils and groundwater consistent with Federal and State regulations in a manner and time frame described in the Hanford Federal Facility Agreement and Consent Order (Tri-Party Agreement) (Ecology et al. 1992), and (3) final decontamination and decommissioning of the facility.

Deactivation of N Reactor is planned to take place from 1994 through 1997 at an estimated cost of $\$ 270 M$. A significant reduction to the estimated deactivation costs is expected to be realized. The effort to revise the cost estimate is ongoing because the deactivation program is refined and optimized based on the outcome of value engineering analyses and other planning activities that are currently being performed. Remediation of the 
contaminated soils and groundwater is scheduled from 1999 through 2006 at an estimated cost of $\$ 400 M$, based on removing contaminated soil. Decontamination and decommissioning, after completion of future National Environmental Policy Act of 1969 documents, is assumed to consist of the complete dismantling of the facility by 2016 at an estimated cost of over \$720M.

Cessation of production operations has also identified certain institutional issues concerning potential long-term obligations with the Washington Public Power Supply System's Hanford Generating Plant, which used steam from $N$ Reactor to generate 860 MW of electrical power. Obligations will entail environmental restoration and dismantling of the facility. Hanford Generating Plant bonds have already been retired.

All $N$ Reactor fuel has been removed from the facility and is being stored for the near term in fuel storage basins on the Hanford Site. Unencapsulated fuel will be encapsulated. The final disposition of over 1,800 metric tons of unirradiated nuclear fuel and 2,100 metric tons of irradiated nuclear fuel will be addressed subsequent to the completion of DOE complex-wide material disposition studies.

The environmental restoration and cleanup activities are assigned to DOE's Office of Environmental Restoration within the Office of Environmental Restoration and Waste Management through which they wi11 be assigned priorities and budgeted. 
DOE/RL 91-59

This page intentionally left blank. 
DOE/RL 91-59

\section{CONTENTS}

1.0 PURPOSE AND OBJECTIVES ................... . . . . . .

2.0 SETTING . . . . . . . . . . . . . 2-1

2.1 LOCATION AND DEMOGRAPHY .............. 2-1

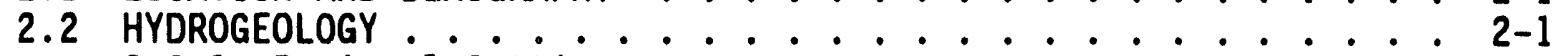

2.2.1 Regional Setting ................ 2-1

2.2 .2 Local Setting.......................

2.3 METEOROLOGY .................. . . 2-5

2.3.1 Precipitation . . . . . . . . . 2-5

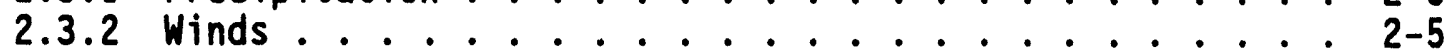

2.3.3 Temperature . . . . . . . . . . 2-6

2.3.4 Evapotranspiration ............. 2-6

2.4 ENVIRONMENTAL RESOURCES ................. $2-6$

2.4.1 Flora and Faund . . . . . . . . . . . . 2-6

2.5 LAND AND WATER USE and Endangered Species ....... $2-7$

2.5.1 Sensitive Environments . . . . . . . 2-8

2.5.2 Land Use ................. 2-. . .

2.5.3 Water Use . . . . . . . . . . 2-9

2.6 ARCHAEOLOGY AND HISTORICAL RESOURCES .......... 2- 29

3.0 FACILITY DESCRIPTIONS . . . . . . . . . . . . . 3-1

3.1 INTRODUCTION . . . . . . . . . . . .... 3-1

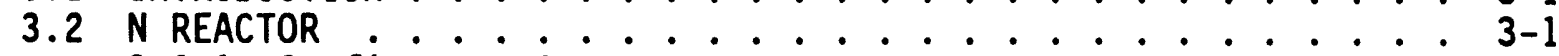

3.2 .1 Confinement System ................. 3-2

3.2.2 Reactor Coolant System ........... 3-2

3.2.3 Nuclear Fuel System ............. 3-3

3.2.4 Heat Dissipation System ............ 3-3

3.2.5 Water Supply System .............. 3-3

3.2 .6 Emergency Cool ing Systems . . . . . . . . 3-6

3.2 .7 Process Decontamination . . . . . . . . 3-6

3.2.8 Plant Service Boilers . . . . . . . . . . . . 3-7

3.2.9 Diesel Fuel Storage and Transfer System . . . . . . 3-7

3.2.10 Boiler fuel 0il System . . . . . . . . . 3-7

3.3 WASTE-GENERATING PROCESSES ............. $3-8$

3.3.1 Radioactive Effluents and Wastes . . . . . . . . 3-8

3.3.2 Reactor Primary Cool ant System ... . . . . . 3-8

3.3.3 Spent Fuel Storage Bas in Cooling Water System . . . 3-8

3.3.4 Reactor Periphery Cooling Systems . . . . . . . 3-9

3.3.5 Reactor Primary Coolant Loop Decontamination

System . . . . . . . . 3-9

3.3.6 Drainage From Reactor Support Facilities ...... 3-9

3.3.7 Water Treatment and Demineralization Plants . . . . . 3-10

3.4 RESOURCE CONSERVATION AND RECOVERY ACT FACILITIES

IN THE 100-N AREA ...... 3-11

3.4.1 1301-N (116-N-1) Liquid Waste Disposal Facility . . 3-11

3.4.2 1325-N Liquid Waste Disposal Facility ...... 3-12

3.4.3 1324-N/NA Surface Impoundment/Percolation Pond ... 3-13 
CONTENTS (continued)

3.5 OTHER FACILITIES ................ . . . . . . . . . . . . .

3.5.1 Hanford Generating PI ant ............ 3-13

3.5.2 Bonneville Power Administration Substation ..... 3-13

3.5.3 100 D Area Support Facilities ........... 3-14

3.5.4 100 C Area Support Facilities . . . . . . . . 3-14

4.0 HISTORY . . . . . . . . . . . . . . . . 4-1

4.1 OPERATIONAL HISTORY OF N REACTOR ........

5.0 ENVIRONMENTAL PROBLEMS . . . . . . . . . . . . 5-1

5.1 OPERATIONAL ACTIVITIES AFFECTING THE ENVIRONMENT ...... 5-1

5.1.1 1314-N Liquid Waste Loadout Station .......... . 5-1

5.1.2 119-N Air Sampling and Monitoring Building ..... . 5-2

$5.1 .3166-N$ Facility ............ . . 5-3

5.1.4 116- $\mathrm{N}-1$ Crib and Trench Grouping ........ 5-5

5.1.5 116-N-2 Radioactive Chemical Waste Treatment and Storage Facility ............ 5-7

$5.1 .6116-\mathrm{N}-3$ Crib and Trench . . . . . . . . . . . . 5-9

5.1 .7 128-N-1 Burning Pit . . . . . . . . . . . . . . . . . 5-9

5.1 .8 181-N River Pumphouse . . . . . . . . . . . . . . . 5-9

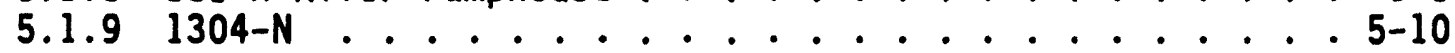

$5.1 .10118-N-1 \ldots . . . . . . . . . . .5-12$

5.1 .11 182-N Underground Storage Tank Area . . . . . . . 5-14

5.1.12 105-N Reactor Spent Fuel Storage Basin . . . . . . 5-14

5.1 .13 182-N High Lift Pumphouse . . . . . . . . . . . 5-15

5.1.14 Acid/Caustic Storage and Transport System . . . . . . 5-16

5.1.15 116-N-8 Hazardous and Mixed Waste Storage Area . . . . 5-21

$5.1 .16184-N$. . . . . . . . . . . . 5-21

5.1.17 Decontamination Drainline Leak ....... . 5-23

$5.1 .18120-\mathrm{N}-4$. . . . . . . . . . . . . 5-23

5.1.19 Regeneration/Filter Backwash Waste Disposal Area . . . 5-24

5.1.20 Office Septic Tank Area . . . . . . . . . . . 5-26

5.1.21 N-17 Paint Shop ............... 5-28

5.1.22 1120-N Septic Tank Grouping . . . . . . . . . . . 5-28

$5.1 .23100-\mathrm{N}-10$ Sewer System Grouping ... . . . . . 5-28

5.2 AFFECTED ENVIRONMENT - GROUNDWATER . . . . . . . . . . 5-29

5.2.1 116-N-1 and 116-N-3 Crib and Trench Areas . . . . . . 5-29

5.2.2 1304-N Emergency Dump Tank and 118-N-1 Spacer

Storage Silos Area ........ 5-33

5.2.3 120-N-1 Percolation Pond $/ 120-\mathrm{N}-2$ Surface

Impoundment Area ......... . . 5-34

5.2.4 Summary of Groundwater Contamination . . . . 5-34

5.3 AFFECTED ENVIRONMENT - SOIL .......... . . 5-38

5.3.1 Surface Soil Contamination ............. 5-38

5.3.2 Vadose Zone Soil Contamination ........ 5-38

5.4 AFFECTED ENVIRONMENT - BIOTA . . . . . . . . . 5-40

5.4 .1 flora . . . . . . . . . . . . . . 5-40

5.4.2 Fauna ............. . . 5-40

5.5 AFFECTED ENVIRONMENT - RADIATION . . . . . . . 5-42

5.6 NET EFFECT ON THE ENVIRONMENT . . . . . . . . . 5-42 
DOE/RL 91-59

CONTENTS (continued)

6.0 N REACTOR CLOSURE SCOPE .................. 6-1

6.1 N REACTOR DEACTIVATION ......... 6-1

6.2 RESOURCE CONSERVATION AND RECOVERY ACT CLOSURES $\ldots \ldots$. . $6-2$

6.3 SITE REMEDIATION ............. . . 6-3

6.4 DECONTAMINATION AND DECOMMISSIONING . . . . . . . 6-5

7.0 PROPOSED ClEANUP SCHEDULE . . . . . . . . . . . . 7-1

8.0 ESTIMATED COST FOR CLOSURE . . . . . . . . . . . . . 8-1

8.1 N REACTOR DEACTIVATION . . . . . . . . . . . 8-1

8.2 RCRA FACILITY CLOSURE . . . . . . . . 8-1

8.3 RCRA PAST PRACTICE SITE REMEDIATION ......... 8- .

8.4 DECONTAMINATION AND DECOMMISSIONING $\ldots \ldots . \ldots . \ldots$

9.0 REgULATORY AND U.S. DEPARTMENT OF ENERGY ORDER COMPLIANCE . . . . . 9-1

10.0 NATIONAL ENVIRONMENTAL POLICY ACT STRATEGY . . . . . . . . 10-1

10.1 GENERAL ................ 10-1

10.2 EXISTING NATIONAL ENVIRONMENTAL POL ICY ACT DOCUMENTATION $: 10-1$

10.3 FUTURE NATIONAL ENVIRONMENTAL POLICY ACT DOCUMENTATION . . . . 10-1 10.3.1 N Reactor Deactivation ............. 10-1 10.3.2 Environmental Remediation .......... 10-2 10.3.3 Decontamination and Decommissioning ...... 10-2

11.0 DISPOSITION OF SPECIAL NUCLEAR MATERIAL . . . . . . . . . 11-1

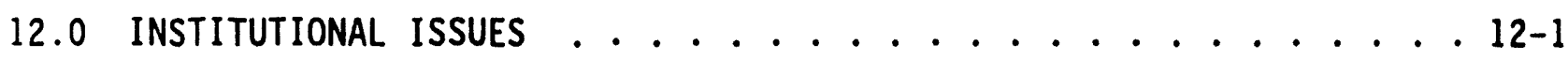

13.0 ClOSURE MANAGEMENT AND PROGRAMMATIC INTERFACE . . . . . . . 13-1

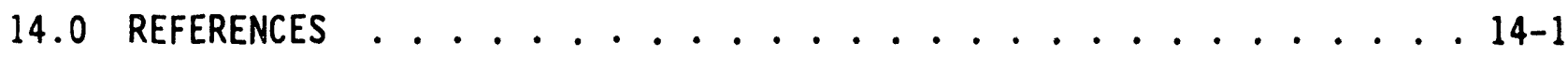


DOE/RL 91-59

\section{LIST OF FIGURES}

2-1 The Hanford Site ................. . F2-1

2-2 Structural Geology of the Pasco Basin . . . . . . . . . F F2-2

2-3 Generalized Stratigraphic and Hydrogeologic Column for 100-N . . F2-3

2-4 Geologic Cross Section ............... . F2-4

2-5 Artificial Groundwater Mounding at N Reactor . . . . . . . F2-5

2-6 Water Table and Inferred Flow Directions in the Northern Hanford Site, December 1990 . . . . . . . . . . . F2-6

2-7 Water Table and Inferred Flow Directions in the 100-N Area and Vicinity, December 1990 .............. . F2-7

2-8 Wind Roses for the Hanford Site . . . . . . . . . . . . . F2-8

2-9 Land Use of Area Within the Hanford Site . . . . . . . . . . . F2-9

3-1 100-N Area Facilities and Structures ............ . F3-1

3-1A Enlarged Legend for Figure 3-1 . . . . . . . . . F3-3

3-2 Legend for Figures 3-3 through 3-13 . . . . . . . . . . F3-5

3-3 Plan - Elevation - Minus 16' 0 ", $105 \mathrm{~N} \ldots \ldots$. . . . . . . F3-7

3-4 Plan - Elevation - 0' 0", 105 N . . . . . . . . . . F3-9

3-5 Plan - Elevation - 14' 6", $105 \mathrm{~N} \ldots \ldots$.......... F3-11

3-6 Plan - Elevation - 28' 3", $105 \mathrm{~N} \ldots \ldots$.......... F3-13

3-7 Plan - Elevation - 40' 0", $105 \mathrm{~N} \ldots \ldots$. . . . . . . F3-15

3-8 Plan - Elevations 51' $0 "$ and 60' 0", $105 \mathrm{~N} \ldots \ldots$. . . . . F3-17

3-9 Elevations I, II, and XI, 105 N ............ F3-19

3-10 Elevations III through VI, $105 \mathrm{~N} \ldots \ldots$. . . . . . . F3-21

3-11 Elevations VII through $X, 105 \mathrm{~N} \ldots \ldots$. . . . . . . . F3-23

3-12 Plan at Elevations $0^{\prime \prime} 0^{\prime \prime}$ and 16' $0 "$, 109-N Building . . . . . . F3-25

3-13 Elevations 109-N Building . . . . . . . . . . . . . F3-27

3-14 N Reactor Confinement System . . . . . . . . . . . . F3-29

3-15 N Reactor Coolant Flow ................. F3-30 


\section{LIST OF FIGURES (continued)}

3-16 Water Supply System . . . . . . . . . . . . F F3-31

3-17 163-N Facility . . . . . . . . . . . . F3-32

3-18 163-N Demineralization Process ............. F3-33

3-19 Layout of the 1301-N Liquid Waste Disposal Facility . . . . . . F3-34

3-20 Layout of tirk 1325-N Liquid Waste Disposal Facility . . . . . . . F3-35

3-21 1324-NA Facility Diagram with Dimensions, 1977 to 1983 . . . . F3-36

3-22 1324-N Surface Impcundment Physical Dimensions . . . . . . . F3-37

3-23 Liner Construction Details . . . . . . . . . . . F F3-38

3-24 1324-N/NA Surface Impoundment/Percolation Pond 1986 to 1988 . . . F3-39

5-1 N Reactor Operational Area . . . . . . . . . . . . . . F5-1

5-2 Waste Sources ................... F5-3

5-3 Original 116-N-1 Crib Layout . . . . . . . . . . . F5-5

5-4 Regeneration Waste Transport System, 1977-1983 . . . . . . . . F5-6

5-5 Regeneration Waste Transport System, 1983-1986 . . . . . . . F5-7

5-6 Regeneration Waste Transport System, 1986-1988 . . . . . . . . F5-8

5-7 120-N-1 Percolation Pond Area as it existed from 1977 to 1983 . . . F5-9

5-8 120-N-1 Percolation Pond/120-N-2 Surface Impoundment Area as they existed from 1986 to 1988 . . . . . . . . . . . F5-10

5-9 120-N-2 Surface Impoundment Schematic. . . . . . . . . . F5-11

5-10 Tritium Activity Levels in N Springs During 1988 . . . . . . . F5-12

5-11 ${ }^{90}$ Sr Activity Levels in N Springs During 1988 . . . . . . . . . F5-13

5-12 ${ }^{90}$ Sr Activity in Wells Near 116-N-1 . . . . . . . . . . . . F5-14

5-13 ${ }^{90}$ Sr Activity in Wells Near 116-N-3 ............ F5-15

5-14 Tritium Activity in Wells Between $116-\mathrm{N}-3$ and the

5-15 Tritium Activity in 100-N Area Groundwater During September-October $1989 \ldots \ldots$ F5-17 


\section{LIST OF FIGURES (continued)}

5-16 Tritium Activity in 100-N Area Groundwater During November-December 1989 . . . . . . . . . . . . . F5-18

5-17 Tritium Activity in 100-N Area Groundwater During February 1990................... F5-19

5-18 ${ }^{90}$ Sr Activity in 100-N Area Groundwater During September-October 1989 ................ . . F5-20

5-19 ${ }^{90}$ Sr Activity in 100-N Area Groundwater During November-December 1989 ................... F5-21

5-20 ${ }^{90}$ Sr Activity in 100-N Area Groundwater During February 1990

5-21 Sodium Concentration in 100-N Area Groundwater During September-October 1989 F5-23

5-22 Sulfate Concentration in 100-N Area Groundwater During September-October 1989 ............... . . F5-24

5-23 Soil Sampling Locations in the 100-N Area . . . . . . . . . . F5-25

5-24 Location of Monitoring Wells . . . . . . . . . . . . . F5-26

5-25 Areas of Inferred Soil Contamination in the 100-N Area . . . . F5-27

5-26 Results of January 10, 1990, Survey along the Top of the 1301-N LWDF Trench Sections................ F5-28

5-27 Net Exposure Rate (above local background) along the Columbia River Shoreline near 100-N Springs . . . . . . . . . F5-29

6-1 RCRA Closures ....................... . . F6-1

6-2 Operable Unit Designations . . . . . . . . . . . . . . . F6-2

6-3 Comparison of Resource Conservation and Recovery Act Corrective Action and Comprehensive Environmental Response, Compensation, and Liability Act Remedial Processes . . . . . . . . . . . F6-3

7-1 N Reactor Deactivation Program Schedule. . . . . . . . . . . F7-1

7-2 Site Remediation and Decontamination and Decommissioning Schedule...................... . . . F7-4

8-1 N Reactor Closure Costs . . . . . . . . . . . . . . F8-1

8-2 105N and 109N Decontamination and Decommissioning . . . . . . . F8-2 
DOE/RL 91-59

\section{LIST OF FIGURES (continued)}

11-1 Irradiated N Reactor Fuel Canisters . . . . . . . . . . F11-1

13-1 Hanford Environmental Restoration MSA Work Breakdown

Structure ..................... F13-1 


\section{LIST OF TABLES}

2-1 List of Endangered and Threatened Washington State Species

Having the Potential to Occur on the Hanford Site . . . . . . . T2-1

3-1 Facilities in the 100-N Area . . . . . . . . . . . . . T3-1

3-2 100-N Area Waste Stream Information . . . . . . . . . . . . T3-7

3-3 Treatment, Storage, and Disposal Units .......... . T3-10

4-1 Overview of Significant Dates for 100-N Area Operation . . . . . T4-1

5-1 100-N Area Sources .................... . T5-1

5-2 Selected Radionuclide Inventory for 116-N-1 Crib and Trench for the 1301-N Liquid Waste Disposal Facility . . . . . . . . . T5-10

5-3 Dangerous Waste Inventory for the 116-N-1 Crib and Trench for the 1301-N Liquid Waste Disposal Facility ...... . . . T5-10

5-4 Selected Radionuclide Inventory from October 1983 to January 1, 1988 for 116-N-3 Crib and Trench for the 1325-N Liquid Waste Disposal Facility . . . . . . . . T5-11

5-5 Dangerous Waste Inventory for 116- $\mathrm{N}-3 \mathrm{Crib}$ and Trench for the 1325-N Liquid Waste Disposal Facility ... . . . . . . T5-11

5-6 Average Annual Concentrations of Radionuclides ( $\mathrm{pC} i / \mathrm{L}$ ) Detected in Water Samples from the 1300-N EDB for 1978 through 1985 . . . T5-12

5-7 163-N Demineralization Plant Regeneration Effluent Waste Analysis Cation Regeneration Cycle ........... T5-13

5-8 163-N Demineralization Plant Regeneration Waste Analysis Anion Regeneration Cycle ............. . . T5-15

5-9 183-N Filtered Water Plant Backwash Effluent Analysis . . . . . T5-17

5-10 Average Radionculide Concentrations ( $\mathrm{PC} / \mathrm{L}$ ) Detected in Groundwater Samples Collected in the 100-N Area During 1981 . . T5-19

5-11 Radionuclide Concentrations ( $\mathrm{PCi} / \mathrm{L}$ ) in Wells 199-N-8P Through 199-N-8S . . . . . . . . . . . . . . T5-19

5-12 Selected Constituents Detected Above Drinking Water Standards in the 100-N Area Between April 1987 and November 1989 . . . . . T5-20

5-13 Constituents Detected Above Drinking Water Standards in the 100-N Area During July/August 1989 . . . . . . . . . . T5-22

5-14 Estimated Background Levels for Selected Constituents in Hanford Site Groundwater 


\section{LIST OF TABLES (continued)}

5-15 1980 Select Radionuclide Concentrations Detected in Soi?

Samples Adjacent to the 116-N-1 Facility and in the

116-N-1 Trench Sediment Samples $(\mathrm{pCi} / \mathrm{g})$. . . . . . . . . T5-25

5-16 Average Radionuclide Concentrations ( $\mathrm{pC} / \mathrm{g}$ ) Detected in Soil

Samples near the 116-N-1 Crib and Trench from

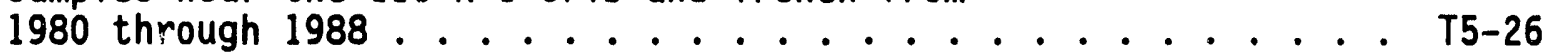

5-17 Hydrophilic Organics in Trench Sediments . . . . . . . . . . T5-27

5-18 Average Radionuclide Concentrations ( $\mathrm{pC} i / \mathrm{g}$ ) Detected

in 100-N Area Vegetation Samples . . . . . . . . . . T5-28

5-19 Average Radionuclide Concentrations ( $\mathrm{pC} i / g$ ) Detected in Vegetation Samples near 116-N-1 Crib and Trench . . . . . . T5-28

5-20 Average Radionuclide Concentrations ( $\mathrm{pC} i / g$ ) Detected in $N$ Springs Vegetation Samples ..... . . . . . . . T5-28

5-21 Sampling Results of $\mathbf{N}$ Springs Vegetation . . . . . . . . . T5-29

5-22 1979 Concentrations of Radionuclides in Swallow Nests Compared to $N$ Area and Hanford Soil Sample (pCi/g) . . . . . . T5-30

5-23 1985 Radionuclide Concentrations ( $\mathrm{pCi} / \mathrm{g}$ ) of Cliff Swallow Nests, Excrement Samples, and Shell/Embryo Samples Collected Near the 1304-N EDB During 1985 . . . . . . . . . . . . T5-30

5-24 Radionuclides Detected in Rabbits Collected Around the 116-N-Facility, 1981 Concentrations in $\mathrm{pCi} / \mathrm{g}$ (wet weight) . . . T5-31

5-25 Concentrations of Gamma-Emitting Radionuclides Detected in Deer Mice Collected Around the 116-N-1 Trench, 1981 $(\mathrm{pCi} / \mathrm{g}-\mathrm{wet} / \mathrm{wt})$................. . . . T5-33

5-26 Deer Mice Collected at N Springs, 1982 . . . . . . . . . . . T5-34

5-27 Whole Body Radionuclide Concentrations (nCi/g, wet wt.) Detected in Deer Mice Collected Near the 1301-N LWDF . . . . . . T5-35

6-1 Summary of Major $N$ Reactor Deactivation Activities . . . . . . . T6-1

7-1 RCRA Facility Closure Schedule . . . . . . . . . . . . . T7-1

8-1 N Reactor Deactivation Cost Estimate Summary for Tasks Planned Between FY 1992 and FY 1999 ............... . . T8-1

8-2 100-N Area RCRA Facility Closure Cost Estimate Summary . . . . . T8-1

8-3 N Reactor RCRA Past Practice Site Remediation . . . . . . . . . T8-1 
DOE/RL 91-59

\section{LIST OF TABLES (continued)}

8-4 N Reactor Decontamination and Decommissioning Cost Estimate Summary ........................ . . T8-2

9-1 Potential Contaminant-Specific ARARs . . . . . . . . . . . T9-1

9-2 Potential Action-Specific ARARs . . . . . . . . . . . . . T9-5

9-3 Potential Location-Specific ARARs . . . . . . . . . . . . T9-8

9-4 Decontamination and Decommissioning Potential ARARs . . . . . . T9-13 


\section{LIST OF COLOR ILLUSTRATIONS}

2-1 Aerial View of 100-N Area . . . . . . . . . . . CI2-1

2-2 N Reactor Monitoring Well Locations............. CI2-3

3-1 N Reactor Operating Components. . . . . . . . . . . . . CI3-1

3-2 Reactor Core . . . . . . . . . . . . . CI3-3

3-3 N Reactor fuel . . . . . . . . . . . . CI3-5

3-4 Aerial View of the 1301-N and 1325-N Liquid Waste Disposal Facilities ..................... CI3-7

5-1 116-N-2 Radioactive Chemical Waste Treatment and Storage Facility. ............... CI5-1

5-2 116-N-4 Emergency Dump Basin . . . . . . . . . . . . CI5-3

5-3 1304-N Emergency Dump Tank . . . . . . . . . . . . . CI5-5

5-4 105-N Reactor Spent Fuel Storage Basin (Above Water) . . . . . CI5-7

5-5 105-N Reactor Spent Fue! Storage Basin (Below Water) . . . . . CI5-9

5-6 120-N-2 Surface Impoundment. . . . . . . . . . . CI5-11

5-7 100-N Regulatory-driven Milestones . . . . . . . . . . . CI5-13

6-1 Strontium-90 Concentrations for 1990 . . . . . . . . . C16-1

6-2 Projected Strontium-90 Concentrations for 2020 . . . . . . CI6-3 
DOE/RL 91-59

\section{LIST OF TERMS AND ACRONYMS}

ACL
ADM
AEC
ALARA
ANPRM
ARAR
BAT
BDL
BPA
BSL
CERCLA
CPM
CPP
D\&D
DCG
DOE
EA
ECOIOGy
ECS
EDB
EDE
EDT
EIS
ENU
EPA
ER
ERA
HDPE
HEPA
HGP
HRA-EIS
HSWA
LERF
LWDF
LWLS
MCL
MCLG
MSL
MTCA
MTU
NESHAP
NEPA
NPDES
NPL
NPRM
NRC
NTU
NUREG
OSHA

alternate concentration 1 imit action description memorandum Atomic Energy Commission As Low as Reasonably Achievable Advanced Notice of Proposed Rulemaking applicable or relevant and appropriate requirements best available technology below detectable limit Bonneville Power Administration below sea level

Comprehensive Environmental Response, Compensation, and Liability Act of 1980

counts per minute

CERCLA past-practice

decontamination and decommissioning

Derived Concentration Guide

U.S. Department of Energy

environmental assessment

Washington State Department of Ecology

emergency cooling system

emergency dump basin

effective dose equivalent

emergency dump tank

environmental impact statement

elementary neutralization unit

U.S. Environmental Protection Agency

Environmental Restoration

expedited response actions

high-density polyethylene

high-efficiency particulate air (filter)

Hanford Generating Plant

Hanford Remedial Action EIS

Hazardous and Sol id Waste Amendments to RCRA

liquid effluent retention facility

liquid waste disposal facility

liquid waste loadout station

maximum contaminant leve 1

maximum contaminant level goal

Mean Sea Level

Model Toxics Control Act

metric ton units

"National Emission Standards for Hazardous

Air Pollutants"

National Environmental Policy Act of 1969

National Pollutant Discharge Elimination System

National Priorities List

Notice of Proposed Rulemaking

Nuclear Regulatory Commission

nephelometric turbidity unit

identifier on Nuclear Regulatory Commission documents

Occupational Safety and Health Administration 


\section{LIST OF TERMS AND ACRONYMS (continued)}

PCB
POTW
PUREX
RCRA
RCS
RCW
RFI/CMS
RI/FS
RL
ROD
RPP
S\&M
SNF
SNM
SUPp Iy System
SWP
TBC
Tri-Party Agreement
TRU
TSCA
TSD
TSDF
WAC
WDOH
WHC
WIDS

polychlorinated biphenyl

Public Owned Treatment Works

Plutonium-Uranium Extraction (Plant)

Resource Conservation and Recovery Act of 1976

reactor coolant system

Revised Code of Washington

RCRA Facility Investigation/Corrective Measures Study remedial investigation/feasibility study

U.S. Department of Energy, Richland Field Office record of decision

RCRA Past Practice

Surveillance and Maintenance

spent nuclear fuel

special nuclear materials

Washington Public Power Supply System

Special Work Permit (protective clothing)

to be considered (materials)

Hanford Federal Facility Agreement and Consent Order transuranic waste

Toxic Substances Control Act of 1976

treatment, storage, and disposal

Treatment, Storage, and Disposal Facility

Washington Administrative Code

Washington State Department of Health

Westinghouse Hanford Company

Waste Information Data System 
DOE/RL 91-59

This page intentionally left blank. 


\subsection{PURPOSE AND OBJECTIVES}

The U.S. Department of Energy (DOE) ordered the permanent cessation of production operations of $N$ Reactor in February of 1991 . The facility was dedicated to the irradiation of nuclear fuel on the Hanford Site in south-central Washington State.

$N$ Reactor is a 4,000-MW thermal nuclear reactor used to produce special nuclear materials (SNM) and byproduct steam that provided electricity to the Washington Public Power Supply System's (Supply System's) 860-MW Hanford Generating Plant (HGP) located adjacent to N Reactor. N Reactor is situated on 640 acres of 1 and along the Columbia River. The facility was built in 1963 and operated until 1986 when DOE initiated a series of safety enhancements. The reactor was placed in a standby configuration in 1988, and in $1991 \mathrm{DOE}$ ordered that preservation activities cease and steps be taken to place the facility in a condition requiring only minimal surveillance and maintenance (S\&M) until ultimate decontamination and decommissioning (D\&D).

Pursuant to the above action and to Public Law 101-i89, Section 3156(b), "Reports in Connection with Permanent Closures of Department of Energy Defense Nuclear Facilities," this $N$ Reactor Closure Report has the purpose and objective to provide Congress the following information.

- A complete survey of environmental problems at the facility.

- Budget quality data indicating the cost of environmental restoration (ER) and other remediation and cleanup efforts at the facility.

- A discussion of the proposed cleanup schedule.

Section 3156(a) of Public Law 101-189 was satisfied in a letter dated August 1, 1991, from the Secretary of Energy to the Committees on Armed Services of the Senate and the House of Representatives. This letter discussed the training and job placement services concerning $\mathrm{N}$ Reactor (Watkins 1991).

This closure report has been organized into the following sections to provide a comprehensive description of the facility, its environmental problems, and regulatory and institutional considerations in a manner that provides a basis for the estimated cost and schedule presented for cleanup actions.

- 2.0 Setting--This section presents a general overview of the $N$ Reactor area and describes the location and demography. Color Illustration 2-1 is an aerial view of the $N$ Reactor area that can be useful to identify facilities described in the closure report. Figures 2-3 and 2-4 show the general geology and hydrology beneath $\mathrm{N}$ Reactor. Figures 2-5, 2-6, and 2-7 illustrate groundwater movement in the area. These figures, in conjunction with Section 5.0, will give the reader a picture of the environmental problems relating to $\mathrm{N}$ Reactor closure. 
- 3.0 Facility Description--This section gives information about $N$ Reactor operations such as facility process descriptions, waste-generating processes, Resource Conservation and Recovery Act of 1976 (RCRA), treatment, storage, and disposal (TSD) units at the $N$ Reactor facility and other facilities outside of the $N$ Reactor area. Figures 3-2 through 3-13 are sectional drawings of $\mathrm{N}$ Reactor itself, which provide the reader with an idea of the size and elevation of this structure.

- 4.0 History--This section provides a brief summary of $N$ Reactor's operational history. Table 4-1 provides an overview of significant dates.

- 5.0 Environmental Problems--This section describes $\mathbf{N}$ Reactor operational activities that affected the environment. The affected environs are groundwater contamination (radionuclides, volatile organics, polychlorinated biphenyls (PCB), metals, and other inorganics); soil contamination (surface and vadose zone contamination from radionuclides and organic compounds); biota contamination (flora [vegetation] and fauna [animals] from radionuclide uptake by plants or ingestion by animals); and evaluated radiation at the Columbia River (unshielded sediments in the 1301-N Liquid Waste Disposal Facility).

Table 5-1 and Figure 5-2 show the waste or release sites at the $N$ Reactor facility. Other tables and figures in this section provide an in-depth look at the environmental contamination that must be handled before closurs of $N$ Reactor can take place.

- 6.0 N Reactor Closure Scope--This section describes the deactivation of $N$ Reactor, closure of RCRA TSD facilities, site remediation, and D\&D. N Reactor deactivation entails removal of $200,000 \mathrm{lb}$ of radiologically contaminated hardware and over $1,000,000$ gal of radiologically contaminated water currently used for shielding. Table 6-1 shows the major deactivation activities for $N$ Reactor. The four RCRA facilities have Part A Applications completed, with the Closure/Post-Closure Plans to be submitted by 1994. Site remediation entails the cleanup of past spills and releases to the soil and groundwater. N Reactor has two aggregate areas, 100-NR-1 Source Operable Unit and 100-NR-2 Groundwater Operable Unit, as illustrated by Figure 6-2. Color Illustrations $6-1$ and $6-2$ show computer-generated images of ${ }^{90} \mathrm{Sr}$ in the soil and groundwater beneath the 1301-N and 1325-N Liquid Waste Disposal Facilities. The D\&D of $N$ Reactor will isolate any remaining radioactive or hazardous waste in a manner that will minimize environmental impact. D\&D can be accomplished through ongoing S\&M of the facility, dismantling and burial at another location onsite, or dismantling and burial at $100 \mathrm{~N}$. The scope of work for purposes of this closure report is assumed to be dismantling of all 100- $\mathrm{N}$ facilities and burial at another location onsite, based on Decommissioning of Eight Surplus Production Reactors at the Hanford Site, Richland, Washington (DOE/EIS 1993). The actual means of decommissioning the reactor and other facilities 
at the 100-N site will depend on future engineering studies and the National Environmental Policy Act of 1969 (NEPA) process, which will evaluate alternative actions followed by a Record of Decision (ROD).

- 7.0 Proposed Cleanup Schedule--This section is an integrated schedule aimed at satisfying the objectives of this facility closure. The schedule highlights those cleanup activities that have milestone dates associated with consent order agreements between DOE, the U.S. Environmental Protection Agency (EPA), and the Washington State Department of Ecology (Ecology).

- 8.0 Estimated Cost for Closure--This section estimates the cost for $N$ Reactor deactivation, RCRA facility closures, RCRA past-practice (RPP) site remediation, and D\&D. The level of confidence behind these cost estimates is commensurate with engineering and planning currently available. Cost estimates for near-term activities are based on more detailed planning than outyear activities, such as site remediation after 2005 and D\&D by 2016 .

- 9.0 Regulatory and U.S. Department of Energy Order Compliance--This section briefly describes those promulgated regulations, DOE orders, and consent order agreements between DOE, EPA, and Ecology that are considered applicable or relevant and appropriate requirements (ARAR) to the Hanford Site remediation and cleanup activities. These statutes and regulations are coded by scope of activity and degree of applicability. The potential ARARs are grouped by topic:

- Contaminant-, action-, or location-specific

- DOE orders and directives related to facilities and operations, including deactivation implementation actions

- Decontamination and decommissioning, to provide a logical sequence for review.

- 10.0 National Environmental Policy Act Strategy--This section describes the manner in which NEPA will be applied to the ER and cleanup activities. This discussion includes NEPA documentation directed at both $N$ Reactor-specific closure activities and Hanford Site-wide remediation activities.

- 11.0 Disposition of Special Nuclear Material--This section describes the quantity of uranium billets, unirradiated nuclear fuel elements, and irradiated nuclear fuel elements that are being stored and includes a strategy for their disposition.

- 12.0 Institutional Issues--This section describes unique issues directed at future land use and means of dispositioning facilities owned by the Supply System, and the Bonneville Power Administration (BPA) that are built on land leased from DOE. 
- 13.0 Closure Management and Programmatic Interface--This section provides a work breakduwn structure for $\mathrm{N}$ Reactor closure and describes the organizational responsibilities for its implementation and means of performance monitoring together with the budget and cost-control process.

- 14.0 References--This section 1ists documents used or referred to in preparing this closure report.

In 1991, DOE, EPA, and Ecology entered into the Hanford Federal Facility Agreement and Consent Order, popularly known as the Tri-Party Agreement (Ecology et al. 1992). While this agreement sets forth the schedule for site remediation activities required under RCRA and the Comprehensive Environmental Response, Compensation, and Liability Act of 1980 (CERCLA), at this time, the Tri-Party Agreement does not include requirements for the D\&D of the $\mathrm{N}$ Reactor. For the purpose of this report, D\&D of the $N$ Reactor is assumed to be performed based on dismantling it and transporting it to the burial site in pieces as described in Decommissioning of Eight Surplus Production Reactors at the Hanford Site, Richland, Washington (DOE/EIS 1993). 
DOE/RL 91-59

\subsection{SETTING}

\subsection{LOCATION AND DEMOGRAPHY}

The Hanford Site is a $1,450-\mathrm{km}^{2}\left(560-\mathrm{mi}^{2}\right)$ tract of 1 and located in Benton, Franklin, and Grant Counties in south-central Washington State. Figure 2-1 is a layout of the Hanford Site; Color Illustration 2-1 shows an aerial view of the $100-N$ Area where $N$ Reactor is located. The 2.6-km (640-acre) $100-N$ Area is situated along the Columbia River. The 100-D/DR Area is northeast of the 100-N Area and the 100-K Area is southwest. The 200 Area is southeast of $100-N$ and was used for the disposal of solid and liquid wastes from $N$ Reactor. The city of Richland is approximately 43 air and 61 river $\mathrm{km}$ (27 air and 38 river $\mathrm{mi}$ ) south of the 100-N Area. Approximately 258,000 people live within an $80-\mathrm{km}(50-\mathrm{mi})$ radius of the $100-\mathrm{N}$ Area. The cities of Richland, Pasco, and Kennewick, located southeast of the Hanford Site, are the main population centers. Many other smaller communities surround the Hanford Site. The nearest residences are farm homes about $9.7 \mathrm{~km}$ ( $6 \mathrm{mi}$ ) north of the 100-N Area. No residences remain on the Hanford Site.

\subsection{HYDROGEOLOGY}

\subsubsection{Regional Setting}

The Hanford Site is located within a natural depression called the Pasco Basin, as shown in Figure 2-2. The Pasco Basin is part of the larger Columbia Plateau, which includes most of southeastern Washington and northeastern Oregon, a province that is roughly defined by the extent of Columbia River basalt flows. The Pasco Basin is bounded on the north by the Saddle Mountains and on the south by Rattlesnake Mountain. To the west lie anticlines of the Yakima Fold Belt, including Umtanum Ridge, Yakiina Ridge, and the Rattlesnake Hills. The eastern boundary is formed by the Palouse siope, a west-dipping monocline. Within the Pasco Basin lie several northwest-southeast trending synclines; the Wahluke Syncline crosses the northern portion of the basin.

Saturated sediments about the Columbia River Basalt form a series of aquifers (underground beds of gravel or porous stone containing water) and aquitards (confining layers), collectively called the uppermost aquifer system. The shallowest aquifer in the system is unconfined over most of the Hanford Site, and is made up of Hanford and Ringold fluvial (streambed) sediments. It is commonly termed the uppermost or unconfined aquifer. The uppermost aquifer is naturally recharged by runoff from surrounding highlands. Recharge via precipitation on the Hanford Site is negligible because of the desert climate (see Section 2.3). Some areas show evidence of leakage upward from confined aquifers into the uppermost aquifer. Artificial recharge also occurs from irrigation on lands adjacent to the Hanford Site and has occurred in the past from liquid waste disposal on the Site. Groundwater in the uppermost aquifer primarily discharges to the Columbia River, and to a lesser extent, the Yakima River. Recharge to the underlying confined aquifers in the northern portion of the Pasco Basin primarily comes from the southwest, west, and north, with flow generally to the east-southeast across the 100 Areas, as suggested by the structural trend of the Wahluke Syncline (DOE-RL 1990). 
The uppermost aquifer system is dominated by gravelly, sandy, and silty sediments associated with fluvial channel and overbank deposits of the ancestral Columbia River. Known groundwater contamination plumes are typically contained in the uppermost aquifer, which comprises sand and gravel. Generally upward hydraulic movement prevents downward migration of contamination. Flow direction is generally toward the Columbia River. Liquid effiuent disposal in the past created groundwater mounds, which caused some migration inland as well. Flow rates are in the range of 0.5 to $4.5 \mathrm{~m} /$ day (1 to $15 \mathrm{ft} /$ day) under natural conditions. When groundwater mounds were present, flow rates of $15 \mathrm{~m} /$ day ( $50 \mathrm{ft} / \mathrm{day}$ ) were observed. These higher flow rates were caused by unnaturally steep gradients and thermally hot recharge sources (DOE-RL 1991). Short-term flow near the river is strongly influenced by daily fluctuations in river stage, which may be in the range of 2 to $2.5 \mathrm{~m}$ (6 to $8 \mathrm{ft}$ ) daily and 2.5 to $3 \mathrm{~m}(8$ to $10 \mathrm{ft}$ ) seasonally.

The following description of hydrostratigraphy for the 100 Areas is from Peterson (1992) and was developed from more detailed descriptions in the RCRA Facility Investigation/Corrective Measures Study (RFI/CMS) for the 100-NR-1 Operable Unit (DOE-RL 1990). A generalized stratigraphic and hydrogeologic column is shown in Figure 2-3. Six hydrostratigraphic units are identified: Unsaturated (vadose) zone, Ringold/Hanford producing layer (i.e., uppermost aquifer), Ringold confining layer, Ringold confined aquifer, another Ringold confining layer, and another Ringold confined aquifer. Beneath this sequence, which in total is usually referred to as the unconfined aquifer system, lie Columbia River basalt flows, which contain their own series of confined aquifers. The uppermost confined aquifer in the basalt sequence may be formed either by flow tops in the Elephant Mountain Member or by the Rattlesnake Ridge interbed and underlying Ponoma flowtop zone.

The unsaturated (vadose) zone is contained in Hanford Formation sediments. This zone is approximately $9 \mathrm{~m}(30 \mathrm{ft})$ thick. These sediments typically are open-framework pebble- to boulder-sized gravels. Interstitial sand content is generally low and mud-sized sediment is limited to coatings on individual grains and rip-up clasts. Interstratified lenses of sand and mud may be encountered, but they are very localized. Drilling of one well at the 100-N Area near an active liquid waste disposal facility in 1984 led to the only known occurrence of a perched water table within the 100 Areas.

The fluvial sediments of Ringold gravel unit $E$ make up the uppermost aquifer through most of the 100 Areas. At some locations, the uppermost aquifer includes the bottom few feet of the Hanford sediments. Erosional features, such as channels, are present at the unconformity between the Ringold Formation and overlying Hanford sediments. The more transmissive Hanford deposits that fill these channels may act as preferred pathways for groundwater movement.

Underlying the uppermost aquifer is a confining bed that consists of interbedded clays, silts, and a few thin sand layers. These strata represent river overbank deposits. This interval ranges in thickness from 3 to $15 \mathrm{~m}$ $(10$ to $50 \mathrm{ft})$. 
Layers of silty sand to sandy silt that are equivalent to Ringold gravel units $B$ and $C$ form the fourth hydrogeologic unit, a confined aquifer. This unit is 53 to $61 \mathrm{~m}$ (175 to $200 \mathrm{ft}$ ) thick. Alternating lithologies in the unit suggest the possibility of alternating producing and confining layers.

Another confining layer underlies the sand and silt aquifer. It consists of 30 to $46 \mathrm{~m}$ (100 to $150 \mathrm{ft}$ ) of interbedded clay and silt assigned to the lower mud sequence of the Ringold Formation. These fine-grained sediments are predominantly lacustrine.

The lowest unit of the unconfined aquifer system consists of fluvial sediments of Ringold gravel unit $A$. This unit lies unconformably over the Elephant Mountain Member of the Saddle Mountains Basalt. Unit A consists of interbedded sands and pebble- to cobble-sized gravels, with some caliche layers. It ranges in thickness from 5.5 to $20 \mathrm{~m}(18$ to $65 \mathrm{ft})$. Unit $A$ is present near the 100-N Area.

Within the Columbia River Basalt Group, the Rattlesnake Ridge Interbed and underlying Pomona flowtop zone form the uppermost confined aquifer in most locations. The Rattlesnake Ridge Interbed consists of 14 to $18 \mathrm{~m}$ (45 to $60 \mathrm{ft}$ ) of tufaceous silt, stone, and sandstone. The Elephant Mountain Member, which is found throughout the 100 Areas, forms the confining layer above the Rattlesnake Ridge Interbed.

\subsubsection{Local Setting}

Figure 2-4 shows a generalized northwest to southeast geologic cross section. Approximately 70 wells, between 7.5 and $40 \mathrm{~m}$ ( 25 and $132 \mathrm{ft}$ ) deep, (shown in Color Illustration 2-2) have been drilled in the 100-N Area since 1962. These wells were installed for several purposes, including the following:

- Monitoring radionuclide migration from soil column disposal facilities used for liquid effluents

- Monitoring for oil and grease from past spills and leaks

- Monitoring for hazardous waste constituents upgradient and downgradient at each of three RCRA landfills.

The geologic units in the 100-N Area include the Ringold Formation and the overlying glaciofluvial sediments informally referred to as the Hanford Formation.

The Ringold Formation in the 100-N Area is composed of five geologic units that are illustrated in Figure 2-3. The units are described as follows, in ascending order: (1) indurated sandy gravel (unit A); (2) lower mud sequence; (3) silty sand to sandy silt (units $B$ and $C$ ); (4) overbank deposits; and (5) fluvial gravels (unit E).

The indurated sandy gravel unit rests unconformably on basalt. It consists of interbedded sand and cobbles with some caliche, and ranges in thickness from 5.5 to $20 \mathrm{~m}$ (18 to $65 \mathrm{ft}$ ). The second unit, the lower mud 
sequence, comprises interbedded clay and silt. It is 30 to $46 \mathrm{~m}$ (100 to $150 \mathrm{ft}$ ) thick. The third unit is made up of silty sand to sandy silt, possibly in alternating fine-grained and coarser-grained layers. This unit is 53 to $61 \mathrm{~m}$ (175 to $200 \mathrm{ft}$ ) thick. The fourth unit, overbank deposits, comprises clay and silt with a few thin sand layers. The fourth unit is 3 to $15 \mathrm{~m}$ ( 10 to $50 \mathrm{ft}$ ) thick. The uppermost unit in the Ringold Formation is composed of fluvial gravels, and is approximately $15 \mathrm{~m}(50 \mathrm{ft}$ ) thick in the 100-N Area.

The Ringold is differentiated from the glaciofluvial sediments by an increase in calcium carbonate with a zone of increased consolidation and cementing, a decrease in the percentages of basalt clasts, and an increase in the quartz/silica-rich materials. These changes are reflected in a distinct color change from the olive gray of the glaciofluvial sediments to a dark grayish brown of the Ringold. This sand gravel unit of the Ringold is heterogenous, varying in texture both laterally and vertically (DOE-RL 1990).

Overlying the Ringold Formation are flood deposits that were laid down when ice-dammed lakes within the Columbia River drainage were periodically breached during Pleistocene glaciation (Waite 1980). Deposits of glaciofluvial sediments from these floods have been informally referred to as the Hanford Formation. These deposits are highly variable both laterally and vertically, reflecting the dynamic nature of deposition. These cataclysmic flood deposits have been divided into two facies: a coarse-grained channel facies (the Pasco gravels) and a fine-grained slackwater facies (the touchet beds). The Pasco gravels predominate at the 100-N Area. These gravels are composed of poorly sorted coarse sand and gravel that were deposited in flood bars along high-energy flood channels (DOE 1988).

The unconfined aquifer in the 100-N Area is contained in the sand/gravel unit of the Ringold Formation with its lower boundary being the mud unit. The water table occurs about $6 \mathrm{~m}(20 \mathrm{ft})$ below the contact with the glaciofluvial sediments. The general groundwater flow direction is northwest, toward the Columbia River, based on interpretation of groundwater gradients. The elevation of the water table at the site is affected by the level of the nearby Columbia River, to which the unconfined aquifer is hydraulically connected by groundwater entering the 100-N Area from other parts of the Hanford Site and, in the past, by discharge of wastewater to the disposal facilities. Near the 1301-N facility during high river levels, bank storage along the river is increased. Groundwater levels near the river rise in response (Newcomer 1988).

During $N$ Reactor operation over 7.5 billion $L$ ( 2 billion gal) of effluent were discharged to the soil column, which caused artificial groundwater mounding on the site, as shown in Figure 2-5. Since the shutdown of $N$ Reactor and the corresponding reduction in effluent discharged to the soil column, the groundwater levels have returned to a natural level leaving older groundwater monitoring wells dry. Figures 2-6 and 2-7 show more recent groundwater table maps of the northern Hanford Site and the 100-N Area. 
DOE/RL 91-59

\subsection{METEOROLOGY}

The Hanford Site weather is monitored at the Hanford Meteorology Station and at other points situated across the Site, including Station 13 of the Hanford Telemetry Network located at the 100-N Area. The Cascade Mountains, to the west of the Site, greatly affect the local climate. The Hanford Site is situated in the rainshadow of the Cascade Mountains.

\subsubsection{Precipitation}

The Hanford Site receives an average of $16 \mathrm{~cm}(6.25 \mathrm{in.})$ of precipitation annually. Precipitation falls mainly in the winter months, with almost half of the annual precipitation occurring between November and February. Rainfall from July through September makes up only 10 percent of the annual precipitation (DOE-RL 1990).

Precipitation of $1.3 \mathrm{~cm}$ (0.5 in.) or more within a 24-hour period occurs only twice yearly on the average. Instances of $2.5 \mathrm{~cm}(1.00 \mathrm{in}$.) or more of precipitation within a 24-hour period are rare; only four occurred between 1946 and 1980. One of these events was the record storm of October 1-2, 1957, when $4.8 \mathrm{~cm}$ (1.88 in.) of rainfall occurred in 12 hours (DOE-RL 1990).

Winter monthly average snowfall ranges from $13.5 \mathrm{~cm}(5.3 \mathrm{in.})$ in January, to $0.8 \mathrm{~cm}(0.31 \mathrm{in.})$ in March. The record snowfall of $62 \mathrm{~cm}(24.41 \mathrm{in.})$ occurred in February 1916. Between December and February, snowfall accounts for about 38 percent of all precipitation (DOE-RL 1990).

The average yearly relative humidity at the Hanford Site for the period from 1946 to 1980 was 54.4 percent. Humidity is higher in winter than in summer. The monthly averages range from 32.2 percent for July to 80 percent in December. The lowest monthly average (21.9 percent) occurred in July 1959 and the highest monthly average ( 90.5 percent) occurred in December 1950 (DOE-RL 1990).

\subsubsection{Winds}

By serving as a source of cold air drainage, the Cascade Mountains greatly affect the wind regime at the Hanford Site. This gravity drainage, plus topographic channelling, results in a northwest to west-northwest prevailing wind direction at the Site (DOE-RL 1990). The average mean monthly speed for the period from 1945 to 1980 was $12.4 \mathrm{~km} /$ hour $(7.7 \mathrm{mi} /$ hour), with monthly means ranging from $9.8 \mathrm{~km} /$ hour $(6.1 \mathrm{mi} / \mathrm{hour}$ ) in December to $14.8 \mathrm{~km} /$ hour ( $9.2 \mathrm{mi} /$ hour) in June (DOE-RL 1990). Peak gust speeds range from 101 to $129 \mathrm{~km} /$ hour (63 to $80 \mathrm{mi} /$ hour) and are generally southwest or west-southwest winds (DOE-RL 1990).

Figure 2-8 presents wind roses for the Hanford Telemetry Network (DOE-RL 1990). The Columbia River exerts a strong local channelling effect on the local wind regime. This channelling, along with the gravity drainage from the Cascades, produces a prevailing west-southwest wind at the 100-N Area. In addition, diurnal fluctuations in wind speeds are common during the summer months. In July, hourly average wind speeds range from a low of $8.4 \mathrm{~km} / \mathrm{hour}$ 
(5.2 mi/hour) from 9:00 to 10:00 a.m. to a high of $21 \mathrm{~km} / \mathrm{hour}(13.0 \mathrm{mi} / \mathrm{hour}$ ) from 9:00 to 10:00 p.m. The diurnal fluctuation is less in the winter months, ranging from $8.8 \mathrm{~km} /$ hour $(5.5 \mathrm{mi} /$ hour) in the morning to $10 \mathrm{~km} /$ hour (6.3 $\mathrm{mi} /$ hour) in the evening (DOE-RL 1990).

\subsubsection{Temperature}

Based on data from 1914 to 1980 , winter minimum temperatures vary from -33 to $-5.6{ }^{\circ} \mathrm{C}\left(-27\right.$ to $\left.+22{ }^{\circ} \mathrm{F}\right)$, and summer maximums vary from 38 to $46{ }^{\circ} \mathrm{C}$ $\left(100\right.$ to $\left.115^{\circ} \mathrm{F}\right)$. Temperatures reach $32{ }^{\circ} \mathrm{C}\left(90^{\circ} \mathrm{F}\right)$ or above an average of 55 days a year; however, minimum temperatures reach $21^{\circ} \mathrm{C}\left(70^{\circ} \mathrm{F}\right)$ or above an average of only 8 days per year. Half of all winters are free of temperatures below $-18{ }^{\circ} \mathrm{C}\left(0^{\circ} \mathrm{F}\right)$. Between 1914 and 1980,16 days with temperatures $-29{ }^{\circ} \mathrm{C}$ $\left(-20^{\circ} \mathrm{F}\right)$ or below were recorded. Ten days are on record when the maximum temperature failed to exceed $-18{ }^{\circ} \mathrm{C}\left(0^{\circ} \mathrm{F}\right)$. The winter of 1925-1926 is the mildest winter on record, with $-5.6{ }^{\circ} \mathrm{C}\left(22^{\circ} \mathrm{F}\right)$ recorded as the lowest temperature. In 1954 , only one day reached $38^{\circ} \mathrm{C}\left(100^{\circ} \mathrm{F}\right)$. Before 1980 , there were three summers on record when the temperatures were $38^{\circ} \mathrm{C}\left(100^{\circ} \mathrm{F}\right)$ or above for 11 consecutive days (DOE-RL 1990).

\subsubsection{Evapotranspiration}

Mean annual loss of water from the soil (evapotranspiration) for the Tri-Cities area immediately southeast of the Hanford Site has been estimated to be about $74 \mathrm{~cm}$ ( $29 \mathrm{in.}$.). The actual annual evapotranspiration rate under current conditions in the northern portion of the Hanford Site is estimated to be about $15.5 \mathrm{~cm}$ (6.1 in.) (DOE-RL 1990).

\subsection{ENVIRONMENTAL RESOURCES}

The Hanford Site has a cool desert or a shrub-steppe type of environment and supports a biological community typical to this setting.

\subsubsection{Flora and Fauna}

More than 240 species of plants have been identified on the Hanford Site. Plants likely to be present at the 100-N Area include the gray rabbitbrush (Chrysothamnus nauseosus), cheatgrass (Bromus tectorum), tumbleweed (Salsola kali), yarrow (Achillea millefolium), yellow salsify (Tragopogon dubius), false yarrow (Chaenactis douglasii), and tumble mustard (Sisymbrium altissimum). Cheatgrass and riparian plants are the most prevalent (DOE-RL 1990).

More than 300 species of terrestrial and aquatic insects have been found on the Hanford Site. Specific insects likely to be found in fresh water in the 100-N Area include water striders (Gerridae), backswimmers (Notonectidae), water boatman (Corixidae), and diving beetles (Dytiscidae). Seasonal inhabitants would include larvae of the cadisfly (Trichoptena), the mosquito (Culicidae), and the manfly (Ephemeroptera) (DOE-RL 1990). 
Approximately 16 species of amphibians and reptiles have been observed on the Hanford Site. The side-blotched lizard is the most abundant reptile. Toads (family: Bufonidae) and frogs (family: Ranidae) are found along the Columbia River (DOE-RL 1990).

More than 125 species of birds have been identified on the Hanford Site; the horned lark and western meadowlark are the most abundant nesting birds. Wastewater ponds at the Hanford Site are important habitats for songbirds, shore birds, ducks, and geese. The most abundant nesting bird at these sites is the American coot. Waterfowl frequently use the ponds during fall migration. The Hanford Site is located in the Pacific Flyway. The resident waterfowl include the Canada goose, whose nesting habitat includes islands in the Columbia River. Birds identified at the 100-N Area include swallows and robins (DOE-RL 1990 ).

Of the approximately 30 species of mammals that have been identified on the Hanford Site, most are small and nocturnal. Muskrats and porcupines have been observed along the shorelines of the ponds and ditches, and beavers are resident in the sloughs along the Columbia River. Mule deer are found mostly along the Columbia River and in the Rattlesnake Hills (DOE-RL 1990).

The Columbia River supports a large diverse community of planktonic and benthic invertebrates, fish, and other communities (DOE-RL 1990).

Phytoplankton and periphyton are abundant in the Columbia River.

Phytoplankton and zooplankton populations in the river are largely transient, flowing from one area to another. However, characteristic endemic groups of plankton generally have insufficient time to develop in the Hanford Reach (DOE-RL 1990).

Forty-four species of fish have been identified in the Hanford Reach of the Columbia River. The chinook salmon (Oncorhynchus tshawytscha), sockeye salmon (Oncorhynchus nerka), coho salmon (Oncorhynchus kisutch), and steelhead trout (Salmo gairdneri) use the river as a migration route to and from spawning areas farther upstream. The fall chinook salmon and the steelhead trout also spawn in the Hanford Reach (DOE-RL 1990).

\subsubsection{Threatened and Endangered Species}

Table 2-1 includes State-designated endangered and threatened flora and fauna that could potentially occur at the Hanford Site. State designations are at least as strict as Federal designations. No plants on the Federal Endangered and Threatened Wildlife and Plants 1 ist are known to occur on the Hanford Site.

Two species of plants found at the Hanford Site are identified on the Washington State list of threatened or endangered species: the Columbia milk-vetch (Astragalus columbianus barneby), listed as threatened, and persistentsepal yellowcress [Rorippa columbiae (Suksde)], designated endangered. Columbia milkvetch occurs on dry land benches of the Columbia River in the Priest Rapids Dam, Midway, and Vernita vicinity. Persistentsepal yellowcress occurs in the wetted zone of the water's edge along the Columbia River (DOE-RL 1990). Both species may exist along the 100-N Area shore, but neither have been specifically identified. 
The Federal Government lists the American peregrine falcon ( $F$ alco peregrinus anatum) as endangered and the bald eagle (Haliaeetus leucocephalus) as threatened. The Washington State list includes these two birds and also identifies the white pelican (Pelecanus erythrorhynchos) and sandhill crane (Grus canadensis) as endangered, and the ferruginous hawk (Buteo regalis) as threatened. The peregrine falcon does not nest at the Hanford Site but is a casual migrant. The bald eagle is a regular winter resident in areas where it forages on dead salmon and waterfowl along the Columbia River. "Washington State Bald Eagle Protection Rules" were issued in 1986 (WAC 232-12-292) pursuant to which DOE will prepare a management plan to mitigate eagle disturbance (DOE-RL 1990). The frequency of these birds visiting the 100-N Area is unpredictable, but they visit the Hanford Site between October and March.

The pygmy rabbit (Sylvilagus idahoensis), identified as threatened, is the only mammal species listed as endangered or threatened having the potential to occur on the Hanford Site.

Critical Habitats. It is not known if bald eagles or ferruginous hawks roost or forage in the 100-N Area. If roost trees or forage areas for these birds do exist on Site, such areas would be critical habitat. It is also not known if the endangered persistentsepal yellowcress or the threatened eatonella are present on the Site, but such occurrences would also constitute critical habitat.

\subsection{LAND AND WATER USE}

\subsubsection{Sensitive Environments}

The Columbia River's importance as a recreational resource and a regional source of drinking and irrigation water, as well as a productive habitat for waterfowl, economically important fish species, and transitory endangered and threatened wildlife, could merit special concern for this environment during implementation of the remedial activities at the 100-N Area. If critical bald eagle habitats exist on the Site, they may be regarded as sensitive environments as defined in Appendix A of 40 CFR Part 300.

The Columbia River is regarded as an important environment with respect to the 100-N Area. The Hanford Reach is the only significant stretch of the Columbia River within the United States that is not impounded by a dam (DOE-RL 1990). The reach has also been designated a class $A$ (excellent) surface water by Washington State (WAC 173-201). This designation requires that water quality be maintained for the following uses.

- Domestic, industrial, and agricultural water supply

- Stock watering

- Fish and shel'fish migration, rearing, spawning, and harvesting

- Wildlife habi:at

- Recreation (i icluding primary-contact recreation)

- Commerce and navigation. 
DOE/RL 91-59

\subsubsection{Land Use}

Land use in the area surrounding the Hanford Site consists primarily of irrigated and dry-land farming, livestock grazing, and urban and industrial development. Principal agricultural crops include hay, wheat, potatoes, corn, apples, soft fruit, hops, grapes, and vegetables. Most industrial activities are associated with either agriculture or energy production (DOE-RL 1990).

Immediately north and across the river from the 100 Areas are the $130-\mathrm{km}^{2}$ (32,100-acre) Saddle Mountain National Wildlife Refuge and the $225-\mathrm{km}^{2}$ (55,600-acre) Washington State Department of Wildlife Reserve. These lands provide a buffer around the reactor sites and are owned by the DOE

(DOE-RL 1990). Figure 2-9 shows the use of lands within the Hanford Site.

\subsubsection{Water Use}

Surface Water Use. The Hanford Reach of the Columbia River forms the northern boundary of the 100-N Area. The entire Hanford Reach is used for boating and fishing. River water is used onsite and at downstream communities for drinking water.

Five management alternatives for future use of the Hanford Reach are being analyzed as part of an environmental impact study being prepared by DOE, the National Park Service, and the U.S. Fish and Wildlife Service (DOE/EIS 1992):

- National wildlife refuge with national wild and scenic river overlay

- Water resource development projects prohibited

- National conservation area

- National river

- No action.

Columbia River water was the source of cooling water at $N$ Reactor until 1987. Water for the heat dissipation system was drawn from the river through a shoreline intake system, circulated through various condensers and heat exchangers, and discharged to the center of the river through the $260-\mathrm{cm}$ (102-in.) discharge line. The normal pumping rate was $1,100,000 \mathrm{~L} / \mathrm{min}$ $(290,000 \mathrm{gal} / \mathrm{min})$ (DOE-RL 1990).

Groundwater Use. No production wells exist at the 100-N Area. The nearest reported domestic groundwater well is located near the Vernita Bridge, over $16 \mathrm{~km}$ (10 mi) west (upgradient) of the 100-N Area (DOE-RL 1990).

\subsection{ARCHAEOLOGY AND HISTORICAL RESOURCES}

Knowledge about the archaeology of the 100-N Area is largely based on reconnaissance-level archaeological surveys. Within $2.4 \mathrm{~km}(1.5 \mathrm{mi})$ of the 100-N Area are 8 archaeological sites. Several of these are located north of 
the Columbia River; the other sites situated on the south shore make up the Ryegrass Archaeological District. No known sites of religious importance actually lie within the 100-N Area (DOE-RL 1990).

The most common evidence of historic activity now found near the 100-N Area is gold mine tailings on river banks and archaeological sites where homesteads once stood. Few of these vestiges of the early years remain. 
DOE/RL $91-59$

Color Illustration 2-1. Aerial View of 100-N Area.

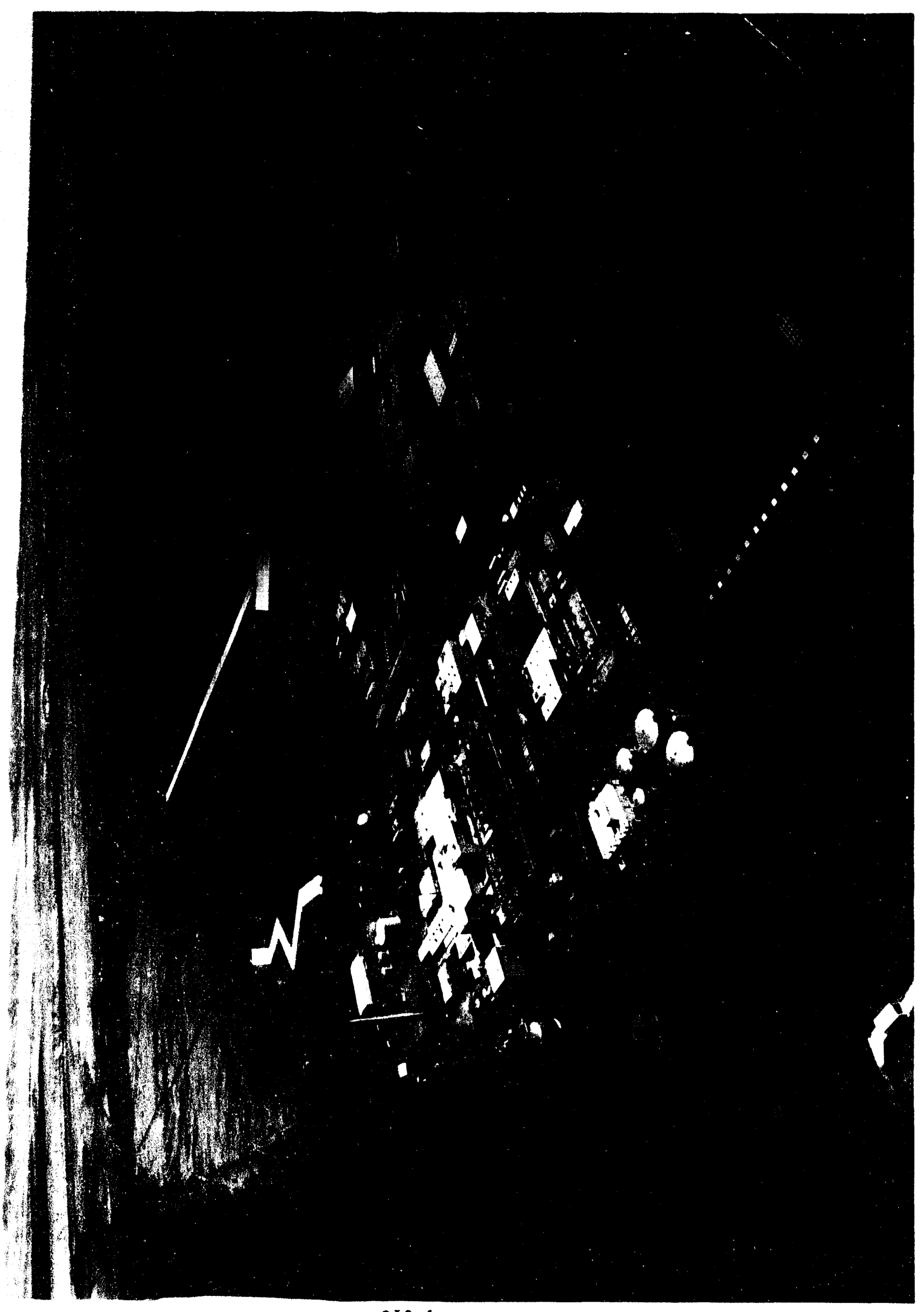


DOE/RL 91-59

Color Illustration 2-2. N Reactor Monitoring Well Locations.

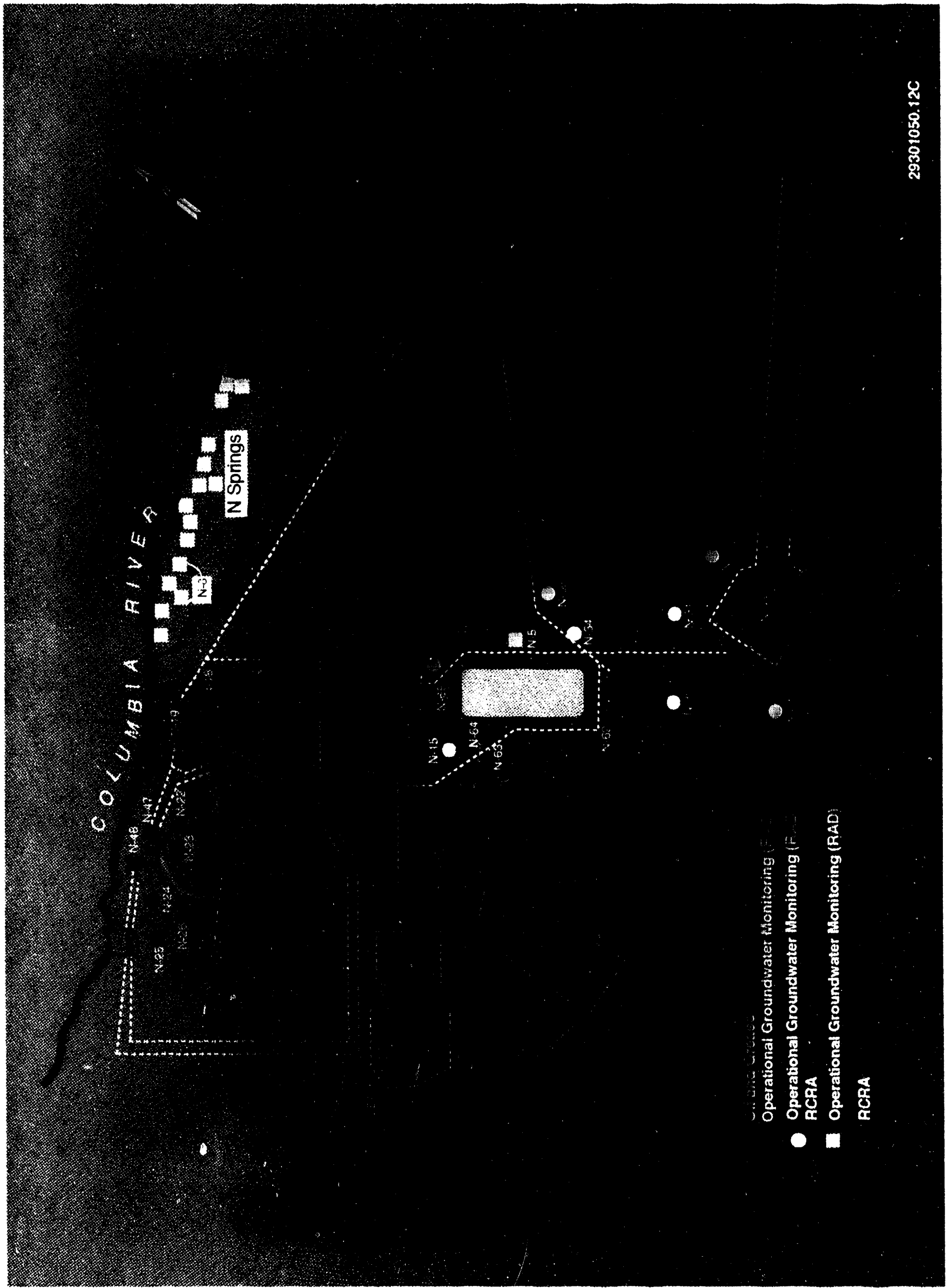


Figure 2-1. The Hanford Site.

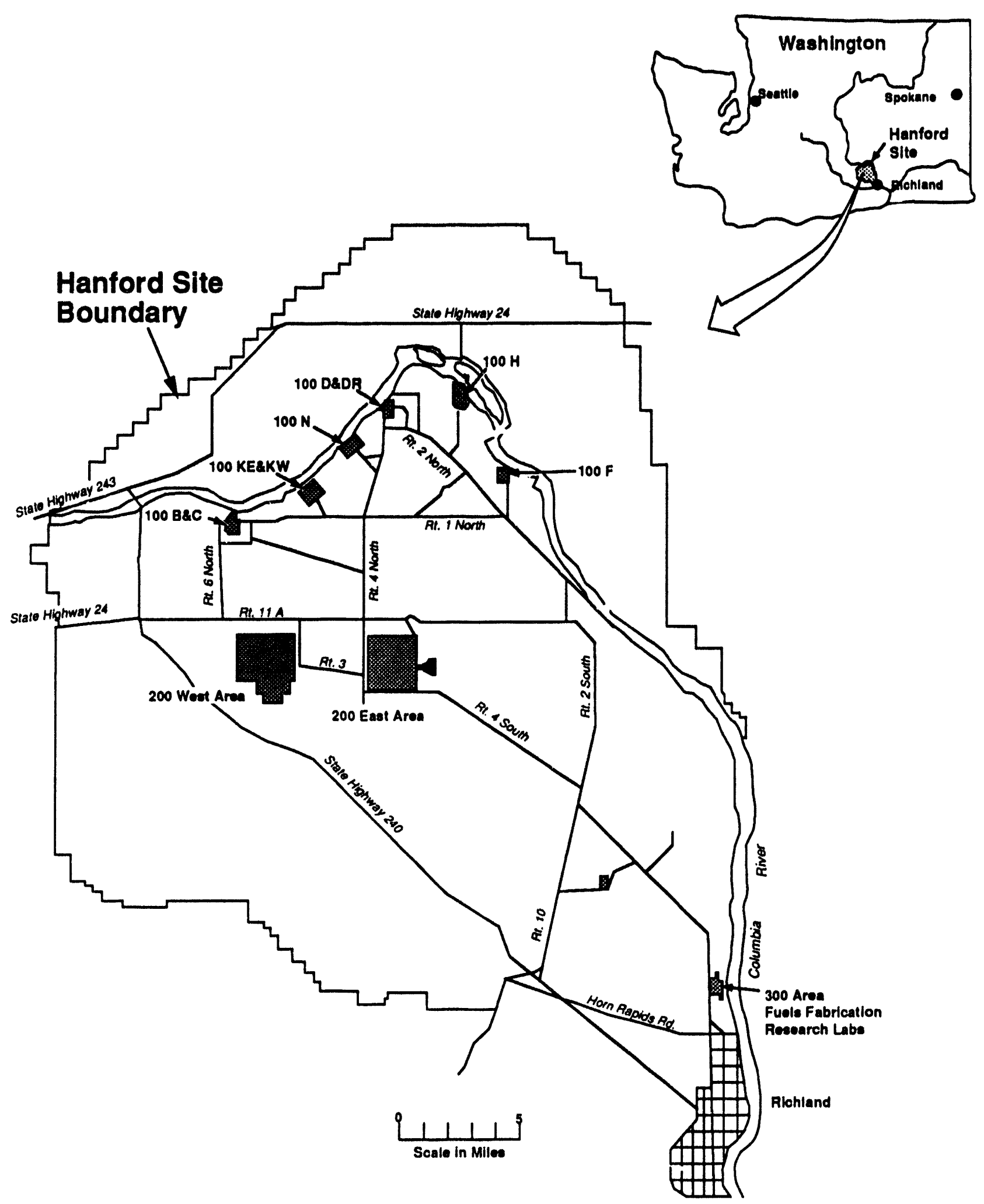


DOE/RL 91-59

Figure 2-2. Structural Geology of the Pasco Basin.

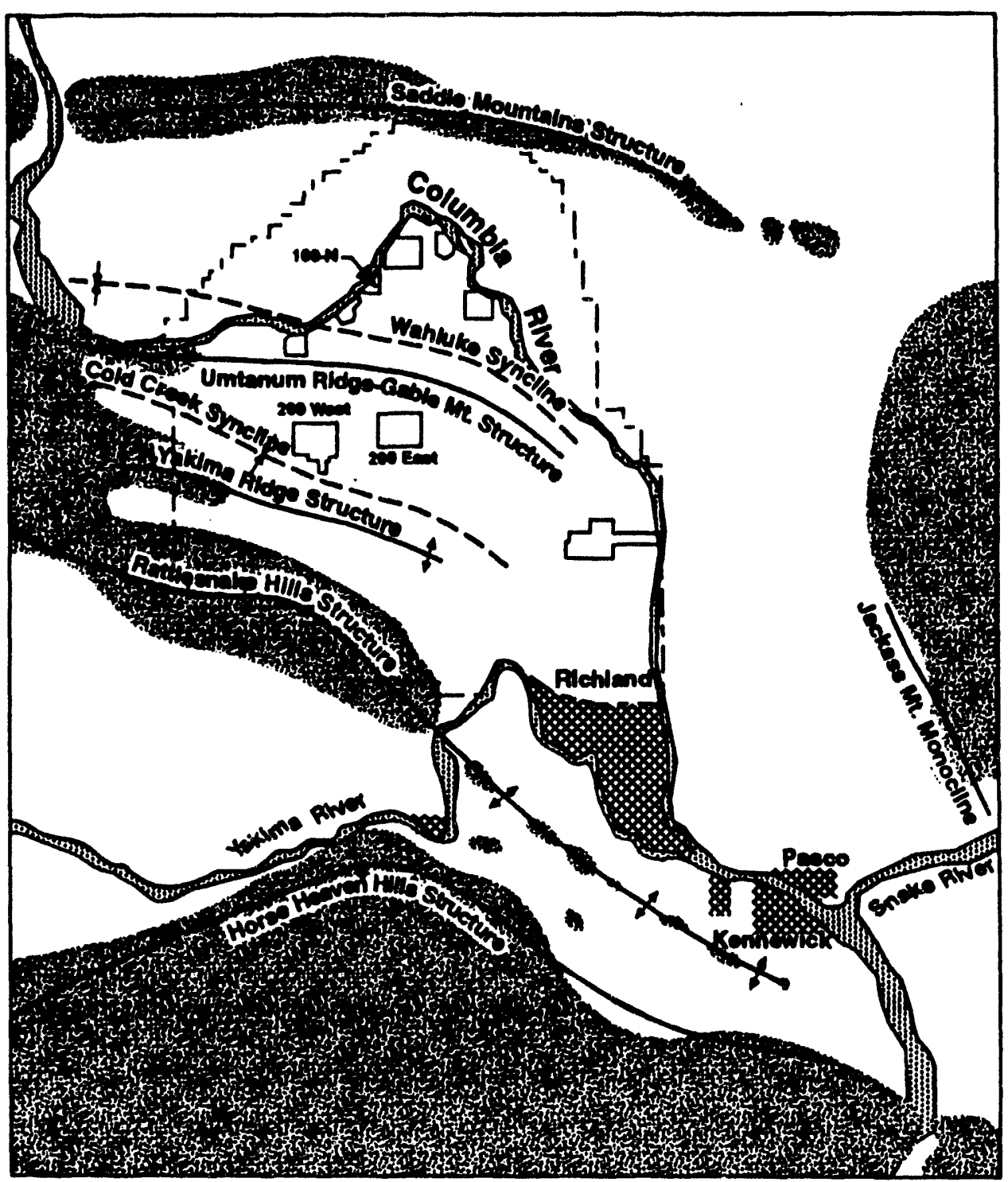

\section{LEGEND:}

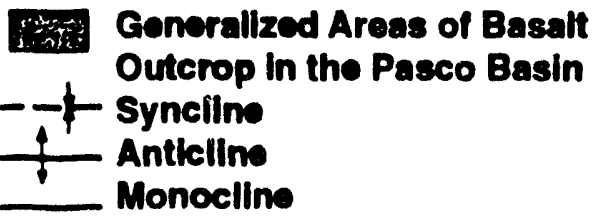

Source: Myere ot di. 1979.

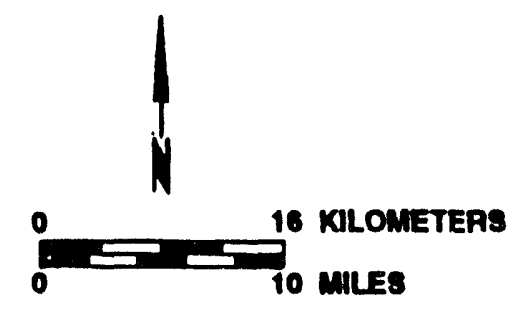

29212025.53 
Figure 2-3. Generalized Stratigraphic and Hydrogeologic Column for $100-\mathrm{N}$.

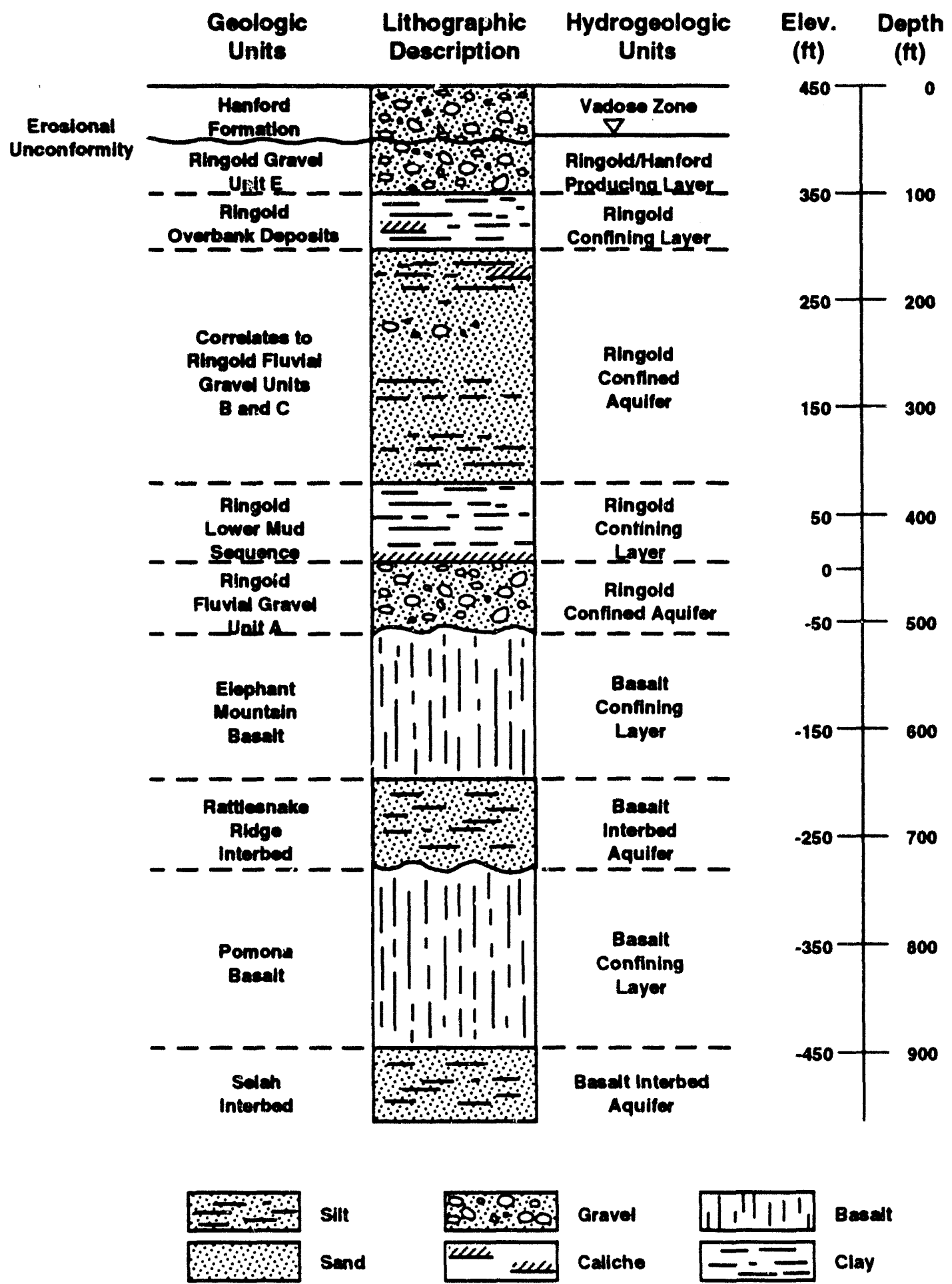

H9102029.2 
DOE/RL 91-59

Figure 2-4. Geologic Cross Section.

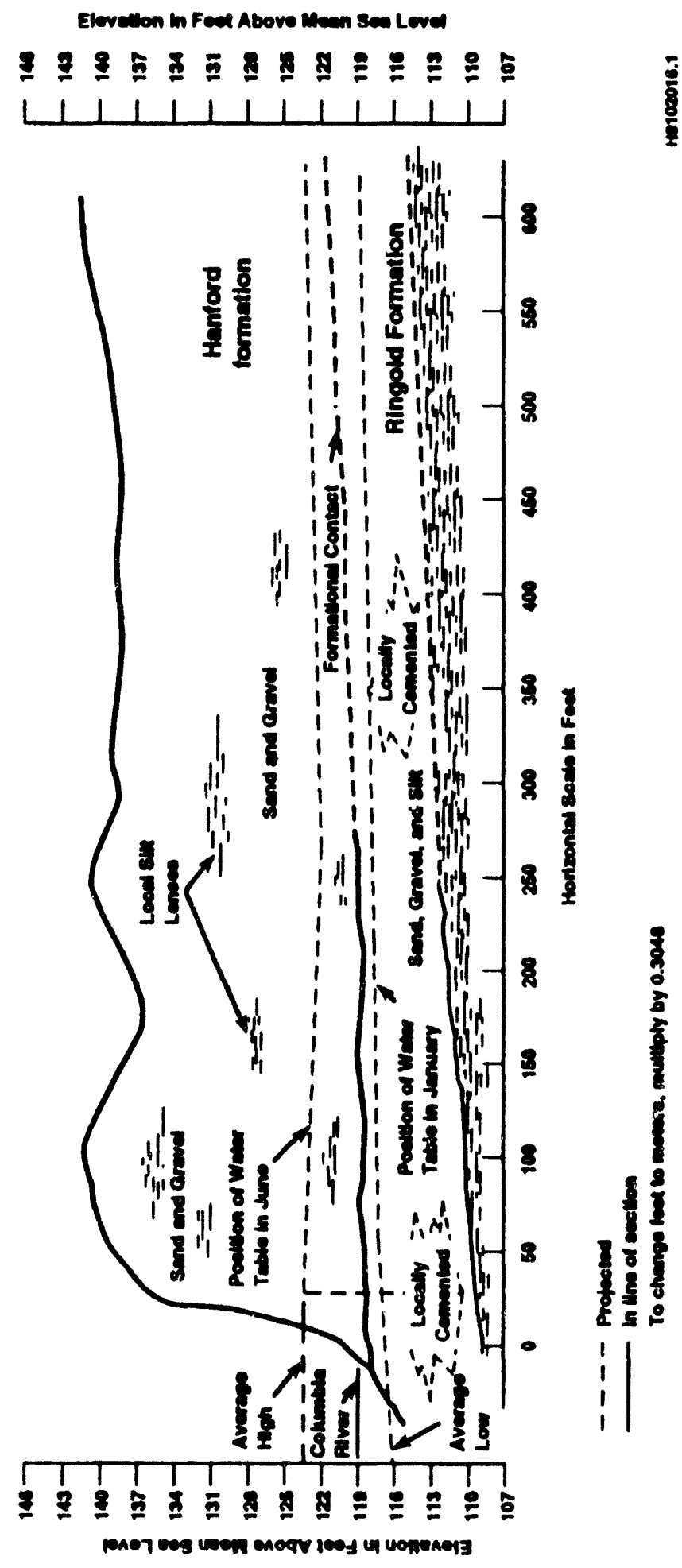




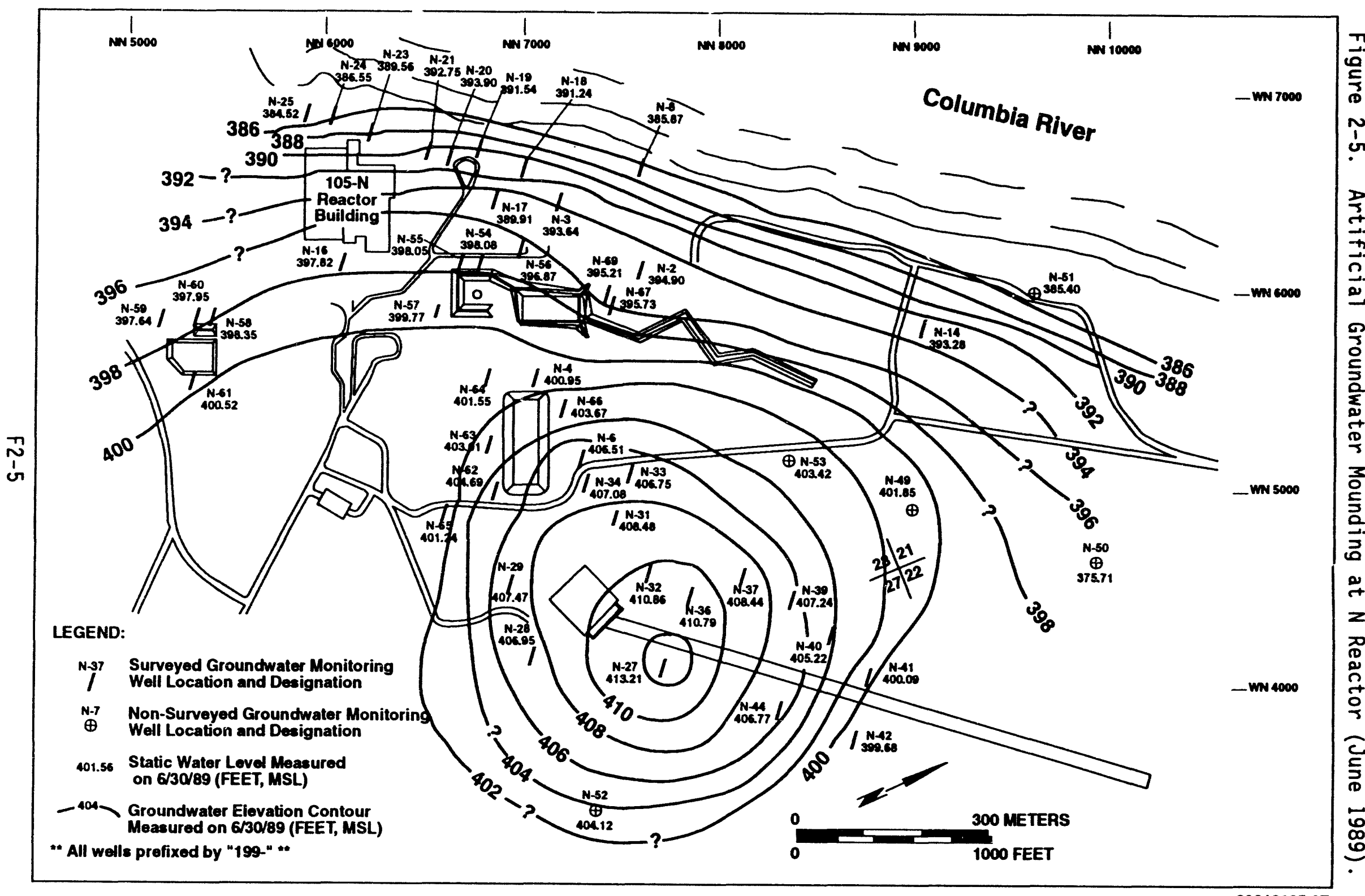




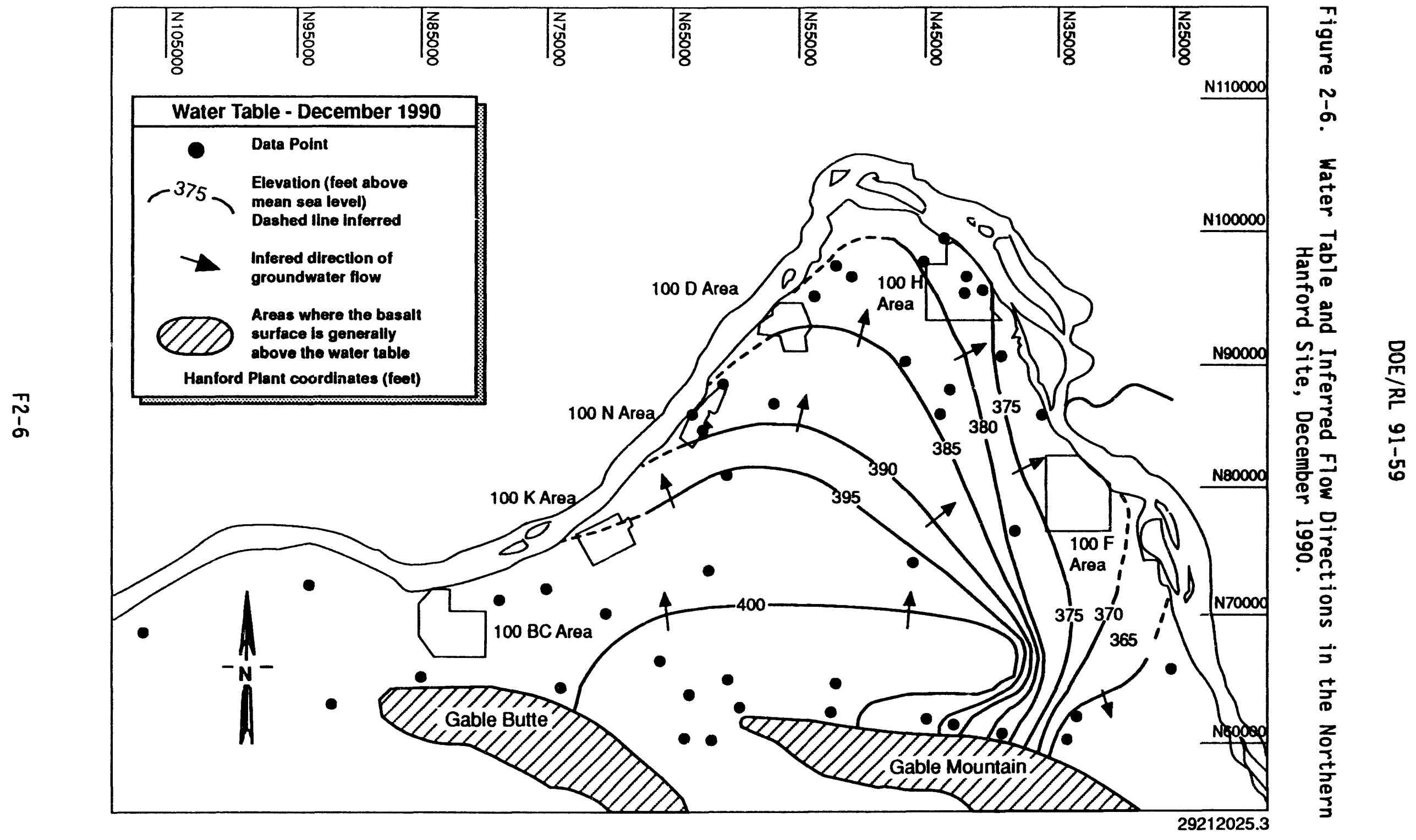


Figure 2-7. Water Table and Inferred Flow Directions in the 100-N Area and Vicinity, December 1990.

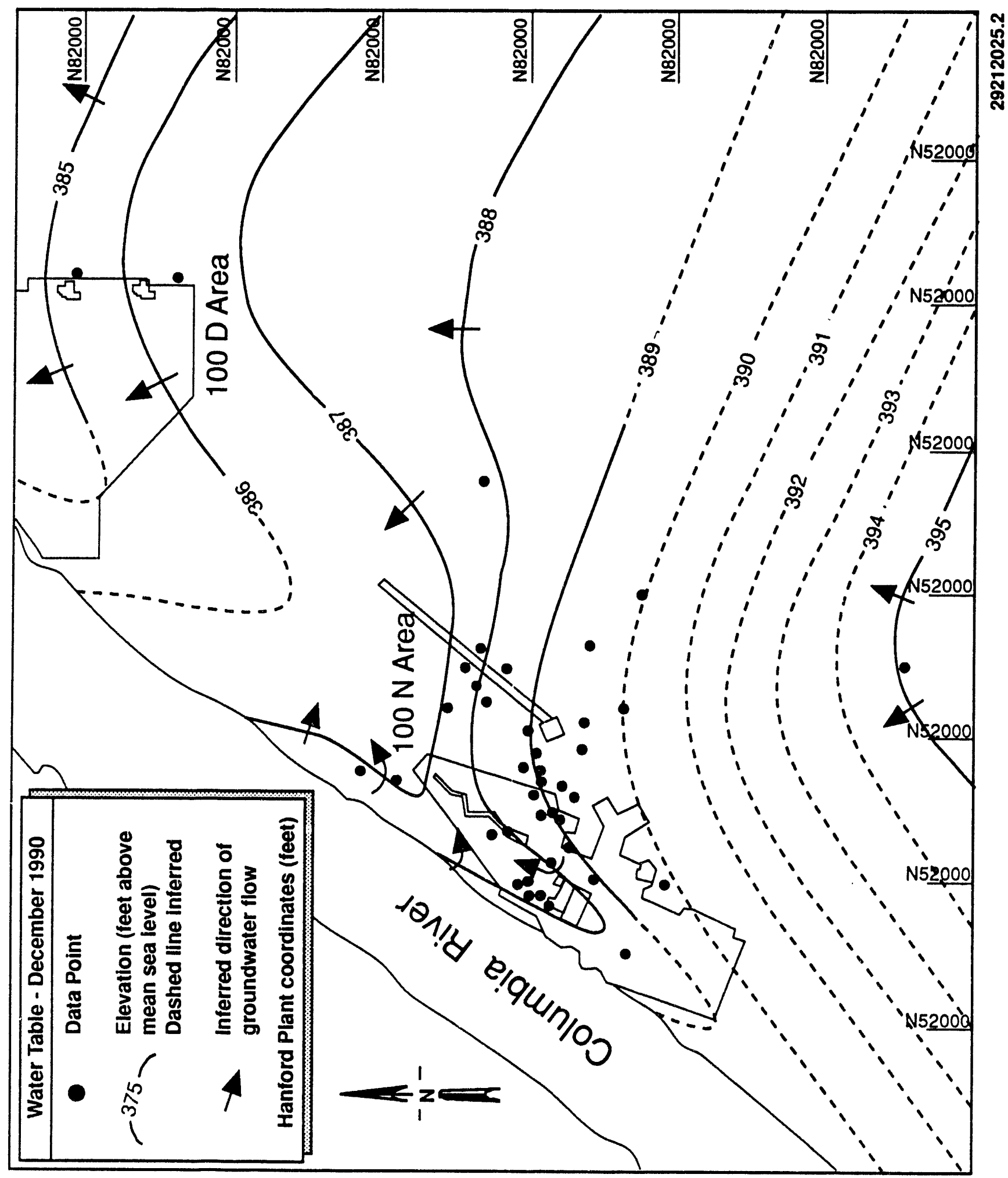


Figure 2-8. Wind Roses for the Hanford Site.

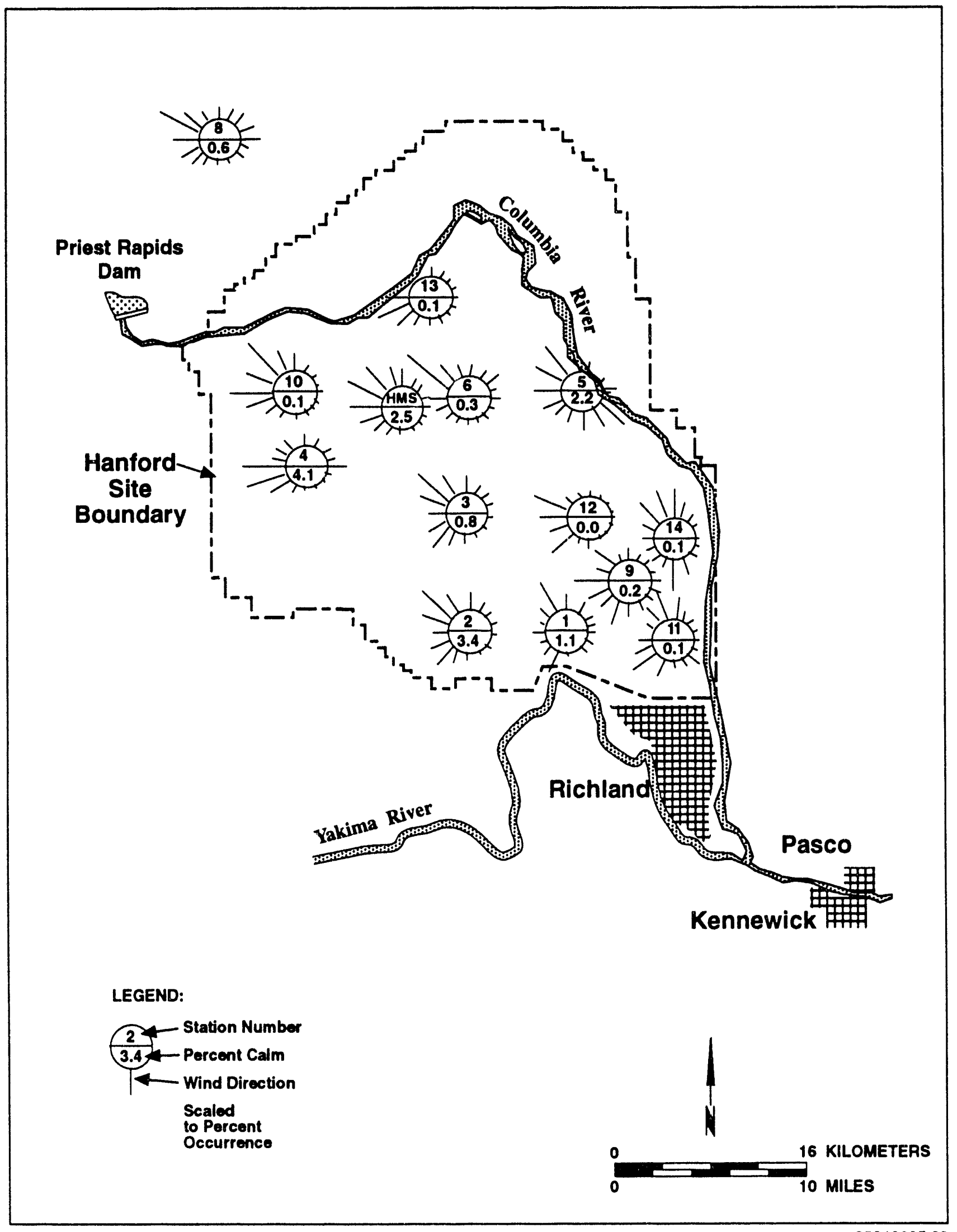


Figure 2-9. Land Use of Area Within the Hanford Site.

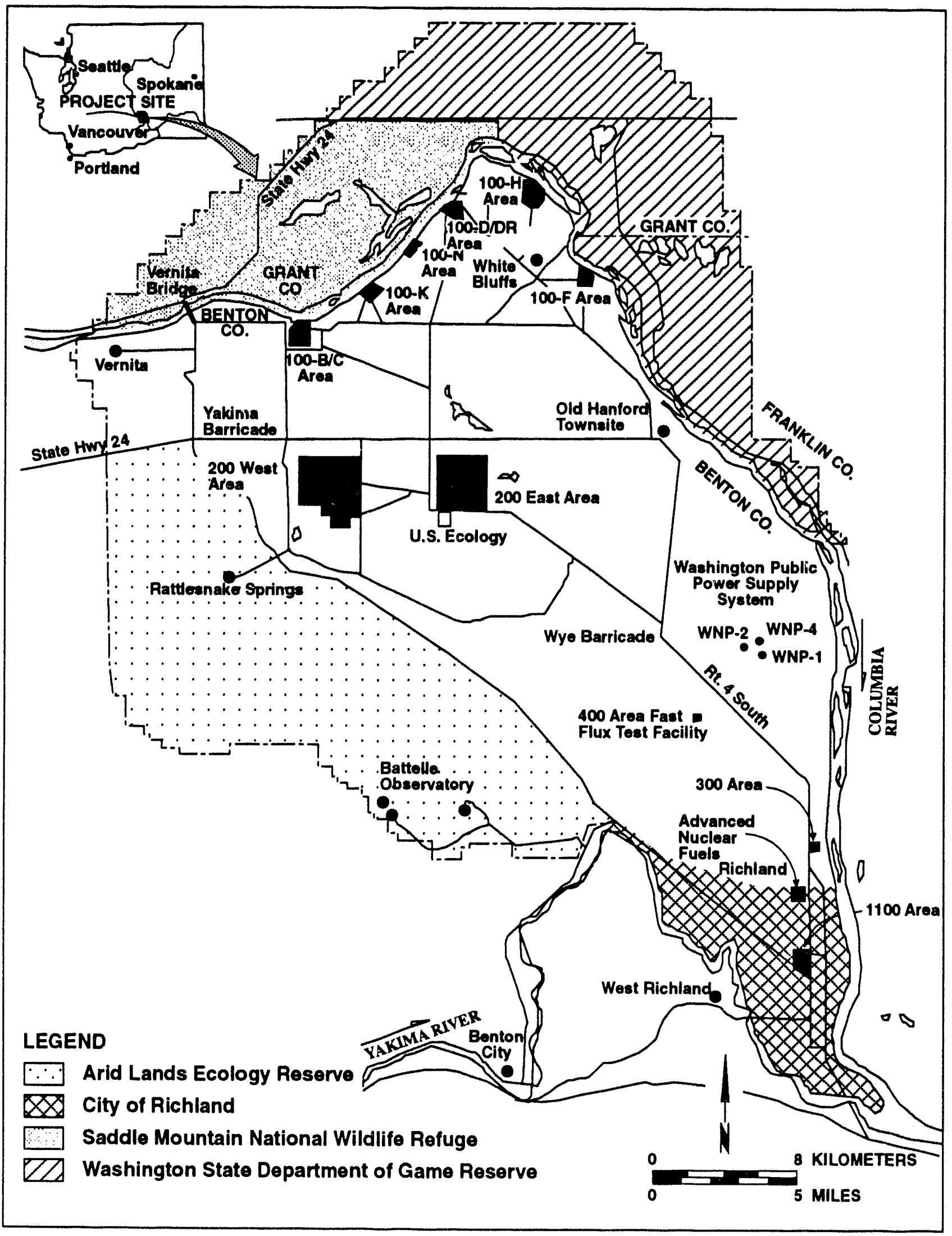


DOE/RL 91-59

This page intentionally left blank. 
Table 2-1. List of Endangered and Threatened Washington State Species Having the Potential to Occur on the Hanford Site. (2 sheets)

\section{Endangered Vascular Plants}

Persistentsepal yellowcress (Rorippa columbiae): Known to have a scattered distribution because of specialized habitat requirements or habitat loss; generally occurs in moist to marshy places and is known to inhabit the wetted shoreline of the Hanford Reach of the Columbia River in Benton County.

\section{Threatened Vascular Plants}

Columbia milk-vetch (Astragalus columbianus): Endemic to the area in the immediate vicinity of Priest Rapids Dam, including a portion of Benton County; could occur along the Columbia River in the northwestern portion of the Hanford Site.

Eatonella (Eatonella nivea): Known to occur along the Columbia River in Grant County; could occur along the river in the northern portion of the Hanford Site.

Hoover's desert parsley (Lomatium tuberosum): Endemic to south-central Washington, including Benton County; known to inhabit rocky hillsides.

\section{Endangered Birds}

Aleutian Canada goose (Branta canadensis leucopareia): Nests in the Aleutian Islands of Alaska and winters in California; has been occasionally sighted as a migrant in Benton County; a potential seasonal user of the Columbia River valley, feeding on grasses, sedges, and berries.

American white pelican (Pelecanus erythrorhynchus): Winters along the southern Pacific Coast and the Gulf Coast and nests in northern prairie and intermontane lakes; no longer nests in Washington State; migrates through eastern Washington State; flocks are common in the Columbia Basin during the summer; known to occasionally winter on the Columbia River, foraging on fish, amphibians, and crustaceans and roosting on islands.

Peregrine falcon (Falco peregrinus): Breeds and winters in eastern Washington State, inhabiting open marshes, river shorel ines, wide meadows, and farmlands; nests on undisturbed cliff faces; an erratic visitor at the Hanford Site, feeding on songbirds, shorebirds, and waterfowl. 
Table 2-1. List of Endangered and Threatened Washington State Species Having the Potential to Occur on the Hanford Site. (2 sheets)

Endangered Birds (continued)

Sandhill crane (Grus canadensis): Inhabits open prairies, grain fields, shallow lakes, marshes, and ponds, nesting in drier grassy and marshy areas; common migrant during the spring and fall in Washington State; some known and suspected nesting sites in eastern Washington State; unlikely visitor at the Hanford Site.

Upland sandpiper (Bartramia longicauda): Inhabits ungrazed and lightly grazed prairies, upland meadows, and fields that are usually located near lakes or rivers; breeds in the northern and central portions of North America and winters in South America; uncommon in eastern Washington State; a potential migratory visitor at the Hanford Site, feeding on insects, worms, and some vegetation.

Western snowy plover (Charadrius alexandrus): A coastal species rarely observed in eastern Washington State.

Threatened Birds

Bald eagle (Haliaeetus leucocephalus): A regular winter visitor to the Columbia River, feeding on spawning salmon and perhaps waterfowl and small mammals; roosting areas are known to exist in the 100 Areas of the Hanford Site (roost sites and winter feeding areas constitute critical habitats for this species).

Ferruginous hawk (Buteo regalis): Inhabits open prairies and sagebrush plains, usually with rocky outcrops or scattered trees, located well away from human disturbance; known to nest in Benton and Frankl in counties, with Frankl in County possessing the majority of the nests within Washington State; known to nest in the Hanford Site on the Arid Lands Ecology Reserve; rarely winters in Washington State; known to occasionally forage on small mammals, birds, and reptiles on sagebrush plains on the Hanford Site.

\section{Threatened Mammals}

Pygmy rabbit (Sylvilagus idahoensis): May be extinct in Washington State; inhabits undisturbed areas of sagebrush having soils soft enough in which to dig burrows; once known to exist on the Hanford Site near springs in the Snively Basin, west of the 200 Areas plateau, al though there have been no documented sightings. 


\subsection{FACILITY DESCRIPTIONS}

\subsection{INTRODUCTION}

This section describes the $N$ Reactor facility and its major processes. These facilities are summarized in Table 3-1, which includes a brief description of their use and purpose. The facilities and structures are shown in Figure 3-1. Color Illustration 3-1 is a diagram of $\mathrm{N}$ Reactor operating components, and Figures 3-2 through 3-13 show floor plans that detail the size and elevation of the facility that houses the reactor.

Two primary numbering systems were used in the 100-N Area and several buildings, structures, and waste units have two number designations. Under the original Hanford Site numbering system, buildings, structures (such as river outfalls), and some waste handling units (such as retention basins) were given a unique number (e.g., 105-N for the $N$ Reactor). Most waste units were not assigned a unique number but were instead referred to by the number of the nearby building (e.g., 163-N waste container storage pad). Under the current Hanford Site Waste Information Data System (WIDS) (DOE-RL 1991), most of the waste units and some buildings and structures were assigned site designation numbers (e.g., $116-N-8$ for the $163-N$ storage pad).

\subsection{N REACTOR}

The $N$ Reactor is a graphite-moderated, light-water-cooled, horizontalpressure-tube nuclear reactor (see Color Illustration 3-2). The reactor piping was designed for $13,000 \mathrm{kPa}\left(1,825 \mathrm{lbs} / \mathrm{in}^{2}\right)$ and $320^{\circ} \mathrm{C}\left(600^{\circ} \mathrm{F}\right)$ maximum operating conditions. Normal operating parameters were $11,000 \mathrm{kPa}$ $\left(1,600 \mathrm{lbs} / \mathrm{in}^{2}\right)$ and approximately $290^{\circ} \mathrm{C}\left(550^{\circ} \mathrm{F}\right)$. The reactor coolant circulating pumps are single-stage, horizontal, centrifugal pumps with high-pressure water-injection seals to prevent reactor coolant loss (WHC 1989a).

The $N$ Reactor was designed for two modes of operation: (1) production of SNM only and (2) production of SNM and byproduct steam used by the HGP to generate electricity. For SNM production only, steam from the secondary side of 10 steam generators was routed through 16 river-water-cooled dump condensers. Condensate from the dump condensers was routed back to steam generators for regeneration. For dual-purpose operation, byproduct steam from the steam generators was supplied to the HGP to produce 860 MW (electrical) (WHC 1989a).

For either single- or dual-purpose operation, there were 12 steam generators. During dual-purpose operation, most of the steam produced was available for use by HGP to generate electricity, with some reserved to power the reactor coolant system (RCS) pump drive turbines and the in-plant turbine generator (WHC 1989a). 
DOE/RL 91-59

\subsubsection{Confinement System}

The $N$ Reactor used a confinement system based on the concept of releasing the initial burst of steam resulting from a postulated reactor-coolant-pipe break. When the confinement pressure subsided, the steam vents were closed and ventilation valves opened. The ventilated steam was filtered through charcoal and high-efficiency filters to prevent any release of fission products from fuel fallure (WHC 1989a). Figure 3-14 11lustrates the confinement system process.

The 1312-N Liquid Effluent Retention Facility (LERF) was constructed as part of the safety enhancement program initiated in 1987. This facility served as a backup to the existing containment system and was designed to receive primary cooling water during an emergency, such as fuel failure. The LERF consists of a high-density polyethylene (HDPE) bladder contained with in a lined and bermed impoundment. The facility has never been used.

\subsubsection{Reactor Coolant System}

The portion of the RCS within the N Reactor building consists of 16 parallel lines that conducted cooling water from an inlet water manifold in the 109-N heat exchanger building to the reactor. Each of these 161 ines terminates in a vertical header that has 54 to 66 individual pressure tube header-to-inlet nozzle connectors attached. Similar outlet risers and parallel lines conducted the coolant from the pressure-tube-outlet nozzle-to-header connectors to an outlet water manifold (WHC 1989a). Figure 3-15 diagrams the RCS flow at $N$ Reactor.

In the 109- $N$ heat exchanger building, which is immediately adjacent to the $N$ Reactor building, the RCS consisted of six cells in parallel, each containing two steam generators (in parallel), a circulating pump and associated valves and instrumentation. Valves could be used to isolate piping and steam generators in each of the six cells from the main header piping (WHC 1989a).

Reactor coolant pressure and temperature were controlled to prevent boiling at any point in the system. A surge vessel (pressurizer) controlled system pressure and volume surges resulting from normal coolant density changes during reactor transient heat output conditions, The pressurizer consists of a cylindrical pressure vessel of about $34 \mathrm{~m}^{3}\left(1,200 \mathrm{ft}^{3}\right)$ useful volume directly connected to the reactor outlet piping. Two electric immersion heater systems maintained the pressurizer at saturation temperature and pressure with about 40 percent useful surge volume during normal operations. During outsurges of water from the pressurizer, flashing steam from the saturated water compensated for the drop in pressure. Increases in system pressure were limited by injecting a water spray into the steam space to condense some of the steam. This spray was reactor coolant fed from either reactor inlet water or from the high-pressure injection pumps (WHC 1989a). 
DOE/RL 91-59

\subsubsection{Nuclear Fuel System}

The $N$ Reactor fuel was slightly enriched $U(0.94$ percent to 1.25 percent), clad with a zirconium alloy. When last operated in 1986, a concentric tube-in-tube fuel design was in use for coproduction of SNM and byproduct steam. In the past, other materials have been used as a target in connection with an enriched uranium driver fuel element to produce useable isotopes such as ${ }^{3} \mathrm{H}$ and ${ }^{238} \mathrm{Pu}$. The fuel cladding is Zircaloy-2 metallurgically bonded to the uranium by a co-extrusion process. The fuel elements used in N Reactor were manufactured by United Nuclear Corporation and ranged from 38 to $66 \mathrm{~cm}$ (15 to $26 \mathrm{in}$.) long (WHC 1989a). A photograph of a fuel element is presented in Color Illustration 3-3.

\subsubsection{Heat Dissipation System}

The secondary steam system for the $N$ Reactor removed the reactor heat from the RCS by boiling secondary water in the shell side of the steam generator. During operation solely for the production of SNM, the major fraction of this steam was routed to 16 dump condensers arranged in parallel and cooled by untreated Columbia River water. These condensers operated at a pressure near that of the steam generators and el iminated the need for steam pressure reducing stations. Condensate was pumped from the dump condensers back to the steam generators for recycling. To achieve maximum single-purpose production operation, the steam temperature and pressures were maintained as low as practicable. A portion of the steam generated was used by the coolant pump drive turbines and by the turbine generator for local station service (WHC 1989a).

During dual-purpose operation, the major fraction of steam generated was routed to the HGP (Figure 3-15). A portion of the steam generated was used to drive the reactor coolant pumps and the onsite turbine generator and keep the dump condensers warm so they would be ready to accept a full steam load if an HGP turbine generator shut down (WHC 1989a).

\subsubsection{Water Supply System}

Untreated water from the Columbia River was supplied as coolant to the dump condensers, as well as to the reactor coolant pump drive turbine surface condensers and the local turbine generator condenser. This condenser cooling water was then returned to the river. Untreated water was also supplied to the water treatment facility for the filtered water, sanitary water, and demineralized water systems (see Figure 3-16). The entire untreated water system was supplied by four pumps each with a capacity of 400,000 L/min $(105,000 \mathrm{gal} / \mathrm{min})$. Water was supplied from the $181-\mathrm{N}$ river pumphouse to points of process use through two separate headers (WHC 1989a).

The 183-N filtration plant supplied the filtered and potable water needs of the 100-N Area. Filtered water was used for producing demineralized water. Raw water from the Columbia River was treated with chlorine gas (a biocide) and alum (a coagulant) in a mixing tank. From there, it was piped to a coagulator, where a polyelectrolyte was added as a coagulation aid, and then piped to the sand filters where filtration took place. The filtered water was 
pumped to the 3,200,000-L (850,000-gal) filtered-water storage tank from a clear well south of the 163-N facility (DOE-RL 1990). This tank supplied water for the following uses:

- Horizontal control rod backup cooling

- Fuel-element storage-basin cooling and cleanup

- Area service water

- Potable water system

- Demineralization plant influent (WHC 1989a).

The sand filter backwash was discharged to the 183-N filter backwash pond.

The 163-N facility (Figure 3-17) produced high-quality, demineralized makeup water from filtered river water for the major coolant systems of the $\mathrm{N}$ Reactor. Demineralized water has virtually all dissolved and suspended matter removed by ion exchange. Demineralized water was used to prevent mineral deposits that would foul piping systems and to limit the generation of radioactive waste through neutron activation of dissolved and suspended matter (DOE-RL 1990).

The 163-N facility contains demineralization equipment, including ion exchange units, regeneration tanks, treatment tanks (for $\mathrm{pH}$ adjustment) that are part of the elementary neutralization unit (ENU), acid and caustic-materials storage tanks, a heater, and a degasifier (DOE-RL 1990). The basic components of the plant and the demineralization process (Figure 3-18) are described in the following paragraphs.

Primary Cation Exchange Units. The 163-N facility has four primary cation exchange units, which are the top portions of four large tanks (or ion exchange columns). They contain ion exchange resins saturated with hydrogen ions to displace cation impurities (e.g., calcium, sodium, manganese, and iron) in the water. At the same time, the displaced cations accumulate on the resins and the resins eventually become "exhausted," losing their capacity to absorb more cations. When this occurs, the resins are sent to a regeneration tank, where they are again saturated with hydrogen ions while the cation impurities are removed and discharged to the 1324 NA percolation ponds (DOE-RL 1990).

Primary Anion Exchange Units. The four primary anion exchange units are the bottom sections of the tanks that contain the primary cation exchange units. These primary anion units contain ion exchange resins saturated with hydroxide ions. The hydroxide ions displace anion impurities (e.g., chlorides, fluorides, and sulfates) in the water. The resins eventually become exhausted in the process and require regeneration (DOE-RL 1990). When this occurs, the resins are sent to a regeneration tank where they are again saturated with hydroxide ions, while the anion impurities are removed and discharged to the 1324 NA percolation pond (DOE-RL 1990).

Degasifier. Also referred to as the deaerator, this device used heat and vacuum to remove noncondensible gases (e.g., nitrogen and oxygen) from the cation effluent water. The degasifier has two vacuum systems: a steam jet air ejector system that uses medium-pressure steam to create a vacuum and a system that consists of three vacuum pumps (DOE-RL 1990). 
Heater. Also referred to as the heat exchanger, this device used medium-pressure steam to warm up the water after it exited the primary cation units. The water was heated to reduce the solubility of gases and make the degasifier more efficient (DOE-RL 1990).

Secondary Cation and Anion Exchange Units. The four secondary cation units and four secondary anion units are in four tanks in the top and bottom sections, respectively. They are arranged in a manner similar to the primary units. The secondary units contained the same ion exchange resins as the primary units. The secondary units were to remove any impurities remaining in the water following treatment in the primary units. Because the water treated in the secondary units had already been largely deionized in the primary units, the resins in the secondary units were less exhausted and required regeneration less frequently (DOE-RL 1990).

Demineralized Water Storage Tank. This tank stored water from the 163-N facility before the water was used at $N$ Reactor. It is a $3.8 \mathrm{M}-\mathrm{L}$ (1 M-gal) -capacity tank, located with other water storage tanks southwest of the 163-N facility (DOE-RL 1990).

Regeneration Tanks. The four regeneration tanks were used to regenerate the cation and anion exchange resins when they became exhausted. The regeneration tank for the primary cation units and the regeneration tank for the primary anion units each has an upper compartment where regeneration occurred and a lower compartment where a spare resin charge was stored. The spare resin was sent to the primary units at the same time as the depleted resin charge was sent to the regeneration tank, allowing near-continuous operation of the primary units (DOE-RL 1990).

A sulfuric acid solution was used to regenerate cation resins; a sodium hydroxide solution was used to regenerate anion resins. The solutions were pumped through the resins in the regeneration tanks and drained to the 1324-N Surface Impoundment until 1988, when a spent regenerant surge tank and ENU were installed (DOE-RL 1990).

Acid and Caustic Storage Tanks. Located along the west inside wall of the 163-N facility, acid and caustic-materials storage tanks contained solutions of sulfuric acid $\left(\mathrm{H}_{2} \mathrm{SO}_{4}, 93\right.$ percent by weight) and sodium hydroxide ( $\mathrm{NaOH}, 50$ percent by weight), respectively. These solutions were used to regenerate the resins and neutralize the spent regenerant (i.e., the wastewater from regeneration). The storage tanks are surrounded by curbs for spill control. The storage tanks were filled, as needed, through below-grade pipelines that run through concrete trenches from larger tanks located at the 108-N chemical unloading facility east of the 163-N facility (DOE-RL 1990).

Spent Regenerant Surge Tank. The $454,000 \mathrm{~L}(120,000$ gal) capacity spent regenerant surge tank was installed in 1988 and is located outside on the north side of the 163-N facility. The surge tank was designed to store spent regenerant until it could be neutralized in the ENU or to function as an ENU. During normal operation, the surge tank discharged to the ENU system where the effluent stream was then neutralized and discharged to the 1324-NA (120-N-1) percolation pond (DOE-RL 1990). 
An alternative mode of operation allowed the waste stream to be neutralized in the surge tanks by recirculation and $\mathrm{pH}$ adjustment. On reaching proper $\mathrm{pH}$, the liquid was sent to the $1324 \mathrm{NA}(120-\mathrm{N}-1)$ Percolation Pond through a $20-\mathrm{cm}(8-i n$.$) chemical waste pipeline. Analyses have confirmed$ that after neutralization the effluent stream no longer exhibited the dangerous waste characteristic of corrosivity. The surge tank is surrounded by a concrete berm capable of containing the entire volume of the tank if a spil1 occurred (DOE-RL 1990).

Elementary Neutralization Unit. The ENU was installed in 1988 to replace the 1324-N Surface Impoundment. It consists of three upright tanks adjacent to the acid and caustic-materials storage tanks inside the 163-N building. Spent regenerant is piped from the surge tank to the ENU. After adding the proper amount of acid or caustic solution, the tank contents were mechanically agitated to ensure mixing and $\mathrm{pH}$ neutralization. Neutralized spent regenerant was discharged from the ENU to the 1324-NA Percolation Pond (120-N-1) when its $\mathrm{pH}$ fell within the range from 6.0 to 9.0. Spent regenerant having a $\mathrm{pH}$ outside this range was piped back to the surge tank until it could receive additional treatment in the ENU. The ENU is surrounded by a curb for spill control (DOE-RL 1990).

\subsubsection{Emergency Cooling Systems}

The emergency cooling system (ECS), an engineered safety system, provided a separate independent water system for once-through cooling of the reactor. Three diesel-driven high-lift pumps were provided to deliver the cooling water to the bottom of the inlet risers where it entered the RCS through check valves when the system pressure fell below approximately $2,000 \mathrm{kPa}$

$\left(300 \mathrm{lbs} / \mathrm{in}^{2}\right)$. The high-lift pumps were initially supplied with treated water from the demineralized water storage tank, which was maintained with a minimum of $1,700,000 \mathrm{~L}(452,000 \mathrm{gal})$ for ECS use only. If this supply was depleted, untreated river water was automatically supplied from two diesel-driven pumps located in a separate section of the river pumphouse (WHC 1989a).

A similar diesel-driven pumping system provided emergency once-through couling to the graphite moderator and shields. This coolant provided backup to the ECS in the event of long-term failure of the ECS (WHC 1989a).

Both ECSs were intended for use only if the normal circulating-water heat-removal systems were disabled. Activation of the ECSs would be triggered by loss of all normal pumping power caused by a major break in the RCS, which would result in depressurizing the RCS (WHC 1989a).

\subsubsection{Process Decontamination}

A process decontamination system used phosphoric acid for chemical decontamination of the entire RCS or for any of several major portions of the system, including the individual heat exchangers. The graphite and shield cooling system could also be chemically decontaminated. Equipment was included for storing and preparing the necessary chemicals and piping for injecting them at appropriate points. Mixed wastes from decontamination, 
along with rinse waters, were routed to the 1310-N (116-N-2) storage tank, then shipped by tank truck or rail car to the 200 Area for disposal

(WHC 1989a).

\subsubsection{Plant Service Boilers}

The onsite plant service boilers provided steam for the reactor coolant pump drive turbines and for the onsite turbine generator as a backup power supply (WHC 1989a).

One boiler was housed in the 184-N plant service powerhouse and two boilers were housed in the 184- $N$ building annex. During reactor operation, the boilers were maintained in a condition capable of being brought on 1 ine to carry the shutdown load. These boilers could supply approximately $150,000 \mathrm{~kg}$ $(340,000 \mathrm{lb})$ of steam per hour (WHC 1989a).

Fuel for the boilers was piped from the 166-N tank farm to the 184-N day tanks, located north of the 184-N building. Two 130,000-L (35,000-gal) No. 6 fuel $0 i 1$ tanks and one 30,000-L (8,000-gai) No. 2 diesel oil tank provided fuel to the boilers. Underground piping connects the 166-N tank farm, the :84- $\mathrm{N}$ day tanks, and the 184- $\mathrm{N}$ boilers. These tanks are empty.

\subsubsection{Diesel Fuel Storage and Transfer System}

The diesel fuel oil storage and transfer system supplied diesel oil to the seven ECS and fog spray diesel engines, the 182-N high lift pumphouse, the diesel fire pump, and the diesel-driven electrical generator located in the 182-N building. The 105-N 1 ift station sump pump diesel engine had an independent underground storage tank. The total storage capacity of the diesel oil storage system is $1,800,000 \mathrm{~L}(465,700 \mathrm{gal})$, consisting of four $400,000-\mathrm{L}(105,000-\mathrm{gal})$ bulk storage tanks and 5 day tanks (three $60,000 \mathrm{~L}$ [15,000 gal], which have been removed, and two $1,400 \mathrm{~L}$ [360 gal]). The unloading station for the four diesel bulk storage tanks is located west of the 166-N building adjacent to the No. 6 fuel oil unloading station at the 166-N tank farm (WHC 1989a). These tanks are empty.

\subsubsection{Boiler Fuel 0il System}

The boiler fuel oil system supplied No. 6 fuel oil to the plant service boilers. The total capacity of the system is $5,500,000 \mathrm{~L}(1,445,000 \mathrm{gal})$. The single bulk storage tank volume is $5,200,000 \mathrm{~L}(1,375,000 \mathrm{gal})$; each of the two day tanks contained $130,000 \mathrm{~L}(35,000 \mathrm{gal})$ of fue $10 \mathrm{il}$. The unloading station for the bulk fuel oil storage tank is located west of the 166-N building adjacent to the diesel oil system unloading station. This unloading system is a concrete trench containing six tank car connections and four tank truck connections (WHC 1989a). These tanks are empty except for some residual oil in the bottom of the single bulk storage tank. 
DOE/RL 91-59

\subsection{WASTE-gENERATING PROCESSES}

Radioactive and hazardous wastes were generated in various processes that supported $\mathrm{N}$ Reactor. In some cases, wastes were considered both hazardous and radioactive and were referred to as mixed wastes. The main waste-generating processes are discussed in the following subsections. The waste streams are summarized in Table 3-2.

\subsubsection{Radioactive Effluents and Wastes}

Radioactive effluents and wastes were generated in a variety of process systems. These radioactive wastes included reactor primary coolant water, spent fuel storage basin cooling water, reactor periphery systems cooling water, reactor primary-coolant-loop decontamination and rinse solution, and miscellaneous drainage from reactor support facilities (WHC 1987a).

Primarily, radioactive effluents and wastes were generated within the 105-N reactor building and the 109-N heat transfer building. The radioactive process effluent and waste streams ultimately were sent to either the $116-\mathrm{N}-1 \mathrm{crib}$ and trench, the 116-N-3 crib and trench, or the 1314-N Iiquid waste loadout station (LWLS).

\subsubsection{Reactor Primary Coolant System}

The reactor primary coolant system was supplied by demineralized water from the 163-N demineralization plant. Ammonium hydroxide and hydrazine were introduced into the primary coolant system to control water $\mathrm{pH}$ and oxygen, respectively. Normal operation of the reactor primary coolant system resulted in approximately $760 \mathrm{~L} / \mathrm{min}(200 \mathrm{gal} / \mathrm{min})$ of bleed off and leakage, which were discharged to the 116-N-1 and/or the 116-N-3 crib and trench via the $91-\mathrm{cm}$ (36-in.) radioactive drainline (WHC 1987a). Analysis of primary coolant wastewater in 1985 indicated that it did not exhibit any of the characteristics of a regulated dangerous waste (WHC 1987a) per waste stream designation based on dangerous waste regulations contained in Washington Administrative Code (WAC) 173-303.

\subsubsection{Spent Fuel Storage Basin Cooling Water System}

Until December 1984 , essentially all the ${ }^{90} \mathrm{Sr}$ and ${ }^{137} \mathrm{Cs}$ discharged to the $116-N-1$ crib originated in the $N$ Reactor spent fuel storage basin. The spent fuel storage basin operated in a single-pass cooling mode from the time of initial $N$ Reactor operation until a recirculation system was installed in December 1984 (DOE-RL 1990).

The single-pass cooling system operated on the basis that every 6 weeks, $N$ Reactor fuel elements were discharged to the spent fuel basin via a large tunnel-like canal located at the outlet face of the $N$ Reactor. During this transfer process, a large quantity of reactor primary-cooling-circuit water, containing considerable amounts of suspended and soluble metals and metal oxides was added to the spent fuel storage basin. Excess basin water was routed to the $116-\mathrm{N}-1$ and/or $116-\mathrm{N}-3$ cribs via the basin overflow weirs and a 
91-cm (36-in.) drainline (WHC 1987a). Between reactor fuel element transfers, the spent-fuel-basin water level was maintained by adding treated water from the 183-N filtration plant (DOE-RL 1990).

Starting in December 1984, the 107-N spent fuel storage basin cooling water recirculation system was operated. The 107-N basin recirculation facility cleaned $\mathrm{N}$ Basin in a closed-10op system. Mixed wastes were routed to the 190,000-L (50,000-gal) tank, where they were neutralized and then pumped to the 1314-N LWLS (DOE-RL 1990).

\subsubsection{Reactor Periphery Cooling Systems}

Three reactor periphery cooling systems, the graphite and shield cooling system, the reactor control rod cooling system, and the reactor secondary coolant loop system, were closed systems using demineralized water from the 163-N demineralization plant. As with the primary coolant system, bleed off and spillage from the periphery cooling systems resulted in small continuous discharges to the $116-N-1$ (1301-N) and/or $116-N-3$ (1325-N) liquid waste disposal facilities. Periphery cooling water also had a variety of chemicals added, including ammonium hydroxide and morpholine for $\mathrm{pH}$ control and hydrazine for oxygen control.

\subsubsection{Reactor Primary Coolant Loop Decontamination System}

Approximately every 3 to 5 years, the reactor primary coolant loop was decontaminated with a 70 percent phosphoric acid/diethylthiourea solution diluted to an 8 percent solution by weight. This solution was mixed in the 109-N mix tank, then piped to the RCS manifold in the $N$ Reactor. From the manifold, the solution entered the primary piping through the $V-3$ valves. The decontamination solution removed the radioactive oxides (containing activation and fission products) that had built up as residues in the piping. The solution exited the primary coolant loop through the $V-4$ valves and was then routed to the $116-\mathrm{N}-2(1310-\mathrm{N})$ radioactive chemical waste storage facility tank (DOE-RL 1990). Decontamination solution and subsequent rinsate were pumped into this tank. This volume was approximately $2,300,000 \mathrm{~L}$ $(600,000 \mathrm{gal})$ of mixed waste. Another $380,000 \mathrm{~L}(100,000 \mathrm{gal})$ of rinsate was pumped through the primary cooling system and discharged to $116-\mathrm{N}-1$ and/or 116-N-3 (WHC 1987a). The acidic decontamination solution in the 116-N-2 storage tank was neutralized with sodium hydroxide to a pH greater than 7.0 and pumped to the 1314-N LWLS. Before 1987, the $\mathrm{pH}$ was required to be between 9.0 and 11.0. From the 116-N-2 tank the waste solution was sent to the 200 Area via rail tank car for disposal in accordance with the Hanford Site Radioactive Solid Waste Acceptance Criteria (Willis and Tiner 1991).

\subsubsection{Drainage From Reactor Support Facilities}

The radioactive drain system is a network of floor drains that collected radioactive water from throughout the $N$ Reactor building and 109-N building. In addition to pump leakage and system bleed off from reactor primary and 
periphery cooling systems, spills and waste streams may also have originated at several locations (WHC 1987a,b; DOE-RL 1990), including the following:

- Five wet laboratories that performed chemical analyses on cooling water to $116-\mathrm{N}-1$ and/or $116-\mathrm{N}-3$

- Auxiliary power battery lockers (potential spills) to $116-\mathrm{N}-1$ and/or $116-\mathrm{N}-3$

- Hydrazine mixing and injection area to the Columbia River

- Room 11 decontamination station to $116-\mathrm{N}-2$

- Room 307 decontamination station to $116-\mathrm{N}-2$

- Room 191 decontamination station to 116-N-2.

\subsubsection{Water Treatment and Demineralization Plants}

The configuration of the 163-N mineralization plant regeneration waste stream that existed before facility shutdown is shown in Figure 3-17. Before 1977, the nonneutralized spent regenerant was discharged from the 163-N facility to the Columbia River, as was common industry practice at the time. The resulting dilution was relied on to mitigate the corrosive nature of this wastewater. Beginning in 1977, spent regenerant from the 163-N facility was discharged to the unlined 120-N-1 (1324-NA) percolation pond. The alternate addition of acidic cation regenerant and alkaline anion regenerant served to neutralize the $\mathrm{pH}$ of the pond contents over time. In addition, the buffering capacity of the calcareous soil underlying the pond assisted the neutralizing process. Waste from the 163-N facility was treated in situ at the 120-N-1 percolation pond by a combination of pH neutralization and eventual percolation or evaporation of the wastewater (DOE-RL 1990).

Since early 1986, the 120-N-1 (1324-NA) percolation pond has received only neutralized wastewater because construction of the lined $120-\mathrm{N}-2$ surface impoundment in that year provided a means of neutralizing and mixing the spent regenerant (in batches from successive regenerations) from the 163-N facility before discharging it to $120-\mathrm{N}-1$. The neutralizing process involves treating individual batches with either sulfuric acid or sodium hydroxide, as appropriate. From $120-\mathrm{N}-2$, the neutralized wastewater was then piped to the $120-N-1$ for disposal (DOE-RL 1990).

In November 1988 use of the 120-N-2 (1324-N) surface impoundment was discontinued when the newly constructed ENU was put on line inside the 163-N facility. The ENU neutralizes the spent regenerant before it is discharged to the 120-N-1 percolation pond, and does so with greater efficiency and operator control than was possible in the $120-N-2$ facility (DOE-RL 1990). 
DOE/RL 91-59

\subsection{RESOURCE CONSERVATION AND RECOVERY ACT FACILITIES IN THE 100-N AREA}

Table 3-3 lists the active or recently active TSD units in the 100-N Area that must be operated, permitted, and/or closed in compliance with RCRA and the State dangerous waste program regulations identified in Appendix $B$ of the Tri-Party Agreement Action Plan within the 100-NR-1 Operable Unit (DOE-RL 1990).

The 100-N Area TSD units historically received hazardous or dangerous wastes, but are either out of service or have stopped receiving such wastes. The 100-N Area TSD units will be closed in accordance with the State dangerous waste program as specified in WAC 173-303-610 (DOE-RL 1991). Draft closure plans have already been prepared for these units, but are scheduled to be submitted to Ecology in 1994 in accordance with the Tri-Party Agreement (Ecology et al. 1992).

\subsubsection{1-N (116-N-1) Liquid Waste Disposal Facility}

The 1301-N Liquid Effluent Disposal Facility (LWDF), shown in Figure 3-19, was the primary receiving facility for $N$ Reactor effluent primarily contaminated with corrosion products and fission products. From the operations start of $N$ Reactor in 1963 until September 1985, the 1301-N LWDF was the primary liquid radioactive waste disposal facility for the $N$ Reactor. The nominal effluent flow rate was $6,435 \mathrm{~L} / \mathrm{min}(1,700 \mathrm{gal} / \mathrm{min})$. Wastes disposed of in the 1301-N LWDF consisted of RCS bleed off, spent fuel storage basin bleed off, periphery cooling systems bleed off, reactor primary coolant loop decontamination rinse solution, and discharges from building drains containing radioactive wastes generated in reactor support facilities. This effluent was contaminated chiefly with corrosion products and fission products.

The 1301-N LWDF is an inactive land disposal facility that used the filtration properties of soil to remove radioactive material from effluent water. Radioactive materials included in the discharged wastes were retained in the soil by the filtration, absorption, and ion exchange that occurred in the soil column underlying the 1301-N LWDF (DOE-RL 1990).

The facility is located approximately $18 \mathrm{~m}(60 \mathrm{ft})$ above and $262 \mathrm{~m}$ $(860 \mathrm{ft})$ east of the shore of the Columbia River, see Color Illustration 3-4. The original facility consisted of a $16-\mathrm{m}$ by $3.5-\mathrm{m}(52-\mathrm{ft}$ by $12-\mathrm{ft}$ ) concrete trough (weir box) and a $38-\mathrm{m}$ by $88-\mathrm{m}$ (125-ft by 290-ft) rectangular basin (crib). The crib was constructed by excavating existing soil and then surrounding the excavation with a soil and gravel embankment. The bottom of the crib was filled with a $1-m(3-f t)$ layer of large stones (WHC 1987b).

After only a few years of reactor operation, an extension trench was added to the crib. The trench is an excavated ditch approximately $487 \mathrm{~m}$ long, $15 \mathrm{~m}$ wide, and $3.5 \mathrm{~m}$ deep $(1,600 \mathrm{ft}$ long, $50 \mathrm{ft}$ wide, and $12 \mathrm{ft}$ deep), extending in a zigzag pattern. In 1982, the trench was covered with precast concrete panels to prevent animals from coming in contact with the wastes (WHC 1987b). 
The radionuclide inventory under 1301-N LWDF has been estimated to $6,800 \mathrm{Ci}$ based on radiological analyses of the effluent stream. Present radiation dose rates on the surface of the facility range from $10 \mathrm{mrem}$ to $1,000 \mathrm{mrem}$ (WHC 1987b).

This facility was replaced in 1985 by the 1325-N LWDF because elevated levels of radionuclides were released via the N-Springs (DOE-RL 1987a).

\subsubsection{5-N Liquid Waste Disposal Facility}

The 1325-N LWDF, shown in Figure 3-20, started receiving part of the $N$ Reactor liquid radioactive effluent in 1985. In September 1985, the 1325-N LWDF became the primary liquid radioactive waste disposal facility for the $\mathrm{N}$ Reactor. The nominal effluent flow rate during reactor operation was $6,345 \mathrm{~L} / \mathrm{min}(1,700 \mathrm{gal} / \mathrm{min})$ and is now 1 ess than $7.5 \mathrm{~L} / \mathrm{min}(2 \mathrm{gal} / \mathrm{min})$. Wastes disposed of in the 1325-N LWDF consist of RCS bleed off, spent fuel storage basin bleed off, periphery cooling systems bleed off, reactor primary coolant loop decontamination rinse solution, and discharges from building drains containing radioactive wastes generated in reactor support facilities. Hazardous wastes generated by laboratories, decontamination activities, and other routine activities at $N$ Reactor were discharged to the building drains. Through laboratory waste collection, additional secondary containment, and application of administrative controls, hazardous wastes are no longer discharged to the 1325-N LWDF (WHC 1987a).

The 1325-N LWDF is a land-disposal facility that also makes use of the filtration properties of soil to remove radioactive material from effluent water. Radioactive materials included in the discharged wastes are retained by the soil and the filtration, absorption, and ion exchange that occur in the soil column underlying the 1325-N LWDF (DOE-RL 1990).

The facility is located approximately $18 \mathrm{~m}(60 \mathrm{ft})$ above and $732 \mathrm{~m}$ $(2,400 \mathrm{ft}$ ) east of the shore of the Columbia River (see Color Illustration 3-4). The original facility consisted of a reinforced concrete header box and a $73-\mathrm{m}$ by $76-\mathrm{m}$ (240-ft by $250-\mathrm{ft}$ ) rectangular basin (crib). The crib was constructed by excavating existing soil and then covered with precast, prestressed concrete panels sealed with grout. The crib cover is approximately $3 \mathrm{~m}(10 \mathrm{ft})$ lower than the surrounding ground and less than $1.5 \mathrm{~m}(5 \mathrm{ft}$ ) above the percolation surface (WHC 1987a).

In 1985, after 2 years of operation, an extension trench was added to the $1325-\mathrm{N}$ crib because it was unable to handle the flow rate of the original design. The trench is an excavated ditch approximately $914 \mathrm{~m}$ long, $17 \mathrm{~m}$ wide, and $2 \mathrm{~m}$ deep $(3,000 \mathrm{ft}$ long, $55 \mathrm{ft}$ wide, and $7 \mathrm{ft}$ deep). The trench ties into the crib at two points (from the crib's northern and eastern corners) with the effluent from each combining in a common weir box. The tie-in is composed of rubber gasket-joined, precast, reinforced, concrete box sections. The trench was covered with precast concrete panels that were placed close together, left unsealed, and contain lifting lugs to facilitate panel removal. The $81-\mathrm{cm}$ $(32-i n$.$) wide centra-core concrete panels were placed (unsealed) along the$ side of the trench, mating with the trench cover panels. The sides of the trench were then backfilled (WHC 1987a). 
The 2-m $(7-\mathrm{ft})$ deep trench is divided into 4 equal sections by 3 dams (in addition to the weir box) composed of structural fill and concrete. A 1 ayer of riprap was added to the downstream side of each dam to prevent scouring. The top $60 \mathrm{~cm}$ ( $24 \mathrm{in.}$ ) of the trench bottom were screened to remove fines to aid in preventing trench plugging. effluent.

Only the first of the four trench sections have received $\mathrm{N}$ Reactor

\subsubsection{4-N/NA Surface Impoundment/Percolation Pond}

The 1324-NA Percolation Pond, shown in Figure 3-21, was constructed in 1977 to receive neutralized regeneration waste water initially from the north and south settling ponds. In 1986 this pond received waste from the 1324-N Surface Impoundment, which was replaced by an ENU in 1988. The 1324-NA is an unlined excavation $82 \mathrm{~m}$ by $24 \mathrm{~m}$ by $4.5 \mathrm{~m}$ deep ( $270 \mathrm{ft}$ by $80 \mathrm{ft}$ by $15 \mathrm{ft}$ deep) (WHC 1986).

The 1324-N Surface Impoundment shown in Figures 3-21, 3-22, and 3-23 was constructed in 1986 and was used to neutralize corrosive ion exchange regeneration waste water from the 163-N Demineralization Facility. The surface impoundment is a double-lined pond with leak-detection capability between the two liners. The surface impoundment is situated over a previously used unlined north settling pond, Figure 3-24, that had received unneutralized regeneration waste water from the 163-N Demineralization Facility from 1977 to 1986. The 1324-N Surface Impoundment is $23 \mathrm{~m}$ by $43 \mathrm{~m}$ by $4.5 \mathrm{~m}$ deep (75 ft by $140 \mathrm{ft}$ by $15 \mathrm{ft}$ deep).

\subsection{OTHER FACILITIES}

\subsubsection{Hanford Generating Plant}

The HGP, owned by the Supply System, received steam via the steam piping system from the $N$ Reactor. The HGP consists of two 430-MW (electrical) low-pressure turbine generator systems with associated auxiliary equipment normally found in a steam power station. The HGP is operated by the Supply system. The HGP condensers and auxiliary cooling systems were supplied by raw water pumped from the Columbia River and discharged back to the river approximately $90 \mathrm{~m}(300 \mathrm{ft})$ upstream from the $\mathrm{N}$ Reactor raw water intake structure.

\subsubsection{Bonneville Power Administration Substation}

The BPA Hanford switching substation was built in 1968 to supply electrical power produced by the HGP to the Supply System's main electrical grid. The substation consists of a switch yard and control house on approximately $0.1 \mathrm{~km}^{2}$ (30 acres). The control house contains the communication equipment used to run the substation remotely. The control house is also used to store maintenance equipment. 


\subsubsection{D Area Support Facilities}

Three interconnected buildings at the deactivated $D$ Reactor were used to support $\mathrm{N}$ Reactor. parts.

185-D Building. This building was a warehouse for equipment and spare

189-D Building. An engineering laboratory was operated in the 189-D building to support $N$ Reactor when it was operating.

190-DR Building. This building was a warehouse for equipment and spare parts.

\subsubsection{C Area Support Facilities}

190-C Building. The 117-N filter building used charcoal filters to absorb airborne radioactive materials emitted from $N$ Reactor. The filters were transported to the 190-C building where the spent charcoal was removed and taken to the 200 East burial ground. New charcoal was then added and the filter was placed back in the 117-N building. 


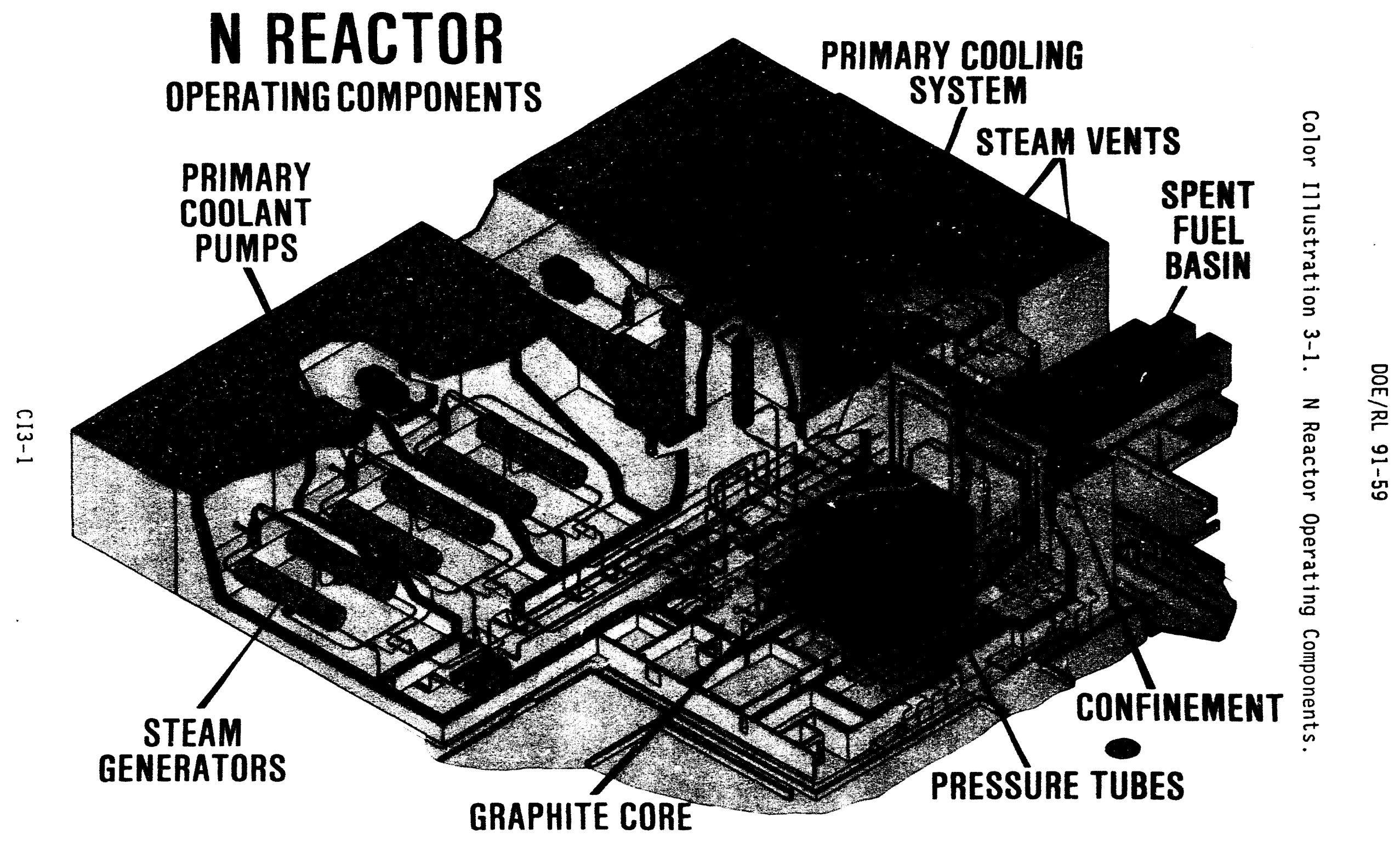


Color Illustration 3-2. Reactor Core.

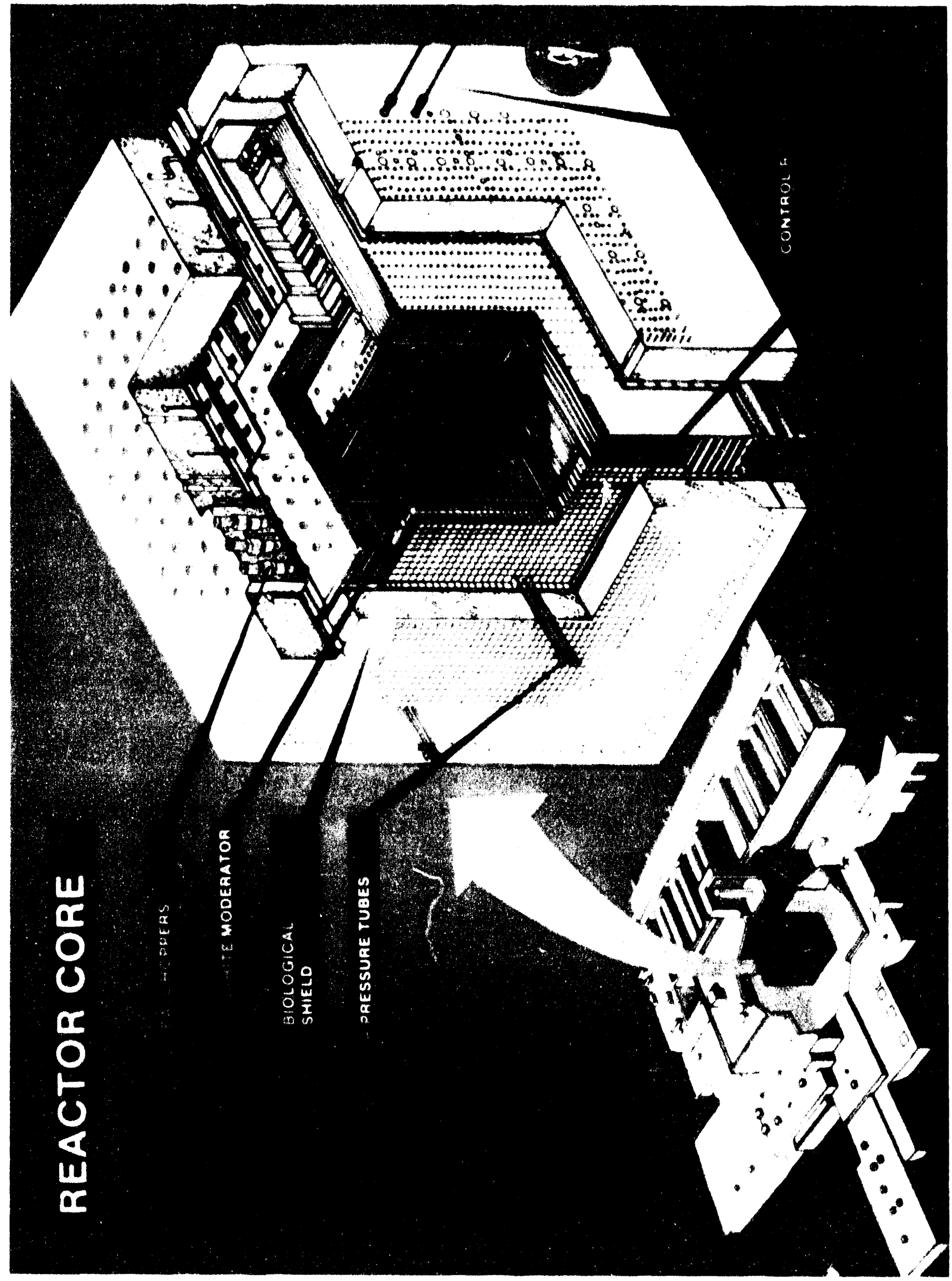


DOE/RL 91-59

Color Illustration 3-3. N Reactor Fuel.

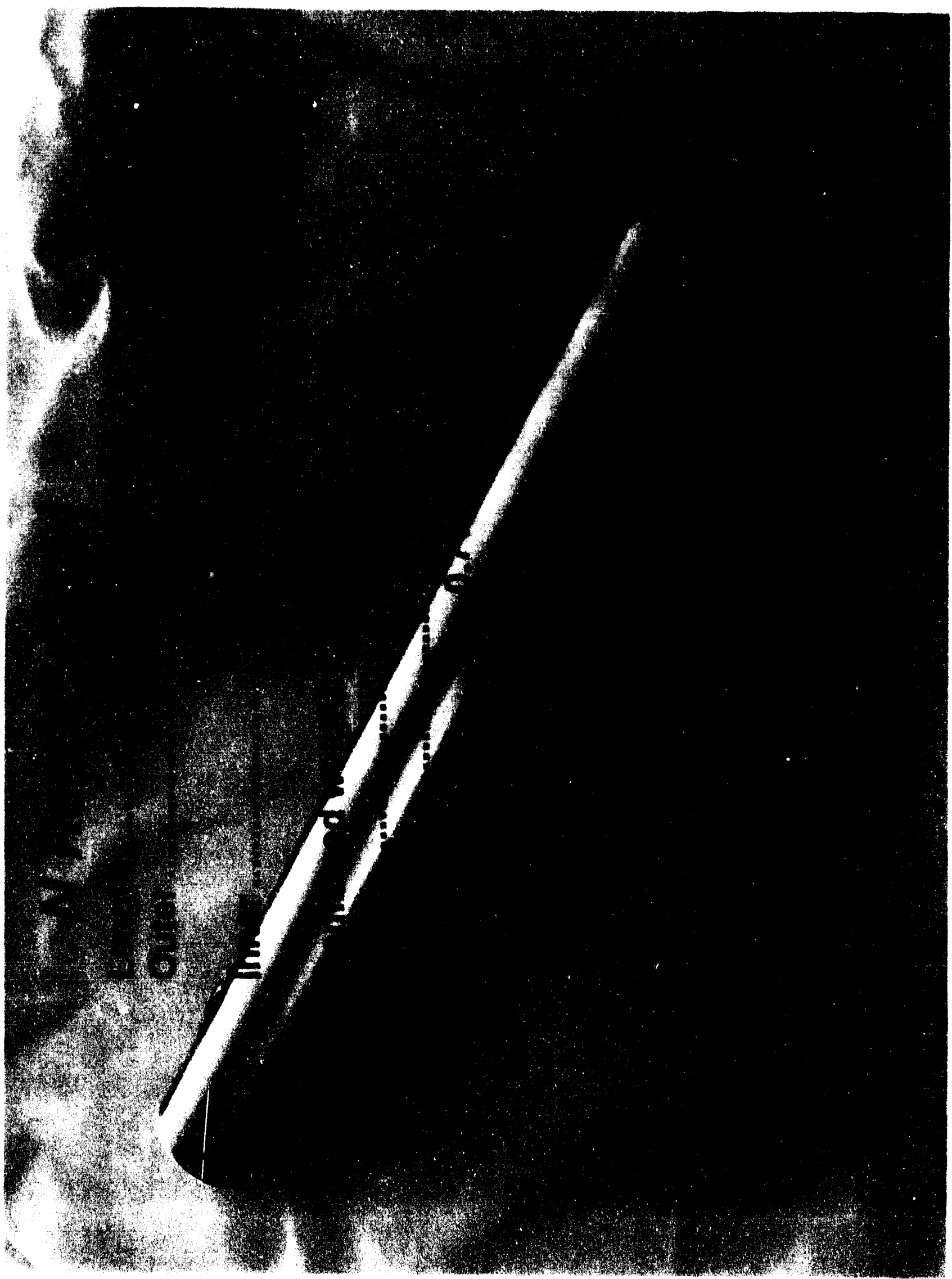


Color Illustration 3-4. Aerial View of the $1301-\mathrm{N}$ and $1325-\mathrm{N}$ Waste Disposal Facilities.

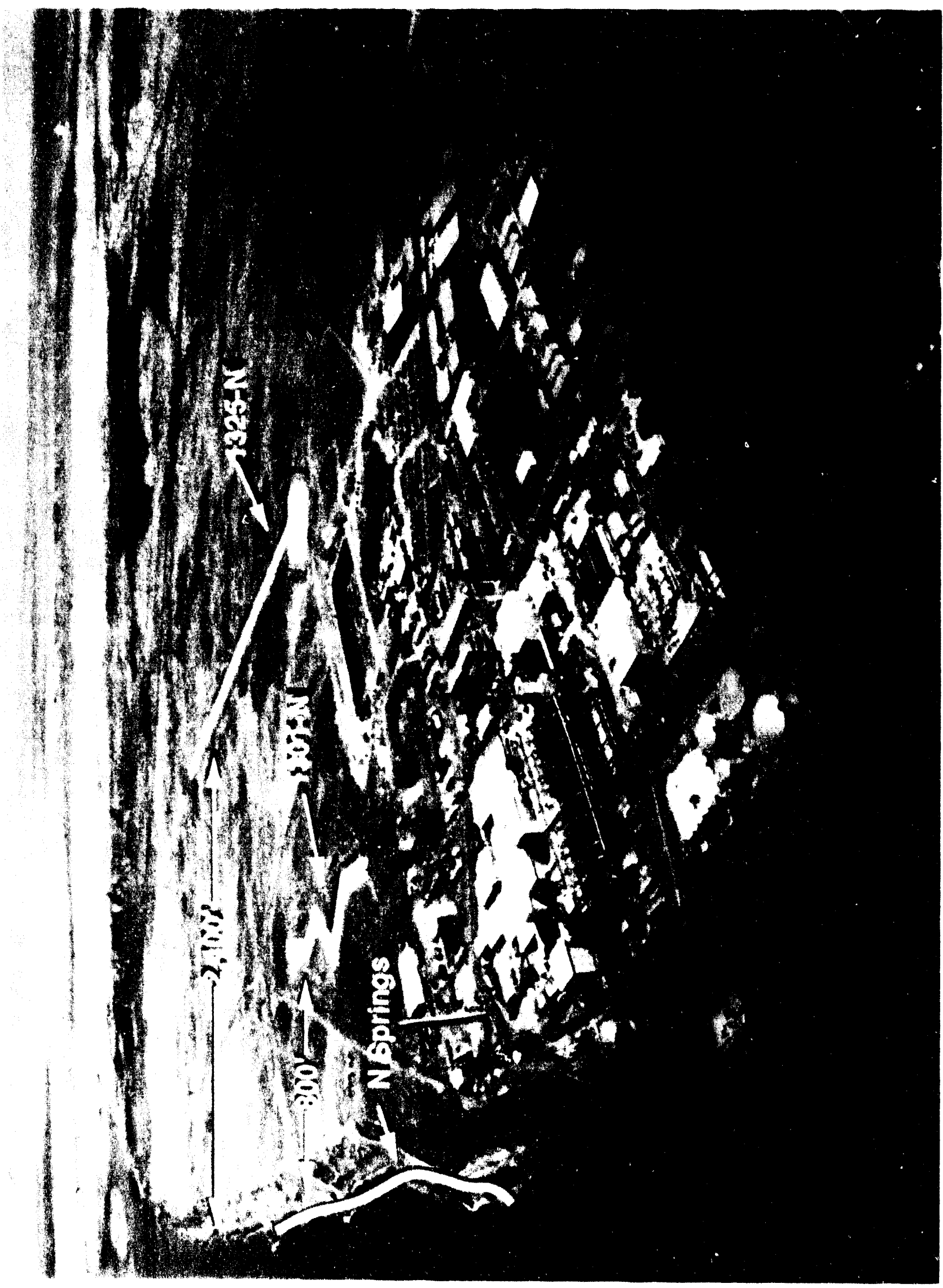




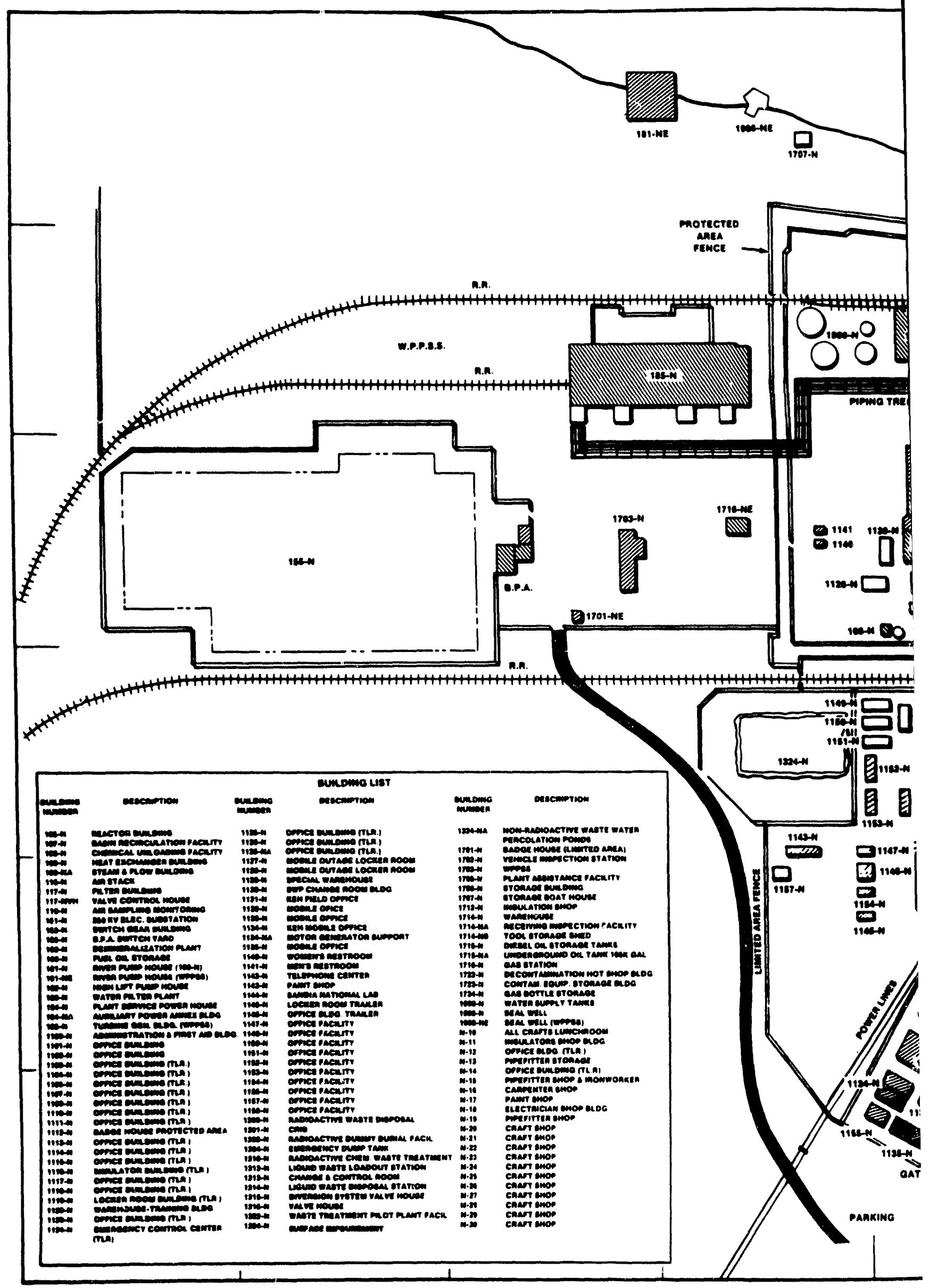


DOE/RL $91 / 59$

Figure 3-1. 100-N Area

Facilities and Structures

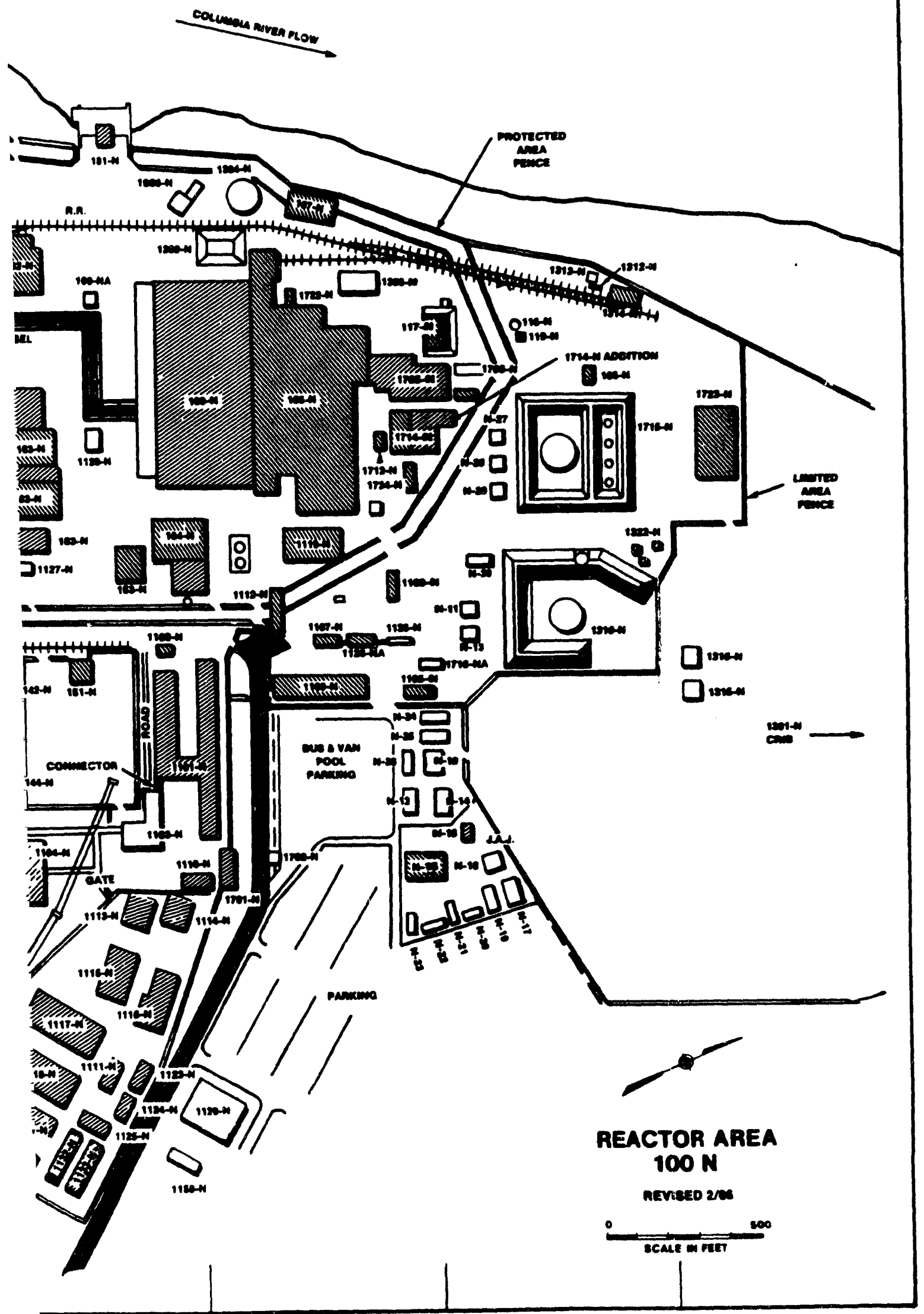




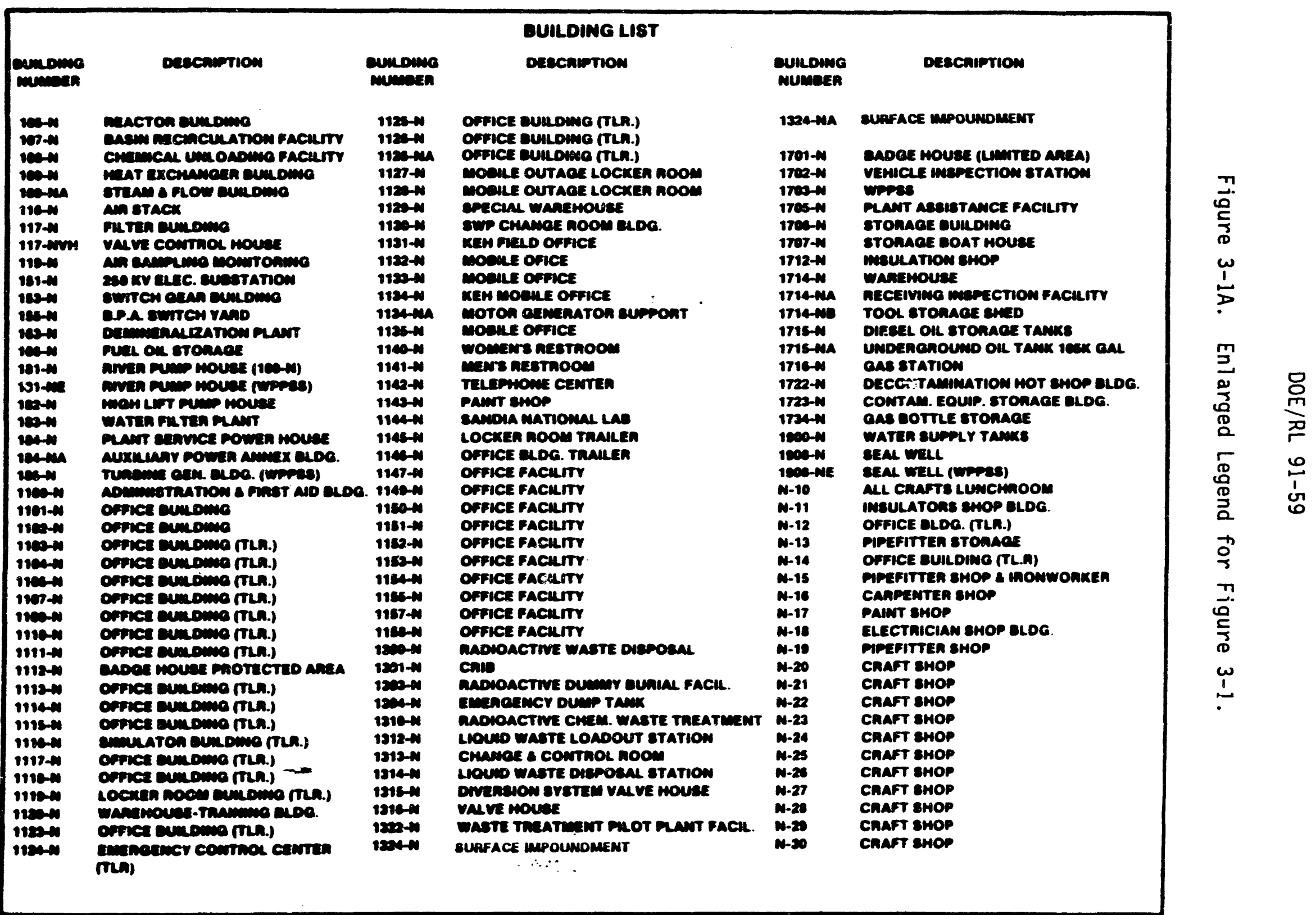


DOE/RL 91-59

This page intentionally left blank. 


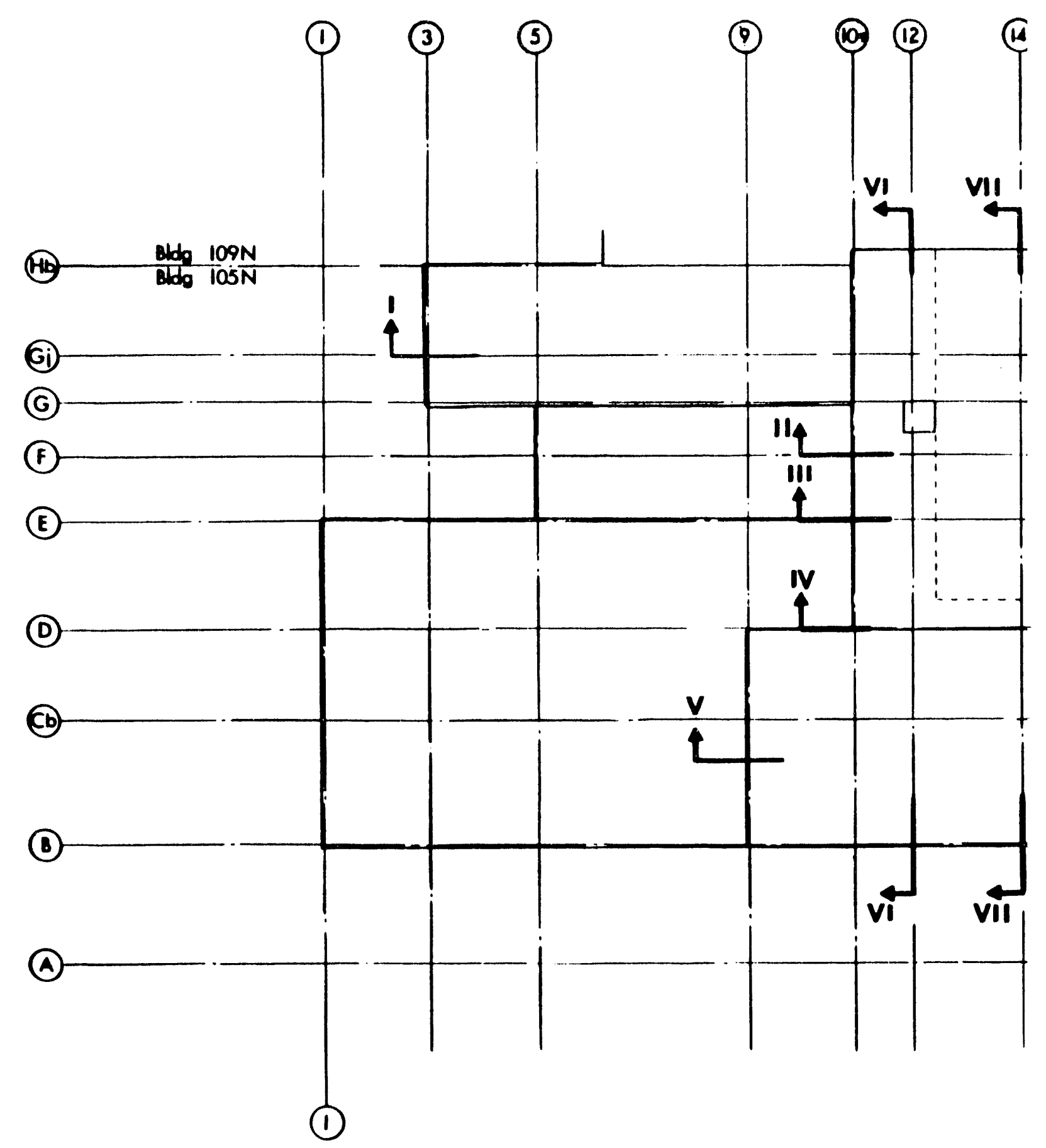

LEGEND

(1) Zone 1 Primary Confinement Zone

(i1) Zone II Secondary Confinement Zone

(iii) Zone III Confinement Buffor Zone

(1V) Zone $N$ Personnel Zone

(V) Zone V Reoctor Control \& Aersonned Zone 


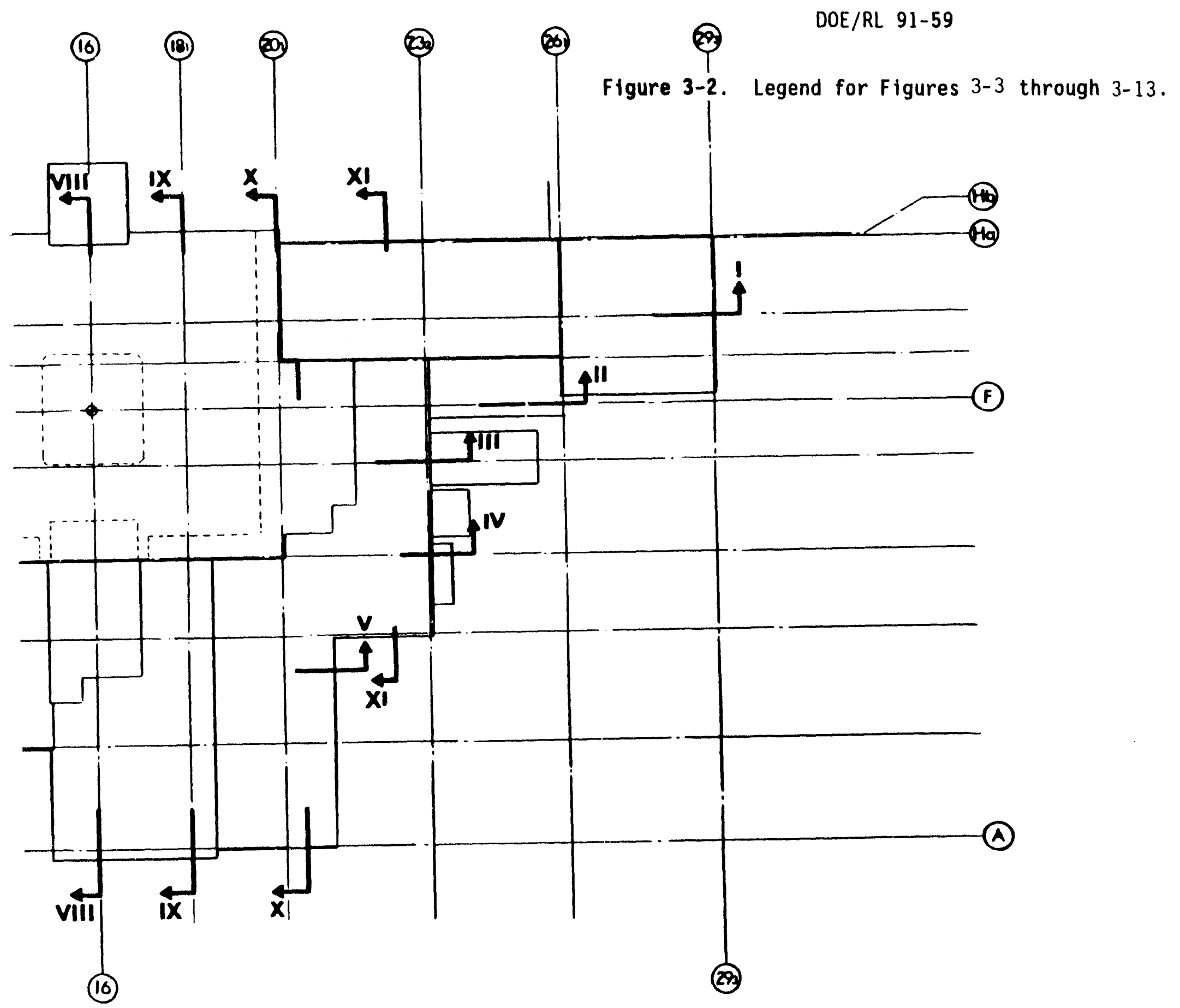

\footnotetext{
His:- Zone 1 Porimelor

Page Rodiation Zone Boundry

- Conter of Reoctor
} 


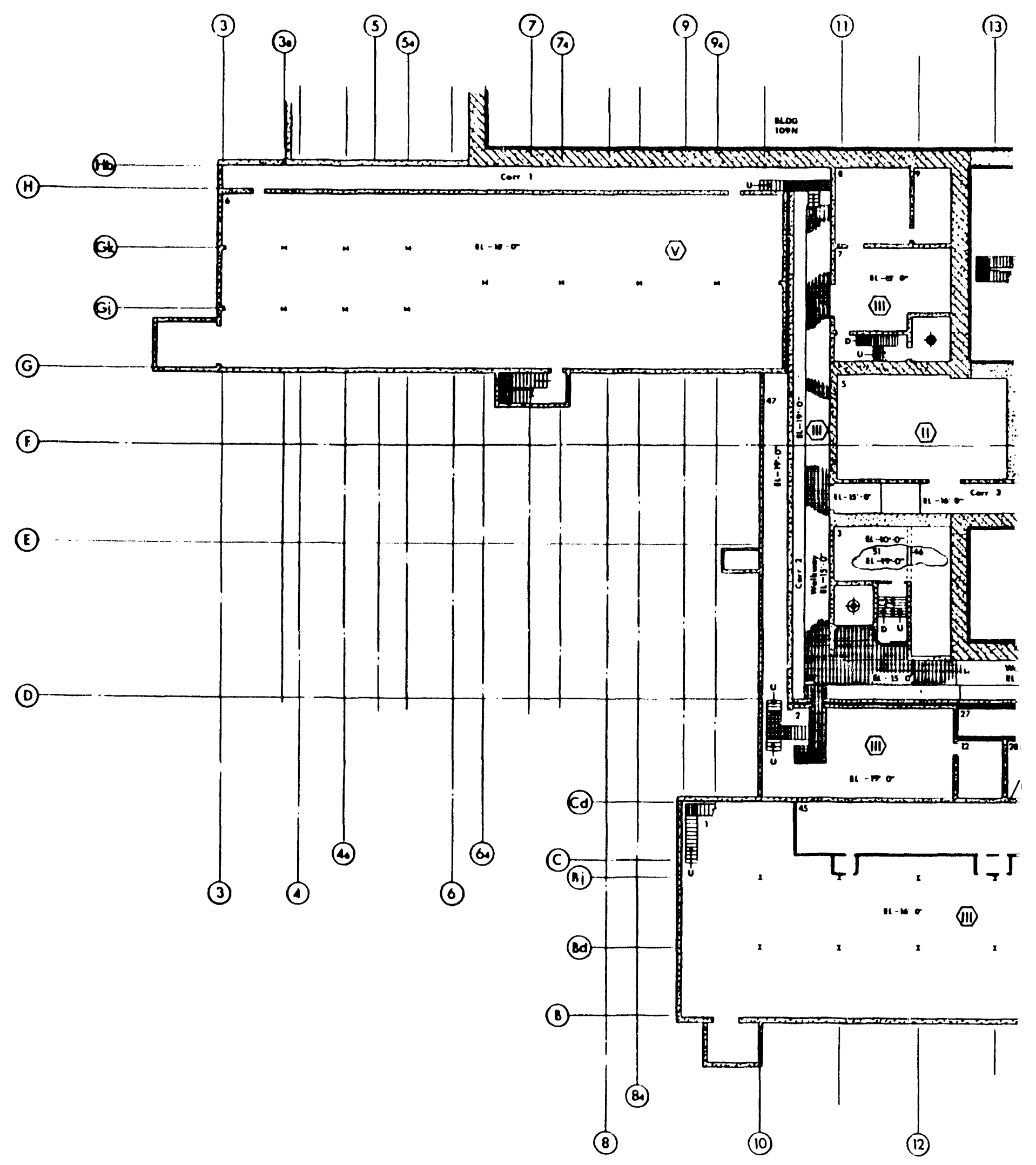




\section{$[-\varepsilon\rfloor$}

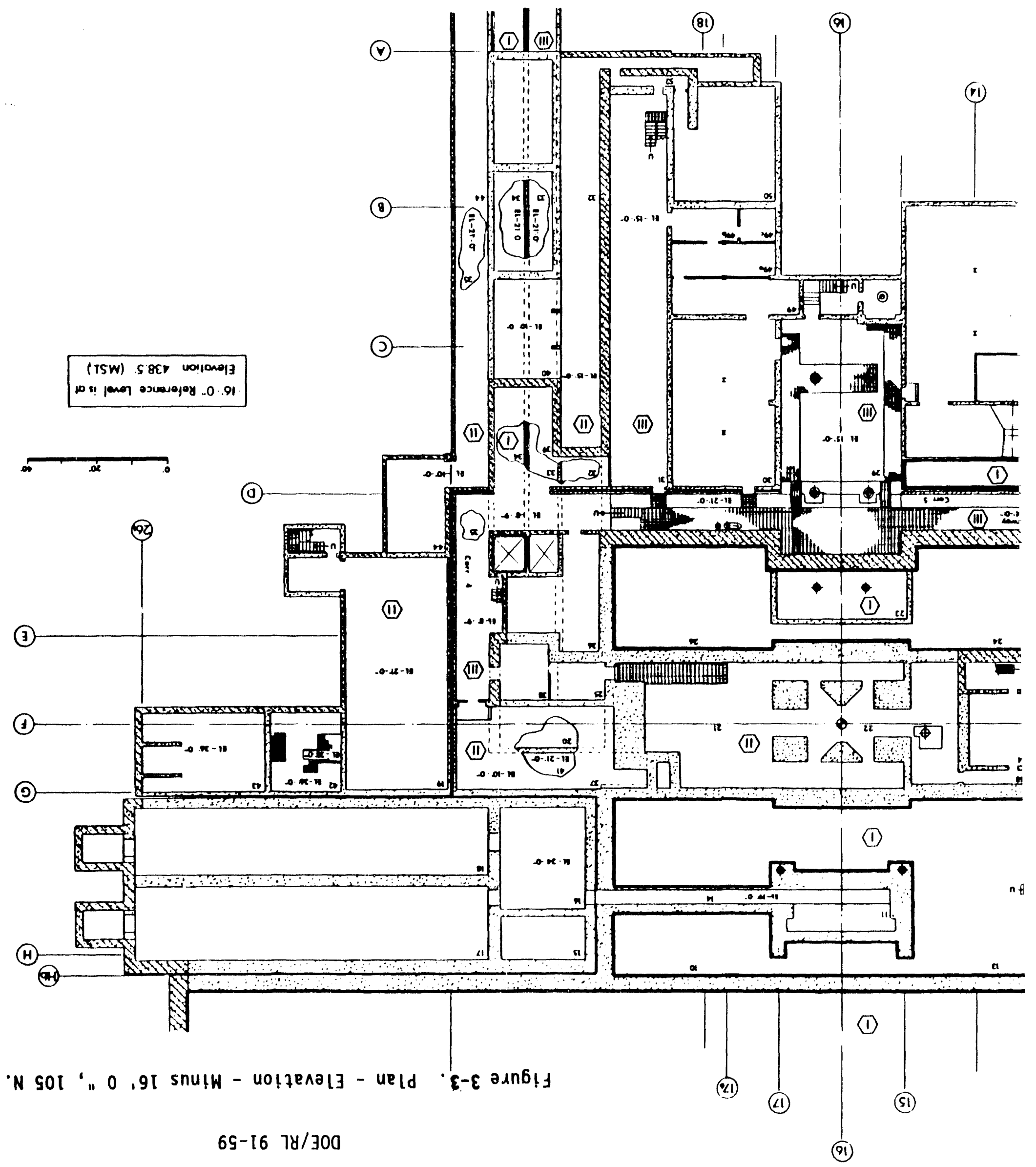




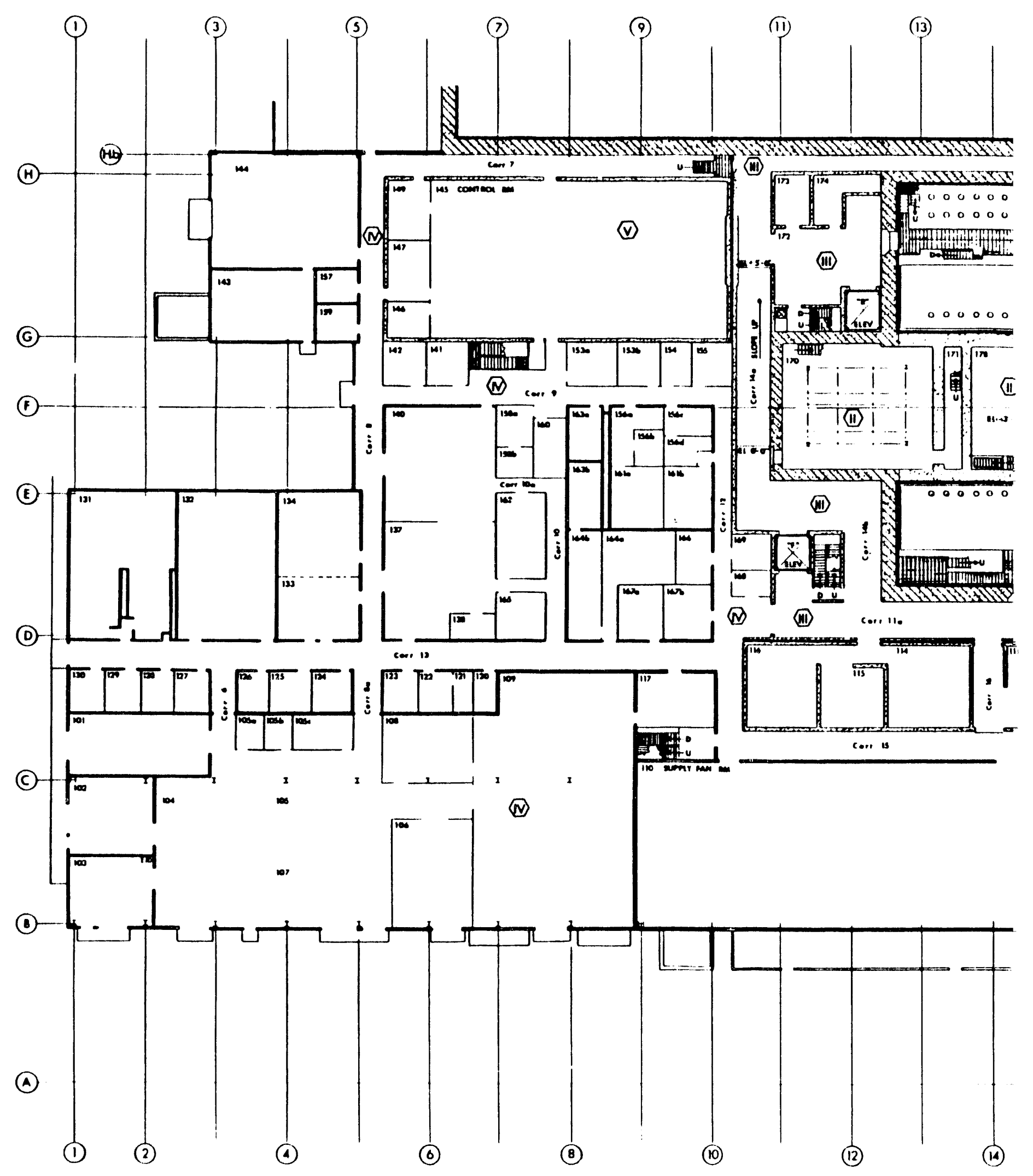




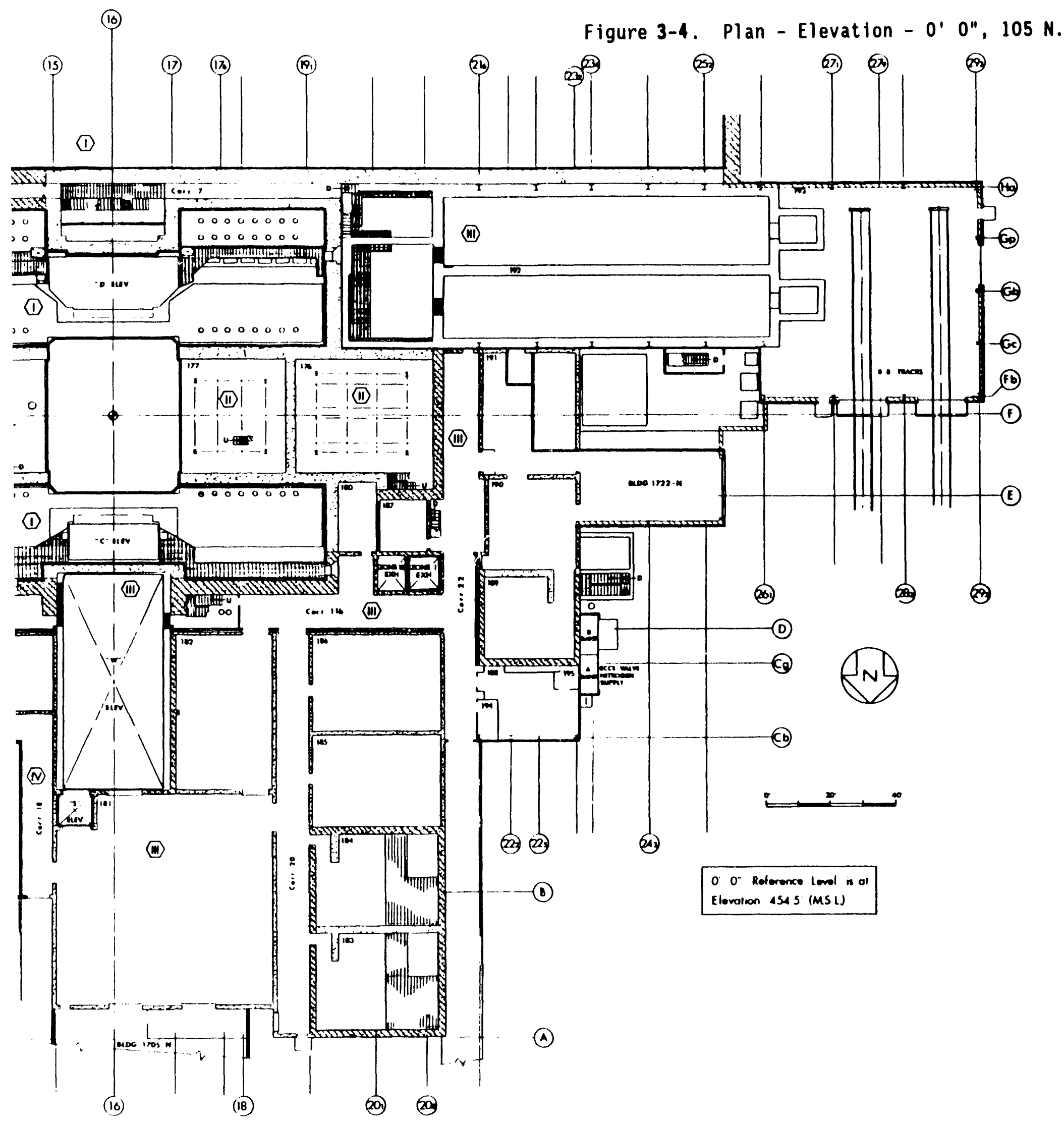




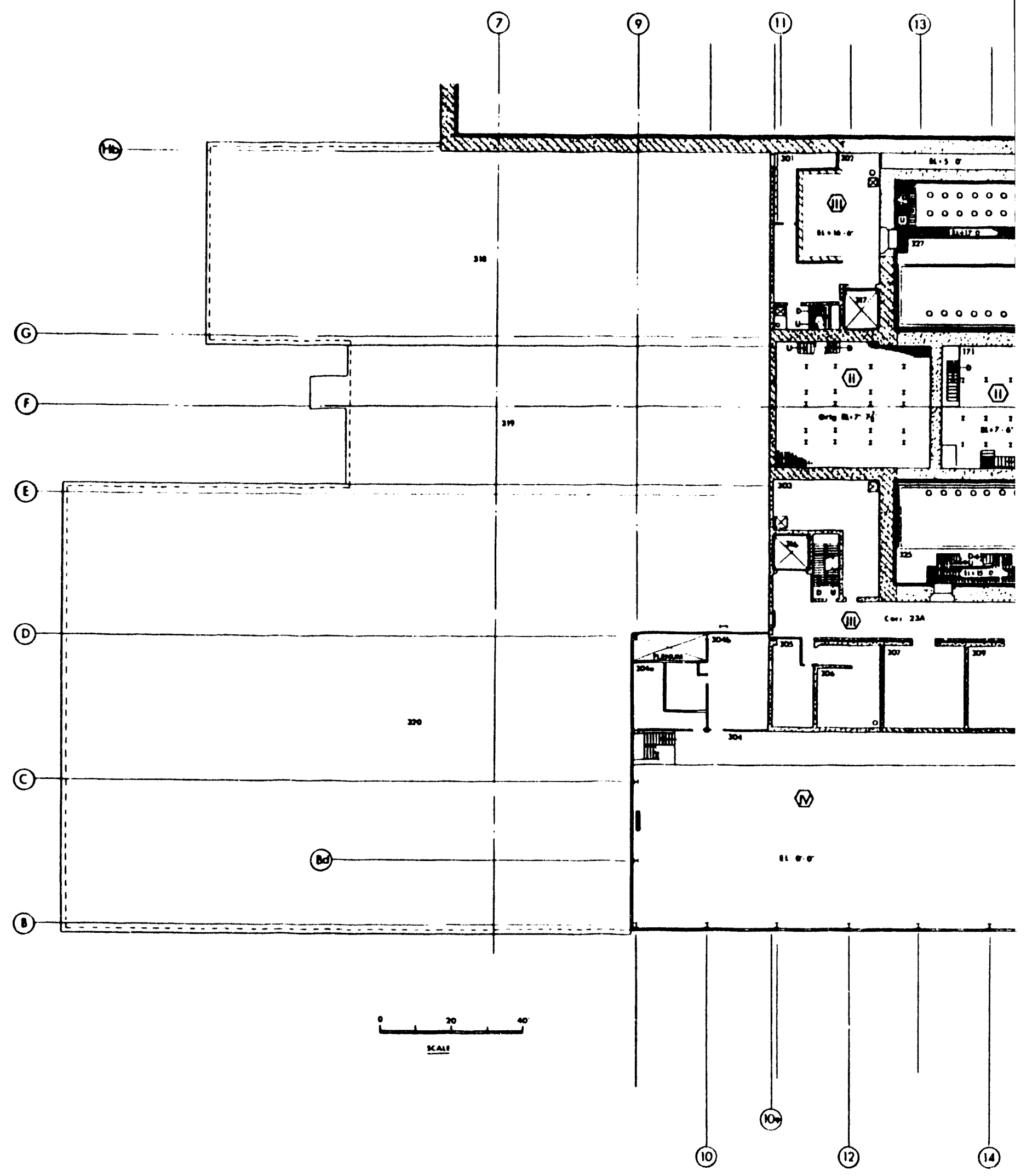




\section{$\mid I-\varepsilon\rfloor$}

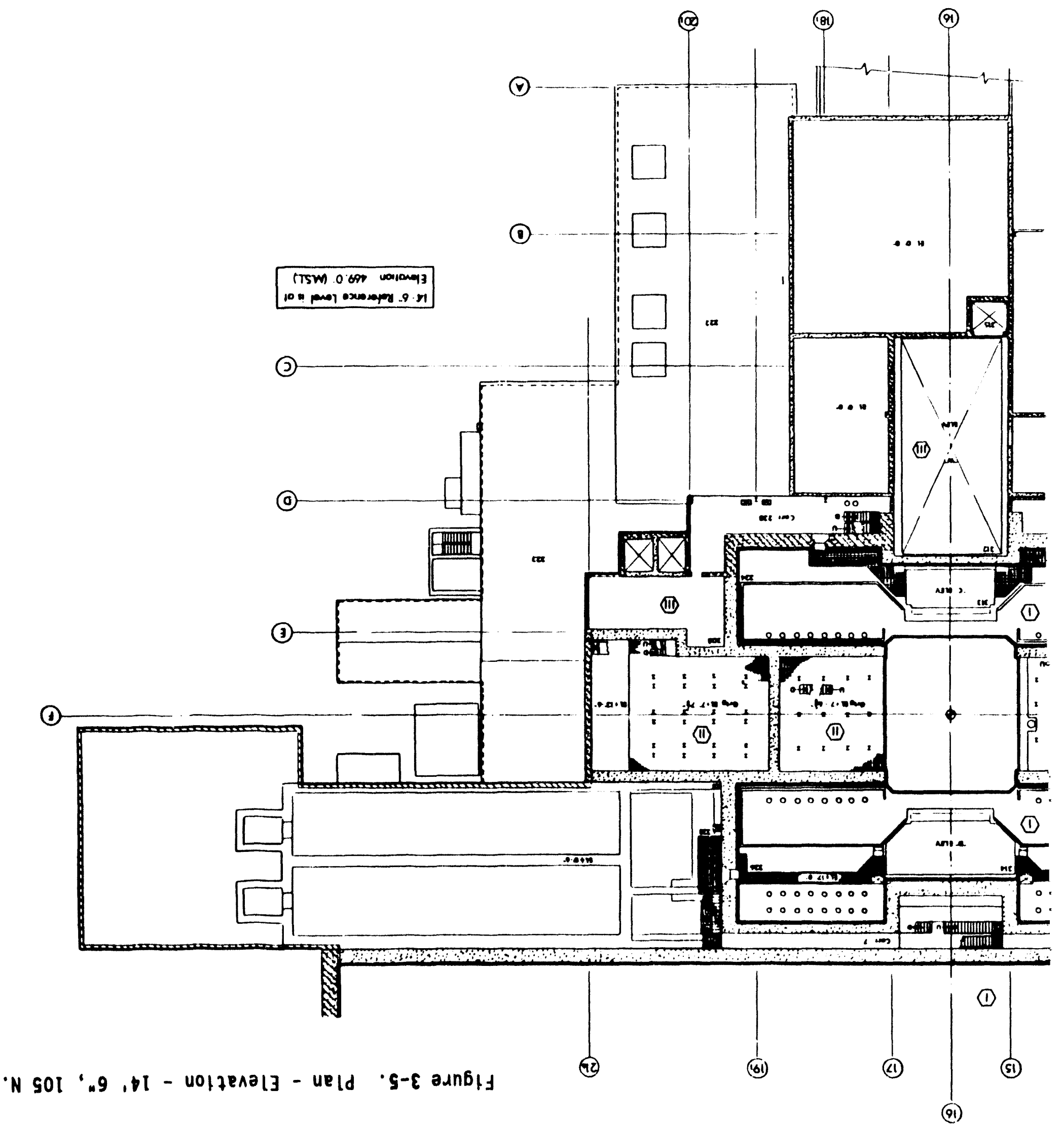





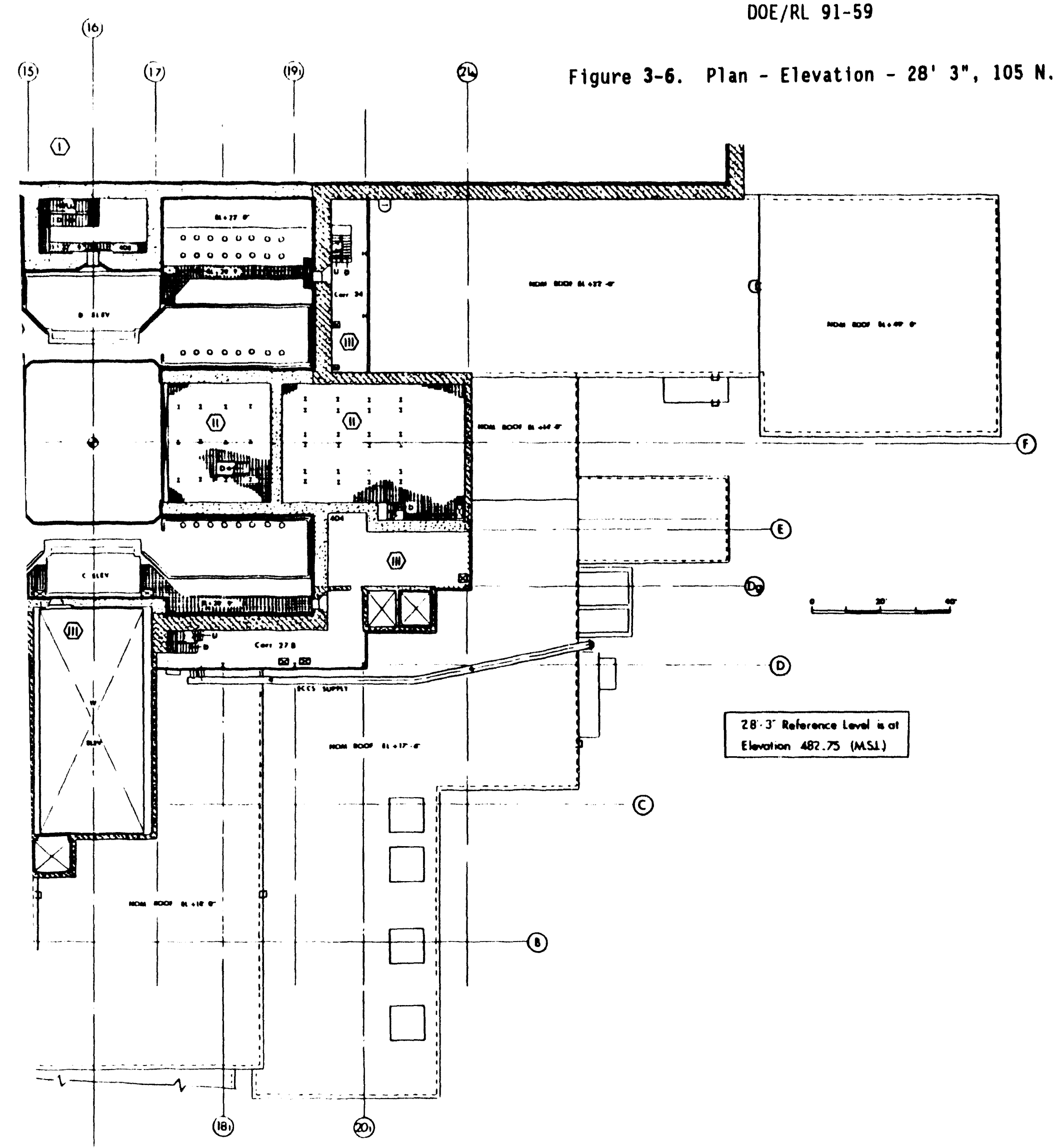

(16) 


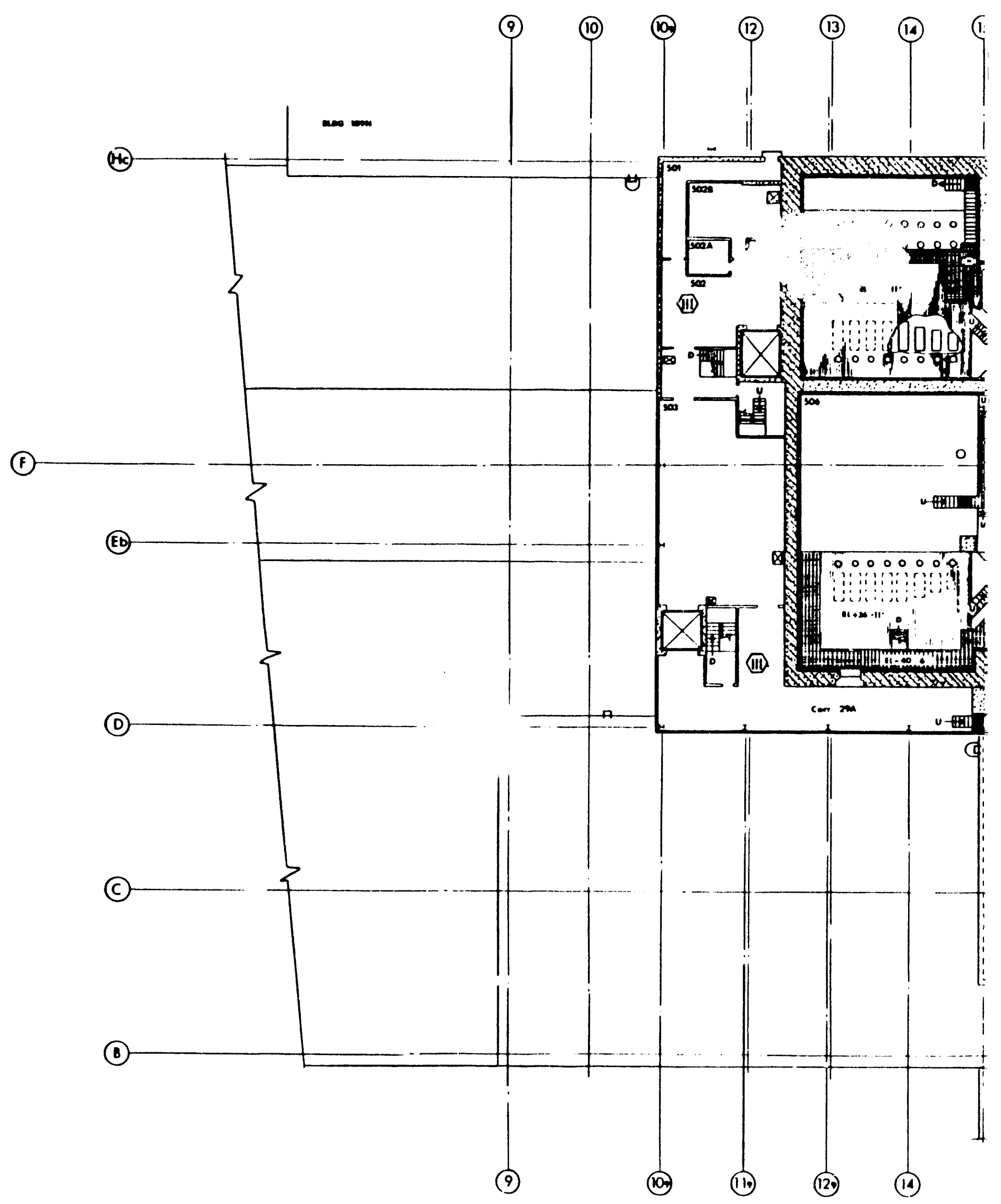


(16)

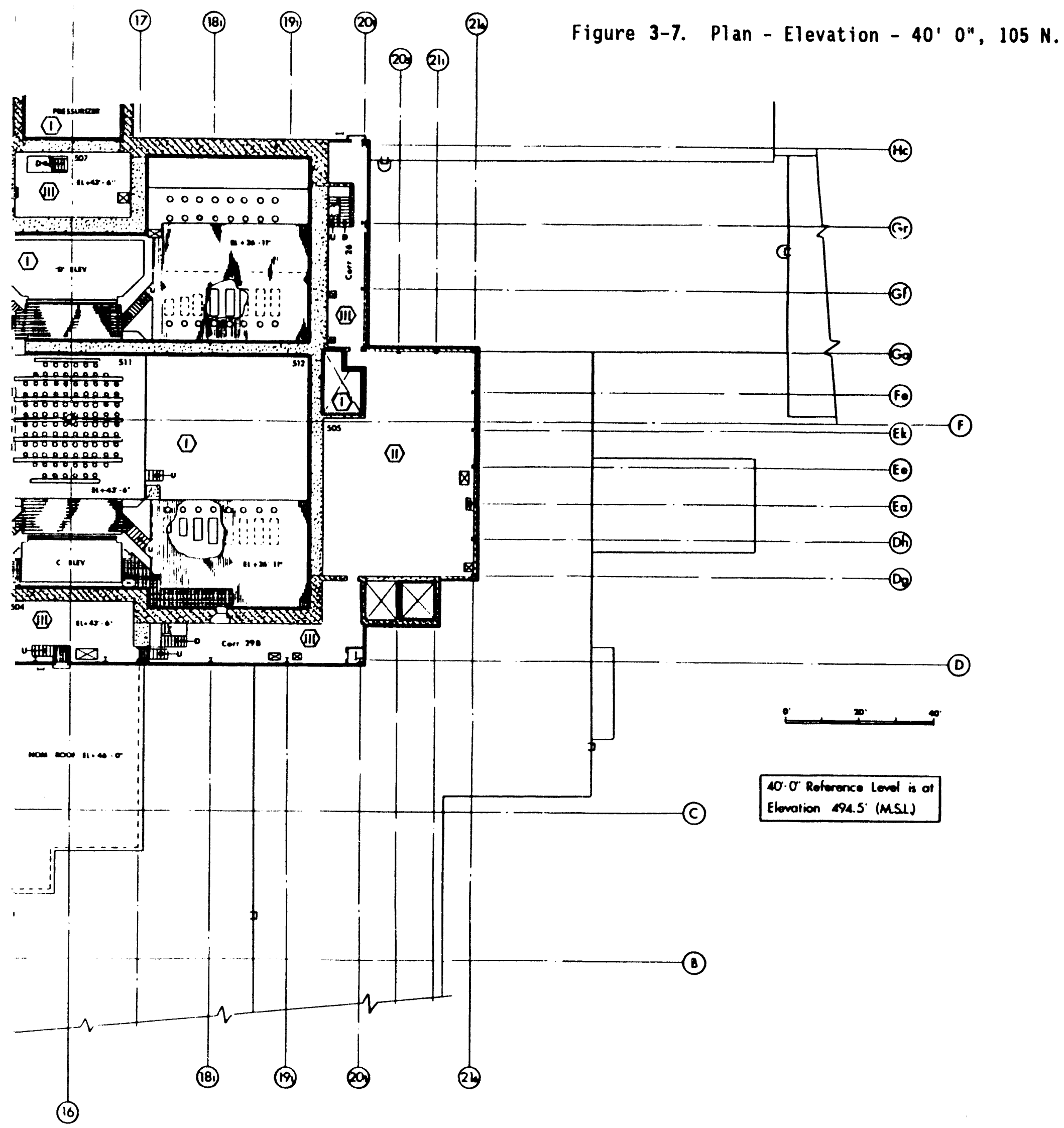




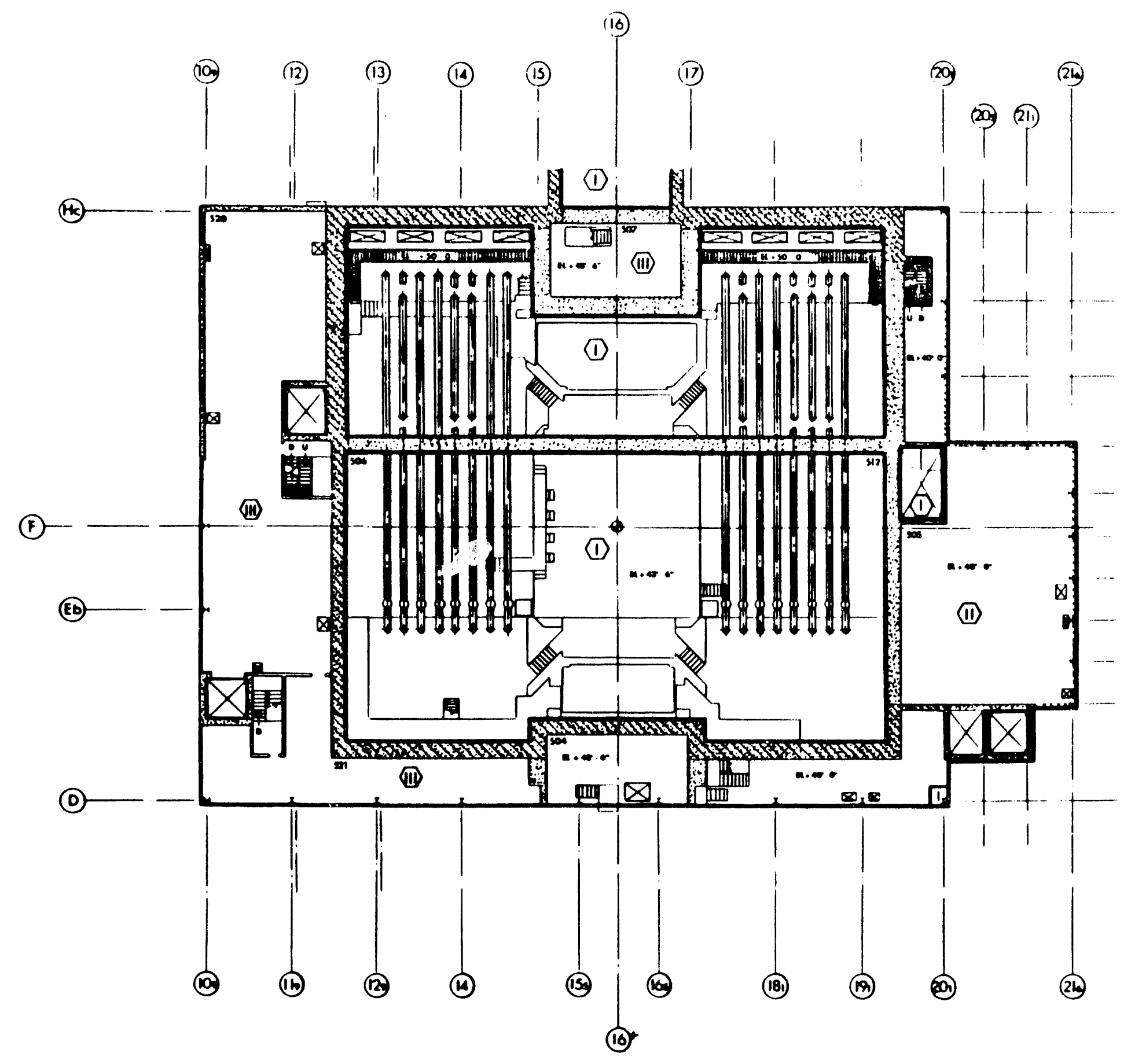

FlOOR Than of EL SH-O"

(E. 505.5 mst) 
Figure 3-8. Plan - Elevations $51^{\prime} 0^{\prime \prime}$ and $60^{\prime} 0^{\prime \prime}, 105 \mathrm{~N}$.

(10)

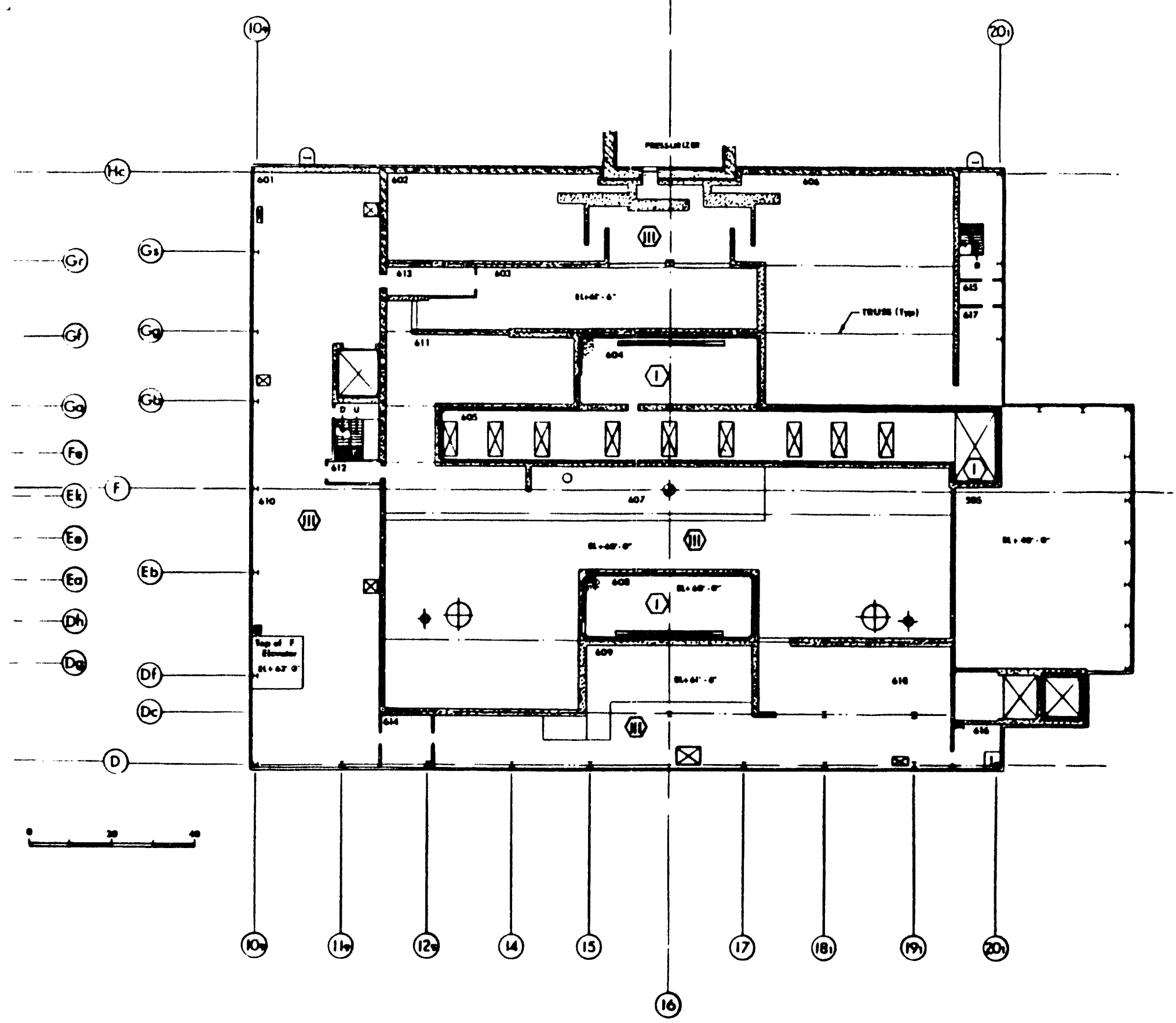

FlOOR MaN of a $60^{\circ}-6^{\prime \prime}$

(A. S15. O Msis) 

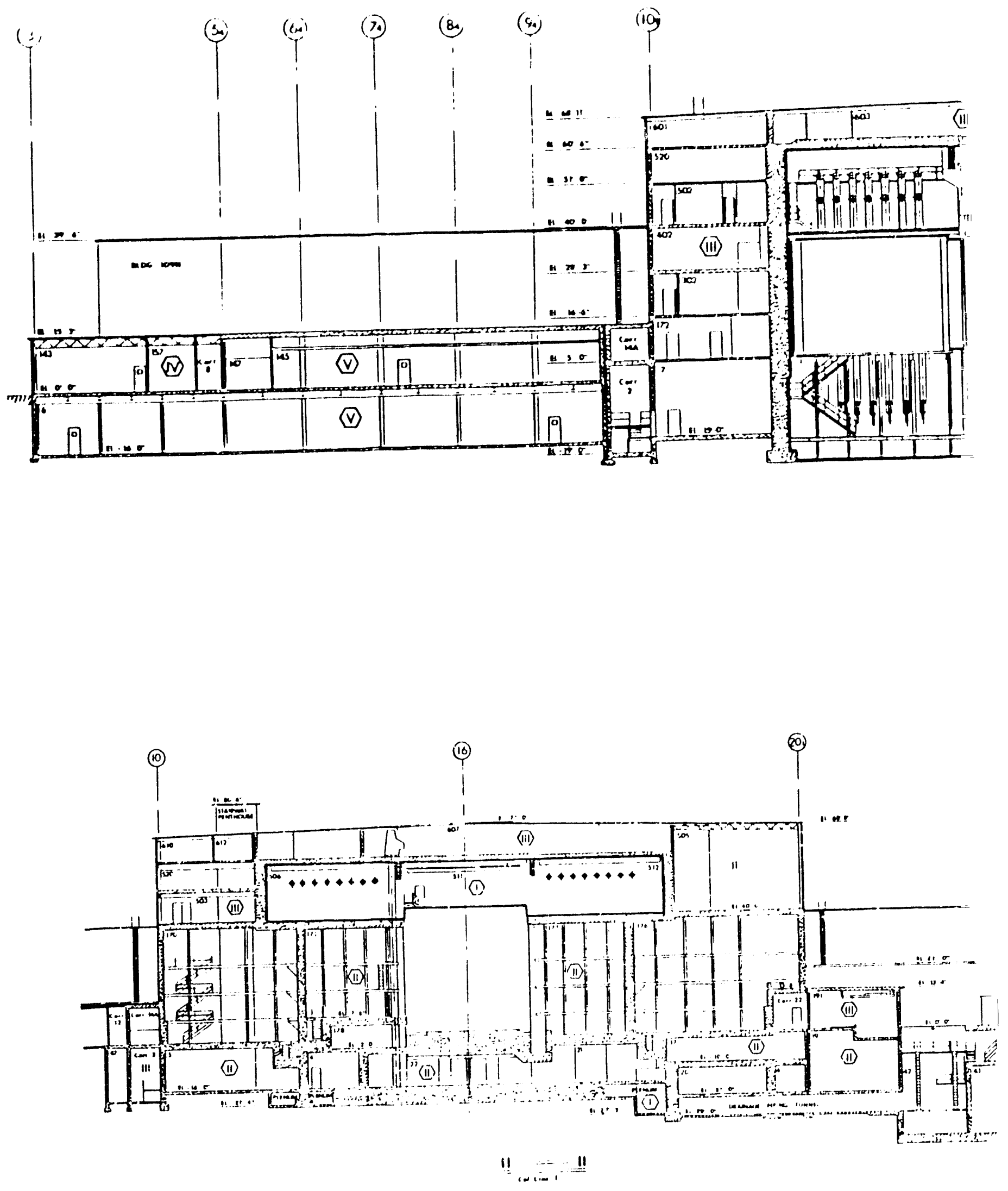


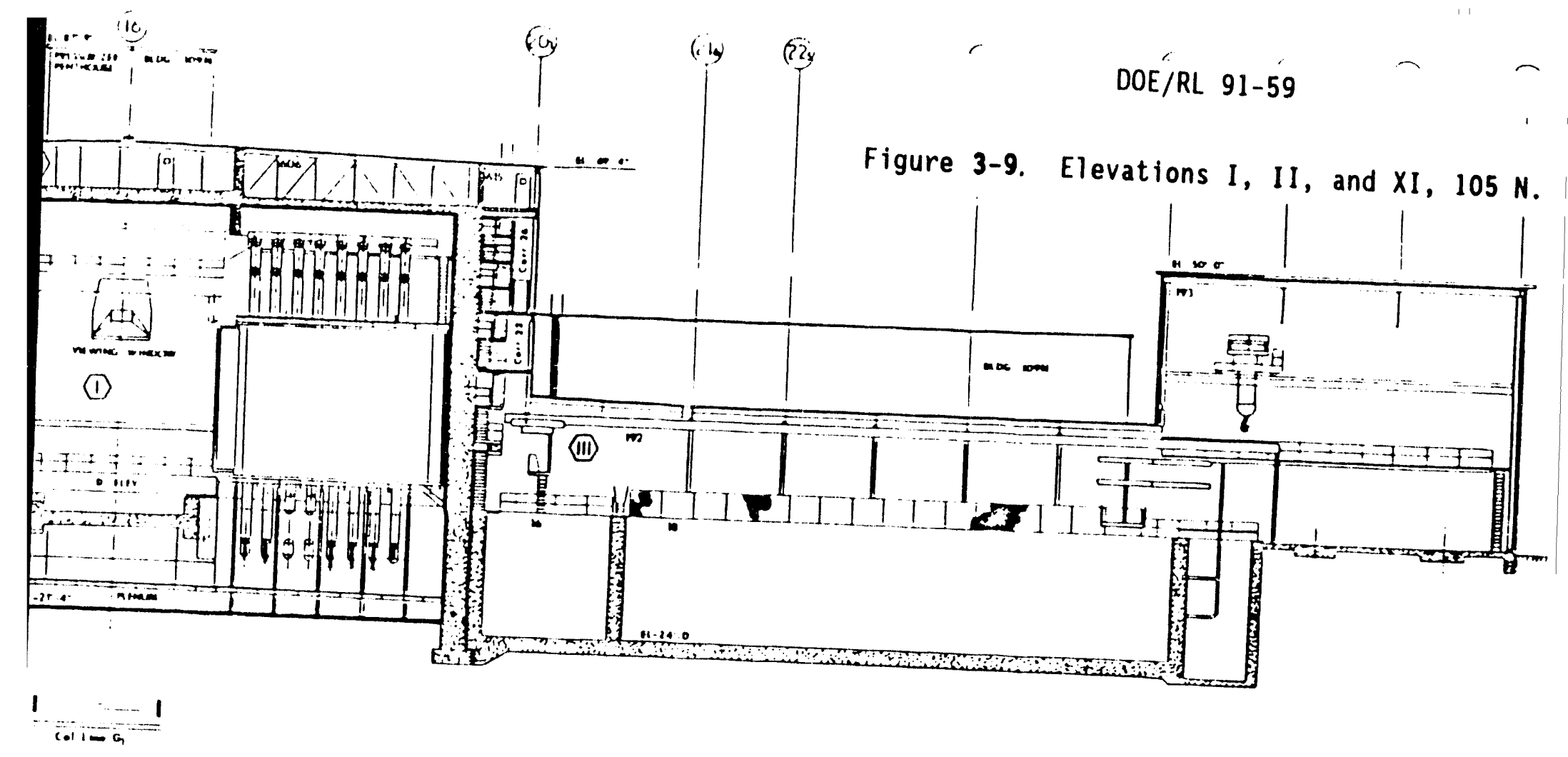

(D)
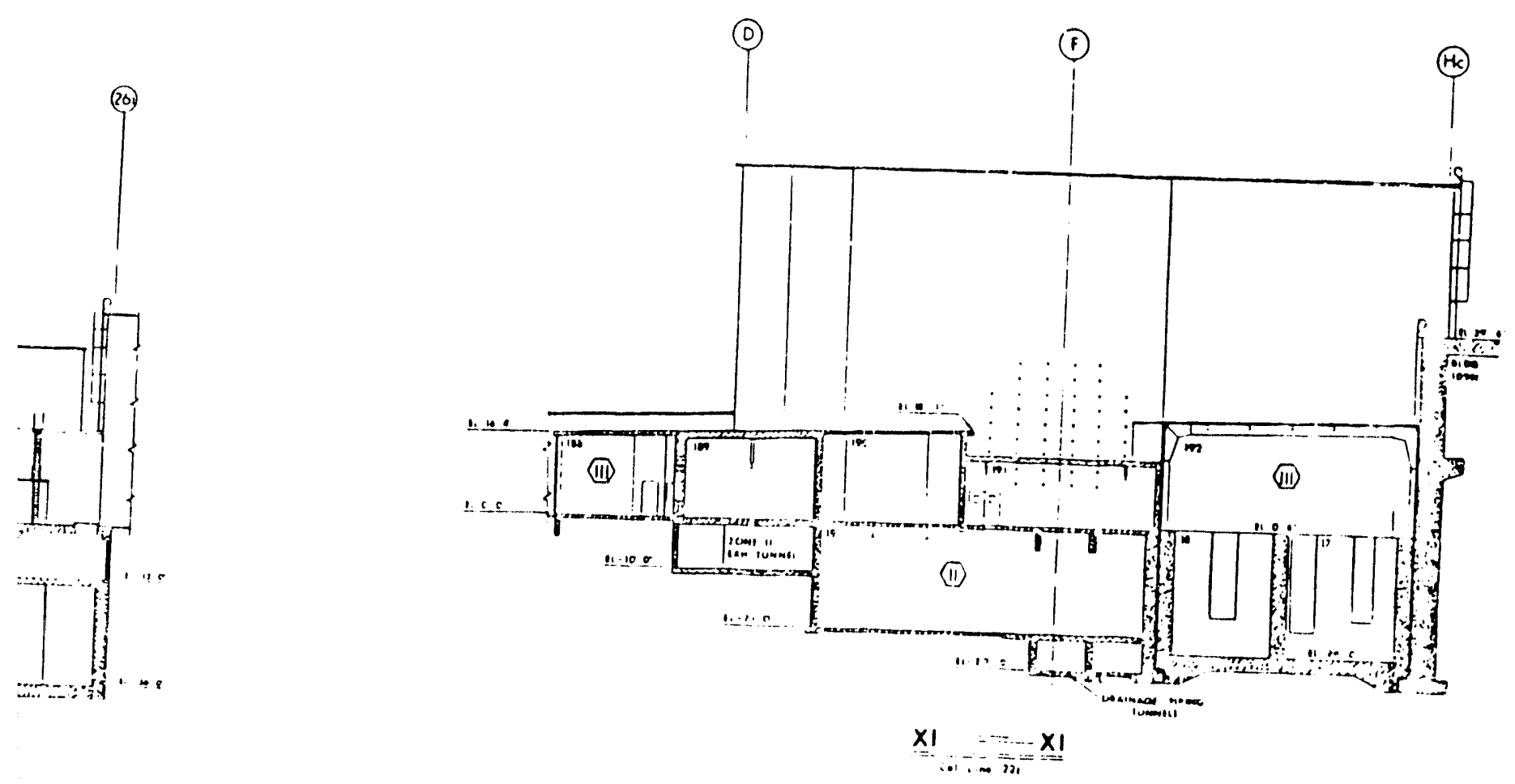


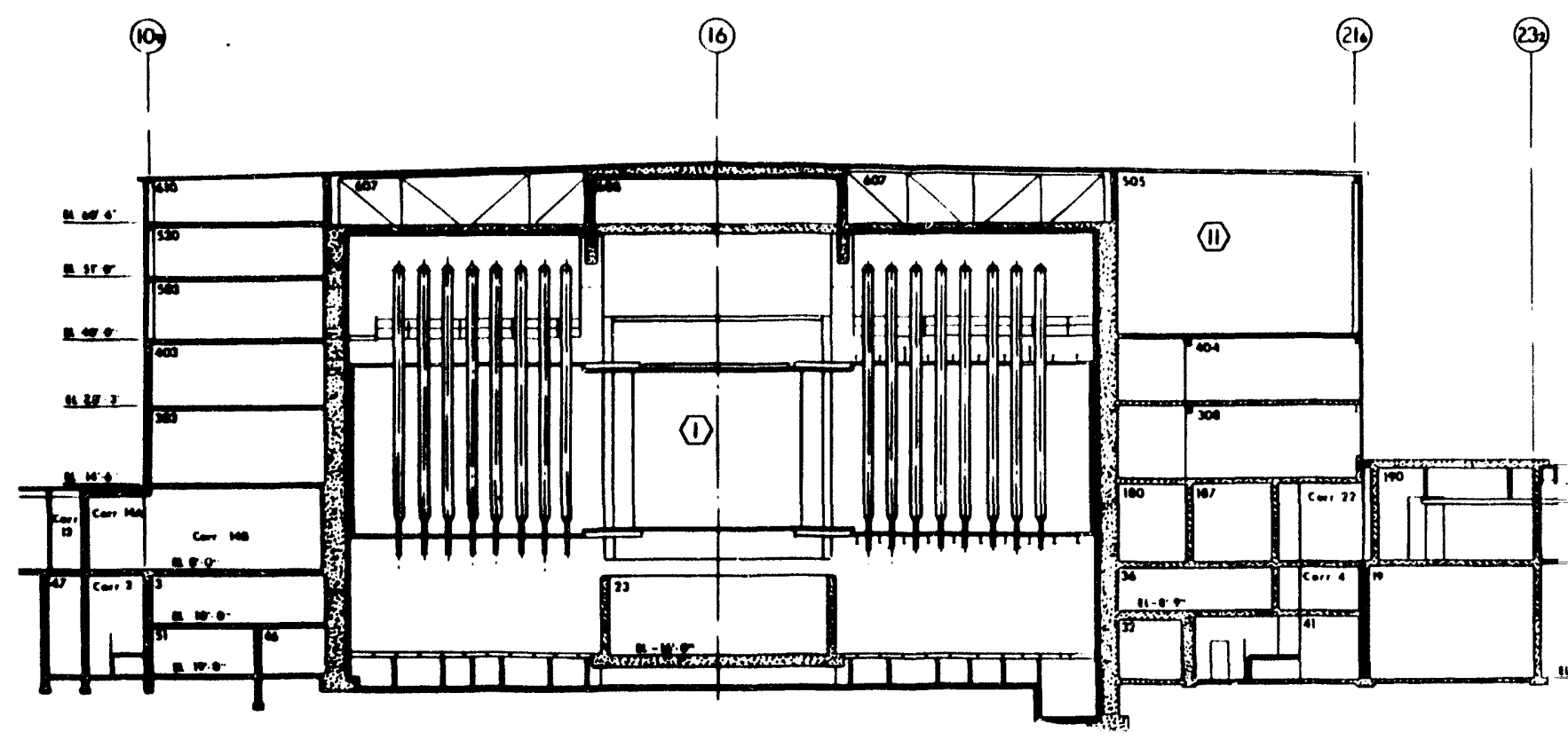

$\frac{\text { III }=\text { III }}{\text { col Lin- }}$

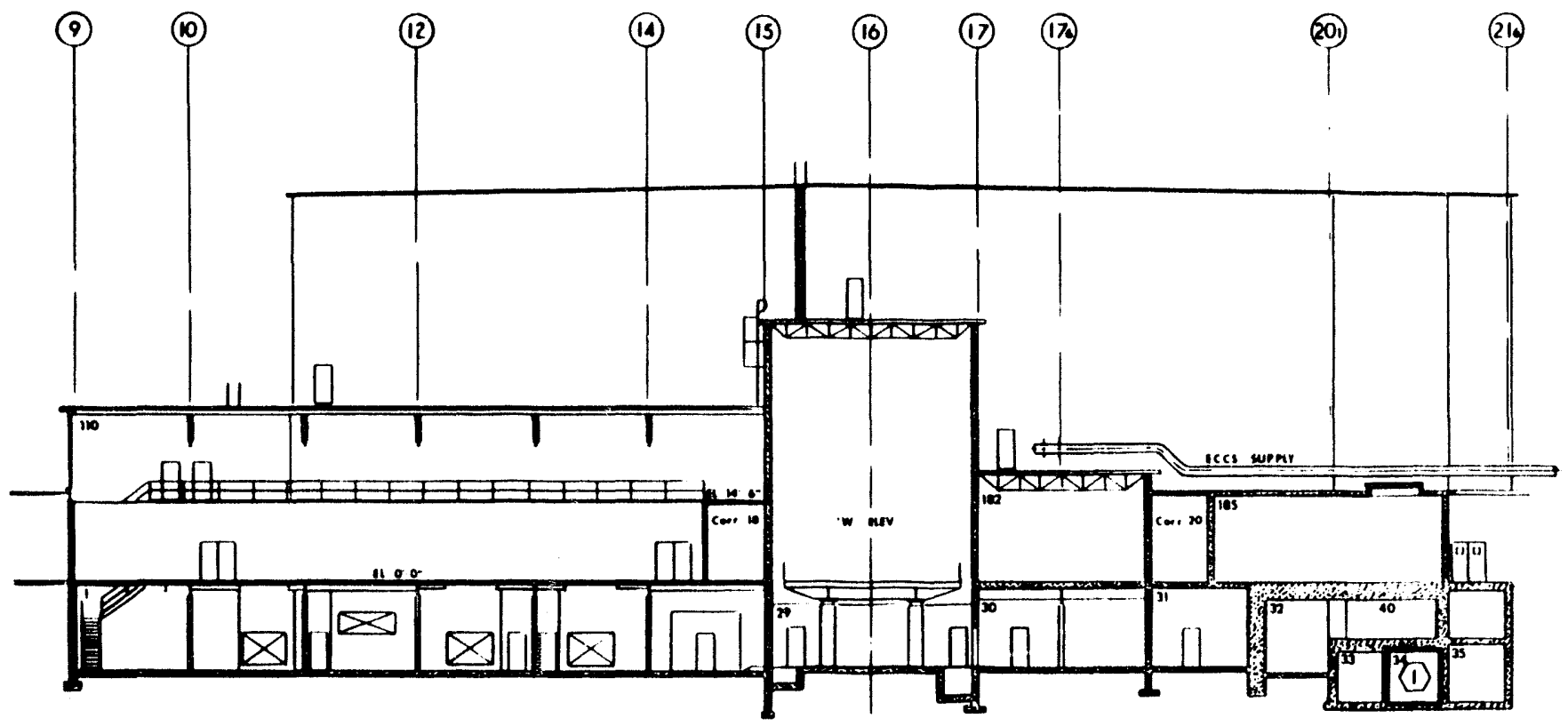

$\frac{V=V}{\cot \operatorname{tin} 4}$ 
DOE/RL 91-59

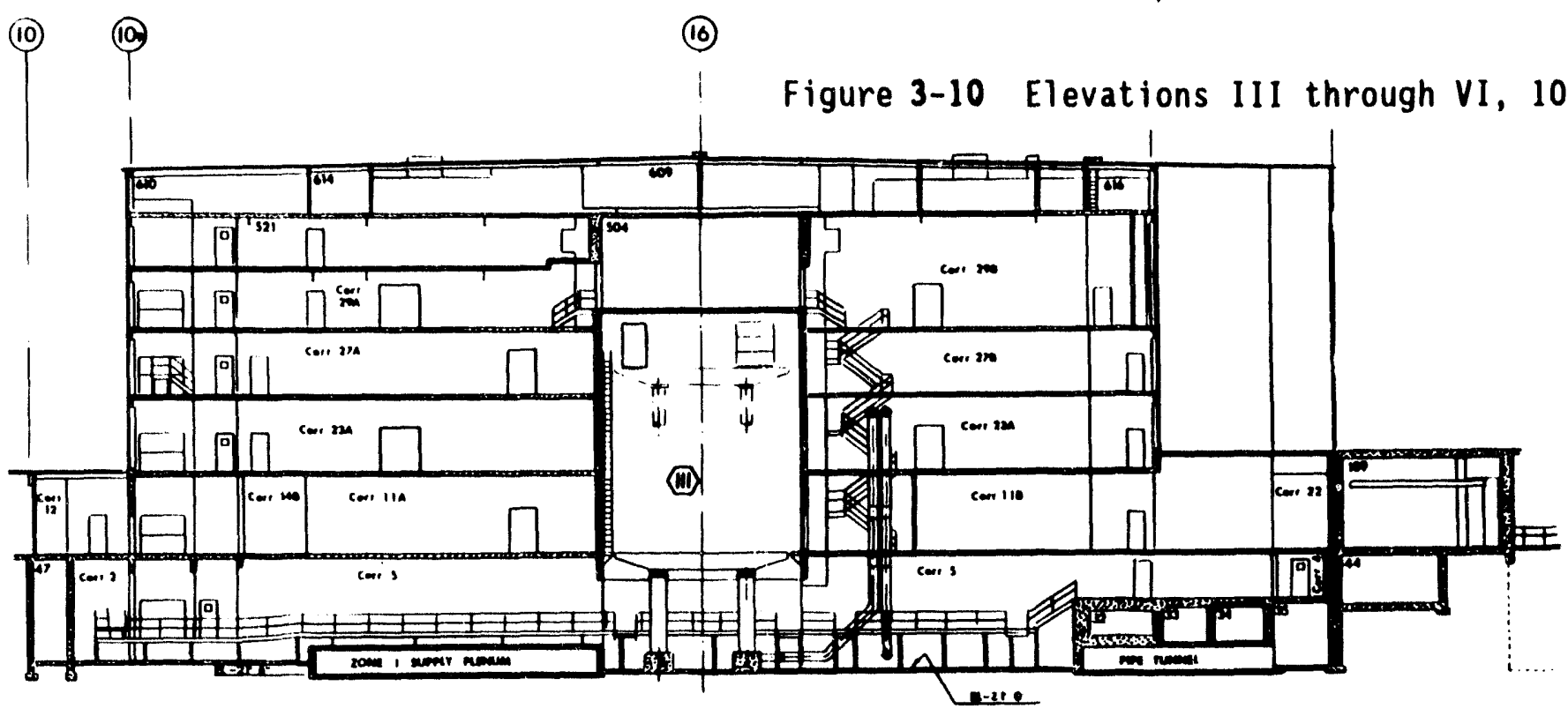

$\frac{\text { IV }=\text { IV }}{\operatorname{cotho} D}$

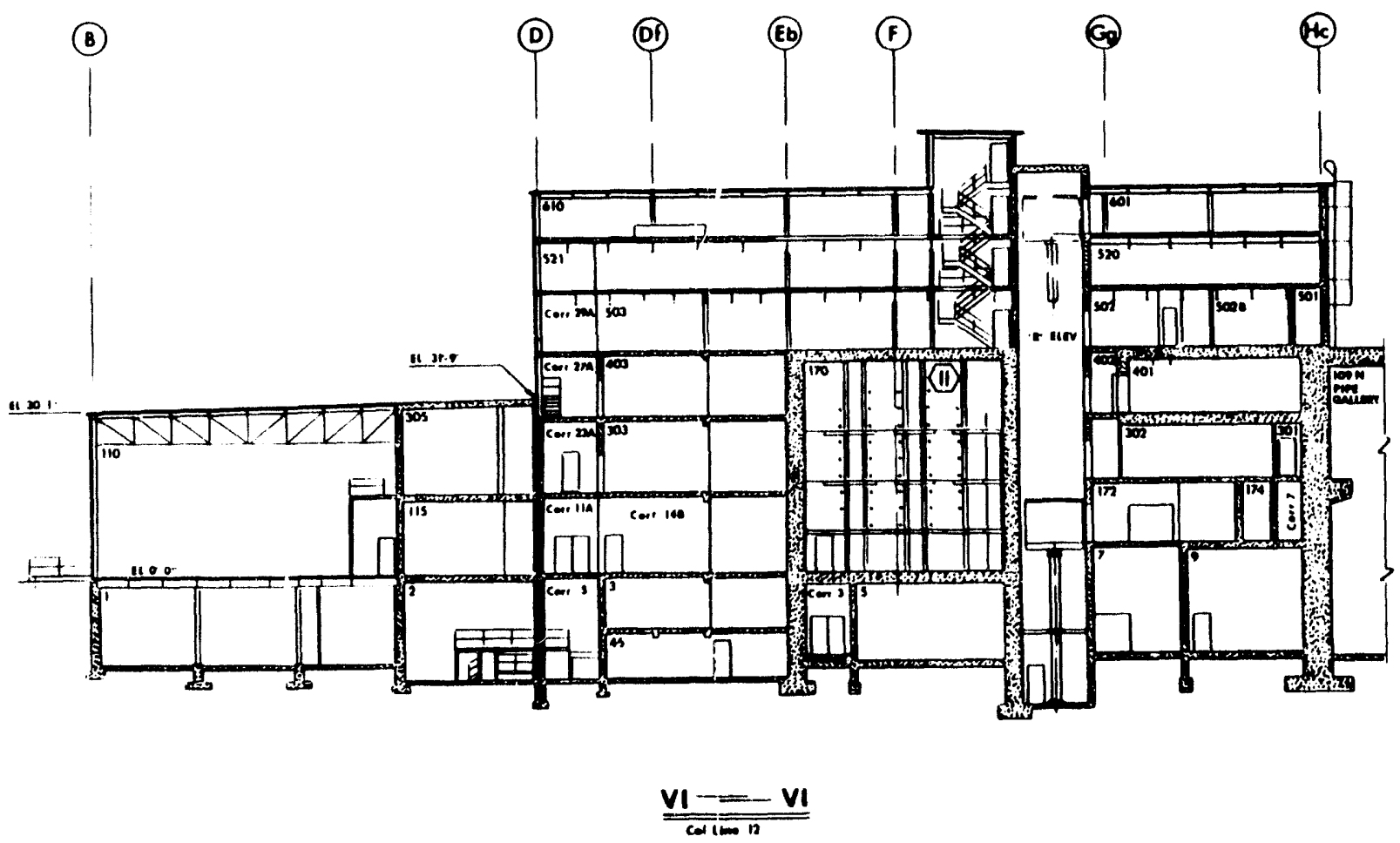



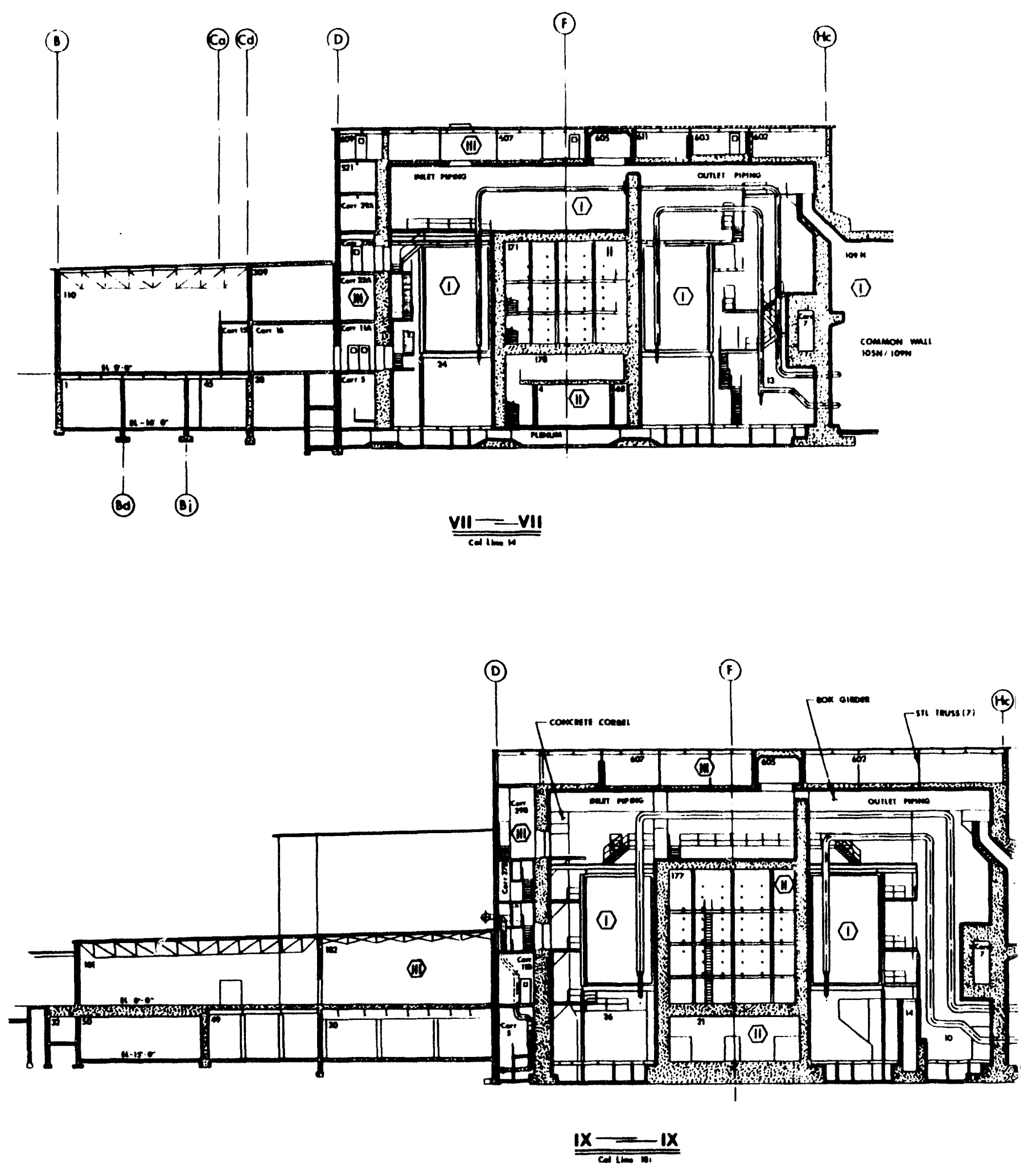

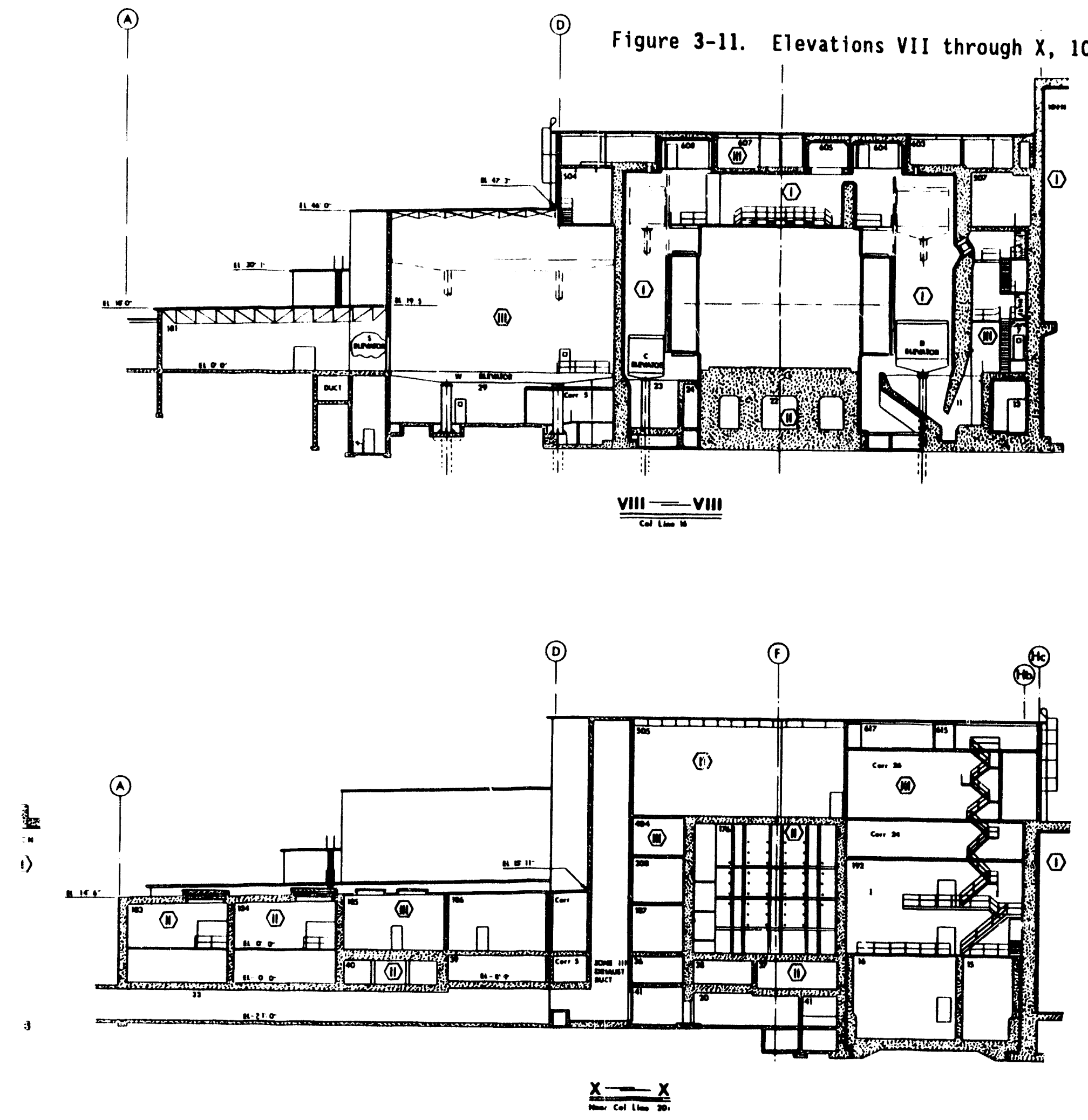


\section{$\sqrt{0-.9-13 \text { to NVId POOIJ }}$}

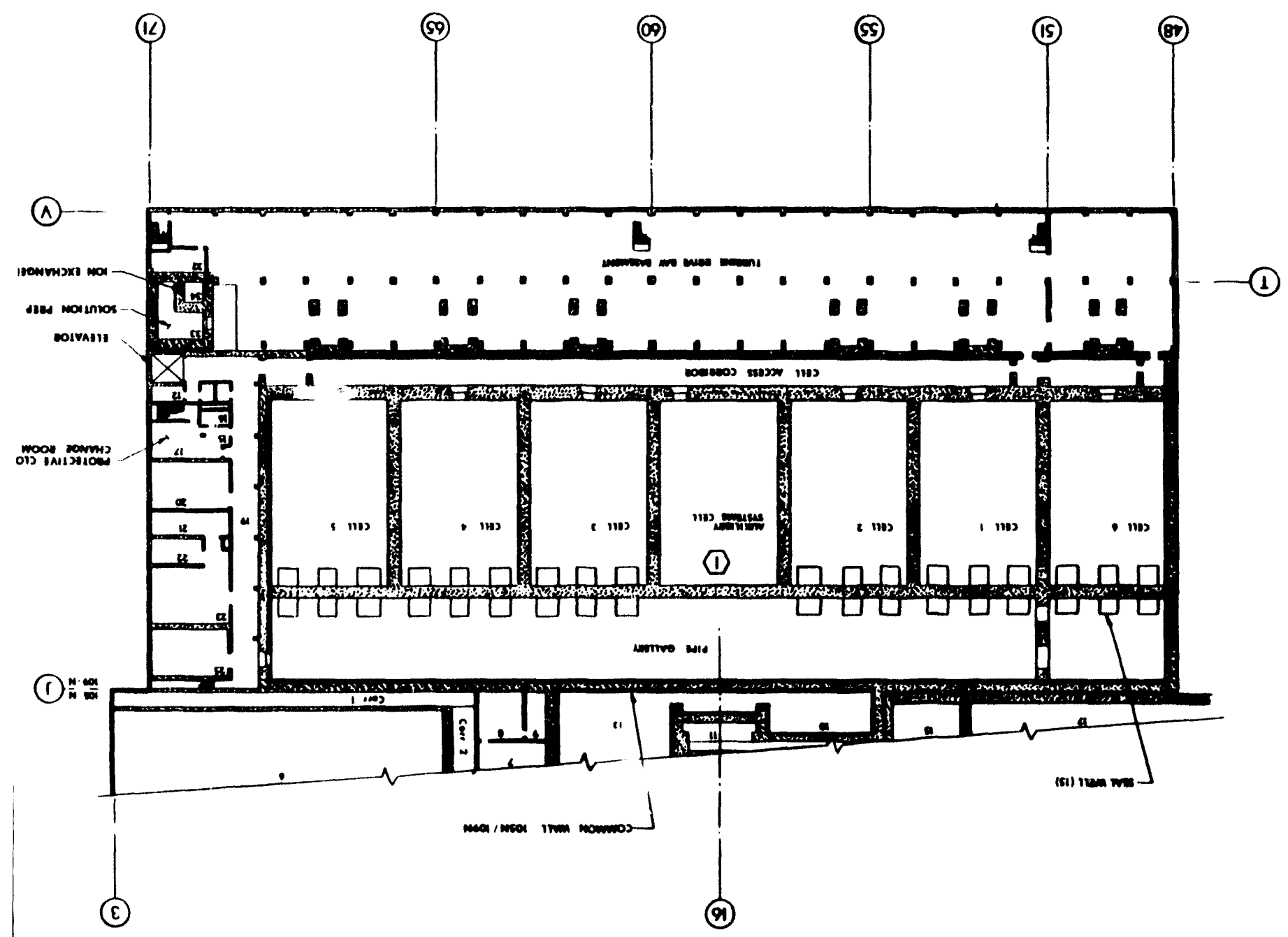


Figure 3-12. Plan at Elevations $0^{\prime \prime} O^{\prime \prime}$ and 16' $0^{\prime \prime}, 109-N$ Building.

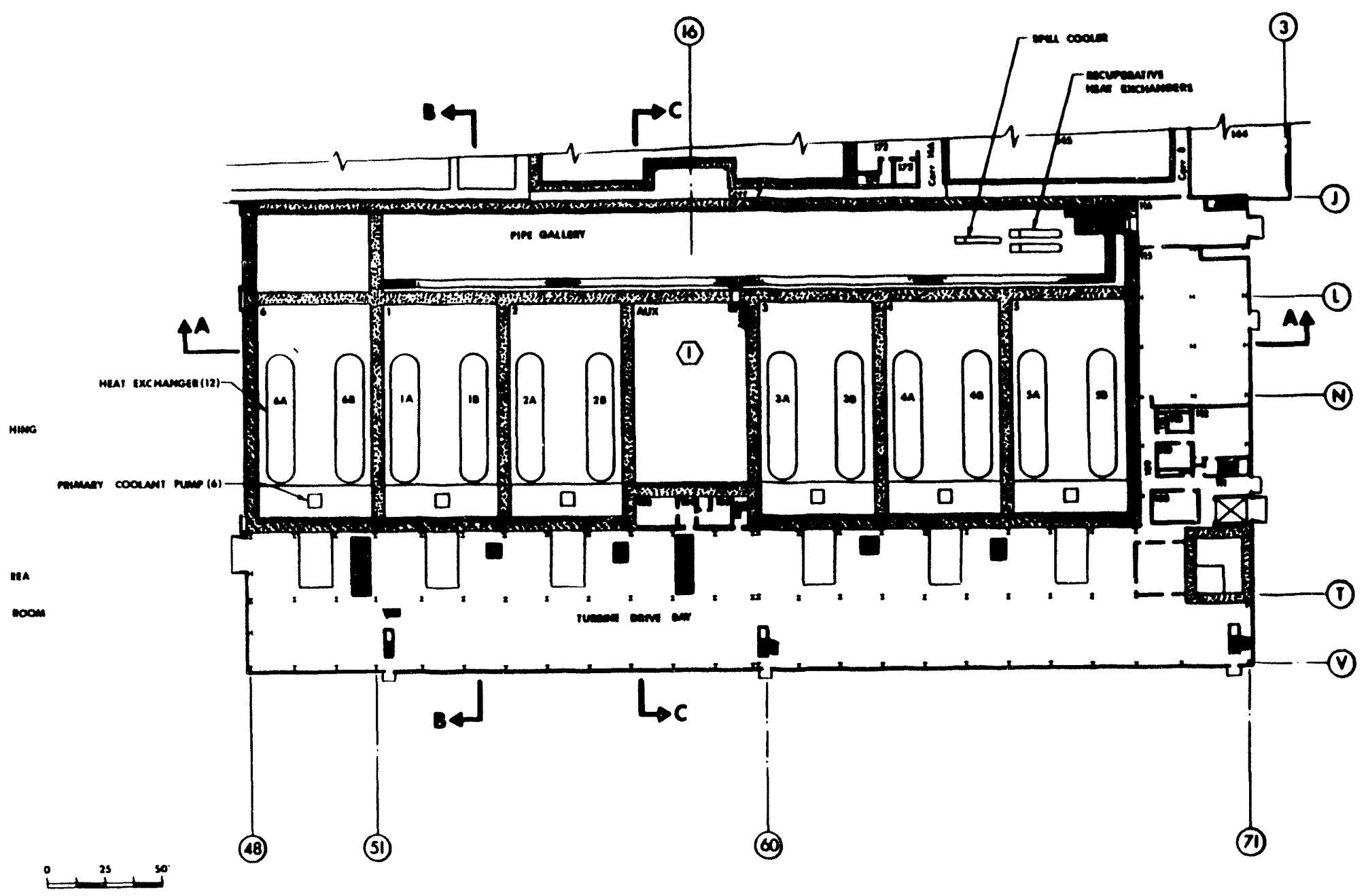

FLOOR PLAN of EL O'-O' 

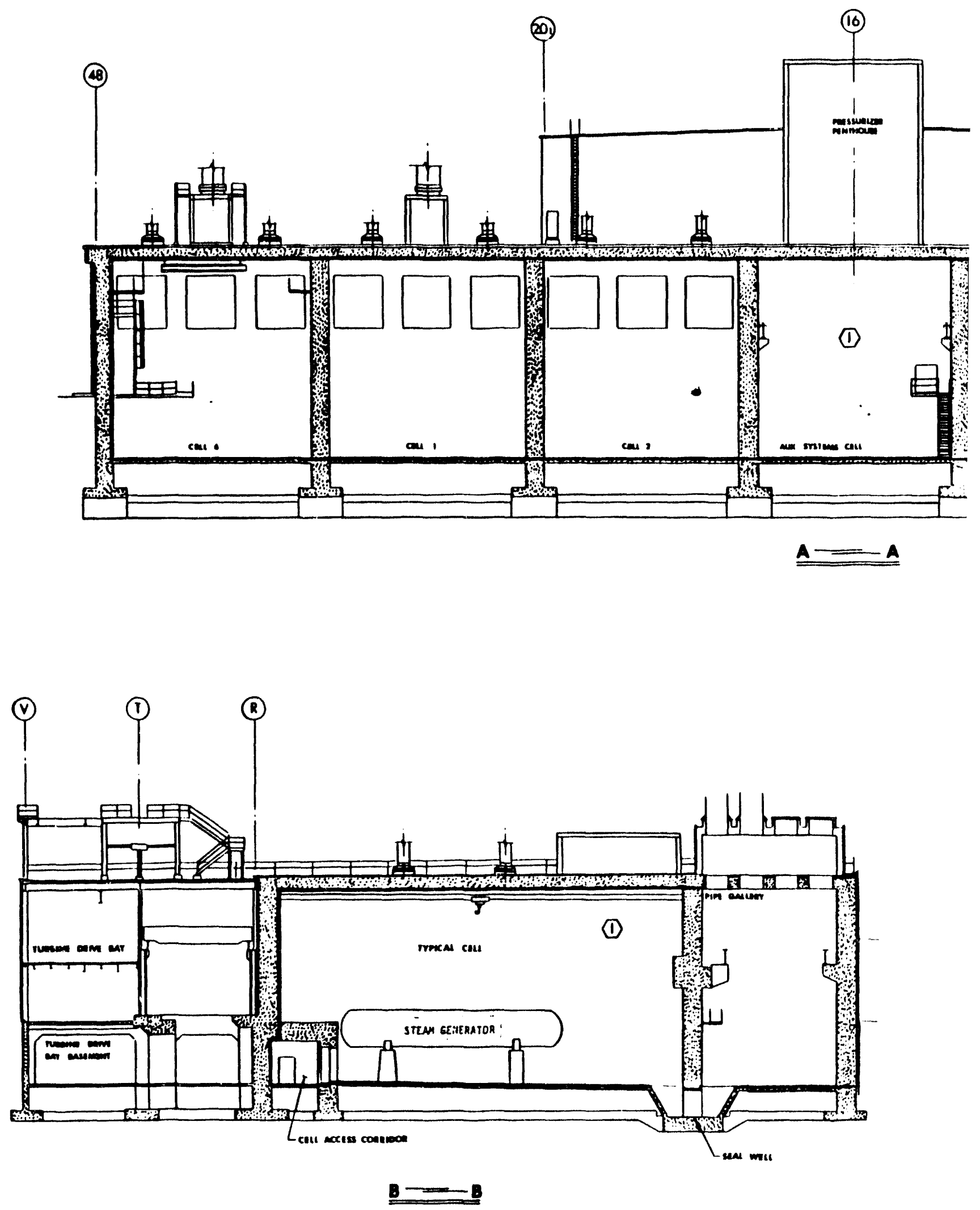
DOE/RL 91-59
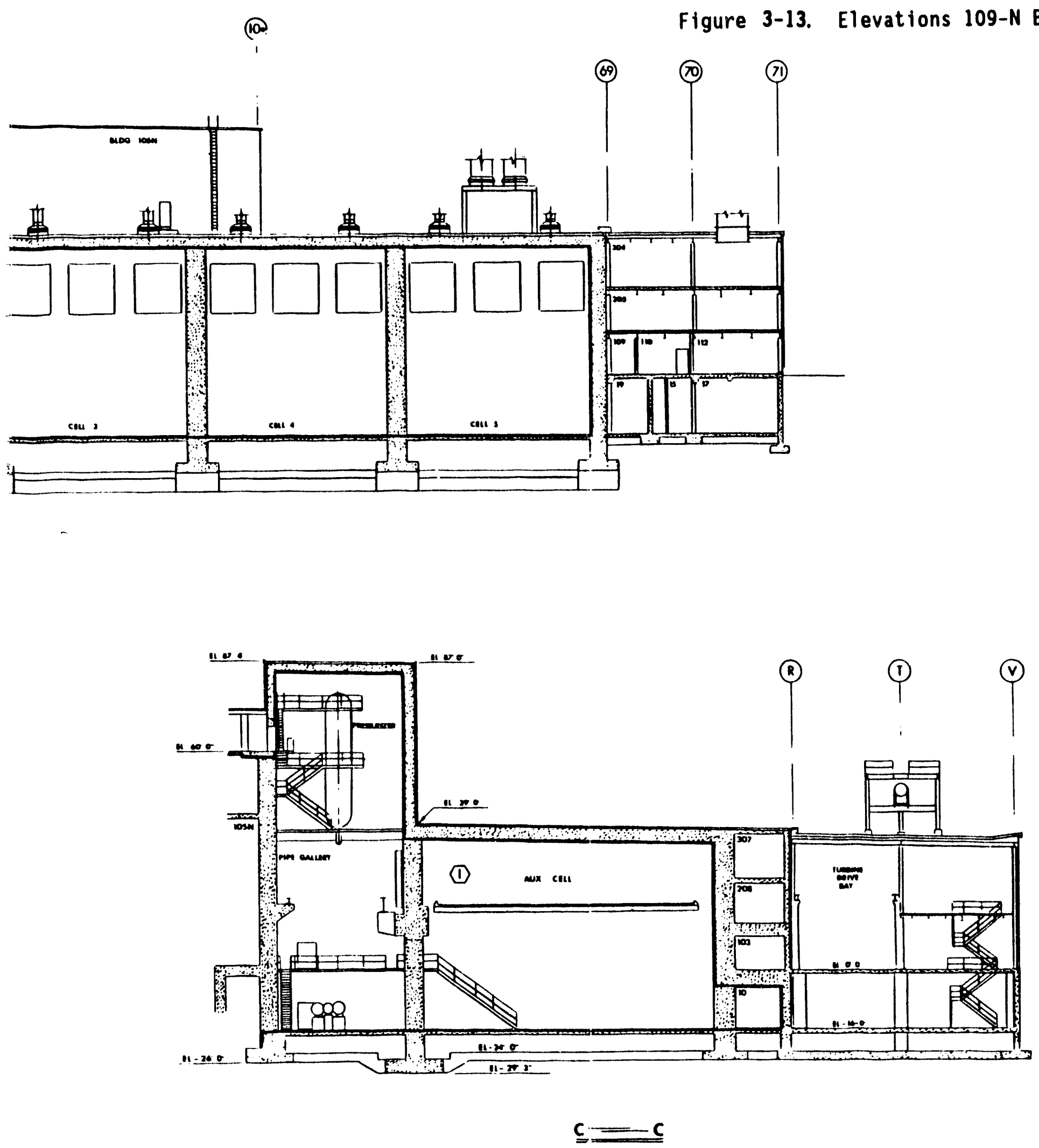
1. Marmoi Oporallon

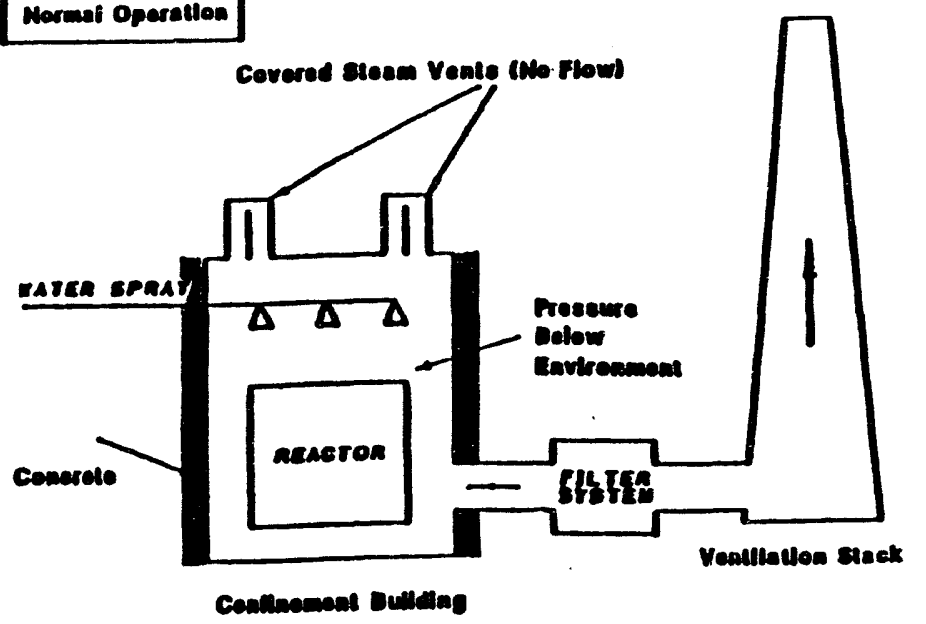

$\underset{\tilde{\omega}}{\tilde{\omega}}$

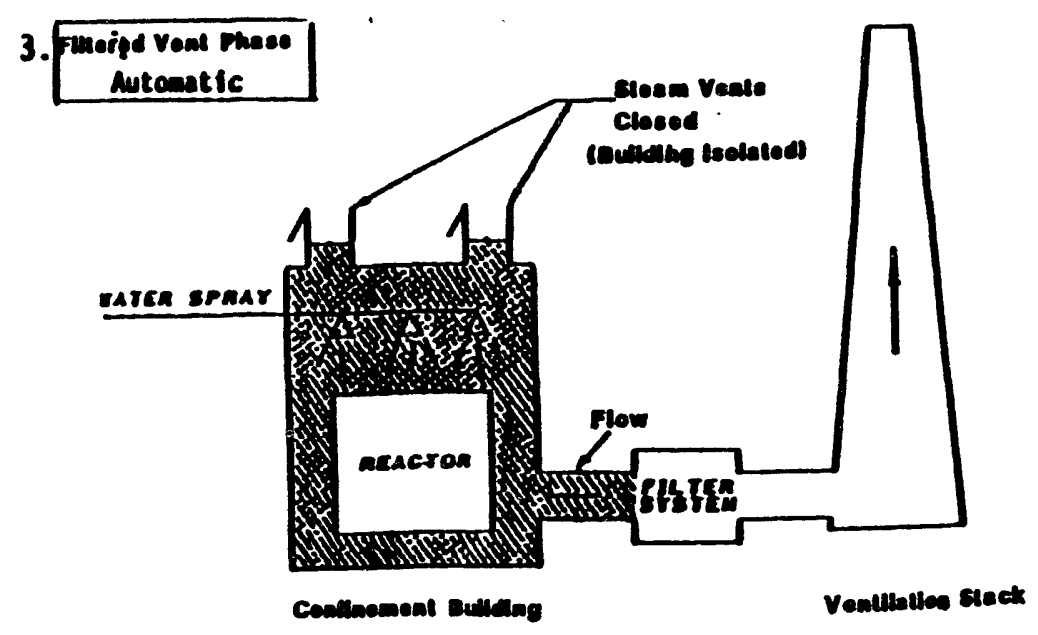

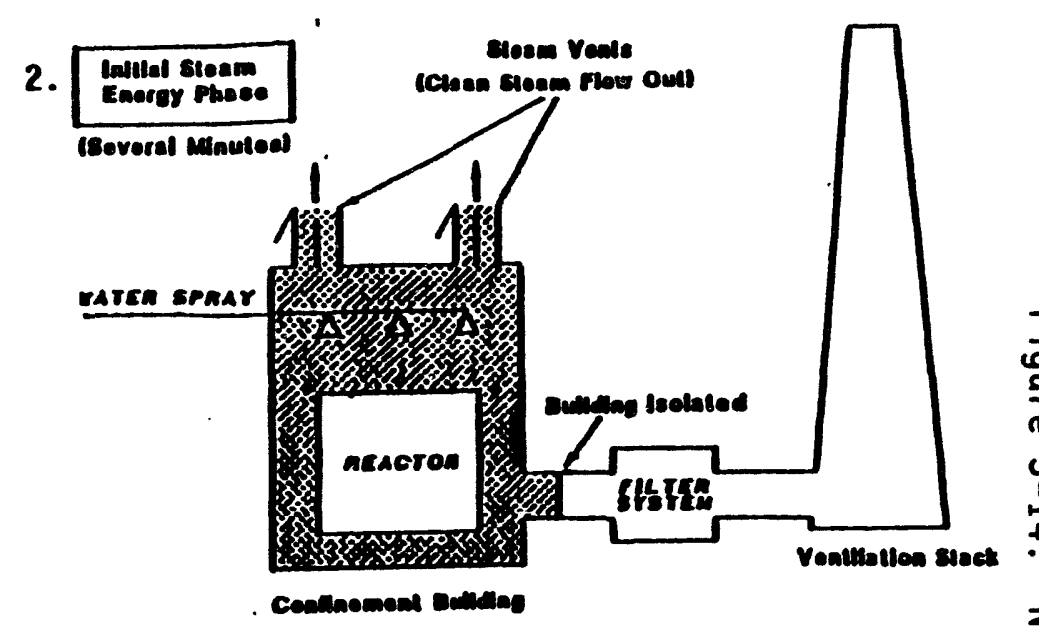

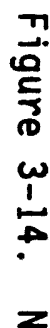

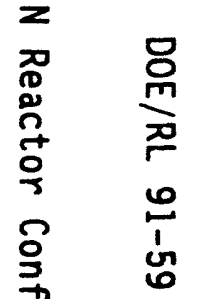

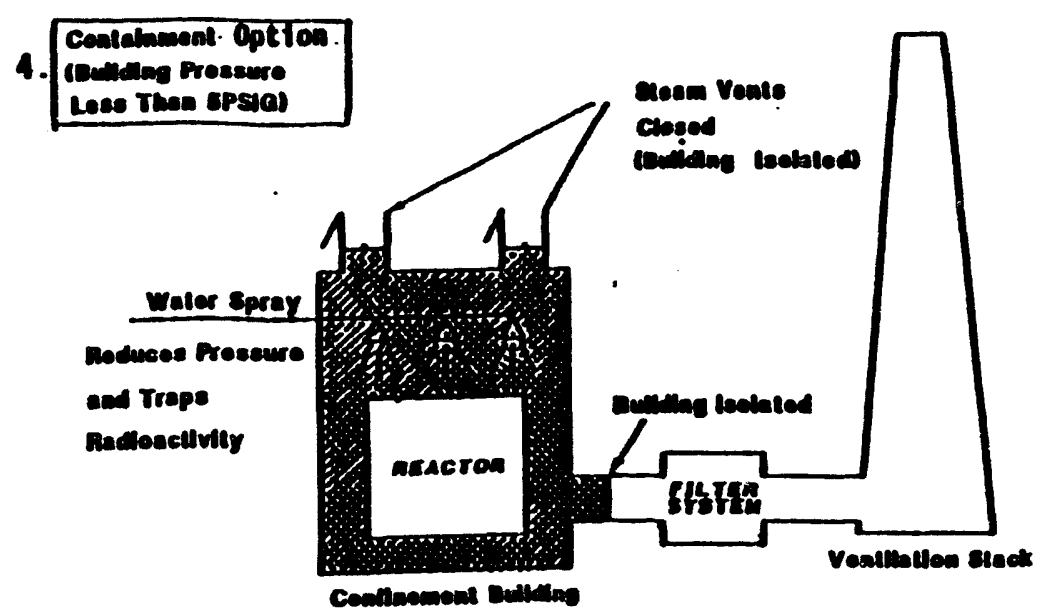




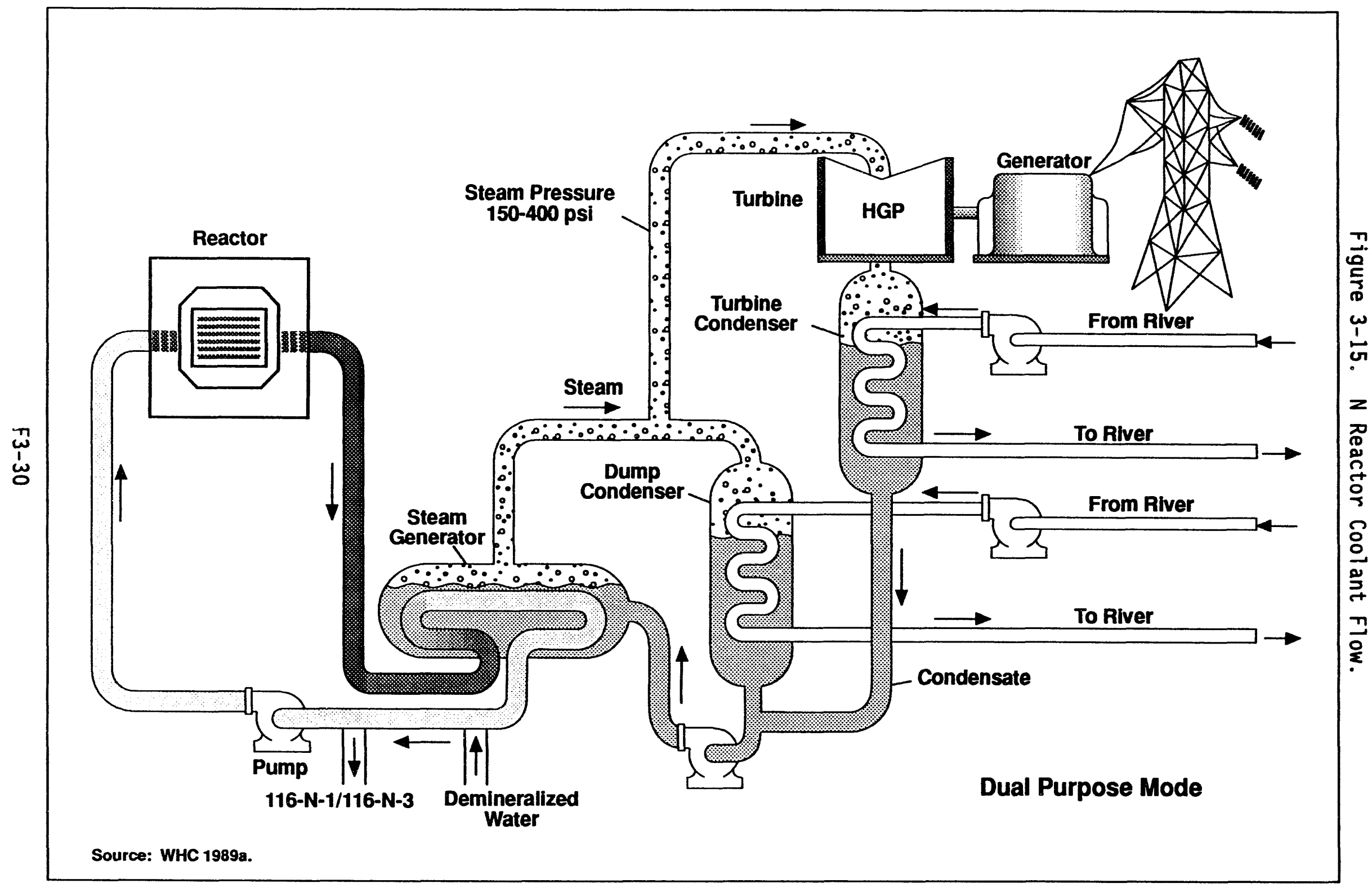

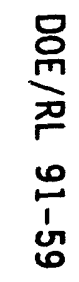


DOE/RL 91-59

Figure 3-16. Water Supply System.

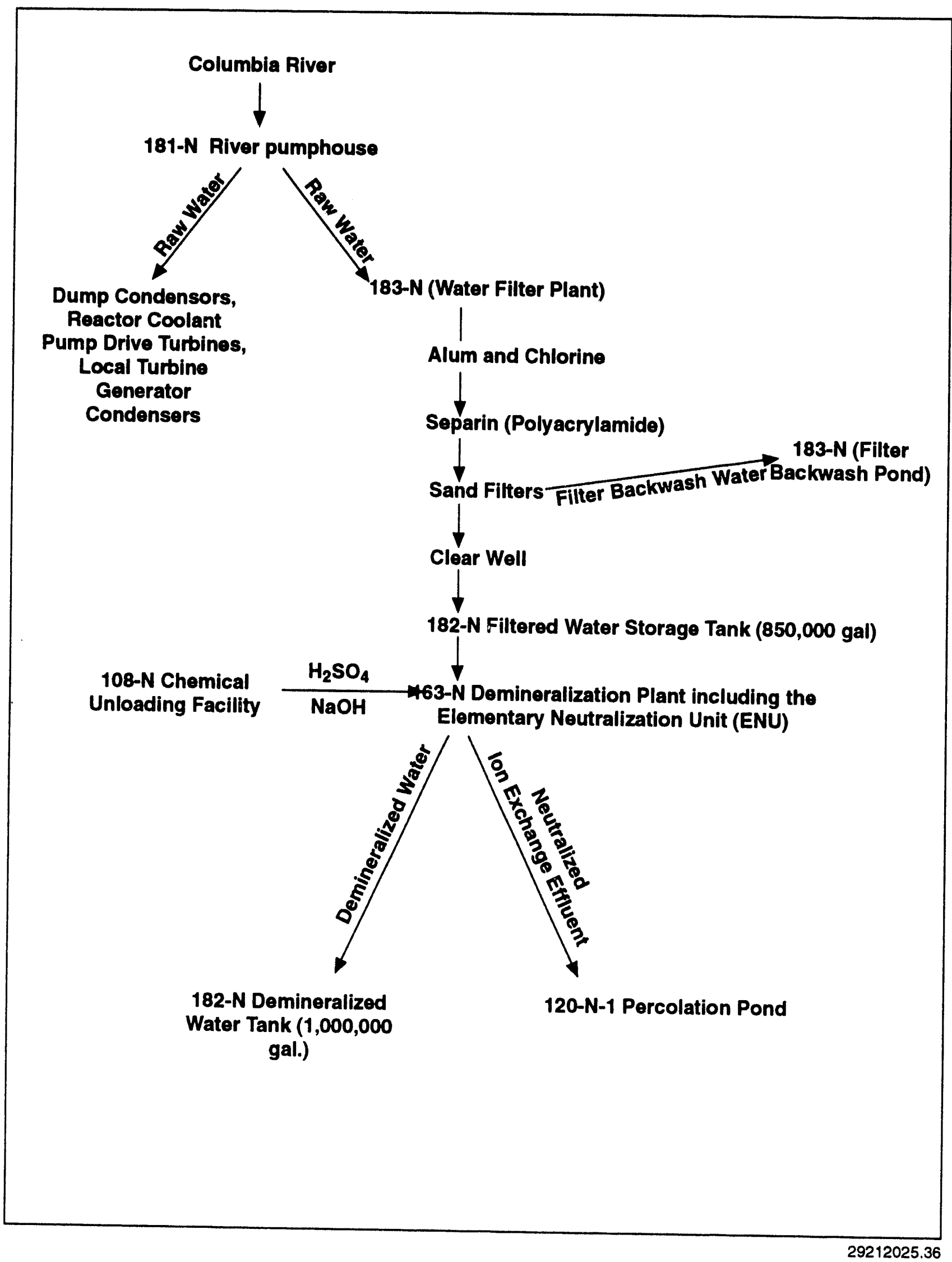




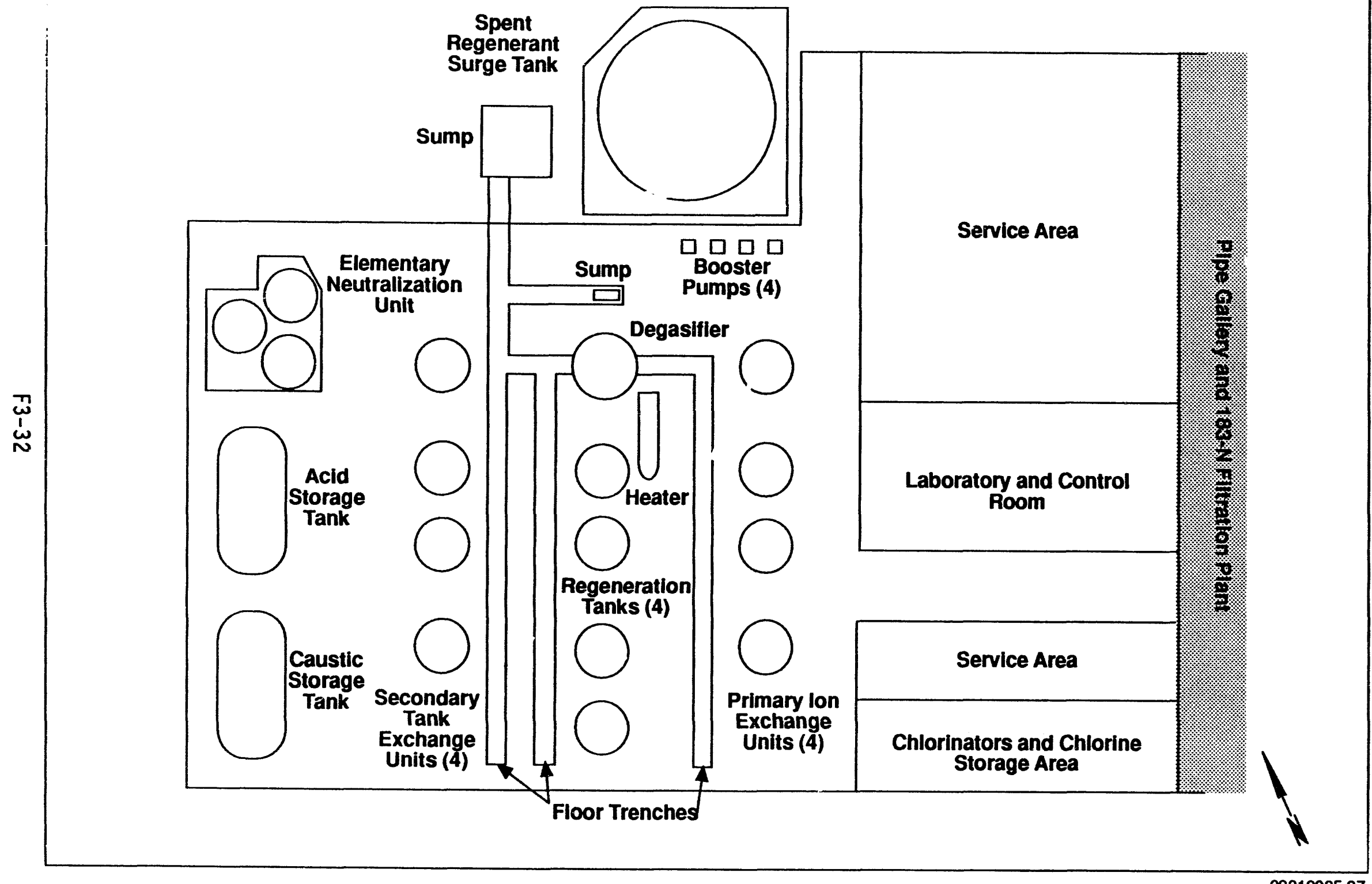

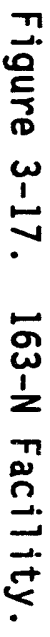




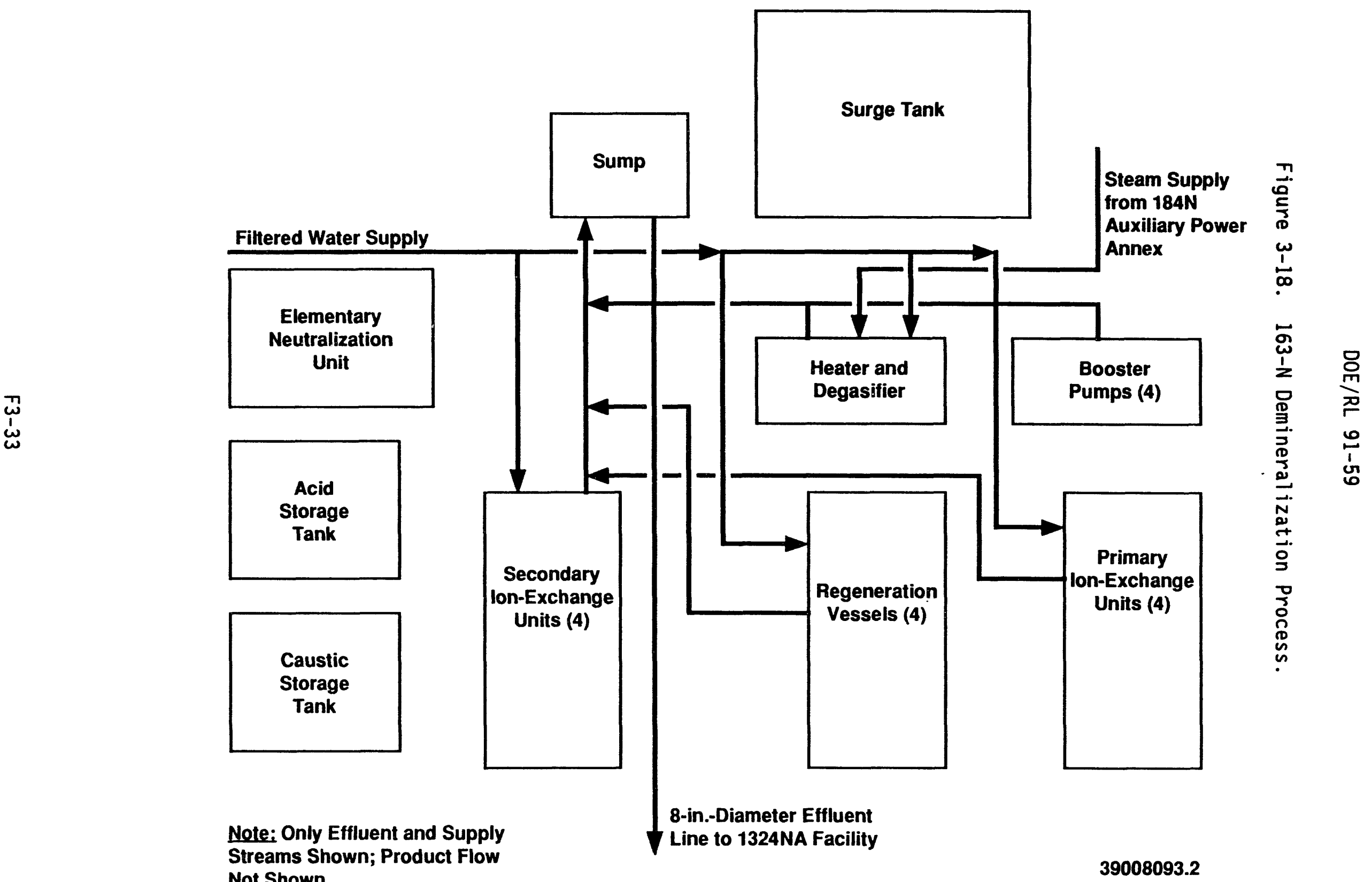


Figure 3-19. Layout of the 1301-N Liquid Waste Disposal Facility.

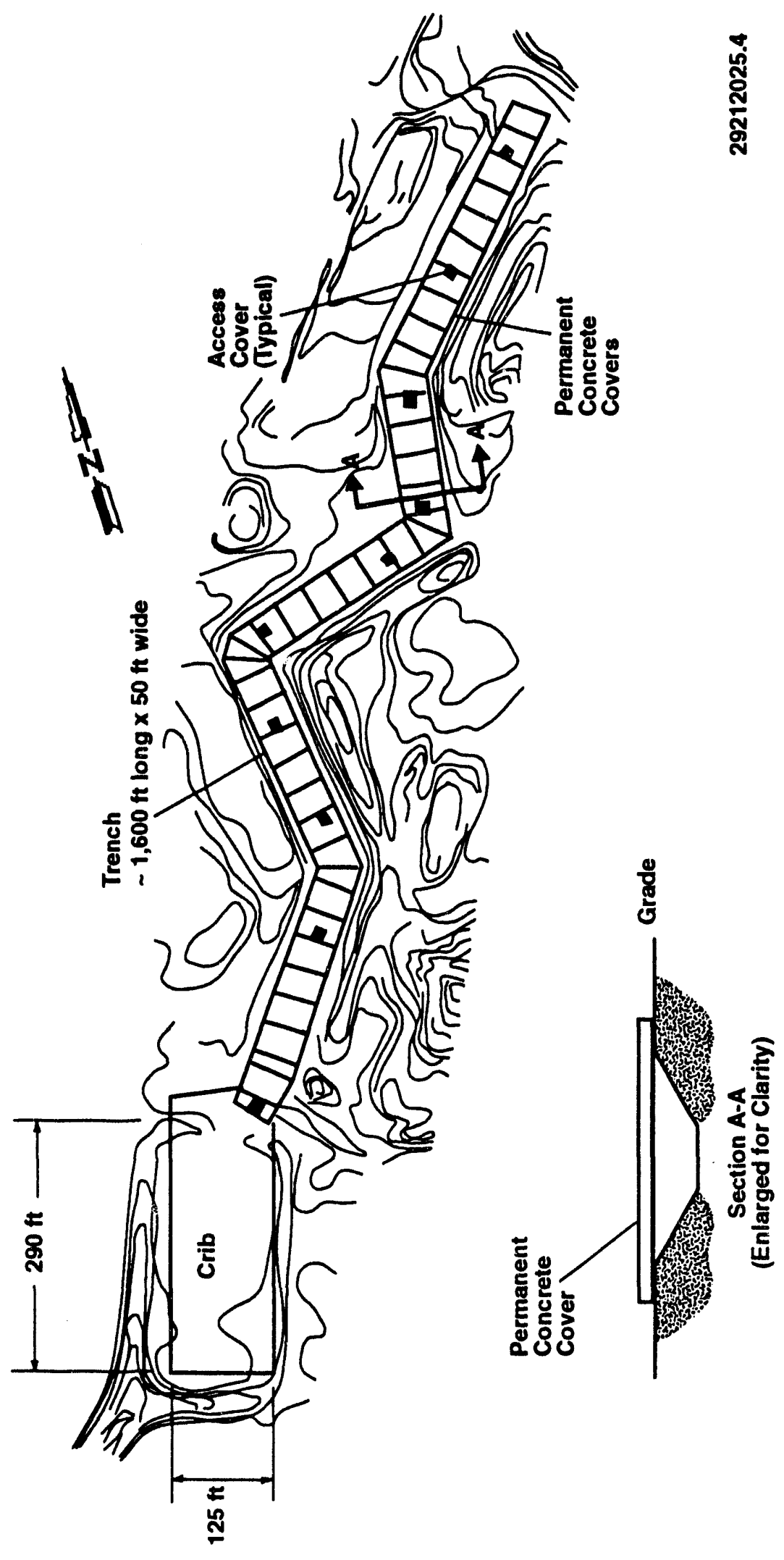




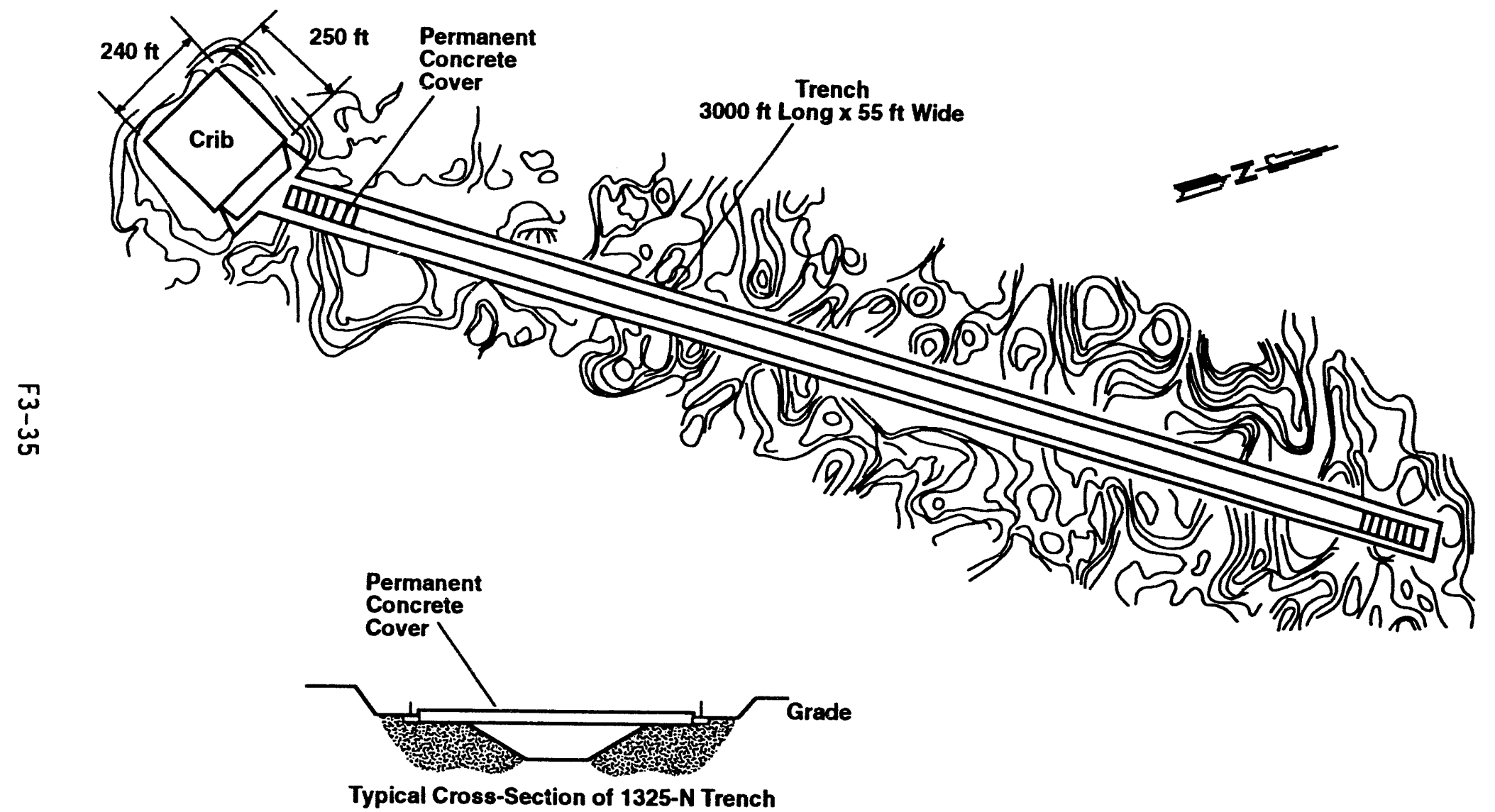

29212025.5

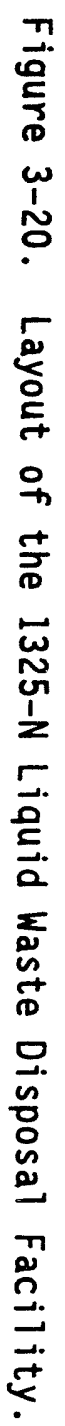


DOE/RL $91-59$

Figure 3-21. 1324-NA Facility Diagram with Dimensions, 1977 to 1983.

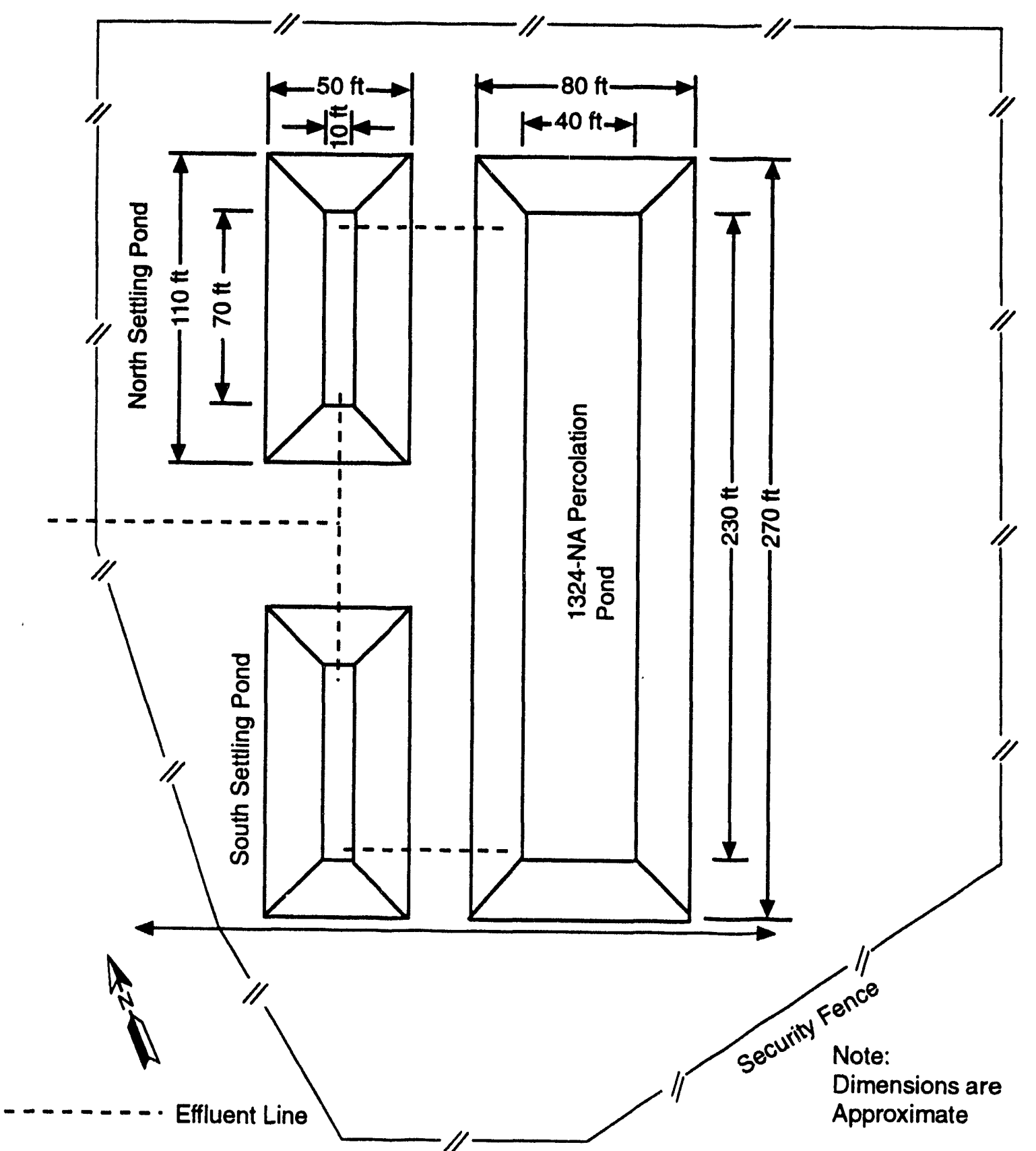

29212025.9 
Figure 3-22. 1324-N Surface Impoundment Physical Dimensions.

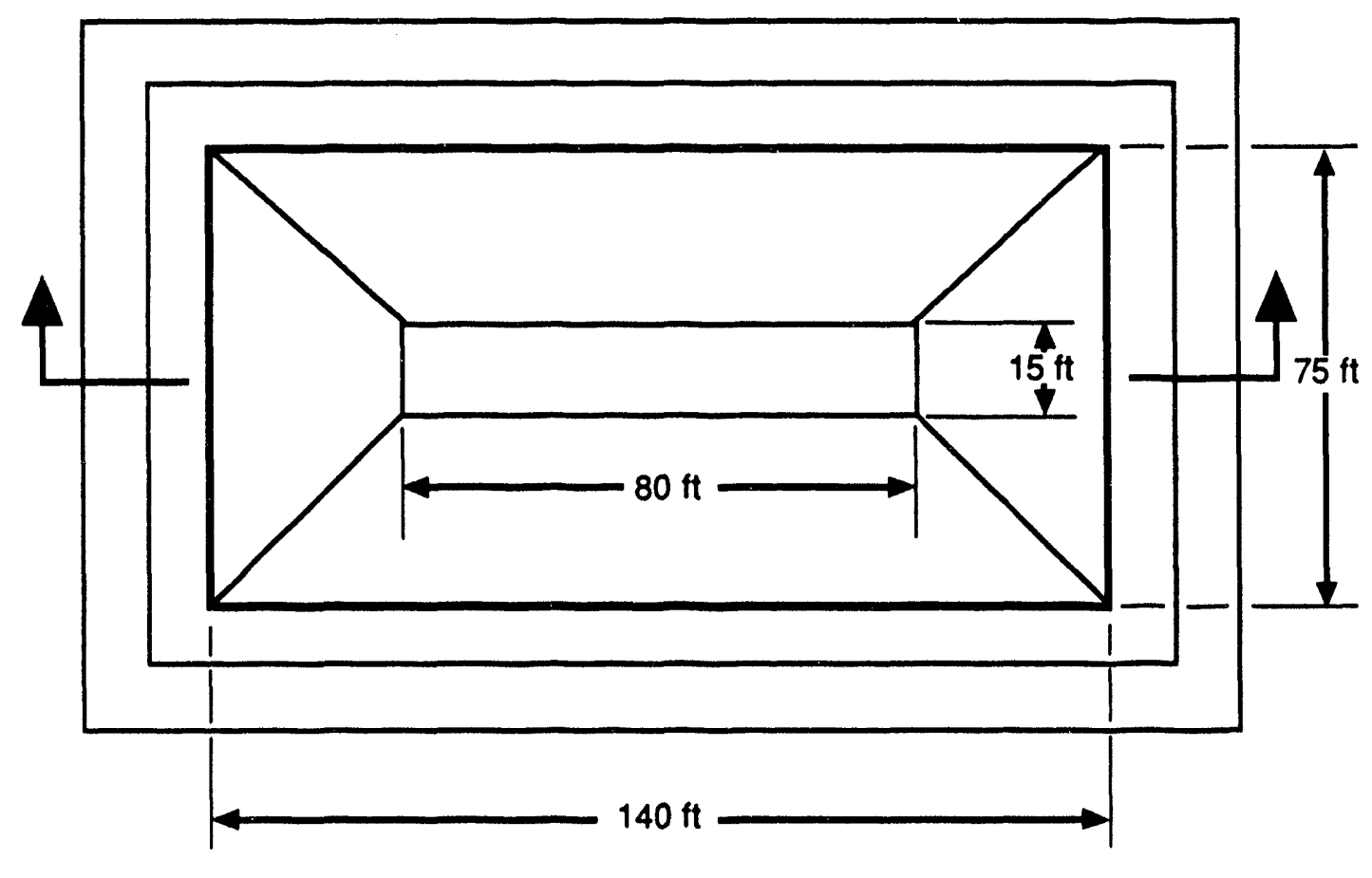

Plan View

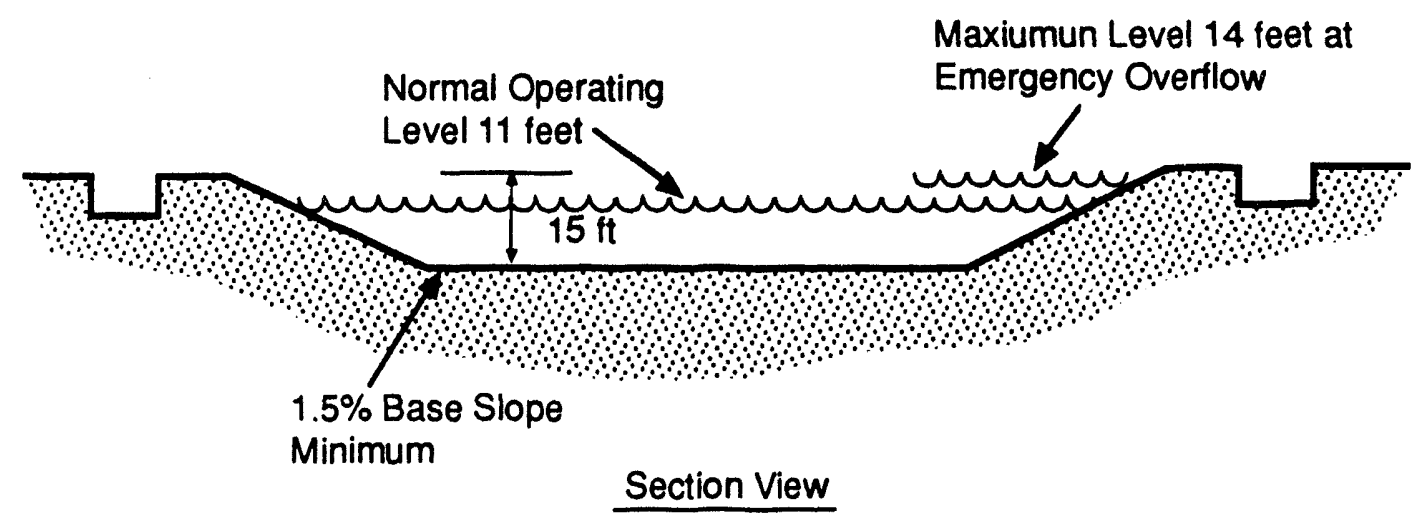

29212025.8 


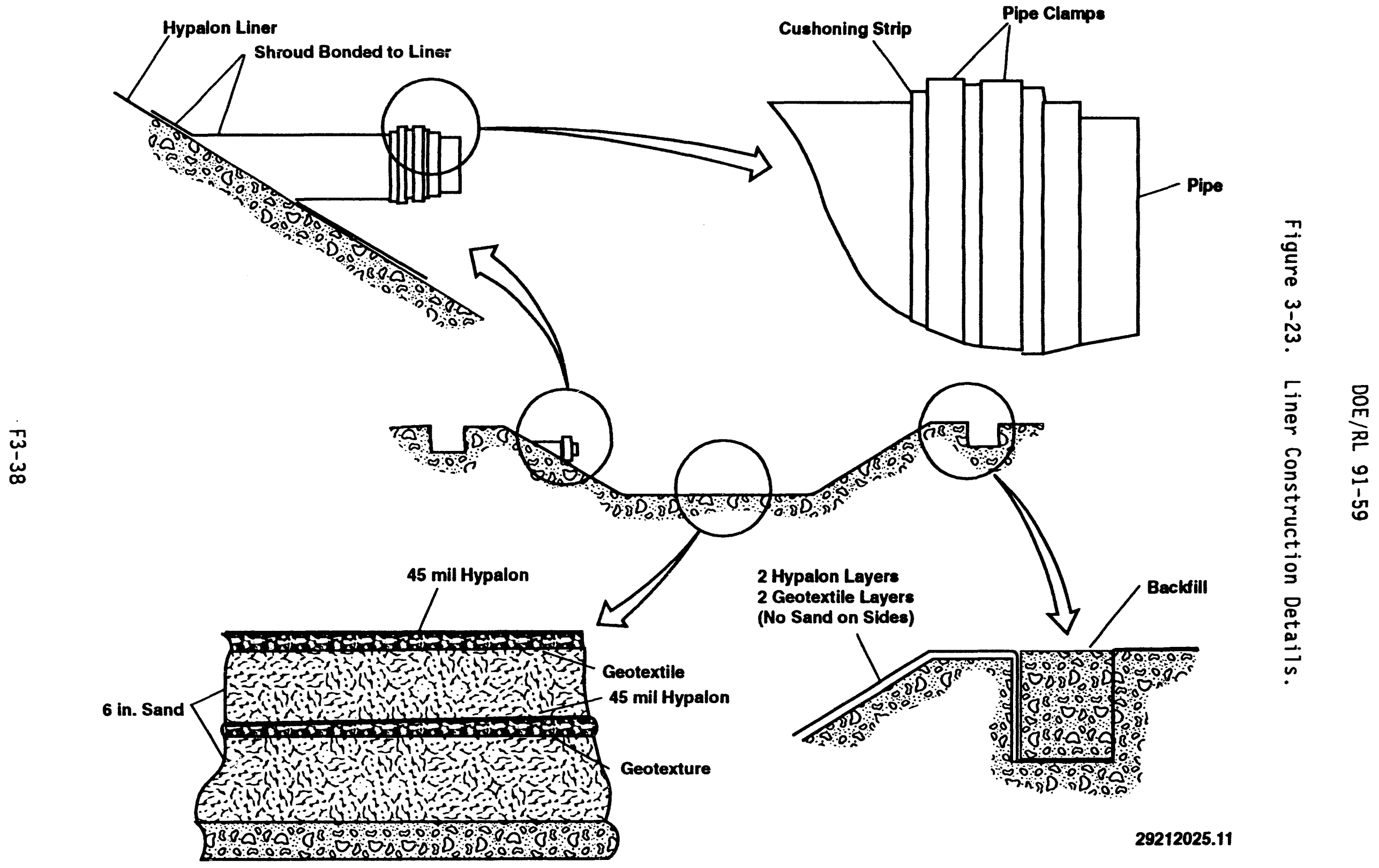




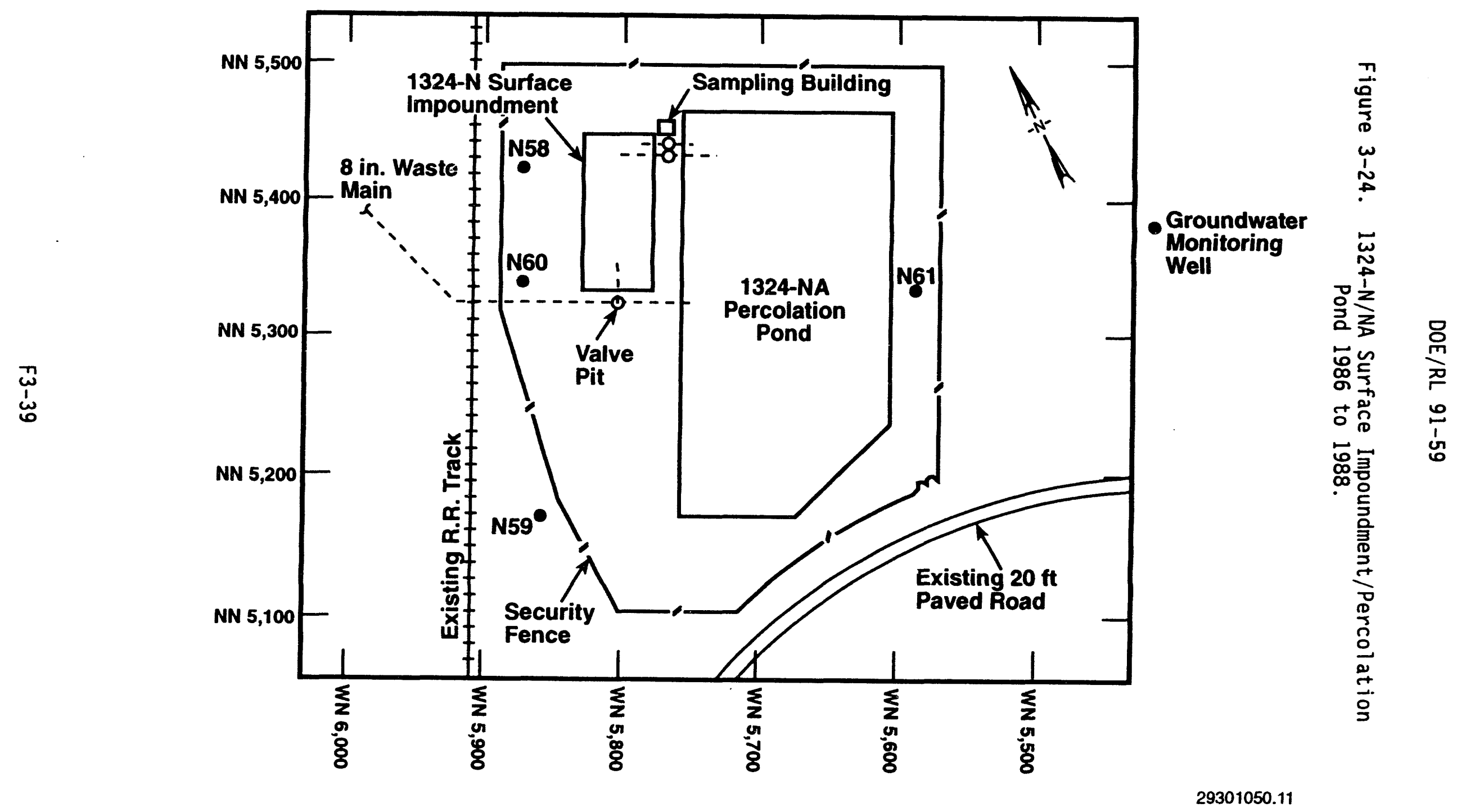


DOE/RL 91-59

This page intentionally left blank. 


\begin{tabular}{|c|c|c|c|}
\hline \begin{tabular}{|c|}
$\begin{array}{c}\text { Current Designation } \\
\text { (Alias) }\end{array}$ \\
\end{tabular} & Mame & Facility Purpose & Facility Description \\
\hline 105-N (N Reactor) & Reactor building & $\begin{array}{l}\text { Provides housing for } N \text { Reactor and } \\
\text { ancillary facilities. }\end{array}$ & $\begin{array}{l}\text { Monairtight reinforced concrete and steel } \\
\text { structure. Houses reactor, fuel storage, } \\
\text { reactor work areas, instrumentation room, and } \\
\text { transfer area. }\end{array}$ \\
\hline $107-n$ & $\begin{array}{l}\text { Basin recirculation } \\
\text { facility }\end{array}$ & $\begin{array}{l}\text { Contains fil tration system used to } \\
\text { remove radionucl ides from the } \\
\text { N Reactor basin water. }\end{array}$ & $\begin{array}{l}\text { Reinforced concrete structure. Chemical } \\
\text { storage tanks located outside building. }\end{array}$ \\
\hline $\begin{array}{l}108-N \\
(1106-N)\end{array}$ & $\begin{array}{l}\text { Chemical unloading } \\
\text { facility }\end{array}$ & $\begin{array}{l}\text { Unloading area for trucks and tank } \\
\text { cars conteining sul furic acid or } \\
\text { sodium hydroxide. }\end{array}$ & $\begin{array}{l}\text { Three abovegrourid sul furic acid tanks, one } \\
\text { aboveground sodium hydroxide tank and six } \\
\text { french drains. }\end{array}$ \\
\hline $109-N$ & Heat exchange building & $\begin{array}{l}\text { Provides means for dissipation of } \\
\text { reactor process heat. }\end{array}$ & $\begin{array}{l}\text { A reinforced concrete, structural steel } \\
\text { building with channeled steel siding. Shares } \\
\text { compon wall with south wall of the I Reactor } \\
\text { building. Houses six heat exchanger cells. } \\
\text { associated pumps, piping, laboratory, offices } \\
\text { and shop areas. }\end{array}$ \\
\hline$\vec{\omega}$ & $\begin{array}{l}\text { Steam and flow } \\
\text { building }\end{array}$ & $\begin{array}{l}\text { Houses hydraul ic power packs used for } \\
\text { supplying hydraul ic control power to } \\
\text { stean valves located in the } \\
109-1 \text { building. }\end{array}$ & steel butler-type building. \\
\hline $116-N$ & Air stack & $\begin{array}{l}\text { MEPA filtered exhaust air and gas from } \\
\text { the } N \text { Reactor ventilation system. }\end{array}$ & Reinforced concrete stack. \\
\hline $\begin{array}{l}116-N-1 \\
(1301-N)\end{array}$ & Crib and trench & $\begin{array}{l}\text { Received radioactive effluent from } \\
\text { weactor and } 109-\mu \text {. Water contained } \\
\text { activation and fission products and } \\
\text { small quantities of corrosive liquids } \\
\text { and laboratory wastes. }\end{array}$ & $\begin{array}{l}\text { Rectangular basin: } 290 \mathrm{ft} \text { long, } 125 \mathrm{ft} \text { wide, } \\
12 \mathrm{ft} \text { deep. The bottom is covered with } 3 \mathrm{ft} \text { of } \\
\text { large stones. An extension trench measured } \\
50 \text { by } 1000 \mathrm{ft} \text {. Trench surface covered by } \\
\text { concrete slabs. }\end{array}$ \\
\hline $\begin{array}{l}116-N-2 \\
(1310-N, \text { golf ball })\end{array}$ & storage tank & $\begin{array}{l}\text { Collection tank for } N \text { Reactor primary } \\
\text { piping decontamination wastes. }\end{array}$ & $\begin{array}{l}\text { Spherical steel structure uith storage capacity } \\
\text { of } 900,000 \mathrm{gal} \text {. }\end{array}$ \\
\hline $\begin{array}{l}116-M-3 \\
(1325-M)\end{array}$ & Crib and trench & $\begin{array}{l}\text { Receives radioactive activation and } \\
\text { fission products and small quantities } \\
\text { of corrosive liquids and laboratory } \\
\text { chemicals. }\end{array}$ & $\begin{array}{l}\text { Rectangular concrete diversion box } \\
(250 \times 240 \mathrm{ft} \text {, a header box, and }-1,200 \mathrm{ft} \text { of } \\
36-\text { in. di ameter pipel ine. covered with precast } \\
\text { concrete panels. Extension trench is } 3,000 \mathrm{ft} \\
\text { long, } 55 \mathrm{ft} \text { wide, and } 7 \mathrm{ft} \text { deep. }\end{array}$ \\
\hline
\end{tabular}




\begin{tabular}{|c|c|c|c|}
\hline $\begin{array}{c}\text { Current Designation } \\
\text { (Alias) }\end{array}$ & Mame & Facility Purpose & Facility Description \\
\hline $\begin{array}{l}116-N-4 \\
(1300-N)\end{array}$ & EDB & $\begin{array}{l}\text { Received steam blowdoun from N Reactor } \\
\text { steam generators containing low levels } \\
\text { of radioactive activation and fission } \\
\text { product. Also received water from } \\
\text { w Reactor lift station and possible } \\
\text { conteminated low-point drains. }\end{array}$ & $\begin{array}{l}\text { A steel-l ined storage pond with a } 650,000-\mathrm{gal} \\
\text { capacity. }\end{array}$ \\
\hline $\begin{array}{l}116-N-8 \\
\text { (163-N mixed waste } \\
\text { and hazardous waste } \\
\text { container storage } \\
\text { pad) }\end{array}$ & Storage ped & $\begin{array}{l}\text { Orum and containerized storage for } \\
\text { mixed and miscellaneous hazardous } \\
\text { process chemicals. }\end{array}$ & $\begin{array}{l}\text { A 152- } \times 60-f t \text { curbed and fenced concrete ped } \\
\text { located south of the 163-w building in a } \\
\text { three-sided building }\end{array}$ \\
\hline $117-N$ & $\begin{array}{l}\text { Exhaust air filter } \\
\text { building }\end{array}$ & $\begin{array}{l}\text { Filters ventilation air from the } \\
\text { confinement zone of the } \mathrm{NR} \text { Reactor } \\
\text { building before it is discharged to } \\
\text { the atmosphere. }\end{array}$ & $\begin{array}{l}\text { Reinforced concrete structure, olmost } \\
\text { completely below grade, } 4,290 \mathrm{ft}^{2} \text { of enclosed } \\
\text { space. }\end{array}$ \\
\hline 117-MVH & Valve control house & $\begin{array}{l}\text { Valve house for instrumentation and } \\
\text { controls. }\end{array}$ & $\begin{array}{l}\text { Small sheet metal building of opproximately } \\
80 \mathrm{ft}^{2} \text {. }\end{array}$ \\
\hline $\begin{array}{l}118-N-1 \\
(1303-N)\end{array}$ & Spacer storage silos & $\begin{array}{l}\text { Used for storing irradiated fuel } \\
\text { spacers from the N Reactor. }\end{array}$ & $\begin{array}{l}\text { The area consists of three silos, two } \\
\text { approximately } 16 \mathrm{ft} \text { in diameter and } 20 \text { ft deep. } \\
\text { The third smaller silo has a concrete floor; } \\
\text { the other two are open to the soil. All three } \\
\text { sil os are covered by concrete caps. }\end{array}$ \\
\hline $119-N$ & \begin{tabular}{|l} 
Air sampl ing and \\
monitoring
\end{tabular} & $\begin{array}{l}\text { Building houses air sampl ing equipment } \\
\text { used for monitoring airborne } \\
\text { emissions. }\end{array}$ & Prefabricated metal building. \\
\hline $\begin{array}{l}120-M-1 \\
(1324-M A \\
\text { percolation pond) }\end{array}$ & Percolation pond & $\begin{array}{l}\text { Received corrosive regeneration waste } \\
\text { and filter backwash water. }\end{array}$ & $\begin{array}{l}\text { Unl ined pond, } 29,000 \mathrm{ft}^{2} \text { with a total volume of } \\
\text { approximately } 2 \text { Mgal. }\end{array}$ \\
\hline $\begin{array}{l}120-N-2 \\
(1324-N \text { surface } \\
\text { impoundnent) }\end{array}$ & Surface impoundnent & $\begin{array}{l}\text { Received corrosive waste from } \\
163-n \text { building. }\end{array}$ & $\begin{array}{l}\text { Lined pond (1986-1988) } 140 \times 75 \times 15 \text { ft deep } \\
\text { with an approximate capacity of } 424,000 \text { gal. } \\
\text { Before } 1983 \text { was unl ined settl ing pond. }\end{array}$ \\
\hline $\begin{array}{l}128-N-1 \\
(100-M \text { burning pit })\end{array}$ & Burning pit & $\begin{array}{l}\text { Used to dispose conbustible materials, } \\
\text { such as muisance vegetation, office } \\
\text { wastes, tools, hardware, and possibly } \\
\text { paints and solvents. }\end{array}$ & $\begin{array}{l}\text { Ares located approximately } 1,500 \mathrm{ft} \text { northeast } \\
\text { of } 1120-N \text { building, southeast of } 116-\mu-3 \text {. }\end{array}$ \\
\hline $\begin{array}{l}130-N-1 \\
(183-N \text { backwash } \\
\text { discharge pond) }\end{array}$ & Backwash disposal pond & Percolation pond for filter backwash. & Unl ined pond \\
\hline
\end{tabular}




\begin{tabular}{|c|c|c|c|}
\hline $\begin{array}{c}\text { Current Designation } \\
\text { (Alias) }\end{array}$ & Name & Facility Purpose & Facility Description \\
\hline $143-N$ & Snubber shop & $\begin{array}{l}\text { stored paint, miscellaneous products, } \\
\text { and small power equipment. }\end{array}$ & Hood structure \\
\hline $151-N$ & $\begin{array}{l}250 \mathrm{KV} \text { electric } \\
\text { substation }\end{array}$ & $\begin{array}{l}\text { Supplies electrical power to } \\
100-N \text { Area }\end{array}$ & Located north of $120-N-1$ percolation pond \\
\hline $153-n$ & Switch gear building & Houses a transformer/substation. & Block building of approximately $2,300 \mathrm{ft}^{2}$ \\
\hline $155-n$ & BPA switch yard & $\begin{array}{l}\text { Electrical power line junction and } \\
\text { switch yard }\end{array}$ & $\begin{array}{l}\text { Large area containing electrical energy } \\
\text { transmission equipment and support building }\end{array}$ \\
\hline $163-N$ & Demineral ization plant & $\begin{array}{l}\text { Provided demineral ized water for } \\
\text { reactor primary coolant system. }\end{array}$ & $\begin{array}{l}\text { Reinforced concrete and structural steel, } \\
82 \times 78 \times 40 \mathrm{ft} \text { high immediately west of } \\
183-\mathrm{M} \text { building with which it shares a common } \\
\text { wall }\end{array}$ \\
\hline $166-\mathrm{N}$ & $\begin{array}{l}\text { Oil pump house and } \\
\text { storage tank }\end{array}$ & Fuel oil transfer. & $\begin{array}{l}\text { Includes a } 1,375,000-\text { gal tank immediately } \\
\text { adjacent to the i715-w Facility and a two-story } \\
\text { block building }\end{array}$ \\
\hline $181-n$ & River pump house & $\begin{array}{l}\text { Provided raw Columbia River water to } \\
\text { various facilities. }\end{array}$ & Reinforced concrete structures \\
\hline $181-$ ME & HGP river pump house & $\begin{array}{l}\text { Provided raw Columbia River water to } \\
\text { Supply System facilities. }\end{array}$ & Reinforced concrete structure \\
\hline $182-n$ & High-lift pump house & $\begin{array}{l}\text { Provided cool ing water to } N \text { Reactor } \\
\text { and } 109-N \text { systems. }\end{array}$ & $\begin{array}{l}\text { Reinforced concrete building } 107 \times 97 \times 20 \mathrm{ft} \\
\text { high with chameled steel walls. Houses pumps } \\
\text { used to inject demineral ized water into cool ing } \\
\text { systems }\end{array}$ \\
\hline $183-N$ & Water filter plant & $\begin{array}{l}\text { Provided treated and fil tered water } \\
\text { for process applications. }\end{array}$ & $\begin{array}{l}\text { Consists of a chemical treatment facility, } \\
102 \times 84 \times 20 \mathrm{ft} \text { high flocculation basins, } \\
\text { filter system, and clearwell storage }\end{array}$ \\
\hline 184-N Day tanks & Storage tanks & Diesel and black oil storage. & $\begin{array}{l}\text { Two } 35,000 \text { gal fuel tanks and one } 8,000 \mathrm{gal} \\
\text { diesel oil tank }\end{array}$ \\
\hline $184-N$ & Power house & $\begin{array}{l}\text { Provided electrical power for routine } \\
\text { and emergency use and process steim. }\end{array}$ & $\begin{array}{l}\text { Reinforced concrete and structural steel } \\
\text { building } 112 \times 96 \times 70 \mathrm{ft} \text { high with channeled } \\
\text { steel siding }\end{array}$ \\
\hline 184-MA, NB, MC & Annex & $\begin{array}{l}\text { Houses two boilers that provided } \\
\text { auxil iary and additional process } \\
\text { steam. }\end{array}$ & Prefabricated metal buildings \\
\hline $185-\boldsymbol{N}$ & $\begin{array}{l}\text { HGP turbine generator } \\
\text { building }\end{array}$ & $\begin{array}{l}\text { Main building that houses turbine } \\
\text { generators used for electrical - energy } \\
\text { production. }\end{array}$ & $\begin{array}{l}\text { This Supply System facility used surplus } \\
\text { Reactor steam to generate electricity from } \\
1964 \text { to } 1987 \text {. }\end{array}$ \\
\hline
\end{tabular}




\begin{tabular}{|c|c|c|c|}
\hline \begin{tabular}{|c|} 
Current Designation \\
(Al ias)
\end{tabular} & Mame & Facility Purpose & Facility Description \\
\hline $1100-n$ & $\begin{array}{l}\text { Administrotion and } \\
\text { first-aid building }\end{array}$ & Vacant & $\begin{array}{l}\text { Two-story frame construction } 199 \times 35 \times 28 \mathrm{ft} \\
\text { high on a concrete foundation and floor }\end{array}$ \\
\hline $1101-N$ & Office building & Vacant & \\
\hline $\begin{array}{l}1102-N \text { to } 1105-N, \\
1107-N, 1109-N \text { to } \\
1111-N,\end{array}$ & office buildings & $\begin{array}{l}\text { Provides office space for } \\
\text { actministrative and technical staff. }\end{array}$ & Variable construction \\
\hline $1112-n$ & Badge house & Telecomminications & $\begin{array}{l}\text { Concrete block building with a glass and steel } \\
\text { walk-through }\end{array}$ \\
\hline $\begin{array}{l}1113-N \text { to } 1115-N, \\
1117-N \text { to } 1118-N,\end{array}$ & Office buildings & Provides office space. & Trailers \\
\hline $1116-N$ & simulator building & Control room training (formerly) & Trailer \\
\hline $119-n$ & Locker room building & Provides locker space & Irailer \\
\hline $1120-n$ & $\begin{array}{l}\text { Warehouse and training } \\
\text { building }\end{array}$ & Provides office space & Prefabricated metal building \\
\hline $1123-N$ & office building & Provides office space & Trailer \\
\hline $\mid 1124-N$ & $\begin{array}{l}\text { Office and records } \\
\text { control center }\end{array}$ & Provides office and storage space & Trailer \\
\hline $1125-N, 1126-N$ & Office buildings & Provides office space & Trailers \\
\hline $1127-N$ & $\begin{array}{l}\text { Mobile outage locker } \\
\text { room }\end{array}$ & Provides office space and change room & Trailer \\
\hline $1129-N$ & Special warehouse & Vacant & Trailer \\
\hline $1130-n$ & $\begin{array}{l}\text { sup change room } \\
\text { building }\end{array}$ & $\begin{array}{l}\text { Used as a changing and storage } \\
\text { facility }\end{array}$ & Trailer \\
\hline $\begin{array}{l}1131-N, \\
1133-N, \\
1135-N\end{array}$ & $\begin{array}{l}\text { Mobile office } \\
\text { buildings }\end{array}$ & Provides office space & Irailers \\
\hline $1140-N, 1141-N$ & Restrooms & Provided restroom space & Trailer \\
\hline $1142-N$ & Telephone center & Telecomminications/storage & Trailer \\
\hline $1143-N, 1143-M A, M B$ & $\begin{array}{l}\text { Paint shop and storage } \\
\text { buildings }\end{array}$ & Provides maintenance space & $\begin{array}{l}\text { Prefabricated metal buildings, paint shop, and } \\
\text { carpenter shops are approximately } \\
1,450 \mathrm{ft}^{2} \text { each }\end{array}$ \\
\hline
\end{tabular}




\begin{tabular}{|c|c|c|c|}
\hline $\begin{array}{c}\text { Current Designation } \\
\text { (Al ias) }\end{array}$ & Name & Facility Purpose & Facility Description \\
\hline $\begin{array}{l}1144-N \text { to } 1145-N, \\
1147-N, 1149-N \text { to } \\
1158-N, 1163-N\end{array}$ & |offices & General office space & Trailers \\
\hline $\mid 1304-N$ & EDT & $\begin{array}{l}\text { Received emergency blowdown of } \\
\text { thermally hot primary reactor cool ant } \\
\text { water }\end{array}$ & $\begin{array}{l}1,300,000-\text { gal storage tank located west of the } \\
\text { w Reactor building. }\end{array}$ \\
\hline $1312-N$ & LERF & $\begin{array}{l}\text { Backup to existing containment systems } \\
\text { to receive primary cool ing water }\end{array}$ & $\begin{array}{l}\text { HDPE bladder within a lined and bermed } \\
\text { impoundhent. This facility has never been } \\
\text { used. }\end{array}$ \\
\hline $1313-n$ & $\begin{array}{l}\text { Change and control } \\
\text { room }\end{array}$ & $\begin{array}{l}\text { Remote instrumentation control for } \\
\text { waste transfers to } 116-\mathrm{N}-2\end{array}$ & Small metal building of approximately $140 \mathrm{ft}^{2}$ \\
\hline $\mid \begin{array}{l}116-N-4 \\
(1300-N)\end{array}$ & EDB & $\begin{array}{l}\text { Received emergency blowdown of } \\
\text { thermal ly hot primary reactor cool ant } \\
\text { water. Replaced by } 1304-N \text {. }\end{array}$ & $\begin{array}{l}1,000,000 \text { gal storage basin located west of the } \\
\text { w Reactor building. }\end{array}$ \\
\hline $\mid 1314-N$ & LLLLS & $\begin{array}{l}\text { Received spent radioactive } \\
\text { decontamination liquid from 116- } N-2 \\
\text { facility and } 107-N \text { facility for } \\
\text { transfer to railway tank farms }\end{array}$ & $\begin{array}{l}\text { Curbed pad with prefabricated metal building. } \\
\text { Before } 1976 \text { the site consisted of concrete ped. }\end{array}$ \\
\hline $1315-n$ & $\begin{array}{l}\text { Diversion system valve } \\
\text { house }\end{array}$ & $\begin{array}{l}\text { Houses valves that control discharge } \\
\text { to the crib }\end{array}$ & A $130-\mathrm{ft}^{2}$ steel building \\
\hline $1316 \mathrm{~N}$ & Valve house & $\begin{array}{l}\text { Houses valves that control discharges } \\
\text { to cribs }\end{array}$ & Small steel building \\
\hline$\left|\begin{array}{l}1322-\mathrm{N} \text { and } 1322-\mathrm{MA}, \\
\mathrm{NB}, \mathrm{NC}\end{array}\right|$ & $\begin{array}{l}\text { Pilot plant treatment } \\
\text { facility/sample } \\
\text { building and valve } \\
\text { house }\end{array}$ & $\begin{array}{l}\text { Sample station. Buildings contained } \\
\text { automated sampling equipment used to } \\
\text { sample the } N \text { Springs and radioactive } \\
\text { drainl ines and the } 36 \text { in. and } 12 \text { in. } \\
\text { effluent lines. }\end{array}$ & Small prefabricated metal building \\
\hline $1701-N, 1701-$ ME & Badge house & Security & Trailer \\
\hline $1702-N$ & Vehicle inspection & Security & Small portable $6 \times 8 \mathrm{ft}$ shed \\
\hline $1703-N$ & HGP offices & Offices & \\
\hline $1705-N$ & $\begin{array}{l}\text { Instrument and } \\
\text { electrical facility }\end{array}$ & $\begin{array}{l}\text { Provides space for instrutzant and } \\
\text { electrical shops. }\end{array}$ & Large steel building with offices and shops \\
\hline $1706-N$ & Shop area & Provide shop and storage space. & Prefabricated metal building \\
\hline $1707-N$ & Storage boat house & Provides storage space. & Metal \\
\hline $1712-N$ & Insulation shop & Provides maintenance space. & Hood \\
\hline
\end{tabular}




\begin{tabular}{|c|c|c|c|}
\hline $\begin{array}{c}\text { Current Designation } \\
\text { (Al ias) }\end{array}$ & Name & Facility Purpose & Facility Description \\
\hline $1714-N$, and $1714-M A$ & $\begin{array}{l}\text { Warehouse and } \\
\text { receiving facility }\end{array}$ & Provides storage space. & Prefabricated metal building \\
\hline $1715-\mu$ & $\begin{array}{l}\text { Diesel oil storage } \\
\text { tanks }\end{array}$ & $\begin{array}{l}\text { Storage of diesel oil used by pumping } \\
\text { systems. }\end{array}$ & Four 105,000-gal aboveground diesel oil tanks \\
\hline $1722-\mu$ & $\begin{array}{l}\text { Decontamination hot } \\
\text { shop building }\end{array}$ & $\begin{array}{l}\text { Provides decontamination space and } \\
\text { area for working wi th contaminated } \\
\text { equipment. }\end{array}$ & |Transite- and steel-sided buildings \\
\hline $\mid 1723-\mu$ & $\begin{array}{l}\text { Contaminated equipment } \\
\text { storage building }\end{array}$ & $\begin{array}{l}\text { Provides storage space. } 1980 \text { to } 1984 \\
\text { stored conteminated equipment. }\end{array}$ & $\begin{array}{l}\text { In } 1984 \text { the area was decontaminated and mode } \\
\text { into shipping and receiving warehouse }\end{array}$ \\
\hline $1734-N$ & Gas bottle storage & Store pressurized bottles. & Block, transite, and steel construction \\
\hline $1900-\mu$ & Hater supply tanks & Meets the plant's water storage needs. & Steel tanks \\
\hline |1908-N & Seal well & $\begin{array}{l}\text { Provides access to the } 102 \text { in. } \\
\text { discharge line for sampl ing, etc. }\end{array}$ & Concrete-reinforced wier box \\
\hline 1908-NE & HGP seal well & $\begin{array}{l}\text { Provides access for the river } \\
\text { discharge for sampl ing, etc. }\end{array}$ & Concrete-reinforced wier box \\
\hline$N-1$ to $M-30$ & \begin{tabular}{|l|} 
Craft shops and of fice \\
buildings
\end{tabular} & Provides office and shop space. & $\begin{array}{l}\text { Trailers, wood, and steel buildings. Man } \\
\text { being removed. }\end{array}$ \\
\hline
\end{tabular}

Sources: DOE-RL 1991, AEC-GE 1964, and UHC personnel.

$B P A=$ Borneville Power Administration

$E D B=$ emergency dimp basin

EDT = emergency dump tank

HDPE $=$ high-density polyethylene

MEPA = high-efficiency particulate air (filter)

HGP = Hanford Generating Plant

LERF = Liquid Effluent Retention Facility

LELS = Liquid Maste Loadout Station

Supply System = Mashington Public Power Supply System

$\begin{aligned} \text { SWP } & =\text { Special Work Permit (protect ive clothing) }\end{aligned}$

WHC = Westinghouse Hanford Company

*and the 36" and 12" effluent lines 


\begin{tabular}{|c|c|c|c|c|c|}
\hline Maste Stream & Origin & Type & Volume. & Disposal Location & $\begin{array}{c}\text { Maste } \\
\text { Characteristics }\end{array}$ \\
\hline \multicolumn{6}{|l|}{ Primary Process Systems } \\
\hline $\begin{array}{l}\text { Reactor } \\
\text { Decontamination yaste }\end{array}$ & 105-N Zone I & Sol id & $\begin{array}{l}600,000 \text { gal per } \\
\text { decontamination } \\
\text { event }\end{array}$ & $\begin{array}{l}\text { Shipped to } 200 \text { Area } \\
\text { tank farm }\end{array}$ & Low level* \\
\hline $\begin{array}{l}\text { 163-N } \\
\text { Demineral ization } \\
\text { Backwash Maste }\end{array}$ & $\begin{array}{l}163-N \\
\text { demineral ization } \\
\text { water plant }\end{array}$ & Sol id & $-400,000 \mathrm{gal} / \mathrm{d}$ & $\begin{array}{l}\text { Neutral ized at } \\
1324-M \text { then } \\
\text { discherged to } \\
1324-\text { ma }\end{array}$ & Corrosive liquid \\
\hline $\begin{array}{l}\text { 107-N Demineralizer } \\
\text { Colum Regeneration } \\
\text { Maste }\end{array}$ & $107-\mu$ & Solid & ma & $\begin{array}{l}\text { Neutral ized at } \\
107-\mu \text { tank then } \\
\text { shipped to } 200 \text { Area } \\
\text { tank farm }\end{array}$ & $\begin{array}{l}\text { Low-level } \\
\text { redioact ive waste, } \\
\text { corrosive }\end{array}$ \\
\hline \multicolumn{6}{|l|}{ Sol id Maste } \\
\hline $\begin{array}{l}\text { 1143-M Paint Shop } \\
\text { Mastes }\end{array}$ & $1143-N$ point shop & Solid & $-55 \mathrm{gal}$ in 2 months & $\begin{array}{l}\text { Drum transferred to } \\
116-M-8 \text { storage pad } \\
\text { then to } 616 \text { blda } \\
\end{array}$ & \begin{tabular}{|l} 
Mazardous waste \\
including MEK, \\
ignitable
\end{tabular} \\
\hline $\begin{array}{l}\text { Maintenance Shop } \\
\text { Conteminated Rags }\end{array}$ & $105-\mu$ & Sol id & -55 gal in 4 months & $\begin{array}{l}\text { Drum transferred to } \\
163-N \text { storage pad } \\
\text { then to } 616 \mathrm{bldg}\end{array}$ & Igni table \\
\hline $\begin{array}{l}\text { 105-N Battery Locker } \\
\text { Wastes }\end{array}$ & $105-n$ & Solid & $\begin{array}{l}\text { Variable, } \\
<100 \mathrm{gal} / \mathrm{yr}\end{array}$ & $\begin{array}{l}\text { Acid collected in } \\
13-g a l \text { carboys and } \\
\text { transferred to } \\
116-N-8 \text {, then to } \\
616 \mathrm{bldg}\end{array}$ & $\begin{array}{l}\text { Toxic (cadmium, } \\
\text { lead), corrosive }\end{array}$ \\
\hline $\begin{array}{l}\text { Water Oual ity } \\
\text { Laboratory Mastes }\end{array}$ & $109-N$ & Solid & $\begin{array}{l}300 \mathrm{ml} / \mathrm{d} \text { when } \\
\text { reactor not } \\
\text { operat ing }\end{array}$ & $\begin{array}{l}116-N-8 \text { storage pad } \\
\text { then } \\
\text { non-radioactive } \\
\text { transferred to } 616 \\
\text { bldg }\end{array}$ & $\begin{array}{l}\text { Low-level*", } \\
\text { corrosive, toxic } \\
\text { (mercury) }\end{array}$ \\
\hline $\begin{array}{l}116-N-8(163-N \\
\text { Storage Pad) }\end{array}$ & 100-M/K Areas & Solid & $\begin{array}{l}\text { 1,000 drums } \cdots \text { can be } \\
\text { stored at one time }\end{array}$ & $\begin{array}{l}616 \mathrm{bldg} \text { or } \\
\text { radioactive } \\
\text { retrievable storage } \\
\text { units }\end{array}$ & $\begin{array}{l}\text { Hazardous and } \\
\text { low-level } \\
\text { radioact ive waste }\end{array}$ \\
\hline 1310-N Storage Pad & 100-N/K Areas & Solid & $\begin{array}{l}200 \text { drums ' can be } \\
\text { stored at one time }\end{array}$ & MA & Maste oil \\
\hline
\end{tabular}




\begin{tabular}{|c|c|c|c|c|c|}
\hline Maste Stream & Origin & Type & Volume & Disposal Location & $\begin{array}{c}\text { Maste } \\
\text { Characteristics }\end{array}$ \\
\hline \multicolumn{6}{|l|}{ Liquid Mastes } \\
\hline 183-N Filter Backwash & $\begin{array}{l}\text { 183-N Filtered Water } \\
\text { Plant }\end{array}$ & Liquid & $\begin{array}{l}300,000 \text { to } \\
470,000 \mathrm{gal} / \text { day } \\
(0,30 \mathrm{to} / \mathrm{day}) \\
0.47 \mathrm{mgal} / \text { day })\end{array}$ & $\begin{array}{l}\text { Fil ter backwash } \\
\text { pond }\end{array}$ & Sand filter backwash \\
\hline 163-N Process Water & $163-N$ & Liquid & 200 to $300 \mathrm{gal} / \mathrm{min}$ & $1324-M / M A$ & $\begin{array}{l}\text { Acid and Caustic } \\
\text { liquid waste }\end{array}$ \\
\hline $\begin{array}{l}\text { 105/109-N Radioactive } \\
\text { Liquid Discharges }\end{array}$ & $105 / 109-N$ & Liquid & $\begin{array}{l}1,300 \text { to } 2,000 \\
\mathrm{gal} / \mathrm{min}\end{array}$ & $1325-M$ and $1301-M$ & $\begin{array}{l}\text { Low level liquid } \\
\text { effluent** }\end{array}$ \\
\hline $\begin{array}{l}\text { 182-N Tank Farm } \\
\text { Overflow }\end{array}$ & $182-M$ & Liquid & $\begin{array}{l}4 \text { to } 7.2 \text { mod without } \\
\text { low lifts; } 50 \mathrm{mg} \text { S } \\
\text { with low i ifts }\end{array}$ & Columbia River & $\begin{array}{l}\text { t\#MPDES permit } \\
\text { ma-000374-3 }\end{array}$ \\
\hline 182-N Drain System & $182-N$ & Liquid & $\begin{array}{l}0.29 \text { mod wi thout fog } \\
\text { spray; } 5.4 \text { to } 26 \mathrm{mgd} \\
\text { with fog spray }\end{array}$ & Columbia River & $\begin{array}{l}\text { \#MPDES permit } \\
\text { MA-000374-3 }\end{array}$ \\
\hline $\begin{array}{l}\text { 181- } N \text { Inlet Screen } \\
\text { Backwash }\end{array}$ & $181-n$ & Liquid & 0.31 to $0.48 \mathrm{mgd}$ & Columbia River & $\begin{array}{l}\text { *MPDES permit } \\
\text { MUA-000374-3 }\end{array}$ \\
\hline 102 in. Outfall & $105 / 109-N$ & Liquid & 390 to $610 \mathrm{mgd}$ & Columbia River & $\begin{array}{l}\text { twPDES permit } \\
\text { mu-000374-3 }\end{array}$ \\
\hline M-Springs & $\begin{array}{l}\text { 1325-N and 1301-N } \\
\text { (via soil column) }\end{array}$ & Liquid & 0.96 to $1.4 \mathrm{mgd}$ & Columbia River & $\begin{array}{l}\text { *MPDES permit } \\
\text { MA-000374-3; plus } \\
\text { radioactive } \\
\text { const i tuents }\end{array}$ \\
\hline \multicolumn{6}{|l|}{ Airborne Emissions } \\
\hline $105 / 109-N$ & $105 / 109-N$ & Gas & $210,000 \mathrm{ft}^{3} /$ min & 116-N stack & $\begin{array}{l}\text { Radioactive } \\
\text { constituents }\end{array}$ \\
\hline $109-N$ & $\begin{array}{l}\text { Zone } 1 \\
\text { Cell } 1 \text { to } 5\end{array}$ & Gas & $120,000 \mathrm{ft}^{3} / \mathrm{min}$ & $\begin{array}{l}109-\mu \text { Zone I } \\
\text { cell } 1 \text { to } 6 \text { Vents }\end{array}$ & $\begin{array}{l}\text { Radioactive } \\
\text { constituents }\end{array}$ \\
\hline $109-N$ & $\begin{array}{l}\text { Zone } 1 \\
\text { cell } 6\end{array}$ & Gas & $28,000 \mathrm{ft}^{3} / \mathrm{min}$ & 109-cell 6 vent & $\begin{array}{l}\text { Radioactive } \\
\text { constituents }\end{array}$ \\
\hline $109-N$ & Zone 11 & Gas & $23,000 \mathrm{ft}^{3} / \mathrm{min}$ & $\begin{array}{l}\text { 105/109-N Zone II } \\
\text { exhaust fan }\end{array}$ & $\begin{array}{l}\text { Radioactive } \\
\text { constituents }\end{array}$ \\
\hline $109-N$ & Zone III & Gas & $130,000 \mathrm{ft}^{3} / \mathrm{min}$ & $\begin{array}{l}\text { Zone } 111 \text { exhaust } \\
\text { fan } 10\end{array}$ & $\begin{array}{l}\text { Radioactive } \\
\text { constituents }\end{array}$ \\
\hline
\end{tabular}




\begin{tabular}{|c|c|c|c|c|c|}
\hline Waste Stream & Origin & Type & Volume & Disposal Location & $\begin{array}{c}\text { Waste } \\
\text { Characteristics }\end{array}$ \\
\hline $109-N$ & Zone IV & Gas & $1,600 \mathrm{ft}^{3} / \mathrm{min}$ & $\begin{array}{l}\text { Zone IV exhaust } \\
\text { fans } 14 \text { and } 15\end{array}$ & $\begin{array}{l}\text { Radioact ive } \\
\text { const ituents }\end{array}$ \\
\hline 105-N Transfer Area & $105-N$ & Gas & $28,000 \mathrm{ft}^{3} / \mathrm{min}$ & $\begin{array}{l}\text { 105- } N \text { transfer area } \\
\text { exhaust fans }\end{array}$ & $\begin{array}{l}\text { Radioact ive } \\
\text { cons t i tuents }\end{array}$ \\
\hline $\begin{array}{l}\text { 105-N Spacer } \\
\text { Decontamination } \\
\text { Facility }\end{array}$ & $105-N$ & Gas & $4,800 \mathrm{ft}^{3} / \mathrm{min}$ & $\begin{array}{l}105-N \text { spacer } \\
\text { decontamination } \\
\text { facility exhaust } \\
\text { fan }\end{array}$ & $\begin{array}{l}\text { Radioactive } \\
\text { constituents - uses } \\
\text { HEPA filter }\end{array}$ \\
\hline $\begin{array}{l}105-\mathrm{N} 14 \mathrm{ft} \\
\text { Decontamination } \\
\text { Facility }\end{array}$ & $105-N 14 \mathrm{ft}$ & Gas & $6,400 \mathrm{ft}^{3} / \mathrm{min}$ & $\begin{array}{l}105-N 141 \\
\text { decontanination } \\
\text { facility exhaust } \\
\text { fan }\end{array}$ & $\begin{array}{l}\text { Radioactive } \\
\text { const ituents - uses } \\
\text { HEPA filter }\end{array}$ \\
\hline 107-N Exhaust & $107-N$ & Gas & $7,300 \mathrm{ft}^{3} / \mathrm{min}$ & $107-M$ exhaust vent & $\begin{array}{l}\text { Radioactive } \\
\text { const ituents - uses } \\
\text { HEPA filter }\end{array}$ \\
\hline
\end{tabular}

*At various times these waste streams may have received mixed waste. Sources: ICf Technology, Inc. and Ebasco Services, Inc. 1988; MHC personnel.

**Pollutants permitted per MPDES permit.

***0rums $=55$ gallon drums

HEPA = hioh-efficiency particulate air

MEK = methylethyl ketone

$M A=$ Mot available.

MPDES = Mational Pollutant Discharge Elimination System

WHC = Mestinghouse Hanford Company 
Table 3-3. Treatment, Storage, and Disposal Units.

\begin{tabular}{|c|l|c|}
\hline WIDS No. & \multicolumn{1}{|c|}{ TSD unit } & Year last operated \\
\hline $116-\mathrm{N}-1$ & $\begin{array}{l}1301-\mathrm{N} \text { Liquid Waste } \\
\text { Disposal Facility }\end{array}$ & 1985 \\
\hline $116-\mathrm{N}-3$ & $\begin{array}{l}1325-\mathrm{N} \text { Liquid Waste } \\
\text { Disposal Facility }\end{array}$ & Active \\
\hline $120-\mathrm{N}-1$ & $1324-\mathrm{NA}$ percolation pond & 1991 \\
\hline $120-\mathrm{N}-2$ & $1324-\mathrm{N}$ surface impoundment & 1988 \\
\hline
\end{tabular}

WIDS = Waste Information Data System

TSD = treatment, storage, and disposal 
DOE/RL 91-59

\subsection{HISTORY}

\subsection{OPERATIONAL HISTORY OF N REACTOR}

In 1953, studies were conducted by the U.S. Atomic Energy Commission (AEC) to show the technical and economic feasibility of constructing a dual-purpose reactor capable of producing SNM for weapons and research and steam for electricity. In 1957, Congress authorized the AEC (now a part of DOE) to build a special reactor on the Hanford Site. Construction of the New Production Reactor, now simply called $N$ Reactor, began May 1, 1959. It took 4.5 years to complete; initial criticality was achieved December 31, 1963. Table 4-1 provides an overview of significant dates for major activities occurring at $100-\mathrm{N}$.

$N$ Reactor's purpose was to produce weapons-grade plutonium, but between 1966 and 1967 a special program successfully showed that tritium could be produced as well.

To aid in generating electricity for the region, the Supply System constructed the HGP on 1 and leased from DOE adjacent to $\mathrm{N}$ Reactor. There, two turbine generators converted steam from $N$ Reactor to 860 MW of electrical power making $N$ Reactor the first dual purpose reactor. HGP became operational in 1966. During $N$ Reactor's operations, the revenues received by the government from selling the steam to HGP for the production of electrical power totaled $\$ 6.7$ bilition.

In January 1967, $N$ Reactor was shut down because its plutonium production capability was not needed, but in April 1967 DOE agreed to operate $N$ Reactor solely in a power-production mode for supplying steam to HGP in response to electrical power needs in the Northwest. On June 21, 1967, a fire in the 109-N Steam Generator Building Cell 3 of the reactor resulted in a temporary shutdown. The $N$ Reactor was restarted on July 21 of the same year.

In 1980, in response to a mission to upgrade the nation's nuclear weapons, $N$ Reactor began producing plutonium again. In 1986, following the Chernobyl accident, $N$ Reactor's safety was studied to determine if it could incur a similar accident. In 1987, the reactor was shut down to perform a number of safety reviews and facility upgrades as part of the Safety Enhancement Program. In 1988, DOE announced that $N$ Reactor would be placed in a cold standby preservation mode while the nation's needs for further plutonium production were evaluated. In 1989, all fuel elements were removed from the Reactor core and on October 1, 1990, the program to place the facility in a preserved standby state was declared complete. In 1991, DOE directed the cessation of preservation activities directed at maintaining the facility in condition for possible future use and to initiate steps to prepare the facility for eventual D\&D.

$N$ Reactor facilities are currently being maintained in an environmentally safe condition awaiting funding to commence the stajilization program for transition to decommissioning. The $N$ Reactor Deactivation Program is a 4-year effort required to remove all hazardous and radioactive wastes including over $306 \mathrm{~m}^{3}\left(400 \mathrm{yd}^{3}\right)$ of material and sediment and $4.5 \mathrm{ML}$ (1.2 Mgal) 
DOE/RL 91-59

of contaminated water from the fuel storage basins, and to stabilize all radioactively contaminated surfaces. Table 6-1 identifies key activities of the stabilization programs. 
Table 4-1. Overview of Significant Dates for 100-N Area Operation.

\begin{tabular}{|l|l|}
\hline \multicolumn{1}{|c|}{ Date } & \multicolumn{1}{|c|}{ Activity } \\
\hline May 1959 & Construction of N Reactor begins \\
\hline September 1963 & Construction of HGP begins \\
\hline October 1963 & Construction of N Reactor completed \\
\hline December 1963 & N Reactor achieves initial criticality \\
\hline November 1964 & N Reactor reaches 4,000 MW (thermal) \\
\hline April 1966 & HGP completed; production of electricity begins \\
\hline December 1966 & HGP output reaches 800 MW (electrical) \\
\hline June 1975 & $\begin{array}{l}\text { N Reactor irradiated fuel storage begins in the 105 KE } \\
\text { fuel storage bas in }\end{array}$ \\
\hline February 1981 & $\begin{array}{l}\text { N Reactor irradiated fuel storage begins in the 105 KW } \\
\text { fuel storage bas in }\end{array}$ \\
\hline December 1986 & N Reactor shutdown for safety upgrades \\
\hline February 1988 & $\begin{array}{l}\text { DOE directive to prepare N Reactor for cold standby } \\
\text { issued }\end{array}$ \\
\hline January 1989 & Defuel ing of reactor core begins \\
\hline April 1989 & Defuel ing completed \\
\hline October 1990 & Layup completed; cold standby begins \\
\hline October 1991 & N Reactor deactivation/preparation for D\&D ordered \\
\hline
\end{tabular}

HGP = Hanford Generating Plant 


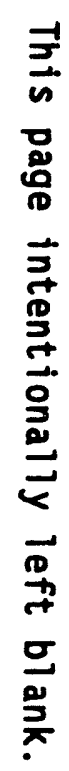




\subsection{ENVIRONMENTAL PROBLEMS}

This section provides a description of environmental problems at the $100-N$ site linked to $N$ Reactor operations. The operational activities affecting the environment are described first, followed by an assessment of the affected environments (groundwater, soil, and biota). The net effect of $N$ Reactor operations on the environment is also included.

\subsection{OPERATIONAL ACTIVITIES AFFECTING THE ENVIRONMENT}

The 100-N Area has 109 discrete sources of known or potential contamination. Table 5-1 contains brief background information on these sources: location, waste description, and unit/release description.

The sources are placed together into logical groupings, either according to geographic location or process and waste-handling similarities. Figure 5-1 shows the area affected by $N$ Reactor operations and Figure 5-2 shows the locations of waste source units within the 100-N Area (WHC 1989d).

All the currently available information for the 100-N Area sources is discussed below. No data concerning unplanned releases are available from before 1973, but releases probably did occur. All historical records are being reviewed as part of the source record compilation task, as discussed in Chapter 5 of the RCRA Facility Investigation/Corrective Measures Study Work Plan for the 100-NR-: Operable Unit, Hanford Site, Richland, Washington (DOE-RL 1990). If additional information becomes available for any of the sources or unplanned releases, it will be incorporated in the RFI/CMS as appropriate. At present, no cleanup lnvels have been negotiated for the unplanned releases.

\subsubsection{4-N Liquid Waste Loadout Station}

This grouping is located at the 1314-N LWLS near the west fence, north of the $N$ Reactor building. The grouping consists of one waste management unit and two unplanned releases that occurred at this unit.

The 1314-N LWLS is a transfer station consisting of numerous valves, pumps, underground and overhead piping and couplings, and underground tanks at the north end of the $100-\mathrm{N}$ Area, approximately $90 \mathrm{~m}$ (300 ft) from the Columbia River. The unit received liquid radioactive waste from the $116-\mathrm{N}-2$ radioactive chemical waste treatment and storage facility and the 107-N spent fuel basin recirculation facility. The waste was transferred into railway tank cars and transported to the 200 Area for processing and disposal.

The dimensions of valve pits and the concrete spill catch basin below the railroad tank car loading station are unknown. Valve pit floors are soil. Two drains in the catch basin connect to a separate catch tank and a 3,800-L (1,000-gal) transfer tank located inside the 1314-N building. 
The radioactive effluent piped from the $116-\mathrm{N}-2$ storage tank was internal decontamination solution from cleaning the primary coolant loop in $\mathrm{N}$ Reactor and various waste decontamination solutions from small decontamination jobs. The primary loop was decontaminated every 3 to 5 years. The radioactive wastewater resulting from this procedure contained phosphoric acid and diethylthiourea. The wastewater was neutralized in the $116-\mathrm{N}-2$ facility.

Two unplanned releases associated with the 1314-N LWLS have been documented. The releases are described in the following paragraphs.

- September 24, 1973 (UN-100-N-13) - Operations personnel were filling a railroad tank car with waste material from $1314-N$ when the solution began overflowing from the tank car fittings. The tank car loading pump was turned off. Solution flowed up through the drain in the catch basin beneath the car and overflowed into the dry we11, which also filled and overflowed. The catch basin and dry well are located within the 1314-N building. Approximately $380 \mathrm{~L}$ (100 gal) of solution flowed out of the dry well, covering a $6-\mathrm{m}$ by $6-\mathrm{m}(20-\mathrm{ft}$ by $20-\mathrm{ft}$ ) section of ground inside the radiation zone at the loading station. Total activity in approximately $280 \mathrm{~L}(75 \mathrm{gal})$ released to ground through the dry well was estimated at $0.011 \mathrm{Ci}$. Total activity in the $6-\mathrm{m}$ by $6-\mathrm{m}(20-\mathrm{ft}$ by $20-\mathrm{ft})$ area on the ground surface was estimated at $0.015 \mathrm{Ci}$. An unknown amount of crntaminated soil was packaged and shipped to the 200 Area burial ground (DOE-RL 1990).

- December 7, 1978 (UN-100-N-26) - The second unplanned release was a 3,800-L (1,000-gal) spill of reactor decontamination solution. The solution backflowed while being pumped into a tank car and contaminated the valve pit. Most of the solution was pumped back into the tank car. The remaining wastewater was absorbed by commercially available material and sent to the 200 Area burial ground (DOE-RL 1990).

\subsubsection{9-N Air Sampling and Monitoring Building}

This grouping is located in the vicinity of the 119-N air sampling and monitoring building, which is $150 \mathrm{~m}(500 \mathrm{ft})$ north of the $N$ Reactor building and directly south of the 1314-N LWLS grouping. The grouping consists of one waste management unit and two unplanned releases listed in this paragraph. All sources are adjacent to the 119-N building.

- UN-100-N-14 unplanned release

- UN-100-N-9 unplanned release

- 116-N air stack.

119-N Air Sampling and Monitoring Building Unplanned Releases. The 119-N building houses the equipment used to sample effluent gases and particulates in the adjacent 116-N air stack. One sample collection device used clean water as the coolant for a condenser. When originally installed, the coolant drain pipe was improperly connected to the nearby $91-\mathrm{cm}(36-\mathrm{in}$. low-pressure flush line that carried irradiated reactor cooling water from the 
105-N lift station to the 116-N-1 crib and trench. The improper connection caused the 119-N drain to become pressurized whenever the 105-N lift station pumps were operating.

The first unplanned release occurred when 119-N plumbing repairs were being attempted. The repairs were being done without knowledge of the improper connection, which was not shown on plans.

- August 5, 1974 (UN-100-N-14) - Maintenance personnel were working on the 119-N drain system to correct loss of coolant flow in a condensate collection sampler. When this opened the 5-cm (2-in.)-diameter drain system to the atmosphere, a backflow from the drain occurred, causing an unplanned release. Intermittent flow was observed from the opened line, until the drain system was reconnected. Approximately $265 \mathrm{~L}(70 \mathrm{gal})$ of effluent covering an area of $74 \mathrm{~m}^{2}$ ( $800 \mathrm{ft}^{2}$ ) was released to the ground near the 119-N sample building. Total activity was estimated at $0.8 \mathrm{mCi}$ of beta/gamma activity. The contaminated soil area was covered with plastic sheeting and the area barricaded. An unknown amount of soil was excavated and moved to the 200 Area burial ground. The area was backfilled with clean soil (DOE-RL 1990).

The second unplanned release occurred during attempts to find the improper connection point. The drain line was eventually disconnected permanently and routed to a soil column disposal pit (DOE-RL 1990).

- October 14, 1974 (UN-100-N-9) - A 8,300-L (2,200-gal) leak from the same $5-\mathrm{cm}(2-$ in.)-diameter cooling water drain line connected to the 91-cm (36-in.) low-pressure flush line occurred when a backhoe hooked onto a buried $5-\mathrm{cm}(2-i n$.$) valve in the drain line (depth$ unknown). Contaminated water immediately flowed into the excavation hole around the valve. To facilitate inspection of the valve, excess water was pumped from the hole into a nearby load lugger for temporary storage and the excavation was enlarged. Inspection showed water leaking from the valve bonnet because the valve bonnet bolts failed. The leak was repaired by replacing the valve bonnet bolts. The excavation site, including the backhoe, was roped off as a radiation zone. Total activity was approximately $4 \mathrm{mCi}$. An unknown amount of contaminated soil was excavated and removed to the 200 Area burial ground (DOE-RL 1990).

\subsubsection{6-N Facility}

166-N Fuel Unloading and Storage Area. This grouping is located near the $116-N$ air stack and the $116-N-2$ radioactive chemical waste treatment and storage facility and includes the following potential sources:

- Fuel oil unloading station

- 166-N Tank Farm

- UN-100-N-17 unplanned release

- UN-100-N-20 unplanned release

- UN-100-N-24 unplanned release. 
Fuel oil Unloading Station. The fuel oil unloading station is a concrete-lined containment structure containing $30-\mathrm{cm}$ (12-in.) fuel oil supply piping that loads to the No. 6 fuel oil storage tank at the 166-N Tank Farm. The unit is located east of the 1314-N LWLS. Tanker railcars unloaded No. 6 fuel oil at the station and oil was transferred via underground piping to the tank farm. Small amounts of diesel oil were periodically spilled at the 166-N diesel oil loadout station. The spills ( 3.75 to $7.5 \mathrm{~L}$ [1 to 2 gal] each) occurred when the tanker gas truck fill hoses were disconnected from the diesel oil receiving line (WHC 1989b).

166-N Tank Farm. The 166-N Tank Farm is an earthen-bermed tank farm containing one aboveground $5,200,000-\mathrm{L}(1,375,000-\mathrm{gal})$ No. 6 fuel oil tank and four aboveground $400,000-\mathrm{L}(105,000-\mathrm{gal})$ diesel oil storage tanks. The earthen berm has a total containment capacity of $11,500,000 \mathrm{~L}(3,030,000 \mathrm{gal})$ (WHC 1989b). The tank farm is located between the 116-N air stack and the 116- $\mathrm{N}-2$ radioactive chemical waste treatment and storage facility. It has been in operation since 1963.

No. 6 fuel oil was unloaded at the fuel oil unloading station from rail tanks and piped via underground lines to the storage tank. A $20-\mathrm{cm}(8-\mathrm{in}$. underground transfer line runs from the storage tank to the 184-N day tank, where the oil was stored before being used in the boilers (WHC 1989b).

Diesel oil was unloaded from tanker trucks into the diesel storage tanks. The oil was pumped from the storage tanks via a $10-\mathrm{cm}(4-\mathrm{in}$.$) underground$ transfer line to the 184-N day tanks and gravity-fed through a separate $10-\mathrm{cm}$ (4-in.) line to the 182-N underground storage tanks (WHC 1989b).

Three documented unplanned releases have been associated with the 166-N Tank Farm:

- August 1966 (UN-100-N-17) - One unplanned release at the unit was caused by the external corrosion of a $10-\mathrm{cm}$ (4-in.) supply line between the diesel $0 i 1$ storage tank and the west dike, detected when a discrepancy in the diesel oil inventory was discovered. By that time, $300,000 \mathrm{~L}(80,000 \mathrm{gal})$ of diesel oil had been released. The diesel oil drained through the soil to groundwater, then migrated to the Columbia River. A trench was excavated along the river bank in an attempt to intercept the oil before it could reach the river. $0 i 1$ exposed in the trench was ignited and burned periodically through 1967 (WHC 1989b). No contaminated soil was removed from the site, and the supply line was repaired in September 1966 (DOE-RL 1990).

- June 1985 (UN-100-N-20) - External corrosion reportedly caused a leak in a $5-\mathrm{cm}(2-i n$.$) diesel oil return pipeline within the bermed$ area containing the four diesel oil tanks. The release of $760 \mathrm{~L}$ (200 gal) ran onto the soil. No cleanup occurred (DOE-RL 1990).

- February 1, 1987 (UN-100-N-24) - A leak was caused by external corrosion brought on by a leaking heat transfer line. The leak occurred on the west side of the No. 6 fuel oil tank outside of the bermed area. The release consisted of an unknown amount of No. 6 
fuel oil that ran onto the soil. No information is available concerning the cleanup of this spill. A portion or all of this spill penetrated the soil (DOE-RL 1990).

\subsubsection{6-N-1 Crib and Trench Grouping.}

This grouping is located directly east of the $166-\mathrm{N}$ fuel storage area and consists of the following sources.

- 116-N-1 crib and trench

- UN-100-N-31 unplanned release

- 1322-N and 1322-NA sampling buildings

- UN-100-N-8 unplanned release

- UN-100-N-4 unplanned release.

116-N-1 $\mathrm{Crib}$ and Trench. The $116-\mathrm{N}-1 \mathrm{crib}$ and trench is a major inactive waste management unit located approximately $300 \mathrm{~m}(1,000 \mathrm{ft})$ east of the $N$ Reactor building. The system is commonly referred to as the 1301-N LWDF. The 116-N-1 crib and trench was used from 1964 until September 1985. This ground disposal facility made use of the filtration and ion exchange properties of soil to remove radioactive material from radioactive liquid effiuents from $N$ Reactor (DOE-RL 1990). No liquid remains in the crib and trench; however, approximately $7,000 \mathrm{Ci}$ of radionuclides remain in the soil column. The crib is $88 \mathrm{~m}(290 \mathrm{ft})$ long and $38 \mathrm{~m}(125 \mathrm{ft})$ wide. The walls of the crib are sloped soil and gravel embankment and approximately $3.7 \mathrm{~m}$ (12 ft) deep from floor to ground surface (DOE-RL 1990). The crib outline is shown in Figure 5-3.

A zig-zag extension trench, $15 \mathrm{~m}(50 \mathrm{ft})$ wide and $3.7 \mathrm{~m}(12 \mathrm{ft})$ deep, extends $490 \mathrm{~m}(1,600 \mathrm{ft})$ from the crib. The $116-\mathrm{N}-1$ extension trench was built in 1965 because wastewater volume exceeded the capacity of the crib. A $0.9-\mathrm{m}(3-\mathrm{ft})$ layer of boulders was placed in the crib. Precast concrete cover panels were placed over the trench in 1982 to minimize wildlife access and airborne contamination (DOE-RL 1990).

Pipelines that discharged directly into the $116-\mathrm{N}-1$ crib and trench through a $16-\mathrm{m}$ by $3.7-\mathrm{m}(52-\mathrm{ft}$ by $12-\mathrm{ft})$ concrete weir box included an underground $91-\mathrm{cm}(36-\mathrm{in}$.$) main effluent line from the 105-N lift station, an$ underground $30-\mathrm{cm}(12-\mathrm{in.})$ effluent drainline from the 109-N steam generator building, and a 15-cm (6-in.) effluent drain line from the 109-N floor drains.

The 116-N-1 crib and trench is a dangerous waste disposal facility under RCRA interim status. The cumulative inventory (accounting for decay to September 1985) of major long-lived radionuclides disposed of in the 116-N-1 crib and trench from 1964 to September 1985 is presented in Table 5-2. The dangerous wastes disposed in the 116-N-1 crib and trench are listed in Table 5-3 (WHC 1987b). 
One unplanned release (other than systematic releases of radionuclides to the soil and groundwater) is associated with the 116-N-1 crib and trench.

- July 22, 1974 (UN-100-N-31) - While sample lines were being installed in a 15-cm (6-in.) steel casing through the berm on the west side of the $116-N-1$ crib, the water level in the crib was raised 38 to $46 \mathrm{~cm}$ (15 to $18 \mathrm{in.}$ ) as a result of an emergency dump tank (EDT) drawdown test being conducted. The increased water level allowed an unplanned release of approximately 3,800 L $(1,000 \mathrm{gal})$ of effluent water to flow through the casing. This release contaminated $190 \mathrm{~m}^{2}\left(2,000 \mathrm{ft}^{2}\right)$ of soil. Sand and fines were used to stabilize soil contamination (DOE-RL 1990). The extent of remediation instituted is unknown.

1322-N and 1322-NA Sample Buildings. These buildings contain the liquid effluent waste treatment facility pilot plant. They are located immediately north of the 116-N-2 tank. Building 1322-N contains a drainage tank. Building 1322-NA contains automatic sequential sampling equipment for the $30-\mathrm{cm}(12-i$. $)$ and $91-\mathrm{cm}(36-\mathrm{in.})$ radioactive drainlines and the riverbank springs (DOE-RL 1990).

Two documented unplanned releases have occurred and are described below:

- May 11, 1975 (UN-100-N-8) - An operator on routine patrol at 4:00 a.m. noticed a small but steady spray of contaminated water coming from the $91-\mathrm{cm}(36-i n$.$) radioactive drain return line sampler$ located near the back of the building. The operator stopped the leak by shutting down the sample pumps. Contaminated water was observed to cover the floor and various pieces of equipment in the rear of the building. Subsequent contamination surveys showed that the water had run out the back door and contaminated approximately $2.3 \mathrm{~m}^{2}\left(25 \mathrm{ft}^{2}\right)$ of ground. The amount of water released was estimated to be 190 to $380 \mathrm{~L}$ ( 50 to 100 gal). All contaminated areas were roped off, covered with plastic, and identified as a radiation zone. Total activity was estimated to be less than $0.5 \mathrm{mCi}$. An unknown amount of the contaminated soil was removed, transported to the 200 Area, and replaced with clean fill (DOE-RL 1990).

- May 7, 1977 (UN-100-N-4) - A contaminated water leak in the 1322-N and 1322-NA buildings was discovered at 1:00 a.m. An operator on routine patrol found water running out from under the doors in the 1322-NA building. He then checked the adjacent 1322-N building and found water spraying out of the top vent on the drainage tank. The sump pump was not operating at the time, and the water left the tank via the vent, backing up the sink drain in the 1322-NA building. The water flowed out of the drains and over the $15-\mathrm{cm}(6-\mathrm{in}$.$) curb$ onto the ground in front and back of the building. The amount of water released is unknown. All electrical equipment in the buildings was shut off, preventing further leaks. The contaminated soil outside the front and rear doors, an area of approximately $140 \mathrm{~m}^{2}\left(1,500 \mathrm{ft}^{2}\right)$, was covered with plastic, roped off, and 
controlled as a radiation zone. Total activity was $0.5 \mathrm{mCi}$. An unknown amount of contaminated soil was removed and transported to the 200 Area (DOE-RL 1990).

\subsubsection{6-N-2 Radioactive Chemical Waste Treatment and Storage Facility}

These potential sources are grouped together because they are all located at or near the 116-N-2 radioactive chemical waste treatment and storage facility. See Color Illustration 5-1 for a photograph of the facility.

- 116-N-2 radioactive chemical waste treatment and storage facility

- UN-100-N-5 unplanned release

- UN-10C-N-25 unplanned release

- Spring, 1983 unplanned release

- 124-N-4 septic tank and drainfield.

116-N-2 Radioactive Chemical Waste Treatment and Storage Facility. The 116-N-2 facility is a waste management unit complex consisting of piping, pumps, a transfer tank commonly referred to as the silo, and a large treatment and storage tank referred to as the golf ball $(1310-N)$. The treatment tank is a spherical steel tank with a capacity of $3,400,000 \mathrm{~L}(900,000 \mathrm{gal})$. It is partly buried in the ground and surrounded by a 7.6-m (25-ft) high compacted soil radiation barrier on three sides. The unit operated from 1968 until 1987.

The 116-N-2 facility was used to neutralize and temporarily store radiologically contaminated phosphoric acid waste used in the internal decontamination of the $N$ Reactor. Chemical wastes from the internal decontamination of the primary loop of the reactor were transferred to the $116-\mathrm{N}-2$ silo by a $15-\mathrm{cm}(6-\mathrm{in}$.$) underground transfer line. The silo is a$ transfer station for materials going into or out of the 116-N-2 storage tank. An additional 3.8- $\mathrm{cm}(1.5-$ in.) decontamination line enters the silo from the $\mathrm{N}$ Reactor building. This line was for transporting small-scale decontamination solutions. Decontamination chemical solutions could be transferred to the 116-N-1 crib and trench, if necessary, via a $61-\mathrm{cm}$ (24-in.) aboveground transfer 1 ine from the $116-\mathrm{N}-2$ storage tank.

The silo routed the acidic decontamination solutions into the 116-N-2 storage tank for neutralization. The dangerous wastes in the decontamination solution included approximately $80,000 \mathrm{~L}(21,000 \mathrm{gal})$ of 70 percent phosphoric acid and 140 to $180 \mathrm{~kg}$ (300 to $400 \mathrm{lb}$ ) of diethylthiourea. The primary loop of the reactor was decontaminated every 3 to 5 years. Approximately $2,300,000 \mathrm{~L}(600,000 \mathrm{gal})$ of solution were generated during each decontamination event.

From 1968 until 1972, the neutralized decontamination solution was routed from 116-N-2 directly to tanker trucks parked east of the containment area. Trucks would then take the waste from the containment area to the 200 Area for disposal. After 1972, the neutralized decontamination solution from $116-\mathrm{N}-2$ was sent to the 1314-N LWLS for transfer to rail cars (DOE-RL 1990). 
Three documented unplanned releases associated with the 116-N-2 radioactive chemical waste treatment and storage facility have occurred. These releases are described in the following paragraphs.

- June 27, 1972 (UN-100-N-5) - An unplanned release occurred from a leak in the piping between the recirculation pump and the 116-N-2 tank. Evidence indicated that a failure occurred in the underground section of this pipe, causing discharge of approximately $340,000 \mathrm{~L}(90,000 \mathrm{gal})$ of radioactive chemical waste to the ground. The low-level radioactive wastewater contained decontamination chemicals used in the decontamination of the $N$ Reactor primary loop. The waste contained $35 \mathrm{Ci}$ of activity, $26 \mathrm{Ci}$ of which were ${ }^{60} \mathrm{Co}$. The $\mathrm{pH}$ of the solution was about 9. An unknown amount of contaminated soil was removed and sent to the 200 Area burial ground. The excavation was then backfilled with clean soil (DOE-RL 1990).

- May 15, 1975 (UN-100-N-25) - An unplanned release occurred during transfer of chemical decontamination solution into the 116-N-2 tank. A surge of contaminated water sprayed out an open access hole on the tank. An estimated 1,900 L (500 gal) of primary loop decontamination solution containing phosphoric acid and diethylthiourea was released to the ground inside the radiation zone surrounding the tank. A radiation survey outside the posted zone showed no contamination after cleanup (DOE-RL 1990).

- Spring, 1983 - An unplanned release of caustic sodium hydroxide occurred in the spring of 1983 in association with the reactor decontamination event taking place at that time. A tanker truck was offloading sodium hydroxide to the silo when a fitting came loose and spilled approximately $380 \mathrm{~L}$ (100 gal) of liquid to the open soil. No remediation was conducted.

124-N-4 Septic Tank and Drainfield. The 124-N-4 septic tank and drainfield was the primary septic system that served the majority of the $100-N$ buildings from 1963 until 1987. It is located east of the 116-N-2 facility, and consists of two septic tanks (the second tank was installed in 1975) with a total fluid capacity of $53,000 \mathrm{~L}(14,000 \mathrm{gal})$. The septic tank effluent drained into a large drainfield. The drainfield is divided into four sections, with a distribution box in each section feeding eight drainlines. The drainfield provided approximately $830 \mathrm{~m}^{2}\left(8,900 \mathrm{ft}^{2}\right)$ of infiltrative surface area. The unit received approximately $110,000 \mathrm{~L} /$ day $(30,000 \mathrm{gal} / \mathrm{day})$ of sanitary sewage (DOE-RL 1990).

No releases to or from the unit other than sanitary sewage are documented. In addition, this unit is near the 116-N-2 radioactive chemical waste treatment and storage facility where tanker trucks were loaded with irradiated, neutralized decontamination solutions from 1968 until 1972. The close proximity of the units suggests that the possibility of contamination from small releases during tanker loading may exist here. 


\subsubsection{6-N-3 Crib and Trench}

The 116-N-3 crib and trench (1325-N LWDF) waste management unit is located approximately $300 \mathrm{~m}(1,000 \mathrm{ft})$ east of the $116-\mathrm{N}-1$ facility. The crib is $76 \mathrm{~m}(250 \mathrm{ft})$ long and $73 \mathrm{~m}(240 \mathrm{ft})$ wide and provides $5,600 \mathrm{~m}^{2}$ $\left(60,000 \mathrm{ft}^{2}\right)$ of percolation surface. An extension trench $914 \mathrm{~m}$ long by $17 \mathrm{~m}$ wide by $2 \mathrm{~m}$ deep $(3,000 \mathrm{ft}$ by $55 \mathrm{ft}$ by $7 \mathrm{ft})$ was added to augment the operational capacity of the crib.

The 116-N-3 crib was constructed as a replacement liquid radioactive waste disposal facility for the $116-\mathrm{N}-1$ crib and trench and first received $N$ Reactor effluent in 1983. The 116-N-3 trench was put in full service in September 1985 (DOE-RL 1990). Between these two dates, both the 116-N-1 and the 116-N-3 facilities were in service. Both the 116-N-3 crib and trench are covered by precast concrete panels to prevent access by the local fauna to contaminated water.

The 116-N-3 system's reinforced concrete header box is tied in to the 116-N-1 weir box, with $366 \mathrm{~m}(1,200 \mathrm{ft})$ of 91-cm (36-in.) diameter piping. The 116-N-3 facility, like the 116-N-1 facility, received radioactive liquid effluents from the RCS, spent fuel storage basin, periphery coolant systems, and various radioactive drain systems located throughout the reactor facility. The average monthly flow rate into the $116-\mathrm{N}-3$ facility during 1983 through 1986 , when the $N$ Reactor was in normal operation, was approximately $5,300 \mathrm{~L} / \mathrm{min}(1,400 \mathrm{gal} / \mathrm{min})$ (DOE-RL 1990).

The cumulative inventory of select radionuclides disposed of in the 116-N-3 crib from October 1983 to January 1,1988 is shown in Table 5-4 (WHC 1987a). An estimate of other wastes annually disposed of in the 116-N-3 crib (1984 through 1986) is shown in Table 5-5.

\subsubsection{8-N-1 Burning Pit}

The 128-N burning pit is located directly east of the $116-\mathrm{N}-3 \mathrm{crib}$ and trench grouping. The unit was used primarily for burning nonhazardous waste (e.g., paper, wood, and trash) generated at the 100-N Area. The amount of waste managed by the unit is unknown. There is no documented evidence of release of dangerous or radioactive wastes from the unit. The unit size and location changed during the period of operation (1962 to 1986) (DOE-RL 1990).

\subsubsection{1-N River Pumphouse}

The 181-N river pumphouse grouping consists of two potential sources that are grouped based on their location at or near the 181-N river pumphouse. The grouping is located between the railroad tracks and the Columbia River and includes the following potential sources.

- April 18, 1986 unplanned release (WHC 1987d)

- Aboveground waste oil tank (DOE-RL 1990). 
DOE/RL 91-59

Aboveground Waste 011 Tank. An aboveground steel waste ofl tank is located at the base of the bluff near the 181-N river pumphouse. The capacity of the tank is $980 \mathrm{~L}(260 \mathrm{gal})$. The tank was designed to store waste oil from drip pans used to catch oil from the river pumps, but was never used (DOE-RL 1990).

\section{$5.1 .9 \quad 1304-N$}

1304-N Emergency Dump Tank Grouping. The 1304-N EDT grouping consists of two waste management units and six associated unplanned releases. These sources are grouped together because they are close geographically and manage waste similarly. The potential sources in this grouping are:

- $1304-N$ EDT

- UN-100-N-1 unplanned release

- UN-100-N-29 unplanned release

- UN-100-N-30 unplanned release

- UN-100-N-32 unplanned release

- UN-100-N-2 unplanned release

- UN-100-N-7 unpl anned release

- 116-N-4 emergency dump basin (EDB).

1304-N Emergency Dump Tank. The 1304-N EDT is a 4.9-ML (1.3 Mgal) steel aboveground pressure vessel (see Color Illustration 5-3). It is located south of the 107-N basin recirculation building and west of the 1300-N EDB. It replaced the EDB as a quench tank for emergency blowdown of thermally hot, pressurized reactor primary coolant water. The tank maintained a constant volume of 2,600,000 $L(680,000 \mathrm{gal})$ of unheated water for quenching the hot water to prevent it from fiashing to steam. Because a small flow of primary coolant was maintained to the EDT to keep interconnecting piping thermally warm, overflow from the tank, normally containing a smali inventory of radioactive materials, was discharged to the $116-\mathrm{N}-3 \mathrm{crib}$. The unit was used from 1973 until reactor shutdown in 1987.

Several documented unplanned releases have been associated with the 1304-N EDT:

- March 27, 1974 (UN-100-N-1) - The EDT 76-cm (30-in.) overflow line developed a leak through a $76-\mathrm{cm}(30-i n$.$) inflatable pipe flow$ stoppage device during maintenance operations. In addition, a fill valve on the EDT began leaking at the same time. Approximately $110,000 \mathrm{~L}(30,000 \mathrm{gal})$ of irradiated cooling water was spilled on the ground near the tank and flowed over the bank to an area near the 181-N river pumphouse. The spilled material did not reach the river. Analyses of samples taken at the time of the leak indicate that $0.2 \mathrm{Ci}$ was released in the $110,000 \mathrm{~L}$. (30,000 gal) spill. Contaminated soil reading greater than 1,000 counts per minute (cpm) was removed and transported to the 200 Area for burial. The remaining contaminated soil was covered using 10 to $15 \mathrm{~cm}$ (4 to 6 in.) of clean soil (DOE-RL 1990).

- April 23, 1974 (UN-100-N-29) - A faulty check valve in the EDT bypass line allowed approximately $380 \mathrm{~L}$ (100 gal) of irradiated primary coolant water to leak to the ground. The water ran out from 
under a concrete ground cover on the slope above the tank and covered an area of approximately $1.2 \mathrm{~m}$ by $9.1 \mathrm{~m}(4 \mathrm{ft}$ by $30 \mathrm{ft}$ ). A sample of the mud and water yielded a reading of $5,000 \mathrm{cpm}$. Approximately $0.1 \mathrm{Ci}$ of radioactive material, mostly ${ }^{56} \mathrm{Mn}$ and ${ }^{24} \mathrm{Na}$, was released. The contaminated soil was removed and taken to the 200 Area for disposal (DOE-RL 1990).

- July 27, 1974 (UN-100-N-30) - While filling the EDT in preparation for a drawdown test of the system, the EDT overflowed through a vacuum breaker vent in the top of the tank. Approximately 9,500 L $(2,500 \mathrm{gal})$ of water spilled to the ground around the tank and contaminated an area of approximately $230 \mathrm{~m}^{2}\left(2,500 \mathrm{ft}^{2}\right)$ to a maximum of $500 \mathrm{cpm}$. No water reached the river. Sands and fines were used to stabilize the soil contamination. It is unknown if the contaminated soil was removed (DOE-RL 1990).

- September 16, 1974 (UN-100-N-32) - A repeat of the Apri1 23, 1974 incident occurred because of a faulty check valve. Approximately 1,900 to $3,800 \mathrm{~L}$ (500 to 1,000 gal) of irradiated primary coolant water spilled down the slope from the metal culvert housing the check valve and contaminated soil by the south and east walls of the tank. A sample of the mud yielded a reading of $20,000 \mathrm{cpm}$. Two days later, an evaporated sample was analyzed and results indicated that less than $10 \mathrm{mC} i$ of radioactive material remained in the ground. An unknown amount of contaminated soil was removed and taken to the 200 Area for disposal. The remaining contaminated soil was covered using clean fill (DOE-RL 1990).

- February 19, 1980 (UN-100-N-2) - A leak in the $2.5-\mathrm{cm}$ (1-in.) valve body relief line occurred. The valve is on the $81-\mathrm{cm}(32-\mathrm{in}$. low-pressure flush line between the 109-N heat exchanger building and the EDT. Approximately 95,000 to $110,000 \mathrm{~L}(25,000$ to $30,000 \mathrm{gal})$ of irradiated water leaked to the ground. Most of the water in the area was transferred to the EDB using a portable pump. Based on sample analysis it is estimated that less than $1 \mathrm{Ci}$ of beta/gamma radioactivity was released to the ground. An unknown amount of contaminated soil was removed and taken to the 200 Area for disposal. The remaining contaminated soil was covered using clean fill (DOE-RL 1990).

- April 29, 1985 (UN-100-N-7) - Approximately 1,900,000 L (504,000 gal) of irradiated water was released to the soil from a leak in the $25-\mathrm{cm}(10-i n$.$) drain line between the N$ Reactor building and the EDT. The cause of the leak is unknown. Approximately $32 \mathrm{~m}^{3}$ $\left(1,130 \mathrm{ft}^{3}\right)$ of contaminated soil was removed and taken to the 200 Area for disposal.

116-N-4 Emergency Dump Basin. The 116-N-4 EDB is a liquid effluent storage basin that was originally designed to receive emergency cooling water from the $\mathrm{N}$ Reactor (see Color Illustration 5-2). It is located northwest of the 109-N building. The unit is a concrete basin with a welded steel liner (DOE-RL 1990), and is approximately $40 \mathrm{~m}$ (130 ft) long by $24 \mathrm{~m}(80 \mathrm{ft})$ wide by $4.6 \mathrm{~m}(15 \mathrm{ft})$ deep and has a storage capacity of $3,800,000 \mathrm{~L}(1,000,000 \mathrm{gal})$ (DOE-RL 1990). 
The EDB was constructed in 1963 to receive single-pass radioactive emergency cooling water. In the late 1960s, the unit was determined to be insufficient for its original use. The bas in did not have the capacity needed to contain the volume of coolant used during an emergency cooling operation. It was replaced by the 1304-N EDT in 1973. From 1973 unt 11 1987, the EDB received contaminated liquid effluent generated during the periodic blowdown of $\mathrm{N}$ Reactor's 12 steam generators located in the 109-N bullding. This condensate contained low levels of radioactive contamination. Contents of the unit were sampled monthly from 1978 to 1985. Table 5-6 shows the average annual concentrations by radionuclide in the 1300-N EDB unit (DOE-RL 1990). At various times, the EDB also received radioactive wastes from the $N$ Reactor lift station. Since the $N$ Reactor was shut down in 1987 , water has been maintained in the EDB [approximately 2,800,000 L (750,000 gal)] so that the bottom sludge layer will not become exposed. Subsequent drying of the sludge could expose receptors to airborne exposure from contaminated particulate matter. Filtered river water has been added as needed to maintain the water leve1. Documentation of the amount of water added to the EDB has not been maintained. There are no documented releases associated with the unit (DOE-RL 1990).

\section{$5.1 .10 \quad 118-N-1$}

118-N-1 Spacer Storage Silos. The $118-N-1$ spacer storage silo grouping consists of three waste management units and three unplanned releases associated with the spacer storage silos. These potential sources are located in the vicinity of the spacer storage silos and include the following locations:

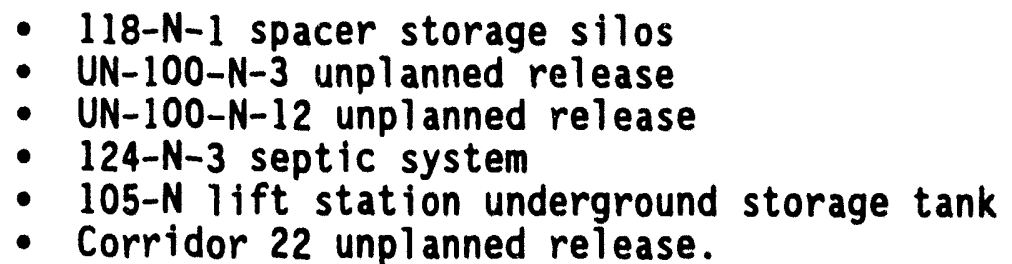

118-N-1 Spacer Storage S110 Group. The three 118-N-1 spacer storage silos were used for the temporary storage of irradiated fuel spacers. The steel spacers came in immediate contact with the fuel elements in the $N$ Reactor. The silos, approximately $4.9 \mathrm{~m}(16 \mathrm{ft})$ in diameter by $6 \mathrm{~m}(20 \mathrm{ft})$ deep, are located north of the $N$ Reactor building. Two of the three silo floors are open to the soil; the third silo has a concrete floor. The silos have approximately 0.5- to 1-m-thick concrete caps covered with soil (DOE-RL 1990).

Releases occurred from 1963 until 1987 when spacers were placed in or removed from the silos. Spacers were transferred to the silos using a buried spacer transfer line connected to the 105-N fuel basin. The depth of the line is variable There was no secondary containment surrounding the line. Small amounts of irradiated water passed through the 1 ine with the spacers and was deposited in the silos. In addition, water was sprayed over the spacers during removal from the top of the silos to eliminate the potential airborne release of radionuclides. In recent years, paint was used as a fixative when spacers were transferred from the silos. The silos currently contain dry 


\section{DOE/RL 91-59}

irradiated spacers. The volume of radiologically contaminated water that reached the soll either through the bottom of the silos or the exposed soil around the silos is unknown.

Two documented releases to the ground have been associated with the spacer storage stlos and associated piping.

- March 8, 1978 (UN-100-N-3) - A leak was detected in the spacer transfer line. The first indication of the leak was the appearance of a $1.2-\mathrm{m}(4-\mathrm{ft})-\mathrm{d}$ ameter by $76-\mathrm{cm}(30-\mathrm{in}$.$) -deep sinkhole between$ the 105-N $11 \mathrm{ft}$ station and the spacer storage silos. Excavation of the sinkhole area revealed a crack in the $7.6-\mathrm{cm}(3-\mathrm{in}$.$) reinforced$ plastic pipe approximately $3.4 \mathrm{~m}(11 \mathrm{ft})$ below grade. It is estimated that approximately $1,400,000 \mathrm{~L}(360,000 \mathrm{gal})$ of irradiated $N$ Reactor fuel storage bas in water entered the ground. Est imated radionuclide releases to the soll were ${ }^{60} \mathrm{Co}(70 \mathrm{mCl}),{ }^{90} \mathrm{Sr}(80 \mathrm{mCl})$, ${ }^{137} \mathrm{Cs}(250 \mathrm{mCl})$, ${ }^{44 \mathrm{CePr}}(140 \mathrm{mCl})$, and ${ }^{239} \mathrm{Pu}(0.4 \mathrm{mCi})$. An unknown amount of soli was removed and disposed of at the 200 Area burial ground (DOE-RL 1990).

- February 27, 1979 (UN-100-N-12) - A leak similar to the March 8,1978 release occurred. A $0.6-\mathrm{m}$ by $0.9-\mathrm{m}$ by $46-\mathrm{cm}(2-\mathrm{ft}$ by 3-ft by 18-in.) sinkhole was discovered at the backfilled location of the previous leak. The pressure of transporting the spacers within the 1 ine apparently caused a rupture. An estimated $950,000 \mathrm{~L}(250,000 \mathrm{gal})$ of irradiated $\mathrm{N}$ Reactor fuel storage basin water was released to the ground. Estimated radignuclide releases to the soil were ${ }^{60} \mathrm{Co}(190 \mathrm{mCi}),{ }^{90} \mathrm{Sr}(126 \mathrm{mCi}),{ }^{137} \mathrm{CS}$ (396 mCi), ${ }^{144} \mathrm{CePr}(34 \mathrm{mCl})$, and $239 / 240 \mathrm{Pu}(0.57 \mathrm{mC} i)$. The plastic pipe was replaced with a stainless steel pipe. Whether the contaminated soil was removed and taken to the 200 Area for disposal or covered with clean fill is not documented (DOE-RL 1990).

124-N-3 Septic Tank. The 124-N-3 septic system is a cesspool that served the $107-N$ building from 1982 to the present. The unit served two to three employees working at the 107-N building, as well as temporary construction workers in the area, and was designed to receive only sanitary sewage. The cesspool includes a $1,900-\mathrm{L}(500$-gal) precast concrete tank, perforated tile pipe, and solid cover with $0.6 \mathrm{~m}(2 \mathrm{ft})$ of crushed stone below the tank. The estimated daily flow was 170 L/day (45 gal/day) (DOE-RL 1990).

105-N Lift Station Underground Storage Tank. The 105-N 1 ift station underground storage tank was a 19,000-L (5,000-gal), single-wall carbon steel tank used for storing diesel ofl (DOE-RL 1990). The tank had no cathodic or interior protection and was approximately 11 to 15 years old when it was removed in December 1990. Soil sampling during tank removal indicated that the site is not contaminated with petroleum derivatives.

Corridor 22 Unplanned Release. In 1984 several hundred liters of radioactively contaminated water were spilled outside the Corridor 22 doorway in the $N$ Reactor building. Scrub water from the fission product filter trap overflowed and was discharged to the ground. The concrete was painted over and an indeterminate amount of soll was removed and taken to the 200 Area for disposal (DOE-RL 1990). 
DOE/RL 91-59

\subsubsection{2-N Underground Storage Tank Area}

This grouping consists of the site where three 182-N underground storage tanks and a septic tank were located south of the $\mathrm{N}$ Reactor building.

Three underground diesel storage tanks were located on the north side of the 182-N high lift pumphouse. These tanks were identified as 182-N-1-DT, 182-N-2-DT, and 182-N-3-DT. All tanks were single-wall carbon steel and each had a capacity of 15,000 gal. They were approximately 16 to 20 years old and had no cathodic or interior protection. The three tanks were pumped dry and removed in December 1990. Soil sampling following tank removal shows the site clean of petroleum derivatives (DOE-RL 1990).

The septic tank and seepage pit are located southeast of the $182-N$ building and were installed in 1963 (DOE-RL 1990). The system is still operating, but only receives sanitary sewage (DOE-RL 1991). The seepage pit for this system provides about $19 \mathrm{~m}^{2}\left(200 \mathrm{ft}^{2}\right)$ of infiltration surface area and $8,540 \mathrm{~L}(2,256 \mathrm{gal})$ of fluid storage. In 1985 the system served 10 personnel and the calculated daily flow was 760 L/day (200 gal/day) (DOE-RL 1990). No remedial activities have taken place.

\subsubsection{5-N Reactor Spent Fuel Storage Basin}

The $N$ Reactor spent fuel storage basin is a concrete containment basin for the storage of process tubes, spent fuel elements, irradiated spacers, and other fuel handling equipment. Underwater transfer carts moved the irradiated fuel elements to the storage basin where they were stored in the temporary storage baskets. Baskets were emptied and fuel elements were placed in storage canisters, sorted by enrichment and discharge dates. The filled canisters were moved by bridge cranes to storage cubicles, formed by a lattice of boron concrete walls. The storage bas in began operating in 1963 and ceased storing irradiated nuclear fuel in 1989 when all fuel was transferred to the 100-K fuel storage basins. However, the basin remains filled with water for shielding and radiological contamination control. Color Illustrations 5-4 and 5-5 show above- and below-water photographs of the basin, respectively.

Two releases near the $\mathbf{N}$ Reactor fuel storage bas in have been documented. These releases are described below.

- May 13, 1975 (UN-100-N-10) - A leak of irradiated water to the ground occurred on May 13, 1975 during preparation for the removal of a check valve from a Zone I gravity drain line to the $105-\mathrm{N}$ lift station. Pumps were used to remove water from the lift station during the shutdown of the lift station pumps. The pumps al so were used to drain the $91-\mathrm{cm}(36-\mathrm{in}$.$) radioactive drain line. The water$ was to be pumped to the EDB during this process. During the drawdown test, approximately $380 \mathrm{~L}$ (100 gal) leaked to the ground through a loose hose fitting between the temporary pump and the EDB. The exact location of the spill is not documented. Approximately $9 \mathrm{~m}^{2}\left(100 \mathrm{ft}^{2}\right)$ of soil were contaminated with $1 \mathrm{mCi}$ of mixed fission and activation products. An unknown amount of contaminated soil was removed and disposed of in the 200 Area burial ground (DOE-RL 1990). 
- February 28, 1986 (UN-100-N-35) - Routine sampling of 100-N Area groundwater monitoring wells revealed elevated levels of ${ }^{131} \mathrm{I}$. Testing showed the leak to be basin water. Investigation showed water leaking through an expansion joint $8.5 \mathrm{~m}(28 \mathrm{ft})$ below ground level. Water was leaking to the lift station and through an expansion joint to the ground. The leaking only occurred when the basin water level was high and water flowed out the overflow weirs. A weir contributing to the leak was located and the leak was determined to be coming fror a cleanout valve. The weir and drain line were grouted and sealed on December 5, 1986 (DOE-RL 1990).

\subsubsection{2-N High Lift Pumphouse}

The 182-N high lift pumphouse grouping consists of four potential sources within the area surrounding the 182-N high lift pumphouse. The grouping includes the water supply tank farm and extends to the Columbia River. The following potential sources are located in this area:

- 124-N-2 septic tank

- 182-N tank farm overflow (Nation Pollutant Discharge Elimination System [NPDES] outfall No. 005)

- 182-N drain outfall (NPDES outfall No. 006)

- February 6, 1987 unplanned release.

124-N-2 Septic Tank. The septic tank and seepage pit making up sewer system II are located southeast of the $182-N$ building and were installed in 1963. The system is still operating and only receives sanitary sewage. The seepage pit for this system provides about $19 \mathrm{~m}^{2}\left(200 \mathrm{ft}^{2}\right)$ of infiltration surface area and 8,540 L (2,256 gal) of fluid storage. In 1985, the system served 10 personnel and the calculated daily flow was $760 \mathrm{~L} /$ day (200 gal/day). No remedial activities have taken place (LOE-RL 1990).

182-N Tank Farm Overflow. This unit is a NPDES-permitted discharge point (Source No. 005) to the Columbia River. It contains drainage (water) from the 182- $\mathrm{N}$ tank farm area. The area discharges to the river by a $91-\mathrm{cm}(36-\mathrm{in}$. raw water return line. The discharge point is located $90 \mathrm{~m}(300 \mathrm{ft})$ upstream of 181-N. In 1987, the average daily discharge to the river via this point was 10 million $L$ ( 2.7 million gal). Currently, discharges from this point are minimal. The startup date for the discharge was 1964 . There is no documented dangerous or radioactive release to the river via this discharge point (DOE-RL 1990).

182-N Drain System. This unit is a NPDES-permitted discharge point (Source No. 006) to the Columbia River. Drainage from the $182-\mathrm{N}$ high lift pumphous " is discharged to the river by a $107-\mathrm{cm}(42-\mathrm{in.})$ raw water return line. $R, I$ and filter water from pump seal leakage is discharged from this point. Westinghouse Hanford Company (WHC) personnel have indicated that low-level radionuclides have been released from reactor emergency core cooling system pumps. When the reactor was operating, small amounts of irradiated water were released through the seals and drained to the river. The discharge 
point is $30 \mathrm{~m}(100 \mathrm{ft})$ upstream of $181-\mathrm{N}$. In 1987, the average daily discharge to the river via this point was $1,000,000 \mathrm{~L}(270,000 \mathrm{gal})$. The startup date for the discharge was 1964. It is currently in use (DOE-RL 1990).

$0 i 1$ Release to the Columbia River. On February 6, 1987, approximately $19 \mathrm{~L}$ ( 5 gal) of turbine oil was discharged to the Columbia River through the 182-N tank farm raw water return line. A small (pin-hole size) leak in a lube oil line in the No. 2 drive turbine allowed oil to enter the secondary steam system. Steam condensate from this system returns to the $100-N$ steam condensate system that drains to the river. The leak in the lube line was repaired (DOE-RL 1990).

\subsubsection{Acid/Caustic Storage and Transport System}

The acid/caustic storage and transport system grouping includes all the process units, waste management units, unplanned releases, and pipelines associated with the storage and transport of acids and caustics used in the 163-N demineralization plant. Because of its location, the 124-N-1 septic tank is also included in this grouping. The following potential sources are included in this grouping.

- 108-N chemical unloading facility

- 120-N-7 unloading station french drain

- 120-N-6 sulfuric acid tank french drains

- 108-N neutralization pit

- UN-100-N-15 unplanned release

- UN-100-N-33 unpl anned release

- December 26, 1987 unplanned release

- 120-N-5 acid/caustic trench and neutralization unit

- UN-100-N-34 unplanned release

- August 7, 1987 unplanned release

- September 2, 1987 unplanned release

- November 9, 1987 unplanned release

- 120-N-3 neutralization pit and french drain

- 120-N-8 sulfuric acid day tank french drain

- Regeneration waste transport system

- June 14, 1986 unplanned release

- June 30, 1986 unplanned release

- 124-N-1 septic tank.

108-N Chemical Unloading Facility. The 108-N chemical unloading facility was used for storage and transfer of 93 percent sulfuric acid and 50 percent sodium hydroxide solutions received by railroad tank car or tank truck. The unloading station french drain (120-N-7) was used for neutralization of small releases from the overhead transfer boom. The french drain was used from 1963 until March 1987 (DOE-RL 1990). The french drain was $0.9 \mathrm{~m}(3 \mathrm{ft}$ ) in diameter by $1.2 \mathrm{~m}(4 \mathrm{ft})$ deep and consisted of a clay pipe filled with i ime (DOE-RL 1990).

There are three 38,000-L (10,000-gal) aboveground steel sulfuric acid storage tanks and one $290,000-\mathrm{L}(76,800-\mathrm{gal})$ sodium hydroxide tank located at the 108-N facility. The tanks began operating in 1964. Adjacent to the 
sulfuric acid tanks are french drains for overflows. The french drains are approximately $0.6 \mathrm{~m}(2 \mathrm{ft})$ in diameter and consist of a clay pipe packed with lime. The french drains operated from 1963 unti1 March 1987 (DOE-RL 1990). The acid transfer system uses a 3,800-L (1,000-gal) steel transfer tank located in a pit west of the 108-N building. The tank is filled with acid via gravity flow from the storage tanks. Air pressure is used to transfer the acid via piping through a trench to the 163-N day tank. No french drains are associated with the sodium hydroxide tank. Transfer pumps located in the 108-N building were used to transfer the sodium hydroxide directly to the 163-N day tank from the storage tank via piping through the trench (DOE-RL 1990).

108-N Neutralization Pit. The brick-lined 108-N neutralization pit is located outside the 108-N building. This facility received drainage from the 108-N floor drains and from the acid transfer tank. The pit was used to manually neutralize waste acid. The neutralized waste was sent via a water jet pump to the 183- $N$ facility where it was then discharged to the river through the $260-\mathrm{cm}(102-i n$.$) outfall line. The unit was in operation since$ 1963. The unit is $1.8 \mathrm{~m}(6 \mathrm{ft})$ wide by $1.2 \mathrm{~m}(4 \mathrm{ft})$ long by $1.8 \mathrm{~m}(6 \mathrm{ft})$ deep. The brick lining has been replaced at least once (DOE-RL 1990).

Various small, intermittent spills have occurred over the years at the 108-N facility that were associated with unloading and transfer operations. Several larger documented spills are described in the following paragraphs.

- March 20, 1981 (UN-100-N-15) - Sulfuric acid and rinsewater were spilled inside the 108-N building. The unknown amount of liquid was transferred to the acid tank french drains for neutralization. The transfer line developed a leak and released acid solution to the ground, The affected area was estimated to be less than $4.6 \mathrm{~m}^{2}$ $\left(50 \mathrm{ft}^{2}\right)$ (DOE-RL 1990). The extent of remedial measures is unknown.

- November 9, 1981 (UN-100-N-33) - Approximately 3,800 L (1,000 gal) of sulfuric acid were spilled during transfer from a rail car to an acid storage tank (DOE-RL 1990). The extent of remedial measures is unknown.

- December 26, 1987 - Approximately 38 L (10 gal) of sodium hydroxide were spilled to the ground during transfer from a rail car to the caustic storage tank. Difficulties during the transfer prompted the operator to disconnect the transfer line and set it on the ground while investigating the problem. At that time the sodium hydroxide leaked from the transfer line. The extent of remediation is unknown (WHC 1989C).

120-N-5 Acid/Caustic Transfer Trench and Neutralization Unit. The unit is a polymer concrete-lined neutralization pit and acid/caustic transfer trench between the 163-N demineralization $\mathrm{plant}$ and the 108-N chemical unloading facility. The neutralization unit consists of two containment vaults: one for sulfuric acid and one for sodium hydroxide. Each containment vault is approximately $1.8 \mathrm{~m}(6 \mathrm{ft})$ long by $1.8 \mathrm{~m}(6 \mathrm{ft})$ wide by $3 \mathrm{~m}(10 \mathrm{ft})$ deep. The trench, containing both acid and caustic piping, slopes toward the neutralization unit so that spills can be contained within the vaults. 
The polymer concrete lining was installed in parts of the trench in 1986. According to personnel, the unit consisted of unsealed concrete from 1963 to 1986 (DOE-RL 1990).

Several documented releases associated with the unit have occurred. These releases are described in the following paragraphs.

- May 12, 1980 (UN-100-N-34) - During the week1y transfer of sulfuric acid from the 108-N storage tank to the 163-N day tank, a rupture in the pipeline occurred. Approximately $13,000 \mathrm{~L}(3,400 \mathrm{gal})$ of sulfuric acid spilled into the containment vault, some of which overflowed to the ground. The acid in the containment vault was neutralized with 50 percent sodium hydroxide and pumped to the clearwell overflow (located south of the unit). The unknown amount of acid that overflowed to the ground was neutralized with soda ash and liquid sodium hydroxide (DOE-RL 1990). No further remediation has been documented.

- August 7, 1987 - Water was found leaking outside the 163-N building north wall to an area of the trench that had not been treated with polymer cement because of clearance restrictions due to the proportions of the trench. Sulfuric acid had corroded away exposed concrete (WHC 1987c). The extent of contamination, amount of sulfuric acid released, and the extent of remediation is unknown.

- September 2, 1987 - During caustic transfer from the 108-N caustic storage tank to the $163-N$ caustic day tank, a leak was noted in the piping and caustic collected in the trench. Transfer was stopped and the pipeline was patched. The amount of caustic released into the trench is unknown and there is no documentation that caustic reached the soil (WHC 1987d).

- November 9, 1987 - A leak of approximately 760 L (200 gal) of sulfuric acid occurred during transfer operations. This leak was cleaned up at the time. On December 4, 1987, it was noticed that the trench was open to the soil at the location where the leak occurred. This open area was found to be a dry well installed in 1986 when the trench was upgraded. The dry well was installed for steam trap drainage, not for containment of acid spills. An estimated 57 to $114 \mathrm{~L}$ (15 to $30 \mathrm{gal}$ ) of sulfuric acid was released to the ground (WHC 1987e). An unknown amount of contaminated soil was removed.

120-N-3 (163-N) Neutralization Pit and French Drain. The unit is a french drain and vault located immediately west of the 163-N demineralization plant. The unit was constructed in 1963 and is still in place (DOE-RL 1990). It serves as a spill containment unit for the two 38,000-L (10,000-gal) acid and caustic day tanks located immediately inside the $163-\mathrm{N}$ building. A drain in the tank area leads to the unit. The vault is approximately $2.4 \mathrm{~m}$ by $7.6 \mathrm{~m}$ ( $8 \mathrm{ft}$ by $25 \mathrm{ft}$ ) in size and approximately $2.4 \mathrm{~m}(8 \mathrm{ft}$ ) deep. The walls of the vault are constructed of concrete and the floor is unlined earthen material. Located in the vault is a 1.2 - to $1.8-\mathrm{m}$ (4- to 6-ft)-diameter french drain made of clay. The depth of the french drain is unknown. No liquid is currently present in the pit and french drain. 
Small intermittent releases of sulfuric acid or sodium hydroxide occurred during transfer operations to or from the 163-N day tanks (DOE-RL 1990). No releases other than the small releases have been documented.

120-N-8 Day Tank Vent French Drain. The unit is a french drain used to receive overflow of sulfuric acid from the $163-N$ demineralization plant sulfuric acid day tank. The unit is 1.2 to $1.8 \mathrm{~m}(4$ to $6 \mathrm{ft}$ ) in diameter and consists of a clay pipe filled with lime to neutralize any sulfuric acid releases. It is located on the north side of the 163-N building. The unit was installed in 1963 and taken out of service on May 13, 1988 (DOE-RL 1990).

The unit received unknown amounts of intermittent sulfuric acid discharges. Each discharge is estimated to have averaged less than $3.8 \mathrm{~L}$ (1 gal) of liquid. There are no specific documented releases associated with the unit (DOE-RL 1990).

Regeneration Waste Transport System. The regeneration waste transport system includes the storage and piping systems that conveyed spent regeneration effluent (either acid, caustic, or neutralized) from the 163-N demineralization $\mathrm{pl}$ ant and routed this effluent to the $120-\mathrm{N}-1$ and 120-N-2 ponds. The system took on various configurations from 1977 until 1990. Before 1977, the spent regeneration waste was discharged to the Columbia River (DOE-RL 1990).

From 1977 until 1983, regeneration effluents flowed through lined concrete trenches, $0.3 \mathrm{~m}(1 \mathrm{ft})$ deep by $0.6 \mathrm{~m}(2 \mathrm{ft})$ wide, in the 163-N demineralization $\mathrm{plant}$. The trenches were covered with a metal grating. The trenches carried the effluent to a sump located near the northwest corner of the 163-N building. There the sump pumps transferred the effluent in an underground $20-\mathrm{cm}(8-i n$.$) epoxy resin waste transfer line with filter backwash$ water effluent from the 183-N water filter plant to the north and south settling ponds and subsequently the 120-N-1 percolation pond (WHC 1987f). Figure 5-4 shows the regeneration waste transfer system configuration from 1977 unti1 1983.

In 1983, the piping was modified to deliver the acid and/or caustic effluent from 163-N directly to the 120-N-1 percolation pond, bypassing the inactive settling ponds. In addition, the filter backwash water was no longer combined with the regeneration effiuent, but was piped to the new 130-N-1 filter backwash discharge pond. The regeneration waste transport system operated in this configuration from 1983 until 1986 (WHC 1987f). Figure 5-5 shows the design of this system for that time period.

In 1986, the 120-N-2 surface impoundment was put into service. At that time, the acid and/or caustic regeneration effluent was routed directly to this unit, where the effluent was neutralized. The neutralized effluent was then piped to the 120-N-1 (1324-NA) percolation pond (WHC 1987f). Figure 5-6 shows the design of the regeneration waste transport system between 1986 and 1988 .

In 1988, an ENU was installed within the 163-N demineralization plant. At that time, the 120-N-2 surface impoundment was taken out of service. From 1988 until 1990, spent regeneration effluent was routed via the same trenches mentioned previously to the spent regeneration surge tank, located on the 
north exterior of 163-N building. The tank discharged to the ENU. After adding the proper amount of acid or caustic solution, the contents were mechanically agitated. When the $\mathrm{pH}$ was within the range of 6.0 to 9.0 , the neutralized effluent was piped via the $20-\mathrm{cm}(8-i n$.$) waste transfer 1$ ine to the 120-N-1 percolation pond (DOE-RL 1990).

Low levels of radionuclides have been found in the piping. Based on analyses of piping scale, the material was determined to be naturally occurring thorium found in alum used during the water purification processes (DOE-RL 1990).

Two documented releases have been associated with the regeneration waste transport system. These releases are described in the following paragraphs.

- June 14, 1986 - A leak was detected in the waste transport pipe while wastes from the anion and cation! regeneration process were being routed to the $120-\mathrm{N}-2$ surface impoundment. Once the leak was discovered, regeneration processes were shut down. A sample was collected at the point of the leak and found to have a $\mathrm{pH}$ of 1.4 . It was estimated that approximately $25,000 \mathrm{~L}(6,500 \mathrm{gal})$ of acidic regeneration waste had leaked to the ground and formed a pond in an area south of the 163-N building. Caustic regeneration waste was pumped through the line and allowed to leak into the acidic pond to neutralize the spilled material. Several hours elapsed until the pH of the spilled material reached 6.9. The neutralized liquid was then pumped to the $260-\mathrm{cm}\left(102-\mathrm{in}_{\text {.) }}\right.$ outfall line and released to the Columbia River. An unknown amount of soil around the leak was excavated and disposed of. The pipe was repaired (DOE-RL 1990).

- June 30, 1986 - Approximately 3,800 L (1,000 gal) of acidic (pH of 1.1) cation regeneration waste spilled to the ground in the area of sump No. 1 when a temporary transport line became dislodged from the sump. When this was discovered, the regeneration process was stopped and the hose was placed back in the sump and secured. The $\mathrm{pH}$ was adjusted by adding $82 \mathrm{~kg}$ (180 1b) of caustic soda (soda ash) to the spilled waste. The soil was sampled and the $\mathrm{pH}$ was 10.1; therefore, no further remediation was conducted (DOE-RL 1990).

124-N-1 Septic Tank. The septic tank and seepage pit making up sewer system I are located south of the 163-N building and were installed in 1963 (DOE-RL 1990); the system is still operating (DOE-RL 1990). This unit receives sanitary sewage. It was originally designed to serve only personnel in the $163-N$ and $183-N$ buildings. The seepage pit for this system provides about $19 \mathrm{~m}^{2}$ (200 $\left.\mathrm{ft}^{2}\right)$ of infiltration surface area and $8,540 \mathrm{~L}(2,256 \mathrm{gal})$ of fluid storage. In about 1982, two bathroom utility trailers were hooked up to this sewer system to serve the personnel in the 1127-N and 1128-N buildings. In 1985, the unit served 50 personnel and calculated daily flow was $5,400 \mathrm{~L} /$ day $(1,420 \mathrm{gal} /$ day) (DOE-RL 1990). There are no documented dangerous or radioactive releases associated with the unit. 


\subsubsection{6-N-8 Hazardous and Mixed Waste Storage Area}

The 116-N-8 hazardous and mixed waste storage area is a concrete-paved less-than-90-day waste container storage pad. The pad is curbed and surrounded by a wire mesh fence. The pad is $18 \mathrm{~m}$ by $46 \mathrm{~m}$ (60 ft by $152 \mathrm{ft}$ ) in size. It is located inside the double-fenced reactor area at the southern corner of the fence. The pad is covered by a roof and walled on two sides. The unit has been in operation since December 1986 (DOE-RL 1990).

Drums and containers stored in this area may contain mixed or hazardous wastes. Hazardous or mixed wastes from satellite collection areas from other generation points within the 100-N Area and at the 100-K Areas are stored at the unit. There are no documented releases to the soil from this unit. Before 1986, the area was used as a maintenance storage area (DOE-RL 1990).

\section{$5.1 .16 \quad 184-N$}

184-N Plant Service Power House, Tanks, and Piping System. The 184-N plant service power house consists of three boilers located in the 184-N building and the 184- $N$ annex. The boiler system provides oil-fired boiler-generated steam to the main steam supply system during reactor startup and shutdown periods. This area is distinguished by the significant number of hydrocarbon product releases. The following potential sources are listed.

- 184-N day tank area

- UN-100-N-19 unplanned release

- UN-100-N-21 unplanned release

- October 9, 1987 unplanned release

- 166-N-184-N piping (not shown in Figure 5-2)

- UN-101-N-18 unplanned release

- UN-100-N-22 unplanned release

- UN-100-N-23 unplanned release

- October 14, 1987 unplanned release

- April 26, 1989 unplanned release.

184-N Day Tank Area. The 184-N plant service power house has three aboveground oil day tanks located outside the building on the north side and one $5,678 \mathrm{~L}(1,500 \mathrm{gal})$ diesel fuel underground storage tank. These include two $130,000-L(35,000-g a 1)$ No. 6 (Bunker $C$ ) fuel 011 day tanks and one $30,000-L(8,000-g a l)$ diesel $0 i 1$ day tank. The day tanks are surrounded by a concrete retaining wall with the ground surface exposed inside.

Several documented unplanned releases are associated with the 184-N day tank area. These are described below.

- April 1984 (UN-100-N-19) - Approximately 7,600 L (2,000 gal) of No. 6 fuel oil spilled to the ground when the day tank overflowed during filling. All the fuel oil was contained within the surrounding retaining walls and did not penetrate the hard sand floor of the containment structure. The waste $0 i 1$ was removed and disposed of (WHC 1989b). 
- April 25, 1986 (UN-100-N-21) - Approximately 3,000 L (800 gal) of diesel oil was released to the ground when the day tank overflowed during filling. This was attributed to a fallure of the tank-level annunciator. The annunciator was repaired and the oil removed from the tank impoundment area. Groundwater monitoring wells were sampled and no ofl was detected (DOE-RL 1990).

- October 9, 1987 - The diesel oil day tank overflowed during filling operations because a level indicator was not reading the correct oil level. An unknown amount of oil was cleaned up (WHC 1987g).

166-N - 184-N Piping. The 184-N fuel oil day tanks are connected to the oil storage tank at $166-\mathrm{N}$ by a $20-\mathrm{cm}\left(8-\mathrm{in}_{\text {. }}\right)$ underground supply line. The 184-N diesel oil day tank is connected to the storage tanks at 166-N by a $10-\mathrm{cm}\left(4-\mathrm{in}_{\text {.) }}\right.$ underground supply line (WHC 1989b). Several unplanned releases from the pipelines have been documented. They are described in the following paragraphs.

- August 1973 (UN-100-N-18) - A leak caused by external corrosion occurred in the 10-cm (4-in.) diesel oil supply line between the 166-N storage tanks and the day tank. The leak was detected by a pressure test after approximately $760 \mathrm{~L}(200 \mathrm{gal})$ of diesel oil had been spilled to the ground (WHC 1989b). The line was excavated and repaired (DOE-RL 1990).

- June 23, 1986 (UN-100-N-22) - External corrosion of the diesel oil supply line caused a $3,800 \mathrm{~L}(1,000 \mathrm{gal})$ leak of diesel oil just outside the 184- $\mathrm{N}$ tank area. The line was excavated and rerouted. An unknown amount of contaminated soil was removed. Groundwater Monitoring Well 199-N-16 was sampled and oil was detected in July 1986. Well 199-N-16 is located approximately $9 \mathrm{~m}(30 \mathrm{ft})$ west of the 184-N building. An unknown amount of residual oil was recovered from the groundwater through Well 199-N-16 (WHC 1989b).

- January 10, 1987 (UN-100-N-23) - External corrosion caused a leak in the diesel oil supply line. Approximately $760 \mathrm{~L}$ (200 gal) of diesel oil was released to the soil. The line was isolated, excavated, and repaired. Groundwater Monitoring Well 199-N-16 was sampled and oil was found. Residual oil was recovered from the well (WHC 1989b).

- October 14, 1987 - An unknown amount of fuel oil leaked from a loose pipe fitting at the 184-N annex. 0 il was being transferred from the day tank to the No. 2 boiler. $0 i l$ was contained in the 184-N annex drain trench and cleaned up (DOE-RL 1990).

- April 26, 1989 - The diesel oil supply pipeline developed leaks in three places between $166-N$ and the 184- $N$ day tanks. The specific cause of the leaks was unknown. A minimum of $1,100 \mathrm{~L}(300 \mathrm{gal})$ of diesel oil was released to the soil along the pipeline. Monitoring Wells 199-N-16 and 199-N-17 were sampled. $0 i 1$ was detected in these samples. Forty-six drums and eight dump trucks of contaminated soil were removed (WHC 1990b). 


\subsubsection{Decontamination Drainline Leak}

A mixed waste leak from the $3.8-\mathrm{cm}(1.5-$ in. $)$ decontamination drainline connecting the $\mathrm{N}$ Reactor building to the $116-\mathrm{N}-2$ radioactive chemical waste storage facility distinguishes this small area from the surrounding source unit areas. Decontamination of the $N$ Reactor occurred every 3 to 5 years. The decontamination solution generally contained phosphoric acid and diethylthiourea, but small-scale decontaminations occurred that contained a variety of cleaning solutions. Generally, the $3.8-\mathrm{cm}\left(1.5-\mathrm{in}_{\text {. }}\right)$ chemical decontamination waste drainline transported these smaller scale decontamination solutions to the $116-\mathrm{N}-2$ facility.

On September 10, 1985, a leak of radiologically contaminated water occurred at four locations near the $\mathrm{N}-29$ craft shop along the decontamination waste drain line between the $N$ Reactor building and 116-N-2 facility (UN-100-N-6). Approximately $6,800 \mathrm{~L}(1,800 \mathrm{gal})$ of irradiated water was released. The water contained a total estimated $0.2 \mathrm{Ci}$ of ${ }^{60} \mathrm{Co}, 0.04 \mathrm{Ci}$ of ${ }^{54} \mathrm{Mn}, 0.003 \mathrm{Ci}$ of ${ }^{100 \mathrm{Ru}}$, and $0.003 \mathrm{Ci}$ Of ${ }^{137} \mathrm{Cs}$. Approximately $17 \mathrm{~m}^{3}\left(590 \mathrm{ft}^{3}\right)$ of contaminated soil reading between 7,000 and 25,000 cpm was excavated and removed to the 200 Area burial ground for disposal. No documented sampling was conducted at the base of the excavation. The area was backfilled with clean fill (DOE-RL 1990).

\section{$5.1 .18 \quad 120-N-4$}

120-N-4 Storage Area Grouping. This grouping consists of two potential sources in an area southwest of the 116-N-2 radioactive chemical waste treatment and storage facility.

- 120-N-4 storage area

- 1716-N service station underground storage tanks.

120-N-4 Storage Area. The unit is currently used as a nonhazardous and/or low level radioactive waste storage pad. It is a $30-\mathrm{m}$ by $23-\mathrm{m}(100-\mathrm{ft}$ by $75-\mathrm{ft})$ curbed concrete pad located immediately southwest of the berm surrounding the 116- $\mathrm{N}-2$ radioactive chemical waste treatment and storage facility. The unit has been in its current configuration since November 1985 (DOE-RL 1990).

Before 1985, the unit was unpaved and used as a laydown yard for radioactively contaminated equipment as well as for storage of radioactively contaminated oils. Information regarding types and amounts of wastes stored in this area is unavailable. Aerial photographs before 1985 indicate storage of unknown materials in the area immediately southeast of the current pad. There are no documented releases to the soil from this unit (DOE-RL 1990).

1716-N Service Station Underground Storage Tanks. Two underground storage tanks were located at the 1716-N service station, located south of the $120-\mathrm{N}-4$ storage area. The tanks are identified as 100-N-SS-27 and $100-\mathrm{N}-\mathrm{SS}-28$. Both tanks contained unleaded gasoline. Tank 100-N-SS-27 had a capacity of $11,000 \mathrm{~L}(3,000$ gal) and tank $100-\mathrm{N}-\mathrm{SS}-28$ had a capacity of $7,600 \mathrm{~L}(2,000 \mathrm{gal})$. The tanks were constructed of single-wall carbon steel and neither had cathodic or interior protection. Tank $100-\mathrm{N}-\mathrm{SS}-27$ was installed in 1967 and Tank 100-N-SS-28 was installed in 1976 (DOE-RL 1990). 
Tank 100-N-SS-27 was removed in December 1990 and 100-N-SS-28 was removed in July 1991. Soil sampling during tank removal indicates that the tanks have leaked. Site remediation is in progress.

\subsubsection{Regeneration/Filter Backwash Waste Disposal Area}

The regeneration/filter backwash waste disposal area grouping includes those units that have received corrosive regeneration wastes from the 163-N demineralization $\mathrm{plant}$ and filter backwash water from the 183-N filtered water plant. This area has five potential sources.

- 120-N-1 percolation pond

- South settling pond

- $120-\mathrm{N}-2$ surface impoundment

- 130-N-1 filter backwash discharge pond

- 1143-N paint shop.

The 1143-N paint shop is included in this area because it is located near the $120-N-1$ and $120-N-2$ farilities.

120-N-1 Percolation Pond. The $120-N-1$ percolation pond is a large unlined pond located approximately $910 \mathrm{~m}(3,000 \mathrm{ft})$ southeast of the $N$ Reactor building. The pond was placed in service in August 1977 and was used to treat corrosive regeneration effluent from the 163-N demineralization plant and filter backwash water from the 183-N filtered water plant. The effluent was treated in the 120-N-1 percolation pond by the alternate addition of acidic cation column regeneration effluent and alkaline anion column regeneration effluent. This alternate addition of low- and high-pH effluent served to neutralize the effluents. The 120-N-1 percolation pond also made use of the buffering capacity and calcareous nature of the soil underlying the pond to neutralize these corrosive wastes. Treated effluents were transferred to the north and south settling ponds located directly west of the percolation pond. These settling ponds were used to settle out the solids in the filter backwash water waste stream. The settling ponds were removed from service in early 1983. The 120-N-1 percolation pond managed an average of $600,000 \mathrm{~L} /$ day ( $160,000 \mathrm{gal} / \mathrm{day})$ of corrosive regeneration effluent and $1,100,000 \mathrm{~L} / \mathrm{day}$ (300,000 gal/day) of filter backwash water (WHC 1987f). Figure 5-7 shows the configuration of the 120-N-1 percolation pond area from 1977 to 1983.

Tables 5-7 and 5-8 show representative analyses of cation and anion regeneration effluent cycles, respectively, which were discharged from 163-N to 120-N-1. Table 5-9 shows representative analyses of the filter backwash effluent discharged from $183-N$ to $120-N-1$. The frequency of these analyses is not known.

Low levels of naturally occurring thorium was present in the water purification chemical, alum, and may have been disposed of in this unit. This radionuclide has been found in the water treatment plant piping (DOE-RL 1990).

In the spring of 1983, the $120-N-1$ percolation pond was enlarged from a bottom area of $850 \mathrm{~m}^{2}\left(9,200 \mathrm{ft}^{2}\right)$ with a volume of approximately $4,500,000 \mathrm{~L}$ $(1,200,000 \mathrm{gal})$ to a bottom area of $2,700 \mathrm{~m}^{2}\left(29,000 \mathrm{ft}^{2}\right)$. This enlarged pond was designed to contain up to $11,000,000 \mathrm{~L}(3,000,000 \mathrm{gal})$ of corrosive wastes from the regeneration of ion exchange columns in the 163-N demineralization 
plant. The filter backwash water was routed to the $130-\mathrm{N}-1$ filter backwash disposal pond at this time, and the south settling pond was backfilled to grade (WHC 1987f). The entire bottom area of the 120-N-1 pond has not been covered with wastes since its enlargement (DOE-RL 1991).

Use of the 120-N-1 percolation pond to treat dangerous wastes was discontinued by May 13, 1986 when the 120-N-2 surface impoundment was put into service to treat the corrosive regeneration effluents. The 120-N-2 surface impoundment is a double-lined pond with a leachate collection system that was used to neutralize the wastes before their discharge to the 120-N-1 percolation pond (WHC 1987f). This unit was used from 1986 until 1988, when it was replaced by an ENU located at the 163-N demineralization plant. The 120-N-1 percolation pond began receiving neutralized regeneration effluent in 1986 and continues to receive effluent from the ENU. The enlarged 120-N-1 percolation pond and the 120-N-2 surface impoundment are shown in Figure 5-8.

South Settling Pond. The south settling pond, in conjunction with the north settling pond, received corrosive regeneration effluent and process and cooling water from the 163-N demineralization plant and filter backwash water from the 183-N filtered water plant from 1977 until 1983. The volume of effluent being sent to these ponds is the same as for the 120-N-1 percolation pond. Analyses of effluents discharged to the south settling pond are presented in Tables 5-7, 5-8, and 5-9. Additional information regarding contaminants possibly contained in the $163-\mathrm{N}$ demineralization plant waste waters is presented in the RFI/CMS for the 100-NR-2 Operable Unit (DOE-RL 1991). Low levels of naturally occurring thorium may be present in the pond as a result of using alum in the water treatment process (DOE-RL 1990).

The south settling pond was a rectangular, unl ined basin constructed below grade. The dimensions of the pond were approximately $34 \mathrm{~m}$ by $15 \mathrm{~m}$ $(110 \mathrm{ft}$ by $50 \mathrm{ft}$ ) at grade; the sides sloped to a bottom measuring approximate 1 y $21 \mathrm{~m}$ by $3 \mathrm{~m}$ (70 ft by $10 \mathrm{ft}$ ). Depth is estimated to have been approximately $4.6 \mathrm{~m}$ (15 ft). After solids, primarily from the filter backwash effluent, were settled, the contents of the south settling ponds were transferred to the 120-N-1 percolation pond. Between 1983 and 1986 the north and south settling ponds were closed. The regeneration effluent was then discharged directiy to the 120-N-1 percolation pond and the filter backwash effluent was discharged to the 130-N-1 filter backwash disposal pond. The south settling pond was backfilled (DOE-RL 1990).

120-N-2 Surface Impoundment. The 120-N-2 surface impoundment was constructed and placed in service in 1986 and operated until 1988. It is located approximately $910 \mathrm{~m}(3,000 \mathrm{ft})$ southeast of the $N$ Reactor building in the former location of the north settling pond (WHC 1986). The operating history for the north settling pond is the same as for the south settling pond described above.

The 120-N-2 unit is a double-lined surface impoundment with leak detection equipment. The unit is approximately $43 \mathrm{~m}$ by $23 \mathrm{~m}$ (140 ft by $75 \mathrm{ft}$ ) at grade sloping to $24 \mathrm{~m}$ by $4.6 \mathrm{~m}$ ( $80 \mathrm{ft}$ by $15 \mathrm{ft}$ ) at approximately $4.6 \mathrm{~m}$ $(15 \mathrm{ft})$ below grade. The impoundment was designed to contain a volume of 
$1,600,000 \mathrm{~L}(424,000 \mathrm{gal})$ (WHC 1986). Figure 5-9 and Color I1lustration 5-6 show the physical design of the unit. No leaks have been detected from this unit (DOE-RL 1990).

Acid and caustic regeneration effluent from the 163-N demineralization plant was neutralized in the 120-N-2 surface impoundment. Approximately $1,630,000 \mathrm{~L} /$ day $(430,000 \mathrm{gal} /$ day) were neutralized (DOE-RL 1990). Tables 5-7 and 5-8 show representative analyses of cation and anion regeneration effluents, respectively. The caustic anion regeneration effluent was generally neutralized in the surface impoundment by the addition of the acidic cation regeneration effluent. Acid cation regeneration effluent was similarly neutralized by the addition of caustic anion regeneration effluent. Once neutralization was complete, the neutralized effluent was discharged to the 120-N-1 percolation pond via a $30-\mathrm{cm}(12-\mathrm{in.})$ drainline and $30-\mathrm{cm}(12-\mathrm{in}$. overflow line (WHC 1986). In 1988, the 120-N-2 surface impoundment was taken out of service and replaced by an ENU located at the 163-N demineralization plant.

130-N-1 Filter Backwash Discharge Pond. The 130-N-1 filter backwash discharge pond is a percolation pond used for disposal of effluents generated during backwash of the sand filters in the 183-N filtered water piant. The pond is a natural basin, marsh-like in appearance, located about $0.8 \mathrm{~km}(0.5 \mathrm{mi})$ southeast of the $N$ Reactor building.

The filter backwash discharge pond was placed in service in early 1983 following reconstruction of the $120-N-1$ percolation pond, which was formerly used for disposal of the backwash effluent. The pond is fed via a 25- to $30-\mathrm{cm}$ (10- to 12-in.) buried line from the 183-N filtered water plant. Approximately 1,100,000 L/day (300,000 gal/day) of backwash effluent were disposed of at the unit. The $183-N$ filter backwash effluent has a neutral $\mathrm{pH}$ and contains low concentrations of several anions and cations (DOE-RL 1990). Aluminum sulfate (alum) is used as a flocculent and polyacrylamide is used as a filter aid/coagulant in filtered water production (DOE-RL 1990). Analysis of the filter backwash effluent indicates that it does not contain any 1 isted dangerous wastes or dangerous waste sources, or exhibit any dangerous waste characteristics or criteria per WAC 173-303 (DOE-RL 1990). Table 5-9 shows representative analyses of the filter backwash effluent.

1143-N Paint Shop. The 1143-N paint shop has three waste management units: a water scrubber for paint overspray, a 210-L (55-gal) solvent accumulation drum, and an associated outdoor sandblasting area, the 1143-N blast yard.

Paint wastes were collected, drummed, and transferred offsite for disposal. Overspray collected by the water curtain filtration system was disposed of onsite. The blast yard manages paint chips and spent garnet sand. These waste management units are still active and no remedial activities have taken place.

\subsubsection{Office Septic Tank Area}

The four septic systems in this area are shown near the 1117-N, 1113-N, $1115-N$, and 1134-N buildings in Figure 3-1. The only known waste managed by these units is sanitary sewage. There is no documented information that any 
other wastes were released into the septic systems or evidence that remedial activities were performed. The office septic tank area consists of the following tanks.

- $124-N-5$ septic tank
$: 124-N-6$ septic tank
$: 124-N-7$ septic tank
- $124-N-8$ septic tank.

124-N-5 Septic Tank. The septic tank and drainfield making up Sewer System $V$ are located south of bullding 1117-N and were installed in 1981; the system was taken out of service in February 1987 (DOE-RL 1990). Sewer System V serves buildings 1111-N, 1116-N, 1117-N, 1118-N, 1123-N, 1124-N, 1125-N, and 1131-N. The septic tank has a fluid capactty of 14,000 L (3,677 gal) and a drainfield providing approximately $90 \mathrm{~m}^{2}\left(960 \mathrm{ft}^{2}\right)$ of infiltrative surface area. Fill dirt was placed over the drainfield to a depth of $0.6 \mathrm{~m}$ (2 ft) or more in the early 1980s. In 1985, the unit served 210 personnel and the calculated daily flow was 140,000 L/day (3,780 gal/day) (DOE-RL 1990). The sewer system is still in place; it is unknown if residual liquid is present.

124-N-6 Septic Tank. The septic tank and drainfield making up Sewer System VI are located south of building 1113-N and were installed in 1979-80; the system was taken out of service in February 1987 (DOE-RL 1990). This unit received sanitary sewage. The septic tank has a fluid capacity of $7,600 \mathrm{~L}(2,000 \mathrm{gal})$ and the drainfield has an infiltrative surface area of $74 \mathrm{~m}^{2}\left(800 \mathrm{ft}^{2}\right)$ (DOE-RL 1990). This system is directly hooked up to Sewer system VII just upstream of the septic tank. Sewer System VI serves buildings 1113-N, 1114-N, and 1115-N. In 1984, the septic tank was pumped out and the system was abandoned (DOE-RL 1990).

124-N-7 Septic Tank. The septic tank and drainfield making up Sewer System VII are located south of building 1115-N under the high-voltage power lines and were installed in 1984 (DOE-RL 1990); the system was taken out of service in February 1987 (DOE-RL 1991). This unit received sanitary sewage. The septic tank has a fluid capacity of $28,000 \mathrm{~L}(7,500 \mathrm{gal})$. Sewer System VII serves buildings $1103-N, 1104-N$, and $1145-N$. In 1985, the unit served 290 personnel and the calculated dally flow was $20,000 \mathrm{~L} /$ day $(5,220 \mathrm{gal} /$ day $)$ (DOE-RL 1990). The sewer system is still in piace and it is unknown if residual liquid is present.

124-N-8 Septic Tank. The septic tank and drainfield making up Sewer System VIII are located south of building 1134-N and were installed in 1983. This unit received sanitary sewage. The septic tank has a fluid capacity of $19,000 \mathrm{~L}(5,000 \mathrm{gal})$, and the drainfield has an infiltrative surface area of $150 \mathrm{~m}^{2}\left(1,650 \mathrm{ft}^{2}\right)$. Sewer System VIII served buildings 1132-N, 1133-N, $1134-N$, and 1135-N. In 1985, the unit served 51 personnel and the calculated daily flow was 3,500 L/day (915 gal/day) (DOE-RL 1990). The sewer system is still in place, but is no longer in use. It was replaced by the 124-N-10 sewer system in 1987. 
DOE/RL 91-59

\subsubsection{N-17 Paint Shop}

This grouping includes the entire craft shop area.

Paints, solvents, and oils are used at the $\mathrm{N}-17$ paint shop, which has two waste accumulation drums, one for waste paint and the other for waste 011. A sandblasting area is also associated with the $\mathrm{N}-17$ paint shop. The paint shop is located about $300 \mathrm{~m}(1,000 \mathrm{ft})$ west of the $N$ Reactor facility. This unit is currently active.

An air compressor located east of the paint shop has leaked nonhazardous lubrication oil over the years; the surrounding soil is oil stained. The extent of contamination is unknown. Six drums of contaminated soil were removed (DOE-RL 1990).

\subsubsection{0-N Septic Tank Grouping}

124-N-9 Septic Tank. The septic tank and drainfield making up the 129-N-9 sewer system are located northeast of building 1120-N and were installed in 1985. The septic tank has a fluid capacity of $11,000 \mathrm{~L}(3,000 \mathrm{gal})$ and the drainfield has an infiltrative surface area of $325 \mathrm{~m}^{2}\left(3,500 \mathrm{ft}^{2}\right)$. This unit receives $8,300 \mathrm{~L} /$ day $(2,200 \mathrm{gal} /$ day) of sanitary sewage (DOE-RL 1990). There is no documented information regarding disposal of any other wastes to the unit. The sewer system is still in place. No documented remedial activities have taken place.

\subsubsection{0-N-10 Sewer System Grouping}

124-N-10 Sewer System. The 100-N sewer system was constructed to replace five 100-N Area sewer systems. The system includes a two-pond lagoon facility, a sewer trunk line and other pipelines, three 1 ift stations, service access holes, and associated sewer system instrumentation and annunciation capability. Five sewer systems were replaced (DOE-RL 1990):

- Sewer System IV, which served the 105-N, 109-N, 184-N, 1100-N, $1101-N, 1102-N, 1107-N, 1112-N, 1119-N$, and 1126-N buildings and contractor construction buildings

- Sewer System V, which served the 1111-N, 1116-N, 1117-N, 1118-N, $1123-N, 1124-N, 1125-N$, and 1131-N buildings

- Sewer System VI, which served the 1113-N, 1114-N, and $1115-N$ buildings and was abandoned

- Sewer System VII, which served the 1103-N and 1104-N buildings, and $1113-N, 1114-N, 1115-N$, and $1146-N$ buildings (1152-N, $1153-N$, and $1154-N$ buildings were also connected to this system)

- Sewer System VIII, which served the 1132-N, 1133-N, 1134-N, and 1135-N buildings. 
This unit has been operational since February 1987 and receives $190,000 \mathrm{~L} /$ day ( $50,000 \mathrm{gal} /$ day) of sanitary sewage (DOE-RL 1990). There is no documented information regarding disposal of any other wastes to the unit other than sanitary sewage routinely trucked there from the 200 Area. The central sewer system is still in place. No remedial activities have taken place. Currently this unit does not have a Washington State Permit, but by June 1994, a WAC 173-216 permit for groundwater discharges will be submitted to the state.

UN-100-N-11 Unplanned Release. On October 2, 1975, a radioactively contaminated $230 \mathrm{~kg}$ (500 lb) valve bonnet fell from a truck onto the road and into the adjacent field. This occurred at the corner of Route 4 North and the 100-N Area access road (see Figure 2-1). Cleanup consisted of removal and disposal of $6.1 \mathrm{~m}^{3}\left(8 \mathrm{yd}^{3}\right)$ of contaminated soil and asphalt (DOE-RL 1990).

\subsection{AFFECTED ENVIRONMENT - GROUNDWATER}

Groundwater in the 100-N Area has been monitored for years as part of the Environmental Surveillance Program at the Hanford Site. The location of groundwater monitoring wells in the 100-N Area is shown in Color Illustration 2-2. Since December 1987, selected wells have been monitored as part of the RCRA interim status detection monitoring program. Detection monitoring networks were established for the 116-N-1 crib and trench (1301-N LWDF), 116-N-3 crib and trench (1325-N LWDF), 120-N-1 (1324-NA percolation pond), and 120-N-2 (1324-N Surface Impoundment). Results of chemical and radiological analyses are discussed in this section for samples taken from wells in the vicinity of the $116-N-1$ and $116-N-3$ crib and trench, 118-N-1 spacer storage silos, $120-N-1$ percolation pond/120-N-2 surface impoundment, and the 1304-N EDT. All these sources are either RCRA facilities or high-priority sites as described in the RCRA Facility Investigation/Corrective Measures Study Work Plan for the 100-NR-2 Operable Unit, Hanford Site, Richland, Washington (DOE-RL 1991).

\subsection{1 $116-N-1$ and $116-N-3$ Crib and Trench Areas}

The 116-N-1 and 116-N-3 facilities (1301-N and 1325-N LWDF) have been used primarily to dispose of radioactive effluent originating in $N$ Reactor and the 109-N heat exchanger buildings. These effluents contained low levels of a number of radioactive constituents, including tritium, plutonium, ${ }^{137} \mathrm{Cs},{ }^{90} \mathrm{Sr}$, ${ }^{60} \mathrm{Co},{ }^{106} \mathrm{Pu},{ }^{125} \mathrm{Sn}$, and ${ }^{131} \mathrm{I}$ (WHC 1990a).

Some radionuclides contained in the effluent such as plutonium and ${ }^{137} \mathrm{Cs}$, are held strongly by soil and are virtually immobile. Other radionuclides, such as iodine, are minimally sorbed and are highly mobile. Because tritium is an analog of the water molecule it migrates at the same rate as water. still other constituents, such as ${ }^{30} \mathrm{Sr}$ and ${ }^{60} \mathrm{Co}$, are adsorbed to the soil but not as strongly as radionuclides such as ${ }^{137} \mathrm{Cs}$. The migration of these constituents is retarded, but not as heavily as the more strongly adsorbed constituents. As a result, plumes containing the more mobile radionuclides, such as tritium and ${ }^{131} \mathrm{I}$, had developed in groundwater soon after discharge to the cribs was initiated, while concentrations of less mobile constituents gradually increased in groundwater (DOE-RL 1990). 
Activity levels for selected radionuclides in groundwater measured during 1981 (DOE-RL 1990 and DOE-RL 1991) show the contamination patterns that had developed by that time in the vicinity of the $116-\mathrm{N}-1$ crib and trench (Table 5-10). The tritium data indicate high levels of contamination in the entire area covered by the then-existing monitoring network. These data also demonstrate that the impact of the 116-N-1 crib and trench extended in all directions. The full extent of influence of the 116-N-1 facility at this time, particularly in the southern and eastern directions, cannot be determined because the network of groundwater monitoring wells is limited.

Examination of these data also indicate elevated levels of other radionuclides throughout much of the area, and there appears to be a pattern of decreasing levels of activity of many radionuclides as the distance from the 116-N-1 crib and trench increases. This pattern is consistent with retardation caused by adsorption, as well as radioactive decay and dilution. A similar pattern of radionuclide activity in groundwater was identified by the RFI/CMS for the 100-NR-1 Operable Unit in its study of radionuclide migration from the 116-N-1 crib and trench (DOE-RL 1990).

In addition to the elevated levels of activity directly observed in groundwater, the springs (N Springs) that developed as a result of discharge to $116-N-1$ were al so observed to exhibit elevated levels of radioactivity. To help monitor these releases, a composite sampling system was installed in 1973 at Wel1 199-N-8T, located approximately $15 \mathrm{~m}(50 \mathrm{ft})$ from the largest of the $N$ Springs. Radionuclide activities in this well increased steadily through the year; however, the rate of increase of ${ }^{131} \mathrm{I}$ and ${ }^{90} \mathrm{Sr}$ activities accelerated in 1982. This was taken as evidence that the soil was saturated with radionuclides and was losing its capacity to retain them. This development prompted the building of the 116-N-3 crib and trench (DOE-RL 1990).

The 116-N-3 crib and trench was built in 1983. It operated jointly with the 116-N-1 crib and trench until September 1985 when the 116-N-1 facility was abandoned. This caused a dramatically different flow regime, accompanied by the formation of additional downstream springs, in the 100-N Area. The activity levels in these springs during 1987 and 1988 have been investigated via RFI/CMS (DOE-RL 1990). The activity measurements reported in 1988 in the $\mathrm{N}$ Springs for tritium and ${ }^{90} \mathrm{Sr}$ are shown in Figures $5-10$ and $5-11$, respectively. These measurements indicate a relatively even distribution of tritium along this entire reach of the river, while ${ }^{90} \mathrm{Sr}$ activity is heavily concentrated in the area adjacent to $116-\mathrm{N}-1$. Almost no ${ }^{90} \mathrm{Sr}$ activity is observed downstream from $116-\mathrm{N}-1$.

This pattern in spring activity is thought to result from several different influences (DOE-RL 1990 and DOE-RL 1991). The relatively even distribution of tritium activity along the entire shoreline is thought to result from recent discharge to $116-N-3$. The high ${ }^{90} \mathrm{Sr}$ levels adjacent to the $116-\mathrm{N}-1$ are thought to result from the remobilization of radionuclides previously adsorbed to the soil (during past operation of the $116-\mathrm{N}-1$ ) as water flowing from the $116-\mathrm{N}-3$ passed through this area. The removal of ${ }^{90} \mathrm{Sr}$ from the effluent by adsorption in the immediate vicinity of the $116-\mathrm{N}-3$ is probably responsible for the low levels of this constituent observed in the downstream springs. Analysis of historical trends in ${ }^{90} \mathrm{Sr}$ and tritium levels also support this hypotheses. Strontium trends are presented in Figures 5-12 and 5-13. 
Levels of ${ }^{90} \mathrm{Sr}$ in areas adjacent to $116-\mathrm{N}-1$ are shown to increase until 1985 when discharge from the unit was terminated. After this time, ${ }^{90} \mathrm{Sr}$ levels stabilized and even began to fall in some wells. This pattern is consistent with an increase in adsorbed levels during operation of the 116-N-1 and a stabilization and decrease in levels as adsorbed radionuclides are gradually desorbed and remobilized when discharge to the unit is no longer occurring.

The trends in ${ }^{90} \mathrm{Sr}$ levels adjacent to $116-\mathrm{N}-3$ (Figure 5-13) are not as clear. Wells 199-N-27 and 199-N-29, however, do show a gradual increase in ${ }^{90} \mathrm{Sr}$ levels until a more stable level was reached. This level may represent the point at which adsorbed levels of ${ }^{90} \mathrm{Sr}$ reach equilibrium with levels of ${ }^{90} \mathrm{Sr}$ in the effluent. Consequently, no more adsorption occurs.

Historical trends in tritium levels in groundwater based on data from wells located between $116-\mathrm{N}-3$ and the Columbia River are shown in Figure 5-14. These trends clearly show a significant variability in tritium levels since 1987 when the $N$ reactor changed its operating status. These changes are reflected in all wells but are more attenuated in areas closer to the river. The wells shown in Figure 5-14, however, are generally screened close to the water table. As discussed, there is some evidence indicating that discharge from 116-N-3 may take a slightly deeper pathway and, consequently, the variations in tritium levels adjacent to the river may be more pronounced at depth (DOE-RL 1991).

Contours of tritium activity in the 100-N Area measured during September/October 1989, November/December 1989, and February 1990 are shown in Figures 5-15, 5-16, and 5-17, respectively. These data indicate widespread tritium contamination. An increase in tritium concentrations from September/October 1989 to February 1990 is also evident. This increase was probably caused by an increase in average tritium activities in the reactor effluent discharged to the $116-\mathrm{N}-3 \mathrm{crib}$ and ponds during this period. Release reports indicate that average tritium activities increased from $74,000 \mathrm{pCi} / \mathrm{L}$ in August 1989 to 550,000 pCi/L in November 1989 (DOE-RL 1991).

Contours of ${ }^{90} \mathrm{Sr}$ activity in the $100-\mathrm{N}$ Area measured during September/October 1989, November/December 1989, and February 1990, are presented in Figures 5-18, 5-19, and 5-20 respectively. These data indicate that ${ }^{90} \mathrm{Sr}$ contamination appears confined to the area immediately adjacent to the 116-N-1 and 116-N-3 facilities, although insufficient data are available to characterize contamination northeast of the $116-\mathrm{N}-1$ trench and south and east of the 116-N-3 crib. In addition, a number of anomalous data points appear in the vicinity of 116-N-3. Variations in monitoring-well-screen lengths and depths may be responsible for many of these anomalies (DOE-RL 1991).

Data on ${ }^{90} \mathrm{Sr}$ from Well 199-N-36 may indicate some increase in contaminant levels from September/October 1989 to November/December 1989 in the immediate vicinity of 116-N-3, with a slight fall-off in activity noted by

February 1990. A decrease in ${ }^{90} \mathrm{Sr}$ activities is evident from November/December 1989 to February 1990. This trend is particularly evident in the peak concentrations observed in Well 199-N-29. These changes conform to the pattern of changes observed in the reactor effluent during this period. In September 1989 average ${ }^{90} \mathrm{Sr}$ activities in the reactor effluent increased to 
$25,000 \mathrm{pCi} / \mathrm{L}$ from $2,300 \mathrm{pCi} / \mathrm{L}$ in August 1989 . These levels increased to $210,000 \mathrm{pCi} / \mathrm{L}$ in November 1989. Average activity levels in the reactor effluent dropped back to $4,900 \mathrm{pC} i / L$ in January of $1990 .{ }^{90} \mathrm{Sr}$ levels in the vicinity of $116-\mathrm{N}-1$ remained relatively stable, further verifying that ${ }^{90} \mathrm{Sr}$ in effluent disposed of in $116-\mathrm{N}-3$ is not migrating significant distances.

The presence of large groundwater mounds at various times beneath $116-\mathrm{N}-1$ and 116-N-3 indicate that sigiificant vertical gradients with corresponding migration of contaminants down past the water table could be expected below both these facilities. Only limited data currently are available for characterizing the vertical distribution of contamination at the site; however, these data do seem to indicate that much of the contamination has been limited to a relatively narrow zone centered around the water table.

Currently two well pairs have been routinely monitored and provide some useful data for characterizing the vertical distribution of contaminants. Wells 199-N-67 and 199-N-69 are located adjacent to the 116-N-1 crib and trench. Well 199-N-67 is completed at the current water table (based on May 1990 water levels) with a 4.6-m (15-ft) screen, while the screened zone of Well 199-N-69 ( $3 \mathrm{~m}$ or $10 \mathrm{ft}$ ) is located $4.3 \mathrm{~m}$ (14 ft) below that of Well 199-N-67. Wells 199-N-39 and 199-N-70 are located adjacent to the $116-\mathrm{N}-3$ trench approximately $300 \mathrm{~m}(1,000 \mathrm{ft})$ from the $116-\mathrm{N}-3 \mathrm{crib}$. Well 199-N-39 is completed at the current water table (based on May 1990 water levels) with a 6-m (20-ft) screen, while the screened zone of Well 199-N-70 $(3 \mathrm{~m}$ or $10 \mathrm{ft}$ ) is located $4.9 \mathrm{~m}$ (16 ft) below that of Well 199-N-39.

Water quality data from samples taken in February 1990 indicate a significant difference in ${ }^{90} \mathrm{Sr}$ activities between Wells $199-\mathrm{N}-67$ and $199-\mathrm{N}-69$. Negligible ${ }^{90} \mathrm{Sr}$ was measured in Well 199-N-69, while an activity of $8,980 \mathrm{pCi} / \mathrm{L}$ was measured in We11 199-N-67. It is interesting to note that an activity of $1,960 \mathrm{pCi} / \mathrm{L}$ was measured in nearby Well 199-N-2. The screen in $199-\mathrm{N}-2$ intercepts the water table and extends over approximately $18 \mathrm{~m}(60 \mathrm{ft})$ of saturated aquifer; thus, the activity measured in the well apparently represents some average measurement over the entire unconfined aquifer. Data from November/December 1989 confirm this general pattern of vertical distribution of ${ }^{90} \mathrm{Sr}$.

Activity measurements for ${ }^{90} \mathrm{Sr}$ from samples taken in November/December from Wells $109-\mathrm{N}-39$ and $199-\mathrm{N}-70$ al so indicate a similar pattern. An activity of $490 \mathrm{pCi} / \mathrm{L}$ was measured in Well 199-N-39, while no measurable activity was detected in Well 199-N-70.

Several other studies conducted in the 100-N Area have confirmed this general pattern for the vertical distribution of contaminants. The RFI/CMS for the 100-NR-1 Operable Unit (DOE-RL 1990) demonstrated that adsorbed radionuclides in the vicinity of the $116-\mathrm{N}-1$ crib and trench were concentrated in the soil column at depths close to the water table. In the RFI/CMS, four wells in the 199-N-8 cluster (199-N-8-P, $-Q,-R$, and $-S$ ) were sampled between January 12 and February 18, 1987 to determine the vertical distribution of radionuclides in groundwater at the riverbank immediately adjacent to $116-\mathrm{N}-1$ (DOE-RL 1990); these data are provided in Table 5-11. These data indicate that the radionuclides measured were concentrated in a zone within $10 \mathrm{~m}$ $(32 \mathrm{ft})$ of the water table. 
Recent tritium data from Wells $199-\mathrm{N}-67$ and 199-N-69 (Color Illustration 2-2), however, show a different pattern. These data indicate significant contamination at depth, and in both We11s $199-\mathrm{N}-67$ and $199-\mathrm{N}-69$ higher levels of contamination at depth than at water table. For example, tritium activity observed in Wells $199-\mathrm{N}-67$ and $199-\mathrm{N}-69$ in November/December 1989 was $42,600 \mathrm{pCi} / \mathrm{L}$ and 78,400 $\mathrm{pCi} / \mathrm{L}$, respectively. The higher tritium levels at depth in the vicinity of $116-\mathrm{N}-1$ may indicate a deeper, preferential flow path for discharge from 116-N-3, particularly in the most direct pathway towards the river. The ${ }^{90} \mathrm{Sr}$ activity associated with discharges to $116-N-3$ is presumably not observed at this point in the migration pathway because it was previously removed through adsorption in the immediate vicinity of the crib.

In addition to the extensive radiological monitoring routinely performed in the vicinity of $116-\mathrm{N}-1$ and $116-\mathrm{N}-3$, an extensive program of sampling and analysis for organic constituents was performed during 1989. Many of the wells in this operable unit were included in this program. All of the wells in the basic RCRA monitoring networks for the 116-N-1 and 116-N-3 facilities were sampled in March 1989, and those wells in the extended 116-N-1 assessment monitoring network were sampled in November/December 1989. This program was associated with the Hanford Site liquid effluent study and included analyses for the dangerous waste constituents 1 ist established by Washington State (WAC 173-303-9905). The analyses have indicated no significant difference for upgradient wells for organic contamination. The organic contamination levels reported were less than detection limits. However, the sampling undertaken in March 1989 did ident ify low levels of chloroform in a number of wells, and other organics were observed in Well 199-N-2. In addition, the more recent sampling in November/December 1989 has detected 2-methylnaphthalene at $26 \mu \mathrm{g} / \mathrm{L}$, naphthalene at $12 \mu \mathrm{g} / \mathrm{L}$, and polycyclic aromatic hydrocarbons totaling $28 \mu \mathrm{g} / \mathrm{L}$ in Well 199-N-56 (DOE-RL 1991).

A limited area along the northwest corner of the plumes emanating from $116-\mathrm{N}-1$ and $116-\mathrm{N}-3$ has also been contaminated by the sodium (Figure 5-21), and sulfate (Figure 5-22), and $\mathrm{pH}$ plumes resulting from discharge to the 120-N-1 percolation pond and 120-N-2 surface impoundment. These contaminants may have played an important role in mobilizing radionuclides adsorbed to soil in this area (DOE-RL 1991).

\subsubsection{4-N Emergency Dump Tank and 118-N-1 Spacer Storage Silos Area}

Several wells are located in the vicinity of the 1304-N EDT and the 118-N-1 spacer storage silos. All of these wells are located in the northwestern portion of the operable unit immediately adjacent to the Columbia River. Wells $199-\mathrm{N}-48$ and 199-N-23 through 199-N-25 monitor groundwater quality beneath the 1304-N EDT and wells $199-\mathrm{N}-47$ and $199-\mathrm{N}-20$ through $199-N-22$ monitor beneath the 118-N-1 spacer storage silo. Well 199-N-23 is located directly between the two sources.

With the exception of Well 199-N-48, all of these wells have undergone radiological analyses during 1989. These analyses have indicated relatively low levels of radionuclides. During this period, the highest ${ }^{90} \mathrm{Sr}$ activity was $11.7 \mathrm{PC} / \mathrm{L}$ observed in Well $199-\mathrm{N}-24$. Levels of ${ }^{60} \mathrm{Co}$ have been belcw 
method detection levels, with the exception of Wells $199-\mathrm{N}-20$ and -21 for which activities of 8.4 and $5.7 \mathrm{pCi} / \mathrm{L}$ were reported. Levels of ${ }^{106} \mathrm{Ru}$ have all been below method detection limits. Tritium levels have ranged from below detection limits (BDL) in Well 199-N-25 to a high of $15,800 \mathrm{pCi} / \mathrm{L}$ in Well 199-N-47 (DOE-RL 1991).

Elevated levels of metals have been measured in several wells, most notably We11 199-N-23 (DOE-RL 1990). December 1989 measured concentrations included $348 \mathrm{mg} / \mathrm{L}$ for iron, $1,620 \mu \mathrm{g} / \mathrm{L}$ for barium, $103 \mu \mathrm{g} / \mathrm{L}$ for cadmium, and $529 \mu \mathrm{g} / \mathrm{L}$ for chromium. The samples from which these measurements were made were highly turbid (i.e., more than 200 nephelometric turbidity units [NTU]) and were unfiltered. Consequently, they do not accurately reflect dissolved metal concentrations in water (DOE-RL 1991).

Many of the wells have undergone recent sampling and analysis for an extensive set of organic constituents. This sampling was done during November/December of 1989 as part of the Hanford Site liquid effluent study and included We11s 199-N-21, 199-N-23 through 199-N-25, and 199-N-47. Analyses were performed for the list of dangerous waste constituents established by Washington State 'WAC 173-303-9905) and have indicated no significant organic contamination, with organic contaminant levels generally reported at less than detection limits. Well 199-N-47 did contain bis(2-ethylhexy1)phthalate at $26.0 \mu \mathrm{g} / \mathrm{L}$ and tetrahydrofuran at $60 \mu \mathrm{g} / \mathrm{L}$. Volatile organic analysis also identified acetone in Well $199-\mathrm{N}-25$ at the detection limit of $10 \mu \mathrm{g} / \mathrm{L}$ (DOE-RL 1990).

\subsubsection{0-N-1 Percolation Pond/120-N-2 Surface Impoundment Area}

The 120-N-1 and 120-N-2 facilities have been used to treat and dispose of corrosive wastes from 1977. The waste has been characterized as a hazardous waste based solely on its corrosive nature. The waste exhibited no other dangerous characteristics; analysis indicated that it did not contain any listed hazardous waste or dangerous waste sources (DOE-RL 1991). Groundwater quality data from nearby Wells $199-N-58$ through $199-N-61$ have supported these conclusions. These wells underwent sampling and analysis for this list of dangerous waste constituents established by Washington State

(WAC 173-303-9905) during March 1989. The analys is indicated no significant organic contamination, with organic contaminant levels generally reported at less than detection limits (DOE-RL 1991). A few organic constituents [bis (2-ethylhexy1) phthalate, chloroform, methylene chloride, and acetone] were found at trace levels. Radiological analyses of samples from these wells indicate that key radionuclides $\left({ }^{106} \mathrm{Ru},{ }^{60} \mathrm{Co}\right.$, and ${ }^{90} \mathrm{Sr}$, and tritium) are present at low levels above detection limits, with an activity of $63.5 \mathrm{pCi} / \mathrm{L}$ reported for Well 199-N-59 (DOE-RL 1991). Groundwater quality data indicate that the 120-N-1 and 120-N-2 facilities are responsible for elevated sodium and sulfate plumes in the groundwater.

\subsubsection{Summary of Groundwater Contamination}

Monitoring data have indicated that groundwater in the 100-N Area has been widely contaminated by constituents in wastes disposed of to the ground (DOE-RL 1990). Table 5-12 lists the constituents measured above drinking 
water standards from April 1987 to November 1989 and the number of instances for exceeding standards for each constituent during this period (WAC 248-54, 40 CFR 143). Table 5-13 identifies those individual wells in which drinking water standards were exceeded during a sampling round (July/August 1989).

These constituents include beta activity and concentrations of ${ }^{90} \mathrm{Sr}$, tritium, nitrate, and sulfate. Beta and ${ }^{90} \mathrm{Sr}$ activities exceeded drinking water standards in the same 10 wells. Tritium activities were greater than the standard in eight wells, and nitrate was exceeded in one well. Sulfate was higher than secondary drinking water standards in seven wells (DOE-RL 1991).

A summary discussion of groundwater quality in the 100-N Area beginning in 1980 and extending to November 1989 is provided in the following paragraphs. This discussion is based on review of the Hanford Sitewide groundwater monitoring and RCRA compliance monitoring reports published for this period. An emphasis is placed on identifying peak concentrations for individual constituents within the 100-N Area. The following discussion is brcken into five major constituent groups:

- Radionuclides

- Volatile organics

- PCBs

- Metals

- Other inorganics.

5.2.4.1 Radionuclides. The radiological parameters identified ingroundwater in the $100-N$ Area include gross beta, gross alpha, tritium, ${ }^{60} \mathrm{Co},{ }^{106} \mathrm{Ru},{ }^{90} \mathrm{Sr}$, ${ }^{137} \mathrm{Ce},{ }^{99} \mathrm{TC},{ }^{125} \mathrm{An}$, uranium, and radium.

The maximum activity measured for gross beta in groundwater was $40,800 \mathrm{pCi} / \mathrm{L}$ in Well 199-N-67 (downgradient from the $116-\mathrm{N}-1 \mathrm{crib}$ and trench) on December 19, 1988 (DOE-RL 1991). Activities in this well have ranged from 24,100 to $40,800 \mathrm{pCi} / \mathrm{L}$ (DOE-RL 1991). The next highest beta concentration was $8,150 \mathrm{pCi} / \mathrm{L}$ on August 17,1988 in Well $199-\mathrm{N}-18$ (DOE-RL 1990).

The maximum activity of gross alpha detected in the groundwater during the period was $36.6 \mathrm{pCi} / \mathrm{L}$ in Wel1 $\mathrm{N}-59$ on December 4,1987 (DOE-RL 1990). In a more recent sampling event (June through August 1989), the highest concentration was $2.49 \mathrm{pC} i / L$ in Well 199-N-42 (DOE-RL 1991).

Tritium is found at elevated levels throughout the areas adjacent to the 116-N-1 and 116-N-3 facilities and provides an excellent indicator of the extent of influence of these units. The maximum activity measured for tritium in groundwater during the period from 1980 to 1989 was $457,500 \mathrm{pCi} / \mathrm{L}$ on December 1, 1988 in Well 199-N-45 (adjacent to 116-N-3) (DOE-RL 1990).

The maximum activity of ${ }^{60} \mathrm{Co}$ (a beta and gamma emitter) in groundwater was at $554 \mathrm{pCi} / \mathrm{L}$ in Wel1 199-N-32 on November 1, 1987 (DOE-RL 1991). A11 the wells sampled on that date $(199-N-14,199-N-30,199-N-31,199-N-32,199-N-33$, 199-N-36, and 199-N-37) had activities above $100 \mathrm{pCi} / \mathrm{L}$. Recent values have been considerably lower, ranging from 9.43 to $26.9 \mathrm{pCi} / \mathrm{L}$ in July and August 1989 (DOE-RL 1991). 
The maximum activity of ${ }^{106} \mathrm{Ru}$ (a beta emitter) in groundwater was detected in Well 199-N-29 at $530 \mathrm{pCi} / \mathrm{L}$ on May 29, 1984. The highest value observed in recent sampling was $50.8 \mathrm{pCi} / \mathrm{L}$ in Well $199-\mathrm{N}-60$ on August 1,1989 , but this value is below the laboratory contract detection limit (DOE-RL 1991).

The maximum activity of ${ }^{90} \mathrm{Sr}$ (a beta emitter) measured in groundwater was 23,400 $\mathrm{pCi} / \mathrm{L}$ in Well 199-N-67 on March 8, 1989. Activities in this well have ranged from 11,600 to $23,400 \mathrm{pCi} / \mathrm{L}$. The next highest concentration was observed in Weil 199-N-39 on July 23, 1987 at 10,400 pCi/L (DOE-RL 1991).

The maximum activity measured for ${ }^{137} \mathrm{Cs}$ in groundwater was $31 \mathrm{pCi} / \mathrm{L}$ in Wells 199-N-30 and 199-N-32 in 1986 (DOE-RL 1991). Recent samples have all been below the detection limit of $20 \mathrm{pCi} / \mathrm{L}$ (DOE-RL 1990).

The maximum activity of ${ }^{99} \mathrm{Tc}$ detected in groundwater was $55.8 \mathrm{pCi} / \mathrm{L}$ in Well 199-N-6 on January 29, 1988 (DOE-RL 1990). The maximum detected uranium activity in groundwater was $8.12 \mathrm{pCi} / \mathrm{L}$ in Well 199-N-23 on March 22, 1988 (DOE-RL 1990). Antimony-125 has been detected at a maximum activity of $540 \mathrm{pCi} / \mathrm{L}$ in Well 199-N-37 in 1986 (DOE-RL 1991). Radium has not been detected in groundwater in the 100-N Area above its detection limit of $1 \mathrm{pCi} / \mathrm{L}$.

5.2.4.2 Volatile Organics. Several volatile organic compounds (benzene, toluene, xylene, chlorobenzene, ethyl benzene, three aliphatic hydrocarbons, and three other indeterminate organic compounds) were reported in a sample from Well 199-N-2 collected on December 19, 1988. Two indeterminant organic compounds and an aliphatic hydrocarbon were detected in a sample from

Well 199-N-4 on the same date. Resampling of Well 199-N-2 on March 19, 1989, showed no such contamination, but sample contamination is suspected (DOE-RL 1991). Sampling from March through August 1989 did not show any of these constituents in either Well 199-N-2 or 199-N-4. An unknown aliphatic hydrocarbon was detected in We11 199-N-33 and its duplicate samples on March 6, 1989 (DOE-RL 1991). Samples from June through August 1989 were not analyzed for these constituents (DOE-RL 1991).

Acetone and methylene chloride have been detected occasionally and are probably the result of laboratory and/or sampling contamination (DOE-RL 1991).

Chloroform has been detected in several wells $(199-\mathrm{N}-3,-27,-29,-31$, $-33,-36,-58,-59,-60,-61$, and -70 ) at low concentrations. Concentrations ranged from $5 \mu \mathrm{g} / \mathrm{L}$ (in several wells) to $15 \mu \mathrm{g} / \mathrm{L}$ (in $199-\mathrm{N}-61$ on July 23 , 1989) (DOE-RL 1991). The chloroform may be derived from the disposal of chlorinated water.

5.2.4.3 Polychlorinated Biphenyls. The PCB arochlor 1016 was first detected in Well 199-N-33 on March 6, 1988 at $4 \mu \mathrm{g} / \mathrm{L}$ (DOE-RL 1991). The well was resampled in July 1989 and had arochlor 1016 at concentrations as great as $148 \mu \mathrm{g} / \mathrm{L}$. The source of this contamination is thought to be an oil-1ubricated submersible pump that was leaking. The pump was removed in August 1989 (DOE-RL 1991).

5.2.4.4 Metals. Metals are naturally occurring constituents and have commonly been detected in the groundwater in the 100-N Area. Metals detected include aluminum, arsenic, barium, beryllium, cadmium, calcium, chromium, 
copper, iron, lead, lithium, magnesium, manganese, nickel potassium, sodium, strontium, titanium, uranium, vanadium, and zinc. Of these metals, only cadmium, iron, and manganese have exceeded drinking water standards (DOE-RL 1991).

Unfiltered cadmium has been detected in groundwater in concentrations up to $45 \mu \mathrm{g} / \mathrm{L}$ (Well 199-N-23 on August 22, 1989). In recent sampling (June through August 1989) Wells 199-N-18, and 199-N-23 through 199-N-25 had concentrations above $10 \mu \mathrm{g} / \mathrm{L}$. These wells are located adjacent to the Columbia River. The source of these elevated levels of cadmium is not known (DOE-RL 1991).

Unfiltered iron has been detected at a maximum concentration of $134,000 \mu \mathrm{g} / \mathrm{L}$ in Well $199-\mathrm{N}-23$ on August 22, 1989. The maximum concentration of filtered iron was $659 \mu \mathrm{g} / \mathrm{L}$ in We11 199-N-17 on August 18, 1989 (DOE-RL 1991). The high iron concentrations may be related to the condition of the steel casings, although Wells 199-N-32, 199-N-42, and 199-N-66, which have stainless steel screens, also have been associated with elevated iron concentrations $(2,240,2,070$ and $5,360 \mu \mathrm{g} / \mathrm{L}$, respectively in July and August 1989). Other wells with elevated iron concentrations from June through August 1989 are $1990-\mathrm{N}-4,-14,-16,-17,-18,-21,-23,-24,-25,-26$, and -41 . These wells are scattered throughout the 100-N Area and the source of the elevated levels of iron is not known (DOE-RL 1991).

The maximum observed concentration of unfiltered manganese was $4,380 \mu \mathrm{g} / \mathrm{L}$ in Well 199-N-18. This concentration was measured in a sample taken on June 20, 1989. The maximum concentration of filtered manganese was $1,180 \mu \mathrm{g} / \mathrm{L}$ in Wel1 199-N-17 on August 18, 1989 (DOE-RL 1991). Wells with elevated concentrations observed from June through August 1989 include 199-N-16, -17, $-18,-23,-24,-25,-26,-42,-59$, and -66 . The source of the elevated manganese levels is not known (DOE-RL 1991).

The concentration of sodium in groundwater has been measured at more than five times greater than background (see Table 5-14). The highest observed concentration was $1,010,000 \mu \mathrm{g} / \mathrm{L}$ in Well 199-N-61 on July 28, 1989. Wells 199-N-16, $-21,-23,-24,-25,-47,-58,-59,-60$, and -61 all have had concentrations greater than $10,000 \mu \mathrm{g} / \mathrm{L}$. These wells are all downgradient from the 120-N-1 percolation pond and the 120-N-2 surface impoundment. The source of the sodium appears to be the sodium hydroxide used in the anion regeneration process and then discharged to $120-\mathrm{N}-1$ and $120-\mathrm{N}-2$ (DOE-RL 1991).

5.2.4.5 Other Inorganics. Many naturally occurring inorganics have been detected in the groundwater in the 100-N Area, including nitrate, sulfate, boron, chloride, and fluoride.

The highest observed nitrate concentration was $89,000 \mu \mathrm{g} / \mathrm{L}$ in Well 199-N-30 on May 29, 1984 (DOE-RL 1991). High concentrations al so have been observed in Wel1 199-N-67 at $87,800 \mu \mathrm{g} / \mathrm{L}$ on March 8, 1989 (DOE-RL 1991). This concentration dropped to $23,500 \mu \mathrm{g} / \mathrm{L}$ on August 16,1989 . A high concentration of $63,600 \mu \mathrm{g} / \mathrm{L}$ was observed on June 21,1989 in We11 199-N-56. This concentration dropped to $23,500 \mu \mathrm{g} / \mathrm{L}$ on August 15, 1989 (DOE-RL 1991). The wells with the highest concentrations in August 1989 were 199-N-24 and 199-N-69) (DOE-RL 1991). 
Elevated sulfate concentrations have commonly been observed downgradient from $120-\mathrm{N}-1$. The source of the high sulfate concentrations appears to be the sulfuric acid that was used in the cation regeneration process and then disposed of to the $120-\mathrm{N}-1 / 120-\mathrm{N}-2$ facility. The highest sulfate concentration measured in the groundwater was 2,180,000 $\mu \mathrm{g} / \mathrm{L}$ in Wel1 $199-\mathrm{N}-61$ on August 14,1989 . If those wells immediately surrounding $120-\mathrm{N}-1 / 120-\mathrm{N}-2$ (199-N-58 through 199- $\mathrm{N}-61$ ) are not considered, the highest concentration measured in groundwater in the $100-\mathrm{N}$ Area was $345,000 \mu \mathrm{g} / \mathrm{L}$ in Well $199-\mathrm{N}-24$ on August 21, 1989 (DOE-RL 1991).

\subsection{AFFECTED ENVIRONMENT - SOIL}

This section discusses known and suspected contaminant occurrences in the soil column as a result of releases from potential sources identified at the 100-N Area.

\subsubsection{Surface Soil Contamination}

Surface soil samples have been collected from various locations in the vicinity of $N$ Reactor's $1301 \mathrm{~N}(116-\mathrm{N}-1)$ and $1325 \mathrm{~N}(116-\mathrm{N}-2)$ LWDFs since 1975. From 1975 to 1980 , soil samples were collected at unspecified locations within the 116-N-1 trench. Analytical data from these samples indicate that ${ }^{90} \mathrm{Sr}$ and ${ }^{239 / 240} \mathrm{Pu}$ concentrations increased during this time, while ${ }^{60} \mathrm{CO}_{0}$ and ${ }^{137} \mathrm{CS}$ values varied yearly (DOE-RL 1990).

Beginning in 1980, soil sampling was conducted in the 100-N Area as part of the annual Environmental Surveillance Program. In 1980, nine surface soil samples were collected in areas both north and south of the 116-N-1 crib and trench and nine surface soil samples were collected within the trench. Results of these analyses are presented in Table 5-15. Concentrations in the trench are at least 100,000 times higher than in the adjacent soil surface (DOE-RL 1990).

Relatively consistent surface soil sampling locations were established by the Environmental Surveillance Program in 1981; these locations were then sampled annually. Sampling locations were added periodically (e.g., sampling locations south of $116-\mathrm{N}-1$ on activation of the $116-\mathrm{N}-3$ crib and trench). The sample location map is presented in Figure 5-23. It must be emphasized that each location may not have been sampled each year, and some sample collection locations were not consistent from year to year. The average concentration of select radionuclides over the entire soil sampling area between 1980 and 1988 is presented in Table 5-16. The concentrations of most of the constituents have decreased since 1980 .

\subsubsection{Vadose Zone Soil Contamination}

In addition to surface soil analyses, soil samples were collected within the vadose zone as part of a 1982 research project (DOE-RL 1990). Three wells were installed at varying distances from the 116-N-1 crib and trench, and soils from the borings were analyzed. The locations of these wells are shown in Figure 5-24. Well 199-N-9 was installed $30 \mathrm{~m}(100 \mathrm{ft})$ from the trench, 
while Wells $199-\mathrm{N}-12$ and $199-\mathrm{N}-13$ were installed 46 and $73 \mathrm{~m}$ (150 and $240 \mathrm{ft}$ ), respectively, from the trench. Depth to groundwater varied from 16 to $18 \mathrm{~m}$ ( 53 to $59 \mathrm{ft}$ ). Boreholes were logged using gamma-ray logging tools to assess the occurrence of radionuclides in the borehole (DOE-RL 1990).

Data from these logs indjcate that very low concentrations of radionuclides such as ${ }^{60} \mathrm{Co},{ }^{3} \mathrm{CS},{ }^{125} \mathrm{Sb}$, and ${ }^{106} \mathrm{Ru}$ were present in Well 199-N-9 soils above the water table, al though the concentration increases markedly in soils at the water table. Wells $199-\mathrm{N}-12$ and $199-\mathrm{N}-13$ are more distant from the trench and had lower radionuclide concentrations in the unsaturated zone but also had elevated concentrations at the water table (within the saturated zone). These data indicate that extensive lateral migration of contaminants from the trench within the unsaturated zone is not apparent in these borings (DOE-RL 1990). Mechanisms that control the lateral migration of contaminants through the unsaturated zone include dispersion, diffusion, capillary flow, migration through a low-permeability zone because of increased moisture content and perched water, and the adsorption capacity of the soil.

Because these wells were installed between the trench and the springs, they could be used to assess the chemical speciation of wastewater as it migrates from the trench to the springs. While this is more specific to the groundwater pathway, the 1982 study also sheds light on the retention of specific radionuclides that could also occur in the vadose zone. This study indicates that as the trench water percolates into the soil and moves downward to the water table and on to the springs, selective removal of the cationic and particulate radionuclides occurs, with the more mobile anionic and nonionic species transmitted with groundwater essentially unattenuated (or with low attenuation). For example, ${ }^{137} \mathrm{Cs}$ is the least mobile of the radionuclides in this study; while $16,100 \mathrm{pCi} / \mathrm{L}$ were present in trench water (all as a soluble cation), no ${ }^{137}$ Cs was detected in either the wells or springs, indicating that this radionuclide was probabiy bound in the soil column. On the other hand, 50 percent of the ${ }^{131}$ I in trench water was present as a soluble anion, and this species was relatively mobile in groundwater (present in both the wells and springs) and did not sorb readily as an anionic species to the soil column.

The occurrence of organic compounds within trench sediments was also assessed as part of the DOE-RL study (1990). The presence of organic compounds in sediments/soils is important because they can form complexes with radionuclides, thus enhancing their mobility. Specific compounds were identified, including alkenes, alkanes, alkynes, elemental sulfur, and three cyclic sulfur species. Organic constituent concentrations are presented in Table 5-17.

Most of the unplanned release locations throughout the 100-N Area have not been sampled to determine the extent of contamination. Although screening sampling or radiation surveys were usually performed and exposed surface contamination was excavated, quantitative radionuclide- and chemical-specific analyses were not performed. Based on the areas of known releases

(e.g., 116-N-3 crib and trench) and areas of inferred releases, inferred areas of soil contamination have been identified. These areas are shown in Figure 5-25 and the sources are listed in Table 5-1. 
Estimates of total activity either released or remaining in soil were usually included in unplanned release or "occurrence" reports. The bases for these estimates are uncertain. However, the general extent of contamination at these locations can be inferred from discussions in the 100-NR-1 and 100-NR-2 work plans.

Where a release of a few hundred to a few thousand liters of radionuclide-contaminated wastewater occurred, the unsaturated soll column directly below the release location probably still holds most of the long-lived radionuclides. These locations include the 119-N building and 1322-N/NA sampling butldings.

At locations where more than a few thousand liters of radionuclidecontaminated water were released (particularly with high concentrations), the long-lived radionuclides are expected to be present in the saturated soilis and (to an unknown extent) in the groundwater directly below and downgradient from the release location. These locations include the $116-\mathrm{N}-2$ radioactive chemical waste treatment and storage tank, the $118-\mathrm{N}-1$ spacer storage silos, the 1304-N EDT, and the 1314-N LWLS.

\subsection{AFFECTED ENVIRONMENT - BIOTA}

\subsubsection{Flora}

As part of the annual Environmental Surveillance Program, collection and analysis of vegetation for radionuclides occurred in the 100-N Area at three locations:

- Along the Columbia River at the N Springs

- Near the 116-N-1 crib and trench

- In the southeast section of the 100-N Area. The average concentration of selected radionuclides for the years 1980 through 1988 are presented in Tables 5-18, 5-19, and 5-20.

In 1990, the Columbia River shoreline within the Hanford Site was surveyed for potential edible natural vegetation and samples were collected for radiological analysis. Table 5-21 shows results for samples of mulberry leaves and berries, and one sample of curly dock collected from the $N$ Springs area. DOE initiated removal of all contaminated vegetation from the $\mathrm{N}$ Springs area in September 1990. Mulberry trees and other plants were removed and disposed of in the 200 Area burial grounds.

\subsubsection{Fauna}

The effects of the 100-N Area on animals living near or at the 100-N Area have been investigated since 1979. The predominant area of investigation has been the 116-N-1 crib and trench. Barn swallow nests and excrement were sampled near the 1304-N EDB in 1979. Rabbits were trapped and collected around 116-N-1 in 1981. In 1982 and 1985, deer mice were trapped and collected in the 116-N-1 vicinity. In 1983, deer mice at the $N$ Springs were trapped and collected. 
5.4.2.1 Birds. The first fauna study conducted was an investigation of barn swallow nests and barn swallow excrement in 1979. Samples were collected in the vicinity of the 1304-N EDB. One of the sources of mud used to bulld the nests was the 116-N-1 trench before the precast concrete cover panels were installed. The nests and excrement were sampled to determine the amount of radionuclides in the nests. Two rounds of sampling were performed. The second round of sampling results, including soll sampling results for the same period, are presented in Table 5-22. Nests, excrement, and shell/embryo samples of barn swallows were collected in 1985 near the 1304-N EDB. The results of these analyses are presented in Table 5-23. The lowering of radionuclide concentrations may be the result of installing a cover over the 116-N-1 trench, which prevents the birds from using mud from the unit for nest building or eating insects that live in the unit (DOE-RL 1990).

Investigation of other nests at the 100-N Area noted a contaminated nest on the side of a craft building. The nest was tentatively identified as a robin's nest that was partly built using mud from the 116-N-1 trench. A Cutie Pie reading of $250 \mathrm{mrad} / \mathrm{hr}$ was the highest reading observed for the nest. Subsequent surveys located one contaminated wasp's nest on the craft building and about 15 nests inside. The highest reading of the nests was 15,000 cpm; however, the meter and frequency of monitoring were not described. A sample of either a wasp's nest or a composite sample of several wasp's nests was collected and analyzed. The following results, reported in $\mathrm{pCi} / \mathrm{g}$, are considerably higher than background (DOE-RL 1990):

$$
\begin{aligned}
& { }^{54} \mathrm{Mn} 1.68 \mathrm{E}+5 \\
& -{ }^{60} \mathrm{CO} 1.06 \mathrm{EE}+6 \\
& -{ }^{137} \mathrm{Cs} 1.55 \mathrm{E}+5 \\
& -{ }^{144} \mathrm{CePr} 5.02 \mathrm{E}+4 .
\end{aligned}
$$

5.4.2.2 Cottontail Rabbits and Deer Mice. Cottontail rabbits (Sylvilagus nuttallii) were collected and radioanalyzed in 1981. Five rabbits were collected from inside the 116-N-1 security fence and four rabbits were collected to the northwest of the fenced area. Rabbit feces were also collected around 116-N-1 for analyses. The study found much higher concentrations of radionuclides in rabbits collected with in the 116-N-1 fenced area (Table 5-24). Concentration differences were attributed to rabbits inside the security fence drinking from the 116-N-1 trench and eating vegetation growing near the trench. The rabbits outside the fenced area were found to have radionuclide concentrations at or near background levels (DOE-RL 1990).

Deer mice (Peromyscus maniculatus) were collected near the 116- $\mathrm{N}-1$ trench in 1981 for radioanalysis. Eighteen mice were collected over 3 days from 30 snap traps set along the first leg of the trench. Results of the sampling are presented in Table 5-25. Fission and activation products were detected in the mice. The ${ }^{60} \mathrm{Co}$ concentrations ranged from 54 to $17,000 \mathrm{pCi} / \mathrm{g}$ and ${ }^{13} \mathrm{Cs}$ concentrations ranged from 25 to $2,300 \mathrm{pCi} / \mathrm{g}$. Concentrations of ${ }^{131}$ I were also noted, which indicated the mice were using the 116-N-1 trench as a drinking water supply. Ingestion of vegetation in the area also may have increased the concentrations of radionuclides in the mice. Total gamma activity ranged from about $3,200 \mathrm{pCi}$ to nearly $1 \mu \mathrm{Ci}$ in the mice. Four composite samples of the 18 mice were formed based on the concentration of ${ }^{137} \mathrm{Cs}$ in each mouse. The composite with the highest average ${ }^{137} \mathrm{CS}$ 
$(1,800 \mathrm{pCi} / \mathrm{g})$ also had the highest ${ }^{90} \mathrm{Sr}$ concentration $(190 \mathrm{pCi} / \mathrm{g})$. This study was conducted before a concrete cover was installed on the $116-N-1$ trench (DOE-RL 1990).

During 1985, 16 deer mice again were collected from 50 traps near 116-N-1. Sampling results from whole body analysis of the miçe, presented in Table 5-27, indicated relatively high concentrations of ${ }^{60} \mathrm{Co}$, ${ }^{59} \mathrm{Fe}$, and ${ }^{54} \mathrm{Mn}$. In addition, ${ }^{131} \mathrm{I}$ and ${ }^{137} \mathrm{Cs}$ were detected. These results suggest that the mice were still obtaining radionuclides directly from the effluent to the 116-N-1 trench (DOE-RL 1990).

Deer mice were collected at the $N$ Springs for analysis of gamma-emitting radionuclides in 1982. Twelve mice were collected over 3 days from 30 snap traps. The results of the analyses, presented in Table 5-26, indicate that the levels were probably caused by ingestion of vegetation growing at the springs and drinking $N$ Springs water (DOE-RL 1990).

\subsection{AFFECTED ENVIRONMENT - RADIATION}

Elevated radiation levels above natural background exist along the $100-N$ Columbia River shoreline. The source term for these radiation levels is the 1301-N LWDF (116-N-1), which is described in Section 3.4.1 and shown in Figure 3-19.

During normal operation, sediments in the trench sections of the disposal facility became radiologically contaminated from the billion gallons of effluent that were discharged each year. Radiation emanating from the top of the trench sections during operation of the 1301-N LWDF was not significant because the sediments were normally under several meters of water, which provided the necessary shielding.

During operation, liquid effluent was present in the trench sections; the rate of percolation into the ground was offset by the rate of discharge of liquid effluent into the trench. Following retirement of the facility in 1985, the liquid normally present in the trench sections of the disposal facility percolated into the ground leaving the residual radioactively contaminated sediments unshielded along the bottom and sides of the trench sections. The radioactive constituents of the contaminated sediments include the gamma-emitting isotopes ${ }^{60} \mathrm{Co}$ and ${ }^{137} \mathrm{Cs}$. Figure $5-26$ shows the results of a 1990 radiation survey on the top of the 1301-N LWDF trench. Because of the lack of water covering, some of the gamma photons that are emitted upward above the trench sections are scattered downward because of the Compton interaction of these photons with atmospheric constituents. These downwardscattered gamma rays are present at tha nearby Columbia River shoreline and increase the exposure rate above the ambient background rate as shown in Figure 5-27. This phenomenon is known as "skyshine."

\subsection{NET EFFECT ON THE ENVIRONMENT}

The net effect on the environment is 109 discrete sources of known environmental contamination. The soil is the medium that received most of the radioactive and hazardous waste at $N$ Reactor through direct disposal of 
liquids to the soil in cribs and trenches and spills and leaks from storage tanks. Soil and groundwater currently represent the primary source terms of radionuclide and hazardous substances that exhibit varying forms of mobility.

Radionuclide and hazardous substances in the soil can also be transported to groundwater. Groundwater monitoring has detected radionuclides in the groundwater under the site. Columbia River-groundwater interactions have also influenced contamination transport. Uptake of radionuclides from the soil and groundwater has also occurred, resulting in the transport of contaminants to both plants and animals.

The hazard proposed by radiation exposures depends to a large extent on the combination of radiation quality (alpha, beta, gamma) and the exposure pathway. External exposure to radionuclides is of concern only if it results from gamma emitters because they can penetrate tissue and deliver a deep dose of radiation. Alpha- and beta-emitting radionuclides only pose a significant hazard when inhaled or ingested because their energy is deposited locally in tissues and cells.

The potential for ingestion or inhalation of radiation through one or more of three general exposure pathways (soil, water, or air) compelled the EPA to place the 100 Area (and therefore, $N$ Reactor) on the National Priority List (NPL) near the end of fiscal year 1987. The NPL was established as part of CERCLA ( 40 CFR 300, Appendix B); the 100 Area is within group numbers 6 and 30 listed in the Federal Facility NPL. 
DOE/RL 91-59

This page intentionally left blank. 
Color Illustration 5-1. 116-N-2 Radioactive Chemical Waste Treatment and Storage Facility.

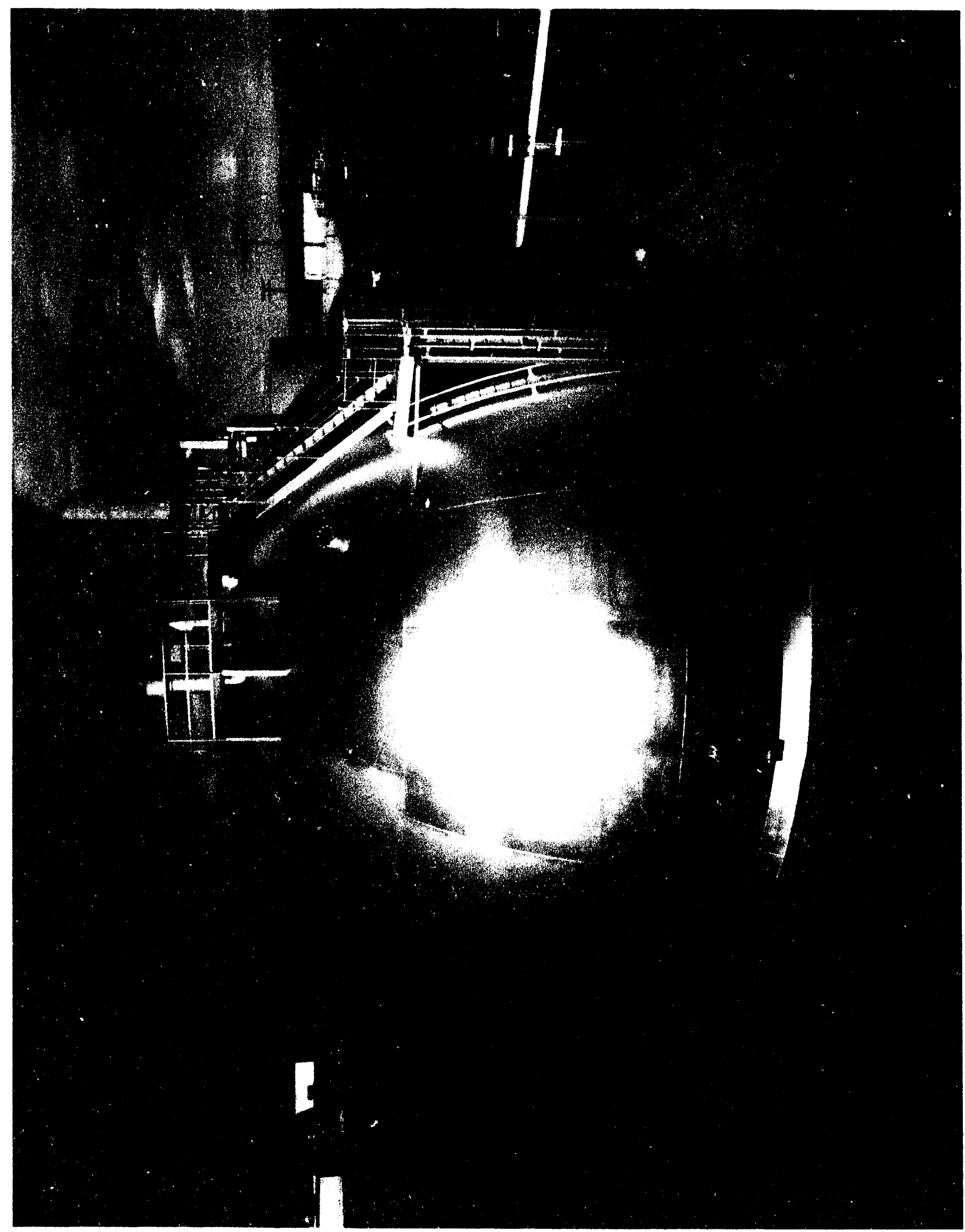




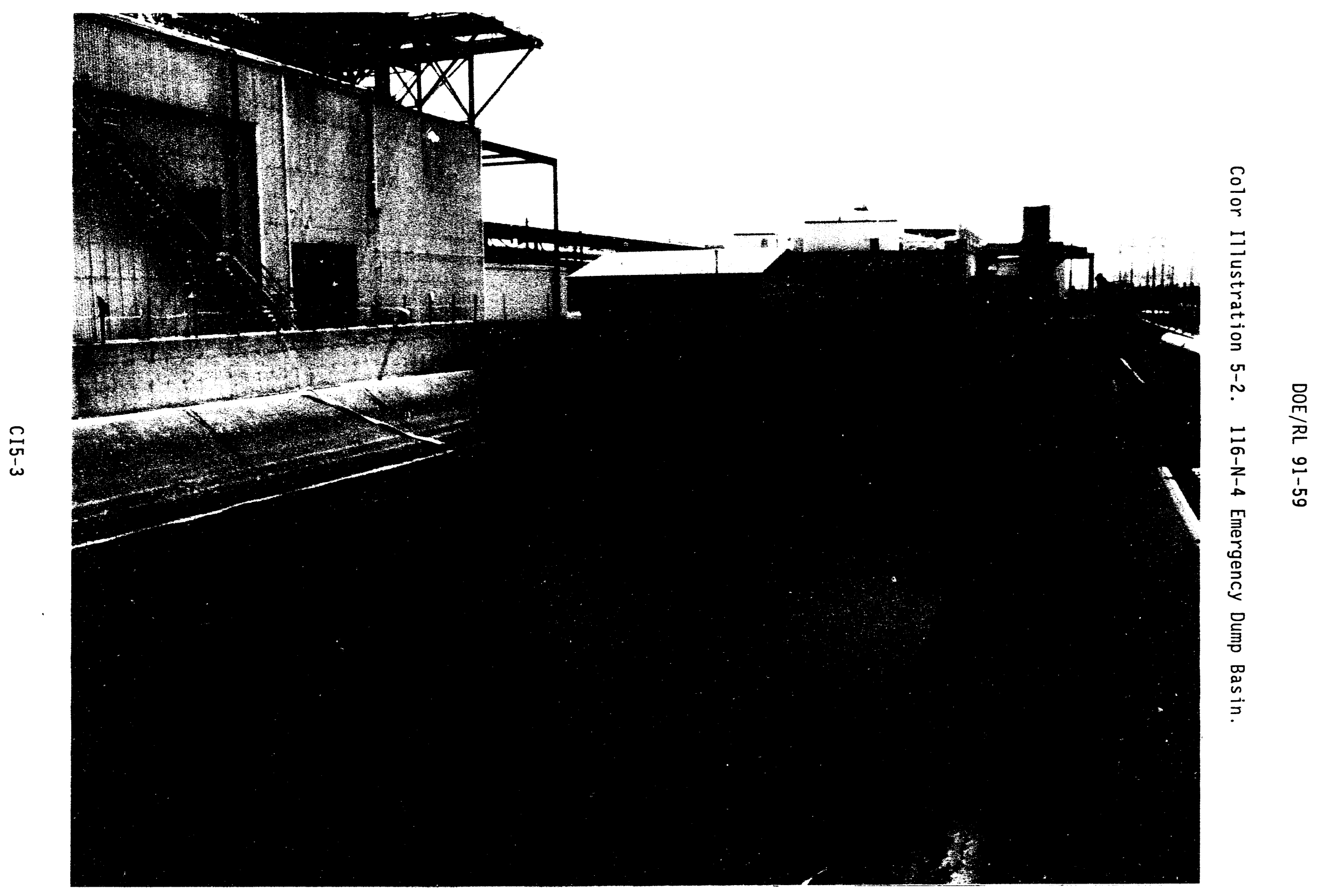




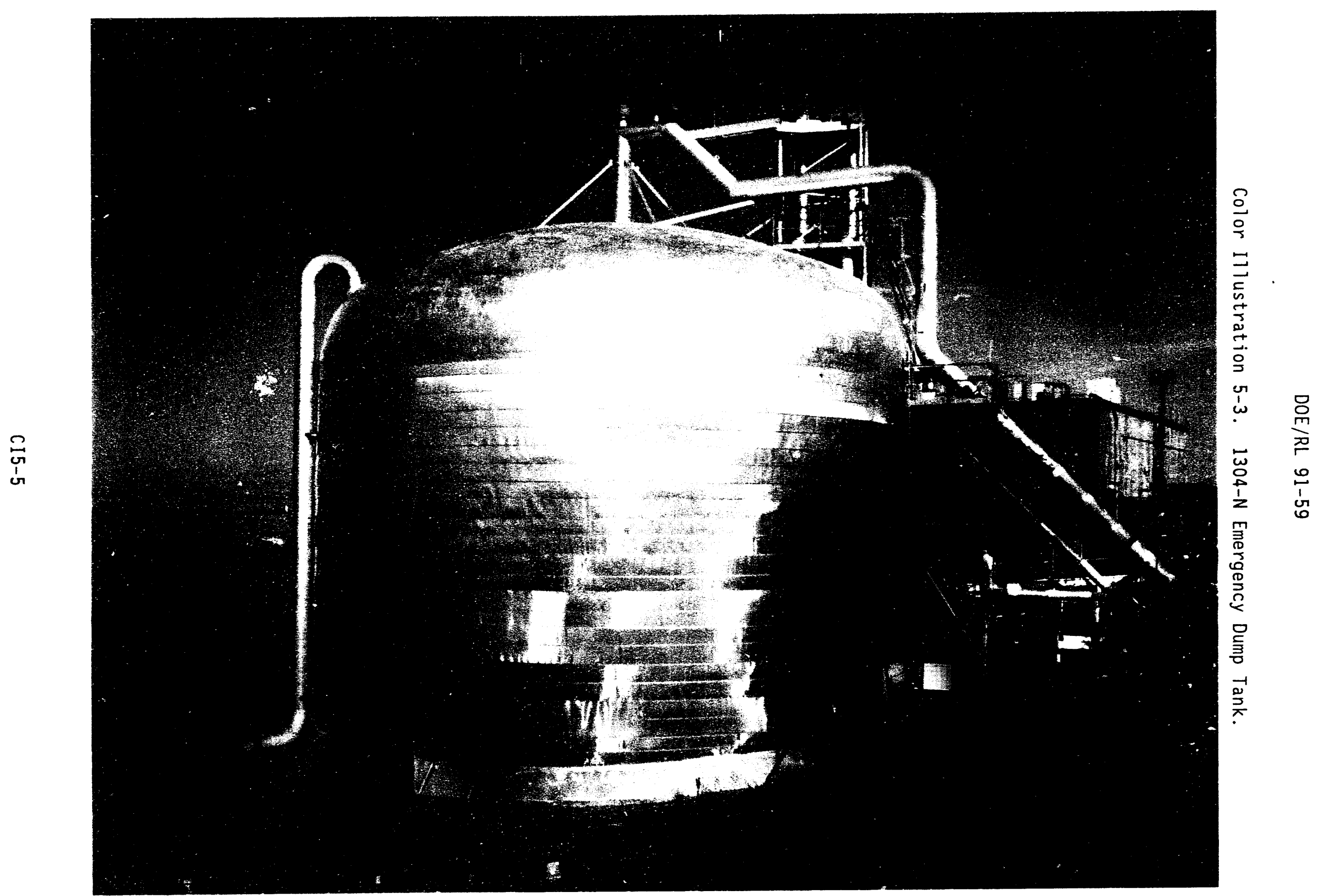


Color Illustration 5-4. 105-N Reactor Spent Fuel Storage Bas in (above water).

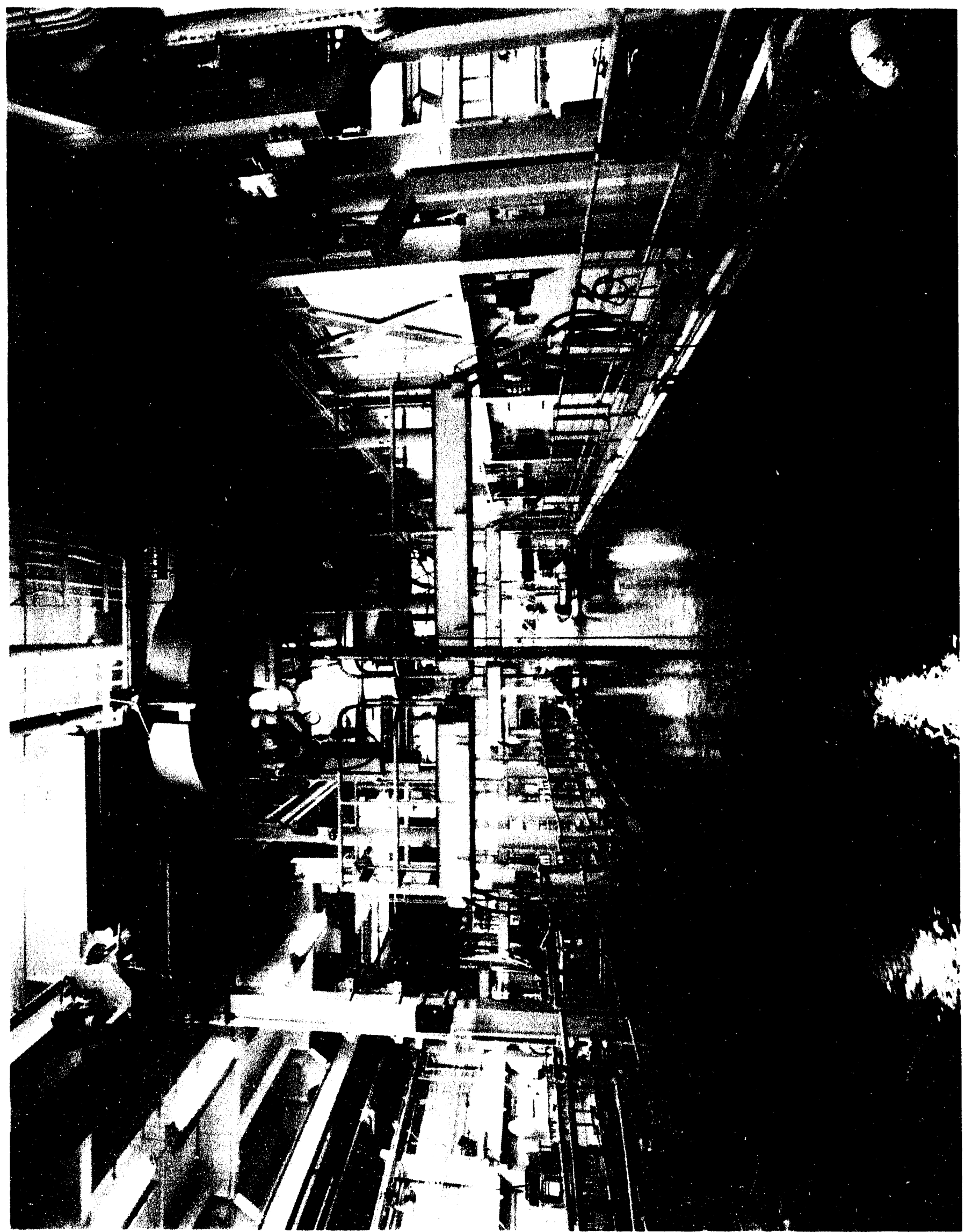


Color Illustration 5-5. 105-N Reactor Spent Fuel Storage Basin (below water).

5

$+4$

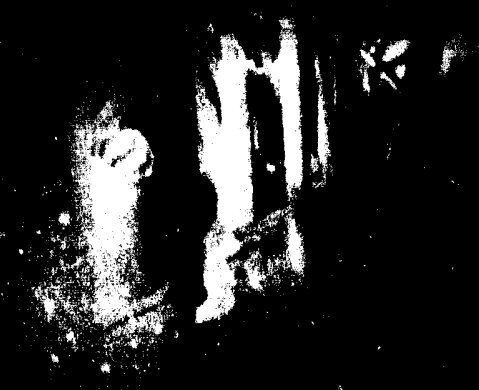

in:

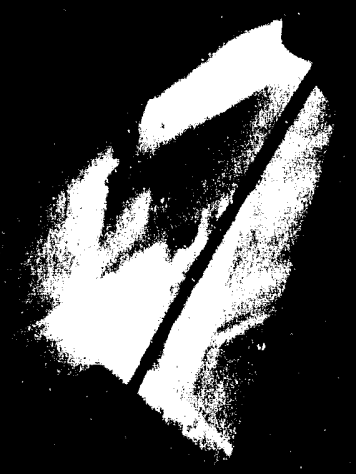




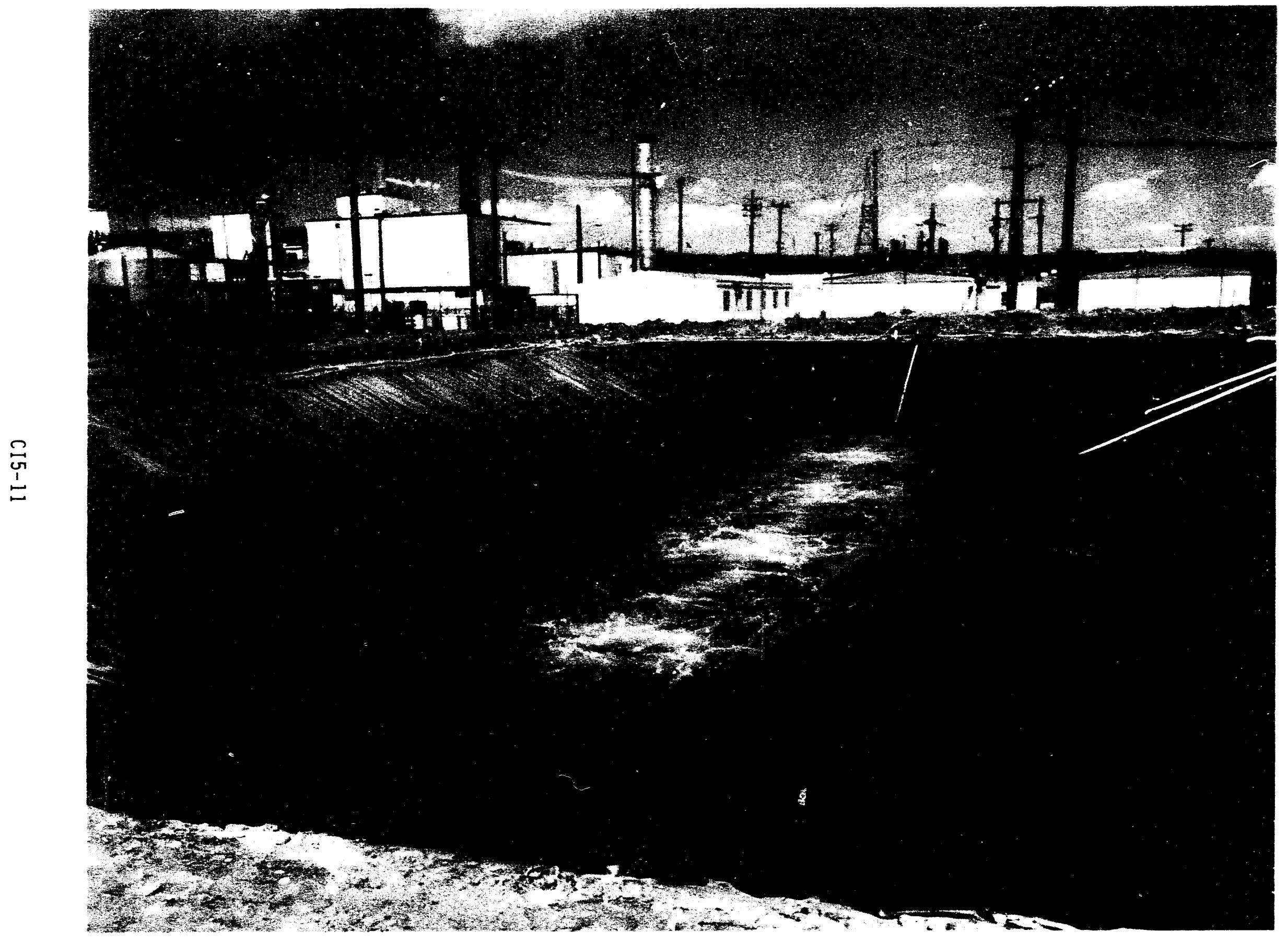


DOE/RL $91-59$

Color Illustration 5-7. 100-N Regulatory-driven Milestones.

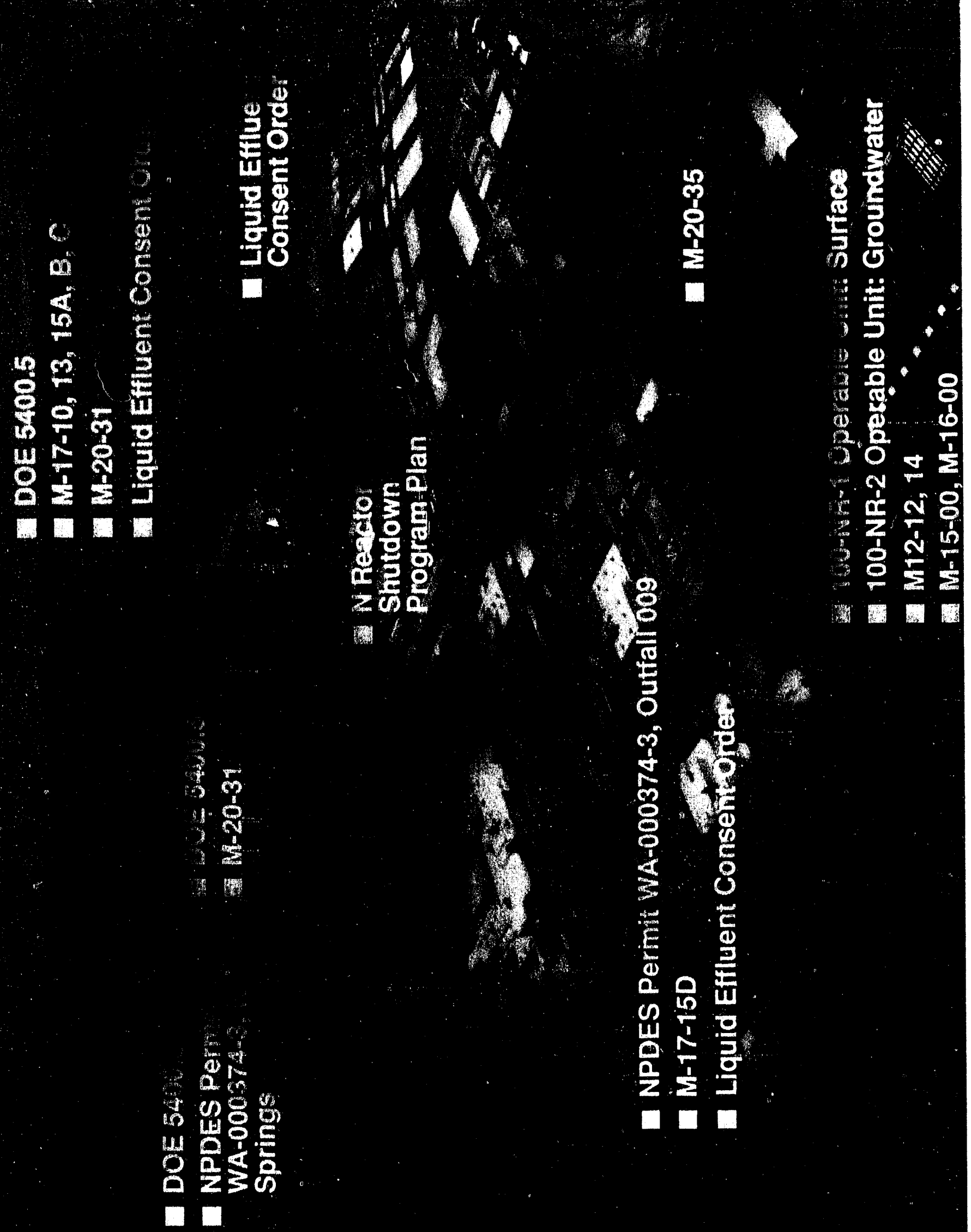




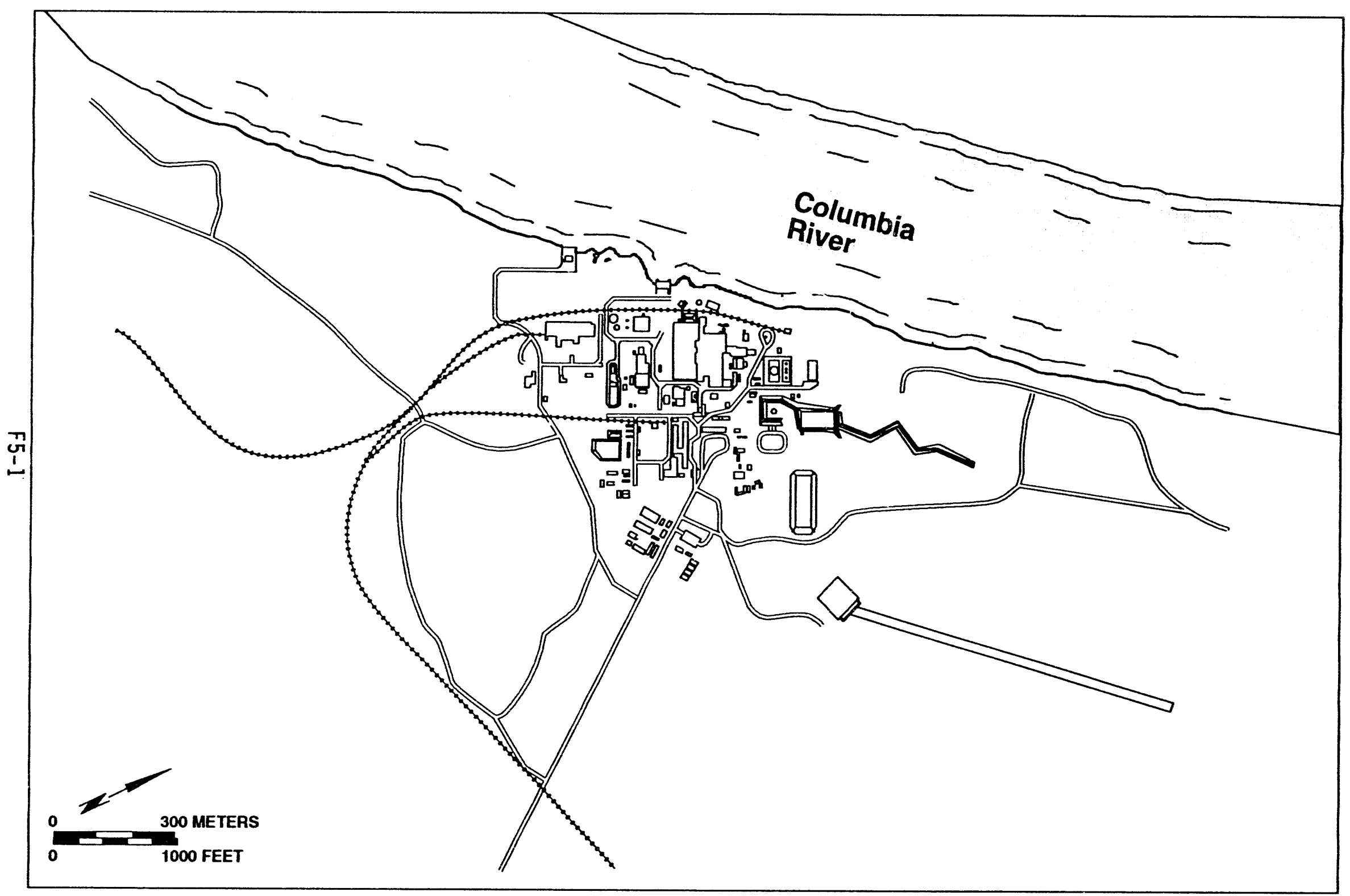

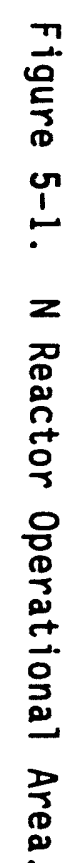

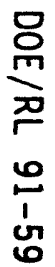


DOE/RL 91-59

This page intentionally left blank.

F5-2 


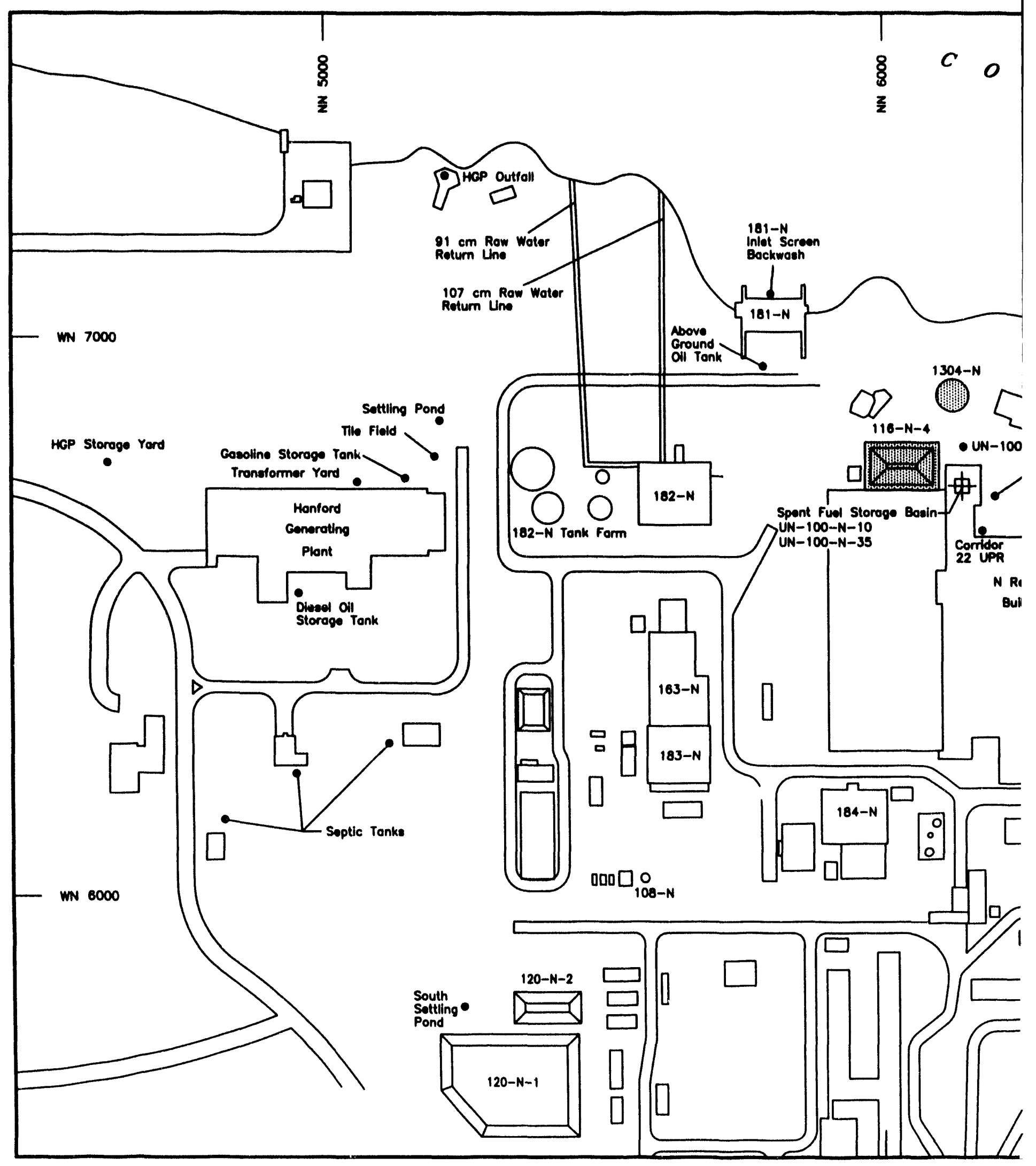


DOE/RL 91-59

Figure 5-3. Original 116-N-1 Crib Layout.

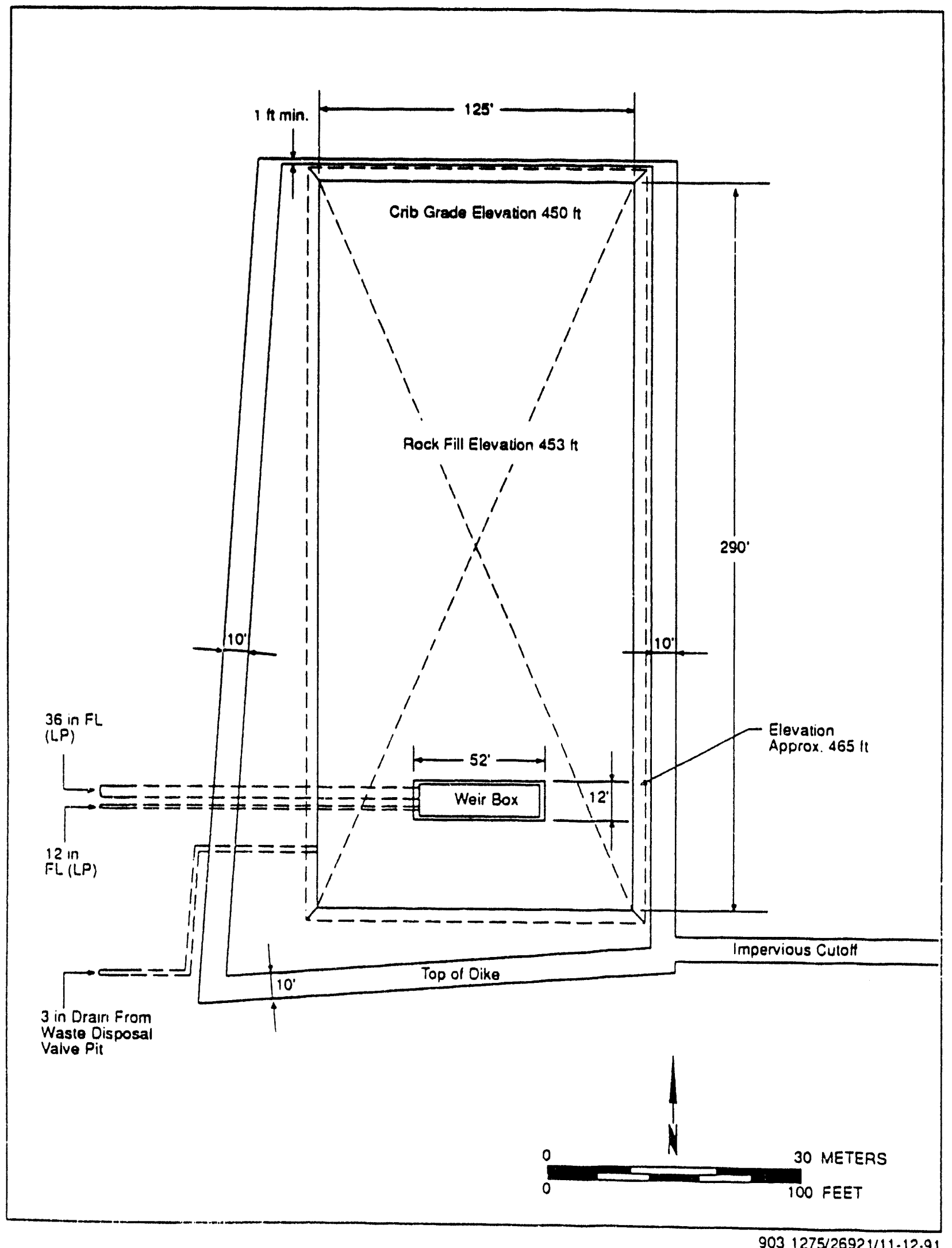


Figure 5-4. Regeneration Waste Transport System, 1977-1983.

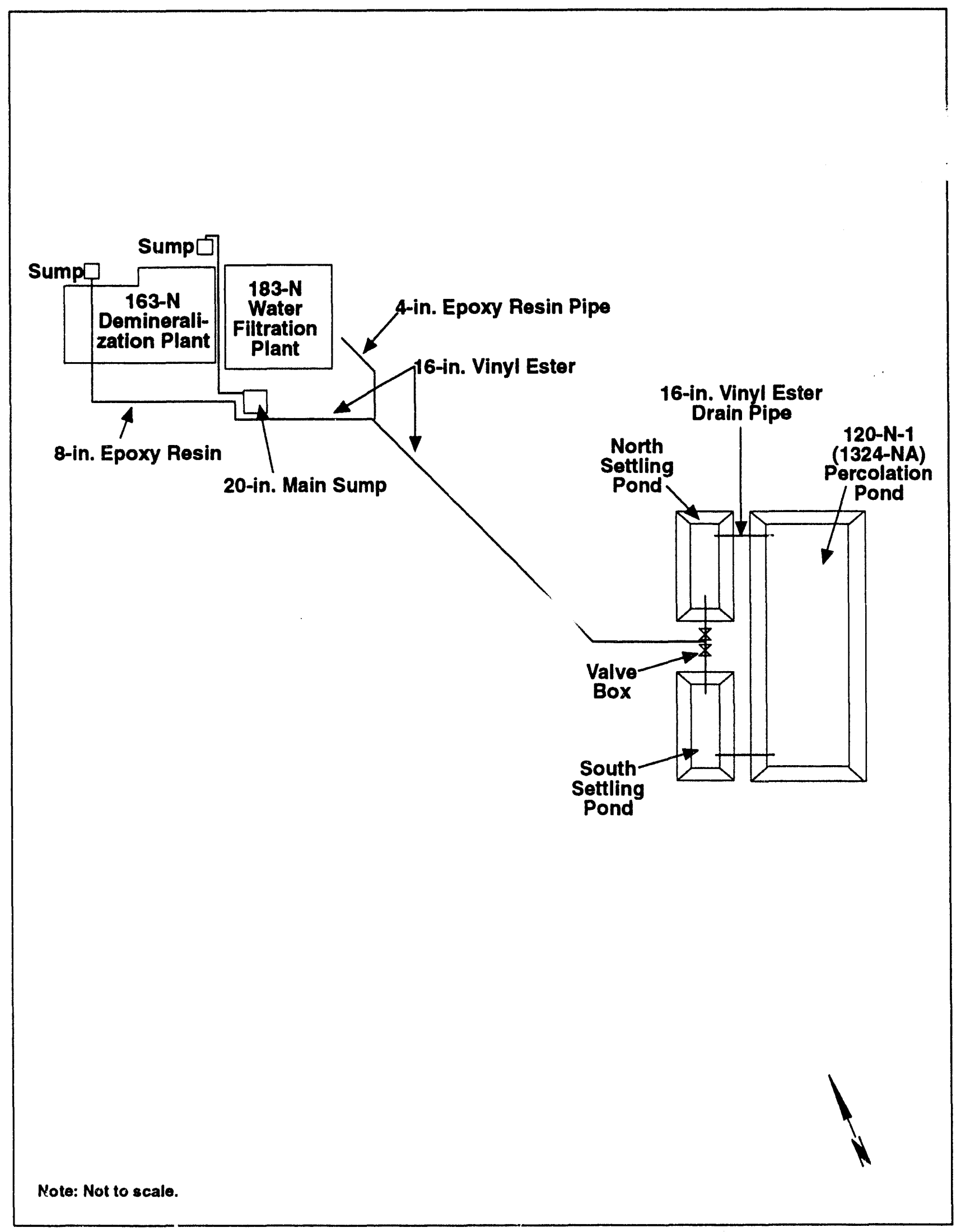


DOE/RL 91-59

Figure 5-5. Regeneration Waste Transport System, 1983-1986.

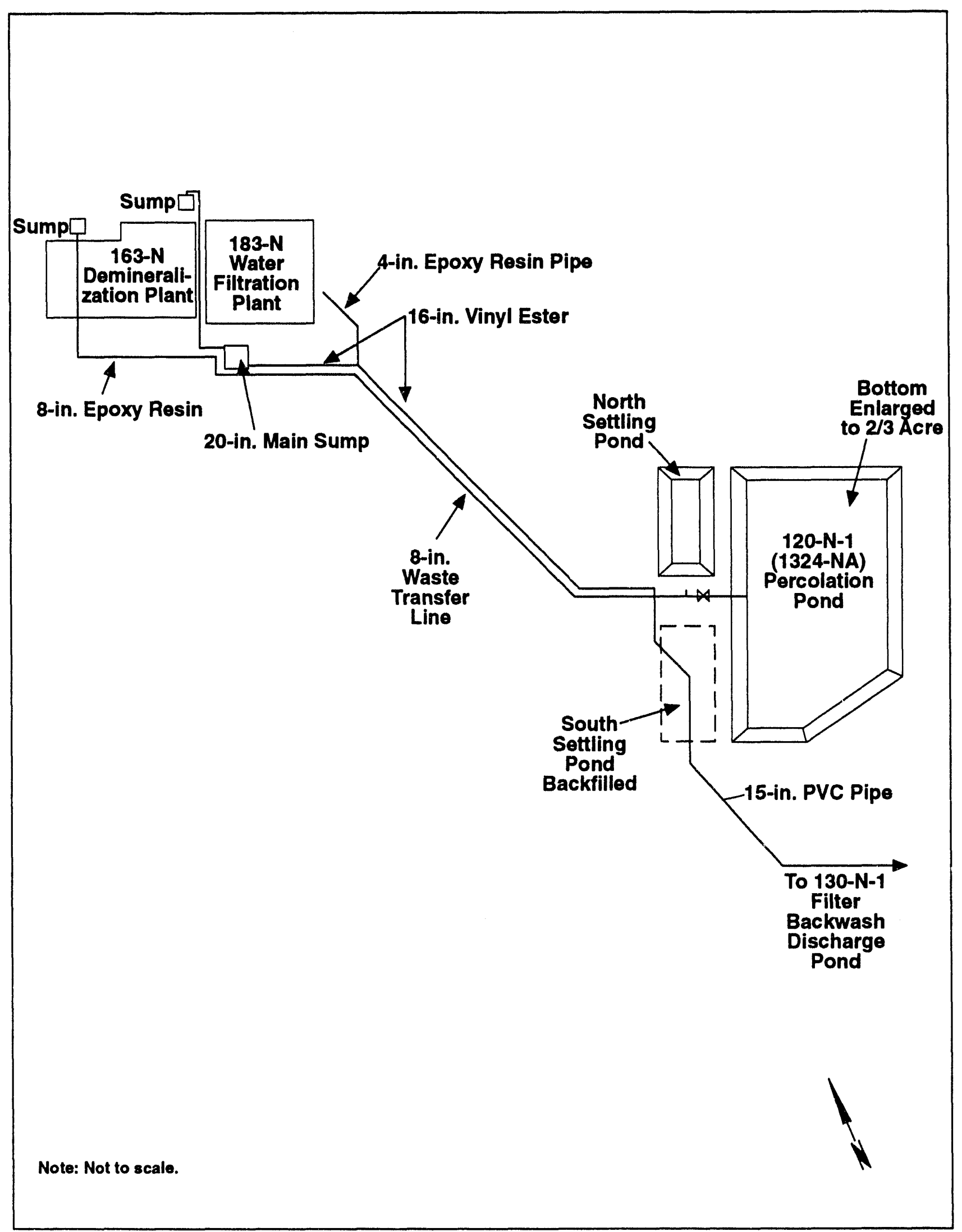


Figure 5-6. Regeneration Waste Transport System, 1986-1988.

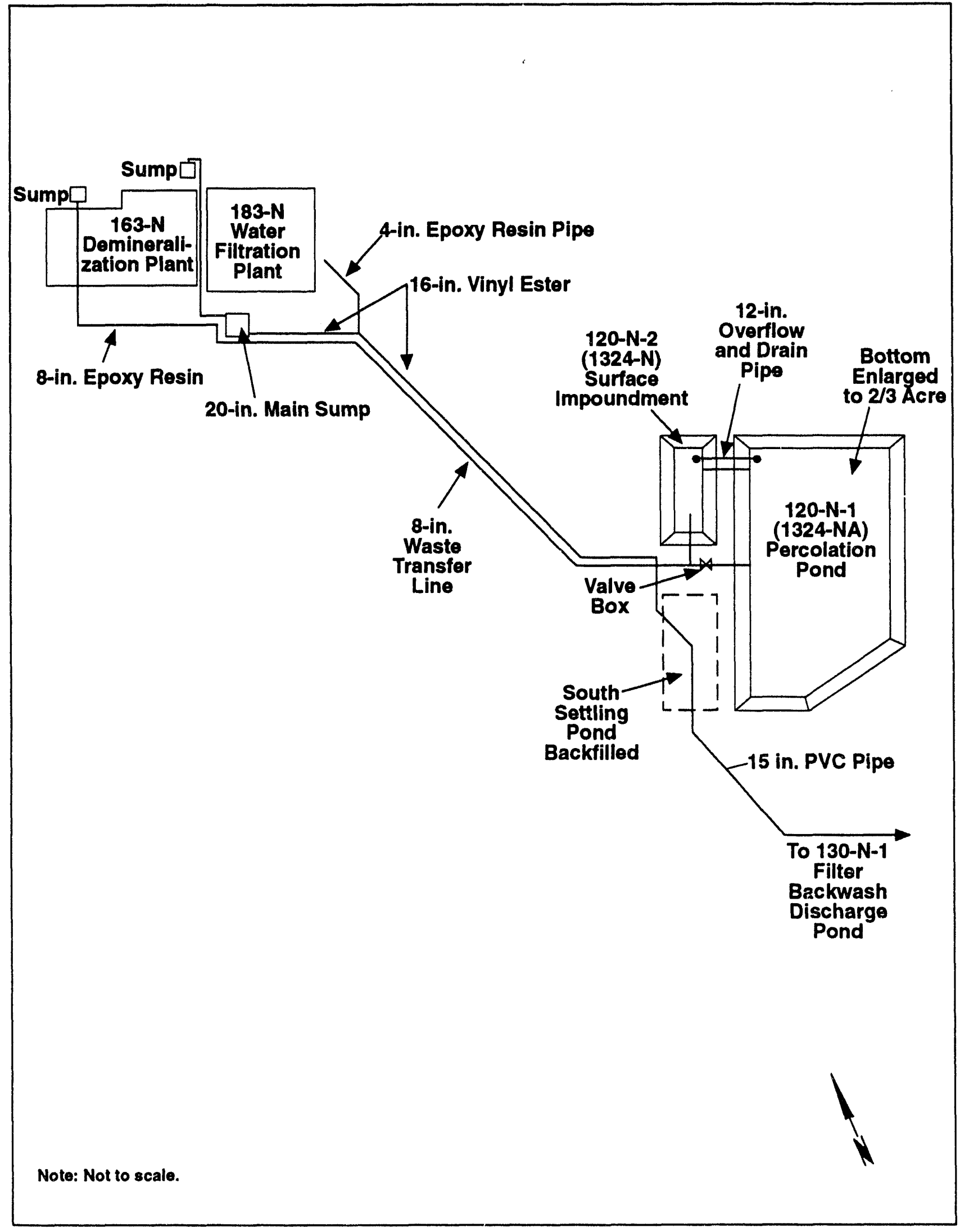


Figure 5-7. 120-N-1 Percolation Pond Area as it existed from 1977 to 1983.

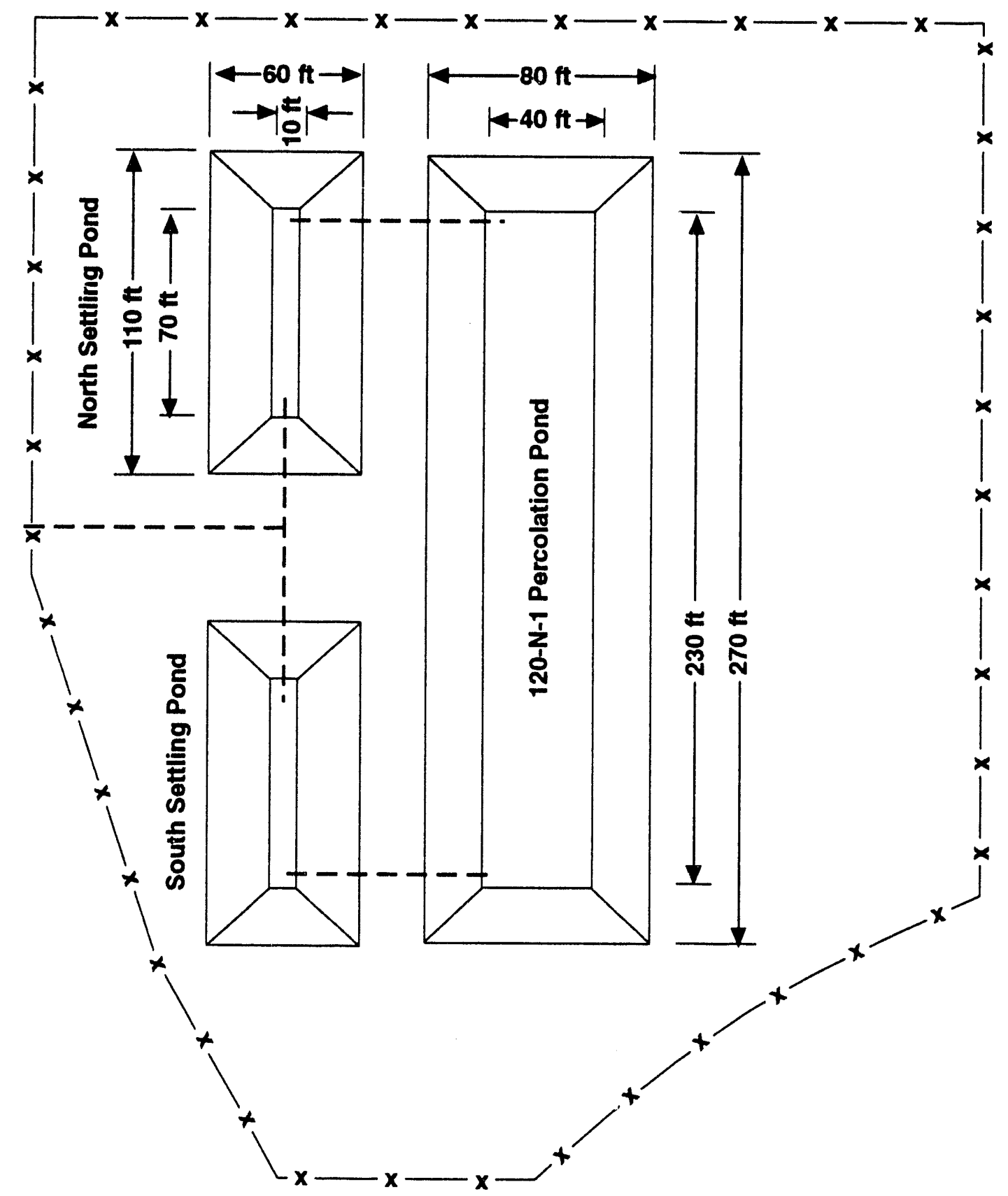

LEGEND

\section{- - - Effluent Line \\ - $\mathrm{X}$ - Security Fence}

Note: Dimensions are approximate. 
Figure 5-8. 120-N-1 Percolation Pond/120-N-2 Surface Impoundment Area as they existed from 1986 to 1988.

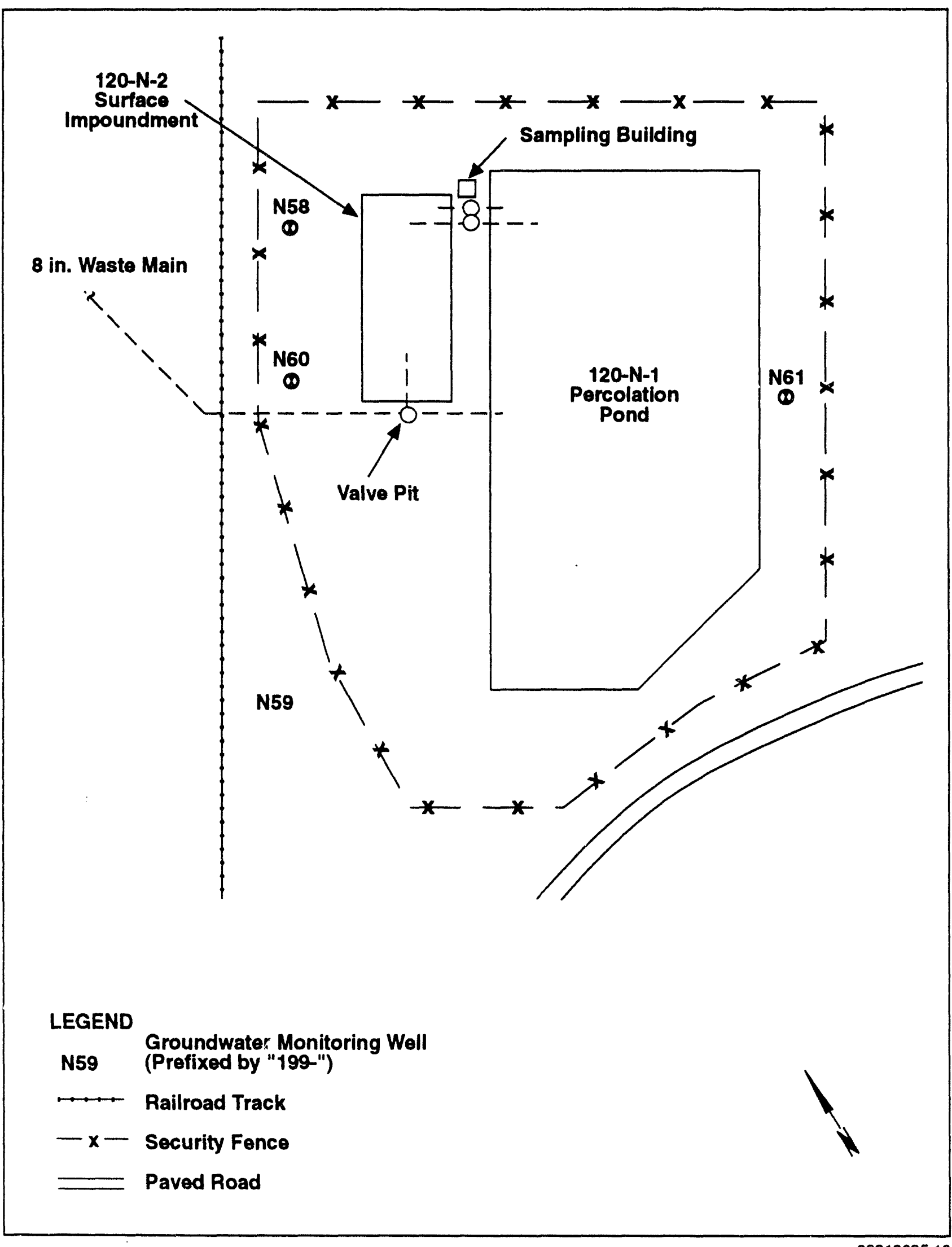


Figure 5-9. 120-N-2 Surface Impoundment Schematic.

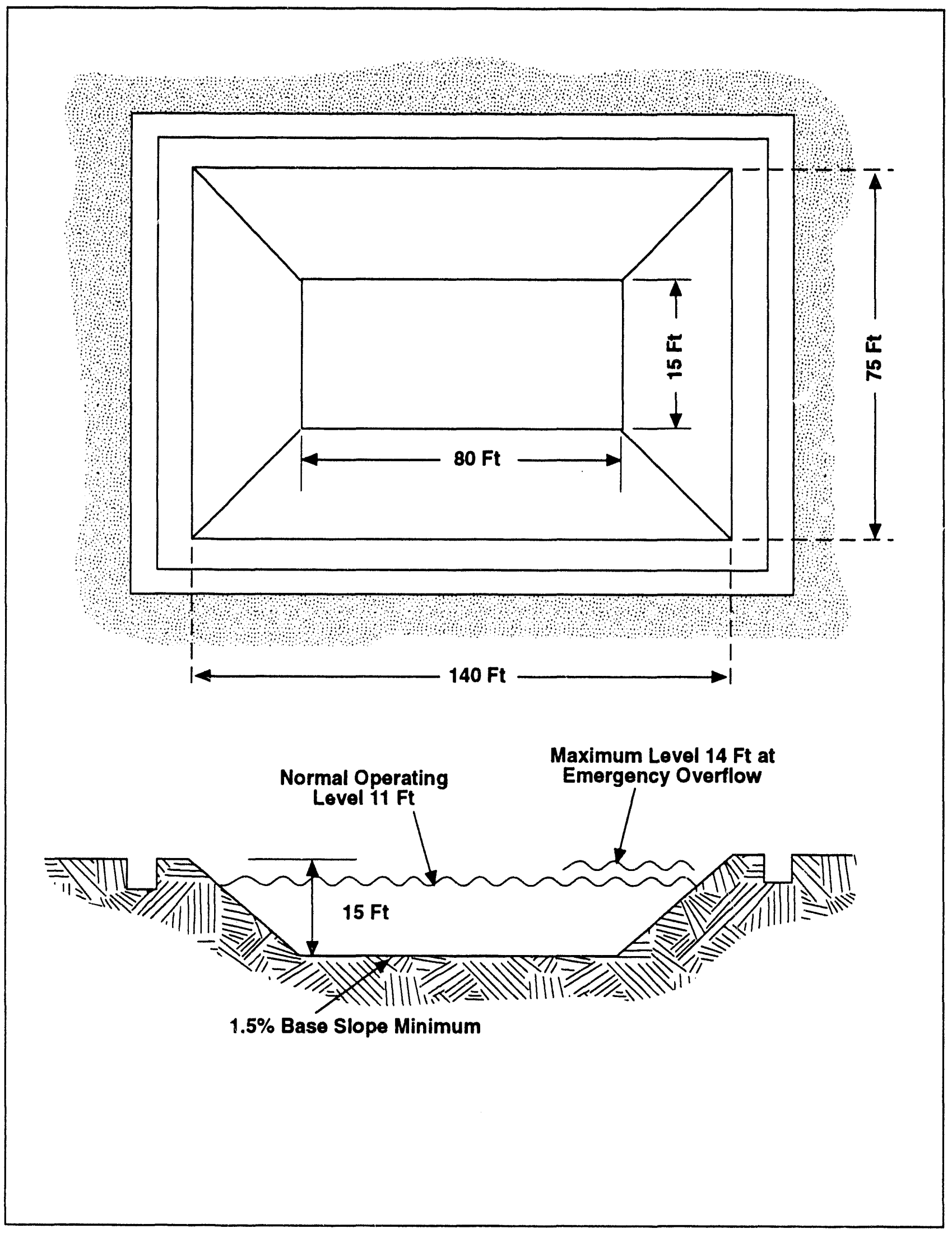


Figure 5-10. Tritium Activity Levels in N Springs During 1988.

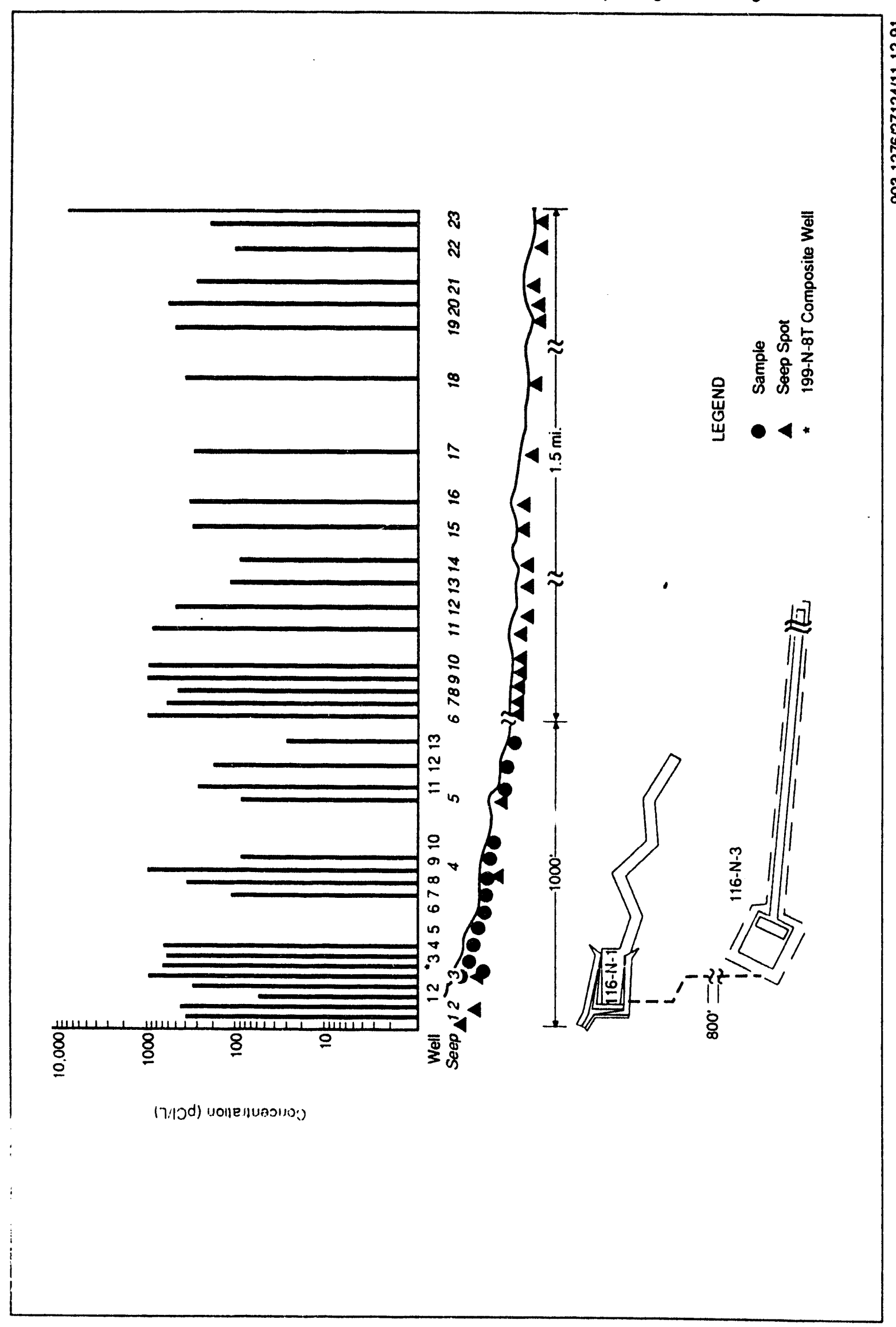




$$
\frac{1}{d u}
$$




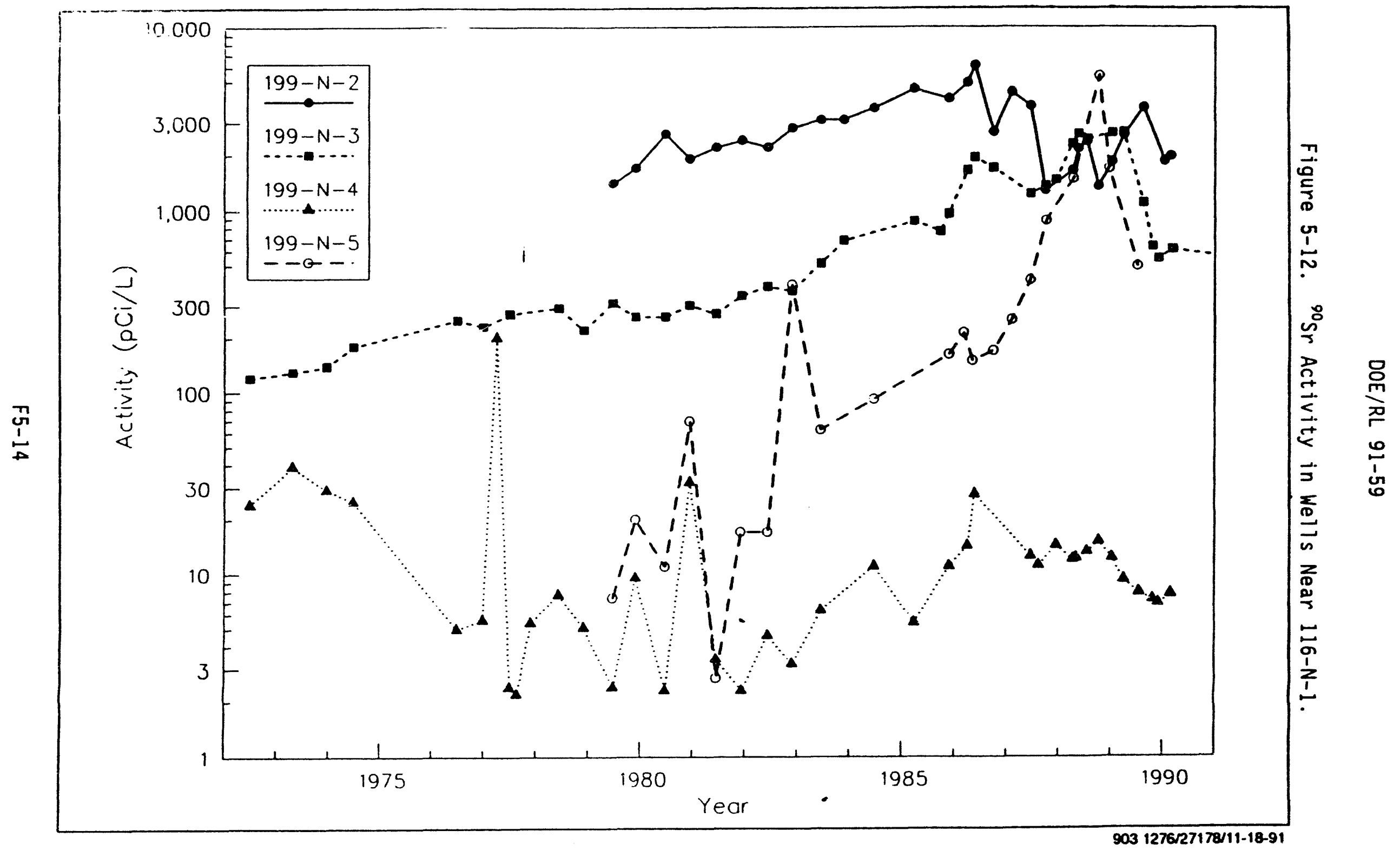




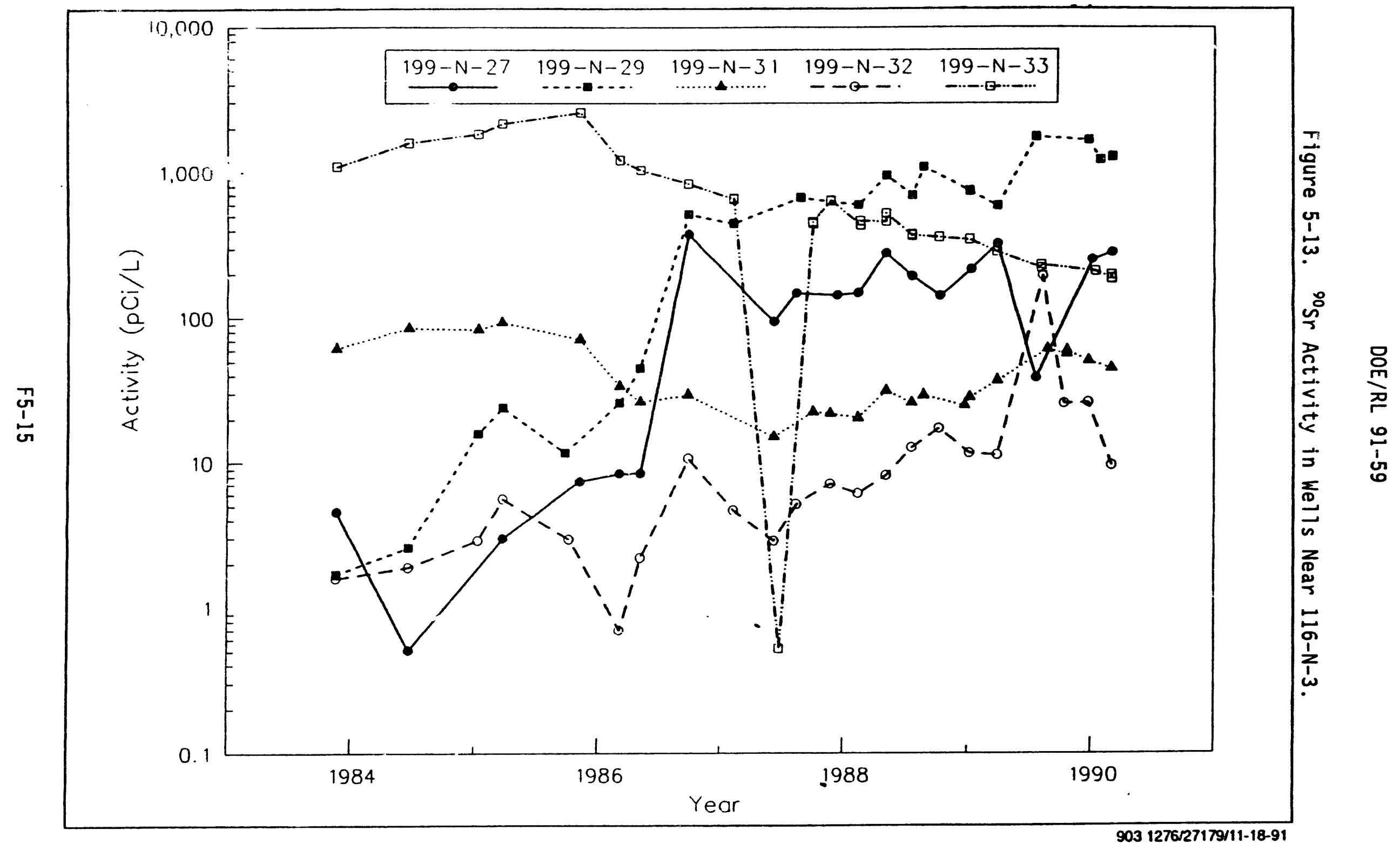




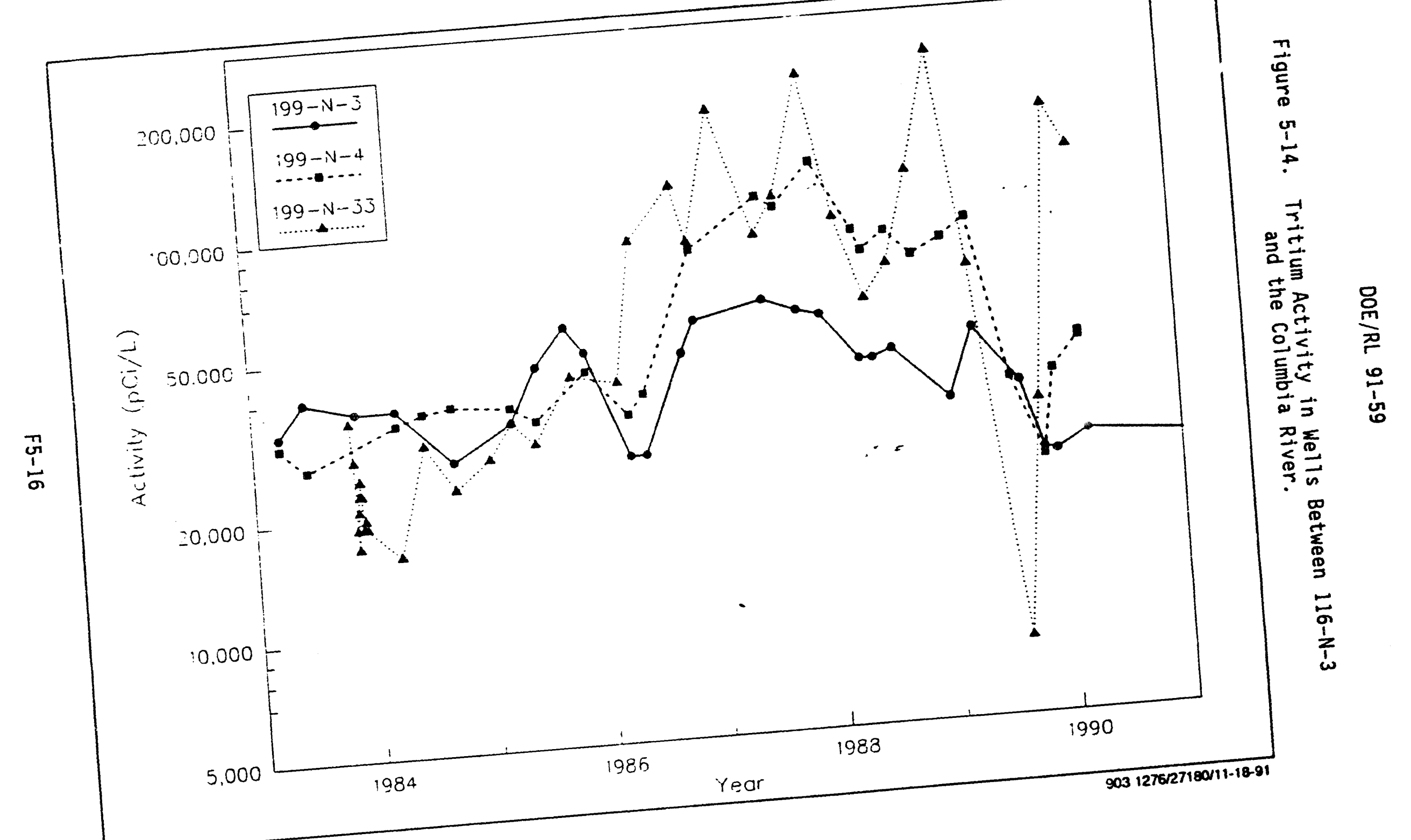


DOE/RL 91-59

Figure 5-15. Tritium Activity in 100-N Area Groundwater During September-October 1989.

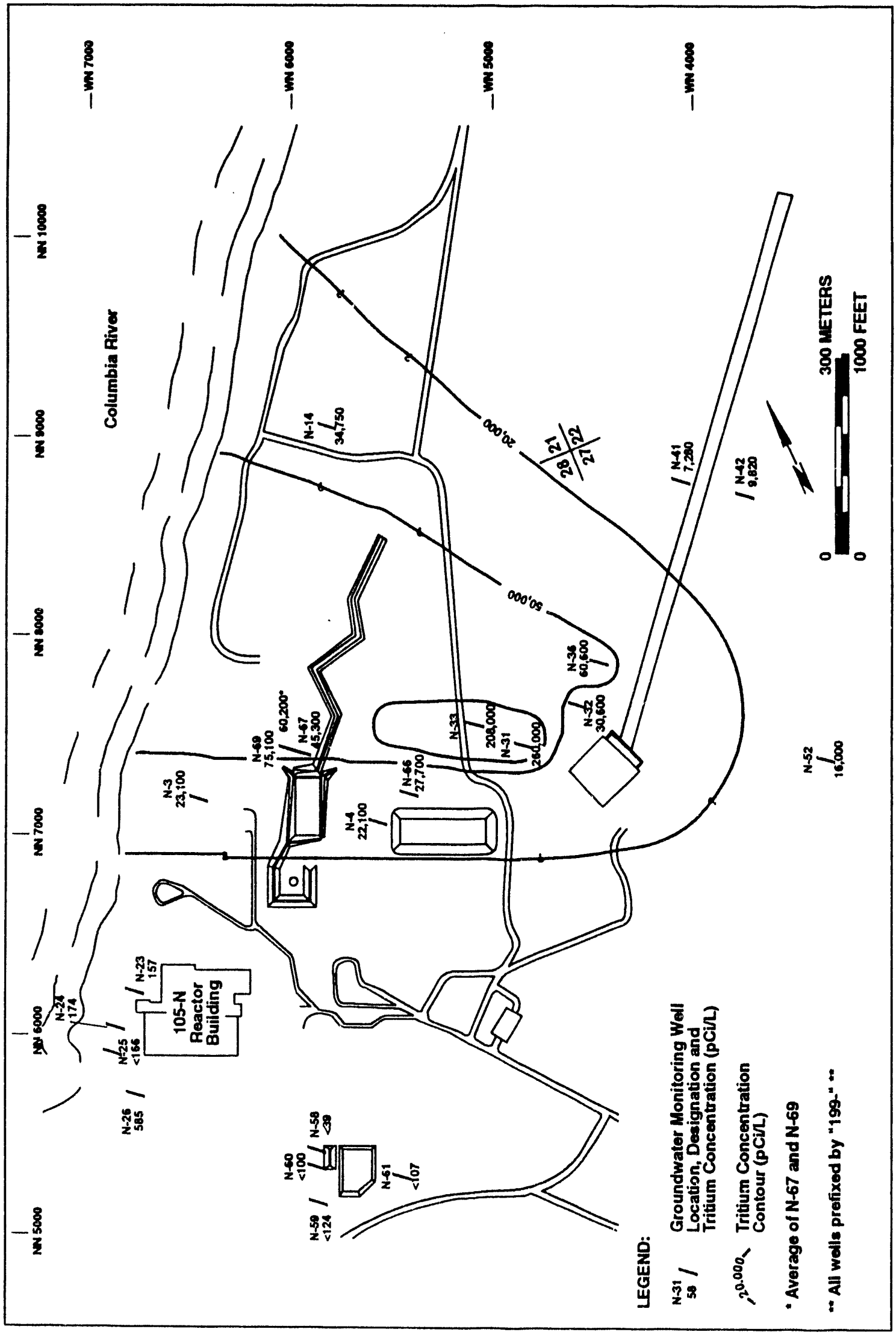




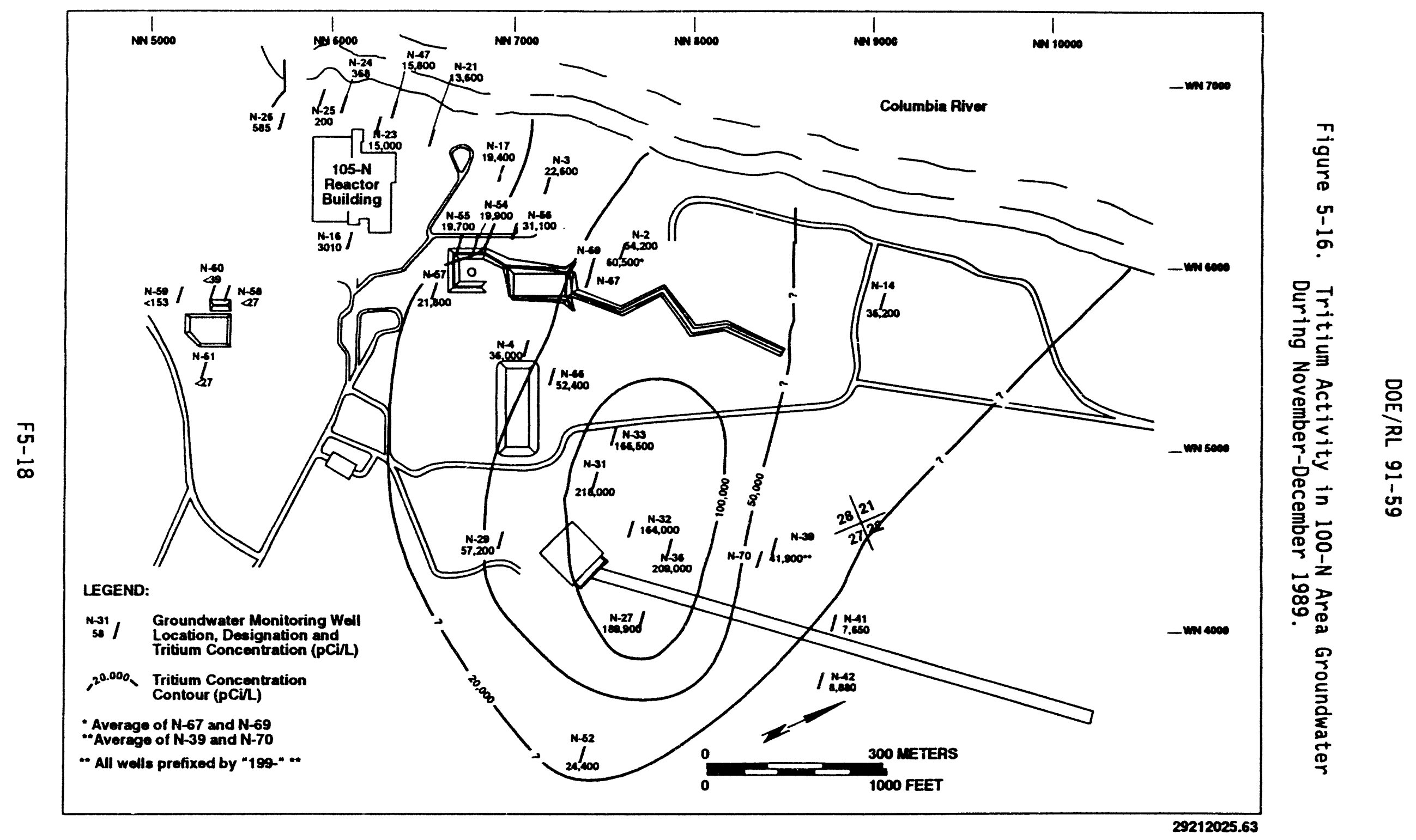




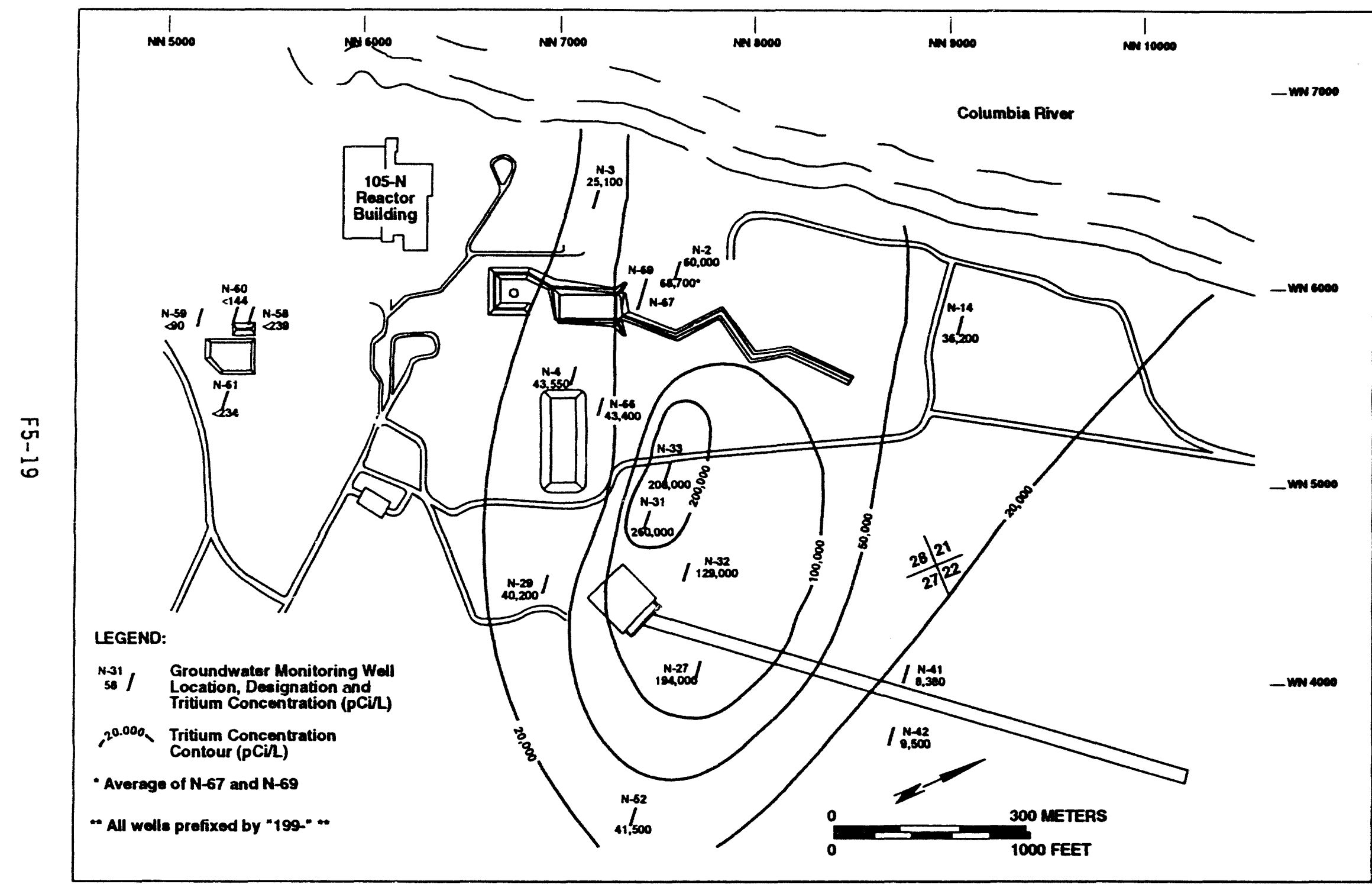

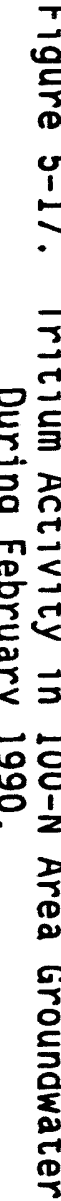




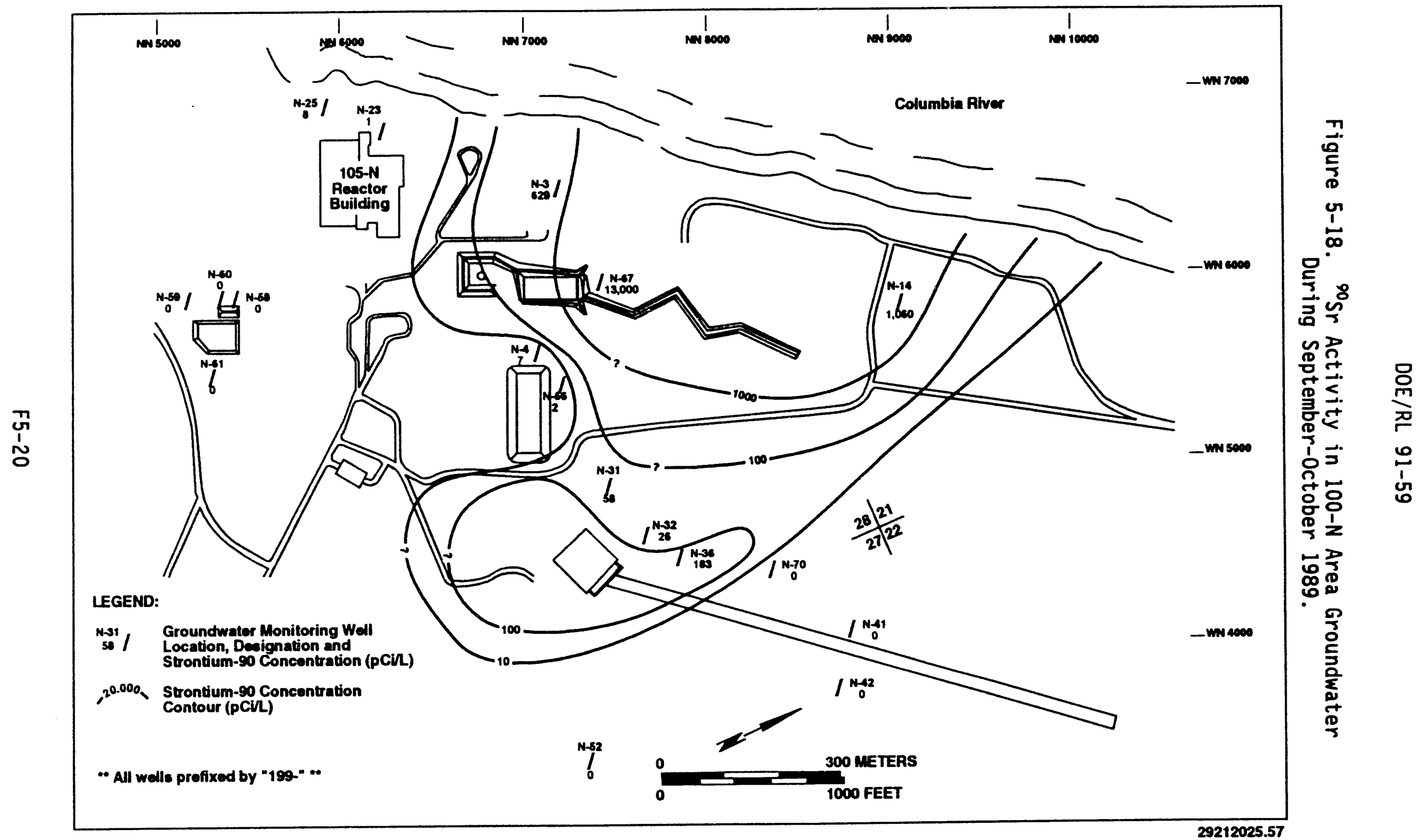




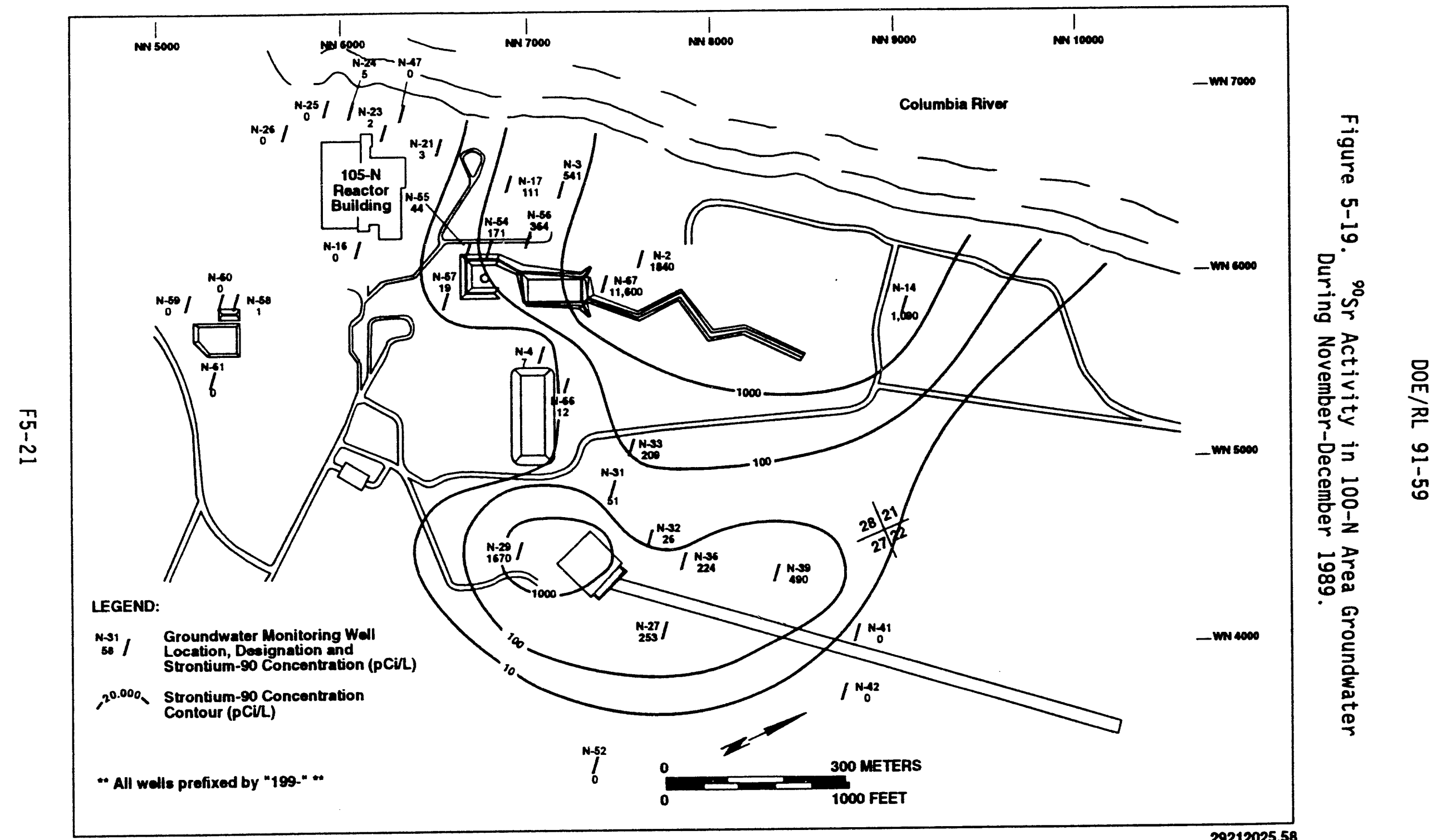




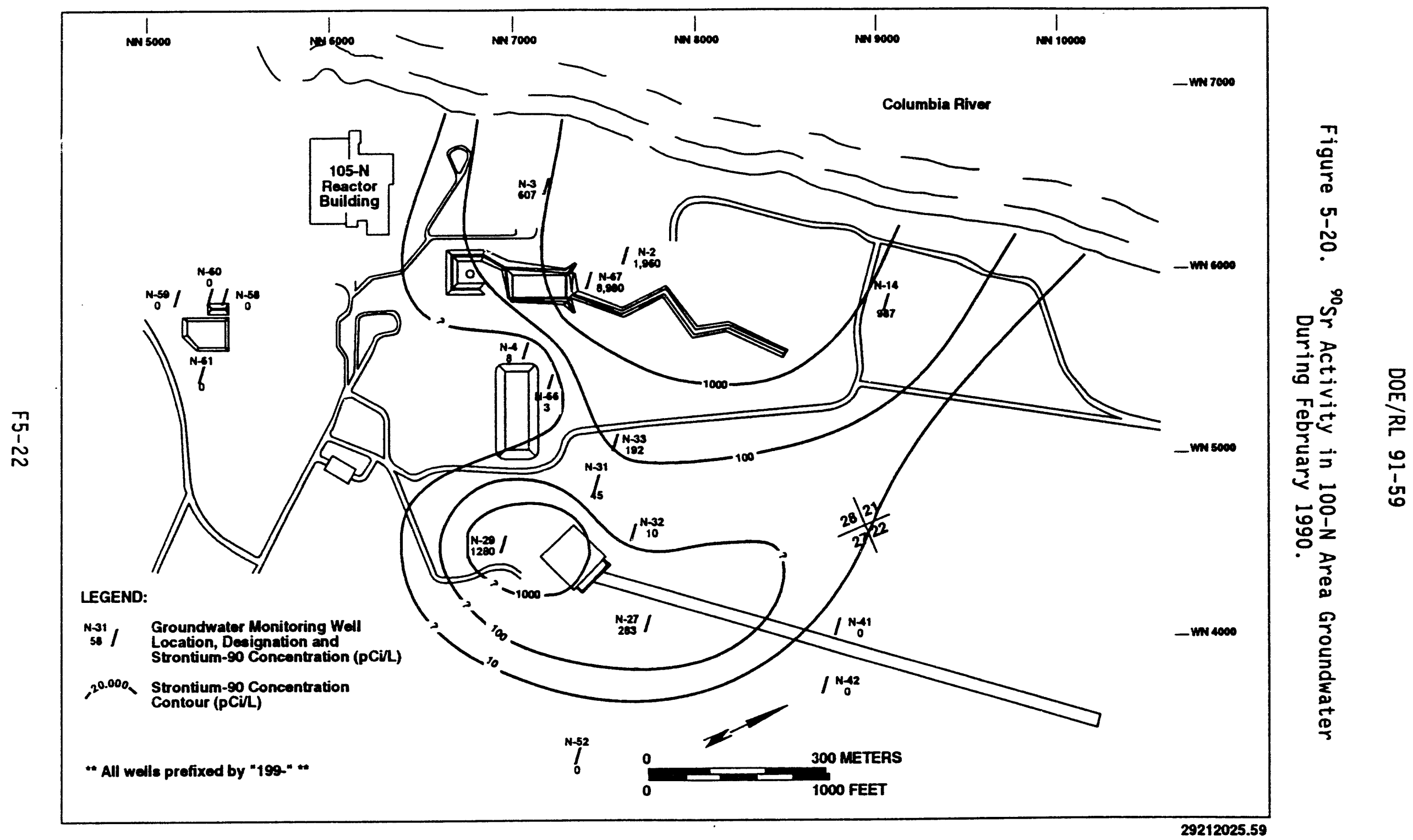




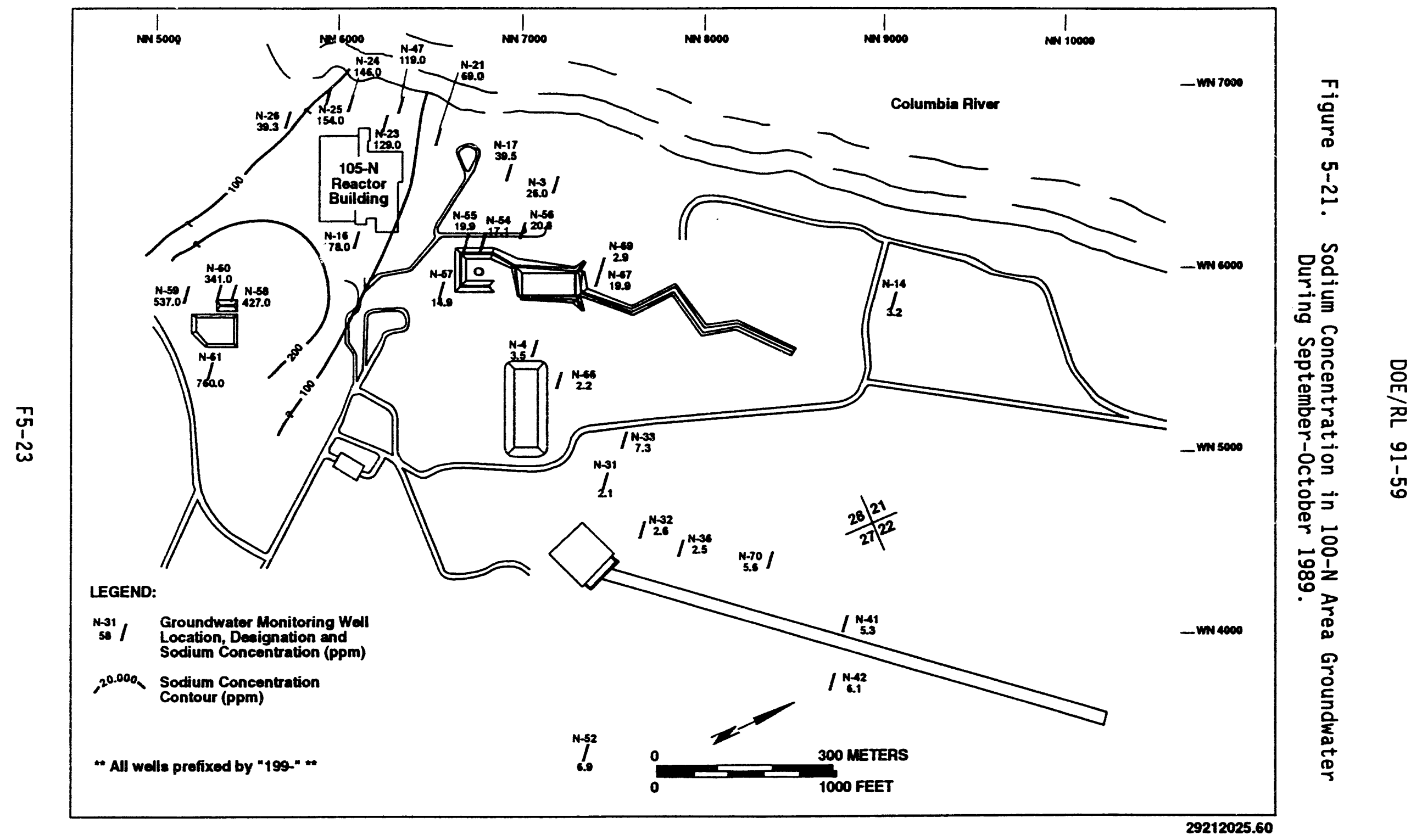




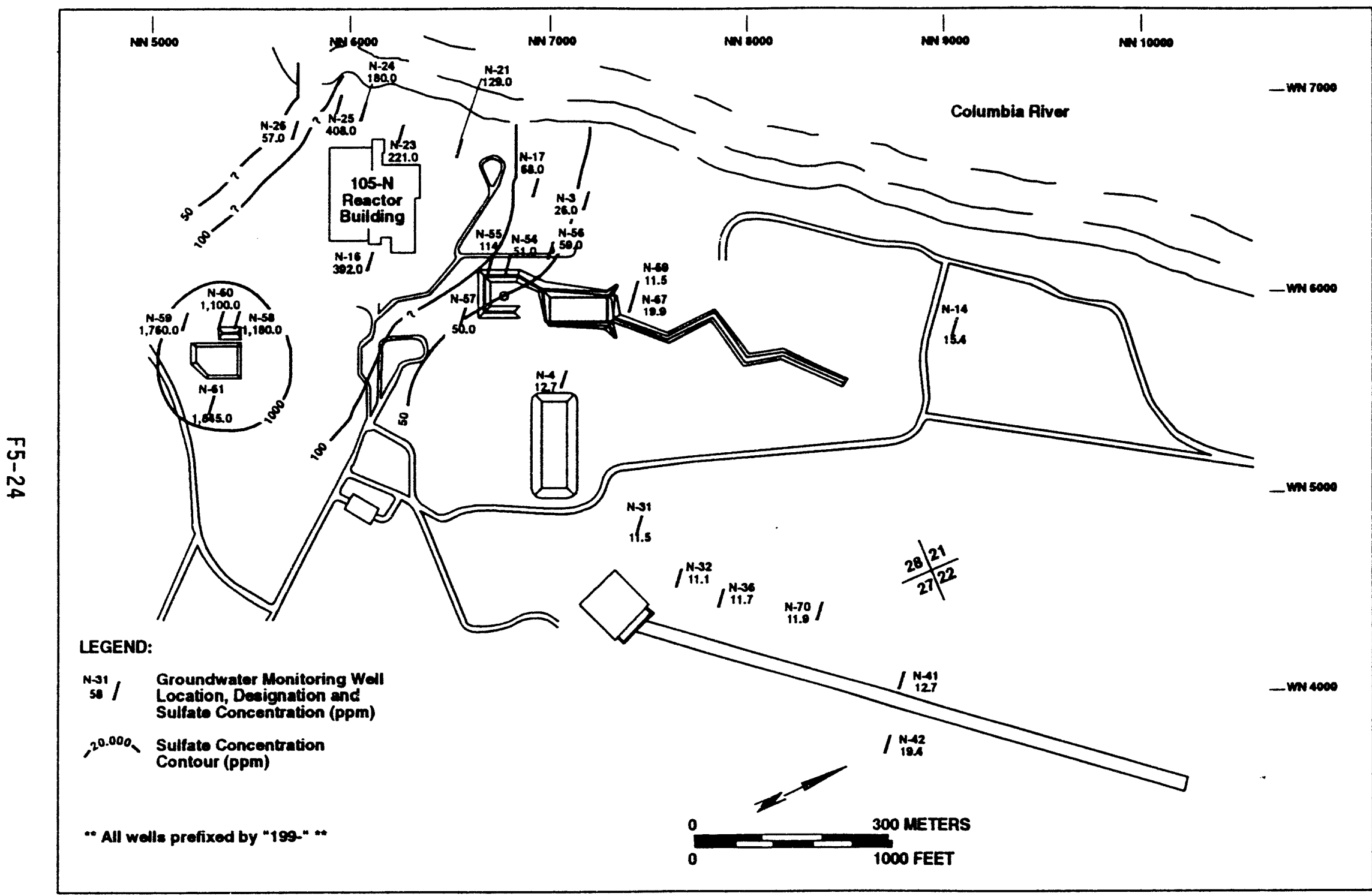

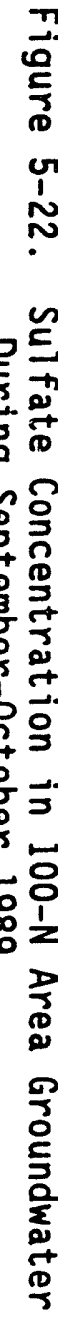


DOE/RL 91-59

Figure 5-23. Soil Sampling Locations in the 100-N Area.

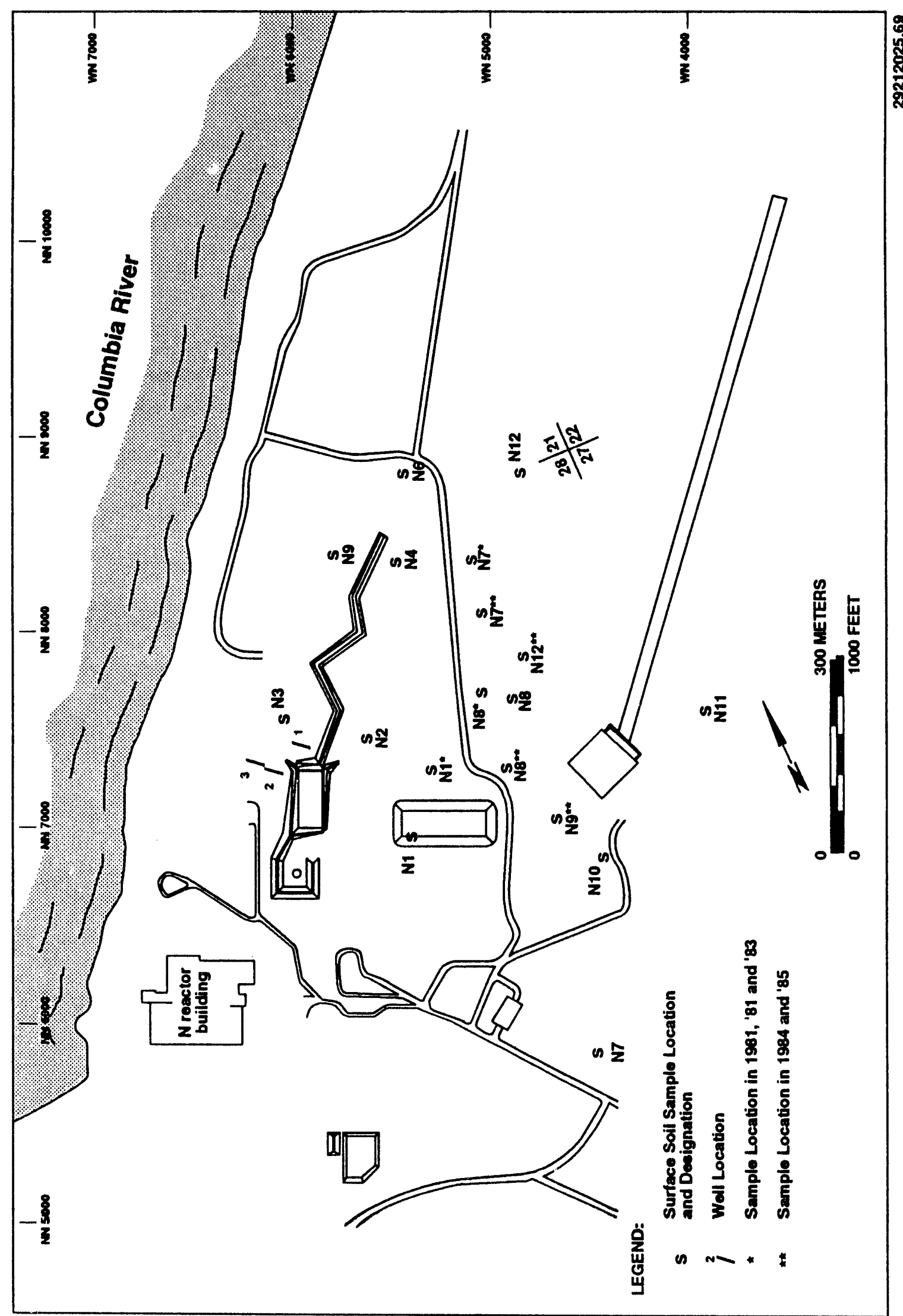




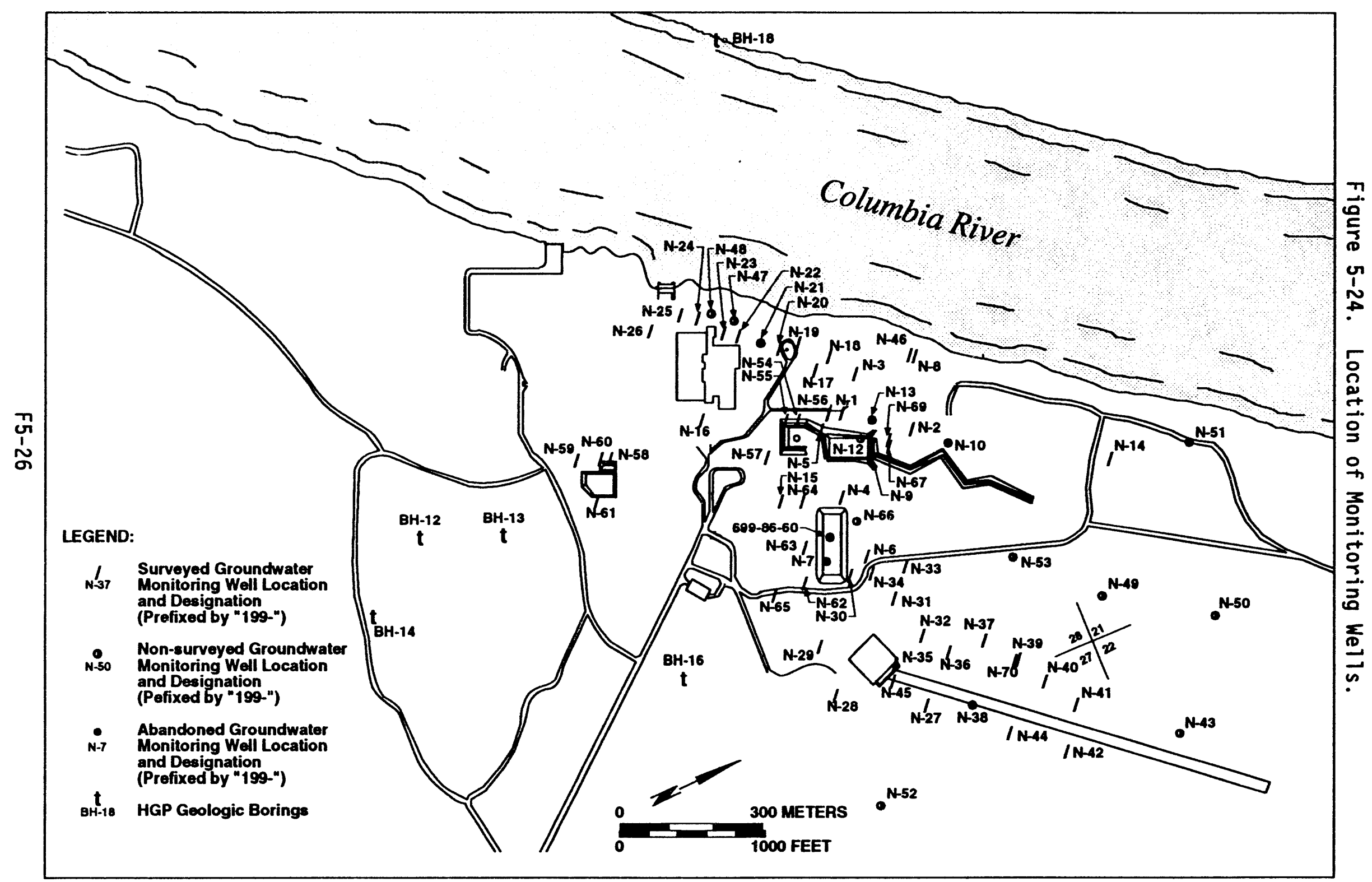

8
学
0
0
$\vdots$
s. 


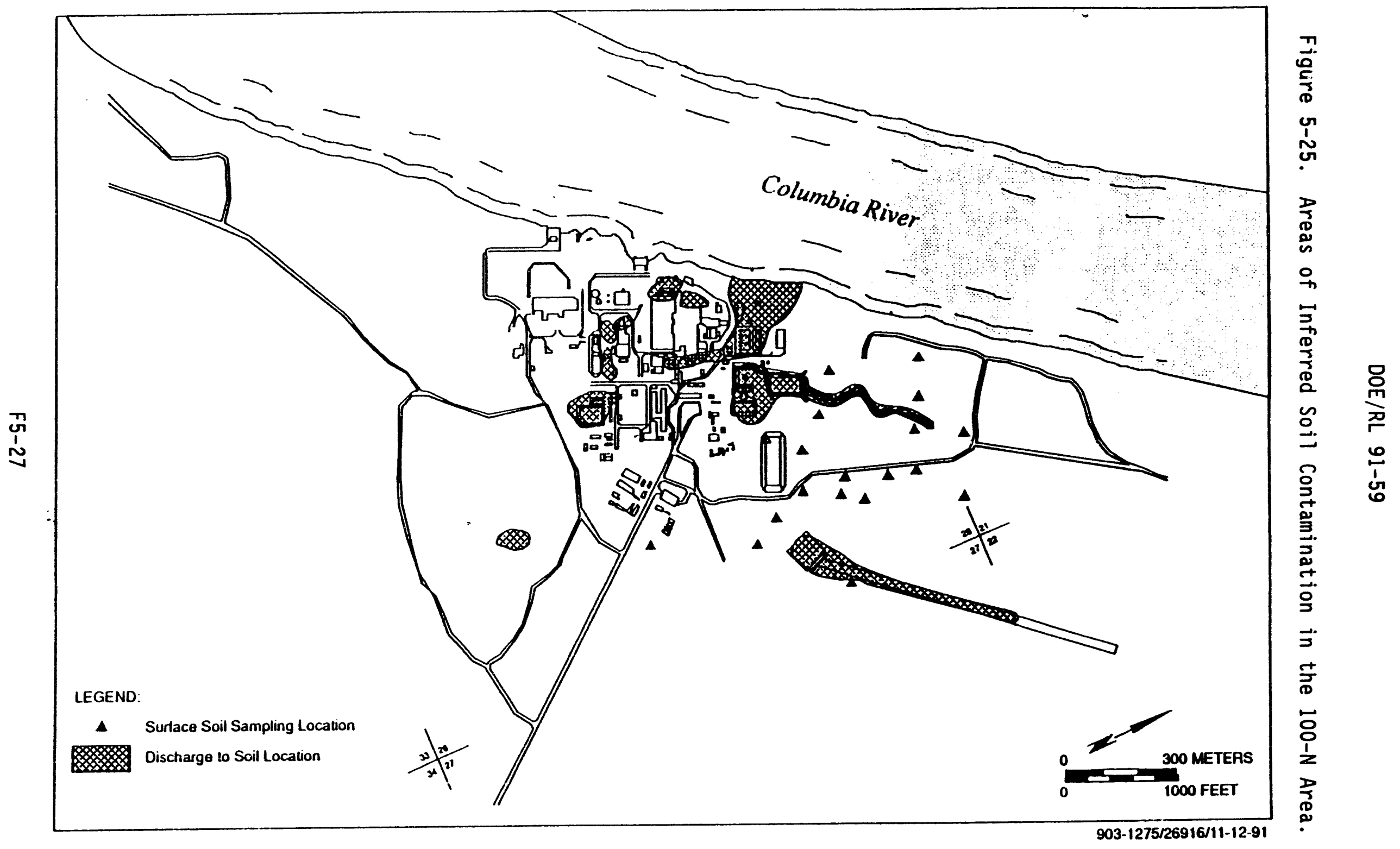


Figure 5-26. Results of January 10, 1990, Survey along the Top of the 1301-N LWDF Trench Sections.

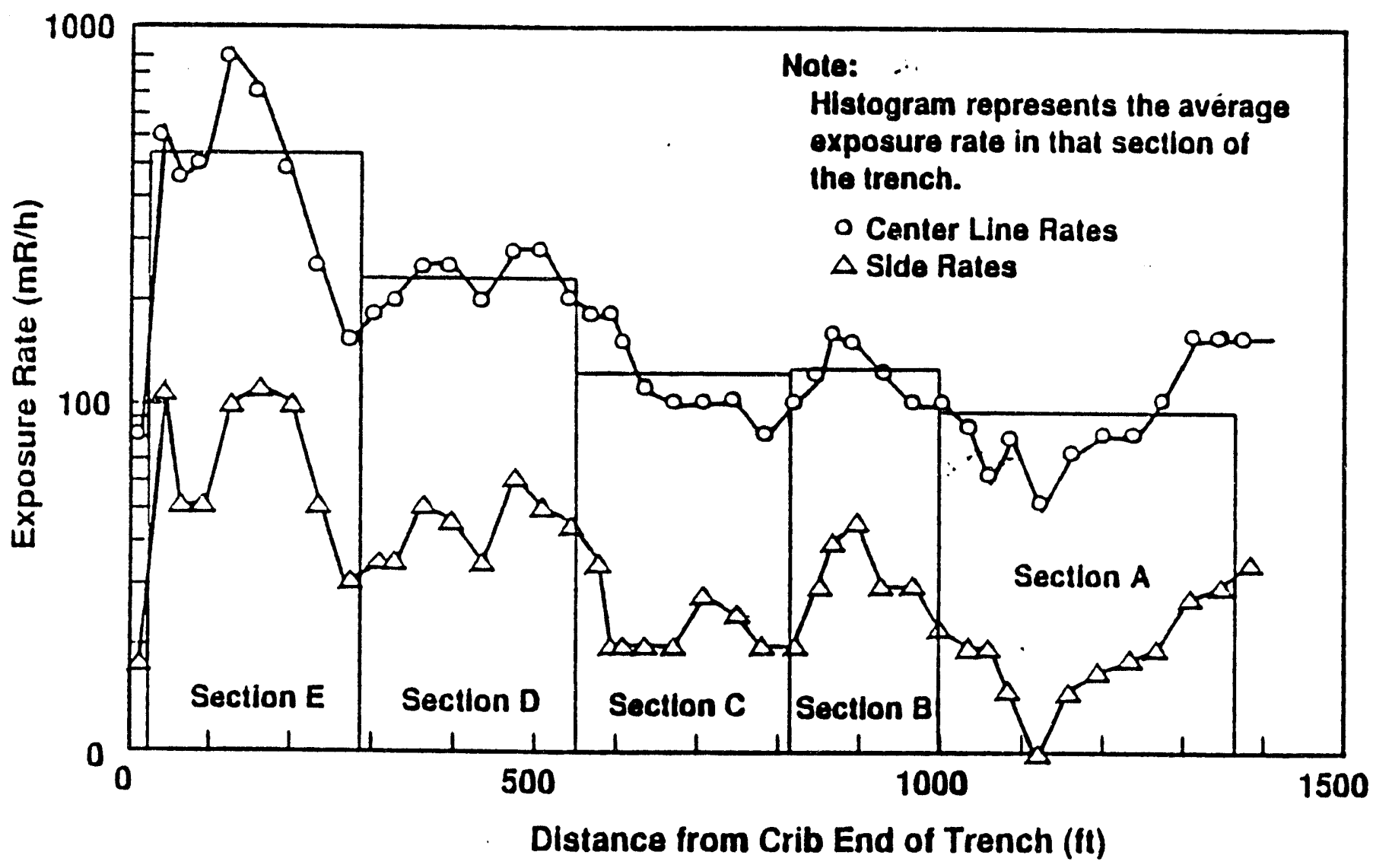

39009058.3 
Figure 5-27. Net Exposure Rate (above local background) along the Columbia River Shorel ine near 100-N Springs.

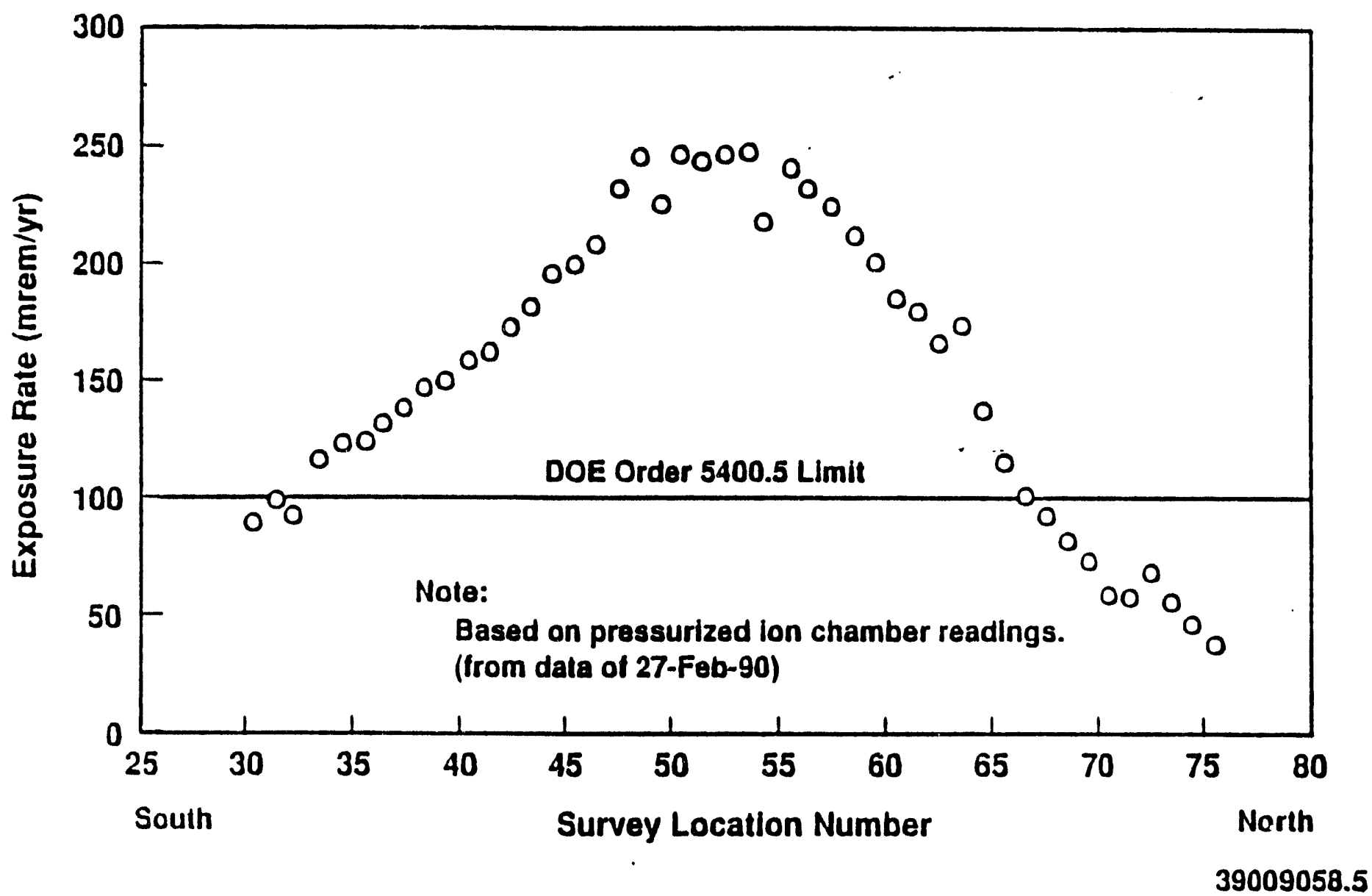


DOE/RL 91-59

This page intentionally left blank. 


\begin{tabular}{|c|c|c|c|}
\hline $\begin{array}{l}\text { HIDS } \\
\text { Designation } \\
\text { Number }\end{array}$ & Alias/Location & Waste Description & Unit or Release Description \\
\hline \multicolumn{4}{|c|}{ 1314-N Liquid Waste Loadout Facility Grouping } \\
\hline$\cdots$ & $1314-N$ & $\begin{array}{l}\text { Receives spent radioactive internal } \\
\text { decontamination solution from the } 116-N-2 \\
\text { radioactive chemical waste storage facility } \\
\text { and the } 107-N \text { basin recirculation facility. } \\
\text { Effluent transferred by railway tank cars to } \\
200 \text { Area tank farms for processing and } \\
\text { disposal. }\end{array}$ & Liquid waste loadout station. \\
\hline UN-100-N-13 & $1314-N$ Loading Station & $\begin{array}{l}100 \mathrm{gal} \text { of spent reactor decontamination } \\
\text { solution containing } 11 \text { mci from } 116-N-2 \\
(1310-W) \text { discharged to ground via dry well. }\end{array}$ & 1314-N dry well overflow. \\
\hline UN-100-N-26 & $\begin{array}{l}\text { Valve Pit at } 1314-N \\
\text { Loading Station }\end{array}$ & $\begin{array}{l}1,000 \text { gal of spent reactor decontamination } \\
\text { solution containing phosphoric acid and } \\
\text { diethyl thiourea backflowed while being } \\
\text { pumped into a tank car. }\end{array}$ & Rail car overflow. \\
\hline \multicolumn{4}{|c|}{ 119-N Air Sampl ing and Monitoring Building Grouping } \\
\hline UN-100-N-9 & $\begin{array}{l}119-N \text { Cool ing Mater Drain } \\
\text { Line }\end{array}$ & $\begin{array}{l}2,200 \text { gal of contaminated water with a total } \\
\text { activity of } 4 \mathrm{mci} \text { released to soil from } \\
\text { punctured drain line. }\end{array}$ & Punctured drain line. \\
\hline UN-100-N-14 & $\begin{array}{l}\text { Drain System at } \\
\text { 119-N Building }\end{array}$ & $\begin{array}{l}70 \text { gal of effluent water containing } 0.8 \mathrm{mci} \\
\text { of beta/gamma activity was released to the } \\
\text { ground. }\end{array}$ & Drain backflow release. \\
\hline \multicolumn{4}{|c|}{ 166-N Fuel Unloading and Storage Area Grouping } \\
\hline$\cdots$ & Fuel oil Unloading & No. 6 fuel oil. & $\begin{array}{l}\text { Tank car unloading area and containment } \\
\text { trench for piping }\end{array}$ \\
\hline$\cdots$ & 166-N Tank Farm & No. 6 fuel oil and Mo. 2 diesel oil. & $\begin{array}{l}\text { One aboveground tank for No. } 6 \text { fuel oil } \\
\text { and four aboveground tanks for No. } 2 \\
\text { diesel oil. }\end{array}$ \\
\hline$U N-100-N-17$ & $\begin{array}{l}\text { 166-N Diesel Oil Supply } \\
\text { Line }\end{array}$ & $\begin{array}{l}80,000 \text { gal of diesel oil was released to } \\
\text { the ground from the external corrosion of } \\
\text { supply line. }\end{array}$ & $\begin{array}{l}\text { oil supply line leak. Trench dug along } \\
\text { river used to intercept and burn off oil } \\
\text { in the groundwater. }\end{array}$ \\
\hline$U N-100-N-20$ & $\begin{array}{l}\text { 2-in Diesel oil Return } \\
\text { Line }\end{array}$ & $\begin{array}{l}200 \text { gal of diesel oil was released to the } \\
\text { line ground as a result of a leak in a } \\
\text { corroded return line. }\end{array}$ & $\begin{array}{l}\text { oil line leak in bermed storage tank } \\
\text { area. }\end{array}$ \\
\hline$U N-100-N-24$ & 166-N oil Supply Line & $\begin{array}{l}\text { Unknown amount of No. } 6 \text { fuel oil was } \\
\text { released to the ground as a result of a leak } \\
\text { in a heat transfer line. }\end{array}$ & $\begin{array}{l}\text { Oil line leak in bermed storage tank } \\
\text { area. }\end{array}$ \\
\hline
\end{tabular}




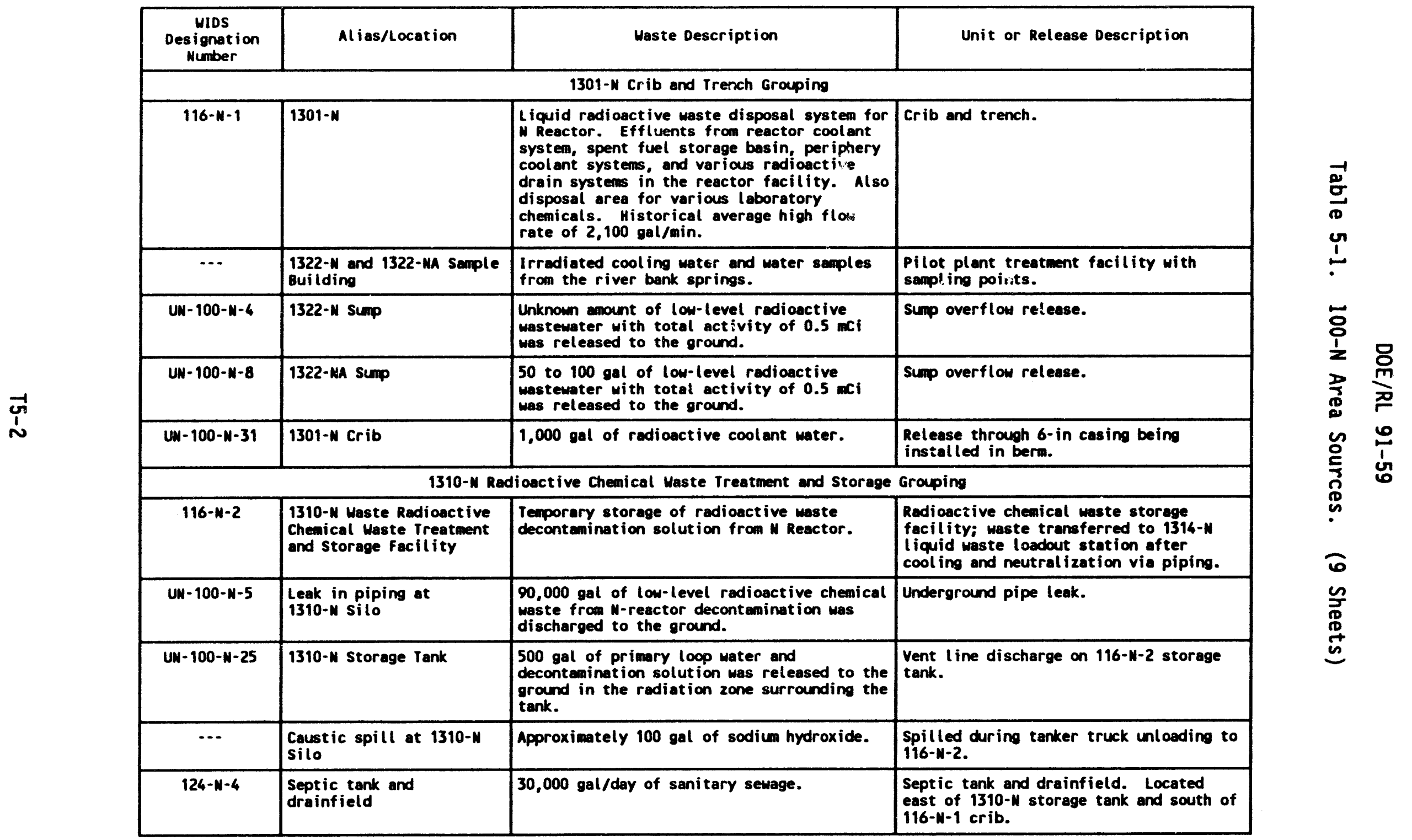




\begin{tabular}{|c|c|c|c|}
\hline $\begin{array}{l}\text { NIDS } \\
\text { Designation } \\
\text { Number }\end{array}$ & Alias/Location & Maste Description & Unit or Release Description \\
\hline \multicolumn{4}{|c|}{ 1325-N Crib and Trench Grouping } \\
\hline $116-N-3$ & $1325-N$ & $\begin{array}{l}\text { Liquid radioactive waste disposal system for } \\
\text { N Reactor after } 116-N-1 \text { ceased operations. } \\
\text { Effluent from reactor coolant system, spent } \\
\text { fuel storage basin, periphery colant } \\
\text { systems, and various radioactive drain } \\
\text { systems in the reactor facility. Also } \\
\text { dispersal area for various laboratory } \\
\text { chemicals. Historical average flow race of } \\
1,300 \text { gal/min. }\end{array}$ & Crib and trench. \\
\hline \multicolumn{4}{|c|}{ Burning Pit Grouping } \\
\hline $128-N-1$ & $\begin{array}{l}\text { Burning Pit South of } \\
1325-N \text { Crib }\end{array}$ & $\begin{array}{l}\text { Office and lunchroom trast, tumbleweeds were } \\
\text { burned using various fuel oils as starters. }\end{array}$ & Burning pit. \\
\hline \multicolumn{4}{|c|}{ 181-M River Pumphouse Grouping } \\
\hline$\cdots$ & 102-in Outfall Line & $\begin{array}{l}\text { Secondary cool ing water containing variety } \\
\text { of radionuclides. }\end{array}$ & $\begin{array}{l}\text { Discharge pipe extending approximately } \\
400 \text { ft into the Colubii River. MPDES } \\
\text { Discharge Point No. 009. }\end{array}$ \\
\hline$\cdots$ & $\begin{array}{l}\text { 102-in outfall pH } \\
\text { Violation }\end{array}$ & Acidic and caustic regeneration wastewater. & $\begin{array}{l}\text { Testing of simultaneous acid and caustic } \\
\text { wastewater releases resulted in MPDES ph } \\
\text { violations. }\end{array}$ \\
\hline$\cdots$ & $\begin{array}{l}\text { Aboveground Maste oil } \\
\text { Tank ot } 181-N \\
\end{array}$ & Maste oil. & Manages waste oil from pump oil changes. \\
\hline$\cdots$ & $\begin{array}{l}\text { 181-N Inlet Screen } \\
\text { Backwash Mater Outfall }\end{array}$ & $\begin{array}{l}\text { Backwash water and sol ids from raw water } \\
\text { intake to } 100-N \text {. Analyzed for total flow } \\
\text { and suspended sol ids per MPDES permit. }\end{array}$ & MPDES D ischarge Point Number 007. \\
\hline \multicolumn{4}{|c|}{ 1304-N Emergency Dump Tank Grouping } \\
\hline$\cdots$ & $\begin{array}{l}1304-N \text { Emergency Dump } \\
\text { Tank (EDT) }\end{array}$ & $\begin{array}{l}\text { Cool ing water containing small amounts of } \\
\text { radionucl ides. }\end{array}$ & $\begin{array}{l}1.3 \text { Mgal storage tank intended to } \\
\text { receive emergency dumpings of thermal ly } \\
\text { hot primary reactor coolant water. }\end{array}$ \\
\hline$U N-100-N-1$ & EDT Overflow Line & $\begin{array}{l}30,000 \text { gal of cooling water containing } \\
0.2 \mathrm{Ci} \text {. }\end{array}$ & $\begin{array}{l}\text { Tenporary flow stoppage device } \\
\text { mal functioned dur ing maintenance. } \\
\text { Contaminated soil removed. } \\
\end{array}$ \\
\hline UN-100-N-29 & EDT Bypass Line & $\begin{array}{l}100 \mathrm{gal} \text { of primary coolant water containing } \\
100 \mathrm{mci} \text { of radioactive materials, mostly } \\
\text { and }{ }^{24} \mathrm{Ha} \text {. }\end{array}$ & $\begin{array}{l}\text { Faulty check valve caused the leak. } \\
\text { Contaminated soil removed. }\end{array}$ \\
\hline
\end{tabular}




\begin{tabular}{|c|c|c|c|}
\hline $\begin{array}{l}\text { MIDS } \\
\text { Designation } \\
\text { Number }\end{array}$ & Alias/Location & Waste Description & Unit or Release Description \\
\hline UN-100-N-30 & EDT Overflow & $\begin{array}{l}\text { 2,500 gal of irradiated water. Maximum } \\
\text { reading of } 500 \text { counts per minute. }\end{array}$ & $\begin{array}{l}\text { Overflowed through vacum breaker vent } \\
\text { at top of EDT during fill ing, stabil ized } \\
\text { the area. No documented removal. }\end{array}$ \\
\hline UN-100-N-32 & EDT Bypass Line & $\begin{array}{l}\text { 500-1,000 gal of primary coolant water. } \\
\text { Sample of contaminated soil vielded 20,000 } \\
\text { counts per minute. }\end{array}$ & $\begin{array}{l}\text { Faulty check valve (similar to } \\
\text { uN-100- } M-29) \text {. Contaminated soil } \\
\text { removed. }\end{array}$ \\
\hline$U N-100-N-2$ & $\begin{array}{l}\text { FLV-858 valve body relief } \\
\text { line }\end{array}$ & $\begin{array}{l}25,000-30.000 \text { gal of } \\
\text { redioactively-contaminated water. Less than } \\
1 \text { ci of radioactivity. }\end{array}$ & $\begin{array}{l}\text { Crack in rel ief line to } 32-\text { in low } \\
\text { pressure flash line between EDT and } \\
109-N \text {. Contaminated soil removed. }\end{array}$ \\
\hline$U N-100-N-7$ & $\begin{array}{l}10-\text { in Drain line between } \\
105-M \text { and EDT }\end{array}$ & $\begin{array}{l}504,000 \mathrm{gal} \text { of irradiated water with unknown } \\
\text { levels of radioactivity. }\end{array}$ & $\begin{array}{l}\text { Leak in drain line. Conteminated soil } \\
\text { removed. }\end{array}$ \\
\hline$\cdots$ & $\begin{array}{l}\text { 1300-N Emergency Dump } \\
\text { Basin }\end{array}$ & $\begin{array}{l}\text { Cool ing and blowdown water and sludge } \\
\text { containing radionucl ides. }\end{array}$ & $\begin{array}{l}\text { One million gal storage basin used for } \\
\text { emergency dmping of thermally hot } \\
\text { primary reactor coolant water and for } \\
\text { blowdoun water form 109- } N \text { stean } \\
\text { generators. }\end{array}$ \\
\hline \multicolumn{4}{|c|}{ Spacer Storage silo Groping } \\
\hline $118-N-1$ & Spacer Storage silos & Irradiated fuel spacers. & $\begin{array}{l}\text { The silos contained irrodiated fuel } \\
\text { spacers thich came in contact with the } \\
\text { reactor fuel rods. }\end{array}$ \\
\hline$U N-100-N-3$ & Spacer Transfer Line & 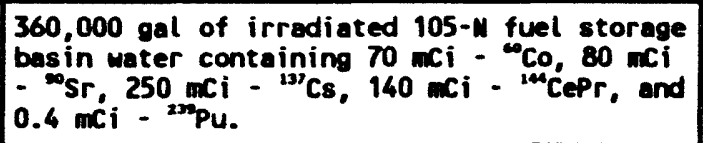 & $\begin{array}{l}\text { Crack in line between } 105-M \text { and silos. } \\
\text { Conteminated soil removed. }\end{array}$ \\
\hline$U N-100-N-12$ & Spacer Transfer Line & 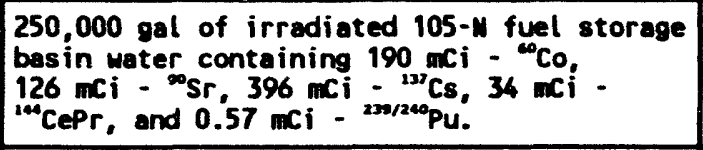 & $\begin{array}{l}\text { Similar to UN-100-N-3. Removal of soil } \\
\text { undocumented. }\end{array}$ \\
\hline $124-N-3$ & 107-M Septic System & Sani tary sewage. & $\begin{array}{l}\text { Sewage system serving } 2-3 \text { employees at } \\
107-\omega \text { plus construction persomel. }\end{array}$ \\
\hline$\cdots$ & Corridor 22 exit & $\begin{array}{l}\text { Several hundred litres of irradiated filter } \\
\text { water. }\end{array}$ & D ischarge to ground. Undocumented. \\
\hline$\ldots$ & $\begin{array}{l}\text { 105-N Lift Station } \\
\text { Underground Station Tank }\end{array}$ & No. 2 diesel oil. & $\begin{array}{l}\text { 5,000 gal steel tank which has been } \\
\text { removed. Wo soil contanination found } \\
\text { during tank removal. }\end{array}$ \\
\hline
\end{tabular}




\begin{tabular}{|c|c|c|c|}
\hline $\begin{array}{l}\text { HIDS } \\
\text { Designation } \\
\text { Number }\end{array}$ & Alias/Location & Maste Description & Unit or Release Description \\
\hline \multicolumn{4}{|c|}{ 182-N Underground Storage Tank Grouping } \\
\hline $\begin{array}{l}182-N-1-D T \\
182-N-2-D T \\
182-N-3-D T\end{array}$ & $\begin{array}{l}\text { 182-N Underground Storage } \\
\text { Tanks (3) }\end{array}$ & Mo. 2 diesel oil. & $\begin{array}{l}\text { Three } 10,000 \text { to } 19,000 \text { gal USTs } \\
\text { containing diesel oil for Emergency } \\
\text { Diesel pumps, 124-N-3, thich have been } \\
\text { removed. The sites are scheduled for } \\
\text { remediation under UST program. }\end{array}$ \\
\hline \multicolumn{4}{|c|}{ 105-N Spent Fuel Storage Basin Grouping } \\
\hline$\cdots$ & $\begin{array}{l}\text { 105-N Spent Fuel Storage } \\
\text { Basin }\end{array}$ & Irradiated cooling water. & $\begin{array}{l}\text { Containment basin for storage of spent } \\
\text { fuel rods and spacers. }\end{array}$ \\
\hline$U N-100-N-10$ & Zone I Gravity Drain Line & $\begin{array}{l}\text { Approximately } 100 \text { gal of irradiated water } \\
\text { containing } 1 \text { mci of radionuclides. }\end{array}$ & $\begin{array}{l}\text { Leaking temporary pump fitting spilled } \\
\text { to the ground. Contaminated soil was } \\
\text { removed. }\end{array}$ \\
\hline$U N-100-N-35$ & $\begin{array}{l}\text { Leak in overflow weir } \\
\text { drain line }\end{array}$ & $\begin{array}{l}\text { Unknown amounts of contaminated water from } \\
\text { the } 105-\mathrm{N} \text { fuel storage basin. }\end{array}$ & $\begin{array}{l}\text { Leaking clean out valve from overflow } \\
\text { weir. Contamination found in } \\
\text { groundwater monitoring wells by the fuel } \\
\text { basin. }\end{array}$ \\
\hline \multicolumn{4}{|c|}{ 182-N High Lift Pumphouse Grouping } \\
\hline $124-N-2$ & 182-N Septic Tank & Sanitary Sewage. & Serves persomel from 182-N building. \\
\hline$\cdots$ & 182-N Tank Farm Overflow & $\begin{array}{l}\text { Overflow water analyzed for temperature, pH, } \\
\text { total suspended sol ids, oil and grease, and } \\
\text { chlorine per MPDES permit. }\end{array}$ & $\begin{array}{l}\text { MPDES Discharge Point Number } 005 \text { via a } \\
\text { 36-in raw water return line. }\end{array}$ \\
\hline$\cdots$ & 182-M Drain system & $\begin{array}{l}\text { Primarily water analyzed for temperature, } \\
\text { pH, total suspended sol ids, oil and grease } \\
\text { per MPDES permit. }\end{array}$ & $\begin{array}{l}\text { MPDES Discharge Point Number } 006 \text { via a } \\
42 \text {-in raw water- return line. Periodic } \\
\text { release of low-level radionucl ides from } \\
\text { emergency core coot ing system pumps. }\end{array}$ \\
\hline$\cdots$ & Lube Oil Line Leak & 5 gal of turbine oil. & $\begin{array}{l}\text { Pinhole leak in lube oil line allowed } \\
\text { oil to enter secondary steam system. } \\
\text { Discharge to river with steam } \\
\text { condensate. }\end{array}$ \\
\hline \multicolumn{4}{|c|}{ Acid/Caustic Storage and Transport System Grouping } \\
\hline$\cdots$ & $\begin{array}{l}\text { 108-N Chemical Unloading } \\
\text { facility }\end{array}$ & $\begin{array}{l}93 \text { percent sulfuric acid and } 50 \text { percent } \\
\text { sodium hydroxide. }\end{array}$ & $\begin{array}{l}\text { Unloading area for trucks or railcars. } \\
\text { Has three aboveground sul furic acid } \\
\text { tanks and one aboveground sodium } \\
\text { hydroxide tank. }\end{array}$ \\
\hline $120-N-7$ & $\begin{array}{l}\text { Unloading Station French } \\
\text { Drain }\end{array}$ & $\begin{array}{l}93 \text { percent sulfuric acid and } 50 \text { percent } \\
\text { sodium hydroxide. }\end{array}$ & $\begin{array}{l}\text { French drain for receiving incidental } \\
\text { spills during railcar or tank truck } \\
\text { unloading. }\end{array}$ \\
\hline
\end{tabular}




\begin{tabular}{|c|c|c|c|}
\hline \multirow{16}{*}{ ज़ } & Alias/Location & Waste Description & Unit or Release Description \\
\hline & $\begin{array}{l}\text { Sulfuric Acid Tank French } \\
\text { Drains (5) }\end{array}$ & 93 percent sulfuric acid. & $\begin{array}{l}\text { French drains surrounding acid tanks for } \\
\text { containment of incidental spills. }\end{array}$ \\
\hline & 108-M Meutralization Pit & Waste sul furic acid. & $\begin{array}{l}\text { The unit was used to neutral ize waste } \\
\text { sulfuric from } 108-\mu \text { floor drains and } \\
\text { acid transfer tank drainage. }\end{array}$ \\
\hline & 108-M Unloading Facility & $\begin{array}{l}\text { Unknown amount of sulfuric acid and } \\
\text { rinsewater. }\end{array}$ & \begin{tabular}{|l} 
Transfer line leak during pumping of \\
liquid from $108-N$ to french drain. \\
\end{tabular} \\
\hline & 108-N Unloading Facility & Approximately 1,000 gal of sulfuric acid. & $\begin{array}{l}\text { Spilled to ground during transfer from } \\
\text { railcar to storage tank. }\end{array}$ \\
\hline & $\begin{array}{l}\text { 108-M Unloading facility } \\
\text { Spill }\end{array}$ & Approximately 10 gal of sodium hydroxide. & $\begin{array}{l}\text { Spilled during transfer from railcar to } \\
\text { storage tank. }\end{array}$ \\
\hline & $\begin{array}{l}\text { Acid/Caustic Iransfer } \\
\text { Trench and Meutralization } \\
\text { Unit }\end{array}$ & sulfuric acid and sodium hydroxide. & $\begin{array}{l}\text { Piping trench between } 108-M \text { and } 163-M \\
\text { and containment vaul ts. }\end{array}$ \\
\hline & $\begin{array}{l}\text { Acid/Caustic Iransfer } \\
\text { Trench and Meutralization } \\
\text { Unit }\end{array}$ & Approximately 3,400 gal of sul furic acid. & $\begin{array}{l}\text { Pipel ine rupture filled containment } \\
\text { vaul ts and spilled to ground. Acid was } \\
\text { neutral ized. }\end{array}$ \\
\hline & $\begin{array}{l}\text { Acid/Caustic Iransfer } \\
\text { Irench }\end{array}$ & Unknown amount of sul furic acid. & $\begin{array}{l}\text { Acid had corroded away exposed trench } \\
\text { area releasing to the soil. }\end{array}$ \\
\hline & $\begin{array}{l}\text { Acid/Caustic Iransfer } \\
\text { Irench }\end{array}$ & Unknown amount of sodium hydroxide. & Leak in piping was contained in trench. \\
\hline & $\begin{array}{l}\text { Acid/Caustic Iransfer } \\
\text { Trench }\end{array}$ & $\begin{array}{l}\text { Approximately } 200 \text { gal of sulfuric scid } \\
\text { spilled and approximately } 15 \text { to } 30 \text { gal } \\
\text { released to ground. }\end{array}$ & $\begin{array}{l}\text { Leak in piping escaped trench through a } \\
\text { dry well. Contaninated soil was } \\
\text { removed. }\end{array}$ \\
\hline & $\begin{array}{l}\text { 163-M Meutralization Pit } \\
\text { and French Drain }\end{array}$ & sul furic acid and sodium hydroxide. & $\begin{array}{l}\text { French drain and vault receiving } \\
\text { drainage from } 163-\Psi \text { acid and caustic day } \\
\text { tank area. }\end{array}$ \\
\hline & $\begin{array}{l}\text { 163-N Sul furic Acid Day } \\
\text { Tank Vent French Drain }\end{array}$ & Sulfuric acid. & $\begin{array}{l}\text { Tank overflows are vented to the french } \\
\text { drain. }\end{array}$ \\
\hline & \begin{tabular}{|l|} 
Regeneration Waste \\
Transport System \\
\end{tabular} & Acid and caustic regeneration wastes. & $\begin{array}{l}\text { Sump and pipeline del ivering wastes from } \\
163-N \text { to } 1324-N \text {. }\end{array}$ \\
\hline & $\begin{array}{l}\text { Regeneration Maste } \\
\text { Iransport System }\end{array}$ & $\begin{array}{l}\text { Approximately } 6,500 \text { gal of acidic } \\
\text { regeneration wastes. }\end{array}$ & $\begin{array}{l}\text { Pipel ine leak during transfer. Spill } \\
\text { was neutral ized and contaminated soil } \\
\text { was removed. }\end{array}$ \\
\hline & \begin{tabular}{|l} 
Regeneration Waste \\
Transport System
\end{tabular} & $\begin{array}{l}\text { Approximately } 1,000 \mathrm{gal} \text { of acidic } \\
\text { regeneration wastes. }\end{array}$ & $\begin{array}{l}\text { Pipel ine leak during transfer. Spill } \\
\text { was neutral ized. }\end{array}$ \\
\hline
\end{tabular}




\begin{tabular}{|c|c|c|c|}
\hline $\begin{array}{l}\text { WIDS } \\
\text { Designation } \\
\text { Number }\end{array}$ & Alias/Location & Maste Description & Unit or Release Description \\
\hline $124-N-1$ & 163-N Septic Tank & Sani tary sewage. & $\begin{array}{l}\text { Serving } 163-N \text {, 183-N, 1127- } N \text {, and } \\
1128-N \text { buildings. }\end{array}$ \\
\hline \multicolumn{4}{|c|}{ Mixed Waste Storage Area Grouping } \\
\hline $116-N-8$ & Mixed Waste Storage Pad & $\begin{array}{l}\text { Radioactively contaminated oil and } \\
\text { miscell aneous process chemicals. }\end{array}$ & $\begin{array}{l}\text { Paved and curbed concrete pad for mixed } \\
\text { waste storage in drums and miscellaneous } \\
\text { containers. }\end{array}$ \\
\hline \multicolumn{4}{|c|}{ 184-M Plant Service Power Mouse } \\
\hline$-\cdots$ & $\begin{array}{l}\text { 184-N Plant Service Power } \\
\text { House }\end{array}$ & $\begin{array}{l}\text { Hydrocarbons, particulates, sulfur dioxide, } \\
\text { sul fur trioxide, carbon monoxide, nitrogen } \\
\text { oxide, and aldehydes. }\end{array}$ & $\begin{array}{l}\text { Rout ine and systematic releases form } \\
\text { boiler stacks. }\end{array}$ \\
\hline$\cdots$ & 184-N Day Tanks & Mo. 6 (Bunker C) fuel oil and diesel oil. & $\begin{array}{l}\text { Two } 35,000 \text { gal fuel oil tanks and one } \\
8,000 \text { gal diesel ofl tank surrounded by } \\
\text { a containment wall. }\end{array}$ \\
\hline UN-100-N-19 & $\begin{array}{l}\text { Fuel Oil Day Tank at } \\
184-N\end{array}$ & Approximately 2,000 gal of fuel oil. & $\begin{array}{l}\text { Tank overflowed during fill ing. Oil } \\
\text { contained with in walls and renoved. }\end{array}$ \\
\hline$U N-100-N-21$ & $\begin{array}{l}\text { Diesel Dil Day Tank at } \\
184-N\end{array}$ & Approximately 800 gal of diesel oil. & $\begin{array}{l}\text { Tank overflowed during filling. Oil } \\
\text { removed from containment area. }\end{array}$ \\
\hline$\cdots$ & $\begin{array}{l}\text { Diesel Oil Day Tank at } \\
184-W\end{array}$ & Unknown amount of diesel oil. & $\begin{array}{l}\text { Tank overflowed during filling. Oil was } \\
\text { renoved. }\end{array}$ \\
\hline$\cdots$ & 166-N - 184-N Piping & No. 6 fuel oil and Mo. 2 diesel oil. & Underground fuel supply piping. \\
\hline$U N-100-N-18$ & $\begin{array}{l}\text { Diesel oil supply line } \\
\text { between } 166-N \text { and } 184-N\end{array}$ & Approximately $200 \mathrm{gal}$ of diesel oil. & Line leak caused by external corrosion. \\
\hline$U N-100-N-22$ & $\begin{array}{l}\text { Diesel oil supply line } \\
\text { near } 184-N\end{array}$ & Approximately $1,000 \mathrm{gal}$ of diesel oil. & $\begin{array}{l}\text { Line leak caused by external corrosion. } \\
\text { Contaminated soil removed. oil detected } \\
\text { in groundwater. }\end{array}$ \\
\hline UN-100-N-23 & $\begin{array}{l}\text { Diesel oil supply line } \\
\text { near } 184-M\end{array}$ & Approximately 200 gal of diesel oil. & $\begin{array}{l}\text { Line leak caused by external corrosion. } \\
\text { oil detected in groundwater. }\end{array}$ \\
\hline$\cdots$ & $\begin{array}{l}\text { Fuel oil pipe fitting at } \\
184-N \text { Annex }\end{array}$ & Unknown amount of fuel oil. & $\begin{array}{l}\text { Oil leaked from loose pipe fitting } \\
\text { during transfer to boiler. oil } \\
\text { contained and removed. }\end{array}$ \\
\hline$--\cdot$ & $\begin{array}{l}\text { Diesel oil supply line } \\
\text { between } 166-N \text { and } 184-N\end{array}$ & A minimm of 300 gal of diesel oil. & $\begin{array}{l}\text { Line leak in three places. } 46 \text { drums of } \\
\text { contaminated soil removed. }\end{array}$ \\
\hline
\end{tabular}




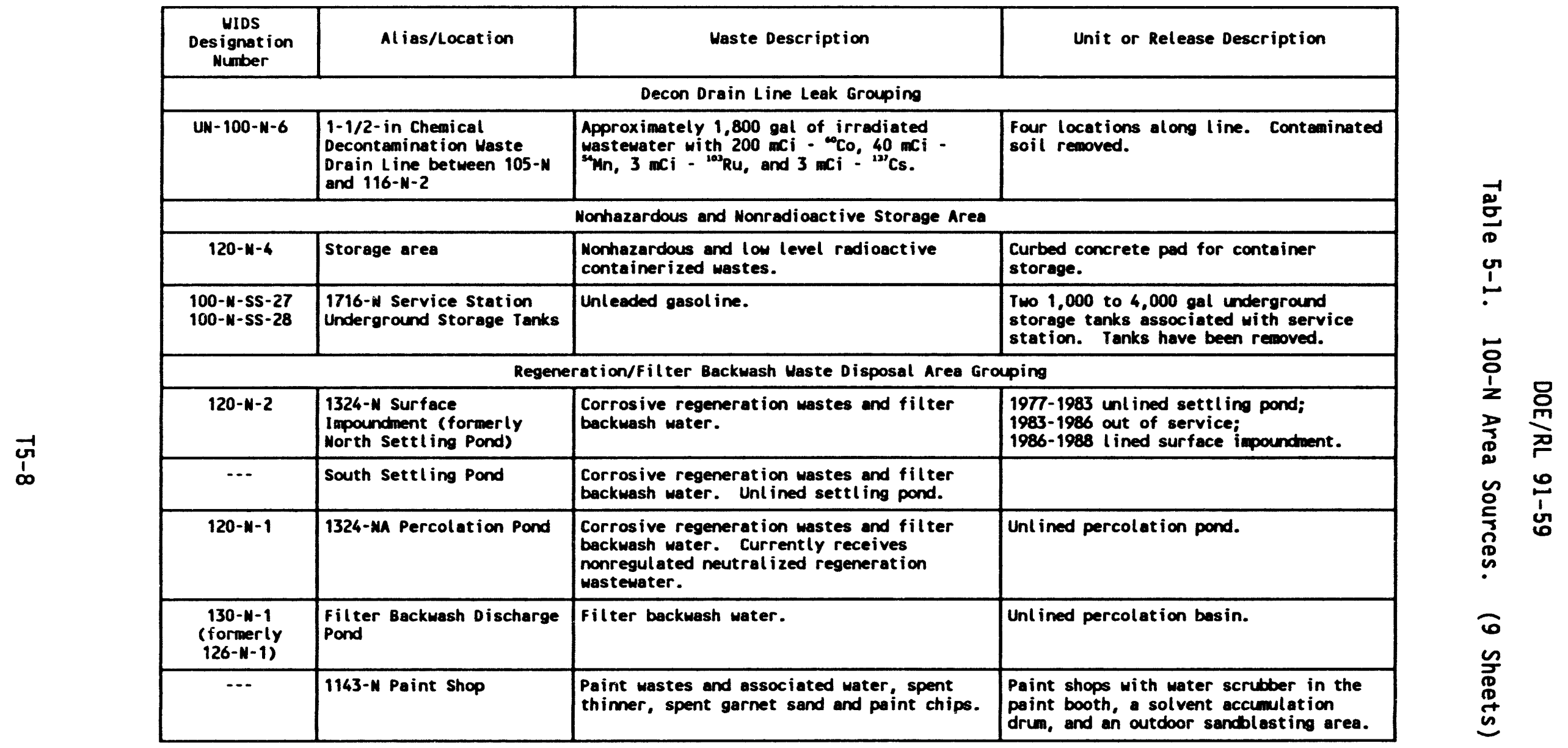




\begin{tabular}{|c|c|c|c|}
\hline $\begin{array}{l}\text { WIDS } \\
\text { Designation } \\
\text { Number }\end{array}$ & Al ias/Location & Waste Description & Unit or Release Description \\
\hline \multicolumn{4}{|c|}{ Office Septic Tank Area Grouping } \\
\hline $124-N-5$ & $\begin{array}{l}\text { 1117-N Septic Tank } \\
\text { (Sewer System V) }\end{array}$ & Sani tary sewage. & Septic tank and drainfield. \\
\hline $124-N-6$ & $\begin{array}{l}\text { 1113-N Septic Tank } \\
\text { (Sewer System VI) }\end{array}$ & Sani tary sewage. & Septic tank and drainfield. \\
\hline $124-N-7$ & $\begin{array}{l}\text { 1115-N Septic Tank } \\
\text { (Sewer System VII) }\end{array}$ & sanitary sewage. & Septic tank and drainfield. \\
\hline $124-N-8$ & $\begin{array}{l}\text { 1134-N Septic Tank } \\
\text { (Sewer System VIII) }\end{array}$ & Sanitary sewage. & Septic tank and drainfield. \\
\hline \multicolumn{4}{|c|}{ N-17 Paint Shop Area Grouping } \\
\hline$\cdots$ & N-17 Paint Shop & Waste paint, solvents, and oils. & $\begin{array}{l}\text { Two waste accumulation drums (one for } \\
\text { waste paint, the other for waste oil); } \\
\text { sandblasting area. }\end{array}$ \\
\hline \multicolumn{4}{|c|}{ 1120-W Septic Tank Grouping } \\
\hline $124-N-9$ & 1120-N Septic Tank & Sanitary sewage. & Septic tank and drainfield. \\
\hline \multicolumn{4}{|c|}{ 100-N Sewer System Grouping } \\
\hline $124-N-10$ & 100-N Sewer System & Sani tary sewage. & $\begin{array}{l}\text { Central sewer system with three } \\
\text { lagoons, sewer trunk line and other } \\
\text { pipel ines, and lift stations. }\end{array}$ \\
\hline$U n-100-N-11$ & $\begin{array}{l}\text { Corner of Route } 4 \text { north } \\
\text { and access road }\end{array}$ & Radioactive soil and asphalt. & $\begin{array}{l}\text { Valve bornet fell from truck onto rood } \\
\text { and rolled into edjacent field. valve } \\
\text { bonnet, asphal t, and soil removed. }\end{array}$ \\
\hline
\end{tabular}

Source: DOE-RL 1990. 
Table 5-2. Selected Radionuclide Inventory for 116-N-1 Crib and Trench for the 1301-N Liquid Waste Disposal Facility (WHC 1987b).

\begin{tabular}{|c|c|c|}
\hline Radionuclide & $\begin{array}{c}\text { Half-Life } \\
\left(Y_{r}\right)\end{array}$ & $\begin{array}{c}\text { Inventory } \\
(\mathrm{C} i)\end{array}$ \\
\hline${ }^{60} \mathrm{Co}$ & 5.3 & 3,800 \\
\hline${ }^{90} \mathrm{Sr}$ & 29 & 1,800 \\
\hline${ }^{106} \mathrm{Ru}$ & 1 & 120 \\
\hline${ }^{134} \mathrm{Cs}$ & 2.1 & 51 \\
\hline${ }^{137 \mathrm{c}}$ & 30 & 2,300 \\
\hline${ }^{239} \mathrm{Pu}$ & 24,000 & 18 \\
\hline
\end{tabular}

Table 5-3. Dangerous Waste Inventory for the 116-N-1 Crib and Trench for the 1301-N Liquid Waste Disposal Facility.

\begin{tabular}{|l|c|}
\hline \multicolumn{1}{|c|}{ Compound } & $\begin{array}{c}\text { Amount, } \\
1 \mathrm{~b} / \mathrm{yr}\end{array}$ \\
\hline Hydrazine Test Solution & 6,100 \\
\hline Ammonia Test Solution & 6,100 \\
\hline Chloride Test Solution & 7,800 \\
\hline Fluoride Test Solution & 3,900 \\
\hline Lead-Acetate Battery Fluid & $630^{\star}$ \\
\hline Nickel-Cadmium Battery Fluid & $270^{\star}$ \\
\hline Hydrazine (Injection System) & 350 \\
\hline
\end{tabular}

* No actual spills reported, but possible because of common floor drains in the storage rooms.

Note: Routine monitoring of the influent to 116-N-1 did not reveal detectable levels of these chemicals (WHC 1987b). 
Table 5-4. Selected Radionuclide Inventory from October 1983 to January 1,1988 for $116-\mathrm{N}-3$ Crib and Trench for the 1325-N Liquid Waste Disposal Facility (WHC 1987a).

\begin{tabular}{|c|c|c|}
\hline Radionuclide & $\begin{array}{c}\text { Half-Life } \\
(Y r)\end{array}$ & $\begin{array}{c}\text { Inventory } \\
(C i)\end{array}$ \\
\hline${ }^{60} \mathrm{Co}$ & 5.3 & 1,300 \\
\hline${ }^{90} \mathrm{Sr}$ & 29 & 200 \\
\hline${ }^{106} \mathrm{Ru}$ & 1 & 66 \\
\hline${ }^{134} \mathrm{Cs}$ & 2.1 & 14 \\
\hline${ }^{137} \mathrm{Cs}$ & 30 & 350 \\
\hline${ }^{239} \mathrm{Pu}$ & 24,000 & 2.6 \\
\hline
\end{tabular}

Table 5-5. Dangerous Waste Inventory for 116-N-3 Crib and Trench for the 1325-N Liquid Waste Disposal Facility.

\begin{tabular}{|l|c|}
\hline \multicolumn{1}{|c|}{ Compound } & Amount, $1 \mathrm{~b} / \mathrm{yr}$ \\
\hline Hydrazine Test Solution & 6,100 \\
\hline Ammonia Test Solution & 6,100 \\
\hline Chloride Test Solution & 7,800 \\
\hline Fluoride Test Solution & 3,900 \\
\hline Lead-Acetate Battery Fluid & $120^{\star}$ \\
\hline Nickel-Cadmium Battery Fluid & $80^{*}$ \\
\hline Hydrazine (Injection System) & 10 \\
\hline
\end{tabular}

- No actual spills reported, but possible because of comnon floor drains in the storage rooms.

Note: Rout ine monitoring of the influent to 116-N-3 did not reveal detectable Levels of these chemicals (WHC 1987a). 
Table 5-6. Average Annual Concentrations of Radionuclides ( $p C i / L$ ) Detected in Water Samples from the 1300-N EDB for 1978 through 1985.

\begin{tabular}{|c|c|c|c|c|c|c|c|c|}
\hline Radionuclide & 1978 & 1979 & 1980 & 1981 & 1982 & 1983 & 1984 & 1985 \\
\hline${ }^{40} K$ & $4.2 \mathrm{El}$ & --- & --- & --- & $--\infty$ & --- & --- & --- \\
\hline${ }^{54} \mathrm{Mn}$ & $5.1 \mathrm{El}$ & $1.5 \mathrm{E} 4$ & 4.1 E3 & $1.0 \mathrm{E3}$ & $2.7 \mathrm{E3}$ & 7.1 E3 & $4.1 E 2$ & --- \\
\hline${ }^{59} \mathrm{Fe}$ & -- & 7.4 E3 & $1.0 \mathrm{E3}$ & --- & --- & --- & -- & --- \\
\hline${ }^{58} \mathrm{Co}$ & --- & $1.1 \mathrm{E3}$ & $4.8 E 2$ & --- & --- & --- & -- & --- \\
\hline${ }^{60} \mathrm{Co}$ & $1.1 E 3$ & $4.8 \mathrm{E4}$ & $2.0 \mathrm{E} 4$ & $1.3 \mathrm{E4}$ & $7.5 \mathrm{E} 4$ & 3.4 E5 & $3.6 \mathrm{E} 4$ & $3.1 \mathrm{E2}$ \\
\hline${ }^{95} \mathrm{ZrNb}$ & -- & $1.6 \mathrm{E3}$ & $2.4 \mathrm{E} 3$ & --- & --- & --- & $9.9 \mathrm{El}$ & --- \\
\hline${ }^{103} \mathrm{Ru}$ & -- & $2.0 \mathrm{E2}$ & $6.6 \mathrm{E2}$ & --- & -- & $3.5 \mathrm{E} 2$ & --- & -- \\
\hline${ }^{124} \mathrm{Sb}$ & -- & $3.4 \mathrm{E2}$ & $-\cdots$ & --- & -- & --- & --- & --- \\
\hline${ }^{131} I$ & $2.1 \mathrm{E2}$ & $3.8 \mathrm{E3}$ & $3.7 \mathrm{E2}$ & $5.5 \mathrm{E2}$ & -- & --- & $2.9 \mathrm{E2}$ & --- \\
\hline${ }^{137} \mathrm{Cs}$ & $1.6 \mathrm{E2}$ & $2.0 \mathrm{E2}$ & $2.4 \mathrm{E2}$ & $4.2 \mathrm{E2}$ & $1.3 \mathrm{E3}$ & $8.8 \mathrm{E3}$ & $1.0 \mathrm{E3}$ & $9.0 \mathrm{El}$ \\
\hline${ }^{140} \mathrm{BaLa}$ & $5.9 \mathrm{E2}$ & $4.7 \mathrm{E3}$ & $1.3 \mathrm{E3}$ & $5.6 \mathrm{E2}$ & $\ldots$ & -- & $3.3 \mathrm{E2}$ & -- \\
\hline Total & $2.2 \mathrm{E3}$ & $8.2 E 4$ & $3.1 \mathrm{E} 4$ & $1.6 \mathrm{E4}$ & $7.9 \mathrm{E4}$ & $3.5 \mathrm{E5}$ & $3.8 \mathrm{E4}$ & $4.0 \mathrm{E2}$ \\
\hline
\end{tabular}

Source: DOE-RL 1990. 
Table 5-7. 163-N Demineralization Plant Regeneration Effluent Waste Analysis Cation Regeneration Cycle. (2 Sheets)

\begin{tabular}{|l|c|c|c|c|}
\hline \multirow{2}{*}{ Parameters (MDL) } & \multicolumn{3}{|c|}{ Sample } & \multirow{2}{*}{ Average } \\
\cline { 2 - 4 } & 1 & 2 & 3 & \\
\hline pH (standard units) & 0.894 & 0.936 & 0.922 & 0.917 \\
\hline Conductivity ( $\mu \mathrm{mhos})$ & 37,000 & 40,100 & 35,000 & 37,367 \\
\hline Mercury $(0.0001 \mathrm{p} / \mathrm{m})$ & LD & LD & LD & LD \\
\hline Ethylene glycol (10 p/m) & LD & LD & LD & LD \\
\hline $\begin{array}{l}\text { Enhanced thiourea } \\
\text { (0.2 p/m) }\end{array}$ & LD & LD & LD & LD \\
\hline TOC (1 p/m) & 0.0013 & 0.0019 & 0.0018 & 0.024 \\
\hline Cyanide $(0.01 \mathrm{p} / \mathrm{m})$ & LD & LD & LD & LD \\
\hline Barium $(0.006 \mathrm{p} / \mathrm{m})$ & 0.030 & 0.023 & 0.020 & 0.024 \\
\hline Cadmium $(0.002 \mathrm{p} / \mathrm{m})$ & 0.003 & 0.002 & 0.003 & 0.003 \\
\hline Chromium $(0.01 \mathrm{p} / \mathrm{m})$ & LD & LD & LD & LD \\
\hline Lead $(0.03 \mathrm{p} / \mathrm{m})$ & LD & LD & LD & LD \\
\hline Silver $(0.01 \mathrm{p} / \mathrm{m})$ & LD & LD & LD & LD \\
\hline Sodium $(0.1 \mathrm{p} / \mathrm{m})$ & 12.2 & 16.5 & 9.6 & 12.8 \\
\hline Nicke1 $(0.01 \mathrm{p} / \mathrm{m})$ & LD & LD & LD & LD \\
\hline Copper $(0.01 \mathrm{p} / \mathrm{m})$ & LD & LD & LD & LD \\
\hline Vanadium $(0.005 \mathrm{p} / \mathrm{m})$ & 0.025 & 0.027 & 0.020 & 0.024 \\
\hline Antimony $(0-1 \mathrm{p} / \mathrm{m})$ & LD & LD & LD & LD \\
\hline Aluminum $(0.15 \mathrm{p} / \mathrm{m})$ & 0.725 & 0.842 & 0.655 & 0.741 \\
\hline Manganese $(0.005 \mathrm{p} / \mathrm{m})$ & 0.027 & 0.035 & 0.027 & 0.030 \\
\hline Potassium $(0.1 \mathrm{p} / \mathrm{m})$ & 12.2 & 15.5 & 14.8 & 14.2 \\
\hline Iron $(0.05 \mathrm{p} / \mathrm{m})$ & 1.1 & 1.2 & 1.0 & 1.1 \\
\hline Bery11ium $(0.005 \mathrm{p} / \mathrm{m})$ & LD & LD & LD & LD \\
\hline Osmium $(0.3 \mathrm{p} / \mathrm{m})$ & LD & LD & LD & LD \\
\hline Strontium $(0.3 \mathrm{p} / \mathrm{m})$ & 1.3 & 1.4 & 1.2 & 1.3 \\
\hline Zinc $(0.005 \mathrm{p} / \mathrm{m})$ & 0.016 & 0.024 & 0.067 & 0.036 \\
\hline Calcium $(0.05 \mathrm{p} / \mathrm{m})$ & 282.6 & 347.4 & 324.92 & 318.3 \\
\hline Nitrate $(0.5 \mathrm{p} / \mathrm{m})$ & 1.0 & 0.5 & 0.8 & 0.8 \\
\hline Sulphate & 2,310 & 4,271 & 2,952 & 3,201 \\
\hline
\end{tabular}


Table 5-7. 163-N Demineralization Plant Regeneration Effluent Waste Analys is Cation Regeneration Cycle. (2 Sheets)

\begin{tabular}{|l|c|c|c|c|}
\hline \multirow{2}{*}{ Parameters (MDL) } & \multicolumn{3}{|c|}{ Sample } & \multirow{2}{*}{ Average } \\
\cline { 2 - 4 } & 1 & 2 & 3 & \\
\hline Fluoride $(0.5 \mathrm{p} / \mathrm{m})$ & LD & LD & LD & LD \\
\hline Chloride $(0.5 \mathrm{p} / \mathrm{m})$ & 2.0 & 1.8 & 1.9 & 1.9 \\
\hline Phosphate (1 p/m) & LD & LD & LD & LD \\
\hline Phosphorus & LD & LD & LD & LD \\
\hline Pesticides (0.005 p/m) & LD & LD & LD & LD \\
\hline $\begin{array}{l}\text { Chlorinated Pesticides } \\
(0.001 \mathrm{p} / \mathrm{m})\end{array}$ & LD & LD & LD & LD \\
\hline Enhanced ABN List & LD & LD & LD & LD \\
\hline Citrus Red (1 p/m) & LD & LD & LD & LD \\
\hline Arsenic (0.005 p/m) & LD & LD & LD & LD \\
\hline Ammonium Ion (0.05 p/m) & LD & LD & LD & LD \\
\hline Coliform (3 MPN) & LD & LD & LD & LD \\
\hline Selenium (0.005 p/m) & LD & LD & LD & LD \\
\hline Thallium (0.01 p/m) & LD & LD & LD & LD \\
\hline Enhanced VOA (10 p/m) & 26 & LD & 26 & 27 \\
\hline
\end{tabular}

$L D=$ less than detectable

MDL = minimum detection limit

MPN = most probable number

Data obtained from samples taken August 1985.

Source: WHC 1987C. 
Table 5-8. 163-N Demineralization Plant Regeneration Waste Analysis Anion Regeneration Cycle. (2 Sheets)

\begin{tabular}{|c|c|c|c|c|}
\hline \multirow{2}{*}{ Parameters (MDL) } & \multicolumn{3}{|c|}{ Sample } & \multirow{2}{*}{ Average } \\
\hline & 1 & 2 & 3 & \\
\hline pH (standard units) & 13.72 & 13.74 & 13.77 & 13.74 \\
\hline Conductivity ( $\mu$ mhos) & 62,000 & 60,000 & 70,000 & 64,000 \\
\hline Mercury $(0.0001 \mathrm{p} / \mathrm{m})$ & 0.00018 & 0.00013 & 0.00019 & 0.00017 \\
\hline Ethylene glycol $(10 \mathrm{p} / \mathrm{m})$ & LD & LD & LD & LD \\
\hline $\begin{array}{l}\text { Enhanced thiourea } \\
(0.2 \mathrm{p} / \mathrm{m})\end{array}$ & LD & LD & LD & LD \\
\hline TOC $(1 \mathrm{p} / \mathrm{m})$ & 462 & 499 & 456 & 472 \\
\hline Cyanide $(0.01 \mathrm{p} / \mathrm{m})$ & 0.010 & 0.015 & LD & 0.013 \\
\hline Barium $(0.006 \mathrm{p} / \mathrm{m})$ & LD & LD & LD & LD \\
\hline Cadmium $(0.002 \mathrm{p} / \mathrm{m})$ & LD & LD & LD & LD \\
\hline Chromium $(0.01 \mathrm{p} / \mathrm{m})$ & LD & LD & LD & LD \\
\hline Lead $(0.03 \mathrm{p} / \mathrm{m})$ & LD & LD & LD & LD \\
\hline Silver $(0.01 \mathrm{p} / \mathrm{m})$ & LD & LD & LD & LD \\
\hline Sodium $(0.1 \mathrm{p} / \mathrm{m})$ & 26,910 & 28,200 & 26,330 & 27,150 \\
\hline Nickel $(0.01 \mathrm{p} / \mathrm{m})$ & LD & LD & LD & LD \\
\hline Copper $(0.01 \mathrm{p} / \mathrm{m})$ & LD & LD & LD & LD \\
\hline Vanadium $(0.005 \mathrm{p} / \mathrm{m})$ & LD & LD & LD & LD \\
\hline Antimony $(0.1 \mathrm{p} / \mathrm{m})$ & LD & LD & LD & LD \\
\hline Aluminum $(0.15 \mathrm{p} / \mathrm{m})$ & LD & LD & LD & LD \\
\hline Manganese $(0.005 \mathrm{p} / \mathrm{m})$ & LD & LD & LD & LD \\
\hline Magnesium $(5 \mathrm{p} / \mathrm{m})$ & LD & LD & LD & LD \\
\hline Potassium $(0.1 \mathrm{p} / \mathrm{m})$ & 26.5 & 27.2 & 26.3 & 26.7 \\
\hline Iron $(0.05 \mathrm{p} / \mathrm{m})$ & LD & LD & LD & LD \\
\hline Beryllium $(0.005 \mathrm{p} / \mathrm{m})$ & LD & LD & LD & LD \\
\hline Osmium $(0.3 \mathrm{p} / \mathrm{m})$ & LD & LD & LD & LD \\
\hline Strontium $(0.3 \mathrm{p} / \mathrm{m})$ & LD & LD & LD & LD \\
\hline $\operatorname{Zinc}(0.005 \mathrm{p} / \mathrm{m})$ & LD & LD & LD & LD \\
\hline Calcium $(0.05 \mathrm{p} / \mathrm{m})$ & LD & LD & LD & LD \\
\hline Nitrate $(0.5 \mathrm{p} / \mathrm{m})$ & 1.0 & 1.4 & 0.9 & 1.1 \\
\hline
\end{tabular}


Table 5-8. 163-N Demineralization Plant Regeneration Waste Analysis Anion Regeneration Cycle. (2 Sheets)

\begin{tabular}{|l|c|c|c|c|}
\hline \multirow{2}{*}{ Parameters (MDL) } & \multicolumn{3}{|c|}{ Sample } & \multirow{2}{*}{ Average } \\
\cline { 2 - 4 } & 1 & 2 & 3 & \\
\hline Sulfate & 30.9 & 30.6 & 30.6 & 30.7 \\
\hline Fluoride (0.5 p/m) & LD & LD & LD & LD \\
\hline Chloride (0.5 p/m) & 2.5 & 2.3 & 2.3 & 2.4 \\
\hline Phosphate (1 p/m) & LD & LD & LD & LD \\
\hline Phcsphorus & LD & LD & LD & LD \\
\hline Pesticides (0.005 p/m) & LD & LD & LD & LD \\
\hline $\begin{array}{l}\text { Phosphorus Pesticides } \\
(0.005 \mathrm{p} / \mathrm{m})\end{array}$ & LD & LD & LD & LD \\
\hline $\begin{array}{l}\text { Chlorinated Pesticides } \\
(0.001 \mathrm{p} / \mathrm{m})\end{array}$ & LD & LD & LD & LD \\
\hline Enhanced ABN List & LD & LD & LD & LD \\
\hline Citrus Red (1 p/m) & LD & LD & LD & LD \\
\hline Arsenic (0.005 p/m) & LD & LD & LD & LD \\
\hline Ammonium Ion (0.05 p/m) & 2.3 & 2.7 & 2.8 & 2.6 \\
\hline Coliform (3 MPN) & LD & LD & LD & LD \\
\hline Selenium (0.005 p/m) & LD & LD & LD & LD \\
\hline Thallium (0.01 p/m) & LD & LD & LD & LD \\
\hline Enhanced VOA (10 p/m) & 26 & 28 & 26 & 27 \\
\hline
\end{tabular}

$L D=$ less than detectable $M D L=$ minimum detection limit MPN = most probable number .

Data obtained from samples taken August 1985.

Source: WHC i 987 c. 
Table 5-9. 183-N Filtered Water Plant Backwash Effluent Analysis. (2 Sheets)

\begin{tabular}{|c|c|c|c|c|}
\hline \multirow{2}{*}{ Parameters (MDL) } & \multicolumn{3}{|c|}{ Sample } & \multirow{2}{*}{ Average } \\
\hline & 1 & 2 & 3 & \\
\hline $\mathrm{pH}$ (standard units) & 7.08 & 7.65 & 7.64 & 7.46 \\
\hline Conductivity ( $\mu$ mhos) & 160 & 150 & 150 & 153 \\
\hline Mercury $(0.0001 \mathrm{p} / \mathrm{m})$ & LD & LD & LD & LD \\
\hline Ethylene glycol $(10 \mathrm{p} / \mathrm{m})$ & LD & LD & LD & LD \\
\hline $\begin{array}{l}\text { Enhanced thiourea } \\
(0.2 \mathrm{p} / \mathrm{m})\end{array}$ & LD & LD & LD & LD \\
\hline TOC $(1 \mathrm{p} / \mathrm{m})$ & 0.00277 & 0.002175 & 0.002257 & 0.002404 \\
\hline Cyanide $(0.01 \mathrm{p} / \mathrm{m})$ & LD & LD & LD & LD \\
\hline Barium $(0.006 \mathrm{p} / \mathrm{m})$ & 0.030 & 0.031 & 0.030 & 0.030 \\
\hline Cadmium $(0.002 \mathrm{p} / \mathrm{m})$ & 0.004 & 0.002 & 0.002 & 0.003 \\
\hline Chromium $(0.01 \mathrm{p} / \mathrm{m})$ & LD & LD & LD & LD \\
\hline Lead $(0.03)$ & LD & LD & LD & LD \\
\hline Silver $(0.01 \mathrm{p} / \mathrm{m})$ & LD & LD & LD & LD \\
\hline Sodium $(0.1 \mathrm{p} / \mathrm{m})$ & 2.202 & 2.287 & 2.186 & 2.225 \\
\hline Nickel $(0.01 \mathrm{p} / \mathrm{m})$ & LD & LD & LD & LD \\
\hline Copper $(0.01 \mathrm{p} / \mathrm{m})$ & LD & LD & LD & LD \\
\hline Vanadium $(0.005 \mathrm{p} / \mathrm{m})$ & LD & LD & LD & LD \\
\hline Antimony $(0.1 \mathrm{p} / \mathrm{m})$ & LD & LD & LD & LD \\
\hline Aluminum $(0.15 \mathrm{p} / \mathrm{m})$ & 0.392 & 0.389 & 0.376 & 0.386 \\
\hline Manganese $(0.005 \mathrm{p} / \mathrm{m})$ & 0.020 & 0.015 & 0.014 & 0.016 \\
\hline Potassium $(0.1 \mathrm{p} / \mathrm{m})$ & 0.799 & 0.814 & 0.762 & 0.792 \\
\hline Iron $(0.05 \mathrm{p} / \mathrm{m})$ & LD & LD & LD & LD \\
\hline Beryllium $(0.005 \mathrm{p} / \mathrm{m})$ & LD & LD & LD & LD \\
\hline Osmium $(0.3 \mathrm{p} / \mathrm{m})$ & LD & LD & LD & LD \\
\hline Strontium $(0.3 \mathrm{p} / \mathrm{m})$ & LD & LD & LD & LD \\
\hline $\operatorname{Zinc}(0.005 \mathrm{p} / \mathrm{m})$ & LD & LD & LD & LD \\
\hline Calcium $(0.05 \mathrm{p} / \mathrm{m})$ & 17.340 & 17.720 & 17.020 & 17.360 \\
\hline Nitrate $(0.5 \mathrm{p} / \mathrm{m})$ & 0.789 & 0.500 & 0.500 & 0.596 \\
\hline Sulphate & 18.900 & 20.980 & 19.110 & 19.663 \\
\hline
\end{tabular}


Table 5-9. 183-N Filtered Water Plant Backwash Effluent Analysis. (2 Sheets)

\begin{tabular}{|l|c|c|c|c|}
\hline \multirow{2}{*}{ Parameters (MDL) } & \multicolumn{3}{|c|}{ Sample } & \multirow{2}{*}{ Average } \\
\cline { 2 - 4 } & \multicolumn{1}{|c|}{1} & 2 & 3 & \\
\hline Fluoride (0.5 p/m) & LD & LD & LD & LD \\
\hline Chloride (0.5 p/m) & 2.846 & 2.671 & 2.901 & 0.2806 \\
\hline Phosphate (1 p/m) & LD & LD & LD & LD \\
\hline Phosphorus & LD & LD & LD & LD \\
\hline Pesticides (0.005 p/m) & LD & LD & LD & LD \\
\hline $\begin{array}{l}\text { Chlorinated Pesticides } \\
\text { (0.001 p/m) }\end{array}$ & LD & LD & LD & LD \\
\hline Enhanced ABN List & LD & LD & LD & LD \\
\hline Citrus Red (1 p/m) & LD & LD & LD & LD \\
\hline Arsenic (0.005 p/m) & LD & LD & LD & LD \\
\hline Ammonium Ion (0.05 p/m) & LD & LD & LD & LD \\
\hline Coliform (3 MPN) & 0.24 & 2.400 & 0.2400 & 1.680 \\
\hline Selenium (0.005 p/m) & LD & LD & LD & LD \\
\hline Thallium (0.01 p/m) & LD & LD & LD & LD \\
\hline Enhanced VOA (10 p/m) & -- & 0.024 & 0.023 & 0.025 \\
\hline
\end{tabular}

$L D=$ less than detectable

$M D L=$ minimum detection limit

MPN = most probable number .

Data obtained from samples taken August 1985.

Source: WHC 1987c. 
Table 5-10. Average Radionculide Concentrations ( $\mathrm{PC} / \mathrm{L}$ ) Detected in Groundwater Samples Collected in the 100-N Area During 1981.

\begin{tabular}{|c|c|c|c|c|c|c|c|}
\hline Well No. & ${ }^{\omega} \mathrm{Co}_{0}$ & ${ }^{103} \mathrm{Ra}$ & ${ }^{100} \mathrm{Ru}$ & ${ }^{126} \mathrm{Sb}$ & 14'Nd & ${ }^{\infty} S r$ & Tritiun \\
\hline $199-N-2$ & 530 & 81 & 370 & 270 & NO & 2,300 & $2.5 \times 10^{3}$ \\
\hline $199-N-3$ & 260 & 49 & 250 & 46 & ME & 305 & $1.9 \times \times 10^{4}$ \\
\hline $199-N-4$ & 43 & ND & $\pi$ & 6 & 570 & 3 & $1.7 \times 10^{4}$ \\
\hline $199-N-5$ & 310 & 26 & 240 & 200 & MD & 3 & $1.8 \times 10^{4}$ \\
\hline $199-N-7$ & 140 & 46 & 140 & 33 & ND & NA & NA \\
\hline $199-N-14$ & 200 & 54 & 150 & 40 & No & 160 & $1.7 \times 10^{4}$ \\
\hline $199-N-15$ & 60 & ND & 72 & 8 & ND & 6 & $2.1 \times 10^{4}$ \\
\hline $199-N-20$ & 10 & No & ND & 9 & ND & 78 & $1.9 \times 10^{4}$ \\
\hline
\end{tabular}

ND a Not detected.

MA = Not available.

Sources: DOE-RL 1990 and DOE-RL 1991

Table 5-11. Radionuclide Concentrations ( $\mathrm{pCi} / \mathrm{L}$ ) in Wells 199-N-8P Through 199-N-8S.

\begin{tabular}{|c|c|c|c|c|c|c|}
\hline We11 & Depth ints & \multicolumn{6}{|c|}{ Radionuclide } \\
& water \\
& table (ft) & ${ }^{60} \mathrm{Co}$ & ${ }^{103} \mathrm{Ra}$ & ${ }^{106} \mathrm{Ru}$ & ${ }^{125} \mathrm{Sb}$ & ${ }^{131} \mathrm{I}$ \\
\hline $199-\mathrm{N}-\mathrm{SP}$ & $74-84$ & 6 & $<6$ & $<46$ & $<19$ & 9 \\
\hline $199-\mathrm{N}-8 \mathrm{Q}$ & $34-44$ & 10 & $<8$ & $<54$ & $<22$ & $<11$ \\
\hline $199-\mathrm{N}-8 \mathrm{R}$ & $22-32$ & 75 & 11 & 95 & $<24$ & $<10$ \\
\hline $199-\mathrm{N}-8 \mathrm{~S}$ & $12-22$ & 78 & 20 & 87 & 66 & $<10$ \\
\hline
\end{tabular}

Note: Samples taken from $1 / 12 / 87$ to $2 / 18 / 87$.

Source: DOE-RL 1991. 
DOE/RL 91-59

Table 5-12. Selected Constituents Detected Above Drinking Water Standards in the 100-N Area Between

April 1987 and November 1989. (2 sheets)

\begin{tabular}{|c|c|c|c|c|c|c|c|c|c|c|c|c|c|c|c|c|c|c|c|c|c|c|c|c|c|c|}
\hline$\underset{E}{E}$ & 0 & $\circ$ & $\simeq$ & 0 & $m$ & $m$ & $n$ & & & ○ & & & & & & & a & $\wedge$ & ㅇ & $\simeq$ & $1=$ & $\div$ & $=0$ & $\checkmark r$ & $\checkmark$ & in \\
\hline$\grave{a}$ & $a$ & $=$ & $\infty$ & 0 & $\bullet$ & $\circ$ & & & - & $\infty$ & 0 & 0 & & ? & 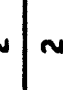 & & $\circ$ & $\wedge$ & $a$ & $m$ & $\infty$ & $\cong$ & $=n$ & $n$ n & & \\
\hline 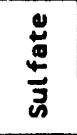 & & & & & & & & $m$ & & & & . & & 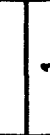 & $-1 \sim$ & & & & & & & & & & & \\
\hline 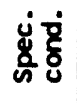 & & $*$ & & & & & & 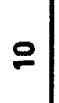 & -1. & - & & $r$ & r & ? & 4 & & -1 & & & & & & - & & & - \\
\hline$\underset{\underline{z}}{\vec{z}}$ & $\sim$ & - & $\sim$ & & $\sim$ & $\sim$ & - & & - & - & . & - & . & 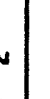 & $\bar{m}$ & & $m$ & & $*-$ & $-\infty$ & n & $\bullet$ & -1 & $-m$ & & \\
\hline 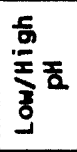 & & & & & & & & & & & & & & & $\stackrel{m}{\sigma}$ & & & & & $\tilde{\Xi}$ & & & & & & \\
\hline 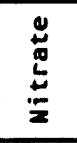 & & & & & & & & & & & & & & & & & & - & & - & 10 & $\sim$ & $-1-$ & - in & N & \\
\hline 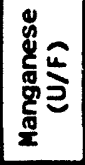 & & in & 3 & & & & & $s$ & 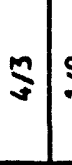 & 9 & & & 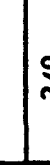 & 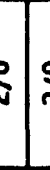 & 9 & $\frac{1}{2}$ & & & & & & & & & & 盆 \\
\hline 登愛 & i & ? & $\stackrel{\infty}{\infty}$ & & & ํ. & & $\cong$ & $\Xi$ & 9 & & 5 & $?$ & $?$ & 2 & is & & & & & & & 9 & $g$ & న & a \\
\hline 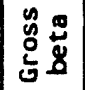 & $a$ & 웅 & $\sim$ & + & $\circ$ & 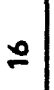 & & & -1 & $n$ & m & & & & & & $\infty$ & 인 & $\simeq 1 n$ & $\sim=$ & : & $=1$ & $\simeq \mathrm{m}$ & $m$ in & in & in \\
\hline 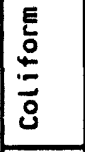 & & - & & & & - & & & & & & & & & & & & & & & $\sim$ & & & - & & \\
\hline 8 & & & $\sim$ & & & $\sim$ & & & & & & & & & & & in & m & $m$ & $n \mathrm{~m}$ & 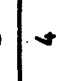 & a & $0 \mathrm{~m}$ & $m=$ & & \\
\hline 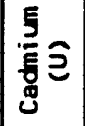 & & & & & & & & -1 & - & - & & & 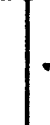 & & $-1-$ & & & & & & & & & & & \\
\hline 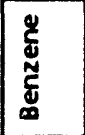 & $1-$ & & & & & & & & & & & & & & & & & & & & & & & & & \\
\hline$\overline{\underline{y}}$ & $\left|\begin{array}{c} \\
\vdots \\
\vdots \\
\delta \\
\delta\end{array}\right|$ & 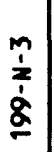 & $\begin{array}{l}\mathbf{1} \\
\dot{z} \\
\dot{\alpha}\end{array}$ & $\begin{array}{l}n \\
\underline{1} \\
\dot{q} \\
\underline{2}\end{array} \mid$ & $\mid \begin{array}{l}0 \\
\vdots \\
\vdots \\
\dot{\alpha} \\
\underline{\alpha}\end{array}$ & 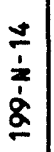 & 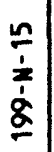 & 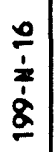 & $\begin{array}{l}\overline{7} \\
\bar{\lambda} \\
\dot{\delta} \\
\end{array}$ & 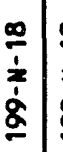 & 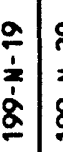 & 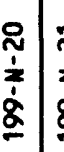 & & $\begin{array}{l}0 \\
\vdots \\
\vdots \\
\vdots\end{array}$ & $\begin{array}{lll}0 \\
\vdots \\
\vdots \\
\vdots \\
\vdots \\
\vdots\end{array}$ & 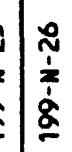 & $\left|\begin{array}{c}\hat{\alpha} \\
\frac{1}{\dot{\alpha}} \\
\underline{\dot{\alpha}}\end{array}\right|$ & 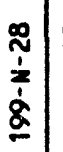 & 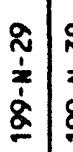 & 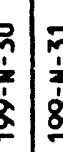 & $: \begin{array}{l}\tilde{N} \\
\tilde{n} \\
\dot{\alpha} \\
\underline{\alpha}\end{array}$ & $\mid \begin{array}{c}\tilde{p} \\
\underline{1} \\
\dot{\alpha} \\
\underline{\underline{\alpha}}\end{array}$ & 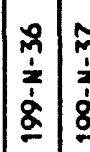 & 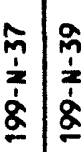 & & 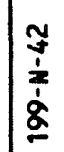 \\
\hline
\end{tabular}




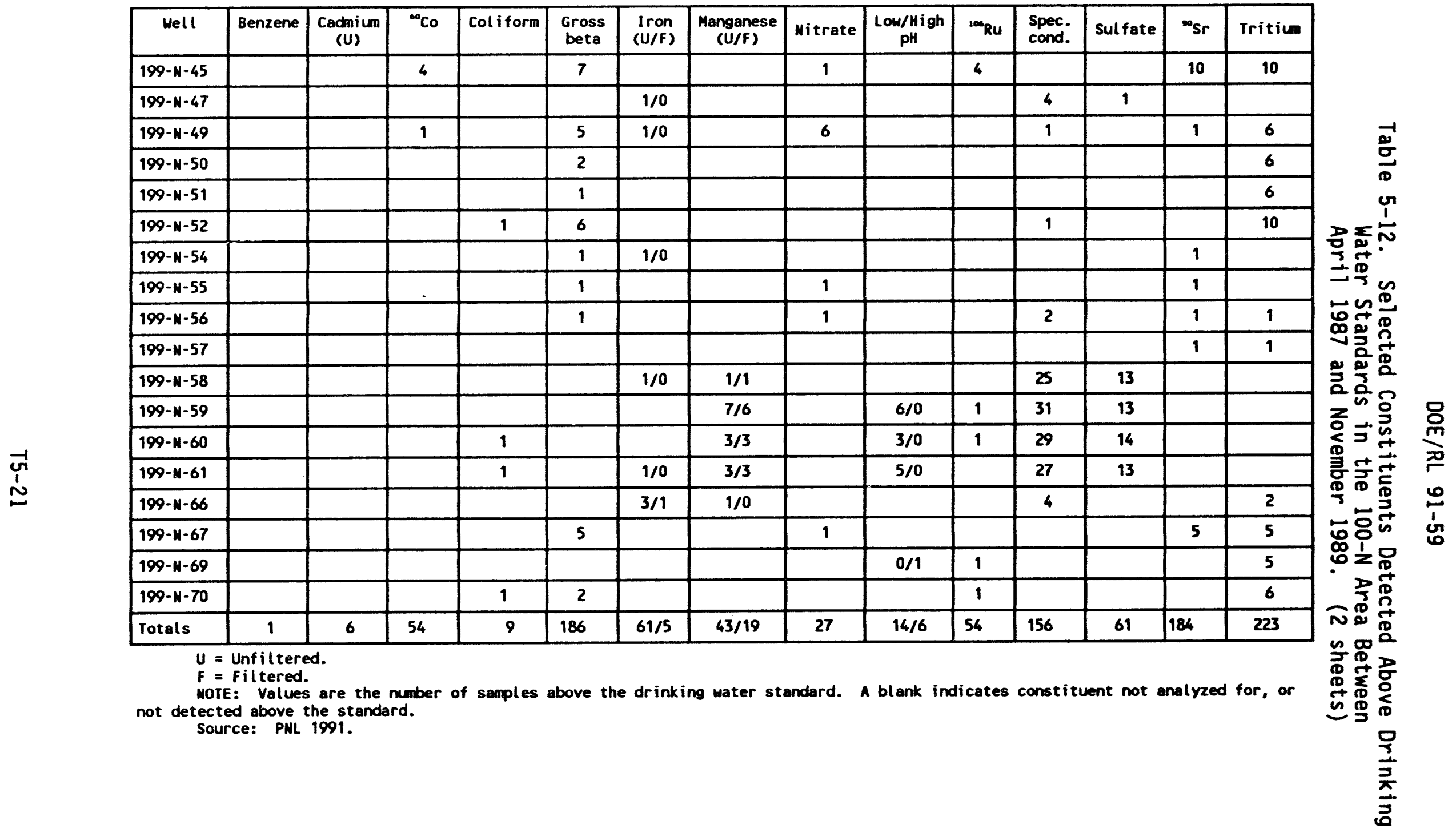


Table 5-13. Constituents Detected Above Drinking Water Standards in the 100-N Area During July/August 1989.

\begin{tabular}{|l|c|l|}
\hline \multicolumn{1}{|c|}{ Parameter } & Standard & \multicolumn{1}{|c|}{ Well in which exceedances occurred } \\
\hline Beta activity & $50 \mathrm{pCi} / \mathrm{L}^{1}$ & $\begin{array}{l}199-\mathrm{N}-2-,-3,-14,-27,-29,-31,-32, \\
-33,-36,-67\end{array}$ \\
\hline${ }^{90} \mathrm{Sr}$ & $8 \mathrm{pCi} / \mathrm{L}^{\circ}$ & $\begin{array}{l}199-\mathrm{N}-2,-3,-14,-27,-29,-31,-32, \\
-33,-36,-67\end{array}$ \\
\hline Tritium & $20,000 \mathrm{pCi} / \mathrm{L}^{\circ}$ & $199-\mathrm{N}-2,-3,-4,-14,-42,-67,-69,-70$ \\
\hline Nitrate & $45 \mathrm{mg} / \mathrm{L}^{\circ}$ & $199-\mathrm{N}-55$ \\
\hline Sulfate & $250 \mathrm{mg} / \mathrm{L}^{\mathrm{b}}$ & $199-\mathrm{N}-16,-21,-47,-58,-59,-60,-61$ \\
\hline
\end{tabular}

"Secondary maximum contaminant levels given in WAC 248-54, "Radioactive Protection Standards."

Secondary maximum contaminant levels given in 40 CFR Part 143

(July 1987) "National Secondary Drinking Water Regulations." 
Table 5-14. Estimated Background Levels for Selected Constituents in Hanford Site Groundwater. (2 sheets)

\begin{tabular}{|c|c|c|c|}
\hline Constituent & Unit & Detection limit & $\begin{array}{l}\text { Background } \\
\text { concentration }\end{array}$ \\
\hline Aluminum & $\mu g / L$ & $2^{\circ}$ & $<2^{\circ}$ \\
\hline Ammonia & $\mu g / L$ & 50 & $<50$ \\
\hline Arsenic & $\mu g / L$ & $0.2^{\circ}$ & $3.9 \pm 24^{\circ}$ \\
\hline Barium & $\mu g / L$ & 6 & $42 \pm 20$ \\
\hline Beryllium & $\mu g / L$ & $0.3^{\circ}$ & $<0.3^{a}$ \\
\hline Bi: nuth & $\mu g / L$ & $0.02^{\circ}$ & $<0.02^{\circ}$ \\
\hline Boron & $\mu g / L$ & $50^{\circ}$ & $<50^{\circ}$ \\
\hline Cadmi um & $\mu g / L$ & $0.2^{\circ}$ & $<0.2^{\circ}$ \\
\hline Calcium & $\mu g / L$ & 50 & $40,400 \pm 10,300$ \\
\hline Chloride & $\mu g / L$ & 500 & $10,300 \pm 6,500$ \\
\hline Chromium & $\mu g / L$ & $2^{\circ}$ & $4.0 \pm 2.0^{\circ}$ \\
\hline Copper & $\mu g / L$ & $1^{a}$ & $<1^{\circ}$ \\
\hline Cyanide & $\mu g / L$ & 10 & $<10$ \\
\hline Fluoride & $\mu \mathrm{g} / \mathrm{L}$ & 500 & $370 \pm 100$ \\
\hline Lead & $\mu g / L$ & $0.5^{8}$ & $<0.5^{a}$ \\
\hline Magnesium & $\mu g / L$ & 10 & $11,800 \pm 3,400$ \\
\hline Manganese & $\mu g / L$ & 5 & $7 \pm 5$ \\
\hline Mercury & $\mu g / L$ & 0.1 & $<0.1$ \\
\hline Nickel & $\mu \mathrm{g} / \mathrm{L}$ & $4^{8}$ & $<4^{\circ}$ \\
\hline Nitrate & $\mu \mathrm{g} / \mathrm{L}$ & 500 & $<500$ \\
\hline Phosphate & $\mu \mathrm{g} / \mathrm{L}$ & 1,000 & 1,000 \\
\hline Potassium & $\mu \mathrm{g} / \mathrm{L}$ & 100 & $4,950 \pm 1,240$ \\
\hline Selenium & $\mu \mathrm{g} / \mathrm{L}$ & $2^{a}$ & $<2^{\circ}$ \\
\hline Silver & $\mu g / L$ & 10 & $<10$ \\
\hline Sodium & $\mu g / L$ & 10 & $18,260 \pm 10,150$ \\
\hline Strontium & $\mu g / L$ & 20 & $236 \pm 102$ \\
\hline Sulfate & $\mu: g / L$ & 500 & $34,300 \pm 16,900$ \\
\hline Tritium & $\underline{p C i / L}$ & 300 & $<300$ \\
\hline Uranium & $\| \mathrm{Ci} / \mathrm{L}$ & 0.5 & $1.7 \pm 0.8$ \\
\hline
\end{tabular}


Table 5-14. Estimated Background Levels for Selected Constituents in Hanford Site Groundwater. (2 sheets)

\begin{tabular}{|l|c|c|c|}
\hline \multicolumn{1}{|c|}{ Constituent } & Unit & Detection limit & $\begin{array}{c}\text { Background } \\
\text { concentration }\end{array}$ \\
\hline Vanadium & $\mu \mathrm{g} / \mathrm{L}$ & 5 & $17 \pm 9$ \\
\hline Zinc & $\mu \mathrm{g} / \mathrm{L}$ & 5 & $6 \pm 2$ \\
\hline Alkalinity & $\mu \mathrm{g} / \mathrm{L}$ & $\mathrm{NA}$ & $123,000 \pm 21,000$ \\
\hline $\mathrm{pH}$ & Unitless & $\mathrm{NA}$ & $7.64 \pm 0.16$ \\
\hline $\begin{array}{l}\text { Total organic } \\
\text { carbon }\end{array}$ & $\mu \mathrm{g} / \mathrm{L}$ & 200 & $586 \pm 347$ \\
\hline Conductivity & $\mu \mathrm{mhos} / \mathrm{cm}$ & 1 & $380 \pm 82$ \\
\hline Gross alpha & $\mathrm{pCi} / \mathrm{L}$ & 0.5 & $2.5 \pm 1.4$ \\
\hline Gross beta & $\mathrm{pCi} / \mathrm{L}$ & 4 & $19 \pm 12$ \\
\hline Radium & $\mathrm{pCi} / \mathrm{L}$ & 0.2 & $<0.2$ \\
\hline
\end{tabular}

${ }^{a}$ Based on inductively coupled plasma/mass spectrometry data.

Average \pm 2 standard errors of the calculated mean.

$N A=$ Not applicable.

Sources: DOE-RL 1990 
Table 5-15. 1980 Select Radionuclide Concentrations Detected in Soil Samples Adjacent to the 116-N-1 Facility and in the

116-N-1 Trench Sediment Samples $(\mathrm{pCi} / \mathrm{g})$.

\begin{tabular}{|c|c|c|c|c|c|c|}
\hline \multicolumn{7}{|c|}{ Soil samples (adjacent areas) } \\
\hline $\begin{array}{c}\text { Sample } \\
\text { Location }\end{array}$ & ${ }^{54} \mathrm{Mn}$ & ${ }^{60} \mathrm{Co}$ & ${ }^{134} \mathrm{Cs}$ & ${ }^{137} \mathrm{Cs}$ & ${ }^{144} \mathrm{CePr}$ & ${ }^{155} \mathrm{Eu}$ \\
\hline $\mathrm{T}-1$ & 0.26 & 2.62 & ND & 0.43 & ND & 0.09 \\
\hline T-2 & 5.17 & 33.10 & 0.29 & 4.76 & 1.73 & ND \\
\hline T-3 & 2.07 & 8.31 & 0.24 & 4.43 & 0.56 & 0.16 \\
\hline$T-4$ & 1.83 & 9.93 & 0.25 & 4.66 & 0.48 & ND \\
\hline T-5 & 2.99 & 11.40 & 0.37 & 6.03 & ND & 0.12 \\
\hline$T-6$ & 0.10 & 1.00 & ND & 0.21 & ND & ND \\
\hline T-7 & 0.37 & 1.70 & ND & 0.74 & ND & ND \\
\hline T-8 & ND & 0.30 & ND & 0.21 & ND & ND \\
\hline T-9 & ND & 0.39 & ND & 0.84 & ND & ND \\
\hline Avg* & 1.83 & 7.64 & 0.27 & 2.48 & 0.92 & 0.12 \\
\hline
\end{tabular}

\begin{tabular}{|c|c|c|c|c|c|}
\hline \multicolumn{6}{|c|}{ Soil samples (trench) } \\
\hline $\begin{array}{l}\text { Sample } \\
\text { Location }\end{array}$ & ${ }^{54} \mathrm{Mn}$ & ${ }^{60} \mathrm{Co}$ & ${ }^{95} \mathrm{~N} 6$ & ${ }^{137} \mathrm{Cs}$ & ${ }^{144} \mathrm{CePr}$ \\
\hline TS-1 & $4.4 E+06$ & $1.3 E+07$ & $3.6 E+06$ & $2.7 E+05$ & $1.1 E+07$ \\
\hline TS-2 & $2.8 E+06$ & $8.8 E+06$ & $1.5 E+06$ & $2.1 E+05$ & $4.1 E+06$ \\
\hline TS -3 & $1.4 E+06$ & $8.4 E+06$ & $2.2 E+05$ & $1.2 E+05$ & $1.1 E+06$ \\
\hline TS-4 & $1.0 E+06$ & $5.1 E+06$ & $2.6 E+05$ & $2.2 E+05$ & $8.0 E+05$ \\
\hline TS-5 & $6.1 E+05$ & $3.1 E+06$ & $1.4 E+05$ & $2.6 E+05$ & $5.1 E+05$ \\
\hline TS-6 & $1.1 E+06$ & $5.6 E+06$ & $2.7 E+05$ & $2.1 E+05$ & $8.6 E+05$ \\
\hline TS -7 & $3.5 E+05$ & $1.7 E+06$ & $9.2 E+04$ & $2.4 E+05$ & $4.1 E+05$ \\
\hline TS-8 & $4.3 E+05$ & $7.6 E+06$ & ND & $6.3 E+05$ & ND \\
\hline is-9 & $7.0 E+05$ & $4.3 E+06$ & $1.2 E+05$ & $3.5 E+05$ & $3.3 E+05$ \\
\hline Avg & $1.4 E+06$ & $6.4 E+06$ & $7.6 E+05$ & $2.8 E+05$ & $2.4 E+06$ \\
\hline
\end{tabular}

ND $=$ Not Detected

*Average determined from samples with detectable results.

Note: In addition to the radionuclides listed, sample TS-1

contained $2.5 \times 10^{5} \mathrm{pCi} / \mathrm{g}$ of ${ }^{58} \mathrm{Co}, 1.98 \times 10^{6} \mathrm{pCi} / \mathrm{g}$ of ${ }^{95} \mathrm{Zr}$, and $2.7 \times 10^{6}$ $\mathrm{pCi} / \mathrm{g}$ of ${ }^{106} \mathrm{Ru}$; sample TS-2 contained $3.3 \times 10^{5} \mathrm{pCi} / \mathrm{q}$ of ${ }^{59} \mathrm{Fe}, 7.9 \times 10^{5}$ $\mathrm{pci} / \mathrm{g}$ of ${ }^{95} \mathrm{Zr}, 1.1 \times 10^{5} \mathrm{pCi} / \mathrm{g}$ of ${ }^{103} \mathrm{Ru}$, and $8.7 \times 10^{5} \mathrm{pCi} / \mathrm{g}$ of ${ }^{106} \mathrm{Ru}$; and sample TS-5 contained $4.1 \times 10^{4} \mathrm{pCi} / \mathrm{g}$ of ${ }^{134} \mathrm{Cs}$.

Source: DOE-RL 1990 
Table 5-16. Average Radionuclide Concentrations ( $\mathrm{pCi} / \mathrm{g}$ ) Detected in Soil Samples near the 116-N-1 Crib and Trench from 1980 through 1988.

\begin{tabular}{|c|c|c|c|c|c|c|}
\hline Year & ${ }^{54} \mathrm{Mn}$ & ${ }^{60} \mathrm{Co}$ & ${ }^{90} \mathrm{Sr}$ & ${ }^{137} \mathrm{Cs}$ & ${ }^{238} \mathrm{Pu}$ & ${ }^{239 / 240} \mathrm{Pu}$ \\
\hline 1980 & $2.5 \mathrm{E}+0$ & $1.3 \mathrm{E}-1$ & $3.5 \mathrm{E}-1$ & $4.1 \mathrm{E}+0$ & NR & $2.5 \mathrm{E}-2$ \\
\hline 1981 & $6.6 \mathrm{E}+0$ & $4.0 \mathrm{E}+0$ & $7.0 \mathrm{E}-1$ & $6.1 \mathrm{E}+0$ & NR & $4.4 \mathrm{E}-2$ \\
\hline 1982 & $6.6 \mathrm{E}-1$ & $6.3 \mathrm{E}+0$ & $2.7 \mathrm{E}-1$ & $2.7 \mathrm{E}+0$ & NR & $1.8 \mathrm{E}-2$ \\
\hline 1983 & $4.1 \mathrm{E}-1$ & $5.4 \mathrm{E}+0$ & $1.3 \mathrm{E}+0$ & $3.8 \mathrm{E}+0$ & NR & $4.3 \mathrm{E}-2$ \\
\hline 1984 & $1.8 \mathrm{E}-1$ & $2.8 \mathrm{E}+0$ & $2.1 \mathrm{E}-1$ & $1.1 \mathrm{E}+0$ & NR & $1.7 \mathrm{E}-2$ \\
\hline 1985 & $1.5 \mathrm{E}+0$ & $1.3 \mathrm{E}-1$ & $6.5 \mathrm{E}-1$ & $3.9 \mathrm{E}+0$ & NR & $3.2 \mathrm{E}-2$ \\
\hline 1986 & $1.6 \mathrm{E}-1$ & $4.5 \mathrm{E}+0$ & $2.2 \mathrm{E}-1$ & $2.5 \mathrm{E}+0$ & NR & $1.7 \mathrm{E}-2$ \\
\hline 1987 & $3.2 \mathrm{E}-1$ & $5.1 \mathrm{E}+0$ & $3.4 \mathrm{E}-1$ & $1.6 \mathrm{E}+0$ & $5.4 \mathrm{E}-3$ & $2.2 \mathrm{E}-2$ \\
\hline 1988 & $1.4 \mathrm{E}-1$ & $7.8 \mathrm{E}+0$ & $3.5 \mathrm{E}-1$ & $2.0 \mathrm{E}+0$ & $2.3 \mathrm{E}-3$ & $1.7 \mathrm{E}-2$ \\
\hline
\end{tabular}

NR $=$ Not Reported 
Table 5-17. Hydrophilic Organics in Trench Sediments.

\begin{tabular}{|c|c|c|c|}
\hline Paraneter & $\begin{array}{l}\text { Sample } \\
\text { No. } 2 \\
\mu g / k g\end{array}$ & $\begin{array}{l}\text { Sample } \\
\text { No. } 6 \\
\mu g / k g\end{array}$ & $\begin{array}{l}\text { Semple } \\
\text { No. } 9 \\
\mu g / k g\end{array}$ \\
\hline \multicolumn{4}{|c|}{ Tricarboxyl le Acids } \\
\hline Citric Acid & 34 & 159 & 80 \\
\hline \multicolumn{4}{|c|}{ Dicarboxylic Acids } \\
\hline Oxalic Acid & 13 & 1,971 & 347 \\
\hline Malonic Acid & 8 & 903 & 141 \\
\hline Malbic Acid & 3 & 375 & 31 \\
\hline Succinic Acid & 33 & 1,992 & 328 \\
\hline 2-Methyl-Succinic Acid & 80 & 94 & BD \\
\hline Pentanedioic Acid & 80 & 282 & BD \\
\hline Hexanedioic Acid & 3 & 193 & 34 \\
\hline Heptenedioic Acid & 20 & 80 & BD \\
\hline Octanedioic Acid & 41 & 592 & 87 \\
\hline Nonanediolc Acid & 91 & 557 & 163 \\
\hline \multicolumn{4}{|c|}{ Carboxylic Acids } \\
\hline Tetradecanoic Acid & 3 & 66 & 34 \\
\hline Hexadecanoic Acid & 49 & 292 & 55 \\
\hline Octadecenoic Acid & 45 & 190 & 39 \\
\hline \multicolumn{4}{|c|}{ Oxygenated Acids } \\
\hline 2-Hydroxy-Propenoic Acid & 80 & 138 & $B D$ \\
\hline 3-Hydroxy-Butanoic Acid & 12 & 80 & 80 \\
\hline 4-Methyoxy-Butanoic Acid & 80 & 18 & 80 \\
\hline 2-Methoxy-2-Butanoic Acid & 49 & BD & BD \\
\hline 4,4-D imethoxy-2-Pentenedioic Acid & 21 & 80 & BD \\
\hline 2-Methoxy-Benzoic Acid & BD & 274 & 80 \\
\hline 2-oxo-Propanoic Acid & 80 & 10 & 3 \\
\hline 4-0xo-Pentanoic Acid & 2 & 169 & 39 \\
\hline \multicolumn{4}{|c|}{ Aromatics } \\
\hline Benzeneacetic Acid & Bo & 222 & 79 \\
\hline Furancarboxylic Acid & 80 & 120 & 20 \\
\hline Benzoic Acid & 80 & 362 & 45 \\
\hline Dimethyl Phthalate & 34 & 188 & 68 \\
\hline Dibutyl Phthalate & 21 & BD & 80 \\
\hline Benzal dehyde & BD & 86 & 14 \\
\hline 2,5-Pyridinecarboxylic Acid & 92 & 80 & BD \\
\hline Pyridinecarboxyiolic Acid & BD & 4 & BD \\
\hline Nitrobenzoic Acid & BD & 115 & BD \\
\hline Nitro-Hydroxybenzoic Acid (Methyl Ester) & 80 & 300 & 57 \\
\hline
\end{tabular}

$B D=$ Below Detection limit.

Note: All samples taken from dried sediments in manways.

Source: DOE-RL 1990 
Table 5-18. Average Radionuclide Concentrations ( $\mathrm{pC} / \mathrm{g}$ ) Detected in 100-N Area Vegetation Samples.

\begin{tabular}{|c|c|c|c|c|c|c|}
\hline Year & $\min$ & ${ }^{m} \mathrm{co}$ & ${ }^{9} 8 r$ & "') Cs & 25u & $200,200 \mathrm{Pu}$ \\
\hline 1990 & $4.8 \mathrm{E}-01$ & $1.0 E+00$ & MR & $2.8 E-01$ & NR & NR \\
\hline 1981 & $1.8 E+\infty$ & $2.5 E+01$ & $5.8 \mathrm{E}-01$ & $7.1 \mathrm{E}-01$ & MR & $2.1 E \cdot 02$ \\
\hline 1982 & $4.9 e-01$ & $1.5 \varepsilon+00$ & $2.0=-01$ & $1.3 E-01$ & MR & $7.8=-03$ \\
\hline 1963 & $3.6 \cdot-01$ & $1.0 E+00$ & $2.9=-01$ & $9.0 \mathrm{E}-02$ & MR & $8.6 E-03$ \\
\hline 1994 & $1.3=-01$ & $4.6 E-01$ & $8.1 E-02$ & $9.0 \mathrm{E}-02$ & MR & $1.3 \mathrm{E}-03$ \\
\hline 1905 & $3.6-01$ & $1.6 E+00$ & $5.1 \in .02$ & $1.6 E-01$ & MR & 8.7 E-04 \\
\hline 1986 & $2.6 E-01$ & $9.5=-01$ & $2.2 E-01$ & $7.9 \mathrm{E}-01$ & MR & $1.1 \mathrm{E}-03$ \\
\hline 1997 & $1.1 E-01$ & $7.0=01$ & $2.6 \varepsilon-01$ & $9.4=-02$ & $1.3=-06$ & $5.8 \mathrm{E}-04$ \\
\hline 1948 & $1.3 \mathrm{E}-01$ & $8.0=-01$ & $2.5 E-01$ & $1.6 E-01$ & $1.7 \mathrm{E} \cdot 06$ & $6.6 E-04$ \\
\hline
\end{tabular}

Source: DOE-RL 1990.

NR a Not Reported.

Table 5-19. Average Radionucl ide Concentrations ( $\mathrm{pCi} / \mathrm{g}$ ) Detected in Vegetation Samples near 116-N-1 Crib and Trench.

\begin{tabular}{|c|c|c|c|c|c|c|}
\hline Year & $\sin n$ & ${ }^{\infty} \mathrm{Co}_{0}$ & ${ }^{m} g r$ & "21'Cs & $250 \mathrm{Pu}$ & $200,200 \mathrm{Pu}$ \\
\hline 1980 & $1.4 E+\infty 0$ & $4.0 E+00$ & MR & $1.1 E-00$ & MR & MR \\
\hline 1981 & $2.5 E+\infty 0$ & $1.2 E+01$ & $1.8 E+\infty$ & $1.8 E+00$ & MR & $7.1 E-03$ \\
\hline 1982 & 4.6 E-01 & $1.6 E+00$ & $1.2 E-01$ & $2.6 t-01$ & MR & $2.6 \mathrm{E}-03$ \\
\hline 1983 & $4.5 E-01$ & $1.9 E+00$ & $6.0 \mathrm{E}-01$ & $3.9 \varepsilon-01$ & MR & 3.2 E-03 \\
\hline 1984 & $2.9 E-01$ & $1.0 E+00$ & $1.2 E-01$ & 8.3 E-02 & NR & 8.5 E-0.4 \\
\hline 1985 & 5.9 E-01 & $1.7 E+\infty$ & $1.9 E+00$ & $1.0 \mathrm{E}-01$ & MR & $1.5 \mathrm{E}-03$ \\
\hline 1986 & $6.8 E-01$ & $3.5 E+00$ & $7.3 \mathrm{E}-02$ & $6.5 E-01$ & MR & $2.6 E-03$ \\
\hline 1987 & $4.9 E-01$ & $2.8 E+00$ & $6.3 E-02$ & $2.0 \mathrm{E}-01$ & $1.2 \varepsilon-03$ & $5.6 E-03$ \\
\hline 1988 & $1.5 E-01$ & $2.0 E+00$ & $1.2 E-01$ & $1.3 E-01$ & $4.3 \mathrm{E}-04$ & $1.7 E-03$ \\
\hline
\end{tabular}

Source: DOE-RL 1990.

NR = Not Reported.

Table 5-20. Average Radionuclide Concentrations ( $\mathrm{PC} / \mathrm{g}$ ) Detected in $N$ Springs Vegetation Samples.

\begin{tabular}{|c|c|c|c|c|c|c|}
\hline Year & shan & ${ }^{\infty} \mathrm{Co}$ & ${ }^{\prime s} s$ & ${ }^{131} \mathrm{Cs}$ & $2 m_{p u}$ & $230,260 \mathrm{Pu}$ \\
\hline 1980 & $1.5 E-01$ & $5.6 E+00$ & NR & $4.4 E-01$ & NR & NR \\
\hline 1981 & MR & $3.3 E+00$ & $2.0 E+02$ & MR & MR & $3.7 E-03$ \\
\hline 1982 & $1.5 E-01$ & $2.8 E+00$ & $4.8 E+02$ & NR & MR & $8.3 E-03$ \\
\hline 1983 & 7.0 E-02 & $3.0 E+\infty 0$ & $3.3 E+02$ & 4.0 E.02 & MR & $8.0 \mathrm{E}-03$ \\
\hline 1924 & MR & NR & MR & Mn & MR & NR \\
\hline 1985 & $7.6 \varepsilon-02$ & $1.2 E+00$ & $4.2 E+02$ & $1.7 E-01$ & MR & 4.6 E-04 \\
\hline 1986 & $1.6 \mathrm{E}-01$ & $1.1 E+00$ & $2.2 E+02$ & $2.1 E-01$ & NR & $4.2 \mathrm{E}-04$ \\
\hline 1987 & $2.0 \mathrm{E}-01$ & $9.0 \mathrm{E}-01$ & $2.9 E+02$ & 1.1 E-01 & $<1.1 \mathrm{E}-04$ & 7.6 E-04 \\
\hline 1988 & $2.4 E-01$ & $1.4 E+00$ & $1.2 E+02$ & $2.0 E-01$ & $8.5 E-05$ & 2.0 E-04 \\
\hline
\end{tabular}

Source: DOE-RL 1990.

NR = Not Reported. 


\begin{tabular}{|c|c|c|c|c|c|c|c|}
\hline \multirow{2}{*}{ Sample Type } & \multirow{2}{*}{ Sample Number } & \multirow{2}{*}{ Location } & \multirow{2}{*}{$\begin{array}{c}90_{2 x} \\
(\mathrm{dpm} / g)^{\circ} \\
\text { wet }\end{array}$} & \multicolumn{4}{|c|}{ Genese Results bq"/g wet $( \pm x)$} \\
\hline & & & & $7 \infty$ & $40 \mathrm{~s}$ & $60_{c o}$ & $137 \%$ \\
\hline \multicolumn{8}{|c|}{ Mulberry Samples: } \\
\hline Mulberries & $9018253289-2$ & $\begin{array}{l}1 \text { (Below } 100-N \text { stack on near } \\
\text { shorel ine) }\end{array}$ & ma & $<0.0085$ & $<0.01$ & $<0.0017$ & $\$ .2 E-4$ \\
\hline $\begin{array}{l}\text { Mulberry } \\
\text { leaves }\end{array}$ & $9018553289-5$ & $\begin{array}{l}2 \text { (Approximately } 50 \mathrm{~m} \text { upstream of } \\
\text { W-8* groundwater well near } \\
\text { shorel ine) }\end{array}$ & $M A$ & $0.024(8.1)$ & $<0.14$ & $4.40 E-4(37)$ & $1.23 E-3(35)$ \\
\hline Mulberries & $9018353289-3$ & 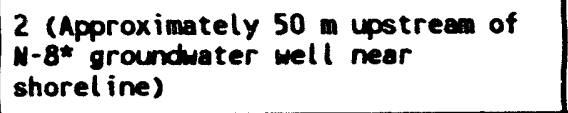 & MA & $0.0047(28)$ & $<0.11$ & $0.0037(5.9)$ & $<3.0 \mathrm{E}-4$ \\
\hline $\begin{array}{l}\text { Mulberry } \\
\text { leaves }\end{array}$ & $9018153289-1$ & $\begin{array}{l}3 \text { (Approximately } 50 \mathrm{~m} \text { downstream of } \\
\text { M-8** groundwater well near } \\
\text { shorel ine) }\end{array}$ & 171 & $0.025(9.8)$ & $0.195(28)$ & $8.59 E-4$ (23) & $8.20 E-4(50)$ \\
\hline Mulberries & $9018453289-4$ & 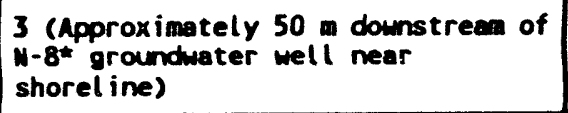 & 41.9 & $0.0046(26)$ & $<0.091$ & $8.42 E-4(16)$ & $5.08 E-4(50)$ \\
\hline \multicolumn{8}{|c|}{ Curly dock sample: } \\
\hline $\begin{array}{l}\text { Curly dock, } \\
\text { plant and root }\end{array}$ & $9018653289-6$ & $\begin{array}{l}\text { (Approximately } 15 \mathrm{~m} \text { dounstream of } \\
M-8^{*} \text { groundwater well at shorel ine) }\end{array}$ & 181 & $0.0035(35)$ & $<0.067$ & $1.21 E-3(18)$ & $<5.0 E-4$ \\
\hline
\end{tabular}

Decays per minute

bq (becquerel) = 1 decay per second

"The analytical uncertainty is the one-sigma value expressed as a percent.

$M A=$ Mot analyzed

* Well number prefixed by "199-" 
Table 5-22. 1979 Concentrations of Radionuclides in Swallow Nests Compared to $N$ Area and Hanford Soil Sample ( $\mathrm{pCi} / \mathrm{g}$ ).

\begin{tabular}{|c|c|c|c|c|c|c|}
\hline \multirow{2}{*}{ Radionuclide } & \multicolumn{5}{|c|}{ Swallow Nest } & \multicolumn{2}{c|}{ Surface Soil } \\
\cline { 2 - 7 } & $\# 1$ & $\# 2$ & $\# 3$ & $\# 4$ & N-Area & Hanford \\
\hline${ }^{54} \mathrm{Mn}$ & 1.9 & 0.14 & -- & 0.27 & 0.90 & -- \\
\hline${ }^{60} \mathrm{Co}$ & 15 & 1.2 & 0.64 & 1.5 & 2.7 & -- \\
\hline${ }^{95} \mathrm{Nb}$ & -- & -- & -- & -- & -- & 0.05 \\
\hline${ }^{137} \mathrm{Cs}$ & 5.5 & 0.28 & -- & 0.19 & 0.61 & 0.70 \\
\hline${ }^{147} \mathrm{Nd}$ & -- & -- & 0.45 & -- & -- & -- \\
\hline $\begin{array}{c}\text { Total } \\
\text { Activity }\end{array}$ & 22 & 1.6 & 1.1 & 2.0 & 4.2 & 0.75 \\
\hline
\end{tabular}

Source: DOE-RL 1990

Table 5-23. 1985 Radionuclide Concentrations $(\mathrm{pCi} / \mathrm{g})$ of Cliff Swallow Nests, Excrement Samples, and Shell/Embryo Samples Collected Near the 1304-N EDB During 1985.

\begin{tabular}{|c|c|c|c|c|}
\hline \multirow{2}{*}{ Nucl ide } & \multicolumn{2}{|c|}{ Swall low Nest } & \multirow{2}{*}{ Excrement } & \multirow{2}{*}{ Shell/Embryo } \\
\cline { 2 - 5 } & $\# 1$ & $\# 2$ & & \\
\hline${ }^{54} \mathrm{Mn}$ & 0.26 & 0.29 & -- & -- \\
\hline${ }^{60} \mathrm{Co}$ & 1.2 & 1.1 & 5.1 & 4.2 \\
\hline${ }^{137} \mathrm{Cs}$ & 0.31 & 0.29 & -- & -- \\
\hline
\end{tabular}

Source: DOE-RL 1990 
Table 5-24. Radionuclides Detected in Rabbits Collected Around the 116-N-Facility, 1981 Concentrations in $\mathrm{pCi} / \mathrm{g}$ (wet weight). (2 Sheets)

\begin{tabular}{|c|c|c|c|c|c|c|c|c|c|}
\hline & Tissue & sting & ${ }^{\infty} \mathrm{Co}$ & ${ }^{45} \mathrm{Zn}$ & ${ }^{134} \mathrm{Cs}$ & ${ }^{13} \mathrm{Cs}$ & ${ }^{\infty} \mathrm{sr}$ & ${ }^{23} \mathrm{Pu}$ & ${ }^{239 / 240} \mathrm{Pu}$ \\
\hline \multicolumn{10}{|c|}{ Inside 1301-N Security Fence } \\
\hline$\because 1$ & Muscle & ND & 0.2 & ND & 0.55 & 8.9 & MA & NA & NA \\
\hline & Bone & NO & no & 0.46 & NO & 3.2 & 73 & ND & NO \\
\hline \multirow[t]{3}{*}{$\# 2$} & Muscte & 6.1 & 13 & 6.2 & 320 & 3,700 & MA & MA & NA \\
\hline & Bone & 46 & 13 & 13 & 98 & 1,100 & 6,000 & ND & 0.14 \\
\hline & Liver & 210 & 120 & ND & 160 & 2,300 & 5.2 & 0.07 & 0.35 \\
\hline \multirow[t]{3}{*}{$\$ 3$} & Muscle & 4.2 & 2.2 & 2.1 & 270 & 3,400 & MA & MA & NA \\
\hline & Bone & 21 & 5.2 & ND & $\pi$ & 1,200 & 1,300 & 0.01 & 0.06 \\
\hline & Liver & 330 & 60 & ND & 190 & 3,400 & 5.6 & 0.005 & 0.02 \\
\hline \multirow[t]{3}{*}{ \#4 } & Muscle & 1.5 & 9.0 & 0.53 & 9.1 & 120 & MA & NA & NA \\
\hline & Bone & 1.4 & 0.57 & ND & 3.0 & 51 & 200 & ND & 0.15 \\
\hline & Liver & 11 & 12 & 8.7 & 9.9 & 160 & 13 & 0.13 & 0.08 \\
\hline \multirow[t]{3}{*}{$\# 5$} & Muscle & 2.2 & 3.4 & 0.42 & 9.4 & 140 & NA & MA & NA \\
\hline & Bone & 2.5 & 1.7 & ND & 3.1 & 43 & 180 & 0.07 & 0.04 \\
\hline & Liver & 26 & 22 & ND & 7.8 & 130 & 1.4 & 0.03 & 0.05 \\
\hline \multirow[t]{3}{*}{ Average } & Muscle & 2.8 & 5.6 & 1.9 & 120 & 1,500 & NA & NA & NA \\
\hline & Bone & 14 & 5.1 & 2.7 & 30 & 480 & 1,600 & 0.02 & 0.08 \\
\hline & Liver & 140 & 54 & 2.2 & 92 & 1,500 & 6.3 & 0.06 & 0.13 \\
\hline \multicolumn{10}{|c|}{ Rock Pile outside Fence } \\
\hline \multirow[t]{3}{*}{$\# 1$} & muscle & ND & 0.36 & ND & ND & 0.07 & WA & NA & NA \\
\hline & Bone & ND & 0.29 & 0.73 & NO & 0.51 & 11 & ND & NO \\
\hline & Liver & ND & 0.36 & ND & ND & 0.25 & NO & ND & 0.005 \\
\hline \multirow[t]{3}{*}{ \#2 } & Muscle & ND & 0.06 & ND & ND & 0.02 & NA & NA & NA \\
\hline & Bone & 1.2 & 0.44 & ND & 1.9 & 0.16 & 1.4 & ND & ND \\
\hline & Liver & NO & 0.22 & ND & 2.7 & 0.13 & ND & ND & ND \\
\hline \multirow[t]{3}{*}{$\$ 3$} & Muscle & ND & 0.07 & ND & No & ND & NA & MA & MA \\
\hline & Bone & ND & 0.22 & 0.16 & No & NO & 4.9 & $\star$ & 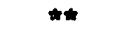 \\
\hline & Liver & ND & 0.13 & ND & 0.33 & 0.15 & ND & $*$ & ** \\
\hline \multirow[t]{3}{*}{$\$ 4$} & Muscle & 2.4 & $\#$ & 0.59 & ND & 9.5 & NA & MA & NA \\
\hline & Bone & ND & 0.72 & ND & No & 0.19 & 1.5 & ND & ND \\
\hline & Liver & ND & 0.18 & No & ND & 0.17 & ND & NO & 0.02 \\
\hline
\end{tabular}


Table 5-24. Radionuclides Detected in Rabbits Collected Around the 116-N-Facility, 1981 Concentrations in $\mathrm{pCi} / \mathrm{g}$ (wet weight). (2 Sheets)

\begin{tabular}{|c|c|c|c|c|c|c|c|c|c|}
\hline & Tissue & stm & ${ }^{\infty} \mathrm{Co}_{0}$ & $4 n$ & ${ }^{130} \mathrm{Cs}$ & ${ }^{13 \prime} \mathrm{Cs}$ & ${ }^{\infty} s r$ & ${ }^{201} \mathrm{Pu}$ & ${ }^{230 / 200} \mathrm{Pu}$ \\
\hline \multirow[t]{3}{*}{ Average } & Muscle & 0.60 & 0.16 & 0.15 & ND & 2.4 & NA & MA & MA \\
\hline & Bone & 0.30 & 0.42 & 0.22 & 0.48 & 0.26 & 1.6 & ND & ND \\
\hline & Liver & NO & 0.22 & No & 0.76 & 0.18 & NO & NO & 0.008 \\
\hline \multicolumn{10}{|c|}{$\begin{array}{l}\text { Radionuclides Detected in Rabbit Feces Collected Around the 1301-N Facility, } 1981 \text { Concentration } \\
\text { in pCi/g (dry weight). }\end{array}$} \\
\hline & stin & \multicolumn{2}{|c|}{${ }^{\infty} \mathrm{Co}$} & \multicolumn{2}{|c|}{${ }^{12 x} \mathrm{cs}$} & \multicolumn{2}{|c|}{${ }^{131} \mathrm{Cs}$} & \multicolumn{2}{|c|}{${ }^{140} \mathrm{Ce}$} \\
\hline $\begin{array}{l}\text { East of } \\
\text { Trench }\end{array}$ & 1,300 & \multicolumn{2}{|c|}{340} & \multicolumn{2}{|c|}{63} & \multicolumn{2}{|c|}{740} & \multicolumn{2}{|c|}{ Not detected } \\
\hline $\begin{array}{l}\text { West of } \\
\text { Trench }\end{array}$ & 650 & \multicolumn{2}{|c|}{870} & \multicolumn{2}{|c|}{19} & \multicolumn{2}{|c|}{270} & \multicolumn{2}{|c|}{120} \\
\hline
\end{tabular}

ND $=$ Not Detected

$\mathrm{NA}=$ Not Analyzed

- Cross Contamination Suspected.

Source: DOE-RL 1990 
등용

串

응

은

㟧安

㝴

可

ن엉

กับ

in

के

รั

음

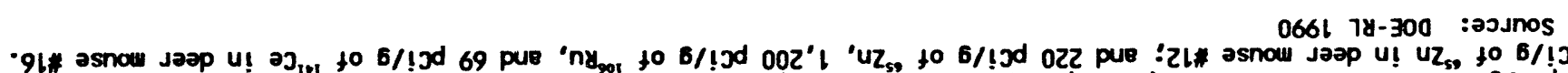
'ny to $6 / 1$ l

\begin{tabular}{|c|c|c|c|c|c|c|c|c|c|c|c|c|c|c|c|}
\hline 19 & 065 & OEI & 019 & 25 & 011 & 62 & 05 & 092 & 092 & $00 y^{\prime} 2$ & 062 & 041 & 085 & 052 & - EAY \\
\hline OA & 091 & 62 & 032 & 92 & 61 & 22 & on & 85 & 27 & $009^{\prime} \downarrow$ & 067 & 95 & $09 \varepsilon$ & 18 & 81 \\
\hline on & on & 22 & 014 & on & 62 & 4 & ON & ON & on & 061 & sL & ON & $\angle \varepsilon$ & on & 21 \\
\hline OSZ & $009^{\prime} \varepsilon$ & 022 & $000^{\prime} 2$ & 002 & 022 & 071 & OSI & $009^{\prime} \mathrm{L}$ & $008^{\prime} \mathrm{L}$ & $000^{\circ} \angle 1$ & 006 & $0<7$ & 000 'ל & 019 & 91 \\
\hline on & 001 & 92 & $00 L^{\prime} l$ & OEL & 32 & on & ON & ON & 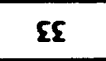 & $00 L^{\prime \prime} 1$ & $08 \varepsilon$ & 82 & $0<2$ & ON & st \\
\hline no & On & on & 021 & 1.2 & El & on & on & on & ON & 86 & 72 & ON & El & ON & 76 \\
\hline no & on & IE & IE & S.E & 8.6 & on & on & ON & on & S5 & 22 & ON & st & ON & $\varepsilon l$ \\
\hline IE & 066 & 021 & $00 \Sigma^{\prime} l$ & 021 & 81 & 15 & 02 & 001 & 26 & OOL'乏 & $008^{\prime} t$ & ont & $000^{\prime} 1$ & OZE & 21 \\
\hline 17 & 022 & 028 & $002^{\prime} 1$ & 011 & OZE & ON & 22 & 091 & 081 & $00 y^{\prime} 2$ & $00 \varepsilon^{\prime} l$ & 021 & $0 \Sigma 9$ & 012 & 11 \\
\hline 62 & OEY & ON & $08 \varepsilon$ & हE & 52 & SI & $\varepsilon 2$ & 58 & 69 & $00 L^{\prime} 2$ & o\&s & 17 & OE' & 011 & OL \\
\hline 001 & $\angle \varepsilon$ & 021 & 76 & on & 81 & 11 & on & 92 & $\varepsilon 2$ & 087 & 082 & 81 & OSL & on & 6 \\
\hline on & on & $1 \cdot 2$ & 52 & on & $7 \cdot 8$ & on & on & ON & ON & 09 & 12 & ON & 21 & on & 8 \\
\hline ON & on & $1 \cdot 2$ & 25 & $8 \cdot 7$ & 22 & ON & on & ON & ON & 75 & IE & ON & 81 & on & 2 \\
\hline on & $009^{\circ} !$ & 8 & $00 \varepsilon^{\prime} 2$ & oघ2 & OSE & on & 15 & OSt & 094 & $000^{\circ} 11$ & $0019^{\prime} 2$ & osz & $009^{\prime} 2$ & $0<4$ & 9 \\
\hline แL & 7 & 58 & $1 L$ & 6.5 & ON & 0.8 & on & s2 & 02 & $03 \varepsilon$ & 051 & on & 06 & on & $s$ \\
\hline on & ON & an & 061 & st & $06 \varepsilon$ & ON & ON & on & ON & 092 & 016 & ON & $2 \varepsilon$ & ON & 7 \\
\hline 22 & OEL & ON & Olt & $s L$ & $07 \varepsilon$ & 0.8 & on & Oll & oEl & $00 L^{\prime} 1$ & 096 & 12 & ols & OS & $\varepsilon$ \\
\hline 6.5 & osl & 031 & 011 & 21 & 65 & 0.8 & ON & 27 & 17 & 025 & 0 ort & $\varepsilon 2$ & 072 & 72 & 2 \\
\hline or & ON & 18 & 82 & 9.8 & 85 & an & On & on & on & 092 & OEL & 21 & §9 & ON & 1 \\
\hline$" 351$ & $\omega^{\infty}+1$ & "0>1 & 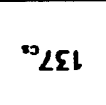 & "דו & ${ }^{x}|\Sigma|$ & ${ }^{*} \geqslant 21$ & "w5Ol & ${ }^{32} 56$ & $" 56$ & ${ }^{30} 09$ & "65 & ${ }^{03} 85$ & S & ${ }^{\circ} 15$ & $\begin{array}{l}\text { Jequinh } \\
\text { asnow } \\
\text { Jazo }\end{array}$ \\
\hline
\end{tabular}




\section{DOE/RL 91-59}

Table 5-26. Deer Mice Collected at N Springs, 1982 (pCi/g-wet wt.).

\begin{tabular}{|c|c|c|c|c|}
\hline Deer Mouse Number & ${ }^{54} \mathrm{Mn}$ & ${ }^{60} \mathrm{CO}$ & ${ }^{35} \mathrm{Cs}$ & ${ }^{155} \mathrm{Eu}$ \\
\hline 1 & ND & 2.1 & ND & ND \\
\hline 2 & ND & 2.6 & ND & 0.59 \\
\hline 3 & ND & 2.9 & ND & ND \\
\hline 4 & 0.78 & 8.1 & ND & ND \\
\hline 5 & ND & 1.0 & 1.5 & ND \\
\hline 6 & ND & 2.4 & 0.55 & 1.2 \\
\hline 7 & ND & 3.0 & ND & ND \\
\hline 8 & ND & 1.4 & ND & 1.4 \\
\hline 9 & ND & 0.35 & ND & ND \\
\hline 10 & ND & 4.6 & 0.39 & 1.5 \\
\hline 11 & ND & 3.5 & ND & ND \\
\hline 12 & 3.7 & 14 & ND & ND \\
\hline
\end{tabular}

ND $=$ Not Detected

Source: DOE-RL 1990 


\begin{tabular}{|c|c|c|c|c|c|c|c|c|c|c|c|c|}
\hline Trap * & $\begin{array}{c}\text { Weight } \\
(g)\end{array}$ & Sex & ${ }^{31} \mathrm{Cr}$ & $59 \mathrm{Mn}$ & soco & ${ }^{59} \mathrm{Fe}$ & ${ }^{\circ} \mathrm{Co}$ & ${ }^{s} 2 r / M b$ & ${ }^{131} \mathrm{I}$ & ${ }^{13} \mathrm{cs}$ & ${ }^{101} \mathrm{Ce}$ & ${ }^{104} \mathrm{Ce}$ \\
\hline 1 & 20.3 & $M$ & 0.82 & 3.3 & 0.25 & 3.1 & 7.1 & 1.1 & 0.29 & 2.5 & 0.035 & 0.49 \\
\hline 6 & 16.3 & $M$ & 0.68 & 1.7 & 0.23 & 2.1 & 5.7 & 0.64 & 0.16 & 0.70 & 0.044 & 0.35 \\
\hline 9 & 19.8 & $F$ & 0.98 & 3.5 & 0.35 & 3.3 & 11 & 1.1 & 0.17 & 0.37 & 0.040 & 0.42 \\
\hline 10 & 16.1 & $\mathbf{F}$ & 0.053 & 0.22 & 0.021 & 0.30 & 0.74 & 0.077 & 0.054 & 0.18 & 0.0028 & 0.046 \\
\hline 11 & 15.8 & F & 0.77 & 2.5 & 0.34 & 2.6 & 9.7 & 0.80 & 0.31 & 0.35 & 0.058 & 0.50 \\
\hline 12 & 21.7 & $M$ & 0.23 & 0.47 & 0.075 & 0.65 & 1.7 & 0.18 & 0.047 & 0.24 & 0.014 & 0.073 \\
\hline 14 & 18.2 & $F$ & 0.26 & 0.95 & 0.081 & 0.75 & 2.6 & 0.52 & 0.19 & 0.23 & 0.037 & 0.29 \\
\hline 25 & 19.6 & $n$ & $<0.014$ & $<0.0019$ & $<0.0017$ & $<0.0029$ & 0.0083 & $<0.0047$ & $<0.0026$ & 0.0022 & MD & $<0.0097$ \\
\hline 30 & 20.1 & $M$ & $<0.021$ & 0.041 & 0.0045 & 0.059 & 0.098 & 0.012 & 0.028 & 0.10 & $<0.0025$ & $<0.011$ \\
\hline 39 & 16.2 & $\mathbf{F}$ & 0.019 & 0.10 & 0.011 & 0.11 & 0.24 & 0.019 & 0.037 & 0.053 & $<0.0041$ & $<0.017$ \\
\hline 40 & 17.4 & $F$ & 1.2 & 4.1 & 0.48 & 4.3 & 15 & 1.4 & 0.20 & 0.97 & 0.15 & 0.86 \\
\hline 42 & 14.0 & $F$ & 2.7 & 12 & 0.99 & 13 & $\underline{36}$ & 3.6 & 0.25 & 1.0 & 0.30 & 1.7 \\
\hline 44 & 19.3 & M & 0.59 & 2.0 & 0.20 & 2.5 & 4 & 0.93 & 0.039 & 0.045 & 0.064 & 0.30 \\
\hline 45 & 14.4 & $F$ & $<.026$ & 0.016 & $<0.0039$ & 0.013 & 0.081 & $<0.0097$ & $<0.0048$ & 0.017 & $<0.0033$ & $<0.015$ \\
\hline 47 & 17.7 & $F$ & 0.93 & 4.5 & 0.30 & 4.7 & 11 & 1.6 & 0.23 & 2.7 & 0.049 & 0.56 \\
\hline$\underline{50}$ & 15.2 & 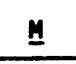 & 2.0 & 9.1 & 0.76 & 11 & 23 & 5.3 & 0.23 & 0.76 & 0.29 & 1.6 \\
\hline Average & 17.6 & & 0.86 & 3.0 & 0.29 & 3.2 & 8.0 & 1.2 & 0.16 & 0.64 & 0.090 & 0.60 \\
\hline S.D. & \pm 2.3 & & \pm 0.77 & \pm 3.5 & \pm 0.29 & \pm 3.9 & \pm 9.9 & \pm 1.5 & \pm 0.10 & \pm 0.83 & \pm 0.10 & \pm 0.54 \\
\hline
\end{tabular}

Underline values indicate the range (high and low value) for each radionuclide.

Source: DOE-RL 1990 
DOE/RL 91-59

This page intentionally left blank. 


\subsection{N REACTOR CLOSURE SCOPE}

The $N$ Reactor closure scope is divided into the following work elements, each with its own criteria and/or cleanup performance levels:

- $\quad$ Reactor deactivation

- RCRA closures

- Site remediation

- D\&D.

\subsection{N REACTOR DEACTIVATION}

According to the $N$ Reactor Deactivation Program Plan, WHC-SP-0615 (WHC 1992a), deactivation includes activities to:

- Deactivate the facility in a manner that will require only minimal S\&M until final D\&D

- Stabilize the facility to preclude any inadvertent environmental releases.

Accomplishment of $N$ Reactor deactivation will entail the following activities ( $A$ through $E$ ):

A. Document the remaining industrial safety, radiological safety, and environmental hazards of the facility.

- Prepare the final radiological/hazardous materials survey records, final configuration and S\&M requirements, and identify available drawings, specifications, procedures, manuals, and unplanned occurrence records applicable to the facility.

- Prepare all available data and reports that describe $N$ Reactor conditions, nature, and extent of contamination. Also, identify any known assessment requirements.

- Prepare or update a S\&M plan or monitoring plan, including a cost estimate, consistent with the final condition of the facility at turnover.

B. Place structures at the facility in a safe condition in compliance with regulatory requirements.

- Maintain structures and existing radiation surveillance systems in a physical condition adequate to contain and detect potential release of any radioactive contamination, in accordance with DOE Order 5400.1, General Environmental Protection Program (DOE 1988). Conduct a radiation contamination/hazardous materials survey of the facility and surrounding areas.

- Maintain security systems and procedures adequate to prevent unauthorized entry to any structures at the facility. 
- Remove all SNM, reactor fuels, high-level waste, contaminated liquid wastes, and hazardous chemicals or materials/wastes that are stored at the facility. The most significant activity in this category is the removal from $\mathrm{N}$ Basin of $90,700 \mathrm{~kg}(200,000 \mathrm{lb})$ of radiologically contaminated hardware and over 3,785,000 L $(1,000,000 \mathrm{gal})$ of radiologically contaminated water currently being used for shielding.

C. Assess the compliance of the facility with respect to environmental, health, and safety regulatory requirements.

- Assess facility for compliance with Occupational Safety and Health Administration (OSHA), RCRA, and Toxic substances Control Act of 1976 (TSCA) requirements and other applicable environmental regulatory requirements.

D. Ensure that any structures at the facility and their required systems are structurally sound to permit deferred final decommissioning of these structures (develop a S\&M plan to ensure facility and environmental safety pending initiation of decommissioning activities).

E. Satisfy specific environmental regulatory requirements.

- Replace the 1325-N LWDF with a waste water treatment system satisfying best available technology (BAT) requirements by 1995 and treat waste water at the Liquid Effluent Treatment Facility in the 200 Area with subsequent disposal of the treated effluent to a soil column disposal site. Alternatively, this waste water may be treated at $N$ Reactor with discharge of the treated effluent to the Columbia River through an NPDES permitted outfall.

- Prepare environmental documentation and secure approvals as necessary (e.g., NEPA and notification to Washington State Department of Health (WDOH) concerning air emissions)

- Upgrade effluent surveillance systems commensurate with the DOE Regulatory Guide, DOE/EH-0173T (DOE 1991).

The criteria governing this work are discussed further in Section 9.0. The major $N$ Reactor deactivation activities are summarized in Table 6-1.

\subsection{RESOURCE CONSERVATION AND RECOVERY ACT CLOSURES}

There are four RCRA facilities at 100-N (see Section 3.0):

- 1301-N LWDF (Mixed Waste)

- 1325-N LWDF (Mixed Waste)

- 1324-N Surface Impoundment (Hazardous Waste)

- 1324-NA Percolation Pond (Hazardous Waste).

Figure $6-1$ is a flowchart of the RCRA closure process. The RCRA closure work element consists of satisfying Ecology's "Dangerous Waste Regulations" (WAC 173-303), which require submitting a RCRA closure plan for each facility 
and maintaining a groundwater monitoring program until closure is complete. The individual RCRA closure plans are required to contain a description of the site characterization process through sampling and analysis of soils and groundwater.

Draft closure plans for the four RCRA facilities were submitted to Ecology in 1987 and are scheduled to be revised and resubmitted in 1994 in accordance with the Tri-Party Agreement (DOE-RL 1991).

Groundwater monitoring began at the four RCRA sites in December 1987 using at least one upgradient and two downgradient wells (see Color I1lustration 2-2). This monitoring is based on requirements for interim-status facilities, as defined by the RCRA and amended by the Hazardous and Solid Waste Amendments of 1984 (HSWA). These regulations are promulgated by the EPA in 40 CFR 265, Subpart F, as implemented by Ecology in WAC 173-303-400. Only at the 1324-N and 1324-NA facilities is there a significant statistical difference between upgradient and downgradient wells. Because of this difference, an assessment monitoring program has begun that will investigate the cause of the elevated total organic halogen and total organic carbon in downgradient wells, as required by 40 CFR 265.93 (D) (5).

The scope of work is to be integrated with other cleanup programs in accordance with the Tri-Party Agreement (Ecology et al. 1992); see Section 6.3. The strategy being used in the RCRA closure process is adopted from the Tri-Party Agreement, which allows for a land disposal unit being closed in conjunction with an operable unit. If initial investigation shows that the unit no longer contains hazardous waste or constituents, the unit may be "clean closed" with no physical closure action. Any remaining CERCLA-only materials (radionuclides) would be addressed as part of the past-practice process as designated for the operable unit.

\subsection{SITE REMEDIATION}

Remediation of the $100-\mathrm{N}$ site entails the cleanup of past spills and releases to the soil and groundwater. Remediation of these waste management units is discussed in the scope of the Tri-Party Agreement (DOE-RL 1991). The Tri-Party Agreement designates the 100-N Area as a RPP site pursuant to HSWA, which addresses releases of RCRA facilities regardless of time of release.

These units include various types of liquid waste disposal sites, underground tanks, and unplanned release sites. The wastes disposed of in these units include nonhazardous solid, hazardous radioactive, and mixed waste. Section 7.0 describes these waste units in more detail. To comply with CERCLA regulations, the four aggregate areas $(100,200,300$, and 1100) were divided into 78 operable units. Two of these units are located at $N$ Reactor (Figure 6-2).

- 100-NR-1 Source Operable Unit

- 100-NR-2 Groundwater Operable Unit.

The Tri-Party Agreement requires that the cleanup programs at the $N$ Reactor integrate the requirements of CERCLA, RCRA, and Washington State's dangerous waste program (the State's RCRA equivalent) (Ecology et al. 1992). 
The EPA maintains authority for CERCLA, and ECology implements RCRA under the authority of the State's dangerous waste program. The State has also received authorization to implement the EPA's radioactive mixed waste program. The State does not yet have authority to implement HSWA, the most recent amendments to RCRA; EPA retains this authority. Corrective action at $N$ Reactor is also subject to the Washington State Model Toxics Control Act (MTCA) [Chapter 70.105D, Revised Code of Washington (RCW)]. Pursuant to the Tri-Party Agreement, the 100-NR-1 and 100 NR-2 source operable units are subject to RCRA corrective action authority. The EPA and Ecology have determined that the EPA guidance for conducting a remedial investigation/feasibility study (RI/FS) under CERCLA may be used at the $N$ Reactor in the performance of a RFI/CMS. Therefore, al though RCRA terminology has been used where appropriate, the content and format of the 100-NR-1 and 100-NR-2 operable units conform to EPA guidance for CERCLA activities.

Cleanup of $N$ Reactor past-practice units will be conducted pursuant to the RPP process. Figure 6-3 highlights the major steps involved in both the CERCLA Past Practice (CPP) and RPP programs and indicates how each of these steps corresponds with the other (i.e., the steps of the RPP program are functionally equivalent to steps in the CPP program).

Before the investigative process starts, an operable unit scoping activity will be used to support the initial planning phase for each operable unit's RFI/CMS. Such activity and planning result in an overall management strategy for each of the $N$ Reactor operable units. The DOE assembled and evaluated existing data and information about the individual waste management units and release sites within each operable unit. This scoping activity was not intended to be a mechanism for generating new information, but a thorough and complete evaluation of existing data. If during the scoping activity, a situation is determined by Ecology to present an imminent and substantial danger to the public health or welfare or the environment because of an actual or threatened release of a hazardous substance or waste at an operable unit, the lead regulatory agency may require DOE to immediately initiate activities to abate the danger or threat. RCRA includes provisions to quickly respond to such situations. DOE is currently preparing a work plan for initiating an ERA. Operable unit work plans have already been prepared for the 100-NR-1 and -2 operable units and were transmitted to EPA and Ecology for approval (DOE-RL 1990 and DOE-RL 1991). Following the RFI/CMS the lead regulatory agency (i.e., Ecology) will issue a ROD, designating what cleanup actions are required.

The EPA is implementing a nationwide program to facilitate response actions at NPL sites through the expedited response actions (ERA-2) process. The purpose of the ERA is to accomplish rapid cleanup by streamlining the RI/FS process for operable units or sites where the most effective mitigation effort is readily evident. The release of ${ }^{90} \mathrm{Sr}$ to the Columbia River along an area of $N$ Reactor's shoreline termed $N$-Springs is a candidate ERA proposed by both EPA and Ecology.

Color Illustrations 6-1 and 6-2 present three-dimensional computerized representations of past and predicted groundwater ${ }^{90} \mathrm{Sr}$ concentration levels that have been and could be released to the Columbia River. 


\subsection{DECONTAMINATION AND DECOMMISSIONING}

The purpose of D\&D is to isolate any remaining radioactive or hazardous waste to minimize environmental impact, especially potential health and safety impacts, on the public. These activities can be accomplished through several actions:

- Ongoing S\&M of the facility

- Dismantling and burial at another Hanford site

- Dismantling and burial at 100-N.

The scope of work for purposes of this Closure Report is assumed to be the dismanting of all 100-N facilities and burial at another Hanford site. This assumption is based on the Hanford Site EIS Decommissioning of Eight Surplus Production Reactors at the Hanford Site (DOE/EIS 1993). The actual means of decommissioning the reactor and other facilities at the 100-N site will depend on future engineering studies and the NEPA process, which will evaluate alternative actions and will be followed by a ROD.

It is estimated that dismantling and burial of $\mathrm{N}$ Reactor will involve more than $9,500 \mathrm{~m}^{3}\left(111,000 \mathrm{yd}^{3}\right)$ of concrete and 9,900 metric tons (11,000 tons) of structural steel. 


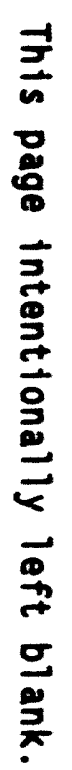

욤

0
1
0

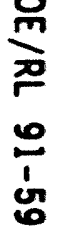


DOE/RL 91-59

Color 1llustration 6-1. Strontium-90 Concentrations for 1990. 
DOE/RL 91-59

Color Illustration 6-2. Projected Strontium-90 Concentrations for 2020.

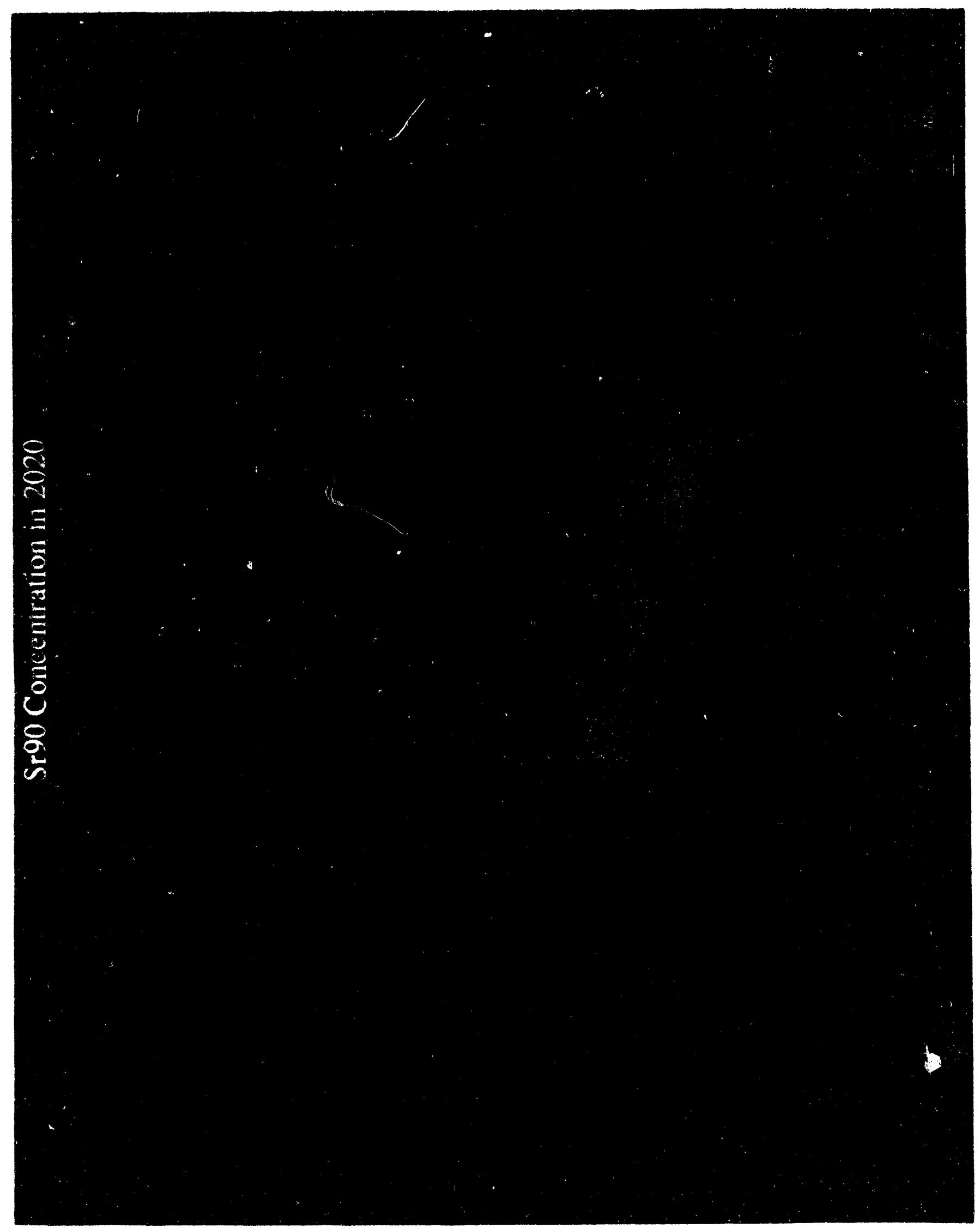


Figure 6-1. RCRA Closures.

\section{Activilies}

1. Submit Part A Application (Completed)

2. Prepare Closure/Post-Closure Plans

\begin{tabular}{|c|c|c|}
\hline \multicolumn{2}{|c|}{ Facility Plan } & \multicolumn{2}{|c|}{ TPA Commitment } \\
\hline $1301-N$ & $\begin{array}{l}\text { Llquild Waste } \\
\text { Dlsposal Facility }\end{array}$ & 1994 M-20-31 \\
$1325-N$ & $\begin{array}{l}\text { Llquid Waste } \\
\text { Dlsposal Facility }\end{array}$ & $1994 \quad$ M-20-31 \\
$1324-N$ & $\begin{array}{l}\text { Surface } \\
\text { Impoundment }\end{array}$ & 1994 M-20-35 \\
$1324-N A$ & Percolation Pond & 1994 M-20-35 \\
\hline
\end{tabular}

3. Submit Environmental Information Check List

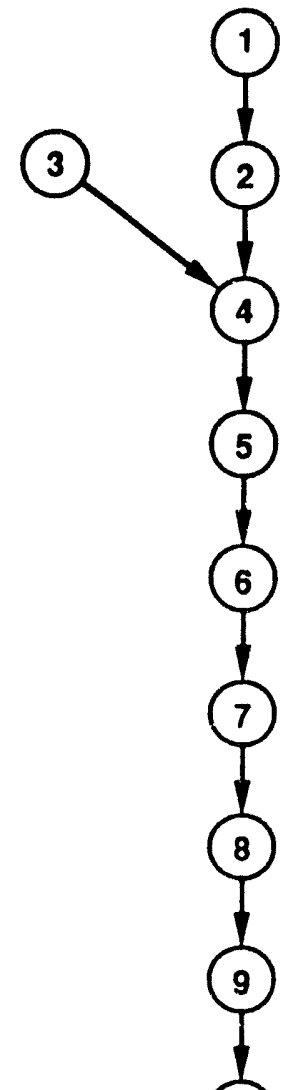

4. Review/Notice of Deficiency Process with Regulators

5. Public Comment/Comment Incorporation Process

6. Closure Plan Approved

7. Implement Closure Activities Described in Closure Plan

8. Clean Closure - No Post-Closure

9. Close with Waste In Place - Prepare/Submit Post-Closure Permit Application

11. Public comment/comment Incorporation process/Public Hearing (as Regulated)

12. Revise Final Status Permit

13. Implement Post-Closure (Install Landfill Cover)

14. Conduct Post-Closure Monitoring 


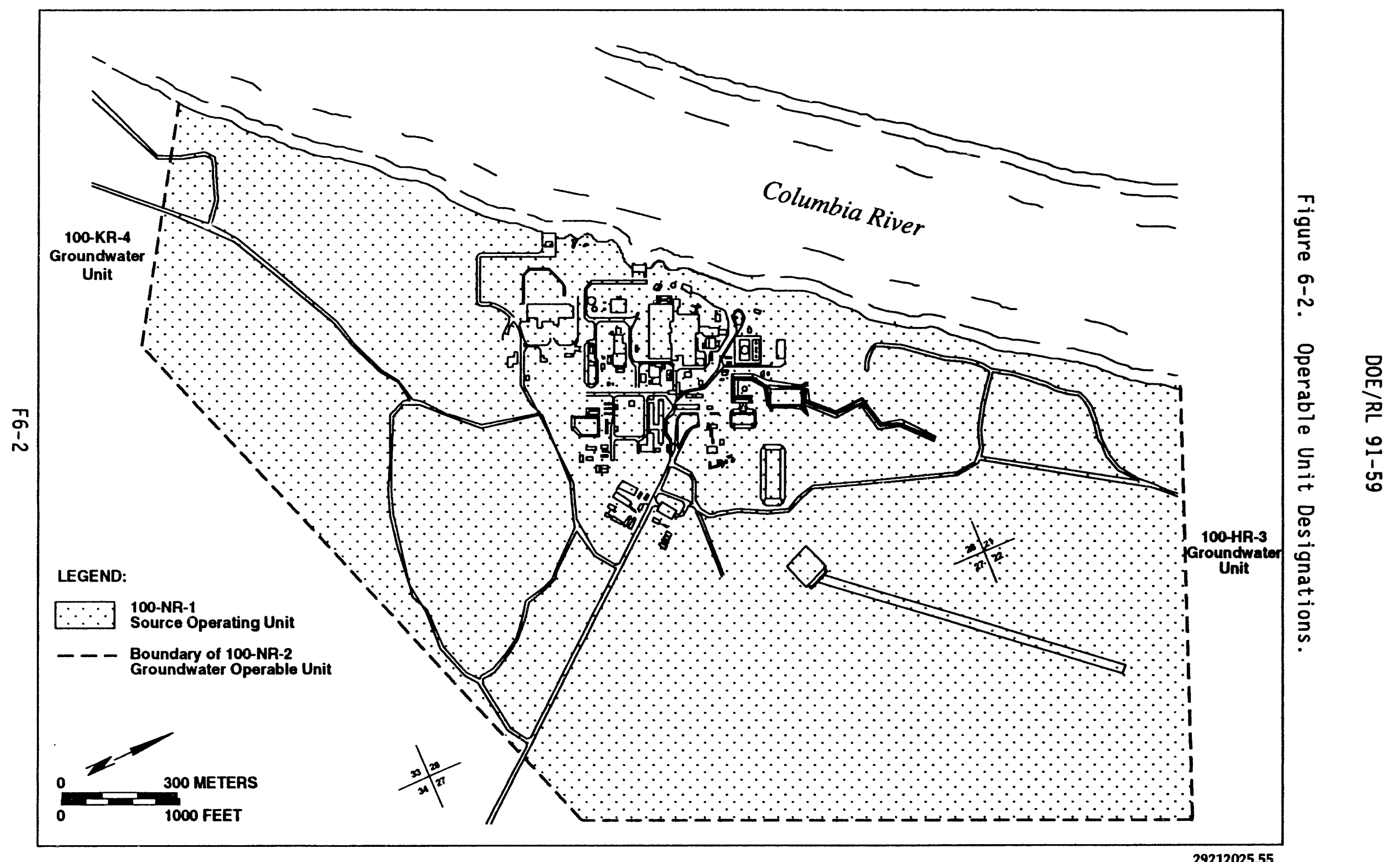


Figure 6-3. Comparison of Resource Conservation and Recovery Act Corrective Action and Comprehensive Environmental Response, Compensation, and Liability Act Remedial Processes.

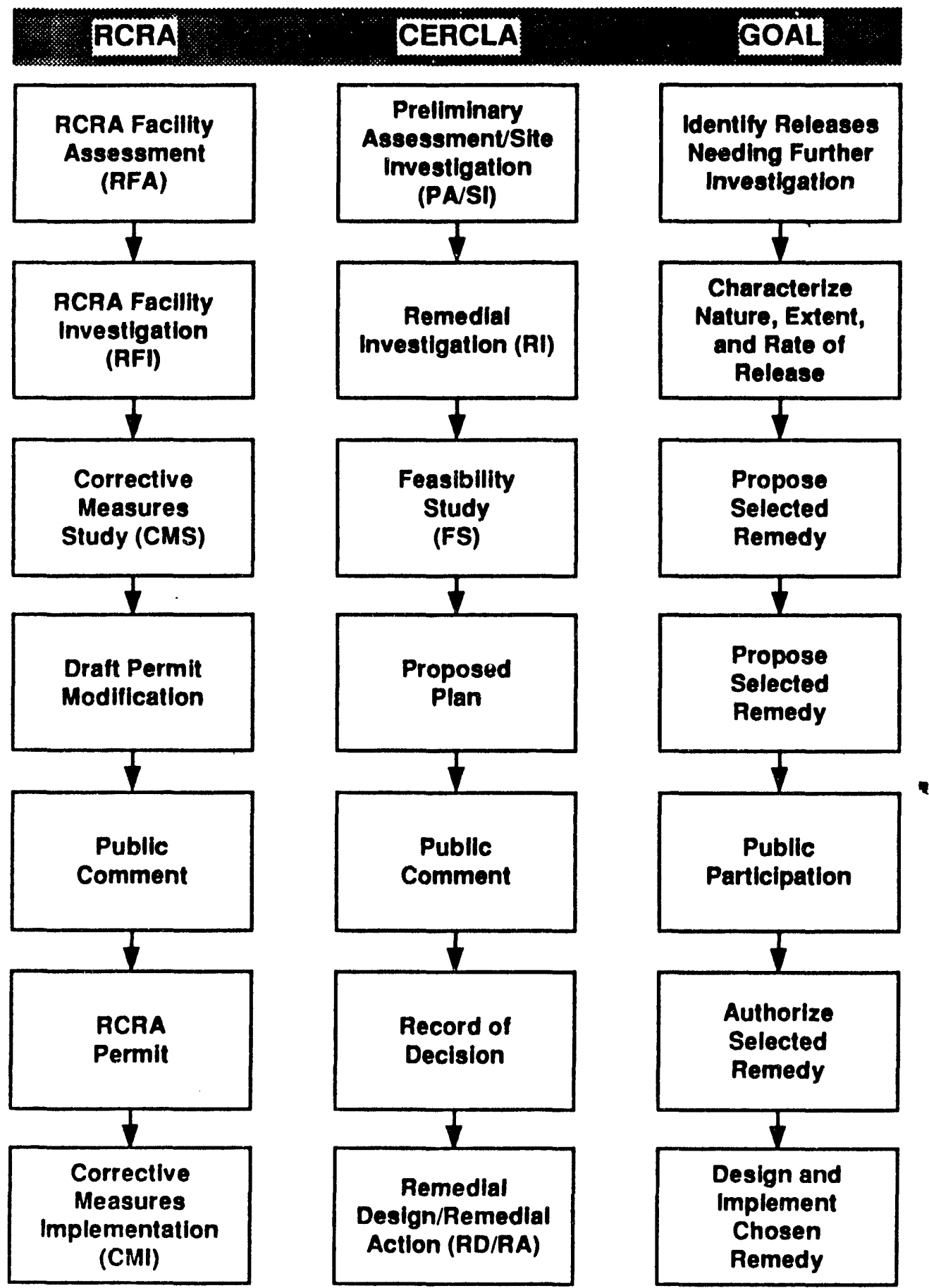

CERCLA = Comprehensive Environmental Response, Compensation, and Liability Act

RCRA = Resource conservation and Recovery Act

Note: Interim remedial actions or interim measures can be performed at any point in the remedial/corrective action process. 
DOE/RL 91-59

This page intentionally left blank.

F6-4 
Table 6-1. Summary of Major N Reactor Deactivation Activities.

(2 Sheets)

Generic Activities for all Facilities

1. Repair any roof leaks and/or deteriorated roof panels.

2. Remove all spare parts/tools/equipment.

3. Remove all unattached hazardous materials.

4. Remove or stabilize loose and/or damaged asbestos.

5. Conduct final radiological status surveys.

6. Drain all tanks, vessels, drums, etc., and characterize for radionuclides and hazardous materials according to applicable Federal and State regulations.

7. Orain elevator systems after they are in their respective bottom position and documented to the type, weight, and class of fluid required to obtain operational status in the event it is desirable for future use.

8. Reduce electrical and water si lly services to that which may be required for surveillance and maintenance.

9. Ensure that zero energy checks were made on all de-energized electrical circuits.

10. Where appropriate, centralize the electrical services left for S\&M to one location.

11. Shut down all air supply and exhaust air systems.

12. Remove all special nuclear material; reactor fuel; high-level waste; contaminated liquids, hazardous chemicals, materials, or wastes; and the combination of hazardous radioactive materials stored at the facility.

13. Reduce surface contamination levels to minimize resuspension and/or migration of loose contamination for all permanent radiation zones outside of 105-N/109-N Zone I (innermost radiation zone around reactor core).

14. Isolate all effluent flow routes to disposal sites by sealing or valving off at the facility and the outlet end of the pipe screened.

15. Seal drains that fed $N$ Reactor NPDES permitted outfall structures and/or valved off to each facility and screen at the outlet for vermin control.

16. Seal all building penetrations (i.e., louvers and open piping) to prevent bird and animal intrusion.

17. Secure all doors by bolting shut from the inside except those required for entrance by S\&M.

18. Dispose of over 500 truckloads of assets, such as nonhazardous salvageable material and equipment and office equipment. 
Table 6-1. Summary of Major N Reactor Deactivation Activities. (2 Sheets) Specific Activities - 105-N Reactor Building (Excluding N Basin)

1. Position all control and safety rods in a full-in position and drain their drive and cooling systems.

2. Provide objective evidence to show that all locations where fuel elements were handled, used, and stored were thoroughly examined (i.e., reactor core, fuel charge/discharge area, charged monotubes, fuel storage basin handling and shipping areas). Also included in this effort is providing documented evidence that the underground dummy storage silos are empty and do not contain any fuel elements.

3. Provide objective evidence that all irradiated lithium slugs have been removed from the building, including any that may have been in the horizontal control rods.

4. Seal the normal exhaust of $105-\mathrm{N} / 109-\mathrm{N}$ Zone I through the $117-\mathrm{N}$ filters and the $116-N$ stack.

5. Install an intrusion alarm on all doors of the $105-\mathrm{N}$ building that are not secured (bolted shut) from the inside.

6. Install a waste water treatment system to process $\mathbf{N}$ Reactor radiologically contaminated effluent before it is discharged to the Coiumbia River.

7. Remove and dispose of spacers used to space fuel elements in the reactor core from three 5.1-m-dia. by 6-m-deep (17-ft-dia. by 20-ft-deep) underground and shielded silos.

Specific Activities - N Reactor Fuel Storage Basins

1. Remove all hardware and debris (approximately $154 \mathrm{~m}^{3}\left[5,400 \mathrm{ft}^{3}\right]$ ).

2. Characterize and remove sediment (approximately $2 \mathrm{~m}^{3}\left[154 \mathrm{ft}^{3}\right]$ ).

3. Remove basin water (approximately $4.5 \mathrm{ML}$ [1.2 M gal]).

4. Stabilize basin surfaces to ensure that no resuspension of contaminants occurs.

5. Ensure no special nuclear material pieces remain. 


\subsection{PROPOSED CLEANUP SCHEDULE}

The $\mathrm{N}$ Reactor cleanup schedule has been prepared with consideration to regulatory-driven milestones, which are contained in either the Tri-Party Agreement, the Liquid Effluent Consent Order, or individual lower-tier documents (e.g., RCRA closure $\mathrm{plans}$ ). Additional related milestones have been proposed in the 100-N Area Operable Unit Work Plans but have not yet been agreed to by the three parties. The applicable milestones for $\mathrm{N}$ Reactor and the 100-N site are listed in Table 7-1.

The $N$ Reactor Deactivation Program schedule is shown in Figure 7-1 (WHC 1992). The schedule for site remediation and the D\&D is shown in Figure 7-2 (DOE-RL 1992). 


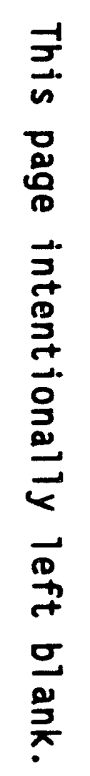




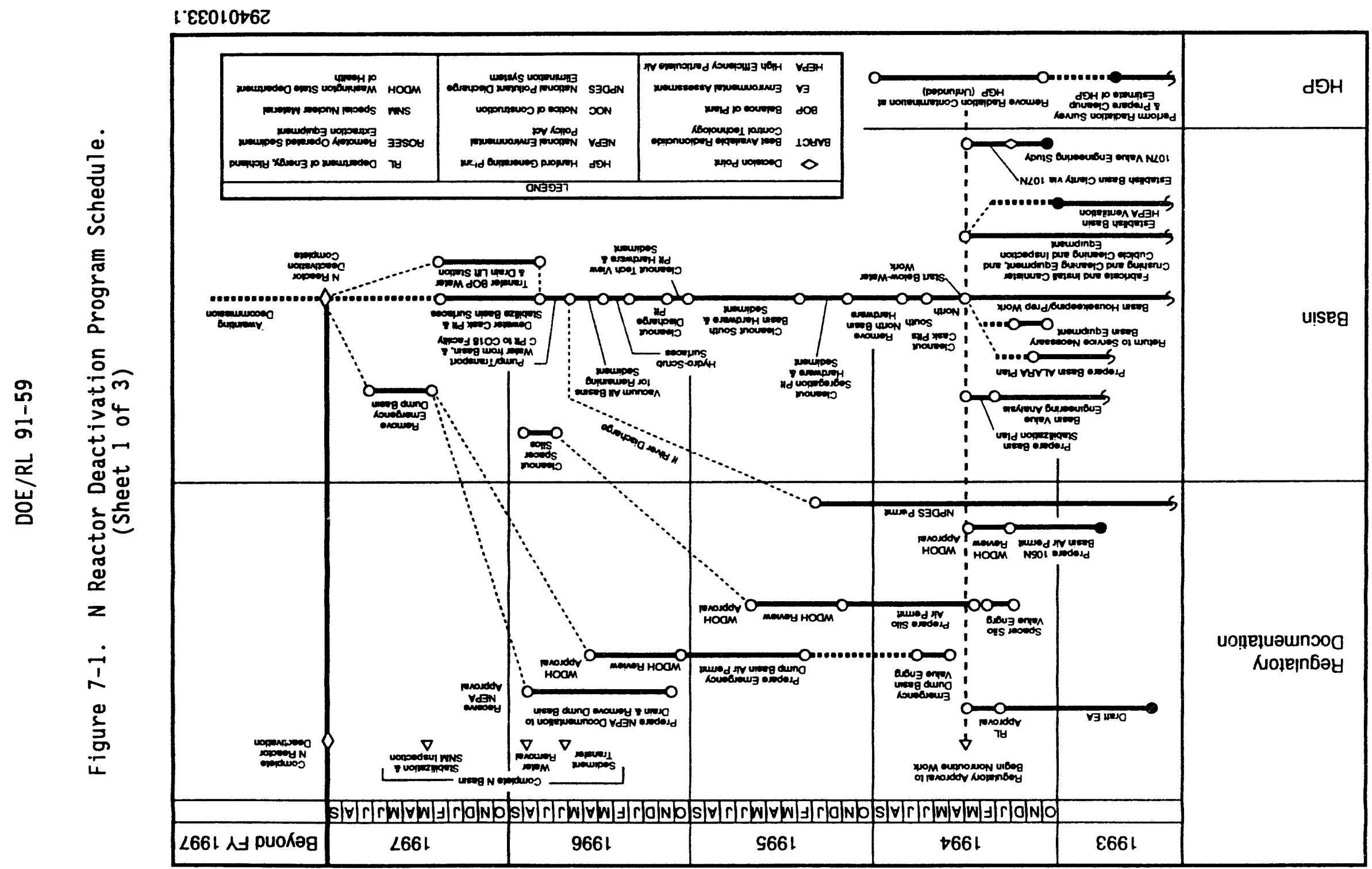




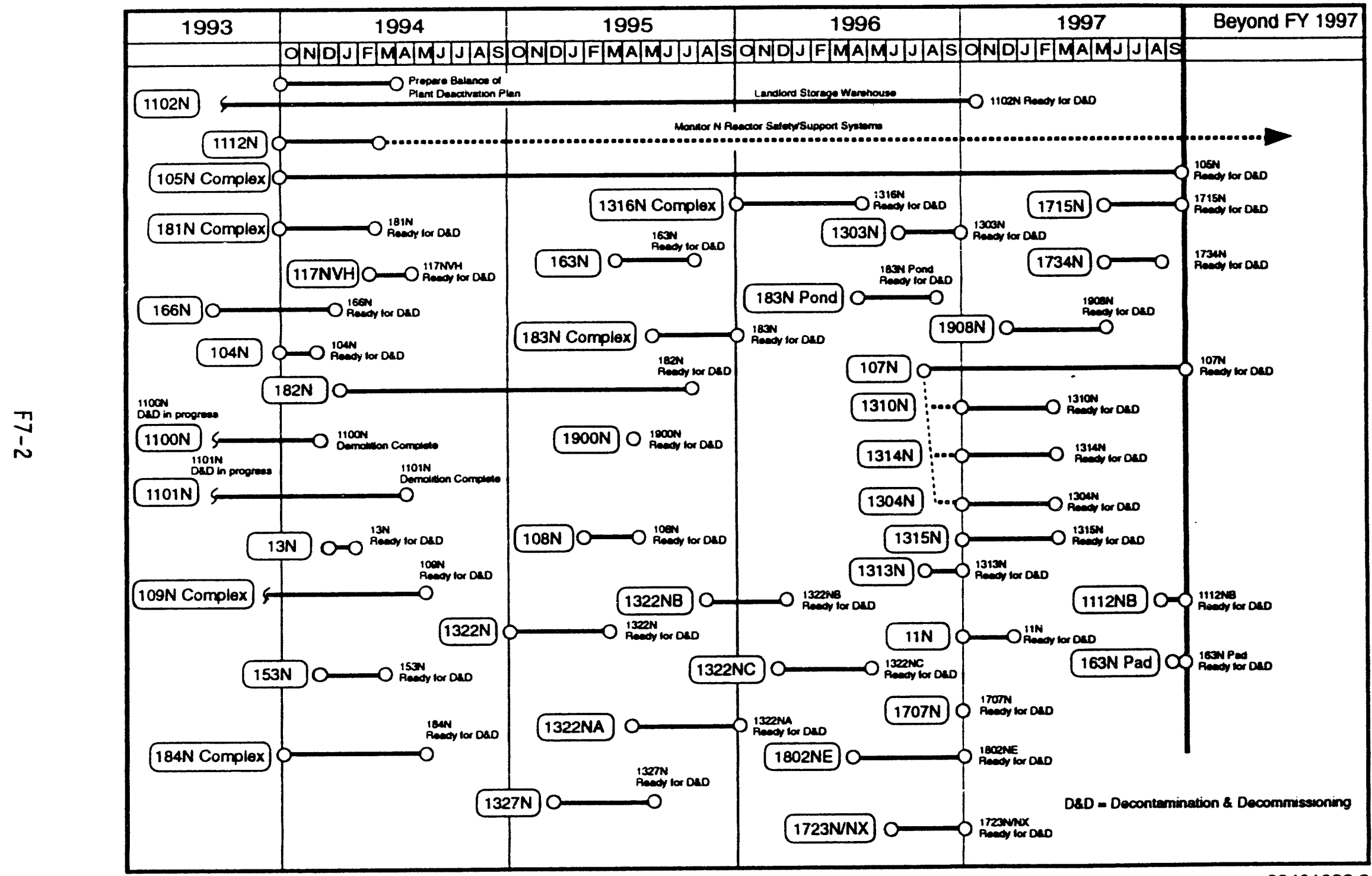

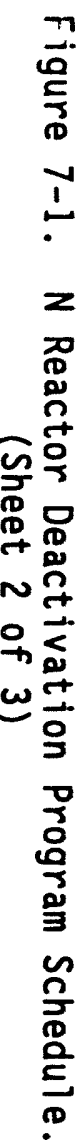




\section{N Facility Deactivation List}

\begin{tabular}{|c|c|c|c|}
\hline $\begin{array}{r}11 \mathrm{~N} \\
13 \mathrm{~N} \\
104 \mathrm{~N} \\
1105 \mathrm{~N} \\
105 \mathrm{NA} \\
105 \mathrm{NB} \\
107 \mathrm{~N} \\
108 \mathrm{~N} \\
109 \mathrm{~N} \\
109 \mathrm{NA} \\
109 \mathrm{NB} \\
117 \mathrm{NVH} \\
153 \mathrm{~N} \\
163 \mathrm{~N} \\
163 \mathrm{NA} \\
166 \mathrm{~N} \\
181 \mathrm{~N} \\
181 \mathrm{NA} \\
181 \mathrm{NB} \\
182 \mathrm{~N} \\
183 \mathrm{~N} \\
183 \mathrm{NB} \\
183 \mathrm{NC} \\
183 \mathrm{~N} P \mathrm{PNO} \\
184 \mathrm{~N} \\
184 \mathrm{NA} \\
184 \mathrm{NB} \\
184 \mathrm{NC} \\
184 \mathrm{ND} \\
185 \mathrm{~N}\end{array}$ & 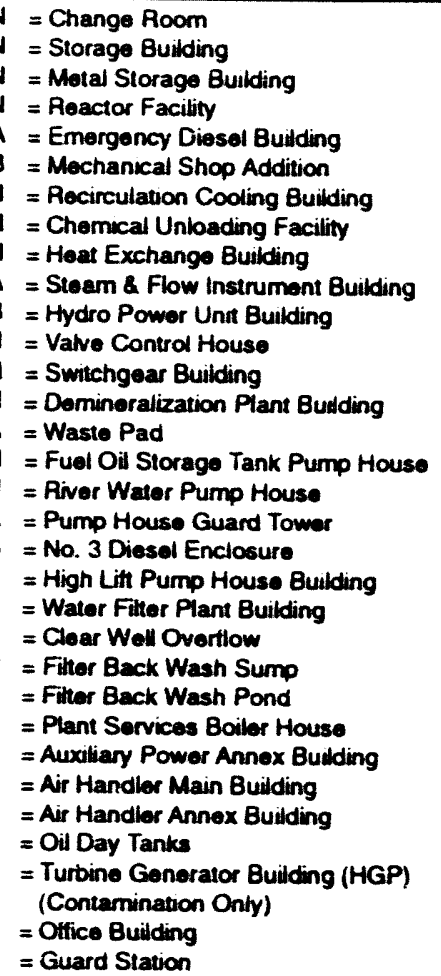 & $\begin{array}{r}1112 \mathrm{NB} \\
1300 \mathrm{~N} \\
11303 \mathrm{~N} \\
\cdot 1304 \mathrm{~N} \\
1310 \mathrm{~N} \\
1313 \mathrm{~N} \\
\cdot 1314 \mathrm{~N} \\
1315 \mathrm{~N} \\
1316 \mathrm{~N} \\
1316 \mathrm{NA} \\
1316 \mathrm{NB} \\
1316 \mathrm{NC} \\
1322 \mathrm{~N} \\
1322 \mathrm{NA} \\
1322 \mathrm{NB} \\
1322 \mathrm{NC} \\
1327 \mathrm{~N} \\
1705 \mathrm{~N} \\
1705 \mathrm{NA} \\
1706 \mathrm{~N} \\
1707 \mathrm{~N} \\
1712 \mathrm{~N} \\
1714 \mathrm{~N} \\
1714 \mathrm{NA} \\
1714 \mathrm{NB} \\
1715 \mathrm{~N} \\
1722 \mathrm{~N} \\
1723 \mathrm{NN} \\
1734 \mathrm{~N} \\
1802 \mathrm{NE} \\
1900 \mathrm{~N} \\
1908 \mathrm{~N}\end{array}$ & 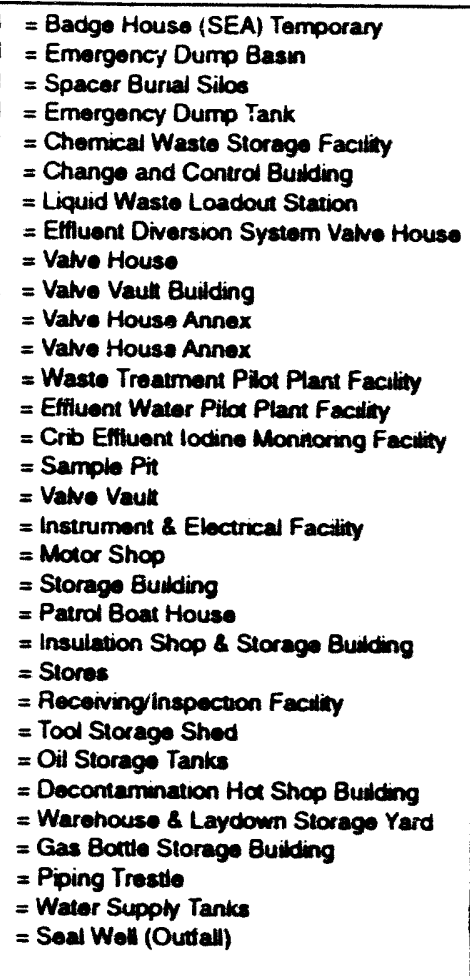 \\
\hline
\end{tabular}

\begin{tabular}{|c|c|}
\hline & Remain in Service \\
\hline $\begin{array}{r}116 \mathrm{~N} \\
117 \mathrm{~N} \\
119 \mathrm{~N} \\
119 \mathrm{NA} \\
151 \mathrm{~N} \\
1112 \mathrm{NA} \\
1120 \mathrm{~N} \\
1143 \mathrm{~N} \\
1702 \mathrm{~N} \\
1904 \mathrm{~N} \\
1904 \text { N } \mathrm{NBNC}\end{array}$ & $\begin{array}{l}=\text { Air Stack } \\
=\text { Air Filter Building Continemont } \\
=\text { Air Sampling Mondor } \\
=\text { Air Sampling Monitor Annex } \\
=230 \text { KV Eloctrical Substation } \\
=\text { Microwave Towor Annex } \\
=\text { Storage \& Training Facility } \\
=\text { Carpenter Shop } \\
=\text { Vohicle inspection Building } \\
=\text { Now Sowage Lagoon } \\
=\text { Sowago Litt Stations No. 1, 2.3 }\end{array}$ \\
\hline
\end{tabular}
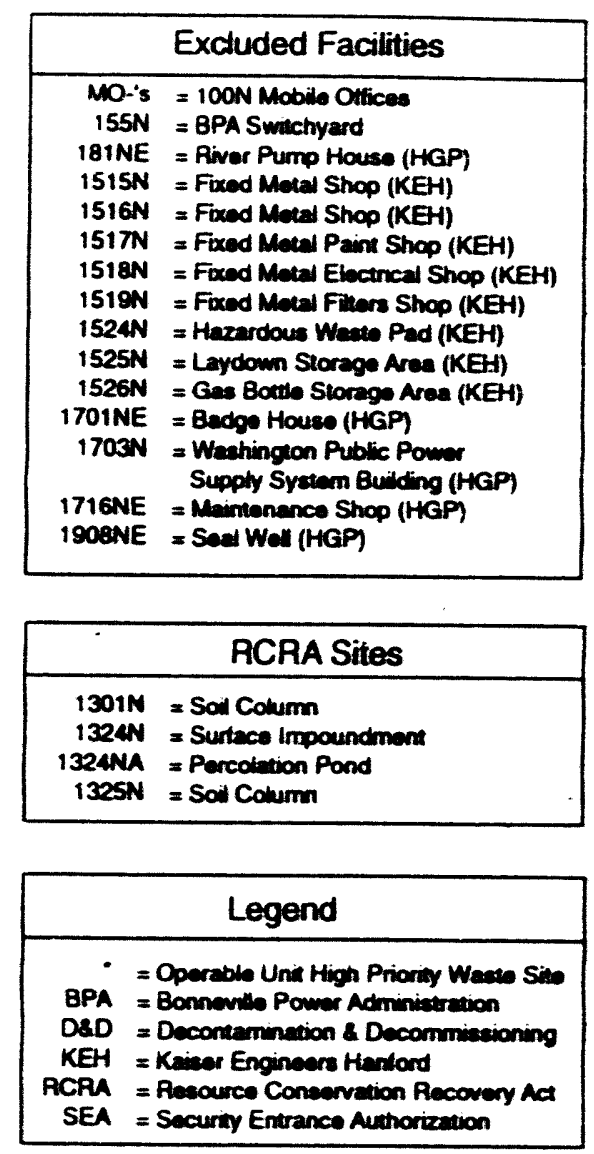

\begin{tabular}{|c|c|}
\hline & Ready for D\&D \\
\hline $\begin{array}{r}128 \mathrm{~N} \\
1100 \mathrm{~N} \\
1101 \mathrm{~N} \\
1312 \mathrm{~N} \\
1706 \mathrm{NA} \\
1903 \mathrm{~N}\end{array}$ & $\begin{array}{l}=100 \mathrm{~N} \text { Burning Pit } \\
=\text { Office Facility - D\&D in Process } \\
=\text { Office Buidding - D\&D in Process } \\
=\text { Liquid Eltluent Retention Facdity } \\
=\text { Old Sower Lit Station } \\
=\text { Old Septic System }\end{array}$ \\
\hline
\end{tabular}

SEA = Security Entrance Authorizatio 


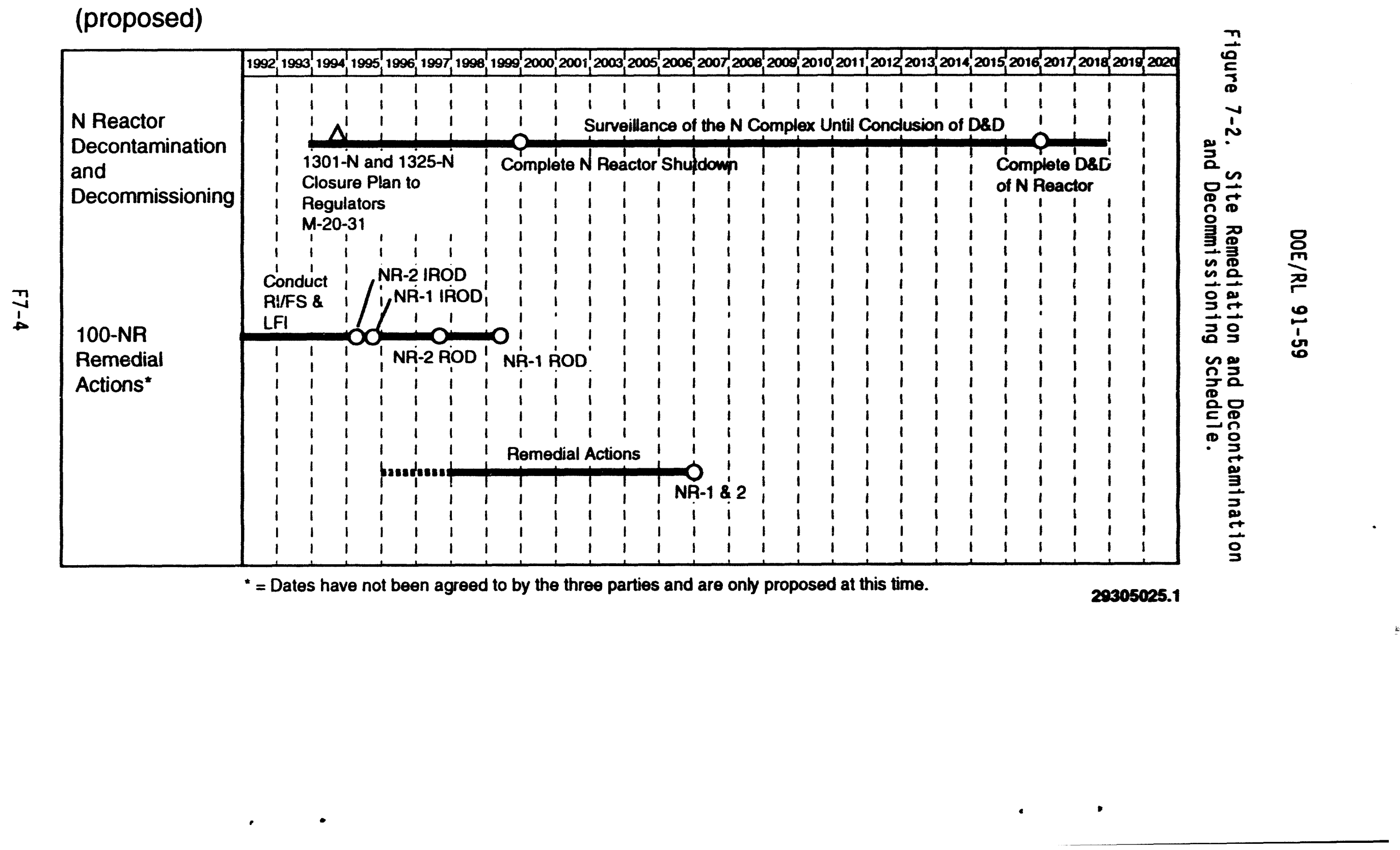




\begin{tabular}{|c|c|c|}
\hline Milestone No. & Milestone Description & Milestone Date \\
\hline$M-20-35$ & $\begin{array}{l}\text { Submit 1301-N LWDF closure plan } \\
\text { Submit 1325-N LWDF closure plan }\end{array}$ & May 1994 \\
\hline$M-20-31$ & Submit 1324-N-NA Surface Impoundment/Percolation Pond closure plan & September 1994 \\
\hline \multicolumn{3}{|c|}{ OPERABLE UNIT REMEDIATION } \\
\hline$M-12-12$ & Submit 100-NR-1 Operable Unit Work Plan & December $1990 c^{*}$ \\
\hline$M-12-14$ & Submit 100-NR-2 Operable Unit Work Plan & December $1990 c^{*}$ \\
\hline$M-15-00$ & Complete RFI/CMS process for all operable units & September 2005 \\
\hline M-16-00 & Complete the remedial actions for all operable units & September 2016 \\
\hline \multicolumn{3}{|c|}{ LIQUID EFFLUENTS } \\
\hline & $\begin{array}{l}\text { Limit discharges to } 1325-\mathrm{N} \text { to less than } \\
2 \mathrm{gpm}\end{array}$ & April 1991c \\
\hline & $\begin{array}{l}\text { Develop a plan to reroute } N \text { Reactor effluent after treatment by BAT } \\
\text { to river }\end{array}$ & January 1992c \\
\hline & $\begin{array}{l}\text { Submit } 216 \text { Application for 100-N Sanitary } \\
\text { Waste Disposal Facility and 183-N Filter Backwash }\end{array}$ & June 1994 \\
\hline \multicolumn{3}{|c|}{ EGULATORY DRIVEN MILESTONES } \\
\hline$M-17-00$ & Complete liquid effluent treatment facilities for all Phase I streams & June 1995 \\
\hline$M-17-10 c$ & $\begin{array}{l}\text { Cease all discharges to hazardous land disposal units unless such } \\
\text { units have been clean closed in accordance with RCRA }\end{array}$ & June $1995 \mathrm{c}$ \\
\hline$M-17-15 a$ & Limit discharges to $1325-N$ to less than $2 \mathrm{gpm}$ & April 1991c \\
\hline$M-17-15 b$ & Submit N Reactor BAT/ACART evaluation & January 1992C \\
\hline$M-17-15 c$ & Submit plan to cease discharge to $1325-\mathrm{N}$ & January $1992 \mathrm{C}$ \\
\hline$M-17-15 d$ & Submit NPDES permit modification & June $1992 c$ \\
\hline
\end{tabular}




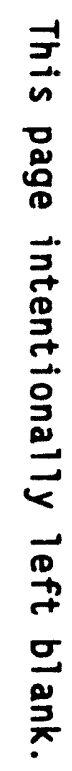

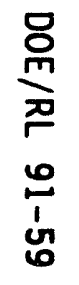

$\frac{1}{n}$ 


\subsection{ESTIMATED COST FOR CLOSURE}

The estimated cost for ER and other remediation and cleanup efforts at the facility are divided into the following work breakdown structure (see Figure 8-1).

- $\quad$ Reactor deactivation

- RCRA facility closures

- RPP site remediation

- D\&D.

The level of confidence behind these cost estimates is commensurate with the engineering and planning currently available. Cost estimates for near-term activities have more detailed planning as a basis than outyear activities (e.g., site remediation and D\&D).

A significant reduction to the estimated deactivation costs is expected to be realized. The effort to revise the cost estimate is ongoing because the deactivation program is refined and optimized based on the outcome of value engineering analyses and other planning activities that are currently being performed (see Table 8-1).

\subsection{N REACTOR DEACTIVATION}

Table 8-1 summarizes the estimated cost for the $N$ Reactor deactivation tasks planned between fiscal year 1992 through fiscal year 1997. A detailed description of the tasks and estimated cost are contained in the $N$ Reactor Deactivation Program Plan (WHC 1992a).

\subsection{RCRA FACILITY CLOSURE}

Table 8-2 summarizes the estimated cost for RCRA closure of the 1301-N LWDF, the 1325-N LWDF, the 1324-N surface impoundment, and the 1324-NA percolation pond as hazardous waste treatment, storage, and disposal facilities (TSDF), pursuant to Washington State "Dangerous Waste Regulations" (WAC 173-300) and the Tri-Party Agreement. If initial investigation shows that the unit no longer contains hazardous waste or constituents, the unit may be clean-closed with no physical closure action. Any remaining materials (radionuclides) would be addressed as part of the past-practices process as designated for an operable unit. The estimated cost for those RCRA closures includes activities such as preparing RCRA closure plans, responding to regulatory comments during the review and approval cycle, and site characterization for determining the absence of hazardous waste to support clean closure under RCRA. This estimate does not include any remedial action, post-closure monitoring, or groundwater monitoring costs. 


\subsection{RCRA PAST PRACTICE SITE REMEDIATION}

Table 8-3 presents the estimated cost for remediation of the 100-NR-1 and 100-NR-2 operable work units, which includes the remedial action and post-closure monitoring at the 1301-N LWDF, 1325-N LWDF, 1324-N surface impoundment, and 1324-NA percolation pond (i.e., the RPP facilities at $100 \mathrm{~N}$ ). Cost estimates for remediation of the RPP sites were developed by the U.S. Corps of Engineers. Estimates assumed complete removal of all contaminated soils and are based on preliminary volume and contaminant data from CPP and RPP sites. These sites include radiologically and chemically contaminated soils from cribs, trenches, ditches, spills, and leaks from past operations. Wastes are assumed to be removed to a disposal facility near the Hanford Site 200 Area. Assessment and remediation costs represent the total estimated costs for site remediation. Estimates for remediation are very preliminary and will be adjusted as more specific data is received to narrow the input parameters to the estimate model.

\subsection{DECONTAMINATION AND DECOMMISSIONING}

Table 8-4 presents the estimated cost for D\&D based on the premise that the $N$ Reactor and all ancillary buildings will be demolished. Figure 8-2 is the cost breakdown for the $105 \mathrm{~N}$ and $109 \mathrm{~N}$ buildings. The cost, which is 89 percent of the total D\&D effort, has been broken into elements to illustrate the impact of radioactive and hazardous waste disposal on the demolition of these structures.

The cost of removing the reactor core is based on dismantling it and transporting it to the burial site in pieces. This method is described in Decommissioning of Eight Surplus Production Reactors at the Hanford Site, Richland, Washington (DOE/EIS 1993).

An alternative method is to move the reactor core in one piece, which is also described in the DOE environmental impact statement (EIS) (1993) and which is the preferred transport method for the eight deactivated reactors at the Hanford Site. However, because the $N$ Reactor sits on a pedestal and the thermal and radiation shielding are not integrated into the core structure, the preferred option may not be feasible. 


\section{DOE/RL 91-59}

Figure 8-1. N Reactor Closure Costs.

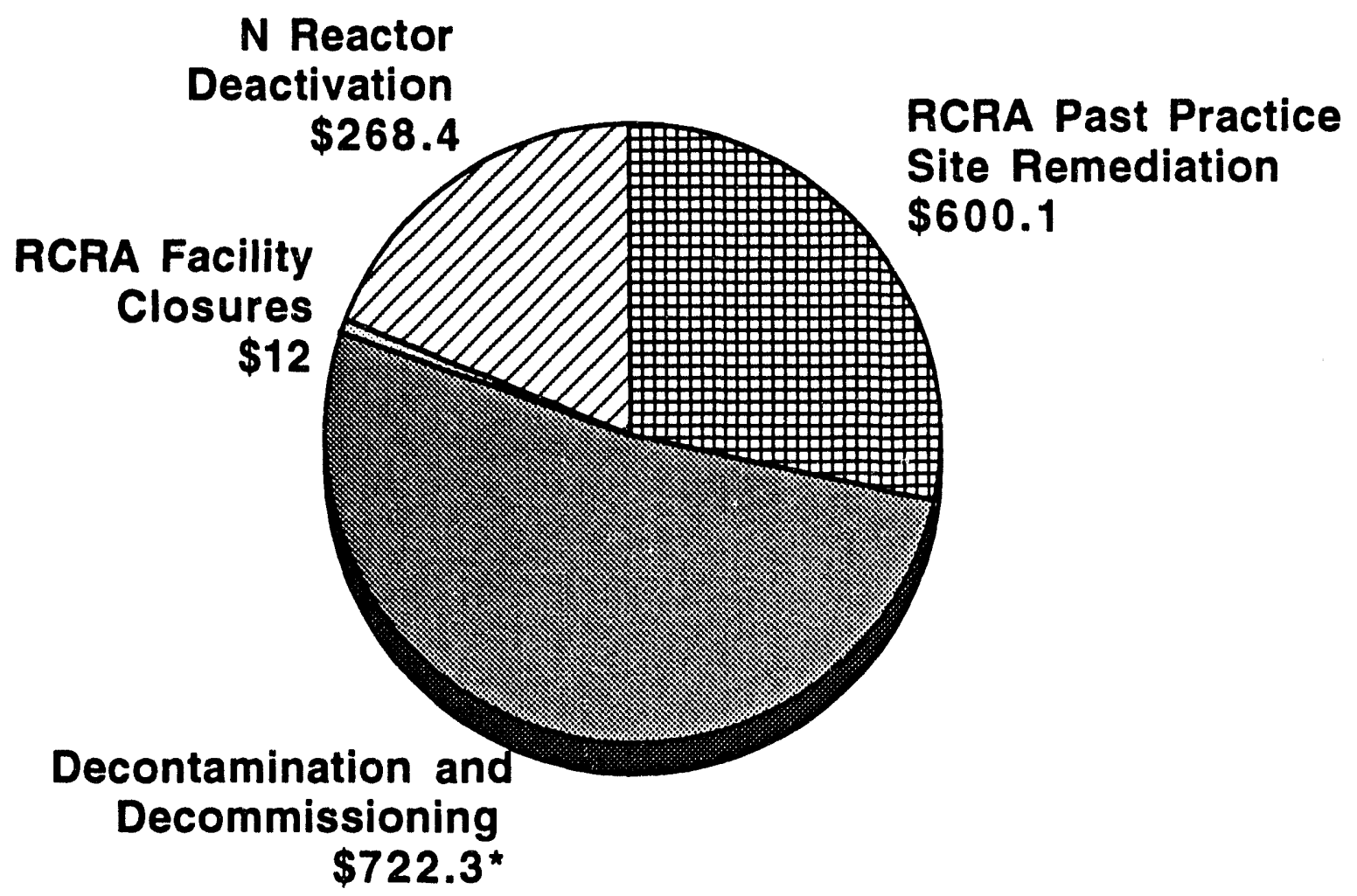

Costs are in millions of dollars

* = not escalated 
Figure 8-2. $105 \mathrm{~N}$ and $109 \mathrm{~N}$ Decontamination and Decommissioning.

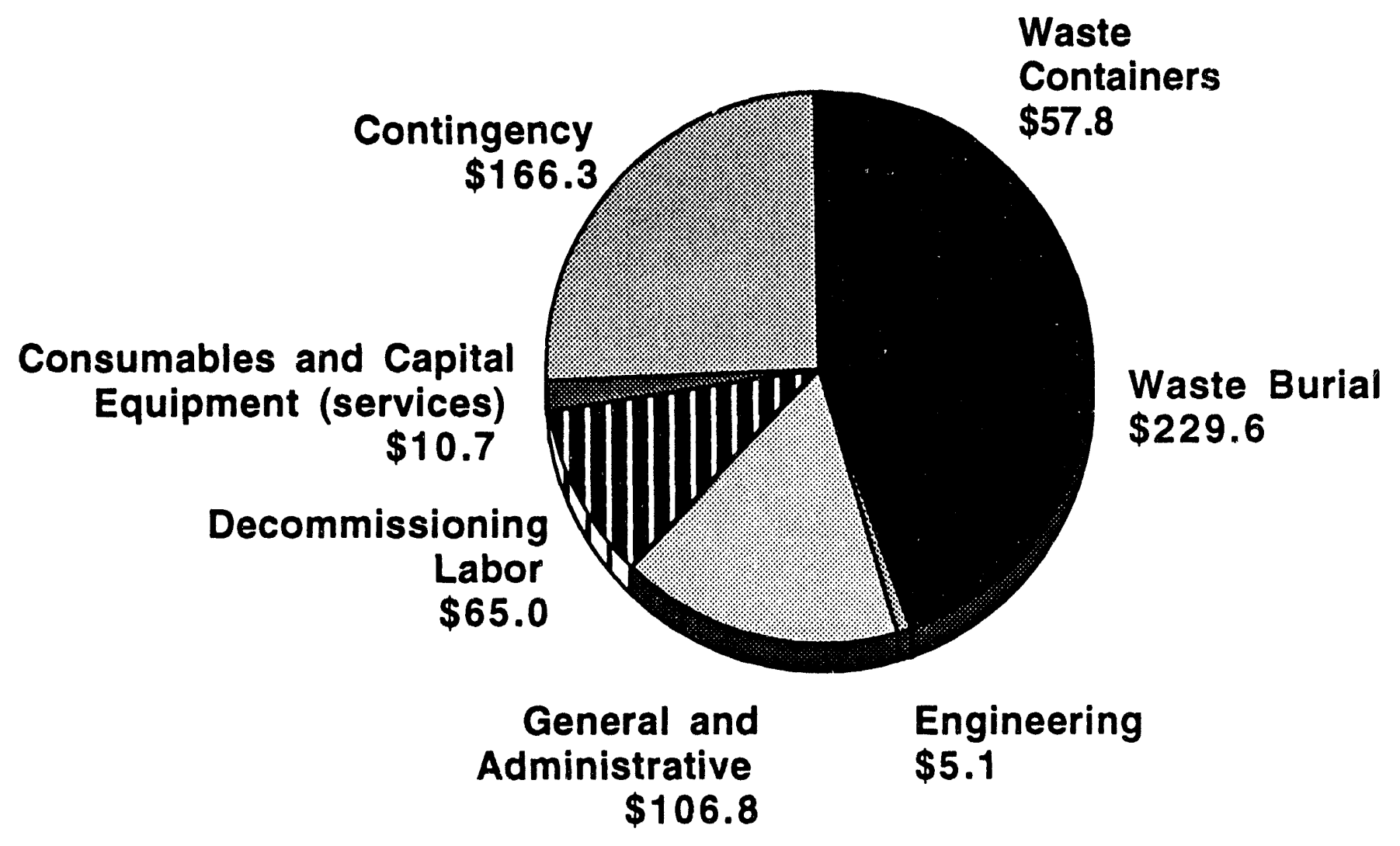

Costs are in million of dollars 
Table 8-1. N Reactor Deactivation Cost Estimate Summary for Tasks Planned Between FY 1992 and FY 1999.

\begin{tabular}{|c|c|c|}
\hline Title & $\begin{array}{l}\text { Subtotals } \\
(\text { (\$M) }\end{array}$ & $\begin{array}{l}\text { Totals } \\
\text { (SM) }\end{array}$ \\
\hline PROGRAM MANAGEMENT & & $\$ 7.9$ \\
\hline SHUTDOWN EXECUTION AND PLANNING & & 218.8 \\
\hline Continuity of Operations & 93.7 & \\
\hline Waste Handling & 12.8 & \\
\hline Waste Disposal & 34.7 & \\
\hline Asset Disposal & 8.7 & \\
\hline Facility Shutdown Execution & 23.8 & \\
\hline Basin Hardware Cleanup & 40.2 & \\
\hline N Reactor Patrol Assessment & 3.8 & \\
\hline N Reactor SAS & 1.1 & \\
\hline REGULATORY RESPONSE & & 41.7 \\
\hline TOTAL & & 268.4 \\
\hline
\end{tabular}

Table 8-2. 100-N Area RCRA Facility Closure Cost Estimate Summary.

\begin{tabular}{|l|c|}
\hline \multicolumn{1}{|c|}{ Facility } & Cost (SM) \\
\hline 1301-N Liquid Waste Disposal Facility & 5 \\
\hline 1325-N Liquid Waste Disposal Facility & 4 \\
\hline $\begin{array}{l}\text { 1324-N/NA Surface Impoundment/ } \\
\text { Percolation Pond }\end{array}$ & 3 \\
\hline
\end{tabular}

Table 8-3. N Reactor RCRA Past Practice Site Remediation.

\begin{tabular}{|l|c|}
\hline \multicolumn{1}{|c|}{ Activity } & Cost (\$M) \\
\hline $100-N$ Operable Unit Assessment & 14,600 \\
\hline $100-N$ Operable Unit Remediation & 585,500 \\
\hline Tota1 & 600,100 \\
\hline
\end{tabular}


Table 8-4. N Reactor Decontamination and Decommissioning Cost Estimate Summary. (2 sheets)

\begin{tabular}{|c|c|}
\hline 105-N Reactor & $\$ 445,600,000$ \\
\hline Asbestos Removal & $2,119,000$ \\
\hline 107-N Basin Waste Recirculation Facility & $20,500,000$ \\
\hline 108-N Chemical Pumping Facility & 639,000 \\
\hline 109-N Heat Exchanger Building & $196,000,000$ \\
\hline 109-NA Steam Flow Instrument Building & 18,000 \\
\hline 109-NB HU building (Hydraulic Power Unit) & 25,000 \\
\hline 1100 Building & 500,000 \\
\hline 1101-N Administrative Office & $1,396,000$ \\
\hline 1102-N Administrative and Training Facilities & 158,000 \\
\hline 1112-N Badge House & 241,000 \\
\hline 1120-N Administrative Training Building & 250,000 \\
\hline 1143-N Carpenter Painters Maintenance Shop & 289,000 \\
\hline 116-N Stack Explosive Demolition & 210,000 \\
\hline 117-N Fiiter Building & $3,726,000$ \\
\hline 117-NVH Valve Control House & 17,000 \\
\hline 1303-N Fuel Spacers Silos (3) & $9,710,000$ \\
\hline 1304-N Dunip Tank & $5,400,000$ \\
\hline 1310-N Liquid Waste Storage Tank & $6,716,000$ \\
\hline 1310-N Chemical Waste Storage Building & 357,000 \\
\hline 1313-N Change and Control Building & 247,000 \\
\hline 1314-N Liquid Waste Loadout & $1,294,000$ \\
\hline 1315-N Effluent Valve Housing & 265,000 \\
\hline 1316-N Valve Housing & 77,000 \\
\hline 1316-NA Valve Vault Building & 77,000 \\
\hline 1316-NB Magnetic Flow Meter & 77,000 \\
\hline 1322-N Waste Treatment Pilot Plant & 543,000 \\
\hline 1322-NA Effluent Water Pilot Annex & 331,000 \\
\hline 1322-NB Crib Effluent Iodine Sample Building & 330,000 \\
\hline 1322-NC Crib Sample Pump Pit & 201,000 \\
\hline
\end{tabular}


Table 8-4. N Reactor Decontamination and Decommissioning Cost Estimate Summary. (2 sheets)

\begin{tabular}{|c|c|}
\hline 151-N Area Electrical Switchgear Building & 183,000 \\
\hline 153-N Area Electrical Switchgear Building & 497,000 \\
\hline 163-N Demineralization Plant & 861,000 \\
\hline 166-N Fuel 0il Storage Pumphouse & 132,000 \\
\hline 1705-N Instrument and Electrical Facility & 152,000 \\
\hline 1705-NA Motor Shop & 194,000 \\
\hline 1707-N Boat House and Boat Launching Facility & 105,000 \\
\hline 1712-N Mechanical Maintenance Insulation Shop & 85,000 \\
\hline 1714-N Warehouse for Smal1 Tools and Parts & 144,000 \\
\hline 1714-NA Receiving and Inspection Building & 75,000 \\
\hline $\begin{array}{l}\text { 1714-NB Tool Storage and Ground Maintenance } \\
\text { Building }\end{array}$ & 136,000 \\
\hline $\begin{array}{l}\text { 1722-N Decon - Hotshop Repair Radioactive Parts } \\
\text { and Equipment }\end{array}$ & 671,000 \\
\hline 1723-N Shipping and Receiving & 230,000 \\
\hline 1734-N Gas Bottle Storage Building & 114,000 \\
\hline 181-N River Pump House & 687,000 \\
\hline 182-N High Lift & $2,678,000$ \\
\hline 183-N Water Filter Plant & 735,000 \\
\hline 184-N Plant Service Power Building & $3,211,000$ \\
\hline 184-NA Auxiliary Power Annex & 388,000 \\
\hline 184-NB Air Handler & 351,000 \\
\hline 1900-N Steamline and Pipe Trestle & $7,304,000$ \\
\hline 1908-N Outfalls & $5,063,000$ \\
\hline Total & $\$ 722,309,000$ \\
\hline
\end{tabular}


DOE/RL 91-59

This page intentionally left blank. 


\subsection{REgULATORY AND U.S. DEPARTMENT OF ENERGY ORDER COHPLIANCE}

This section identifies requirements considered applicable or relevant to the closure of $N$ Reactor and its support facilities. In preparing supporting documents for RCRA and CERCLA activities (e.g., the Hanford Site operable unit work plans [DOE-RL 1990 and 1991]), while ARAR is a term normally associated with environmental remediation documentation, it will be used inclusively for all regulatory and DOE Order requirements associated with the $N$ Reactor closure. The potential ARARs are based on current State and Federal regulations, DOE Orders, and U.S. Nuclear Regulatory Commission (NRC) regulatory guides, NUREGS, and other compliance documents, such as the Tri-Party Agreement and the Liquid Effluent Consent Order.

The identification of potential ARARs will be reassessed during the operable unit RFI/CMS process. As data and information regarding site characterization, future land use, and remedial alternatives become available, the process of identifying potential ARARs will continue.

Tables 9-1 through 9-4 1ist potential ARARs based on whether they are contaminant-specific (Table 9-1), action-specific (Table 9-2), locationspecific (Table 9-3), or pertain to D\&D (Table 9-4). The tables are organized by codes to describe both the type of requirement and to what part of the $\mathrm{N}$ Reactor closure scope they apply.

The first code grouping, ARAR code, defines the potential ARAR in one of four categories of requirements:

ARAR CODES

1. Applicable promulgated regulation

2. Relevant and appropriate promulgated regulation

3. Applicable nonpromulgated requirement (e.g., DOE Orders, Secretary of Energy Directives)

4. Relevant and appropriate nonpromulgated requirement (e.g., NRC Regulatory Guides)

The second code grouping, scope code, defines the part of the $N$ Reactor closure scope to which the potential ARAR applies. Within the scope code grouping are the following categories:

\section{SCOPE CODES}

1. N Reactor deactivation activities

2. RCRA closure activities related to specific treatment and disposal facilities

3. RPP site remediation activities related to past spills and releases to the soil and groundwater

4. D\&D activities related to the dismantling of $\mathbf{N}$ Reactor. 


\section{DOE/RL 91-59}

The tables contain additional information including the number and title of the regulation or requirement (Citation), a summary description of the scope and content of the citation (Requirement), specific or unique requirements that would affect the $N$ Reactor closure (Comment), and applicable actions that must be taken to satisfy the regulation (Resolution). 


\begin{tabular}{|c|c|c|c|c|c|c|}
\hline $\begin{array}{c}\text { ITEM } \\
\text { NUMBER }\end{array}$ & CITATION & $\begin{array}{l}\text { ARAR } \\
\text { COOE } \\
\end{array}$ & REQUIREMENT & COMMENT & RESOLUTIOM & $\begin{array}{c}\text { SCOPE } \\
\text { COOE }\end{array}$ \\
\hline 1 & $\begin{array}{l}\text { Environmental Protection } \\
\text { Agency Rules for controlling } \\
\text { Polychlorinated Biphenyls } \\
\text { under the Toxic Substances } \\
\text { Control Act ( } 40 \text { CFR 761) }\end{array}$ & 1 or 2 & $\begin{array}{l}\text { These regulations control the } \\
\text { manufacture, processing, } \\
\text { storage, disposal, and } \\
\text { cleanup of PCBs present in } \\
\text { quantity greater than } 50 \mathrm{ppm} \text {. }\end{array}$ & $\begin{array}{l}\text { Applicable for spills that } \\
\text { occurred on or after May 4. } \\
\text { 1987. Relevant and } \\
\text { appropriate for spills that } \\
\text { occurred prior to May 4, 1987. }\end{array}$ & $\begin{array}{l}\text { If sampl ing indicates the } \\
\text { presence of PCBs in excess of } \\
50 \text { ppm, and the spill pre--dates } \\
\text { May } 4 \text {. 1987. these regulations } \\
\text { provide guidance for the } \\
\text { clearup and disposal of } \\
\text { PCB-conteminated material. If } \\
\text { the spill post-dates May } 4 \text {, } \\
\text { 1987, these regulations are } \\
\text { appl icable to any cleanup and } \\
\text { disposal activities. }\end{array}$ & $\begin{array}{l}2 \\
3\end{array}$ \\
\hline 2 & $\begin{array}{l}\text { Muclear Regulatory } \\
\text { Cormission Standards for } \\
\text { Protection Against Radiation } \\
\text { (10 CFR 20) }\end{array}$ & 1 & $\begin{array}{l}\text { These regulations apply to } \\
\text { activities licensed by the } \\
\text { Mucl ear Regulatory Comission } \\
\text { and specify radiation dose } \\
\text { standards for individuals in } \\
\text { restricted and unrestricted } \\
\text { areas. } \\
\text { (See Table } 2 \text { for emission } \\
\text { concentration limits.) }\end{array}$ & $\begin{array}{l}\text { Relevant and appropriate } \\
\text { standards for the emission of } \\
\text { specific radionucl ides. }\end{array}$ & $\begin{array}{l}\text { Manajing remediation activities } \\
\text { in a mamer to ensure emission } \\
\text { standards are not exceeded. } \\
\text { Moni toring remediation } \\
\text { activities in a manner to } \\
\text { verify emission standards are } \\
\text { not exceeded. }\end{array}$ & $\begin{array}{l}1 \\
2 \\
3 \\
4\end{array}$ \\
\hline 3 & $\begin{array}{l}\text { Resource Conservation and } \\
\text { Recovery Act of } 1976 \\
\text { (42 USC 6901), subt itle C } \\
\text { Requirements (40 CFR 260-270 } \\
\text { and 280) }\end{array}$ & 1 or 2 & $\begin{array}{l}\text { Governing standards for } \\
\text { oumers and operators of } \\
\text { hazardous waste treatment, } \\
\text { storage, and disposal } \\
\text { facilities, and for } \\
\text { generators and transporters } \\
\text { of hazardous wastes. }\end{array}$ & $\begin{array}{l}\text { Appl icable to any } \\
\text { RCRA-designated hazardous } \\
\text { waste (and the hazardous } \\
\text { components of mixed waste) } \\
\text { generated, transported, } \\
\text { treated, stored, or disposed } \\
\text { of as a result of remedial } \\
\text { actions. specific provisions } \\
\text { also would be appl icable to } \\
\text { any treatment, storage, or } \\
\text { disposal facilities (rsofs) } \\
\text { located in the } 100 \text { areas. }\end{array}$ & & $\begin{array}{l}1 \\
2 \\
3 \\
4\end{array}$ \\
\hline
\end{tabular}




\begin{tabular}{|c|c|c|c|c|c|c|}
\hline $\begin{array}{l}\text { ITEM } \\
\text { NUMBER }\end{array}$ & CITATION & $\begin{array}{l}\text { ARAR } \\
\text { CODE }\end{array}$ & REOUIREMENT & COMMEYT & RESOLUTION & $\begin{array}{l}\text { SCOPE } \\
\text { COOE }\end{array}$ \\
\hline $\begin{array}{c}3 \\
\text { (cont) }\end{array}$ & $\begin{array}{l}\text { Groundwater Protection } \\
\text { Standards ( } 40 \text { CFR 264.94) } \\
\text { Alternate Concentration } \\
\text { Limits (ACLs) }\end{array}$ & 1 or 2 & $\begin{array}{l}\text { ACLs may be used to establ ish } \\
\text { standards for the cleanup of } \\
\text { groundwater under the } \\
\text { foll lowing conditions: } \\
\text { 1. There are known and } \\
\text { projected points of entry of } \\
\text { such groundwater into surface } \\
\text { water. } \\
2 \text {. On the basis of } \\
\text { measurements or projections, } \\
\text { there is or will be no } \\
\text { statist ically significant } \\
\text { increase of such constituents } \\
\text { from such groundwater in such } \\
\text { surface water at the point of } \\
\text { entry or at any point where } \\
\text { there is reason to bel ieve } \\
\text { accumulation of constituents } \\
\text { may occur downstream. } \\
3 \text {. The remedial action } \\
\text { inciudes enforceable measures } \\
\text { that will preclude human } \\
\text { exposure to the contaminated } \\
\text { groundwater at any point } \\
\text { between the facility boundary } \\
\text { and all known and projected } \\
\text { points of entry into surface } \\
\text { water. }\end{array}$ & $\begin{array}{l}\text { ACLs are applicable to } \\
\text { existing RCRA-regulated } \\
\text { operating units, and may be } \\
\text { relevant and appropriate for } \\
\text { non-RCRA sources of } \\
\text { groundwater contamination in } \\
\text { accordance with CERCLA } 121 \\
\text { (d)(2)(B)(ii) }\end{array}$ & $\begin{array}{l}\text { Treatment or stabil ization } \\
\text { (where appl icable) of RCRA } \\
\text { wastes before landfill } \\
\text { disposal. }\end{array}$ & $\begin{array}{l}1 \\
2 \\
3 \\
4\end{array}$ \\
\hline $\begin{array}{c}3 \\
\text { (cont) }\end{array}$ & $\begin{array}{l}\text { Land Disposal Restrictions } \\
\text { ( } 40 \text { CFR 268) }\end{array}$ & 1 & $\begin{array}{l}\text { Regulations contain specific } \\
\text { provisions for restricting } \\
\text { the land disposal of RCRA } \\
\text { hazardous wastes Listed } \\
\text { wastes (def ined in Subpart D } \\
\text { CFR } 261 \text { ) and materials with } \\
\text { the following codes: } \\
\text { F waste code (Part } 261.31 \text { ), } \\
K \text { waste code (Part 261.32), } \\
\text { P waste code (Part } \\
261.33(e)) \text {, } \\
U \text { waste code (Part } \\
261.33(f)) \text {. } \\
\text { Or, the presence of a waste } \\
\text { exhibiting one or more of the } \\
\text { fol lowing characteristics, as } \\
\text { def ined in } 40 \text { CFR } 261 \text { : } \\
\text { ignitability, corrosivity, } \\
\text { reactivity, Extraction } \\
\text { Procedure (EP) }\end{array}$ & $\begin{array}{l}\text { The regulations are appl icable } \\
\text { for intrusive investigation } \\
\text { actions and reaedial actions } \\
\text { requiring the disposal of a } \\
\text { listed RCRA waste. }\end{array}$ & & $\begin{array}{l}1 \\
2 \\
3 \\
4\end{array}$ \\
\hline
\end{tabular}




\begin{tabular}{|c|c|c|c|c|c|c|}
\hline $\begin{array}{l}\text { ITEM } \\
\text { NUMBER }\end{array}$ & CITATION & $\begin{array}{l}\text { ARAR } \\
\text { COOE }\end{array}$ & REQUIREMENT & COMMENT & RESOLUTION & $\begin{array}{l}\text { SCOPE } \\
\text { COOE }\end{array}$ \\
\hline 4 & $\begin{array}{l}\text { Safe Drinking Water Act } \\
\text { ( } 42 \text { U.S.C. (F)) and the } \\
\text { regulations issued pursuant } \\
\text { to the act in } 40 \text { CFR } 141.11 \\
\text { Subpart B and } 40 \text { CFR 143.3; } \\
\text { Maximum Contaminant Levels } \\
\text { (MCLs): Maximum Contaminant } \\
\text { Level Goals (MCLGS) }\end{array}$ & 1 or 2 & $\begin{array}{l}\text { MCLs are applicable at the } \\
\text { tap where the water will be } \\
\text { provided directly to } 25 \text { or } \\
\text { more people or will be } \\
\text { supplied to } 15 \text { or more } \\
\text { service connections. } \\
\text { Otherwise, where surface } \\
\text { water or groundwater is or } \\
\text { may be used for drinking, } \\
\text { MCLs are generally relevant } \\
\text { and appropriate as cleanup } \\
\text { standards for surface water } \\
\text { or the groundwater. } \\
\text { (MCLs and MCLGs are presented } \\
\text { in Table 1.) }\end{array}$ & $\begin{array}{l}\text { Groundwater is not used for } \\
\text { drinking, and institutional } \\
\text { controls can prevent future } \\
\text { use. However, there is } \\
\text { potential for and actual } \\
\text { discharge of contaminated } \\
\text { groundwater to the Columbia } \\
\text { River. which is used as a } \\
\text { drinking water source by } \\
\text { downstream comminities. MCLs } \\
\text { are not legally enforceable; } \\
\text { however, they may be relevant } \\
\text { and appropriate to provide } \\
\text { guidance for cleanup levels. }\end{array}$ & & $\begin{array}{l}2 \\
3 \\
4\end{array}$ \\
\hline 5 & $\begin{array}{l}\text { Clean Air Act } \\
\text { ( } 42 \text { U.S.C. } 7401 \text { ) } \\
\text { a) National Primary and } \\
\text { Secondary Ambient air } \\
\text { Qual ity Standards } \\
\text { ( } 40 \text { CFR 50) } \\
\text { b) New Source Performance } \\
\text { Standards ( } 40 \text { CFR 60) } \\
\text { c) National Emission } \\
\text { standards for Hazardous Air } \\
\text { Pollutants ( } 40 \text { CFR 61) }\end{array}$ & 1 & $\begin{array}{l}\text { a) } 40 \text { CFR } 50 \text { provides for the } \\
\text { establishment of } \\
\text { nonradionuclide air emission } \\
\text { standards. } \\
\text { b) } 40 \text { CFR } 52 \text { provides for the } \\
\text { establishment of air quality } \\
\text { districts and State air } \\
\text { quality plans } \\
\text { c) } 40 \text { CFR } 61 \text { provides for the } \\
\text { establishes air emission } \\
\text { standards for radionuclides } \\
\text { at DOE owned/operated } \\
\text { facilities }\end{array}$ & & & $\begin{array}{l}1 \\
2 \\
3 \\
4\end{array}$ \\
\hline 6 & $\begin{array}{l}\text { Clean Water Act } \\
\text { (33 U.S.C. 1251) }\end{array}$ & 1 & $\begin{array}{l}\text { Establ ishes water quality } \\
\text { standards for surface waters } \\
\text { and pretreatment standards } \\
\text { for waste waters released to } \\
\text { public-owned treatment works } \\
\text { (POTWs). }\end{array}$ & & & $\begin{array}{l}1 \\
2 \\
3\end{array}$ \\
\hline 7 & $\begin{array}{l}\text { DOE Order } 5400.5 \text {, Radiation } \\
\text { Protection of the Public and } \\
\text { the Environment }\end{array}$ & 3 & $\begin{array}{l}\text { Specifies criteria for } \\
\text { protection of the public } \\
\text { (e.g., } 100 \text { mrem } / \text { yr EDE } \\
\text { resulting from DOE operations } \\
\text { for all exposure pathways). } \\
\text { Establishes residual } \\
\text { radioactive materials } \\
\text { criteria. }\end{array}$ & Monpromul gated. & & $\begin{array}{l}1 \\
2 \\
3 \\
6\end{array}$ \\
\hline
\end{tabular}




\begin{tabular}{|c|c|c|c|c|c|c|}
\hline $\begin{array}{l}\text { IIEM } \\
\text { NUMBER }\end{array}$ & CITATION & $\begin{array}{l}\text { ARAR } \\
\text { COOE }\end{array}$ & REQUIREMENT & COMMENT & RESOLUTION & $\begin{array}{l}\text { SCOPE } \\
\text { COOE }\end{array}$ \\
\hline 8 & $\begin{array}{l}\text { Health Advisories, EPA } \\
\text { Office of Drinking Water }\end{array}$ & 3 & $\begin{array}{l}\text { Heal th advisories should be } \\
\text { considered to determine risk } \\
\text { when promulgated standards } \\
\text { are not available or for } \\
\text { multiple contaminants and } \\
\text { routes of exposure then ARARs } \\
\text { are not sufficiently } \\
\text { protective. }\end{array}$ & & $\begin{array}{l}\text { Standards to provide guidance } \\
\text { for development of cleanup } \\
\text { levels. }\end{array}$ & $\begin{array}{l}2 \\
3\end{array}$ \\
\hline 9 & $\begin{array}{l}\text { Washington Standards for } \\
\text { Protection Against Radiation } \\
\text { (HAC 402-24) }\end{array}$ & 1 & $\begin{array}{l}\text { State regulations specifying } \\
\text { radiation dose standards for } \\
\text { permissible levels of } \\
\text { radiation in unrestricted } \\
\text { areas. Table II of } \\
\text { Appendix A itemizes the } \\
\text { allowable concentrations in } \\
\text { air above natural background. } \\
\text { The values in Table II are } \\
\text { the same as the values in } \\
\text { Table II, appendix B, Muclear } \\
\text { Regulatory Commission } \\
\text { Standards (10 CFR 20). }\end{array}$ & & $\begin{array}{l}\text { Managing remediation activities } \\
\text { in a manner to ensure emission } \\
\text { standards are not exceeded. } \\
\text { Monitoring remediation } \\
\text { activities in a manner to } \\
\text { verify emission standards are } \\
\text { not exceeded. }\end{array}$ & $\begin{array}{l}1 \\
2 \\
3 \\
4\end{array}$ \\
\hline 10 & $\begin{array}{l}\text { Washington Ambient Air } \\
\text { Qual ity Standards and } \\
\text { Emission Limits for } \\
\text { Radionucl ides (WAC 173-480) }\end{array}$ & 1 & $\begin{array}{l}\text { Ambient air qual ity standards } \\
\text { and radionuclide emission } \\
\text { limits mandate that } \\
\text { radionuclides in the air mist } \\
\text { not cause a maximm } \\
\text { accumul ated dose equi } \\
\text { of more than } 25 \text { mill } \\
\text { to the whole body or } \\
75 \text { mill irem/year to a } \\
\text { critical organ of any member } \\
\text { of the publ ic (excluding } \\
\text { doses from radon and radon } \\
\text { decay products). (NOTE: } \\
\text { These standards were adopted } \\
\text { by the Washington State } \\
\text { Department of Social and } \\
\text { Heal th Services by reference } \\
\text { to WAC-402-80-050.) }\end{array}$ & & $\begin{array}{l}\text { Managing remediation activities } \\
\text { in a manner to ensure emission } \\
\text { standards are not exceeded. } \\
\text { Monitoring remediation } \\
\text { activities in a manner to } \\
\text { verify emission standards are } \\
\text { not exceeded. }\end{array}$ & $\begin{array}{l}1 \\
2 \\
3 \\
4\end{array}$ \\
\hline 11 & $\begin{array}{l}\text { Washington Model Toxics ACt } \\
\text { (Ch } 70.105 \mathrm{RCW} \text { ) and the } \\
\text { regulations issued pursuant } \\
\text { to it under Model Toxics } \\
\text { control Act Cles. Up } \\
\text { Regulations (Ch } 173-340 \text { WAC) }\end{array}$ & 1 & $\begin{array}{l}\text { Regulations for } \\
\text { contaminated-site cleanup. } \\
\text { Contains standards and } \\
\text { methods for establ ishing } \\
\text { cleanup levels groundwater, } \\
\text { surface water, soil, and air } \\
\text { emissions. }\end{array}$ & & & $\begin{array}{l}1 \\
2 \\
3 \\
4\end{array}$ \\
\hline
\end{tabular}




\begin{tabular}{|c|c|c|c|c|c|c|}
\hline $\begin{array}{l}\text { ITEM } \\
\text { NUMBER }\end{array}$ & CITATION & $\begin{array}{l}\text { ARAR } \\
\text { COOE }\end{array}$ & REQUIREMENT & CONMENT & RESOLUTION & $\begin{array}{l}\text { SCOPE } \\
\text { COOE }\end{array}$ \\
\hline 1 & $\begin{array}{l}\text { Occupational Safety and } \\
\text { Heal th Standards (OSHA) } \\
\text { (29 CFR 1910) }\end{array}$ & 1 & $\begin{array}{l}\text { United States Department of } \\
\text { Labor Standards for Workplace } \\
\text { Heal th and Safety. }\end{array}$ & $\begin{array}{l}\text { Applicable for investigation } \\
\text { and remedial action. }\end{array}$ & $\begin{array}{l}\text { Development of workplans and } \\
\text { methods for site } \\
\text { invest igat ion and remediation } \\
\text { that will meet OSHA standards } \\
\text { for heal th and safety. }\end{array}$ & $\begin{array}{l}1 \\
2 \\
3 \\
4\end{array}$ \\
\hline 2 & $\begin{array}{l}\text { Radiation Protection } \\
\text { Standards for Managing } \\
\text { and Disposing of Spent } \\
\text { Nucl ear Fuel, High-level } \\
\text { and Transuranic } \\
\text { Radi oactive Hastes (40 } \\
\text { CFR 191) }\end{array}$ & 1 & 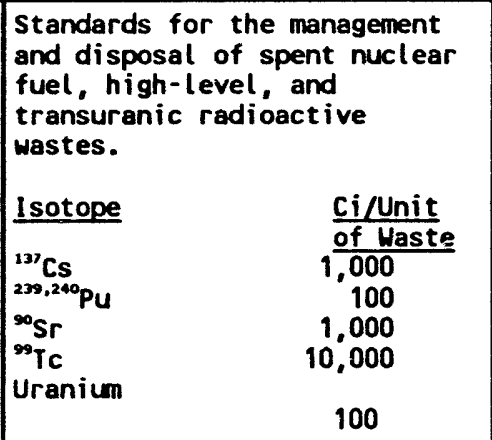 & $\begin{array}{l}\text { Applicable for actions } \\
\text { requiring the management and } \\
\text { disposal of spent nuclear } \\
\text { fuel, high-l evel or } \\
\text { transuranic radioactive } \\
\text { wastes. }\end{array}$ & & $\begin{array}{l}1 \\
3 \\
4\end{array}$ \\
\hline 3 & $\begin{array}{l}\text { Underground Injection } \\
\text { Contro! Regulations (40 } \\
\text { CFR 144-147) }\end{array}$ & 1 & $\begin{array}{l}\text { Define the standards for the } \\
\text { placement of treated } \\
\text { groundwater into an aquifer. }\end{array}$ & $\begin{array}{l}\text { May be applicable for remedial } \\
\text { action requiring the } \\
\text { reinjection of treated } \\
\text { groundwater. }\end{array}$ & & 2 \\
\hline 4 & $\begin{array}{l}\text { Heal th Physics Manual of } \\
\text { Good Practices for } \\
\text { Reducing Radiation } \\
\text { Exposure to Levels that } \\
\text { are ALARA (PNL-65577) } \\
\end{array}$ & 3 & $\begin{array}{l}\text { DOE guidance for the } \\
\text { implementation of As Low As } \\
\text { Reasonably Achievable (ALARA) } \\
\text { policy with respect to } \\
\text { exposure. }\end{array}$ & Nonpromul gated. & & $\begin{array}{l}1 \\
2 \\
3 \\
4\end{array}$ \\
\hline 5 & $\begin{array}{l}\text { DOE Order } 5820.2 \mathrm{~A}, \\
\text { Radioact ive Waste } \\
\text { Management }\end{array}$ & 3 & $\begin{array}{l}\text { Establishes the criteria for } \\
\text { the required radioactive waste } \\
\text { activities associated with a } \\
\text { DOE operation including action } \\
\text { to minimize and stabilize } \\
\text { waste. Addresses the } \\
\text { management, treatment and } \\
\text { disposal of low level } \\
\text { radioactive waste. }\end{array}$ & Nonpromulgated. & • & $\begin{array}{l}1 \\
2 \\
3 \\
4\end{array}$ \\
\hline 6 & $\begin{array}{l}\text { DOE Order } 5480.3 \text {, Safety } \\
\text { Requirements for the } \\
\text { Packaging and } \\
\text { Transportation of } \\
\text { Hazardous Materials, } \\
\text { Hazardous Substances, and } \\
\text { Hazardous Hastes }\end{array}$ & 3 & $\begin{array}{l}\text { Specifies requirements for the } \\
\text { label ing and packaging of } \\
\text { hazardous materials, hazardous } \\
\text { substances and hazardous } \\
\text { wastes in addition to those } \\
\text { specified under } 49 \text { cFR (U.S. } \\
\text { Department of Iransportation } \\
\text { regulations for transportation } \\
\text { of hazardous materials). }\end{array}$ & Nonpromul gated. & & $\begin{array}{l}1 \\
2 \\
3 \\
4\end{array}$ \\
\hline
\end{tabular}




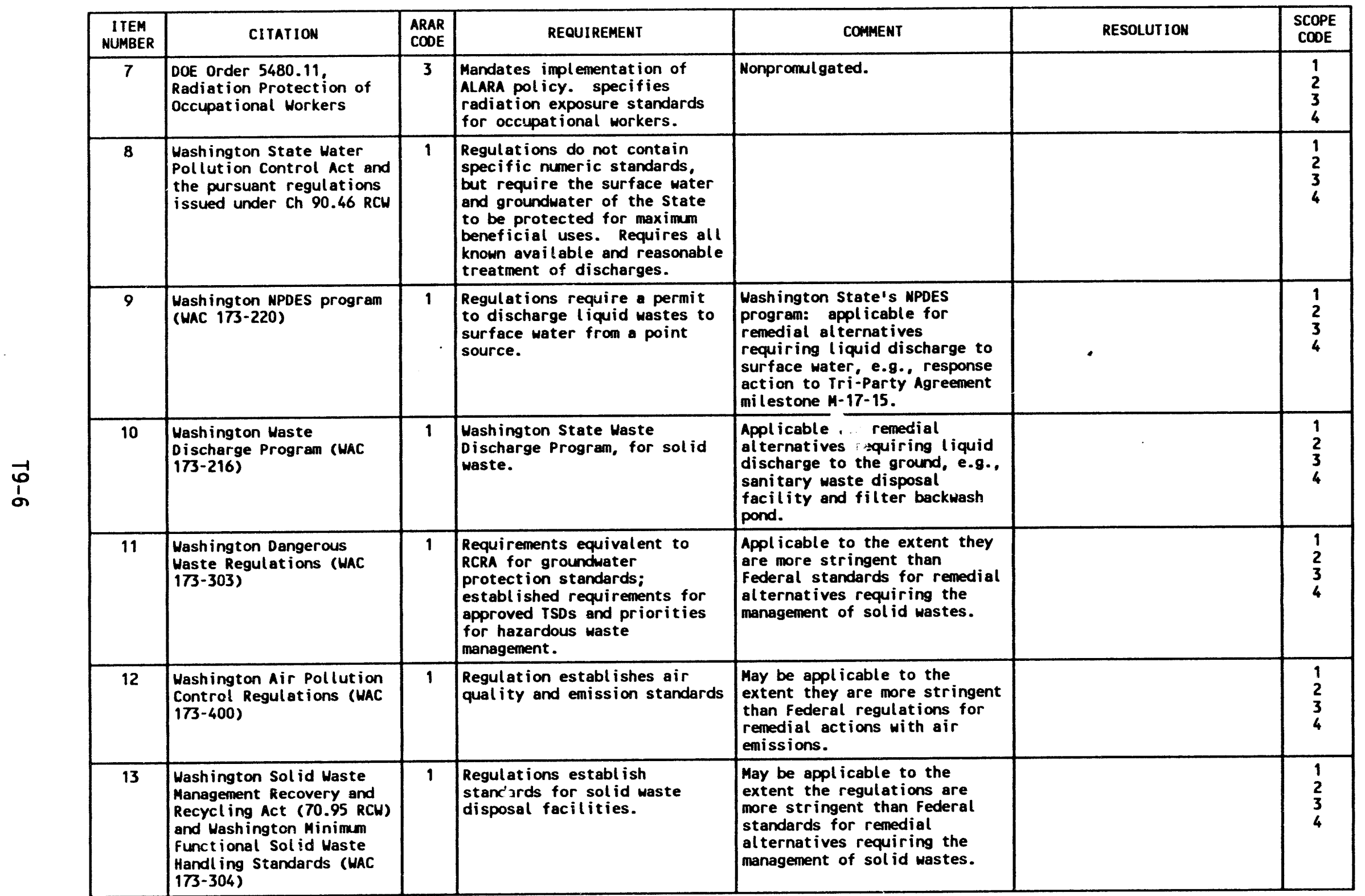




\begin{tabular}{|c|c|c|c|c|c|c|}
\hline $\begin{array}{c}\text { I TEM } \\
\text { NUMBER }\end{array}$ & CITATION & $\begin{array}{l}\text { ARAR } \\
\text { CODE }\end{array}$ & REQUIREMENT & COMMENT & RESOLUTION & $\begin{array}{c}\text { SCOPE } \\
\text { COOE }\end{array}$ \\
\hline 14 & $\begin{array}{l}\text { Washington winimum } \\
\text { Standards for } \\
\text { construction and } \\
\text { Maintenance of Water } \\
\text { Hel ls (WAC 173-160) }\end{array}$ & 1 & $\begin{array}{l}\text { Regulations include minimum } \\
\text { standards for the construction } \\
\text { of resource protection wells } \\
\text { and water supply wells. }\end{array}$ & $\begin{array}{l}\text { Appl icable for monitoring } \\
\text { wells and remedial } \\
\text { al ternatives requiring } \\
\text { extraction wells. }\end{array}$ & $\begin{array}{l}\text { Construction of monitoring } \\
\text { wells and extraction wells to } \\
\text { meet standards set forth in } \\
\text { this regulation. }\end{array}$ & $\begin{array}{l}1 \\
2 \\
3\end{array}$ \\
\hline 15 & $\begin{array}{l}40 \text { CFR } 61 \text { Subpart } M, \\
\text { revised by } 55 \text { FR } 48414 \text {, } \\
\text { November } 20,1990\end{array}$ & 1 & $\begin{array}{l}\text { Regulations contain standards } \\
\text { for the removal and disposal } \\
\text { of asbestos-fiber-containing } \\
\text { material. }\end{array}$ & $\begin{array}{l}\text { Applicable for remedial } \\
\text { actions involving the removal } \\
\text { and disposal of asbestos- } \\
\text { fiber-containing material. }\end{array}$ & & 4 \\
\hline
\end{tabular}




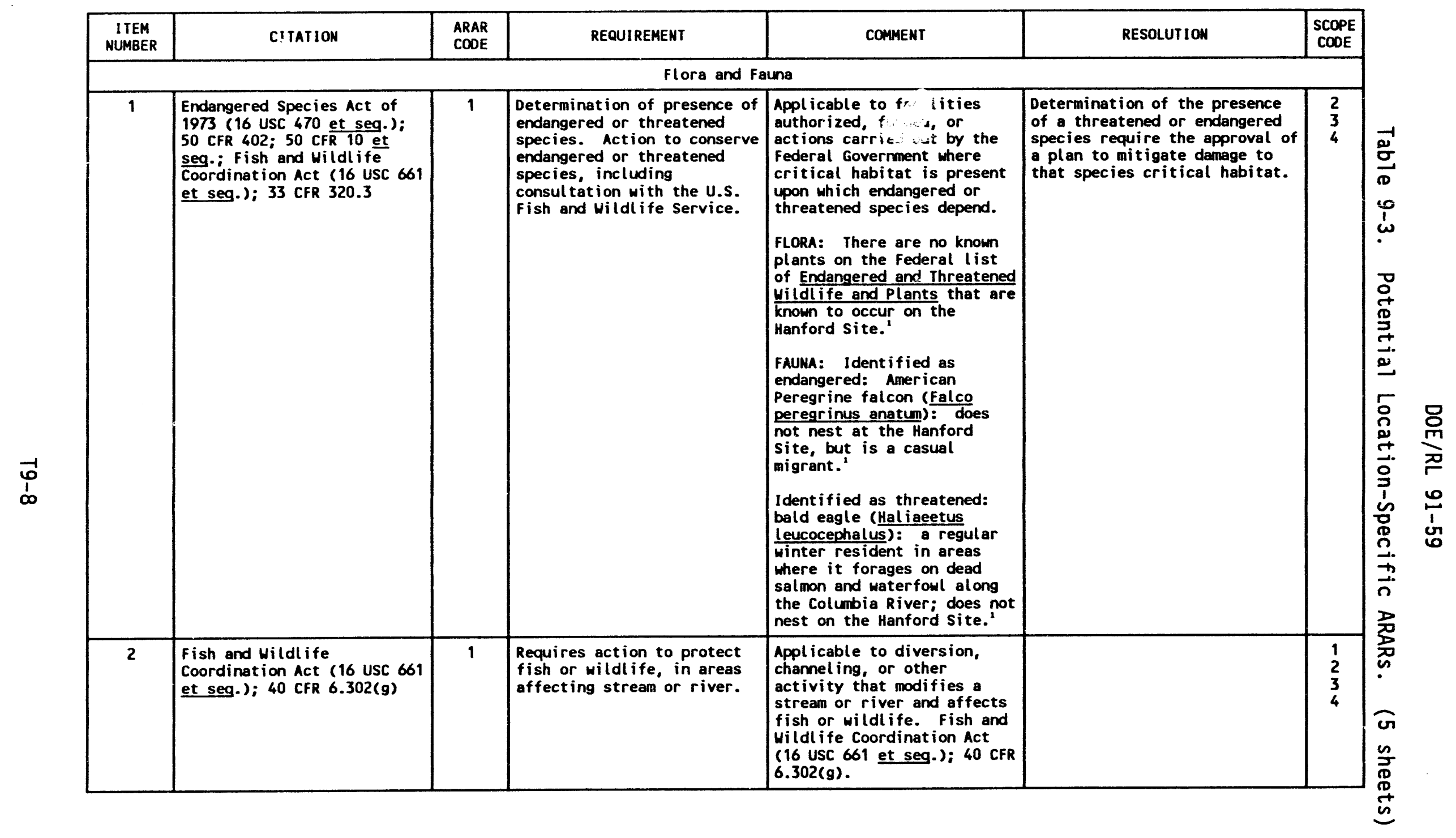




\begin{tabular}{|c|c|c|c|c|c|c|}
\hline $\begin{array}{l}\text { IIEM } \\
\text { NUMBER } \\
\end{array}$ & CITATION & $\begin{array}{l}\text { ARAR } \\
\text { COOE }\end{array}$ & REQUIREMENT & COMMENT & RESOLUTION & $\begin{array}{l}\text { SCOPE } \\
\text { COOE } \\
\end{array}$ \\
\hline 3 & $\begin{array}{l}\text { Wild and Scenic Rivers Act } \\
\text { (16 uSC 1271) }\end{array}$ & 1 & $\begin{array}{l}\text { Protection of rivers } \\
\text { designated as wild or scenic } \\
\text { or designated as a "study" } \\
\text { river on the National Rivers } \\
\text { Inventory. Requires action } \\
\text { to protect the free-flowing } \\
\text { characteristics or scenic, } \\
\text { recreational, or fish and } \\
\text { wildt ife values of a Hild } \\
\text { and Scenic River or Study } \\
\text { River. } \\
\end{array}$ & $\begin{array}{l}\text { Applicable to action } \\
\text { involving construction of a } \\
\text { water-resource project } \\
\text { broadly defined to include } \\
\text { construction or development } \\
\text { on adjacent shorel ines. } \\
\text { Draft EIs has been prepared } \\
\text { for designation of the } \\
\text { Hanford Reach of the } \\
\text { columbia River. }\end{array}$ & & $\begin{array}{l}1 \\
2 \\
3 \\
4\end{array}$ \\
\hline 4 & $\begin{array}{l}\text { Fish and Wildlife } \\
\text { Improvement Act } 16 \text { uSC 742) }\end{array}$ & 1 & $\begin{array}{l}\text { Requires action to conserve } \\
\text { and promote conservation of } \\
\text { fish and wildife and their } \\
\text { habitat. }\end{array}$ & $\begin{array}{l}\text { Applicable to actions that } \\
\text { will have an adverse effect } \\
\text { on fish and wildife or } \\
\text { their habitat. } \\
\end{array}$ & & $\begin{array}{l}1 \\
2 \\
3 \\
4 \\
\end{array}$ \\
\hline 5 & $\begin{array}{l}\text { Fish and Wildlife } \\
\text { Conservation Act (16 USC } \\
\text { 2901) }\end{array}$ & 1 & $\begin{array}{l}\text { Within an area that may } \\
\text { impact nongame fish and } \\
\text { wildt ife, requires action to } \\
\text { conserve and promote } \\
\text { conservation of nongame fish } \\
\text { and wildl ife and their } \\
\text { habitat. }\end{array}$ & $\begin{array}{l}\text { Applicable to actions that } \\
\text { will have an adverse effect } \\
\text { on nongame fish and wildlife } \\
\text { habi tat. }\end{array}$ & & $\begin{array}{l}1 \\
2 \\
3 \\
4\end{array}$ \\
\hline 6 & 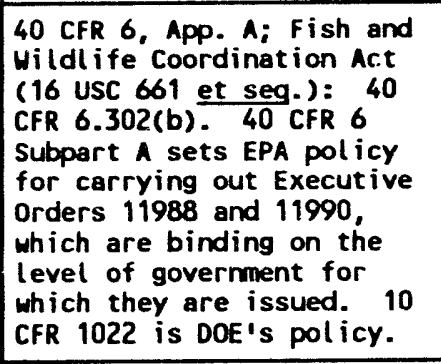 & 1 & $\begin{array}{l}\text { Requires action to avoid } \\
\text { adverse effects, minimize } \\
\text { potential harm, and restore } \\
\text { and preserve natural and } \\
\text { beneficial values within } \\
\text { floodplain. }\end{array}$ & $\begin{array}{l}\text { Applicable to actions that } \\
\text { occur in a floodplain, such } \\
\text { as lowlands and relat ively } \\
\text { flat areas adjoining inland } \\
\text { waters and other flood-prone } \\
\text { areas. }\end{array}$ & & $\begin{array}{l}1 \\
2 \\
3 \\
4\end{array}$ \\
\hline 7 & $\begin{array}{l}40 \text { CFR 6, Appendix } A_{i} \\
\text { Executive Order 11990, } \\
\text { Protection of Wetlands; } 10 \\
\text { CFR } 1022 \text { (DOE pol icy) }\end{array}$ & 1 & $\begin{array}{l}\text { Requires action to the } \\
\text { extent possible to avoid } \\
\text { adverse effect, minimize } \\
\text { potential harm, and preserve } \\
\text { and enhance wetlands. }\end{array}$ & \begin{tabular}{|l|} 
Applicable to action \\
involving construction of \\
facilities or management of \\
property in wetlands, as \\
defined by 40 CFR 6, App. A, \\
Section $4(j)$.
\end{tabular} & & $\begin{array}{l}1 \\
2 \\
3 \\
4 \\
4\end{array}$ \\
\hline
\end{tabular}




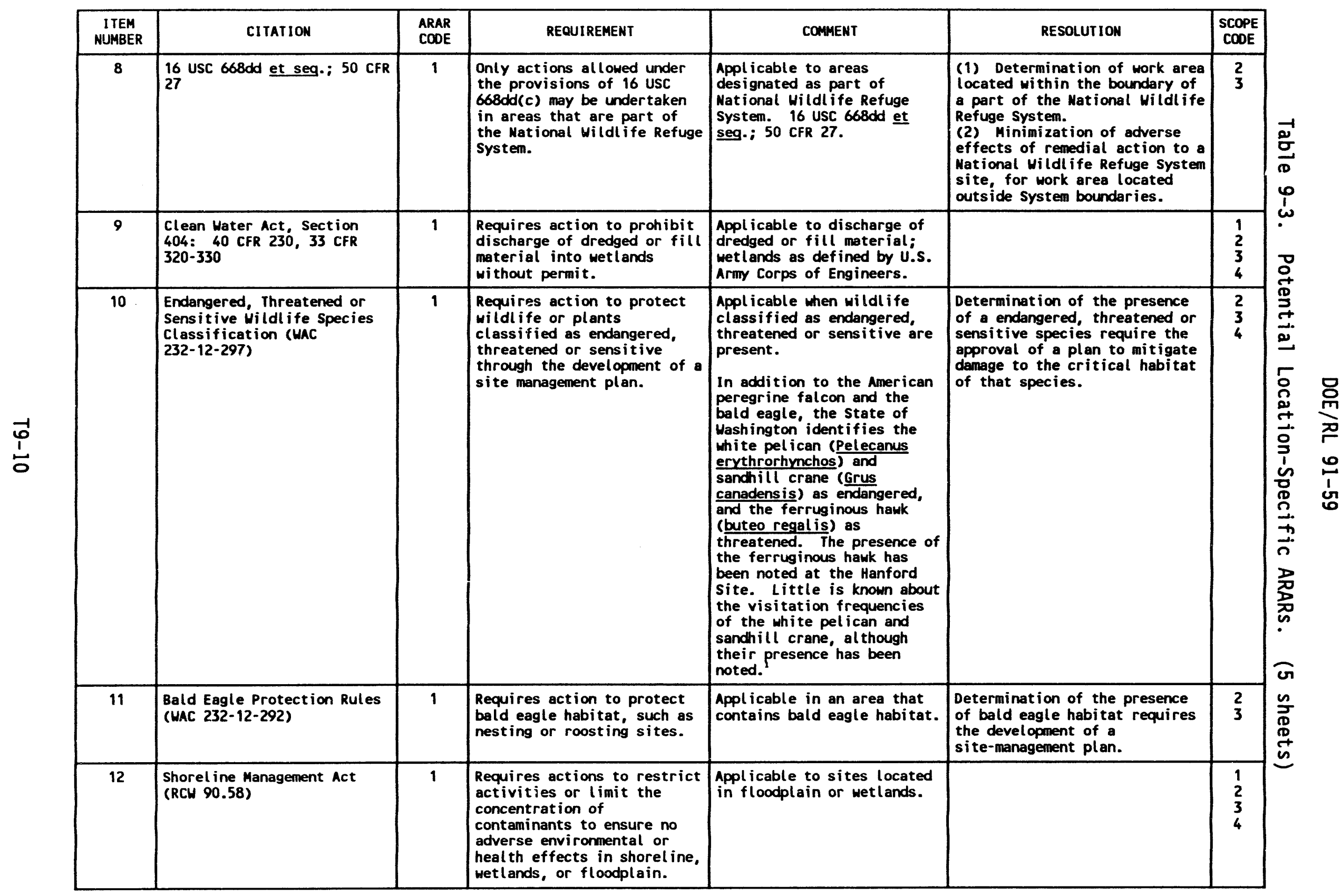




\begin{tabular}{|c|c|c|c|c|c|c|}
\hline $\begin{array}{l}\text { ITEM } \\
\text { NUMBER }\end{array}$ & CITATION & $\begin{array}{l}\text { ARAR } \\
\text { CODE }\end{array}$ & REQUIREMENT & COMMENT & RESOLUTION & $\begin{array}{l}\text { SCOPE } \\
\text { CODE }\end{array}$ \\
\hline \multicolumn{7}{|c|}{ Historical } \\
\hline 1 & $\begin{array}{l}\text { Mational Historical } \\
\text { Preservation Act (16 USC } \\
\text { 469); 36 CFR 65; } 25 \text { CFR 261; } \\
43 \text { CFR 3; } 43 \text { CFR } 7\end{array}$ & 1 & $\begin{array}{l}\text { Action to recover and } \\
\text { preserve artifacts required. }\end{array}$ & $\begin{array}{l}\text { Applicable to actions where } \\
\text { alteration of terrain } \\
\text { threatens significant } \\
\text { scientific, prehistorical, } \\
\text { historical, or } \\
\text { archaeological data, or } \\
\text { where actions may cause } \\
\text { irreparable harm, loss, or } \\
\text { destruction of significant } \\
\text { artifacts. }\end{array}$ & $\begin{array}{l}\text { Cultural Survey to determine } \\
\text { the presence of archeological } \\
\text { artifacts within the subject } \\
\text { work area. }\end{array}$ & $\begin{array}{l}2 \\
3 \\
4\end{array}$ \\
\hline 2 & $\begin{array}{l}\text { National Historic } \\
\text { Preservation Act, Section } \\
106 \text { (16 USC } 470 \text { et seg.); } 36 \\
\text { CFR } 800\end{array}$ & 1 & $\begin{array}{l}\text { Requires action to preserve } \\
\text { historic properties; } \\
\text { planning of action to } \\
\text { minimize harm to National } \\
\text { Historic Landmarks. }\end{array}$ & $\begin{array}{l}\text { Applicable to property } \\
\text { included in or el igible for } \\
\text { the National Register of } \\
\text { Historic Places. }\end{array}$ & $\begin{array}{l}\text { Cultural Survey to determine if } \\
\text { site is subject for eligibility } \\
\text { for inclusion in the Mational } \\
\text { Register of Historical Places. }\end{array}$ & $\begin{array}{l}2 \\
3 \\
4\end{array}$ \\
\hline 3 & $\begin{array}{l}\text { The American Indian } \\
\text { Religious freedom Act (42 } \\
\text { USC 1996) }\end{array}$ & 1 & $\begin{array}{l}\text { Requires action to mitigate } \\
\text { the effects of disturbance } \\
\text { within areas designated as } \\
\text { sacrad lands subject to } \\
\text { recognized spiritual rights } \\
\text { of American Indians. }\end{array}$ & $\begin{array}{l}\text { Applicable to actions that } \\
\text { may occur that restrict or } \\
\text { alter burial grounds or } \\
\text { monuments on land areas } \\
\text { sacred to the religious } \\
\text { practices of tribes } \\
\text { previously inhabiting } \\
\text { subject land. Separate and } \\
\text { distinct from considerations } \\
\text { of archaeological protection } \\
\text { and activities. }\end{array}$ & $\begin{array}{l}\text { Cul tural survey to determine } \\
\text { the presence of land within the } \\
\text { work area that is subject to } \\
\text { recognized spiritual rights of } \\
\text { Native Americans. }\end{array}$ & $\begin{array}{l}2 \\
3\end{array}$ \\
\hline 4 & $\begin{array}{l}\text { Historic Sites, Buildings } \\
\text { and Ant iquities Act (16 USC } \\
461 \text { ) }\end{array}$ & 1 & $\begin{array}{l}\text { Requires action to undertake } \\
\text { the recovery, protection and } \\
\text { preservation of data at } \\
\text { historic sites, buildings, } \\
\text { or objects of national } \\
\text { significance. }\end{array}$ & $\begin{array}{l}\text { Applicable to the alteration } \\
\text { of terrain that threatens } \\
\text { significant scientific, } \\
\text { prehistorical, historical, } \\
\text { or archaeological sites. }\end{array}$ & $\begin{array}{l}\text { Cultural survey to determine } \\
\text { presence of historic, } \\
\text { scientific, or archaeological } \\
\text { sites within the subject work } \\
\text { area. }\end{array}$ & $\begin{array}{l}2 \\
3 \\
4\end{array}$ \\
\hline 5 & $\begin{array}{l}\text { Archeological sites and } \\
\text { Resources (RCH 27.53) }\end{array}$ & $\overline{1}$ & $\begin{array}{l}\text { Requires action to recover } \\
\text { and preserve artifacts, } \\
\text { where action may impact } \\
\text { archeological sites and } \\
\text { resources. }\end{array}$ & $\begin{array}{l}\text { Applicable to any site, } \\
\text { object, artifact, or } \\
\text { location of prehistoric or } \\
\text { archaeologic interest } \\
\text { located in, on, or under } \\
\text { lands of waters under } \\
\text { possession or control of } \\
\text { State, county, city, or } \\
\text { political subdivision. }\end{array}$ & $\begin{array}{l}\text { Cul tural survey to determine } \\
\text { presence of archaeological } \\
\text { sites, relics, or objects } \\
\text { within the subject work area. }\end{array}$ & $\begin{array}{l}2 \\
3 \\
4\end{array}$ \\
\hline
\end{tabular}




\begin{tabular}{|c|c|c|c|c|c|c|}
\hline $\begin{array}{l}\text { ITEM } \\
\text { NUMBER }\end{array}$ & CITATION & $\begin{array}{l}\text { ARAR } \\
\text { CODE }\end{array}$ & REQUIREMENT & COMMENT & RESOLUTION & $\begin{array}{l}\text { SCOPE } \\
\text { COOE }\end{array}$ \\
\hline 6 & $\begin{array}{l}\text { Indian Graves and Records } \\
\text { (RCH 27.44) }\end{array}$ & 1 & $\begin{array}{l}\text { No material may be removed } \\
\text { from the site unless it is } \\
\text { destined for reburial or } \\
\text { perpetual preservation in } \\
\text { recognized archaeological } \\
\text { repository. Permission to } \\
\text { remove material is granted } \\
\text { by the state historic } \\
\text { preservation officer after } \\
\text { notification of the affected } \\
\text { Indian tribe or tribes. }\end{array}$ & $\begin{array}{l}\text { Applicable to intrusive } \\
\text { actions that may occur in } \\
\text { the area of burial grounds } \\
\text { or tribes that previously } \\
\text { inhabited the subject land. }\end{array}$ & $\begin{array}{l}\text { Cultural survey to determine } \\
\text { the presence of Mative American } \\
\text { burial sites within the subject } \\
\text { work area. }\end{array}$ & $\begin{array}{l}2 \\
3 \\
4\end{array}$ \\
\hline \multicolumn{7}{|c|}{ Miscel laneous } \\
\hline 1 & RCRA (40 CFR 264.18(b)) & 1 & $\begin{array}{l}\text { Facility within } 100 \text {-year } \\
\text { floodplain must be designed, } \\
\text { constructed, operated, and } \\
\text { maintained to avoid a } \\
\text { washout. }\end{array}$ & $\begin{array}{l}\text { Applicable to treatment, } \\
\text { storage, or disposal of RCRA } \\
\text { hazardous waste within } \\
100 \text {-year floodplain. }\end{array}$ & & $\begin{array}{l}1 \\
2 \\
3 \\
4\end{array}$ \\
\hline
\end{tabular}




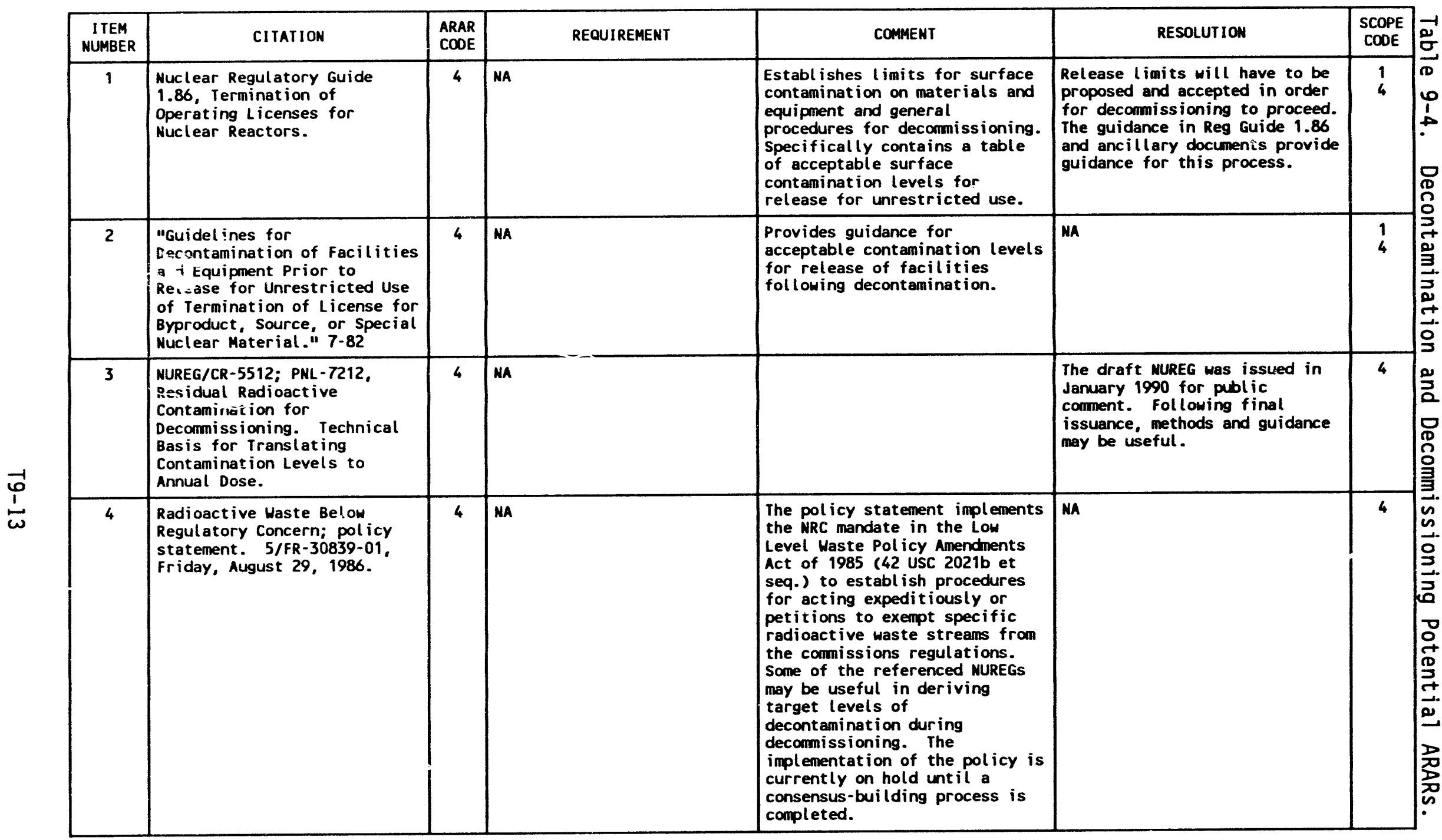




\begin{tabular}{|c|c|c|c|c|c|c|}
\hline $\begin{array}{c}\text { ITEM } \\
\text { NUMBER }\end{array}$ & CITATION & $\begin{array}{l}\text { ARAR } \\
\text { CODE }\end{array}$ & REQUI REMENT & COMMENT & RESOLUTION & $\begin{array}{l}\text { SCOPE } \\
\text { COOE } \\
\end{array}$ \\
\hline 5 & $\begin{array}{l}10 \text { CFR } 20, \text { Standards for } \\
\text { Protection Against Radiation. }\end{array}$ & 2 & MA & 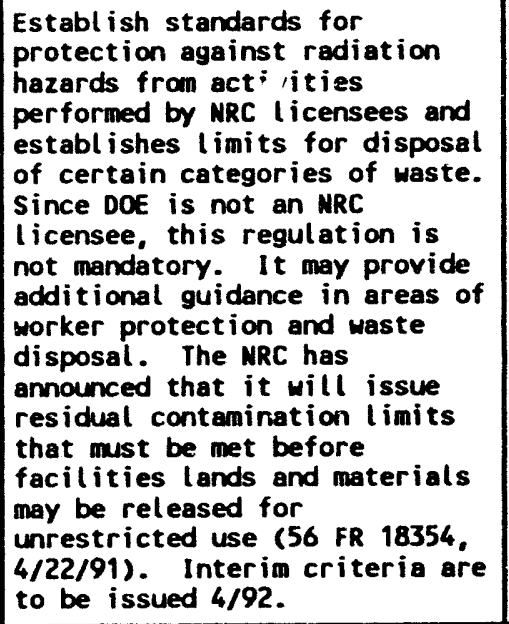 & MA & $\begin{array}{l}1 \\
2 \\
3 \\
4\end{array}$ \\
\hline 6 & $\begin{array}{l}40 \text { Cfr } 61 \text {, Subpart H, } \\
\text { National Emission Standards } \\
\text { for Emissions of } \\
\text { Radionucl ides Other Than } \\
\text { Radon from Department of } \\
\text { Energy Facilities. } \\
\end{array}$ & 1 & $\begin{array}{l}\text { This regulation provides } \\
\text { radioactive emissions } \\
\text { monitoring guidance for DOE } \\
\text { facilities. }\end{array}$ & $\begin{array}{l}\text { The regulation is aimed at } \\
\text { stacks and release points from } \\
\text { DOE facilities. The } \\
\text { application to potential } \\
\text { rel eases dur ing decomissioning } \\
\text { would appear prudent. }\end{array}$ & $\begin{array}{l}\text { Monitor for potential releases } \\
\text { during decommissioning. }\end{array}$ & $\begin{array}{l}1 \\
4\end{array}$ \\
\hline 7 & $\begin{array}{l}40 \text { CFR } 191 \text {, Envirormental } \\
\text { Radiation Protection } \\
\text { Standards for Kanagement and } \\
\text { Disposal of Spent Nuclear } \\
\text { Fuel, High Level and } \\
\text { Transuranic Radioactive } \\
\text { Wastes. }\end{array}$ & 1 & $\begin{array}{l}\text { These regulations supply } \\
\text { envirommental standards for } \\
\text { the management, storage, and } \\
\text { disposal of various classes } \\
\text { of radioactive waste. }\end{array}$ & 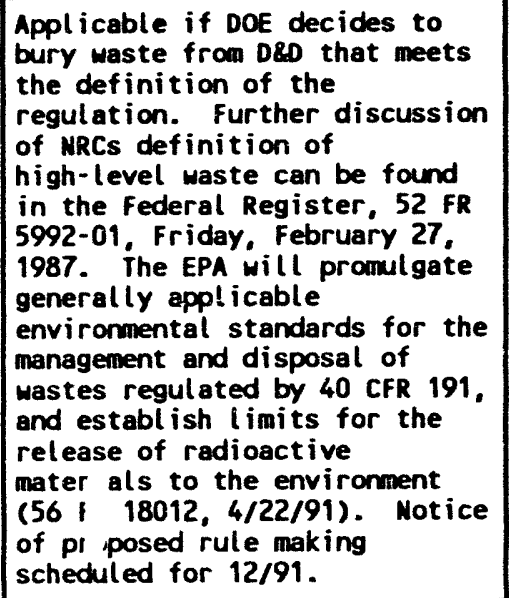 & $\begin{array}{l}\text { Classify the waste to determine } \\
\text { if } 40 \text { C } 191 \text { applies. }\end{array}$ & $\begin{array}{l}1 \\
2 \\
3 \\
4\end{array}$ \\
\hline
\end{tabular}




\begin{tabular}{|c|c|c|c|c|c|c|}
\hline $\begin{array}{c}\text { ITEM } \\
\text { NUMBER }\end{array}$ & CITATION & $\begin{array}{l}\text { ARAR } \\
\text { COOE } \\
\end{array}$ & REQUIREMENT & COMMENT & RESOLUTION & $\begin{array}{l}\text { SCOPE } \\
\text { COOE }\end{array}$ \\
\hline 8 & $\begin{array}{l}\text { MUREG/CR-5517, IMPACTS-BRC } \\
\text { Version 2.0, Program User's } \\
\text { Manual }\end{array}$ & 4 & NA & $\begin{array}{l}\text { The IMPACTS-BRS computer code } \\
\text { user's guide and code are } \\
\text { available. The code provides } \\
\text { capability of catculating } \\
\text { radiation doses to individuals } \\
\text { and target groups as a result } \\
\text { of various Dro activities } \\
\text { (e.g., transportation and } \\
\text { disposal). The code may be } \\
\text { useful in assessing appropriate } \\
\text { levels of cleanup. }\end{array}$ & MA & $\begin{array}{l}1 \\
2 \\
3 \\
4\end{array}$ \\
\hline 9 & $\begin{array}{l}10 \text { CFR } 30 \text {, Rules of General } \\
\text { Applicability to Domestic } \\
\text { Licensing of Byproduct } \\
\text { Material. }\end{array}$ & 4 & & $\begin{array}{l}\text { This regulation sets out } \\
\text { requirements for NRC licensees } \\
\text { with regard to the need for a } \\
\text { license, applying for a } \\
\text { license, recordkeeping, and } \\
\text { decomissioning. Section } 30.36 \\
\text { contains guidance on preparing } \\
\text { a decommissioning plan and } \\
\text { applying for ultimate } \\
\text { termination of the license and } \\
\text { unrestricted release of the } \\
\text { facility or site. The guidance } \\
\text { in this section is not } \\
\text { mandatory but may be useful. }\end{array}$ & ma & 4 \\
\hline 10 & $\begin{array}{l}10 \text { CFR } 61 \text {, Licensing } \\
\text { Requi rements for Land } \\
\text { Disposal of Radioactive } \\
\text { Waste. }\end{array}$ & 1 & $\begin{array}{l}\text { These regulations supply } \\
\text { guidance for land disposal } \\
\text { of radioact ive waste. }\end{array}$ & $\begin{array}{l}10 \text { CFR is appl icable when DOE } \\
\text { would wish to dispose of D\&D } \\
\text { material from } n \text { Reactor and may } \\
\text { be applicable if it is desired } \\
\text { to leave some level of } \\
\text { radioactivity in situ. }\end{array}$ & $\begin{array}{l}\text { Determine if the classification } \\
\text { of waste is low-level (i.e.. } \\
\text { not high-level). }\end{array}$ & $\begin{array}{l}1 \\
2 \\
3 \\
4\end{array}$ \\
\hline 11 & $\begin{array}{l}\text { Regulatory Guide } 3.64 \text {, } \\
\text { Standard Format and Content } \\
\text { of Decormissioning Plans for } \\
\text { Licenses Under } 10 \text { CFR Parts } \\
30,40 \text {, and } 70 .\end{array}$ & 4 & $\begin{array}{l}\text { The guide discusses the } \\
\text { content of a decormissioning } \\
\text { plan. }\end{array}$ & MA & ma & 4 \\
\hline 12 & $\begin{array}{l}\text { General Requirements for } \\
\text { Decormissioning Nuclear } \\
\text { Facilitities (Amendments to } \\
10 \text { CFR } 30,40,50,51,70, \\
72) 53 \text { FR 240i8. }\end{array}$ & 4 & MA & $\begin{array}{l}\text { The amended regulations address } \\
\text { decommissioning, planning, } \\
\text { timing, funding methods, and } \\
\text { envirommental review } \\
\text { requirements. }\end{array}$ & MA & 4 \\
\hline
\end{tabular}




\begin{tabular}{|c|c|c|c|c|c|c|}
\hline $\begin{array}{c}\text { ITEM } \\
\text { NUMBER }\end{array}$ & CITATION & $\begin{array}{l}\text { ARAR } \\
\text { COOE }\end{array}$ & REOUIREMENT & COMMENT & RESOLUTION & $\begin{array}{l}\text { SCOPE } \\
\text { COOE }\end{array}$ \\
\hline 13 & $\begin{array}{l}10 \mathrm{CrR} 60 \text {, Disposal of } \\
\text { High-Level Radioactive Wastes } \\
\text { in Geologic Repositories. }\end{array}$ & 1 & $\begin{array}{l}10 \text { CFR } 60 \text { establishes } \\
\text { guidance for the } \\
\text { establ ishment of High-Level } \\
\text { Radioactive Haste } \\
\text { Repositories and defines the } \\
\text { waste that must be placed } \\
\text { there. }\end{array}$ & $\begin{array}{l}\text { The States of Washington and } \\
\text { Oregon have requested a } \\
\text { revision of the definition of } \\
\text { "high-level radiactive waste" } \\
\text { (56 FR } 51732 \text {, 12/17/90). } \\
\text { A rulemaking decision is } \\
\text { expected by } 12 / 91 \text {. } 10 \text { cFR } 60 \\
\text { is applicable then DOE would } \\
\text { wish to dispose of D\&D material } \\
\text { from W Reactor that meets the } \\
\text { high-level waste criteria. }\end{array}$ & $\begin{array}{l}\text { Deterinine if the classification } \\
\text { of waste is high-level or not. }\end{array}$ & $\begin{array}{l}1 \\
2 \\
3 \\
4\end{array}$ \\
\hline 14 & $\begin{array}{l}\text { Hanford Federal Facility } \\
\text { Agreement and Consent Order } \\
\text { (Tri-Party Agreement or TPA). } \\
\text { Executive Summary, Section } \\
\text { 3.0. }\end{array}$ & 1 & $\mathrm{ma}$ & $\begin{array}{l}\text { The parties agreed that } \\
\text { whenever Dob activities result } \\
\text { in generation of hazardous } \\
\text { waste, it shall be subject to } \\
\text { the Tri-Party Agreement. In } \\
\text { addition, if any contaminated } \\
\text { structure is found to be a } \\
\text { source of a hazardous release } \\
\text { (or substantial threat of a } \\
\text { release), remediation of such a } \\
\text { release ihere subject to RCRA } \\
\text { or CERCLA, shall be subject to } \\
\text { the Tri-Party Agreement. }\end{array}$ & $\begin{array}{l}\text { Assess hazardous const ituents } \\
\text { of waste or structures subject } \\
\text { to D2D and determine if subject } \\
\text { to IPA. }\end{array}$ & $\begin{array}{l}1 \\
2 \\
3 \\
4\end{array}$ \\
\hline 15 & $\begin{array}{l}\text { Joint NRC/EPA Guidance-- } \\
\text { Conceptual Design Approach } \\
\text { for Commercial Mixed Low } \\
\text { Level Radioactive and } \\
\text { Hazardous Haste Disposal } \\
\text { Facil ities - } 1987 \text {. }\end{array}$ & 4 & wA & $\begin{array}{l}\text { Document provides a conceptual } \\
\text { design approach for a disposal } \\
\text { facility handl ing mixed } \\
\text { low-level waste (mixed LLW). }\end{array}$ & $\begin{array}{l}\text { Development of a mixed LLW } \\
\text { disposal facil ity may be } \\
\text { necessary to decomission M } \\
\text { Reactor. }\end{array}$ & $\begin{array}{l}1 \\
2 \\
3 \\
4\end{array}$ \\
\hline
\end{tabular}


DOE/RL 91-59

\subsection{NATIONAL ENVIRONMENTAL POLICY ACT STRATEGY}

\subsection{GENERAL}

The implementing regulations of NEPA were promulgated by the Council on Environmental Quality from 40 CFR, parts 1500 through 1508 , which establish national environmental policy and goals for the protection, maintenance, and enhancement of the environment. It also provides a process for implementing these goals within the Federal agencies (40 CFR 1502.1). For DOE, this process is contained in 10 CFR 1021, "National Environmental Policy Act Implementing Procedures."

\subsection{EXISTING NATIONAL ENVIRONMENTAL POLICY ACT DOCUMENTATION}

The following describes the NEPA documents currently in effect for the performance of $100-\mathrm{N}$ Area activities:

- Memorandum-to-File, dated February 16, 1988, which documents that the proposed action to discontinue $N$ Reactor operations will have a clearly insignificant impact on the environment, and therefore, no environmental assessment (EA) or EIS is required to curtail operations.

- Categorical Exclusion, dated January 15, 1992, which documents that the work performed during the routine maintenance of buildings, facilities, and structures, does not require an EA or an EIS.

\subsection{FUTURE NATIONAL ENVIRONMENTAL POLICY ACT DOCUMENTATION}

\subsubsection{N Reactor Deactivation}

On August 15, 1991 the decision was announced to cease preservation of $N$ Reactor as a defense materials production contingency and to proceed with activities leading to the ultimate decommissioning of the reactor. To comply with this decision, the $N$ Reactor Deactivation Program was established. The primary objective of the program is to place all $\mathrm{N}$ Reactor facilities in an environmentally safe condition for ultimate decommissioning at a later date. The $N$ Reactor Deactivation Program Plan (WHC-SP-0615) defines the basic methodology and identifies the transition activities required to effect the program's objective (see Table 6-1) (WHC 1992a).

The transition activities have been rategorized as being of either a routine or nonroutine nature. Therefore, a NEPA strategy for each activity category will be implemented.

10.3.1.1 Routine Activities. The existing NEPA documentation described above will be used to allow the performance of routine activities that are not part of a set of unique activities that also include nonroutine-type activities. 
10.3.1.2 Nonroutine Activities. An action description memorandum (ADM) was submitted to DOE in December 1992 to determine the appropriate level of NEPA documentation (EA or EIS) required for the program's transition activities. After receiving formal direction on December 7, 1993, an EA is now being prepared.

The proposed actions within the EA include activities such as hardware removal, relocation of the basin's residual materials to a central location for sampling and characterization, packaging and removal of residual materials, draining the basin, and painting. The EA will include an in-depth review and analysis of the nuclear and radiological safety issues associated with the performance of the activities; appropriate alternatives to the proposed actions will be thoroughly evaluated.

\subsubsection{Environmental Remediation}

An ADM prepared in 1989 described the program impacts of the environmental remediation activities required at the Hanford Site. Later, it was determined that an EIS would be the appropriate level of NEPA documentation for these activities.

A Notice of Intent was published in the Federal Register in August 1992 informing the public that the Hanford Remedial Action EIS (HRA-EIS) will be prepared. The HRA-EIS will focus on remediating the Hanford Site to goals that support varying degrees of future use (e.g., restricted or unrestricted use); it will evaluate technologies that are representative of those that could be used for Hanford Site remediation. The HRA-EIS will address the environmental remediation of areas associated with $N$ Reactor and will evaluate alternative classes of future 1 and use for the 100 Area.

\subsubsection{Decontamination and Decommissioning}

An EIS was prepared and issued in 1993 for the D\&D of the surplus reactors located in the 100 Areas of the Hanford Site (DOE/EIS 1993). The $N$ Reactor at the Hanford Site is not within the scope of this EIS because it is currently being shut down and will likely take several years before it is available for decommissioning. Once shutdown is completed, the appropriate NEPA documentation will be developed for decommissioning $N$ Reactor. 


\subsection{DISPOSITION OF SPECIAL NUCLEAR MATERIAL}

An inventory of SNM remains as either unirradiated or irradiated nuclear fuel, also known as spent nuclear fuel (SNF), from operation of $N$ Reactor. The SNM inventory includes 1,800 metric tons of unirradiated uranium and 2,100 metric tons of irradiated uranium materials SNF. The unirradiated uranium consists of billets, unfinished nuclear fuel elements, finished nuclear fuel assemblies (some of which are contaminated), and scrap fuel stored in wooden boxes at the 300 Area Fuel Fabrication Facility. The irradiated uranium materials consist of nuclear fuel assembilies and elements stored in both open and sealed canisters under water in two 100-K Area fuel storage basins, $105 \mathrm{KE}$ and $105 \mathrm{KW}$.

The irradiated $N$ Reactor fuel consists of slightly enriched metallic uranium bonded to a layer of zirconium alloy (Zircaloy-2), hereinafter referred to as the cladding. The cladding provides the primary barrier against the escape of fission products and fissile materials from the fuel assembly. The fuel assembly (see Color Illustration 3-3) includes two components: an inner and an outer tube-shaped element, assembled into a tube-in-tube arrangement.

The irradiated nuclear fuel was discharged from $N$ Reactor between 4 and 21 years ago, and has had sufficient decay time to essentially el iminate ${ }^{131} \mathrm{I}$, as well as other short-half-life radionuclides. Upon its discharge, the fuel was allowed to cool for a minimum of 150 days in the $N$ Reactor fuel storage basin. The fuel was then either placed in open-top (MK 0, I and II) canisters and transported to 105-KE Basin or in sealed (MK I and II) canisters and transported to 105-KW Basin (see Figure 11-1) for temporary storage. Temporary storage was originally planned for no more than 3 years, until the fuel could be processed at the Plutonium-Uranium Extraction (PUREX) PIant. As a result of the discharge and subsequent handling operations, the cladding integrity was occasionally lost. The loss of cladding integrity ranged from a crack to the complete separation of an element (or both elements) into two, or sometimes more parts. If the cladding was intact, radionuclides remained in the fuel assembly. However, once the cladding integrity was lost, the water gained access to the metallic uranium, starting the corrosion of the metallic uranium and the leaching of radionuclides into the basin cooling water. It has been estimated that about 6 percent of the fuel currently stored in the 105-KE and 105-KW Basins has lost its cladding integrity.

The 105-KE Basin currently contains 3,668 open-top canisters filled with $\mathrm{N}$ Reactor fuel and two baskets (the equivalent of five canisters) filled with aluminum-clad fuel assemblies from the retired single-pass reactors. The fuel was not originally encapsulated because the PUREX PIant operations in the 200 Area had been scheduled to process the fuel in the mid 1980s. The shutdown of PUREX precluded the fuel's processing and the existing inventory at 105-KE was never encapsulated. Since the primary barrier (cladding) on approximately 6 percent of the stored fuel had been breached, fission products have escaped from these fuel assemblies, contaminating the basin cooling water and exposing workers to increased amounts of radiation. 
A recent Tiger Team Assessment of the Hanford Site stated that continued storage of fuel in the 105-KE Basin constitutes a safety problem because the fuel is not encapsulated and thus allows fission products to continue to leach out of the damaged fuel elements into the basin cooling water.

The fuel stored in the 105-KE Basin needs to be encapsulated in sealable canisters to provide a new primary barrier that will eliminate the continuing contamination of the basin cooling water. The basin's water filtration and ion exchange systems will then gradually reduce the current contamination level of the basin cooling water, which will result in reduced levels of radiation exposure to the workers. Once the fuel is encapsulated, the basin cooling water will provide a secondary barrier against a potential breach of containment resulting in release of radioactive materials to the environment.

The 105-KW Basin currently contains 3,821 sealed (MK I and II) canisters filled with $N$ Reactor fuel. The 1,773 MK I canisters include 777 aluminum canisters and 996 stainless steel canisters. The MK I and II canisters provide the primary barrier for the fission products escaping from the damaged fuel assemblies, and the basin cooling water provides a secondary barrier to the potential release of radioactive materials to the environment. Because the fuel has been stored in sealed canisters in this basin the contamination of the basins's cooling water has been substantially less than that experienced in the 105-KE Basin where the fuel is stored in open-top canisters.

The strategy for the disposition of the inventory of SNM consists of near-term and long-term actions, which will be commensurate with the Atomic Energy Act of 1954 and applicable DOE Orders.

- Near-Term Actions: Existing SNM inventories will continue to be stored in the 105-KE and 105-KW Basins. The fuel stored in the 105-KE Basin will be encapsulated to provide a new primary barrier between the damaged fuel assemblies and the basin cooling water to eliminate the continuing contamination of the basin's cooling water and to maintain the integrity of present irradiated nuclear fuel storage systems.

Upgrades to the irradiated nuclear fuel storage facilities are planned as part of Project N-032, KE/KW Life Extension because maintaining the existing storage of irradiated nuclear fuel at 105-KE and 105-KW will likely be extended 20 years.

- Long-Term Actions: The SNF inventory described above and other SNF inventories at the Hanford Site and across the DOE complex are the focus of studies that are being conducted to determine how to disposition the material. To implement a fully integrated strategy, a programmatic implementation plan (Idaho EIS) will be prepared to determine the NEPA evaluation alternatives required for dispositioning the SNF at these facilities. 


\section{Irradiated N Reactor Fuel and Canisters}

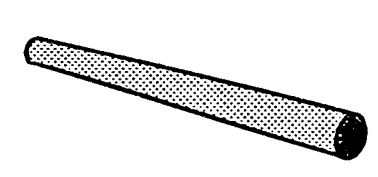

N Reactor Fuel

Length

26 inches (max)

2.435-inch outer

diameter (max)

Weight

52 pounds

Fuel

assemblies

consist of

inner and outer

elements in a

tube-iri-tube

configuration

\section{Fuel}

assemblies per

canister
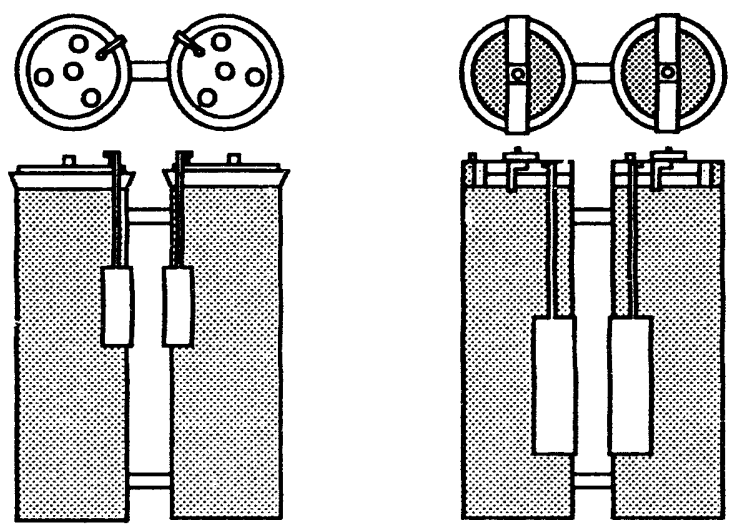

Mark II

Canister

Mark 6

Mark I

Canister

- Open

\begin{tabular}{lll}
\hline -Open & $\cdot$ Closed and open & $\bullet$ Closed and open \\
\hline - Aluminum & - Aluminum & $\bullet$ Stainless steel \\
& - Stainless steel & \\
\hline
\end{tabular}

KE: 1400 (est.)

KW: $\quad 0$

1400 SS (est.)

1800 inc. $800 \mathrm{Al}, 1000 \mathrm{SS}$

900 open

2000 closed

Totals

3700

3800 


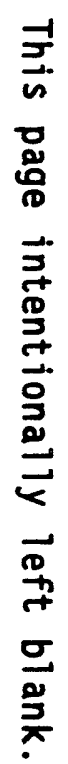




\subsection{INSTITUTIONAL ISSUES}

The HGP is located on the DOE-owned Hanford Site in south-central Washington State adjacent to the south end of the 100-N Area. The HGP was the first nuclear power project in the Pacific Northwest and the fourth in the Nation to generate electricity using a nuclear heat source. At one time, it was the largest nuclear steam electric generating plant in the world, with a capacity of $860,000 \mathrm{~kW}$.

After extensive studies, the AEC in 1961 requested Congress to appropriate funds to add a steam electric generating plant, but the request was denied.

The Supply System proposed to finance and build the steam plant. The proposal was accepted, and a bill authorizing the AEC to sell the steam from $\mathrm{N}$ Reactor, which otherwise would have been dissipated as waste heat, was signed into law on September 26, 1962. The law stipulated that no Federal funds could be spen't to build the plant. It also specified that 50 percent of the electricity produced would be offered for sale to investor-owned utilities and 50 percent would be offered to consumer-owned utilities. With the proceeds from a \$122-million revenue bond issue, the Supply System financed and built the generating facility.

The first nuclear-produced electricity from one of the HGP's two 430,000-kW turbines was synchronized into the Northwest power pool and sent into the BPA transmission grid system. On June 12, 1966, the second turbine generator went into operation, with power generation rising to $800,000 \mathrm{~kW}$.

In August of 1991, DOE decided to cease preservation of $\mathrm{N}$ Reactor as a defense materials production contingency and to proceed with activities leading to the eventual decommissioning of that facility. As part of this process, the U.S. Department of Energy, Richland Operations (RL), was authorized by the Secretary of Energy to take all necessary and appropriate steps to cancel contractual obligations with the Supply System regarding the use of the Supply System's HGP. The Supply System has taken the necessary steps to retire all outstanding $H G P$ revenue bonds that were issued by the Supply System to finance the original construction.

Representatives of RL and the Supply System are holding a series of preliminary discussions regarding the rights and obligations of the parties pertinent to final disposition of HGP. HGP and associated BPA facilities currently occupy land adjacent to the 100-N Area under provisions of a 1962 lease agreement. Of particular concern has been the parties' responsibilities for surveying, cleaning up, and restoring the HGP site. During operation of the $\mathrm{N}$ Reactor, the steam generators suffered tube leaks, which allowed reactor coolant to enter the steam system. The steam generators were retubed, which eliminated the tube leaks, but the leaks did carry radioactive contamination into several portions of the steam system. Radiation surveys indicate that the $96.5-\mathrm{cm}(38-i n$.$) -diameter steam 1$ ines from the $N$ Reactor up to the first steam drain station are contaminated and two steam blowdown tanks and their related sumps are radiologically contaminated. A water disposal pond near the Columbia River shoreline has also been contaminated and will require stabilization and remediation. A radiation 
survey of the HGP facility was performed in fiscal year 1993 to determine the extent of contamination and estimated cost to clean up the affected piping and equipment. This data will be used to finalize liability issues between the DOE and the Supply System. In support of the aforementioned discussions, a preliminary cost estimate was prepared to dismantle the HGP facilities and return the site to its original condition; that estimated cost is $\$ 30,000,000$. For purpose of this closure report, these costs are not included because negotiations between DOE and the Supply System have not been concluded.

BPA occupies a portion of the HGP site, which contains a high-voltage switch yard and a 500-kV powerline transmission corridor. It is planned that this facility will remain in operation for the foreseeable future, and hence discussion of cleanup and D\&D is not included in this closure report. 


\subsection{CLOSURE MANAGEMENT AND PROGRAMMATIC INTERFACE}

The DOE's ER Program was established in 1987 to plan, execute, and control its remediation efforts. This program is assigned to the DOE's Office of Environmental Restoration within the Office of Environmental Restoration and Waste Management.

The ER program for the Hanford Site has been designated as a major system acquisition requiring a project management system pursuant to DOE Order 4700.1, Project Management System. The Hanford ER major system acquisition Work Breakdown Structure is shown in Figure 13-1.

DOE's Office of Environmental Restoration and Waste Management has instituted a planning process for achieving compliance with all applicable laws and regulations, in addition to cleanup of existing waste sites, using agreements with Federal and State agencies as input to the planning process. This planning process is culminated in a 5-year plan based on activity data sheets prepared by the respective field offices.

The activity data sheets provide detailed information describing items such as work scope, funding requirements, and regulatory drivers. The following activity data sheets cover the scope of ER and other remediation and cleanup efforts at $N$ Reactor.

\begin{tabular}{|l|l|}
\hline \multicolumn{1}{|c|}{ ADS } & \multicolumn{1}{|c|}{ SCOPE } \\
\hline RL-4120 & N Reactor Deactivation (FY92) \\
$\mathrm{RL}-3600$ & N Reactor Deactivation (FY93-FY99) \\
$\mathrm{RL}-3600$ & RCRA Facil ity Closures \\
$\mathrm{RL}-5282,5284$ & RCRA Past Pract ice Site Remediation \\
Not Available & Decontamination and Decommissioning \\
\hline
\end{tabular}


DOE/RL 91-59

This page intentionally left blank. 


\section{Hanford Environmental Restoration M!}

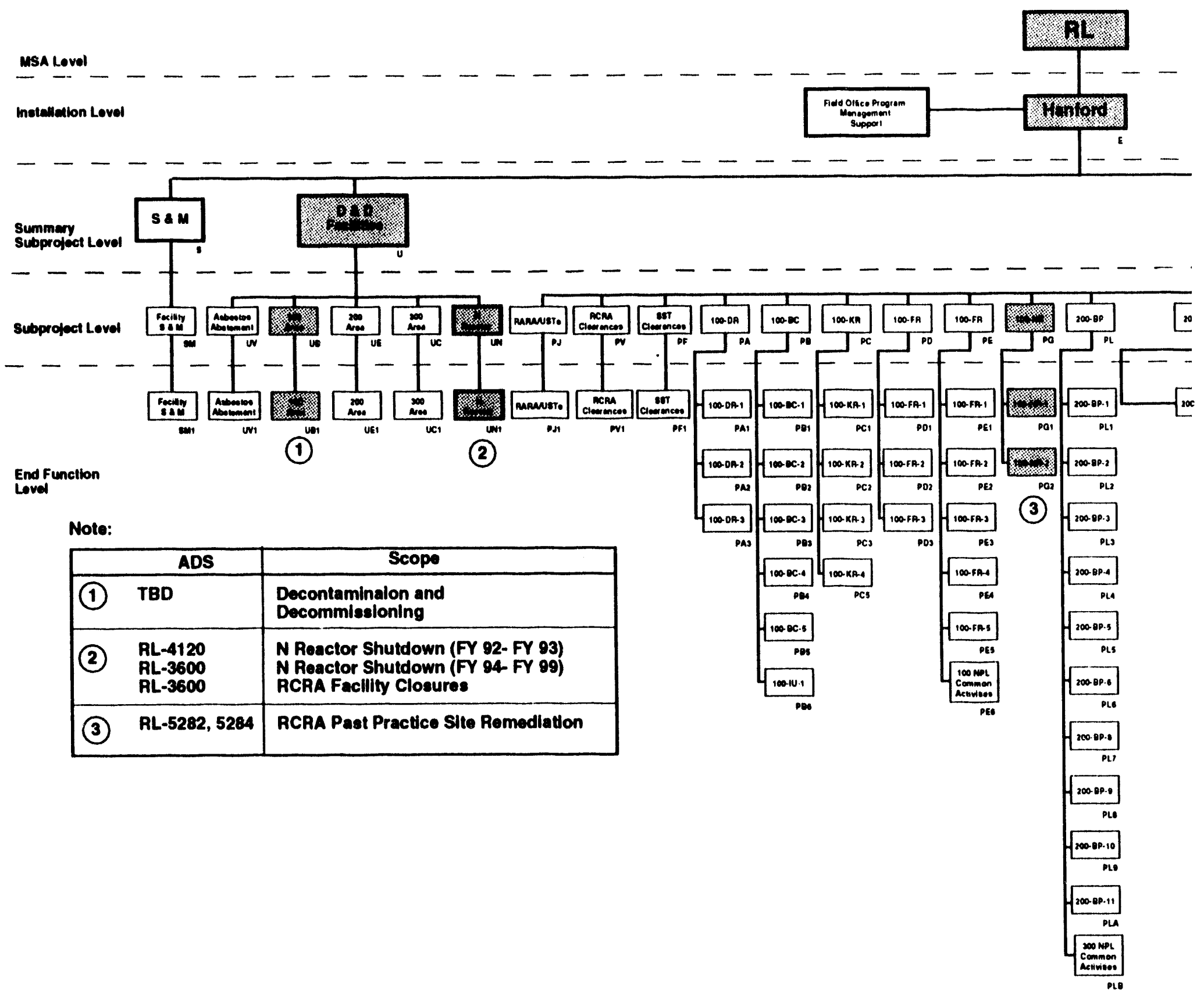




\subsection{REFERENCES}

10 CFR 1021, 1992, "Compliance with National Environmental Policy Act," Code of Federal Regulations, as amended.

40 CFR 52, 1991, "Approval and Promulgation of Implementation Plans," Code of Federal Regulations, as amended.

40 CFR 60, 1991, "Standards of Performance for New Stationary Sources," Code of Federal Regulations, as amended.

40 CFR 61, 1991, "National Emission Standards for Hazardous Air Pollutants," Code of Federal Regulations, as amended.

40 CFP 122, 1991, "EPA Administered Permit Programs: The National Pollutant Discharge El imination System," Code of Federal Regulations, as amended.

40 CFR 141, 1992, "National Primary Drinking Water Regulations," Code of Federal Regulations, as amended.

40 CFR 143, 1991, "National Secondary Drinking Water Regulations," Code of Federal Regulations, as amended.

40 CFR 144, 1991, "Underground Injection Control Program," Code of Federal Regulations, as amended.

40 CFR 145, 1991, "State UIC Program Requirements," Code of Federal Regulations, as amended.

40 CFR 146, 1991, "Underground Injection Control Program: Criteria and Standards," Code of Federal Regulations, as amended.

40 CFR 147, 1991, State Underground Injection Control Programs, " Code of Federal Regulations, as amended.

40 CFR 193, (Proposed), 1991, "Environmental Standards for the Management, Storage, and Land Disposal of Low-Level Radioactive Waste and Naturaliy Occurring Accelerator-Produced Radioactive Waste, " Code of Federal Regulations (Proposed).

40 CFR 260, 1991, "Hazardous Waste Management System - General," Code of Federal Regulations, as amended.

40 CFR 261, 1991, "Identification and Listing of Hazardous Waste," Code of Federal Regulations, as amended.

40 CFR 262, 1991, "Standards Applicable to Generators of Hazardous Waste," Code of Federal Regulations, as amended.

40 CFR 263, 1991, "Standards Applicable to Transporters of Hazardous Waste," Code of Federal Regulations, as amended. 
40 CFR 264, 1991, "Standards for Owners and Operators of Hazardous Waste Treatment, Storage, and Disposal Facilities," Code of Federal Regulations, as amended.

40 CFR 265, 1991, "Interim Status Standards for Owners and Operators of Hazardous Waste Treatment, Storage, and Disposal Facilities," Code of Federal Regulations, as amended.

40 CFR 266, 1991, "Standards for the Management of Specific Hazardous Wastes and Specific Hazardous Waste Management Facilities," Code of Federal Regulations, as amended.

40 CFR 267, 1991, Interim Standards for Owners and Operators of New Hazardous Waste Land Disfosal Facilities," Code of Federal Regulations, as amended.

40 CFR 268, 1991, "Land Disposal Restrictions," Code of Federal Regulations, as amended.

40 CFR 270, 1991, "EPA Administered Permit Programs: The Hazardous Waste Permit Program," Code of Federal Regulations, as amended.

40 CFR 271, 1991, "Requirements for Authorization of State Hazardous Waste Programs," Code of Federal Regulations, as amended.

40 CFR 272, 1991, "Approved State Hazardous Waste Management Programs," Code of Federal Regulations, as amended.

40 CFR 300, 1991, "National 011 and Hazardous Substances Pollution Contingency Plan," Code of Federal Regulations, as amended.

Clean Water Act of 1977, 33 U.S.C. 1251, et seq.

Comprehensive Environmental Response, Compensation, and Liability Act of 1980, 42 U.S.C 9601 , et seq.

DOE, 1988, General Environmental Protection Program, DOE Order 5400.1, U.S. Department of Energy, Washington, D.C.

DOE, 1989, General Design Criteria, DOE Order 6430.1A, U.S. Department of Energy, Washington, D.C.

DOE, 1991, Environmental Regulatory Guide for Radiological Effluent Monitoring and Environmental Surveillance, DOE/EH-0173T, U.S. Department of Energy, Washington, D.C.

DOE/EA, 1992, Environmental Assessment of 105-KE and 105-KW Basins Fuel Encapsulation and Repackaging, 100-K Area, Hanford Site, Richland, Washington, DOE/EA-0535, U.S. Department of Energy, Richland Field Office, Richl and, Washington.

DOE/EIS, 1992, Hanford Reach of the Columbia River, Draft, June 1992, National Park Service, Pacific Northwest Regional Office, Seattle, Washington. 
DOE/EIS, 1993, Decommissioning of Eight Surplus Production Reactors at the Hanford Site, Richland, Washington, DOE/EIS-0119F, U.S. Department of Energy, Washington, D.C.

DOE-RL, 1990, RCRA Facility Investigation/Corrective Measures Study Work Plan for the 100-NR-1 Operable Unit, Hanford Site, Richland, Washington, Draft DOE/RL-90-22, U.S. Department of Energy, Richland Field Office, Richland, Washington.

DOE-RL, 1991, RCRA Facility Investigation/Corrective Measures Study Work Plan for the 100-NR-2 Operable Unit, Hanford Site, Richland, Washington, Draft, DOE/RL 91-46, U.S. Department of Energy, Richland Operations Office, Richland, Washington.

DOE-RL, 1992, Hanford Environmental Restoration Program Roadmap, DOE/RL 92-45, U.S. Department of Energy, Richland, Washington.

Ecology, EPA, and DOE, 1992, Hanford Federal Facility Agreement and Consent Order, Washington State Department of Ecology, U.S. Environmental Protection Agency, and U.S. Department of Energy, 01ympia, Washington.

EPA, 1986, Test Methods for Evaluating Solid Waste: Physical/Chemical Methods, SW-846, 3rd Edition, as revised, U.S. Environmental Protection Agency, Washington, D.C.

EPA, 1988, Guidance for Conducting Remedial Investigations and Feasibility Studies under CERCLA, EPA/53016-89/004, OSWER Directive 9355.03-01, U.S. Environmental Protection Agency, Washington, D.C.

Hazardous and Solid Waste Amendments of 1984, 42 U.S.C. 6912(a), 6921, 6922, $6924,6925,6926,6930,6935,6937,6939,6991$, and 6993.

National Environmental Policy Act of 1969, 42 USC 4832 et seq.

Newcomer, D. R., 1988, Detailed Water Level Data and an Evaluation of Mathematical Approaches for Near-River Monitoring, MS Thesis, Montana College of Mineral Science and Technology, Butte, Montana.

Peterson, R. E., 1992, Hydrologic and Geologic Data Available for the Region North of Gable Mountain, Hanford Site, Washington, WHC-SD-EN-TI-006, Rev 0, Westinghouse Hanford Company, Richland, Washington.

Public Law 101-189, National Defense Authorization Act for Fiscal Years 1990 and 1991.

Price-Anderson Amendments Act of 1988

Resource Conservation and Recovery Act of 1976, 42 USC 6901 et seq.

State of Washington Hazardous Waste Management Act of 1976, Revised Code of Washington, Chapter 70.105 et seq., 01 ympia, Washington.

Superfund Amendments and Reauthorization Act of 1986, 42 U.S.C., 11001 et seq. 
DOE/RL 91-59

Toxic Substances Control Act of 1976, 15 U.S.C. 2601 et seq.

WAC 173-201, 1990, "Water Quality Standards for Surface Waters of the State of Washington," Washington Administrative Code, as amended.

WAC 173-303, 1991, "Dangerous Waste Regulations," Washington Administrative Code, as amended.

WAC 232-12, 1986, "Bald Eagle Protection Rules," Washington Administrative Code, as amended.

WAC 248-54, 1990, "Radioactive Protection Standards," Washington Administrative Code, as amended.

Waite, 1980, "About Forty Lost Glacial Lake Missoula Jakuhlaups Through Southern Washington, "Journal of Geology, volume 88.

Watkins, J. D., 1991, Letter to S. Nunn, Chairman on Armed Services, United States Senate, August 1, 1991, Secretary of Energy, U.S. Department of Energy, Washington, D.C.

WHC, 1986, Part B Permit Application, 1324-N Surface Impoundment, Revision 0, Westinghouse Hanford Company, Richland, Washington.

WHC, 1987a, Closure/Post Closure Plan 1325-N Liquid Waste Disposal Facility, Westinghouse Hanford Company, Richland, Washington.

WHC, 1987b, Closure/Post Closure Plan 1301-N Liquid Waste Disposal Facility, Westinghouse Hanford Company, Richland, Washington.

WHC, 1987c, Leak in Trench, Critique No. WHC-C-87-062, 100N-32, Westinghouse Hanford Company, Richland, Washington.

WHC, 1987d, Release in Excess of pH Limits, UOR 86-09, Westinghouse Hanford Company, Richl and, Washington.

WHC, 1987e, Trench Sump Leakage, Critique No. WHC-C-87-032-100N-15, Westinghouse Hanford Company, Richland, Washington.

WHC, 1987f, Closure Plan 1324-NA Percolation Pond, Revision 0, Westinghouse Hanford Company, Richland, Washington.

WHC, 1987g, Diesel 0il Spill, Fact Sheet No., 100-N-87-91, Revision 0, Westinghouse Hanford Company, Richland, Washington.

WHC, 1989a, (NUSAR) N-Reactor Updated Safety Analysis Report, WHC-SP-0297, Volume 5, Amendment 21, Westinghouse Hanford Company, Richland, Washington.

WHC, 1989b, Oil Spill Prevention Control and Countermeasures Plan, WHC-SP-0437, Westinghouse Hanford Company, Richland, Washington. 
WHC, 1989c, Unplanned Caustic Spill at Unloading Station, Critique No. WHC-C-88-077-100N-03, Westinghouse Hanford Company, Rich1and, Washington.

WHC, 1989d, Preliminary Operable Units Designation Project, WHC-EP-0216, Westinghouse Hanford Company, Richland, Washington.

WHC, 1990a, Diesel 0 il Leak, Critique Report Number WHC-C-89-047-100N-20, Rev. 2, Westinghouse Hanford Company, Richland, Washington.

WHC, 1990b, N Reactor Effluent Stream-Specific Report, WHC-EP-0342, Addendum 3, Westinghouse Hanford Company, Richland, Washington.

WHC, 1992a, N Reactor Deactivation Program Plan, WHC-SP-0615, as amended, Westinghouse Hanford Company, Richland, Washington.

WHC, 1992b, Results of Groundwater Quality Assurance Monitoring at the 1301-N and 1324-N/NA Facilities, WHC-SD-EN-EV-003, Rev. 0, Westinghouse Hanford Company, Richland, Washington.

Willis, N. P., and G. C. Tiner, 1991, Hanford Site Radioactive Solid Waste Acceptance Criteria, WHC-EP-0063-3, Westinghouse Hanford Company, Richland, Washington. 
DOE/RL 91-59

This page intentionally left blank. 
DOE/RL 91-59

DISTRIBUTION

Number of copies

ONSITE

U. S. Department of Energy-Richland Operations office

Public Reading Room A1-65

Pacific Northwest Laboratory

1 Technical Files K1-11

Westinghouse Hanford Company

$\begin{array}{ll}\text { DD Blankenship } & \times 8-29 \\ \text { KE Dodson (10) } & \times 0-57 \\ \text { DW Eide } & \times 0-57 \\ \text { GK Erickson } & \text { A3-30 } \\ \text { GR Frank } & H 6-29 \\ \text { RJ Gimera } & \times 0-57 \\ \text { PW Griffin } & X 5-55 \\ \text { MC Hughes } & X 5-55 \\ \text { GS Hunacek } & X 0-41 \\ \text { KJ Kraemer } & X 0-34 \\ \text { AD Krug } & H 6-02 \\ \text { MA Mihalic } & \times 5-55 \\ \text { PD Mix } & H 6-29 \\ \text { MR Morton } & X 5-55 \\ \text { DL Schilperoort } & \times 8-29 \\ \text { WE Taylor } & H 4-67 \\ \text { RA Toher } & X 0-57 \\ \text { RA Trevino } & X 8-29 \\ \text { FR Turner } & X 1-81 \\ \text { JL Walsh (2) } & X 0-57 \\ \text { DJ Watson } & X 0-41 \\ \text { GT Wells } & X 6-26 \\ \text { Central Files } & L 8-04 \\ \text { Document Processing } & \\ \text { and Distribution (2) } & \text { L8-15 } \\ \text { Information Release } & \\ \text { Administration } & R 1-08\end{array}$


DOE/RL 91-59

This page intentionally left blank. 


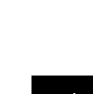

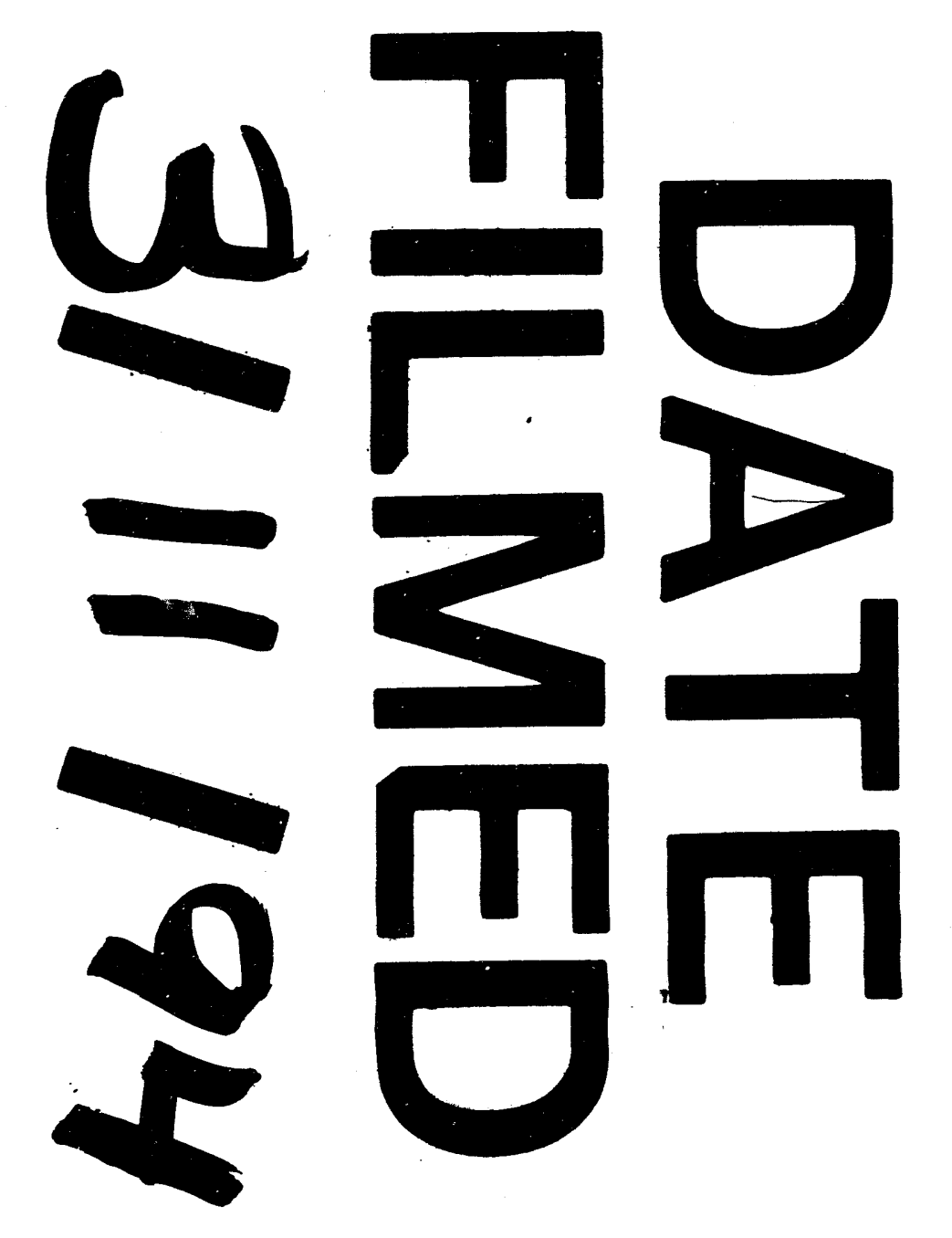

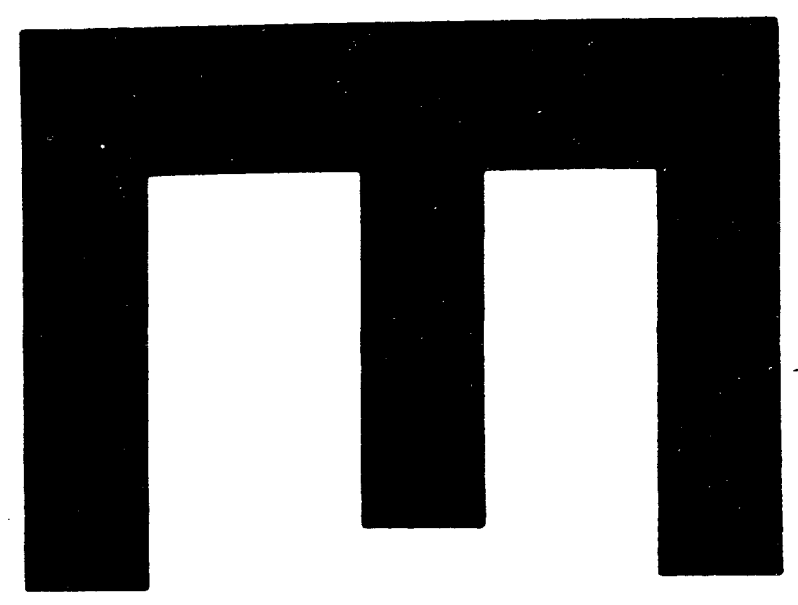
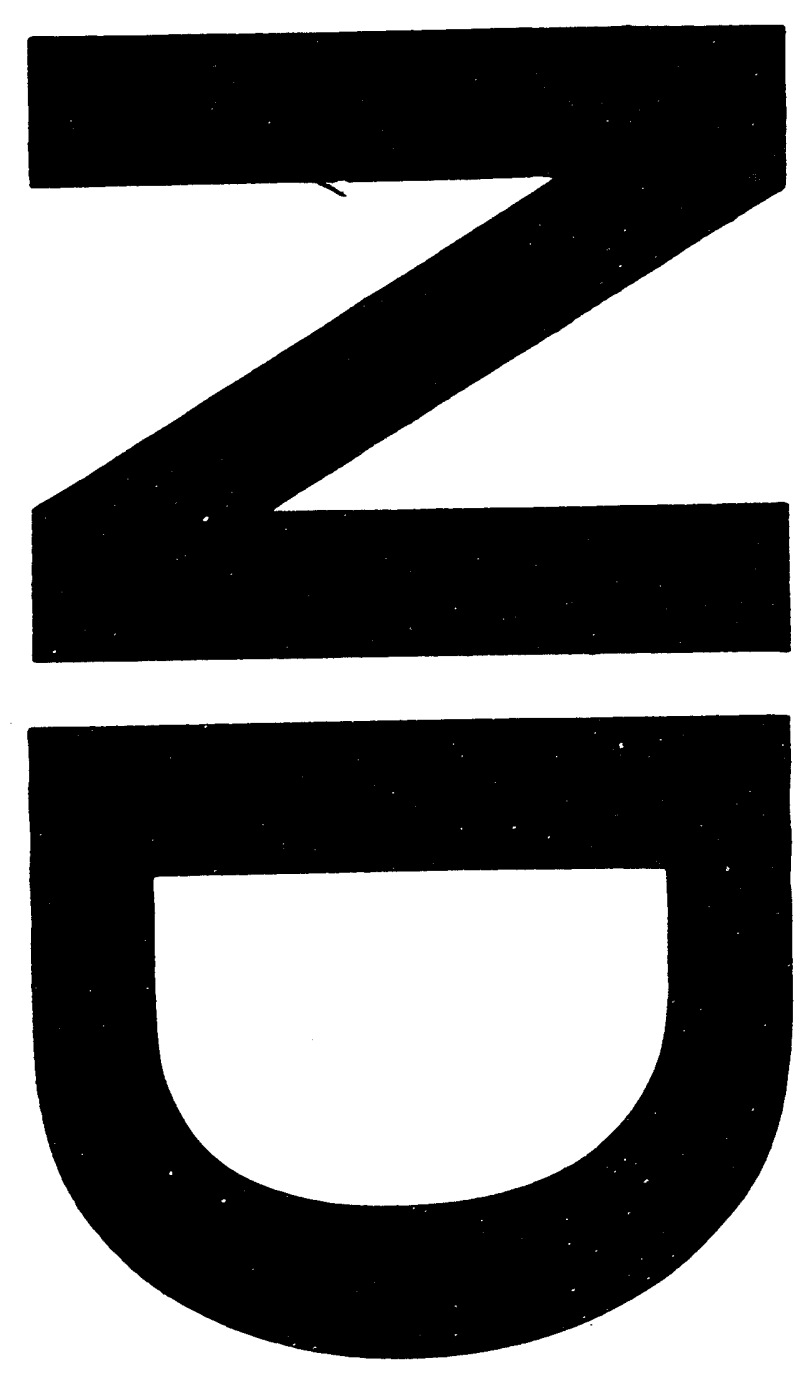
